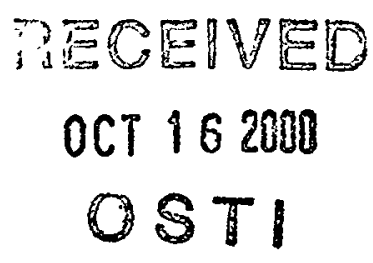

LBNL-45830

A Faint Galaxy Redshift Survey Behind Massive Clusters

\author{
Brenda Louise Frye \\ Ph.D. Thesis \\ Department of Astronomy \\ University of California, Berkeley \\ and \\ Physics Division \\ Ernest Orlando Lawrence Berkeley National Laboratory \\ University of California \\ Berkeley, CA 94720
}

May 1999

This work was supported by the Director, Office of Science, Office of High Energy and Nuclear Physics, Division of High Energy Physics, of the U.S. Department of Energy under Contract No. DE-AC03-76SF00098. 


\section{DISCLAIMER}

This report was prepared as an account of work sponsored by an agency of the United States Government. Neither the United States Government nor any agency thereof, nor any of their employees, make any warranty, express or implied, or assumes any legal liability or responsibility for the accuracy, completeness, or usefulness of any information, apparatus, product, or process disclosed, or represents that its use would not infringe privately owned rights. Reference herein to any specific commercial product, process, or service by trade name, trademark, manufacturer, or otherwise does not necessarily constitute or imply its endorsement, recommendation, or favoring by the United States Government or any agency thereof. The views and opinions of authors expressed herein do not necessarily state or reflect those of the United States Government or any agency thereof. 


\section{DISCLAIMER}

\section{Portions of this document may be illegible in electronic image products. Images are produced from the best available original document.}




\title{
A Faint Galaxy Redshift Survey behind Massive Clusters
}

\author{
by \\ Brenda Louise Frye \\ B.S. (University of Arizona) 1991 \\ B.S. (University of Arizona) 1991 \\ M.A. (University of California at Berkeley) 1995 \\ A dissertation submitted in partial satisfaction of the \\ requirements for the degree of \\ Doctor of Philosophy \\ in \\ Astrophysics \\ in the \\ GRADUATE DIVISION \\ of the \\ UNIVERSITY of CALIFORNIA at BERKELEY
}

Committee in charge:

Professor Hyron Spinrad, Chair

Professor Marc Davis

Professor George Smoot

Professor W. J. Welch 


\author{
Abstract \\ A Faint Galaxy Redshift Survey behind Massive Clusters \\ by \\ Brenda Louise Frye \\ Doctor of Philosophy in Astrophysics \\ University of California at Berkeley \\ Professor Hyron Spinrad, Chair
}

This thesis is concerned with the gravitational lensing effect by massive galaxy clusters. We have explored a new technique for measuring galaxy masses and for detecting high- $z$ galaxies by their optical colors. A redshift survey has been obtained at the Keck for a magnitude limited sample of objects $(I<23)$ behind three clusters, A1689, A2390 and A2218 within a radius of $0.5 \mathrm{Mpc}$. For each cluster we see both a clear trend of increasing flux and redshift towards the center. This behavior is the result of image magnifications, such that at fixed redshift one sees further down the luminosity function. The gradient of this magnification is, unlike measurements of image distortion, sensitive to the mass profile, and found to depart strongly from a pure isothermal halo. We have found that $V R I$ color selection can be used effectively as a discriminant for finding high- $z$ galaxies behind clusters and present five $4.1<z<5.1$ spectra which are of very high quality due to their high mean magnification of $\sim 20$, showing strong, visibly-saturated interstellar metal lines in some cases. Also, We have also investigated the radio ring lens PKS 1830-211, locating the source and multiple images and detected molecular absorption at $\mathrm{mm}$ wavelengths. Broad molecular absorption of width $40 \mathrm{kms}^{-1}$ is found toward the southwest component only, where surprisingly it does not reach the base of the continuum, which implies incomplete coverage of the SW component by molecular gas, despite the small projected size of the source, less than $8 h^{-1} p c$ at the absorption redshift. 


\section{A Faint Galaxy Redshift Survey Behind Massive Clusters}

Copyright $\odot 1999$

by

Brenda Louise Frye

The U.S. Department of Energy has the right to use this document for any purpose whatsoever including the right to reproduce all or any part thereof. 
To my family, 


\section{Contents}

List of Figures vii

List of Tables $\quad$ xix

1 Introduction $\quad 1$

1.1 Dark Matter and Cosmology . . . . . . . . . . . . . . . 1

1.2 Brief History of Lensing . . . . . . . . . . . . . . . . . . . 3

1.3 Thesis Motivation ....................... 4

2 Lensing Theory $\quad 7$

2.1 Deflection Angle . . . . . . . . . . . . . . . . . . . . 7

2.2 Mass Sheet .......................... 8

2.3 Lens Equation $\ldots \ldots \ldots \ldots \ldots \ldots \ldots$

2.4 Einstein Ring Radius . . . . . . . . . . . . . . . . . . . . . . . 12

2.5 Imaging with a Point Mass Lens . . . . . . . . . . . . . . . . 12

2.6 Singular Isothermal Sphere . . . . . . . . . . . . . . . . . . . . 14

2.7 The Lensing Potential . . . . . . . . . . . . . . . . . . . 16

2.8 The Distortion Field . . . . . . . . . . . . . . . . . 18

2.9 The Magnification . . . . . . . . . . . . . . . . . . . . 20

2.10 Conclusions . . . . . . . . . . . . . . . . . 22

3 The Radio Ring Lens PKS 1830-211 24

3.1 Introduction . . . . . . . . . . . . . . . . . 24

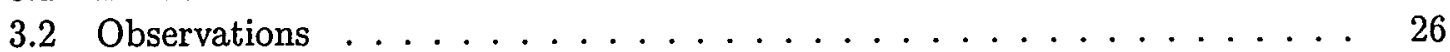

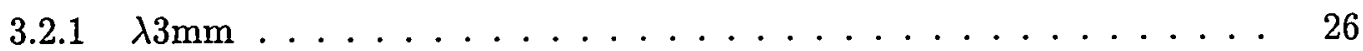

3.2 .2 Optical and Infrared . . . . . . . . . . . . . . . . 29

3.3 Cloud Coverage . . . . . . . . . . . . . . . . . . . 32

3.3.1 Constraints on Source Redshift . . . . . . . . . . . . . . 33

3.3.2 Time Delay from Spectral Monitoring . . . . . . . . . . . 35

3.4 Conclusions . . . . . . . . . . . . . . . . . . 37

4 Spectroscopic Data Reduction $\quad 38$

4.1 Introduction . . . . . . . . . . . . . . . . . . 38

4.2 The Bias and the Gain . . . . . . . . . . . . . . . . 39 
4.3 Flatfielding . . . . . . . . . . . . . . . . . . . 39

4.3 .1 Pixel to Pixel Variations . . . . . . . . . . . . . . . . . . . 39

4.3 .2 Fringing . . . . . . . . . . . . . . . . . . . . 41

4.3 .3 Slit Function . . . . . . . . . . . . . . . . . . . . . . . . 42

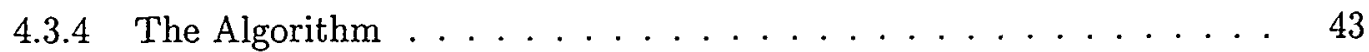

4.4 The Background Subtraction . . . . . . . . . . . . . . . . . . 44

4.4 .1 Signal to Noise . . . . . . . . . . . . . . . . . . . . . 44

4.4 .2 Spatial Distortions . . . . . . . . . . . . . . . . . . . 45

4.4 .3 Median Cosmic Ray Clipping . . . . . . . . . . . . . . . . 47

$4.4 .4 \quad \sigma$-cut Cosmic Ray Clipping . . . . . . . . . . . . . . . . . . . . 48

4.4 .5 Background Subtraction . . . . . . . . . . . . . 48

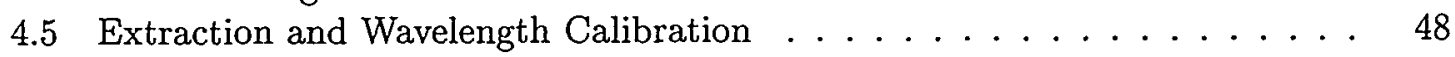

4.5 .1 The Spatial Profile . . . . . . . . . . . . . . . . . . 48

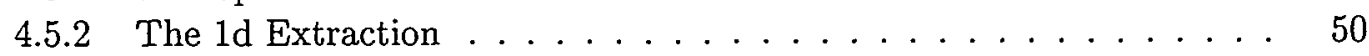

4.5.3 The Wavelength Calibration . . . . . . . . . . . . 51

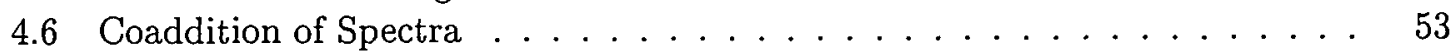

4.7 The Final Spectra . . . . . . . . . . . . . . 56

5 Cluster Redshift Survey $\quad 59$

5.1 The Giant Arcs . . . . . . . . . . . . . . . . . . . . . . 59

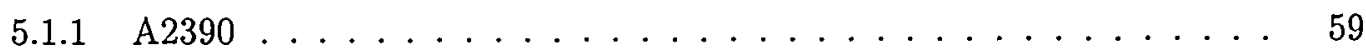

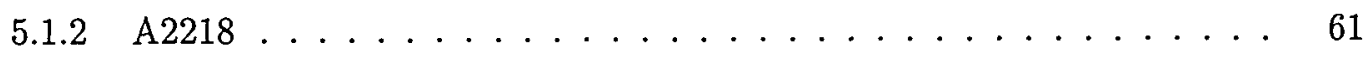

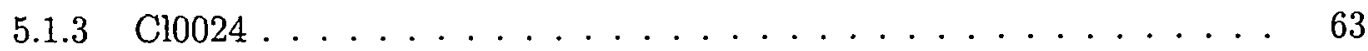

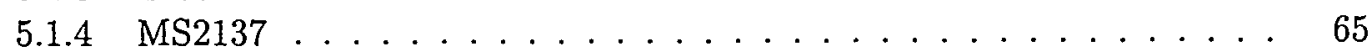

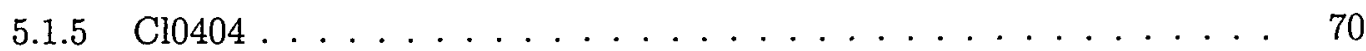

5.2 The Redshift Survey . . . . . . . . . . . . . . . . . . 70

5.2 .1 The Spectra. . . . . . . . . . . . . . . . . . . . 70

5.2 .2 Success Rate . . . . . . . . . . . . . . . . . . . . 75

5.2 .3 Magnitude and Redshift Distributions . . . . . . . . . . . . . . 78

5.2 .4 Measuring Cluster Magnification . . . . . . . . . . . . . . . . 83

5.3 Conclusions . . . . . . . . . . . . . . . . . . . 91

6 High- $z$ Galaxies $\quad 92$

6.1 Introduction . . . . . . . . . . . . . . . . . . 92

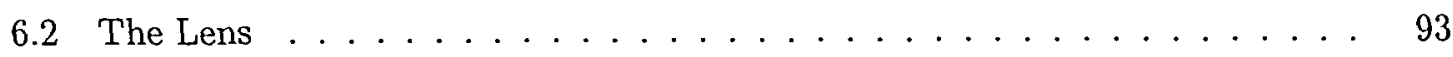

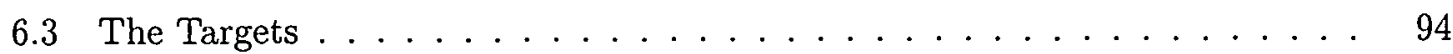

6.4 Observations and Results . . . . . . . . . . . . . . 100

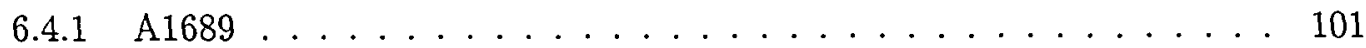

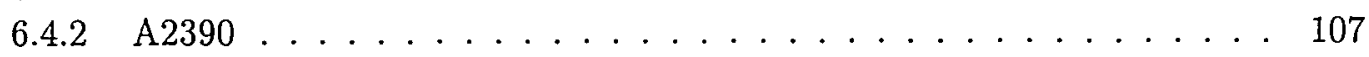

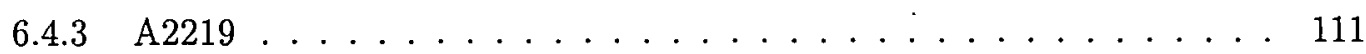

6.4 .4 The Leftovers . . . . . . . . . . . . . . . . . . . . 117

6.4 .5 The Reddest Elliptical Galaxies . . . . . . . . . . . . . . 117

6.5 Discussion . . . . . . . . . . . . . . . . . . . 127

6.5 .1 Continuum fits . . . . . . . . . . . . . . . . 127

6.5 .2 Gas Outflows . . . . . . . . . . . . . . . 127 
6.5 .3 Lensing by $\mathrm{A} 2390 \ldots \ldots \ldots \ldots \ldots \ldots$

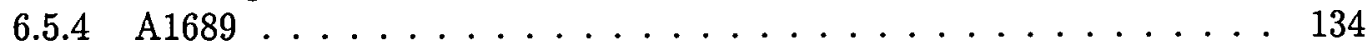

6.5.5 Summary of High- $z$ Properties . . . . . . . . . . . . 135

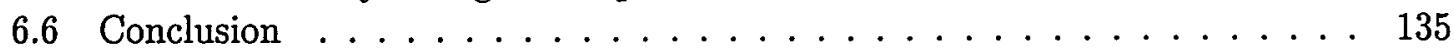

A A1689: 143

A.1 Mask $1 \ldots \ldots \ldots \ldots \ldots \ldots \ldots \ldots \ldots$

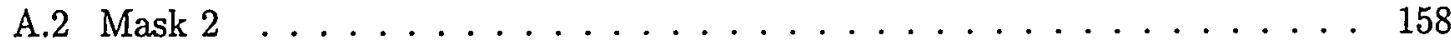

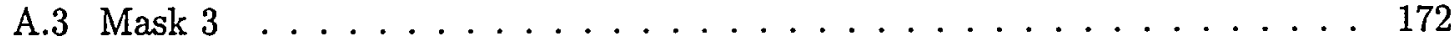

$\begin{array}{ll}B \text { A2390: } & 186\end{array}$

B.1 Mask 1. . . . . . . . . . . . . . . . . . . 186

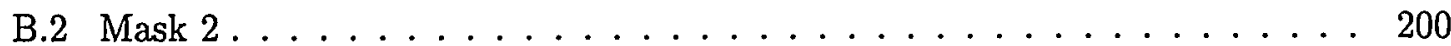

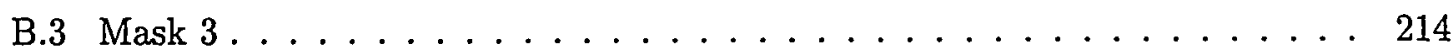

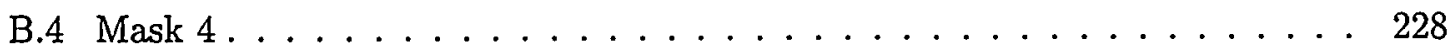

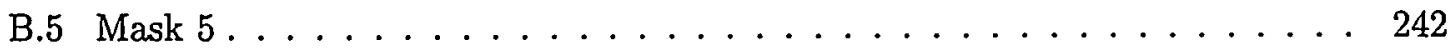

C A2218: 253

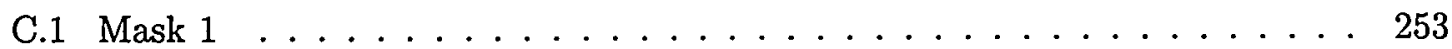

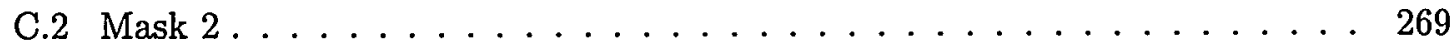




\section{List of Figures}

2.1 Lensing by a point mass. A photon moving with velocity $v$ in direction $\hat{y}$ feels an attractive force towards a mass $M$ a distance $R$ away from it. . . .

2.2 The image plane, where $\vec{\xi}$ is the $2 \mathrm{~d}$ positional vector to the point of intersection with the light rays $I$ and $\vec{\xi}^{\prime}$ is a 2 d positional vector to some arbitrary point $P$. The distance from the light rays to $P$ is $\left|\xi-\xi^{\prime}\right| \ldots \ldots \ldots$

2.3 The lensing diagram. A light ray from source $\mathrm{S}$ is deflected by an angle $\hat{\alpha}$ at point $\xi$ in the lens plane and arrives at $O$. The angles subtended by the optic axis and the source and the optic axis and the image are $\theta_{S}$ and $\theta_{I}$ respectively. The angular difference $\theta_{S}-\theta_{I}$ is the reduced deflection angle $\vec{\alpha}$

2.4 The galaxy luminosity function $\phi(L)$. The $\log$ number of galaxies per unit volume and in luminosity range $L$ and $L+d L$ is plotted against $\log$ Luminosity 21

3.1 Radio image of the radio ring lens PKS 1830-211. Here a quasar with a core plus jet structure is lensed by a foreground galaxy. The two hot spots correspond to the doubly-lensed image of the core, with the jet tail forming the ring. . . . . . . . . . . . . . . . . .

3.2 The $3 \mathrm{~mm}$ continuum map (center) showing the known double lensed structure is well resolved with a separation of $0 ! 98$ and a flux ratio of $1.14 \pm 0.05$. Contour levels are spaced by $0.1 \mathrm{Jy} /$ beam with the lowest value at 0.1 $\mathrm{Jy} /$ beam. The synthesized beamwidth is $0.68 \times 0.45$ (lower left), where the asymmetry is due to the low elevation of the source as seen from the northern latitude of BIMA. The spectral range covers the redshifted $H C N(2-1)$ and $\mathrm{HCO}^{+}(2-1)$ molecular transitions at $5 \mathrm{~km} / \mathrm{s}$ resolution, which are shown (inset) for both images. The molecular absorption is detected only in the SW component and does not reach the base of the continuum. Since the two images are similarly bright, the lack of absorption in the NE spectrum confirms that the spill over between the two images is negligible. . . . . . . . . 
3.3 Top row, from left to right: 1 . Field of $4.0^{\prime \prime}$ around PKS 1830-211 observed with Keck II in the I-band. This image is a stack of 6 frames with a pixel size of 0.215 and seeing of $0^{\prime \prime} 8$. 2. Mean of $5 \mathrm{~K}$-band images obtained with Keck I and NIRC. The pixel size is 0.157 and the seeing is $0^{\prime \prime} 7$. Bottom row, from left to right: 1 . Simultaneous deconvolution of the 6 I-band frames: resolution of $0^{\prime} .215$ and pixel size of $0^{\prime \prime} 1075$. 2. Deconvolution of the mean of 5 NIRC images: resolution of 0.157 and pixel size of 0.0785 . In all the images North is up, East left. The M-star, the NE QSO component candidate and the blended SW component + lensing galaxy candidate are detected. The scale is given in arcseconds relative to the M-star. . . . . . . . . . . . . .

3.4 Positions observed for the different objects detected in the optical and nearIR, relative to the $M$ star (Courbin et al., 1998a). The large open circles show the geometry of the system in the radio. Their radius corresponds to the error bars quoted by S90. The black dots are from the near-IR images while the open circles indicate the result obtained from the $I$ band data. . .

3.5 A geometric model for the lens, image and cloud planes, with two choices for the lens distance, corresponding to the distant molecular absorption at $\mathrm{z}=0.89$ and the nearby $\mathrm{HI}$ absorption at $\mathrm{z}=0.19$. The incomplete absorption of the SW beam is represented as a partially covered arc in the image plane, along with an unabsorbed counter arc to the NW, on either side of the Einstein ring. The spiral arm is shown at two alternative positions in the cloud plane, illustrating the two possible arrangements that give rise to the same $70 \%$ fractional coverage of the resultant lensed image. . . . . . . . . .

3.6 Fluxed spectrum of the source of PKS 1830-211 (Lidman et al., 1999). Regions of strong atmospheric absorption are marked by horizontal lines. They measure $z=2.507$ from the two strong emission lines of $\mathrm{H} \alpha$ and $\mathrm{H} \beta$. They measure $F\left[H_{\alpha}\right] / F\left[H_{\beta}\right]=11 \pm 2$, significantly-redder than measurements of other quasars. . . . . . . . . . . . . . . . . .

4.1 Sample section of a V-band dome flat image. The irregular blotches are CCD defects while the regular pattern of striped rows are inherent to the CCD array. . . . . . . . . . . . . . . . . . .

4.2 Sample section of an object flat from a multislit spectral image. Dispersion direction is left-right and the spatial direction is up-down. Note the interferometric fringes (left), and the wavelength-independent dark and bright rows of pixels, caused by nonuniform illumination of the lamp along the length of the slit. Each spectrum has a unique spatial illumination profile, and a different position in the dispersion direction at which fringing starts to become important. . . . . . . . . . . . . . . . . 
4.3 Example showing how this algorithm follows the curvature of the object. Both panels show the same patch of a fully-reduced $2 \mathrm{~d}$ spectrum, where the dispersion direction is left-right. The two bright vertical lines in each spectrum are skyline residuals. The occasional groups of bright pixels are cosmic rays, which were identified and ignored in finding the best estimate for the sky but not removed from the $2 \mathrm{~d}$ image. The bright horizontal stripe extending through the middle of each spectrum is the object continuum. The two dark lines which frame the top and bottom of the object continuum mark the sptial extraction region. In Panel (a) the curvature of the object is not followed. Note that in going from left to right, towards the blue edge of the chip, increasingly more of the object signal is lost. In Panel (b) the continuum is followed. Note that in this case the continuum signal is fully collected, maximizing on the signal to noise of the extracted object. . . . .

4.4 Comparison of frames before and after background subtraction over the same $2 \mathrm{~d}$ spectral region. Sample regions from four different spectra are shown here, each covering a different wavelength range. The dispersion direction is leftright and the spatial direction is up-down. Note that there is an emission line object in the lower left-hand corner which is only made obvious after the background subtraction. This is Ly $\alpha$ at 7418 Aand $z=5.12$. The redshift of the other emission line object in this field, in the upper right hand side of the image, has yet to be determined. Note the general smoothly-distributed noise of the subtracted background and the inevitable skyline residuals . . .

4.5 Cross-sectional view of the spectrum of a bright object sampled at 20 different positions along the dispersion direction, from pixel number 100 to 2000 . In each panel the spatial profile is plotted up as counts vs. pixels in the spatial direction centered on the object. This is the first plot which appears as part of the 'wavecal' code. Note that the algorithm does a fairly-good job of following the curvature across the full dispersion range. . . . . . . . .

4.6 Another 'wavecal' task output. This is a plot of counts vs. pixel number in the dispersion direction for the 13 skylines which were used to wavelength calibrate this particular spectrum. The wavelengths of the skylines are given as the titles and the position of the line core is given by the solid vertical bar in each panel. . . . . . . . . . . . . . . . . . .

4.7 The 'spadd' spectral coaddition task output. This is a multi-panel plot of counts vs. observed wavelength for each of the 6 exposures of this particular spectrum, and the seventh panel (bottom) gives the result of the coaddition. The continuum level is marked by the solid line, against which high and low deviant points are flagged (diamond points). The dot-dashed line marks the zero level of the continuum. . . . . . . . . . . . . . . . 
4.8 A sample coadded spectrum, in this case of 'Arc 8' in A1689. Counts are plotted against Observed wavelength on the bottom axis and rest wavelength on the top axis, with the middle axis giving the corresponding $2 \mathrm{~d}$ spectrum pixel values. For comparison, the sky spectrum is given in the bottom panel. The redshift, which can be changed on the command line, appears in the upper right-hand corner, and features are both labelled and marked with a dashed vertical line. . . . . . . . . . . . . . . . .

5.1 Keck Spectra taken at two different positions along the Straight Arc. Two unrelated galaxies are found at $\mathrm{z}=0.913$ and $\mathrm{z}=1.033$, corresponding to components $C$ and A respectively (in the notation of Pellò et al. (1991)). In addition interstellar lines of MgII and FeII at the redshift of component $\mathrm{C}$ are seen in absorption towards the higher redshift component A (indicated by dashed lines extending downward into the middle panel), evidence for an extended gaseous halo. . . . . . . . . . . . . . . .

$5.2 V$ and $I$ images were taken at the CFHT and combined to make this $3^{\prime} \times 2.5^{\prime}$ color image of the cluster center of A2218, in $\sim 0.5^{\prime \prime}$ seeing. The giant blue arc seen just to the right of the central cluster galaxy, extending roughly updown in this image orientation, is star-forming galaxy at $z=2.51$ discovered by Ebbels et al. (1996). . . . . . . . . . . . . . . . . .

5.3 Keck spectra of two intermediate- $z$ objects. The lower spectrum is of the giant arc at $z=2.51$ in A2218, first discovered by Ebbels et al. (1996). The upper spectrum is of the giant $\theta$ arc in Cl0024, also shown in Fig. 1.4. The many strong metal lines make this a useful template for galaxies of intermediate redshift. The lower spectrum has been offset by 150 counts, so that the zero-level of the continuum is at $-150 . \ldots \ldots \ldots$

5.4 Keck color image of the cluster center of the cluster Cl0024. . . . . . . . . .

5.5 The upper curve is the fluxed spectrum of the upper HII region of arc C (fig 2b). Many weak absorption lines are visible yielding an unambiguous redshift of $\mathrm{z}=1.675$. Note the similarity with the local "Wolf-Rayet" galaxy (lower curve) NGC4214 (Leitherer et al., 1996). The sky spectrum is also shown, in the lower panel . . . . . . . . . . . . . . . .

5.6 HST $I$ band image of the central region of $\mathrm{Cl0024}$ is shown on the left for comparison with the model on the right. Note the good agreement with the 5 main images (A-E). These may be improved further by hand, incorporating masses local to each image, but will not alter the conclusion that the central mass distribution traces well the location of the brightest ellipticals. A new multiple image pair is identified by the model (i,ii) at a predicted redshift of $z \simeq 1.3$. The contours represent surface mass density from $(0.6-1.2) \Sigma_{\text {crit }}$,

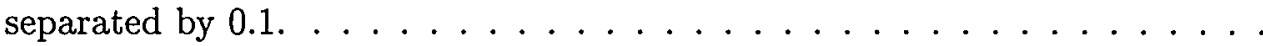

5.7 HST $R$ band image of the central arcminute of the cluster MS2137. Note the radial arcs emanating from the central cluster member, at the pixel coordinates $(380,250)$ and the set of parallel giant tangential arcs $15^{\prime \prime}$ in length centered at pixel coordinates $(320,180) . \ldots \ldots \ldots$ 
5.8 Spectrum of the giant tangential arc in MS2137. This is the first spectrum presented of this object, but there is not yet a convincing redshift for it. The best guess $z=3.02$ template laid down for inspection. Counts are plotted as a function of observed wavelength on the bottom axis and rest wavelength on the top axis. The break at $4900 \AA$ appears to be real but there are no other distinguishing features. More data is required to secure the redshift of this giant arc. The sky spectrum is also shown, in the lower panel, for comparison. 68

5.9 HST $R$ band image (702 nm) of the central arminute of the cluster Cl0404. 69

$5.10 I$ band image of A2390, taken at the CFHT. The pixel scale is 0.206 "/pix. 71

$5.11 I$ band image of A1689, taken at the NTT. The pixel scale is 0.350 "/pix. . 72

$5.12 I$ band image of A2218, taken at the CFHT. The pixel scale is 0.206 "/pix. 73

5.13 3-panel plot showing histograms of the differential counts of the $I$ band apparent magnitude distributions for the individual clusters A1689, A2390, and A2218. In each panel the solid histogram gives the counts for $I<23$, the short-dashed histogram gives the counts for $I<23$ and $z \geq 0$, and the longdashed histogram gives the counts for $I<23$ and $z>0.3$. Note that all three clusters are fairly-complete down to their individual $I$ band limits, which are $I=23$ for A1689 and A2390 and $I=22$ for A2218, and that the surplus of objects at $I \sim 18$ in each panel are cluster members. . . . . . . . .

5.14 Histograms of the $I$ band apparent magnitude distributions plotted as differential counts for the sum total of all objects in the clusters A1689, A2390, and A2218. In each panel the solid histogram gives the counts for $I<23$, the short-dashed histogram gives the counts with $I<23$ and $z \geq 0$, and the long-dashed histogram gives the counts for $I<23$ and $z>0.3$. Note from this distribution that it is fairly-complete down to $I=23$ and that the surplus of objects at $I \sim 18$ are cluster members. . . . . . . . . .

5.15 The $I$ band absolute magnitude distributions for the three clusters A1689, A2390, and A2218, plotted as differential counts. Cuts are made for different radial bins, as labelled. A systematic shift towards higher $z$ is clear for increasing radius. . . . . . . . . . . . . . . .

5.16 The $I$ band absolute magnitude distributions for the three clusters A1689, A2390, and A2218 combined, plotted as differential counts. Cuts are made for different radial bins, as labelled. A systematic shift towards higher $z$ is clear for increasing radius. . . . . . . . . . . . . . .

5.17 The $z$-distributions for the three clusters A1689, A2390, and A2218. Cuts are made for different radial bins for each cluster, as labelled, in arcseconds.

5.18 Histograms of the $z$-distributions for the three clusters A1689, A2390, and A2218 combined. Cuts are made for three different radial bins, as labelled, in arcsec. The two solid curves represent the model field redshift distributions. The low- $z$ curve gives the unlensed redshift distribution and the high- $z$ curve gives the redshift distribution for the best-fit magnification of $\mu \approx 4$. A shift is found behind the cluster relative to the field. . . . . . . . . . 
5.19 This 9-panel plot shows the redshift, absolute $I$-band magnitude, $M_{I}$, and $V-I$ color as a function of radial distance in arcseconds, for the three clusters A1689, A2390, and A2218. The set was selected to have $0.3<z<2$, and to have $I<23$. Overplotted is a boxcar mean. An object with a given $z$ and $M_{I}$ is systematically at higher $z$ and brighter towards the cluster center, showing evidence for cluster magnification. . . . . . . . . . . .

5.20 This 3-panel plot shows the redshift, $I$ band absolute magnitude $M_{I}$, and $V-I$ color as a function of radial distance in arcseconds, for the points in the three clusters A1689, A2390, and A2218 combined. The set was selected to have $0.3<z<2$, and to have $I<23$. Overplotted is a boxcar mean. An object with a given $z$ and $M_{I}$ is systematically at higher $z$ and brighter towards the cluster center, showing evidence for cluster magnification. . . .

5.21 The Canada-France Redshift Survey (CFRS) is plotted as the histogram, with the three model curves showing the expected redshift distributions for all morphological types and in certain $I$ band absolute magnitude ranges, as labelled. . . . . . . . . . . . . . . . . . . .

5.22 The mean $z$ as a function of distance in arcsec away from the cluster centerof-mass appears on the left and the corresponding magnification profile on the right for the cluster A2390. Two models are overlaid for comparison with the data points. They are an isothermal profile with an Einstein radius of $30^{\prime \prime}$ (solid line), and an isothermal model with a radius of $30^{\prime \prime}$ and a power law slope of -0.6 (dashed line). The mean values of the isothermal model summed up over the same intervals as the data is shown by the diamonds. .

5.23 The mean $z$ as a function of radial distance in arcsec away from the cluster center-of-mass appears on the left and the corresponding magnification profile on the right. Two models are overlaid for comparison with the data points. They are an isothermal profile with a mean Einstein ring radius of $30^{\prime \prime}$ (solid line), and a power law model with a radius of $30^{\prime \prime}$ and a power law slope of -0.6 (dashed line). For reference, the integrated model mean in the same radial bins as the data is indicated by the diamond-shaped symbols. . . . .

6.1 Model spectra of the $z=4$ galaxy for a stellar population dominated by B3 stars for three different $z$ 's. $F_{\lambda}$ is plotted against observed wavelength on the lower axis and rest wavelength on the upper axis. The solid line gives the model spectrum, including HI absorption at the source. The model given by the dashed line shows the effect of the $z$-dependent Lyman-series forest opacity. The five dotted continuous curves give the transmission curves for the $U B V R I$ filters, in order of increasing wavelength. . . . . . . . . .

6.2 Model spectra of a $z=4$ galaxy for a stellar population dominated by B3 stars (left-hand panel) or OV stars (right-hand panel). In each case the stellar blackbody (solid line) is attenuated by $\mathrm{HI}$ absorption from the z-dependent Lyman series (dashed line). Note the increased absorption at the observed wavelength of $\mathrm{Ly}-\alpha, 6130 \AA$, from $\mathrm{H}$ absorption intrinsic to the B3 star. . . 
6.3 The blackbody spectra of hot stars with spectral types A0 through O3. Note the increasing strength of $\mathrm{Ly}-\alpha$ absorption in the transition from $\mathrm{O}$ to the $\mathrm{B}$

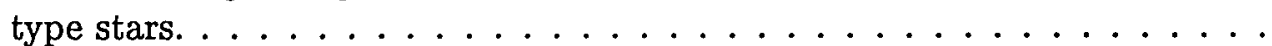

6.4 Color- $z$ plots of high-z spectra, plotted in Vega magnitudes. In both panels models with a stellar population dominated by B0 stars appears as the solid line and the one dominated by B3 stars with a dashed line. In the panel on the right-hand side $V-R$ is plotted against $z$ and in the left-hand panel $R-I$ is plotted against $z$. All four curves have the same general shape, which is roughly-flat until $z=4$, when the Lyman-series forest has redshifted into both the $V$ and $R$ bands. A B3 star has a non-negligible Balmer discontinuity, causing the bump seen in the B3 model curve at $z \sim 1$ in both panels. . . .

6.5 Color-color diagrams of model high- $z$ spectra. The set of models with a stellar population dominated by B0 stars appears on the left-hand side one dominated by B3 stars on the right-hand side. For each plot the Johnson $V-R$ is plotted up against Johnson $R-I$ and redshifts labelled alongside the curves. The general shape of the curves is the same, with the sudden rise in the curve in the blue part of the color-color plane caused by the drop in $V$ band flux when the $V$ band redshifts into the Lyman-series forest. Note that $V-R$ is fairly steep with respect to $R-I$ for $z>4$ but is still useful if the photometry can be measured with reasonably-good accuracy. . . . . . .

6.6 Color images of the center of A1689 $(z=0.18)$ with high- $z$ galaxies labelled. Panel (a) is a ground-based color NTT image of the inner $2^{\prime} \times 3^{\prime}$. Note in the lower left of the image a blue arc subtending 30 degrees, with many other giant arcs visible at this radius. Both of the high- $z$ galaxies on this plot are small, red and unresolved at this resolution ( $\left.0.85^{\prime \prime}\right)$. Panel (b) shows a fairly shallow, high angular resolution Hubble Space Telescope color image of the inner $50^{\prime \prime}$. The galaxy labelled $z=4.88$ is the same galaxy as the one in Panel (a), and is marginally-resolved at this resolution $\left(\sim 0.1^{\prime \prime}\right)$. The J2000 coordinate of the cluster center is $\alpha=13^{h} 11^{m} 28^{s}, \delta=-01^{\circ} 20^{\prime} 48^{\prime \prime} \ldots \ldots$ 102

6.7 Imaging and Spectroscopy of a high-z galaxy in A1689 at $z=4.88$. The spectrum is plotted as $F_{\lambda}$ vs. observed wavelength on the lower axis and rest wavelength on the upper axis. The distinctive features are Ly- $\alpha$ in emission, the Ly- $\alpha$ break, the Ly- $\beta$ break, and the Sill $\lambda 1260$ metal line. Ly- $\alpha$ is shifted redward from SiII by $360 \mathrm{~km} / \mathrm{s}$. The continuum (dashed line) is best fitted by a hot $\mathrm{O}$ stellar population, with attenuation by the Lyman-series forest becoming severe by this $\mathrm{z}$ (solid line). The sky spectrum is shown in the lower panel for comparison. Also included are five ground-based images of this galaxy (circled) in $U B V I J$. There is no emission from this object in $U$ and $B$ 
6.8 Imaging and Spectroscopy of a high- $z$ galaxy in A1689 at $z=5.12$. The spectrum is plotted as Counts vs. observed wavelength on the lower axis and rest wavelength on the upper axis. Ly- $\alpha$ is seen strongly in emission and has an asymmetric profile. The Ly- $\alpha$ break is also clearly-detected. The sky spectrum is shown in the lower panel for comparison. Also included are five ground-based images of this galaxy (circled) in $U B V I J$. There is emission seen only in the $I$ band. . . . . . . . . . . . . . .

6.9 Imaging and Spectroscopy of a high- $z$ galaxy in A1689 at $z=5.12$, centered on the Ly- $\alpha$ emission line. The spectrum is plotted as Counts vs. observed wavelength on the lower axis and rest wavelength on the upper axis. Ly- $\alpha$ is seen strongly in emission and has an asymmetric profile. The sky spectrum is displayed in the lower panel for comparison. . . . . . . . . . . .

6.10 Panel (a) shows the many red and blue arcs around the center of A2390, with the elliptical galaxy centered on the $z=4.04$ arcs indicated. (b) A blow up of the region containing the 4 lensed images of the $z=4.04$ galaxy, with the central elliptical galaxy removed. The locations of the slitlet positions corresponding to Fig. 6.10 are indicated. Also shown is the "straight arc" which spectroscopy resolves into two galaxies: component $A$ (in the notation of Pellò et al. (1991) at $z=1.033$ and component $\mathrm{C}$, at the known redshift of $z=0.913$ (see Fig. 6.11 for the spectra). (c) A simple lens model for the source showing the importance of both the central elliptical and the cluster potential in creating the general image configuration. The parity of the images is also displayed in the model: the brighter part of the images is chosen to match the spatial location of the observed Ly $\alpha$ emission in the $\mathrm{N}$ and $\mathrm{S}$ arcs. These images are overlayed on the lens magnification field, showing clearly the bright asymmetric critical curve. . . . . . . . . .

6.11 Keck spectra of the arcs at $z=4.04$. The upper two panels show fluxed spectra of the Northern and Southern arcs taken with slits aligned normal to the long axes of these arcs. Overlayed is the best fitting B3-star continuum, with the opacity of the forest included, together with Ly $\alpha$ absorption at the galaxy redshift, but no reddening. The middle panel shows the sum of both these arcs with slits aligned along their lengths, revealing clearly metal lines at $z=4.04$, on which the redshift is based. These lines lie blueward of the centroid of the asymmetric Ly $\alpha$ emission by $300 \mathrm{kms}^{-1}$, as discussed in the text. Below this is shown a spectrum centered on the Eastern arc, revealing Ly $\alpha$ emission and the central elliptical $(z=0.223)$. The sky is shown for comparison at the bottom of the panel. 
6.12 Two-dimensional spectrum of two arcs at $z=4.04$, taken along the long axis of the arcs. The northern and southern components are labelled as $\mathrm{N}$ and $S$ corresponding to the image presented in Frye \& Broadhurst 1997. The dispersion direction is in the $\mathrm{x}$-direction with the blue at the left-hand side. The spatial direction is up-down and the pixel scale is 0.21 "/pix, with the northern and southern components extend 5 and $3^{\prime \prime}$ vertically on each side of the central cluster elliptical (the bright white strip). Ly $\alpha$ emission is seen in each component as a bright spot in the blue, and is spatially-separated from the broad clearly visible stellar continuum. . . . . . . . . . . . . .

$6.13 \mathrm{Keck} I$ band image of A2219, taken in in good seeing, 0.7 . The three high- $z$ galaxies marked on the plot were discovered as a part of our survey of faint, red galaxies behind this cluster. The pixel scale is 0.21 "/pix. The cluster center has a J2000 coordinate of $\alpha=16^{h} 38^{m} 54.0^{s}$, dec $=46^{\circ} 47^{\text {arcmin }} 00^{\prime \prime}$. .

6.14 Spectrum and images of a high- $z$ galaxy at $z=4.67$ seen behind A2219. $F_{\lambda}$ is plotted against observed wavelength on the lower axis and rest wavelength on the upper axis. For reference, the middle axis gives the pixel scale for the $2 \mathrm{~d}$ spectrum. the spectrum shows a strong stellar continuum, strong metal lines, Ly- $\alpha$ emission, and a clearly-detected break shortward of Ly- $\alpha$. The dashed line shows the spectrum of a B3 stellar population and the solid line shows the model, which includes Lyman-series opacity plus an HI absorption component at $z_{e m}$ with a column density of $\log N=21.5$. The sky spectrum is plotted in the lower panel for comparison with the data. Two images of this galaxy, in $V$ and $I$ appear on the right-hand side of the spectrum. The $\mathrm{x}$ and $y$ coordinates refer to the coordinates of the larger image of Fig. 6.13. The object is undetected in $V$ and resolved but small in $I$, despite its expected large magnification. . . . . . . . . . . . . . . . .

6.15 Spectrum and images of a high- $z$ galaxy at $z=4.45$ seen behind A2219. $F_{\lambda}$ is plotted against observed wavelength on the lower axis and rest wavelength on the upper axis. For reference, the middle axis gives the pixel scale for the $2 \mathrm{~d}$ spectrum. This is a high quality spectrum with strong stellar continuum, saturated metal lines, and a distinctive Lyman-series break. Ly- $\alpha$ is seen in emission and is asymmetric. The numerous $L y-\alpha$ is shifted redward from the $z$ given by the metal lines by $690 \mathrm{~km} / \mathrm{s}$. The dashed line shows the spectrum of a B3 stellar population and the solid line shows the model, which includes Lyman-series opacity plus a damped HI absorption component at $z_{e m}$ with a column density of $\log N=21.1$. The sky spectrum is plotted in the lower panel for comparison with the data. Two images of this galaxy, in $V$ and $I$ are shown to the right of the spectrum. The $\mathrm{x}$ and $\mathrm{y}$ coordinates refer to the coordinates of the larger image of Fig. 6.13. The object appears at the center of the circle, just to the right of the stellar diffraction spike. It is faint in $V$ and resolved but small in $I$, despite its expected large magnification. . 
6.16 Spectrum and images of a high-z galaxy at $z=4.068$ behind A2219. $F_{\lambda}$ is plotted against observed wavelength on the lower axis and rest wavelength on the upper axis. For reference, the middle axis gives the pixel scale for the $2 \mathrm{~d}$ spectrum. Interestingly, this galaxy displays fully-saturated metal absorption lines. Ly- $\alpha$ is seen in emission above the underlying stellar continuum, and is shifted by $830 \mathrm{kms}^{-1}$ towards the red with respect to the redshift of the metal lines. The Lyman-series break is clearly-detected extending shortward of Ly- $\alpha$. The dashed line shows the spectrum of a B5 stellar population and the solid line shows the model, which includes Lyman-series opacity plus a damped HI absorption component at $z_{e m}$ with column density $\log N=21.1$. The sky spectrum is shown in the lower panel for comparison with the data. Two images of this galaxy, in $V$ and $I$, are shown to the right of the spectrum. The $\mathrm{x}$ and $\mathrm{y}$ coordinates refer to those of the larger image of Fig. 6.13. The object centered in the circle. It is very faint in $V$ and resolved but small in $I .115$

6.17 Spectra of the three high- $z$ galaxies in A2219 and the second highest redshift galaxy in $\mathrm{A} 1689$, as labelled. $F_{\lambda}$ is plotted in arbitrary flux units against observed wavelength. Ly- $\alpha$ is seen in emission above the underlying stellar continuum, and is shifted by varying amounts with respect to the interstellar metal absorption lines (see text). . . . . . . . . . . . . .

6.18 Near infrared image of the elliptical galaxy at $z=1.155$ in A1689, taken with NIRC on Keck 1 in June of 1998. This 30 min exposure in $K$ shows that this

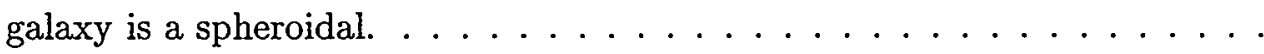

6.19 Spectrum of a elliptical at $z=1.155$ in A1689 with Bruzual-Charlot model overlayed. $F_{\lambda}$ is plotted against observed wavelength on the lower axis and rest wavelength on the upper axis. The model is for a passively-evolved 8 Gyr galaxy with solar metallicity. MgI and MgII are clearly seen. The sky spectrum appears on the bottom panel for comparison. . . . . . . . . .

6.20 Spectrum of an elliptical at $z=0.791$ in A2390 with Bruzual-Charlot model overlayed. $F_{\lambda}$ is plotted against observed wavelength on the lower axis and rest wavelength on the upper axis. The model is for a passively-evolved 8 Gyr galaxy with solar metallicity. $\mathrm{MgI}, \mathrm{MgII}, \mathrm{CaH} \& \mathrm{~K}$, and $\mathrm{H} \delta$ are clearly seen. The sky spectrum appears on the bottom panel for comparison. . . .

6.21 Spectrum of an elliptical at $z=0.783$ in A2390 with Bruzual-Charlot model overlayed. $F_{\lambda}$ is plotted against observed wavelength on the lower axis and rest wavelength on the upper axis. The model is for a passively-evolved 10 Gyr old galaxy with $[\mathrm{Fe} / \mathrm{H}]=-0.33$. MgI, MgII, weak OII, CaH \& $\mathrm{K}$, and $\mathrm{H} \delta$ are clearly seen. The sky spectrum appears on the bottom panel for

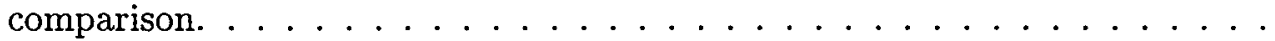


6.22 Generating the fluxing curve. The upper panel shows the spectrum of an early-type cluster member at $z=0.175$ observed simultaneously through the multislit mask (solid line), with the standard empirical E/SO spectrum of Kennicutt (1992) (dashed line). Note that the flux from the template spectrum rises above that of the data at both ends of the spectrum, which if there are minimal differences between the two galaxy spectra are due to the response function of the CCD. The bottom panel shows the ratio of the model template to the cluster elliptical. The 'feature' at $7600 \AA$ is produced by A-band atmospheric absorption. . . . . . . . . . . . . . . .

6.23 Example of fluxing. The upper panel shows the spectrum of the elliptical at $z=0.791$. The middle panel shows the fluxing curve used to flux this spectrum and the bottom panel shows the resulting spectrum. Note that the spectrum has been 'pulled up' at the edges to correct for the declining detector response in the near UV and far red, and made slightly more noisy especially at the blue end, where there were fewer counts. . . . . . . . .

6.24 The left-hand panel shows the family of Bruzual-Charlot spectra for passivelyevolving elliptical galaxies with $[\mathrm{Fe} / \mathrm{H}]=1.0089$ (ten times solar metallicity) and the right-hand panel shows the same for ellipticals with $[\mathrm{Fe} / \mathrm{H}]=-0.3300$ (half solar metallicity). The ages in both cases are are $1 \mathrm{Gyr}, 3 \mathrm{Gyr}, 7 \mathrm{Gyr}$,

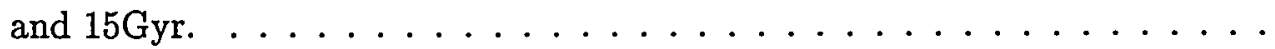

6.25 The left-hand panel shows the family of Bruzual-Charlot spectra for passivelyevolving. elliptical galaxies with $[\mathrm{Fe} / \mathrm{H}]=0.0932$ (solar metallicity) and the right-hand panel shows the same for ellipticals with $[\mathrm{Fe} / \mathrm{H}]=1.0089$ (ten times solar metallicity). The ages in both cases are $1 \mathrm{Gyr}, 3 \mathrm{Gyr}, 7 \mathrm{Gyr}$, and 15Gyr. 126

6.26 Equivalent width measurements of interstellar metal lines in six high- $z$ galaxies. The rest equivalent widths were measured for the lines SiII $1260 \lambda$, OI $1302 \lambda$, SiII1304 $\lambda$, and CII $1336 \lambda$ and the total given in the lower right corner of each box. The dashed lines highlight the line profiles and the solid horizontal lines at the top end of each absorption feature show the extent in wavelength and the continuum level over which the equivalent width was calculated. . . . . . . . . . . . . . . . .

6.27 Equivalent width measurements for interstellar metal lines in high- $z$ galaxies and local starbursting galaxies. The rest equivalent widths were measured for the lines SiII $1260 \lambda$, OI $1302 \lambda$, SiII1304 $\lambda$, and CII $1336 \lambda$ in NGC 1741 (Conti et al., 1996) and in lines OI $1302 \lambda$, SiII1304 $\lambda$ for the other panels. The total rest equivalent width is given in the lower right corner of each box. The dashed lines highlight the line profiles and the solid horizontal lines at the top end of each absorption feature show the extent in wavelength and the continuum level over which the equivalent width was calculated. . . . . 
6.28 The redward velocity shift between the centroid position of Ly- $\alpha$ and the $z$ determined by the metal lines is plotted against the total rest equivalent width of the metal lines. Four different ISM metal lines were summed together. They are: SiII $1260 \lambda$, OI $1302 \lambda$, SiIV $1304 \lambda$, and CII $1336 \lambda$. The different symbols correspond to different sets of data, which are given in the legend in the lower right-hand corner. The high- $z$ points are in general agreement with the local measurement of Heckman et al. (1998) . . . . . .

6.29 The redward velocity shift between the centroid position of Ly- $\alpha$ and $z$ determined from the metal lines is plotted against the total equivalent width of the metal lines. Two different metal lines were summed together. They are: OI $1302 \lambda$ and SiIV 1304 $\lambda$. The different symbols correspond to different sets of data, which are given in the legend in lower right-hand corner. . . . . . . 


\section{List of Tables}

3.1 Summary of the photometry and astrometry the NE component and the SW component of the lensed source (plus lensing galaxy), and photometry for the M-star. . . . . . . . . . . . . . . . .

5.1 Logbook of multislit Keck observations. The columns are: Cluster name, Date of the observation, mask number, number of 20 minute exposures, or equivalent, $I$ band limiting magnitude, and the total number of objects observed on the mask. . . . . . . . . . . . . . . . . .

5.2 Longslit Data for A2390, courtesy of Hy Spinrad, Dan Stern, and Andy Bunker. The columns are: $(x, y)$ pixel position in the $2 \mathrm{~d} I$ image, $I$ magnitude, $V-I$ color, and identifying features. . . . . . . . . . . .

5.3 The success rate for the spectroscopy. The columns are: Cluster name, the number of objects for which $I<I_{\text {lim }}$ the number of objects for which $I<I_{\text {lim }}$ and a redshift is known, $z \geq 0$, the number of objects for which $I<I_{\text {lim }}$ and are behind the cluster, $z>0.3$, the success rate for redshifts, or the fraction of objects with redshifts, and the arclet rate, or the ratio of background objects to the total. . . . . . . . . . . . . . . . .

5.4 I band absolute magnitude distributions for the three radial bins listed, and the total number of arclets in each bin. . . . . . . . . . . . . . . .

5.5 The mean redshifts, $\bar{z}$, magnifications $\mu$, are given for each radial bin of each cluster and for the total. The associated errors are also recorded, as is the number of objects in each bin. . . . . . . . . . . . . . . . .

5.6 The mean redshifts, $\bar{z}$, magnifications $\mu$, are given for 6 different radial bins for the sum total of all three clusters. The associated errors are also recorded, as is the number of objects in each bin. . . . . . . . . . . . .

6.1 Table showing the expected magnification for the isothermal and NFW profiles, given a couple of typical values for $R$ and for an Einstein ring radius of

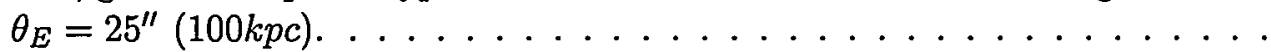

6.2 Properties for three of the best cases for distant ellipticals. The columns are: Cluster, Object Name, $z, I$ band magnitude, $V-I$ color, and age since the last starburst event. 
6.3 Particulars for the high- $z$ galaxies discovered in the survey. The columns are: galaxy cluster, $z, W_{I S}$, the rest equivalent width of SiII $1260 \lambda$, OI $1302 \lambda$, and SiIV $1304 \lambda, \Delta V$, the velocity difference between the $z$ of Ly- $\alpha$ and the $z$ of the interstellar lines, $W_{L y \alpha}$, the rest equivalent width of OI $1302 \lambda$, and SiIV $1304 \lambda$, and the $I$ band magnitude, if known. . . . . . . . . .

6.4 The presence of the Ly $\alpha$ line in high- $z$ galaxies. the columns are: the observing group, the number of high- $z$ objects at $z \geq 4$, and the number of those objects which show $\operatorname{Ly} \alpha$ in emission. . . . . . . . . . . . . 135 


\section{Acknowledgements}

It is a pleasure to acknowledge the advice and guidance offered by my advisor Hy Spinrad and by Jack Welch, Marc Davis, and George Smoot, the other readers of this dissertation. Hy Spinrad has been an inspiration throughout my years here and I am very grateful to him for not sending me off to Tazmania. I am also very grateful for having the pleasure to work with Tom Broadhurst. His good ideas, excitement for science and his encouragement have all been important to me. Steve Kahn and Fritz Paerels I want to thank for introducing me to high energy astrophysics and for good general advice for my first year here. I am grateful for Richard Ellis' guidance and mentorship. Saul Perlmutter has been a recent influence on my scientific outlook and his enthusiasm has been much appreciated. James Graham and Marc Davis have been appreciated sources of sporadic encouragement. I want to thank Raja Guhathakurta for his interest in my work and his solid scientific advice. Carl Heiles provided much needed laughs. Jack Welch has been a mentor, collaborator and friend. I want to thank Jon Arons for his thoughtfulness in his role as departmental chair.

Txitxo Benítez has been most helpful for his enthusiasm for science and good advice. Doug Johnstone helped me adjust to life at Berkeley and to getting through life as a graduate student. Priya Natarajan has been an enthusiastic supportor. Chats with Magdelen Normandeau on life in the sciences have been important to me.

Leonidas Moustakas has been a huge source of continuing support, encouragement, and love. I am grateful for the support of several other people who were graduate students at Berkeley: Young Paik, Jeff Valenti, Luis Ho, Margaret Meixner, Joan Najita, Geoff Bower, Jay Anderson, Ellen Rigsby, and ZiJiang Yang. I would also like to acknowledge current graduate students whose chats and good company I've enjoyed on a daily basis, including Joe Barranco, Jane Burwood-Hoy, Alison Coil, Andrew Cumming, Jim Gibson, Andrea Gilbert, Doug Leonard, Leslie Maxfield, Ben Metcalfe, Andrea Somer, Greg Ushomirsky, and Neal Weiner.

I am also grateful to people from my undergraduate days. Peter Strittmatter gave valued mentorship, Jill Bechtold introduced me to research, and Fred Chaffee and Craig Foltz taught me how to be a good observer. Jennifer Grier, Giselle Sleiman, Jennifer Ash, Joe Tulagen, Mike Salters, and Eric Defonso and I spent much time learning physics together late at night, and I value their continued friendship.

The most important person for me to thank is my brother Brian, who has supported me continuously and with love. I want to thank Rychard Bouwens for his contagious, entertaining, and generous nature, and Dan Stern for removing him from my office when necessary. 


\section{Chapter 1}

\section{Introduction}

\subsection{Dark Matter and Cosmology}

The nature of dark matter remains perhaps the most pressing problem in Cosmology. Dark matter was first inferred by Zwicky in the 1930's from dynamical measurements of galaxies in galaxy clusters (Zwicky, 1933, 1937). He found that the total mass was dominated by some 'unseen' matter, well in excess of sum of luminous cluster galaxies. This conclusion has remained true despite decades of improvements in both the quality of the data and with new methods of detection such as gravitational lensing and X-ray modelling and also despite many theoretical advances. In the case of clusters, we can now quantify accurately the central mass-to light ratio $(\mathrm{M} / \mathrm{L})$ interior to the Einstein Ring of the most massive clusters where the ratio is found to be a remarkably high value $M / L_{B} \sim 200-300 h M_{\odot} / L_{\odot}$ which is completely model independent in the cases of nearly circular rings (see for example the detailed discussion on the giant arc system around CL0024 in Chapter 5). At larger radii weaker gravitational distortions allow the dark matter to be mapped in some detail (Kaiser \& Squires, 1993; Squires \& Kaiser, 1996; Clowe et al., 1998) showing the mass to be irregularly distributed in general. For a constraint on the mass profile gravitational magnification provides the best means, as distortions are sensitive only a differential effect (Chapter 2) indicating the $\mathrm{M} / \mathrm{L}$ may increase with radius, consistent with the general scale dependence of $M / L$ from galaxy to cluster sized systems.

No single experimental result regarding dark matter would be more important than a direct detection of the dark material itself. Solid state 'direct' detection experiments are underway (Sadoulet, 1997) providing a test for the detection of 'cold dark matter' (particle masses in the $\mathrm{Mev}$ range) via the heating of cryogenically cooled and radiation shielded detectors. Bright neutrino sources like SN 1987A have been detected astronomically, with upper limits to the mass of the antineutrino of 3-5 Mev (Lattimer \& Yahil, 1989).

Neutrino mass has also been implied by the detection of a mixing length for the $\mu_{\nu}$ neutrino at the Kamiokande Mine in Japan (Fukuda \& et al, 1999). Mixing between species is understood if neutrinos are massive, leading to a probability of transmutation between 
species. The mixing length is determined to be comparable with the Earth diameter and this sets a constraint on the square of the difference in mass between the muon and most likely the Tau neutrino, $\nu_{\tau}$. The mass is found to be only $\Delta m^{2}<6 \times 10^{-3} \mathrm{eV}^{2}$ (Fukuda \& et al, 1999), too small to be of cosmological significance.

Baryonic dark matter has been shown to be cosmologically insignificant but may be revised in view of some recent developments. The generation of light elements produced during the era of Big Bang nucleosynthesis produces in principle a good constraint on the cosmological baryon-to-photon ratio $\eta$ (Reeves, 1972; Audouze \& Tinsley, 1974; Epstein et al., 1976). In particular a well-constrained measurement of the deuterium-to-hydrogen ratio $(\mathrm{D} / \mathrm{H})$ in absorption systems towards QSOs provides tight constraints on $\eta$. The absorption systems must be carefully selected (Tytler \& Burles 1998) so that confusion with the general forest is reduced, and at high- $z$, to better reflect the primordial value. This experiment gives a value of $\Omega_{B} h_{50}^{2}=0.076 \pm 0.004$ (Tytler \& Burles, 1997), that is, less than $10 \%$ of what is required for the universe to be finite. This small value is usually taken to imply that the mass of the universe is dominated by nonbaryonic matter given the large $\mathrm{M} / \mathrm{L}$ inferred from cluster measurements. However, it does not for example rule out that the haloes of galaxies be composed of dark baryonic matter.

There is now a new check on $\Omega_{m}$ from the important work of Perlmutter \& et al (1999), and Riess \& et al (1998) on the curvature of the redshift-magnitude relation for supernovae of type Ia. In particular the definitive work of Perlmutter \& et al (1999) was the first to claim contributions to the overall cosmological curvature from both matter and cosmological constant from their combined effect on the luminsity distance of standard candles at cosmological distances $(z \sim 0.5)$. Their finding within the Friedmann-Lamaitre model is that $\Omega_{m}$ is well below unity, consistent with dynamics, and that there is a contribution to the stress energy tensor from Einstein's cosmological constant $\Lambda$, such that for a flat model the contributions are $\Omega_{m}=0.2$ and $\Omega_{\lambda}=0.7$. Further detections of distant supernovae will separately constrain these two parameters.

Finally, the distribution of matter on large scales provides a good way to discriminate between 'hot' and 'cold' dark matter. For cold dark matter small scale structure is predicted to form first in a hierarchy. With hot dark matter the scale which collapses first is closest to the free streaming length when the matter becomes non relativistic, leading to a 'pancake' formation of structure and no real prescription for formation of small objects like galaxies. Recent measurements of the power spectrum of galaxies and clusters in the local universe have shown that the relative strength of large to small scale power is surprisingly tipped in favor of large scales, ruling out the so called standard CDM model and inviting compromises such as 'warm' dark matter in which cold and hot contributions combine to generate relatively more variance in the mass distribution on large scales and also small scale potentials becoming galaxies. Large redshift surveys such as the 2Df (Maddox, 1998) or the Sloan survey (Gunn \& et al, 1998) will provide a much improved statistical characterization of large scale structure. 


\subsection{Brief History of Lensing}

The discovery of giant arcs in clusters of galaxies (Soucail et al., 1987b, 1988; Fort et al., 1988; Lynds \& Petrosian, 1989), Einstein rings around galaxies (Hewitt et al., 1988), and the spectroscopic proof that they are produced by gravitational lensing effects (Soucail et al., 1988) have revealed that gravitational distortion can probe the mass distribution of clusters with remarkable detail. Weakly-lensed galaxies were routinely observed in the early 1990's and a theory formulated to relate the distortion to the mass responsible (Kaiser \& Squires, 1993). Of the techniques for measuring masses, the mass inferred from lensing is remarkably-robust, and may be applied over an impressive range of scales, from $\sim 10^{5} \mathrm{~km}<R<\sim 10 \mathrm{Mpc}$. Measuring the mass distribution with gravitational lensing can put important constraints on the history and nature of gravitational history.

Images taken with the first CCD's revealed arc-like features in three different massive clusters in 1976 but the results were not published in a refereed journal until 1989 (Lynds \& Petrosian, 1989). In the interim another group confirmed the discovery in two of the clusters and added that the features appeared to be correlated with the outer edges of the positions of the luminous galaxies in the cluster cores (Soucail et al., 1987a). However, they were thought to be too thin to be viable gravitationally-lensed and so were at first entertained as cluster phenomena. It was suggested that they might be extended regions of a cooling flow made luminous by their associations with intense shock-induced star formation (Begelman \& Blandford, 1987; Soucail et al., 1987a). Measuring a redshift for these arcs would be crucial for determining their origin, but this took time because the arcs were faint and initial attempts on small telescopes gave null results. After a year's worth of effort, high quality spectra taken at three different spatial positions along the arc in A370 using one of the largest telescopes at the time, the $3.6 \mathrm{~m}$ ESO telescope in Chile, showed it to be a single coherent galaxy at twice the distance of the cluster, making it the first confirmed image of lensed galaxy (Soucail et al., 1988; Paczynski, 1987).

It was then found with improvements in image quality that giant arcs were relativelycommon features in massive galaxy clusters and that their shape and position make them sensitive tracers of the underlying mass. The Einstein ring radius, or curve of maximum magnification, can be related to the mass if the geometry is known. Analytical mass models

usually assume circular symmetry and in some cases the addition of some substructure, and give reasonable answers despite this major assumption (Mellier et al., 1993; Kneib et al., 1993, 1995). Model-independent methods have since been explored for the case of weaklensing, and found to be useful in practice (Kaiser \& Squires, 1993; Broadhurst et al., 1995), as shown here in tis thesis.

Smaller, less-distorted versions of the giant arcs first started appearing in deep ground-based images in the late 1980's (Fort et al., 1988). These are examples of weaklylensed galaxies or arclets, in which lensing shears the images into positions and orientations which on average become correlated with the cluster mass. The theory describing the connection between the shear and the mass was first introduced by Tyson et al. (1990) 
but formalized by Kaiser \& Squires (1993) and later extended into the nonlinear regime by Kaiser (1995) and Seitz \& Schneider (1996). The shear field is measured off of the images, by integrating the tangential distortions of background galaxies along a circle in the weak limit, where the shear, a nonlocal quantity is approximately equal to the distortion, an observable local quantity. Then by combining the expressions for the deflection field, the $2 \mathrm{D}$ lensing potential and the magnification field the shear can be expressed as a function of the scaled surface mass density $\kappa$ and inverted to recover $\kappa$ (Kaiser \& Squires, 1993). Careful studies have shown the inversion method to be a highly robust technique for recovering masses, provided that a shear signal is detected (Squires \& Kaiser, 1996).

A characteristic of the shear is that it is invariant to isotropic magnification, so that a shear signal will not be detected if there is no associated change in ellipticity generated by a tidal field. A constant mass sheet or the component of a mass distribution which is a constant mass sheet will do this, making a mass measurement from the inversion method only a firm lower limit.

It has been suggested that this mass-sheet degeneracy can be broken by measuring the magnification (Broadhurst et al., 1995). They noted that the number of background galaxies will be modified by lensing as a result of magnification bias. Magnification imposes two opposing effects on the redshift distribution of background galaxies $N(z)$. The first is magnification, which boosts the brightness of objects above some fixed fluxed limit, increasing the number of objects. The second is field dilation, or an apparent radial stretching of space, which reduces the numbers of objects. The cumulative effect induces a modification in the redshift distribution. A comparison of the lensed to unlensed distributions then gives a measurement of the magnification. This combined with measurements of the distortions can then give unique model-independent measurement of the mass (Broadhurst et al., 1995).

\subsection{Thesis Motivation}

For this thesis a faint galaxy redshift survey was conducted at the Keck in the direction of three well known massive lensing clusters A1689, A2390, and A2218, with the aim of measuring cluster magnification through the modification of the background redshift distribution. The redshift survey is reasonably large, totalling $\sim 300$ weakly-lensed galaxies, and relatively deep, with a flux limit of $I=23$ in order ot obtain redshifts in the range $0.2<z<1.5$ and includes galaxies preselected by color to lie in the background of the cluster.

With high magnification, cluster lensing also provides a viable route to obtaining useful spectroscopic information on high- $z$ galaxies. Using a combination of photometric selection and morphology several good examples of galaxies at $z>4$ have been discovered

in the survey presented in this thesis. The spectra are all of high quality owing to the large cluster magnification, showing stellar continuum, a pronounced Ly- $\alpha$ break, in some cases a Ly- $\beta$ break, and numerous metal absorption lines, useful for exploring internal gas motion. 
The first routine discoveries of $z \sim 3$ galaxies, were found by photometric selection using the Lyman-limit as a discriminator, with follow-up spectroscopy of these faint objects obtained at the Keck. (Steidel et al., 1996a,b; Lowenthal et al., 1997). At higher redshift photometric selection is less reliable because $\mathrm{HI}$ opacity from the intergalactic medium depresses the continuum between Ly- $\alpha$ and the Ly-limit at the redshift of the source, making objects fainter and spectroscopy less practical. To date many galaxies are starting to be reported at $z>4$, (Trager et al., 1997; Franx et al., 1997; Frye \& Broadhurst, 1998; Dey et al., 1998a; Hu et al., 1998), and Chapter 6 of this thesis, many of which are serendipitous discoveries which have been boosted by lensing. For $z>4.5$ the intervening intergalactic HI opacity is known from QSO spectra to be large (Schneider et al., 1989; Storrie-Lombardi et al., 1996), producing a sharp continuum break at Lyman- $\alpha$ (Madau, 1995), and leading to discoveries of galaxies at $z>5$ for the very reddest objects in the Hubble Deep Field (Dey et al., 1998a; Dickinson, 1999; Lanzetta et al., 1996). We make the claim of a galaxy at $z=5.12$ behind the cluster A1689 in this thesis.

A special class of giant arcs around galaxy lenses which form complete 'Einstein rings' is also examined in this thesis. Einstein rings form from a chance alignment of observer, lens, and source for very symmetric mass distributions. Most rings are discovered in the radio. A handful of them have recently been observed in the $\mathrm{mm}$, and their molecular interstellar medium studied via molecular absorption in these galaxies up to $z=0.9$ (Wiklind \& Combes, 1998; Wiklind \& Combes, 1996).

Radio ring lenses are also good systems for measuring the expansion parameter $H_{0}$ through time delays. They are well-chosen systems for measuring time delays because the delays are short, on the order of a few weeks, and the systems are relatively simple to model compared to clusters, usually consisting of a doubly-lensed quasar core and a lensed jet which forms the ring. One such system is the radio ring lens PKS 1830-211, whose location at low latitude near a bright $M$ star have made it unattainable at other wavelengths requiring clever deconvolution (Courbin et al., 1998b).

Three groups have measured time delays towards this object (Wiklind \& Combes, 1998; Lovell et al., 1996; Van Ommen et al., 1995), but the results are of only limited application until the lens geometry and lens characteristics are known. The lens distance was discovered in the $\mathrm{mm}$ in an interesting paper by Wiklind \& Combes (1996). They conducted a blind search of frequency space for strong features, discovering an impressive 12 number of 12 different molecular species in total, all at $z=0.89$. Since then another massive system has been found in absorption in the HI line at $z=0.21$, now considered to be at large impact parameter and not to have an important lensing influence.

The molecular absorption towards PKS1830-211 has been spatially-resolved at the Berkeley-Illinois-Maryland Association Array (BIMA), revealing absorption towards one component, but incomplete absorption towards the other component, requiring the delay measurements to be combined with spatially-resolved imaging in order to distinguish between a flare event in the NW and SW components (Frye, Welch, \& Broadhurst 1997). 
In addition, deep infrared and optical imaging was obtained at the Keck telscopes, which combined with th deconvolution technique pioneered by Courbin et al. (1998b) has revealed the true positions of the lens and lensed images. In Chapter 3 the $\mathrm{mm}$ and optical data for this source will be presented.

The outline of this thesis is as follows. Chapter 2 will give a review of lensing theory, with an emphasis on weaking lensing and the measurement of cluster magnification. Chapter 3 will discuss observations of the radio ring lens PKS1830-211 and the efforts to measure $H_{0}$ through time delay. Chapter 4 will describe the data reduction and analysis, unique in that it is an all-purpose built code optimized for the treatment of multislit spectroscopy. Chapter 5 will present and analyze the redshift survey dataset, and use it to measure cluster magnification. Chapter 6 will present and discuss the galaxies found at high- $z, z>4$, and Chapter 7 will give the conclusions. The data all appear in the Appendices. Appendices $\mathrm{A}, \mathrm{B}$ and $\mathrm{C}$ show the spectra and corresponding images for A1689, A2390, and A2218, respectively. 


\section{Chapter 2}

\section{Lensing Theory}

This chapter is divided up into several sections but there are two primary aims. The first is to review the concepts which are relevant to the study of cluster lensing in the weak lensing regime. The second is to present the theory of how to relate the observables in weakly-lensed fields to important lens quantities such as the total projected cluster mass. A couple of reviews were helpful for this study of lensing theory. They are Narayan \& Bartelmann (1995) and Mellier (1999).

\subsection{Deflection Angle}

A photon moving with velocity $v$ in direction $\hat{y}$ feels an attractive force towards a mass $M$ a distance $R$ away from it. The presence of this mass will cause the photon to accelerate towards it, deflecting the light from $\hat{y}$ by an angle $\alpha$, defined geometrically (Fig. 2.1) as the ratio of the velocities in $\hat{x}$ and $\hat{y}$ :

$$
\alpha=\frac{v_{x}}{v_{y}}=\frac{\int a_{x} d t}{c}
$$

where $a_{x}$ is the rate of change of velocity in $\hat{y}$ and $v_{y}=c+\int a_{y} d t \approx c$ in the nonrelativistic case. We make the substitution $d t=d y / c$ :

$$
\hat{\alpha}=\frac{\int a_{x} d y}{c^{2}}
$$

From the General Relativistic treatment in the weak field limit, $a_{x}=-2 \phi_{, x}$, where $\phi_{, x}$ is the change in the potential in the $\hat{x}$ direction, $\phi_{, x}=\frac{\partial \phi}{\partial x}$. Making this substitution:

$$
\hat{\alpha}=\frac{-2 \int \phi_{, x} d y}{c^{2}}
$$

This expression for $\hat{\alpha}$ is general, depending only on the relevant expression for $\phi_{, x}$. For a point mass $\phi=G M / R$ and $R=\sqrt{x^{2}+y^{2}}$, and $\phi_{, x}$ can be determined by applying the chain 


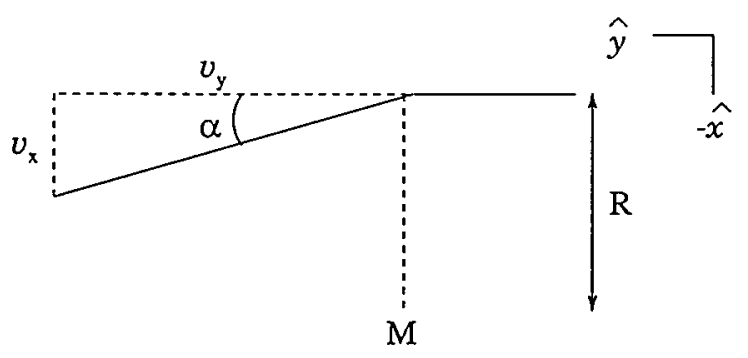

Figure 2.1: Lensing by a point mass. A photon moving with velocity $v$ in direction $\hat{y}$ feels an attractive force towards a mass $M$ a distance $R$ away from it.

rule: $\phi, x=\frac{\partial \phi}{\partial R} \frac{\partial R}{\partial x}=\left(-\frac{G M}{R^{2}}\right)\left(x^{2}+y^{2}\right)^{-1 / 2}=-\frac{G M x}{\left(x^{2}+y^{2}\right)^{3 / 2}}$. Substituting in this expression for $\phi_{x} \hat{\alpha}$ becomes:

$$
\hat{\alpha}=-\frac{2 G M}{c^{2}} \int \frac{d y}{\left(x^{2}+y^{2}\right)^{3 / 2}}
$$

Making the trigonometric substitutions $y=x \tan \theta, d y=x \sec ^{2} \theta d \theta$ and integrating:

$$
\begin{gathered}
\hat{\alpha}=-\frac{2 G M}{c^{2} x} \int \frac{d \theta}{\sec \theta}=-\left.\frac{2 G M}{c^{2} x} \sin \theta\right|_{-\pi / 2} ^{\pi / 2} \\
\hat{\alpha}=-\frac{4 G M}{c^{2} x}
\end{gathered}
$$

where $x$ is the distance of closest approach to $M$ and the minus sign means that the acceleration is downward, towards $M$. The deflection varies inversely with the impact parameter. This expression holds for all lenses which can be treated as point masses. Otherwise, an expression for the mass distribution must be substituted in for $M$.

\subsection{Mass Sheet}

Now consider a lens made up of a sheet of material of surface mass density $\Sigma$. The thickness of the sheet is small compared to the lens and source distances and so can be taken to be the image plane. Fig. 2.2 shows the setup in the image plane, where $\vec{\xi}$ is the $2 \mathrm{~d}$ positional vector to the point of intersection with the light rays and $\vec{\xi}^{\prime}$ is a $2 \mathrm{~d}$ positional 


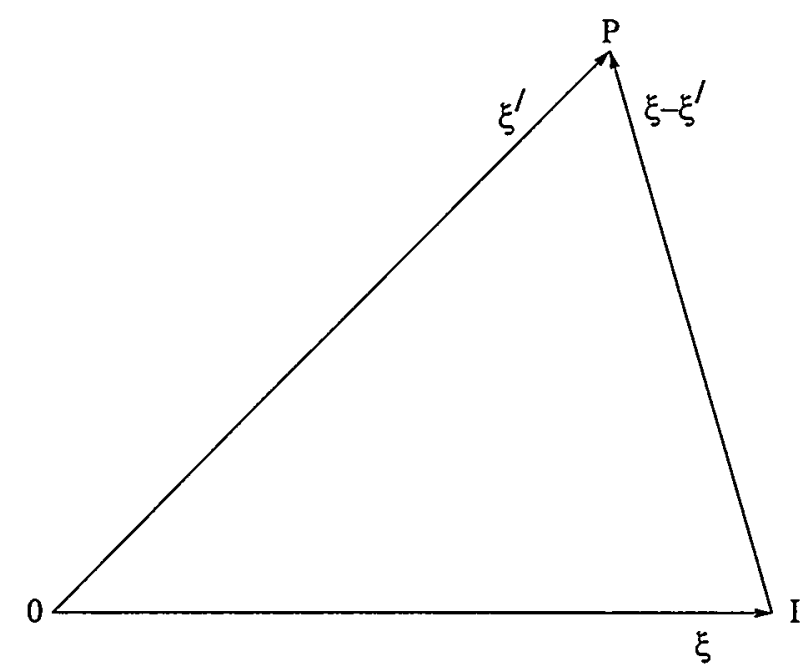

Figure 2.2: The image plane, where $\vec{\xi}$ is the $2 \mathrm{~d}$ positional vector to the point of intersection with the light rays $I$ and $\vec{\xi}^{\prime}$ is a $2 \mathrm{~d}$ positional vector to some arbitrary point $P$. The distance from the light rays to $P$ is $\left|\xi-\xi^{\prime}\right|$.

vector to some arbitrary point $P$. The distance from the light rays to $P$ is $\left|\xi-\xi^{\prime}\right|$. The surface mass density at point $P$ is the mass in the differential area $d \xi^{\prime 2}$, so that the mass distribution is: $M=\Sigma \times d \xi^{\prime 2}$. The total deflection at $\vec{\xi}$ is the sum of the deflections from all the other points in the plane. Substituting in the expressions for $M$ and $x$ into Eq. 2.3 and integrating over the whole plane $\overrightarrow{\hat{\alpha}}$ is:

$$
\overrightarrow{\hat{\alpha}}(\xi)=\frac{4 G}{c^{2}} \int \frac{\left(\vec{\xi}-\vec{\xi}^{\prime}\right) \Sigma}{\left|\vec{\xi}-\vec{\xi}^{\prime}\right|^{2}} d^{2} \xi^{\prime}
$$

For the case of a circularly-symmetric lens, the $2 \mathrm{~d}$ position vectors reduce to $1 \mathrm{~d}$ and the origin is moved to the center of mass of the lens. The mass can now be considered as if it were all located at a single point in the center and $\overrightarrow{\hat{\alpha}}$ becomes:

$$
\overrightarrow{\hat{\alpha}}(\xi)=\frac{4 G M(\xi)}{c^{2} \xi}
$$

where $M(\xi)$ is the mass enclosed within radius $\xi$. The expression in Eq. 2.3 is recovered for this circularly-symmetric case, as is expected via the application of Gauss' law to a spherical distribution.

\subsection{Lens Equation}

The requisite lensing diagram appears in Fig. 2.3. A light ray from source $S$ is deflected by an angle $\hat{\alpha}$ at point $\xi$ in the lens plane and arrives at $O$. The angles subtended 


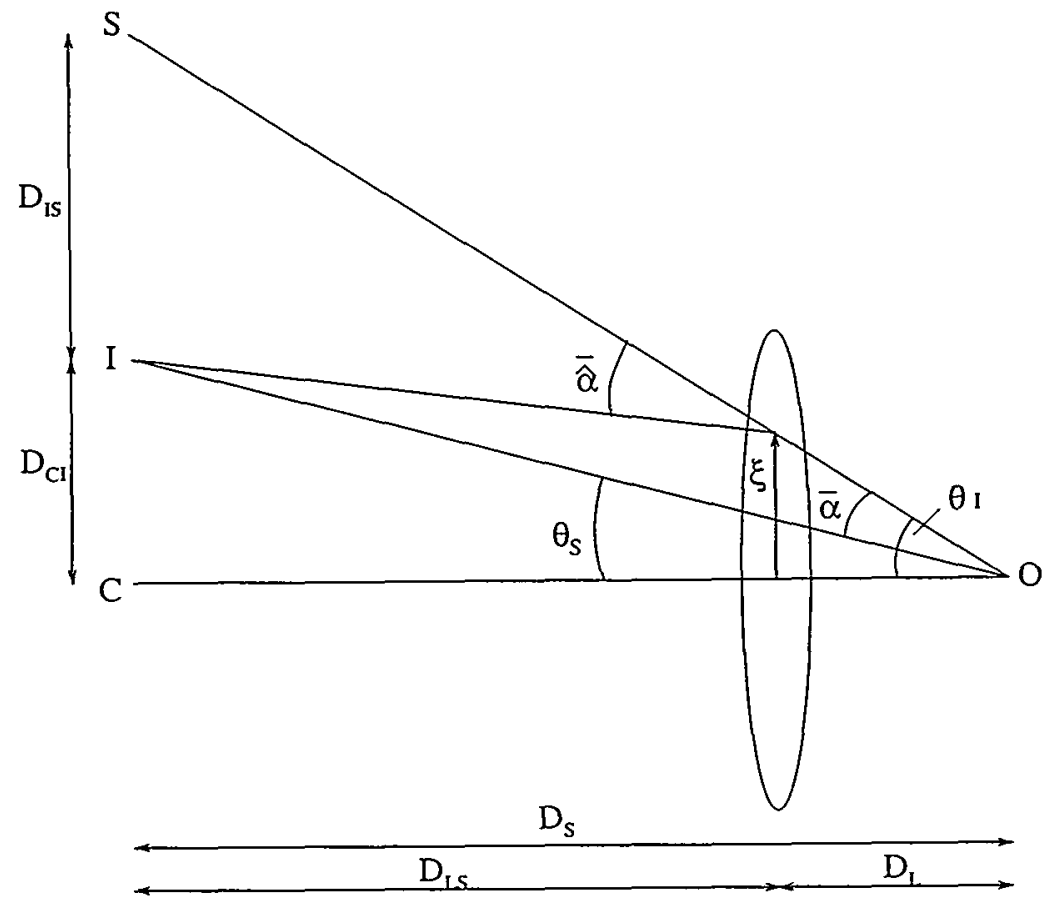

Figure 2.3: The lensing diagram. A light ray from source $\mathrm{S}$ is deflected by an angle $\hat{\alpha}$ at point $\xi$ in the lens plane and arrives at $O$. The angles subtended by the optic axis and the source and the optic axis and the image are $\theta_{S}$ and $\theta_{I}$ respectively. The angular difference $\theta_{S}-\theta_{I}$ is the reduced deflection angle $\vec{\alpha}$ 
by the optic axis and the source and the optic axis and the image are $\theta_{S}$ and $\theta_{I}$ respectively. The angular difference $\theta_{S}-\theta_{I}$ is the reduced deflection angle $\vec{\alpha}$ :

$$
\overrightarrow{\theta_{I}}=\overrightarrow{\theta_{S}}+\vec{\alpha}
$$

This is the lens equation. From the geometry we can relate the deflection angle $\overrightarrow{\hat{\alpha}}$ to the reduced deflection angle $\vec{\alpha}$. This requires doing some simple trigonometry. From Fig. 2.3 the source distance is $D_{S}$ and the lens-source distance is $D_{L S}$. The projected distance between the source and the image is $D_{I S}$ and between the lens center of mass and the image is $D_{C I}$. The distance between the lens center of mass and the source is $\eta=D_{C I}-D_{I S}$. Using the small angle formula (see Fig. 2.3) we can form the relations:

$$
\overrightarrow{\hat{\alpha}}=\frac{D_{I S}}{D_{L S}}, \quad \theta_{I}=\frac{D_{C I}}{D_{S}} \quad \theta_{S}=\frac{\eta}{D_{S}}
$$

Solving the lens equation for $\vec{\alpha}$ and substituting in these relations:

$$
\vec{\alpha}=\theta_{I}-\theta_{S}=\frac{D_{C I}}{D_{S}}-\frac{\eta}{D_{S}}=\frac{D_{I S}}{D_{S}}
$$

Combining now the equations for $\overrightarrow{\hat{\alpha}}$ and $\vec{\alpha}$ :

$$
D_{I S}=\vec{\alpha} D_{S}=\overrightarrow{\hat{\alpha}} D_{L S}
$$

Solving for $\vec{\alpha}$ :

$$
\vec{\alpha}=\frac{D_{L S}}{D_{S}} \overrightarrow{\hat{\alpha}}
$$

From Fig 2.3 and the small angle formula it is straightforward to convert the projected distance $\xi$ to angular distance $\theta: \xi=\theta_{I} D_{L}$. Substituting Eq. 2.5 into Eq. 2.7 and making this conversion $\vec{\alpha}$ becomes:

$$
\vec{\alpha}=\frac{D_{L S}}{D_{L} D_{S}} \frac{4 G}{c^{2} \theta_{I}} M
$$

Here $M=\Sigma \times$ Area $=\Sigma \pi \xi^{2}=\Sigma \pi \theta_{I}^{2} D_{L}^{2}$. Substituting this in:

$$
\vec{\alpha}\left(\theta_{I}\right)=\frac{D_{L} D_{L S}}{D_{S}} \frac{4 \pi G \Sigma}{c^{2}} \theta_{I}=\frac{\Sigma}{\Sigma_{c r} \theta_{I}}
$$

where the critical surface mass density $\Sigma_{c r}$ is defined as:

$$
\Sigma_{c r}=\frac{c^{2}}{4 \pi G} \frac{D_{S}}{D_{L} D_{L S}}=\frac{c^{2}}{4 \pi G} D
$$

and where $D=\frac{D_{L} D_{L S}}{D_{S}}$. The value of $\Sigma_{c r}$ marks a rough dividing line between the strong and weak lensing regimes. The giant arcs and multiple images form in the strong lensing regime, where $\Sigma$ is supercritical somewhere in the lens $\Sigma>\Sigma_{c r}$, but there are exceptions to this rule (Subramanian \& Cowling, 1986). 


\subsection{Einstein Ring Radius}

Substituting Eq. 2.8 into the lens equation yields an expression of $\theta_{S}$ in terms of $\theta_{I}:$

$$
\theta_{S}=\theta_{I}-\frac{D_{L S}}{D_{L} D_{S}} \frac{4 G M(\theta)}{c^{2} \theta_{I}}
$$

For a geometry where the source is coincident with the optic axis, $\theta_{S}=0$ and $\theta_{I}$ becomes:

$$
\theta_{E}=\left[\frac{4 G M\left(\theta_{E}\right)}{c^{2}} \frac{D_{L S}}{D_{L} D_{S}}\right]^{1 / 2}
$$

where $\vec{\alpha}=\theta_{I}=\theta_{E}$ and $\theta_{E}$ is the Einstein ring radius. It gives the angular radius of a circle into which a point source will be imaged if the lens is supercritical $\left(\Sigma>\Sigma_{c r}\right)$ and if there

is a colinear alignment of observer, lens, and source. These requirements are difficult to meet in practice since such a geometry is rare and sources usually have a finite extent, but there are some cases which come close such as PKS1830-211. It is a useful scale to know in any case, as it can tell us important properties of the lens. If there are sources with angular distances $\theta<\theta_{E}$ they will be highly magnified and likely to form multiple images, depending on the exact geometry and the value of $\Sigma$. Also, if multiple images do form then they will be typically separated by $2 \theta_{E}$.

We can write down some typical values for $\theta_{E}$. For a lens which can be considered as a point mass $M\left(\theta_{E}\right) \rightarrow M$ in Eq. 2.11. In this case the value of $\theta_{E}$ for a star of mass $M=1 M_{\odot}$ and $D \sim 10 \mathrm{kpc}$ is $\theta_{E}=0.9$ mas, and for lensing by a galaxy of mass $M \sim 10^{11} M_{\odot}$ and $\sim 1 \mathrm{Gpc}$ is $\theta_{E}=0.9^{\prime \prime}$. Note that $\theta_{E}$ for a galaxy is on the order of the typical value for the seeing at most ground-based observatories, requiring the high angular resolution provided by long baseline radio interferometers and telescopes at good sites to spatially-resolve the image structure. (Frye et al., 1997; Courbin et al., 1998b).

\subsection{Imaging with a Point Mass Lens}

Consider the lens equation for a lens which can be considered as a point mass, Eq. 2.10 , with $M(\theta) \rightarrow M$ :

$$
\theta_{S}=\theta_{I}-\frac{1}{\theta_{I}}\left(\frac{4 G M}{c^{2}} \frac{D_{L S}}{D_{L} D_{S}}\right)
$$

The term in parentheses is just $\theta_{E}$. Making this substitution we have:

$$
\theta_{S}=\theta_{I}-\frac{\theta_{E}^{2}}{\theta_{I}}
$$

Solving for $\theta_{I}$ via the quadratic equation:

$$
\theta_{ \pm}=\frac{1}{2}\left(\theta_{S} \pm \sqrt{\theta_{S}^{2}+4 \theta_{E}^{2}}\right)
$$


There are two solutions here, which tells us that a point mass will image a source twice. In this case the images will appear on opposite sides of the source with one image inside of $\theta_{E}$ and the other outside. The image separation will be $\sim 2 \theta_{E}$. As a source moves away from the optic axis, the image nearer to the source position will be seen to brighten up and approach the source position while the other image will become fainter, and move farther away until eventually the far image disappears and there is only one image left which matches the source in both position and brightness.

Lensing conserves surface brightness, so if an object is magnified then either changes in size or changes in brightness can be used to measure the magnification. The fact that surface brightness is conserved comes from the conservation of phase space density We can write down the magnification as:

$$
\mu=\frac{A_{I}}{A_{S}}
$$

where $A_{S}$ is the area of the source on the sky. Using the small angle formula, it has a projected length of $\theta_{S} D_{S}$ and a projected width of $d \theta_{S}$. Similarly, $A_{I}$ has a projected length of $\theta_{I} D_{S}$ and a projected with of $d \theta_{I}$. Substituting this in for the area:

$$
\mu=\frac{\theta_{I} d \theta_{I}}{\theta_{S} d \theta_{S}}
$$

Differentiating the lens equation for a point mass, Eq. 2.12, $\frac{d \theta_{S}}{d \theta_{ \pm}}=1+\left(\frac{\theta_{E}}{\theta_{ \pm}}\right)^{2}$, and substituting this expression in for $d \theta_{S}$ :

$$
\mu=\frac{\theta_{ \pm} d \theta_{ \pm}}{\theta_{S}\left(1+\left(\frac{\theta_{E}}{\theta_{ \pm}}\right)^{2}\right) d \theta_{ \pm}}
$$

where $\theta_{I} \rightarrow \theta_{ \pm}$refers to the two solutions for the images. Substituting in the lens equation (Eq. 2.12):

$$
\mu=\frac{\theta_{ \pm} d \theta_{ \pm}}{\left(\theta_{ \pm}-\frac{\theta_{E}^{2}}{\theta_{ \pm}}\right)\left(1+\left(\frac{\theta_{E}}{\theta_{ \pm}}\right)^{2}\right) d \theta_{ \pm}}
$$

Expanding the denominator and simplifying:

$$
\mu=\frac{\theta_{ \pm}}{\theta_{ \pm}-\frac{\theta_{E}^{4}}{\theta_{ \pm}^{3}}}=\left[1-\left(\frac{\theta_{E}}{\theta_{ \pm}}\right)^{4}\right]^{-1}
$$

Now substituting in $\theta_{ \pm}$(Eq. 2.13) and simplifying:

$$
\mu_{ \pm}=\frac{u^{2}+2}{2 u \sqrt{u^{2}+4}} \pm \frac{1}{2}
$$

where $u$ is the angular separation of the source from the point mass in units of the Einstein angle, $u=\theta_{S} \theta_{E}^{-1}$. This equation is useful for showing how sources map onto the image 
plane. For an Einstein ring, $\theta_{S}=\theta_{E}, u=1$, and $\mu_{ \pm} \approx \frac{2}{3} \pm \frac{1}{2},>0$ for $\mu$ for both solutions. Otherwise, for $\theta_{S} \neq \theta_{E}$ consider the second or 'negative' solution, $\theta_{-}$. We know from above that $\theta_{-}<\theta_{E}$. This allows for cases where $\mu_{-}<0$, corresponding to images which are parity-flipped with respect to the source. For a more detailed discussion of this topic see Section 5.4.2, where a model is presented for a parity-flip case involving four multiple images of a galaxy observed at $z=4.04$.

\subsection{Singular Isothermal Sphere}

Consider a spherically-symmetric mass $M$ to be made up of particles (stars) which behave like particles in an ideal gas. The particles have isotropic velocity distributions and a velocity dispersion $\sigma$. The gravitational acceleration $g$ is:

$$
g=\frac{G M(<R)}{R^{2}}=\frac{v_{c}^{2}}{R}
$$

Solving for $M(<R)$ :

$$
M(<R)=\frac{v_{c}^{2} R}{G}=\frac{2 \sigma^{2} R}{G}
$$

where $v_{c}^{2}=2 \sigma^{2}$ in blah equilibrium. This is the mass contained within radius $R$. Dividing through by the volume gives the density profile $\rho(R)$ :

$$
\rho(R)=\frac{3 v_{c}^{2}}{4 \pi G R^{2}}
$$

The mass density drops off as $1 / R^{2}$ and the projected mass density drops off as $\Sigma \sim 1 / \xi$, where $\xi$ is the projected radius. The gravitational potential $\phi$ is:

$$
\phi=\int_{0}^{R} \frac{G M}{R^{2}} d R
$$

Substituting in the expression for the enclosed mass, Eq. 2.16, and integrating:

$$
\phi=\int_{0}^{R}\left(\frac{G}{R^{2}}\right)\left(\frac{2 \sigma^{2} R}{G}\right) d R=2 \sigma^{2} \int_{0}^{R} \frac{d R}{R}=2 \sigma^{2} \ln R
$$

Recall that the deflection angle is given by (Eq. 2.2), $\hat{\alpha}=\frac{-2 \int \phi_{x} d y}{c^{2}}$ where by the chain rule $\phi_{x}=\frac{\partial \phi}{\partial R} \frac{\partial R}{\partial x}=\left(\frac{2 \sigma^{2}}{R}\right)\left(\frac{x}{\left(x^{2}+y^{2}\right)^{-1 / 2}}\right)=\frac{2 \sigma^{2} x}{x^{2}+y^{2}}$. Substituting this into Eq. 2.2:

$$
\hat{\alpha}=\frac{2}{c^{2}} \int \frac{2 \sigma^{2} x}{x^{2}+y^{2}} d y=\frac{4 \sigma^{2} x}{c^{2}} \int \frac{d y}{x^{2}+y^{2}}
$$

Making the trigonometric substitutions $y=x \tan \theta, d y=x \sec ^{2} \theta d \theta$ this becomes:

$$
\hat{\alpha}=\frac{4 \sigma^{2} x}{c^{2}} \int \frac{x \sec ^{2} \theta d \theta}{x^{2}+x^{2} \tan ^{2} \theta}=\frac{4 \sigma^{2}}{c^{2}} \int \frac{\sec ^{2} \theta d \theta}{1+\tan ^{2} \theta}=\frac{4 \sigma^{2}}{c^{2}} \int d \theta
$$


Integrating over the limits with $y$ going from $-\infty$ to $\infty$, or equivalently, $\theta$ going from $-\pi / 2$ to $\pi / 2$ we can solve for $\hat{\alpha}$ :

$$
\hat{\alpha}=\left.\frac{4 \sigma^{2}}{c^{2}} \theta\right|_{-\pi / 2} ^{\pi / 2}=\frac{4 \pi \sigma^{2}}{c^{2}}
$$

This result says that the deflection is a constant for the isothermal lens and so $\hat{\alpha}=\theta_{E}$, and all deflections are of magnitude $\theta_{E}$. To put in some numbers here for a typical galaxy which will have $\sigma=220 \mathrm{kms}^{-1}, \alpha=1.4^{\prime \prime}$. The isothermal lens is circularly-symmetric, reducing the lens equation to 1d. The origin shifts to the lens center and the images and the source all become colinear. Multiple images form only when a source lies inside of the Einstein ring radius, $\theta_{S}<\theta_{E}$. When this condition is satisfied the lens equation has two solutions:

$$
\theta_{ \pm}=\theta_{S} \pm \theta_{E}
$$

There is also a third image with 0 flux, which can become nonzero for a nonsingular core (Narayan, 1996?). The magnification produced by the isothermal sphere follows from the expression for $\mu$ in Eq. 2.14, where $\theta_{I}$ and is given by the lens equation Eq. 2.18, and $d \theta_{I}$ is found by differentiating it, $d \theta_{ \pm}=d \theta_{S}$. Making these substitutions we have:

$$
\mu=\frac{\theta_{ \pm} d \theta_{ \pm}}{\theta_{S} d \theta_{S}}=\frac{\theta_{ \pm}}{\theta_{S}}=1 \pm \frac{\theta_{E}}{\theta_{S}}=\left(1 \mp \frac{\theta_{E}}{\theta_{ \pm}}\right)^{-1}
$$

In the general case we consider lensing by a mass sheet with an arbitrary surface mass density distribution, so that $\Sigma \rightarrow \Sigma\left(\vec{\xi}^{\prime}\right)$ and $\xi^{\prime}$ is the 2 d position vector in the image plane to an arbitrary point $P$. The deflection angle is given by Eq. 2.4, and substituting in Eq. 2.7 to relate to the reduced deflection angle we have:

$$
d \vec{\alpha}(\xi)=\frac{4 G D_{L S}}{c^{2} D_{S}} \int \frac{\left(\vec{\xi}-\vec{\xi}^{\prime}\right) \Sigma\left(\vec{\xi}^{\prime}\right)}{\left|\vec{\xi}-\overrightarrow{\xi^{\prime}}\right|^{2}} d^{2} \xi^{\prime}
$$

We can convert the $2 \mathrm{~d}$ positional vectors $\xi$ and $\xi^{\prime}$ into the more standard $2 \mathrm{~d}$ angular vectors, $\theta$ and $\theta^{\prime}$ using the small angle formulas $\vec{\xi}=\vec{\theta} D_{L}$ and $d \xi^{\prime}=D_{L} d \theta^{\prime}$. Making this substitution gives:

$$
d \vec{\alpha}(\theta)=\frac{4 G D_{L S} D_{L}}{c^{2} D_{S}} \int \frac{\left(\vec{\theta}-\vec{\theta}^{\prime}\right) \Sigma\left(\vec{\theta}^{\prime}\right)}{\left|\vec{\theta}-\vec{\theta}^{\prime}\right|^{2}} d^{2} \theta^{\prime}
$$

Substituting in the expression for $\Sigma_{c r}$ from Eq. 2.9:

$$
d \vec{\alpha}(\theta)=\frac{1}{\pi} \int \frac{\left(\vec{\theta}-\vec{\theta}^{\prime}\right) \kappa}{\left|\vec{\theta}-\vec{\theta}^{\prime}\right|^{2}} d^{2} \theta^{\prime}
$$

where $\kappa=\frac{\Sigma}{\Sigma_{c r}}$ and is referred to as the convergence. 


\subsection{The Lensing Potential}

We know that the deflection at each point $\theta$ depends on the sum of the deflections of all the other points in the plane and so is non-local. This can make computing the deflection field by brute force a real chore. A more agreeable approach is to see if the potential, which depends on $\alpha$ (Eq. 2.2), satisfies Poisson's equation. If this is true then we can write down the solution directly. We now introduce the lensing potential $\psi$, which is the $2 \mathrm{~d}$ potential generated by the surface density $\Sigma(\theta)$ and is just the scaled, projected version of $\phi$ :

$$
\psi(\vec{\theta})=\frac{D_{L S}}{D_{L} D_{S}} \frac{2}{c^{2}} \int \phi\left(D_{L} \vec{\theta}, z\right) d z
$$

where $\phi$ is integrated over thickness $z$. By taking the first derivative of $\psi$ with respect to $\theta$, and substituting in the expression for the deflection angle we can write:

$$
\vec{\nabla}_{\theta} \psi(\vec{\theta})=D_{L} \vec{\nabla}_{\xi} \psi=\frac{D_{L S}}{D_{S}}\left(\frac{2}{c^{2}} \int \nabla_{\xi} \phi d z\right)
$$

where from the small angle formula $\vec{\xi}=D_{L} \vec{\theta}$ and $\nabla_{\theta}=\frac{\partial}{\partial\left(\vec{\xi} / D_{L}\right)}=D_{L} \frac{\partial}{\partial(\bar{\xi})}=D_{L} \nabla_{\xi}$. The term in parentheses is just $\overrightarrow{\hat{\alpha}}$, Eq. 2.2. Putting this in gives:

$$
\vec{\nabla}_{\theta} \psi(\vec{\theta})=\frac{D_{L S}}{D_{S}} \overrightarrow{\hat{\alpha}}=\vec{\alpha}
$$

where Eq. 2.7 was used to convert to the reduced deflection angle $\vec{\alpha}$. The result is that the first derivative of the potential yields the deflection angle, consistent with Eq. 2.2. The second derivative of $\psi$ is even more important for our purposes. It is important to note here that the gradient of the potential is what determines the bend angle, and hence the magnification, and not just the total mass. So a cluster with twice the mass does not necessarily give twice the magnification. Now we move on to take the second derivative of $\psi$. We know from the discussion above that $\vec{\nabla}_{\theta}=D_{L} \vec{\nabla}_{\xi}$. Differentiating again gives: $\vec{\nabla}_{\theta}^{2}=D_{L}^{2} \vec{\nabla}_{\xi}^{2}$. If we now take the second derivative of $\psi$, Eq. 2.21 , and substitute in the expression for $\vec{\nabla}_{\theta}^{2}$ we obtain:

$$
\nabla_{\theta}^{2} \psi=D_{L}^{2} \nabla_{\xi}^{2} \psi=\frac{2}{c^{2}} \frac{D_{L} D_{L S}}{D_{S}} \int \nabla_{\xi}^{2} \phi d z=\frac{2}{c^{2}} \frac{D_{L} D_{L S}}{D_{S}} \times 4 \pi G \Sigma
$$

where we solved the integral by making use of Gauss' theorem. Substituting in the expression for $\Sigma_{c r}$, Eq. 2.9, simplifies this expression even further:

$$
\nabla_{\theta}^{2} \psi=2 \frac{\Sigma(\vec{\theta})}{\Sigma_{c r}}=2 \kappa(\vec{\theta})
$$

This is the two-dimensional form of Poisson's equation. We have shown that $\psi$ satisfies Poisson's equation and in this case the Green function gives us the solution to $\psi$ directly: 


$$
\psi(\vec{\theta})=\frac{1}{\pi} \int \kappa\left(\vec{\theta}^{\prime}\right) \ln \left|\vec{\theta}-\vec{\theta}^{\prime}\right| d^{2} \theta^{\prime}
$$

We can make the local mapping from the source to the image plane by taking the Jacobian $A$, which is related to the magnification:

$$
A=\frac{\partial \vec{\theta}_{S}}{\partial \vec{\theta}_{I}}=\left(\delta_{i j}-\frac{\partial^{2} \psi(\theta)}{\partial \theta_{i} \partial \theta_{j}}\right)=M^{-1}
$$

where $\delta \theta_{i}$ and $\delta \theta_{j}$ are the $\mathrm{x}$ and $\mathrm{y}$ components of $\theta_{I}$ and $M$ is the magnification tensor. Written in matrix form:

$$
A=\left[\begin{array}{cc}
1-\frac{\partial^{2} \psi}{\partial x^{2}} & \frac{\partial^{2} \psi}{\partial x \partial y} \\
-\frac{\partial^{2} \psi}{\partial x \partial y} & 1-\frac{\partial^{2} \psi}{\partial y^{2}}
\end{array}\right]
$$

Substituting in Poisson's equation, $\nabla^{2} \psi=2 \kappa, \kappa=\frac{1}{2}\left(\frac{\partial^{2} \psi}{\partial x^{2}}+\frac{\partial^{2} \psi}{\partial y^{2}}\right)$ :

$$
A_{i j}=\left[\begin{array}{cc}
1-2 \kappa+\frac{\partial^{2} \psi}{\partial y^{2}} & -\frac{\partial^{2} \psi}{\partial x \partial y} \\
\frac{\partial^{2} \psi}{\partial x \partial y} & 1-2 \kappa-\frac{\partial^{2} \psi}{\partial y^{2}}
\end{array}\right]
$$

Next come some simplifying definitions. The shear components are given by second derivatives of $\psi$ :

$$
\begin{gathered}
\gamma_{1}=\frac{1}{2}\left(\frac{\partial^{2} \psi}{\partial x^{2}}-\frac{\partial^{2} \psi}{\partial y^{2}}\right) \text { and } \gamma_{2}=\frac{\partial^{2} \psi}{\partial x \partial y} \text { and its magnitude is } \gamma=\gamma_{1}^{2}+\gamma_{2}^{2} \text {. Substituting: } \\
A_{i j}=\left[\begin{array}{cc}
1-\kappa-\gamma_{1} & \gamma_{2} \\
\gamma_{2} & 1-\kappa+\gamma_{1}
\end{array}\right]
\end{gathered}
$$

Diagonalizing the matrix, or equivalently rotating into the direction of the shear, so that the eigenvalues give the stretch factors of major and minor axes:

$$
A_{i j}=\left[\begin{array}{cc}
1-\kappa-\gamma & 0 \\
0 & 1-\kappa+\gamma
\end{array}\right]
$$

and the stretch factors $a$ and $b$ are:

$$
a=\frac{1}{1-\kappa-\gamma} \quad b=\frac{1}{1-\kappa+\gamma}
$$

Recall that the magnification is related to the inverse of the determinant of $A_{i j}$ :

$$
\mu=a \times b=\frac{1}{\operatorname{det} A_{i j}}=\frac{1}{(1-\kappa)^{2}-\gamma^{2}}
$$

The distortion $\delta$ is equal to the ratio of the major and minor axes:

$$
\delta=\frac{a}{b}=\frac{1-\kappa+\gamma}{1-\kappa-\gamma}
$$


The locus of points in the image plane for which $\operatorname{det} A_{i j}=0$ is called the critical curve and corresponds to infinite magnification. The analogous set of points in the source plane is the caustic. We can break up $A_{i j}$ into separate matrix terms:

$$
A_{i j}=(1-\kappa)\left(\begin{array}{ll}
1 & 0 \\
0 & 1
\end{array}\right)-\gamma\left(\begin{array}{cc}
\cos 2 \phi & \sin 2 \phi \\
\sin 2 \phi & -\cos 2 \phi
\end{array}\right)
$$

where $\tan \phi=\frac{\gamma_{1}}{\gamma_{2}}$. This equation gives us a physical intuition into the process of imaging. The first term, the convergence, produces only an isotropic deformation. We know that the contribution from this term is local. The second term, the shear, is external to the beam (nonlocal), and is what introduces the anisotropy. This tidal contribution, deforms the source by magnitude $\gamma$ into orientation $\phi$.

In the external regions, where the weak lensing regime applies, $\operatorname{det}(A)>0$, and the ellipticity of the source, $\epsilon_{S}$ can be related to the ellipticity of the image $\epsilon_{I}$ :

$$
\epsilon_{I}=\frac{1+b / a}{1-b / a} e^{2 i \phi}=\frac{\epsilon_{S}+\mathrm{g}}{1-\mathrm{g} \epsilon_{\mathrm{S}}}
$$

where $\mathrm{g}$ is the reduced complex shear, $\mathrm{g}=\frac{\gamma}{1-\kappa}$.

\subsection{The Distortion Field}

Deep images of galaxy clusters reveal hundreds of faint, weakly-lensed galaxies or arclets. The galaxies are assumed to be randomly placed and oriented behind the clusters but are sheared by weak lensing into new positions and orientations which on average become correlated with the cluster mass. This section reviews the method for recovering the total mass given this field of distorted galaxies.

The theory describing the connection between the shear and the mass was first introduced by Tyson et al. (1990) but formalized by Kaiser \& Squires (1993) and later extended into the nonlinear regime by Kaiser (1995), and Seitz \& Schneider (1996). The technique is based on the fact that both the surface mass density $\kappa$ and the shear $\gamma(\vec{\theta})$ are linear combinations of second derivatives of the lensing potential and so can be related to each other. By combining Eq 2.20, 2.21, and 2.23 they can write down the following relation:

$$
\gamma(\vec{\theta})=\frac{1}{\pi} \int D\left(\vec{\theta}-\vec{\theta}^{\prime}\right) \kappa\left(\vec{\theta}^{\prime}\right) d^{2} \theta^{\prime}
$$

where

$$
D\left(\vec{\theta}-\vec{\theta}^{\prime}\right)=\frac{\left(\theta_{2}-\theta_{2}^{\prime}\right)^{2}-\left(\theta_{1}-\theta_{1}^{\prime}\right)^{2}-2 i\left(\theta_{1}-\theta_{1}^{\prime}\right)\left(\theta_{2}-\theta_{2}^{\prime}\right)}{\left|\vec{\theta}-\vec{\theta}^{\prime}\right|^{4}}
$$

and the subscripts 1 and 2 refer to the different components of the $2 \mathrm{~d}$ angular position vector $\vec{\theta}$. This equation can be inverted to give an expression for $\kappa$ : 


$$
\kappa(\vec{\theta})=\frac{1}{\pi} \int \operatorname{Real}\left[D^{*}\left(\vec{\theta}-\vec{\theta}^{\prime}\right) \gamma\left(\vec{\theta}^{\prime}\right)\right] d^{2} \theta^{\prime}+\kappa_{0}
$$

where the real part has been taken. The integrative constant $\kappa_{0}$ which comes out of the inversion naturally is the only unknown.

The shear is not a measureable quantity but it gives rise to an observable called the distortion $\delta$. The two are related as $\gamma=\frac{\delta}{1-\kappa}$, where the distortion is given by the eigenvalues of the magnification $A_{i j}$ (Eq. 2.27). This theory is restricted to the weak limit, where $\kappa$ is small, so that $\delta \approx \gamma$ and measurements of the distortion give fair estimates of measurements of the shear. The experimental method is relatively straightforward. Tangential distortions of the arclets are simply summed up over a circle at some radius $R$ which is subject to weak lensing and over some annulus which is small compared to the size of the cluster. In the linear regime where $\kappa \ll 1,<\epsilon>\approx|\gamma|$, and the projected mass density can be recovered directly from the measurements of the ellipticities.

There are a few caveats worth mentioning. The first is that the galaxies are not all perfectly round. Fortunately, they are also not terribly complex or amorphous or the method would be made impractical. Instead, they have different intrinsic shapes which work against getting a true distortion measurement and so act as a source of noise. This noise may strongly affect the distortion measurement for a given lensed galaxy, but the measurements taken on average should give a fair estimate so long as there are a sufficiently large number of ellipticities included the average. The second caveat is the finite pixel size of the detector. Ideally, it should be much less than the galaxy size. Otherwise the image will risk being 'squared off' to some extent, depending on the ratio of the sizes. In the extreme case where the pixel size is equal to the object size the arclet will appear as an actual square. Typically the pixels are smaller than the object size but not extremely so, resulting in some amount of an unavoidable rounding off error.

The third source of noise is atmospheric. Flux from the weakly-lensed galaxies gets convolved with the atmospheric seeing, modifying the shapes. The effect depends on the size of the arclet relative to the size of the seeing disk. If the arclet is at or less than the size of the seeing disk, its shape will get smeared out by the larger, circularly-symmetric profile of the seeing, reducing the shear signal. The problem is obviously improved by space-based observing, but the Wide Field Planetary Camera (WFPC2) currently on board the HST does not have a wide enough field of view to be practical for weak lensing studies. The Advanced Camera for Surveys with its $5 x$ improvement in the size of the field of view is ideal for this kind of work and is scheduled to be replace WFPC2 in the year 2001.

By working only with the shear the inversion method is insensitive to isotropic changes in image area generated by a mass-sheet lens. This ambiguity or 'mass-sheet' degeneracy shows up in Eq. 2.29 as the constant of integration $\kappa_{0}$, and means the mass measurements obtained from this method can only be firm lower limits. In the next section it will be shown how the mass-sheet degeneracy can be broken by measuring the magnification. 


\subsection{The Magnification}

Broadhurst et al. (1995) found a unique relation between $\kappa, \delta$, and $\mu$. Combining Eq. 2.26 and 2.27 to eliminate $\gamma$, and solving for $\kappa$ :

$$
\kappa=1 \pm \frac{1 \pm \delta}{2 \sqrt{\mu \delta}}
$$

Once both are known the mass-sheet degeneracy can be broken. The distortion $\delta$ can be measured easily and is just related to the image ellipticities. The measurement of the other observable $\mu$ is the topic of this section. The number density distribution $N(z)$ of background galaxies becomes skewed by lensing, so by comparing $N(z)$ between lensed and unlensed fields $\mu$ can be obtained. However, only the modulus of $\mu$ and $\delta$ can be measured for most arclets, leading to four possible solutions for $\kappa$. This 4-fold redundancy can be broken with a knowledge of the image parity, but in general this is not an observable quantity for arclets. There are two critical curves in the problem. The exterior one is the tangential critical curve, which was discussed above. Analogous to this, the other one is the locus of points over which the radial magnification is infinite and is called the radial critical curve. An image experiences a parity flip each time it crosses a critical curve. There is a locus of points inbetween the two critical curves for which $\kappa=1, \Sigma=\Sigma_{\text {crit }}$, and the images are isotropically-magnified.

The image mapping as follows. The first image lies outside of both critical curves. The second one appears just inside the tangential critical curve. The third image lies somewhere inbetween the $\kappa=1$ and the radial critical curve. Since both the second and third images cross only one critical curve, the outer tangential one, and so experience one tangential parity flip. The fourth image which lies somewhere interior to both critical curves, and so experiences two parity flips, one tangential and one radial. There is a quadruplylensed galaxy in this survey for which the parity has been detected and modelled (See Fig. $6.10)$.

The magnification is measured by the modification of $N(z)$ due to lensing. We start by considering the number of galaxies per unit volume in a luminosity range $L$ and $L+d L$, described analytically by the Schechter luminosity function $\phi(L)$ (Schechter, 1976):

$$
\phi(L) d L=\phi^{*}\left(L / L_{*}\right)^{-\alpha} e^{-L / L *} d L / L^{*}
$$

where $\phi^{*}$ is the number per unit volume, $L_{*}$ is the characteristic luminosity marking roughly the turnover, and the dimensionless parameter $\alpha$ gives the slope on the low luminosity 'flat' part of $\phi(L)$.

The total number of galaxies at a given $z$ is found by integrating $\phi(L)$ over $L>$ $L_{\text {lim }}$. For a sample with an upper luminosity limit of $L_{l i m}(z)$ at $z=0$, by increasing $z$ $L_{\text {lim }}(z)$ is pushed downward along $\phi(L)$ towards higher limiting luminosities (see Fig. 2.4). For the highest redshifts, where $L_{l i m}(z)>L_{*}$, the liimit extends into the exponential cutoff, and only the few, brightests objects are left. At low $z N(z)$ rises along with the total volume, 


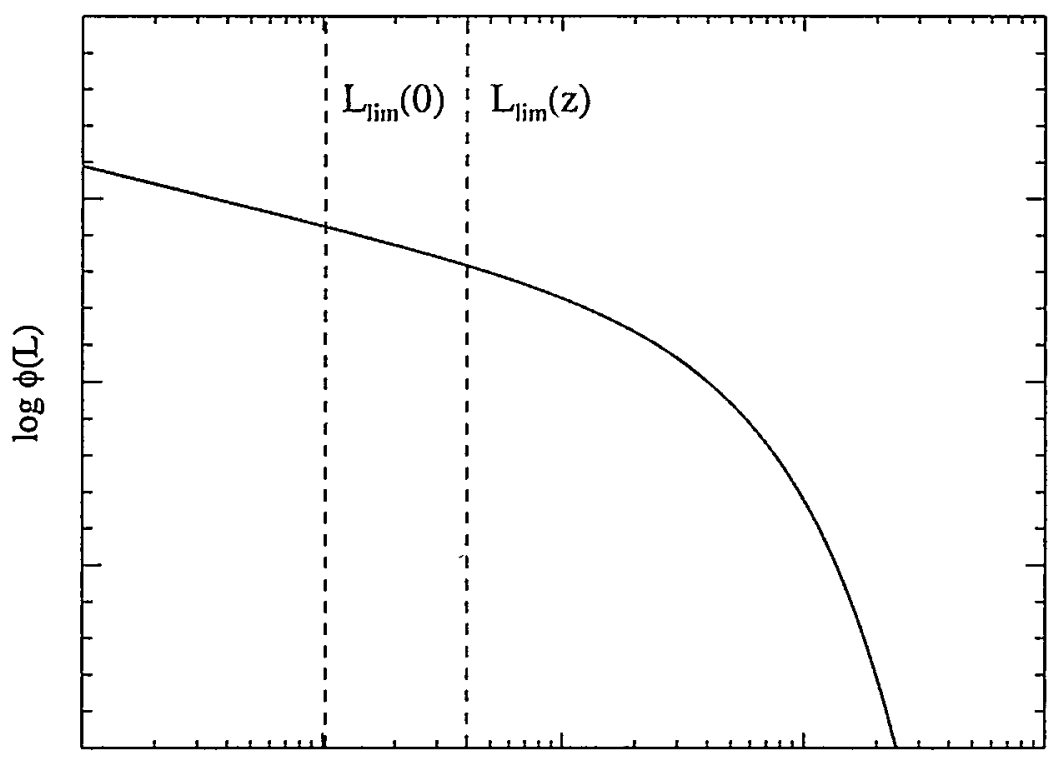

$\log \mathrm{L}$

Figure 2.4: The galaxy luminosity function $\phi(L)$. The $\log$ number of galaxies per unit volume and in luminosity range $L$ and $L+d L$ is plotted against $\log$ Luminosity

reaching some maximum value and then turning over at the $z$ where $L_{l i m}(z)$ reaches $L_{*}$ and dropping off when $L_{l i m}(z)$ is pushed down to the high luminosity exponential tail of $\phi(L)$. $N(z)$ can be written down analytically as:

$$
N(z)=d V(z) \int_{L_{l i m(z)}}^{\infty} \phi(L) d L
$$

where $d V(z)$ is the volume at $z$ and $L_{\text {lim }}(z)$ is the flux limit at $z$. Lensing modifies $N(z)$ in two opposing ways which are collectively known as magnification bias. First of all it boosts the brightnesses of all the objects, allowing one to see further down the luminosity function by a factor of $\mu$, thus increasing the numbers at all $z$ 's. This especially prominent for the highest $z$ 's where the 'boost' takes place on the high-luminosity, exponential part of the luminosity function, increasing the numbers exponentially. Secondly, lensing dilates space with respect to a fixed field of view, apparently pushing objects outward. This causes a reduction in area by a factor of $\mu$ and an associated reduction in number density. This effect is independent of $z$ and opposes the first one but is overshadowed at high- $z$ by the exponential increase in the numbers by luminosity boosting. Thus luminosity boosting dominates at high- $z$ and the field dilation effect at low- $z$, yielding a change in shape for $N(z)$. Taking the two effects of magnification bias into account, the lensed number density distribution $N^{\prime}(z)$ can be written down as: 


$$
N^{\prime}(z)=\frac{d V(z)}{\mu} \int_{L_{l i m}(z) / \mu}^{\infty} \phi(L) d L
$$

where $L$ is not increased by a factor of $\mu$ inside the integrand because it is taken into account in the limits of integration. The ratio of $N^{\prime}(z)$ to $N(z)$ gives the effect of lensing on the redshift distribution:

$$
N^{\prime}(z) d z=\mu^{\beta(z)-1} N(z) d z
$$

where to simplify the analysis $L_{\text {lim }}(z)$ has been approximated by a power law with slope $\beta$ equal to the slope of the luminosity function at $L_{\text {lim }}(z)$ :

$$
\beta=\frac{d \ln \phi(L(z), z)}{d \ln L(z)}
$$

A null result is obtained for $\beta=0.4$, for which the increase in the number of magnified sources is exactly compensated for by the decrease in the number from the reduction of field area. The main limitations of this method are shot noise and galaxy clustering. The shot noise can be brought down by observing more arclets. The galaxy clustering is an additional source of noise, but so far no bonified clusters have been found behind these lenses yet, although a number of galaxy groups have been discovered (See Chapter 6). These groups do not have a significant effect on $N(z)$ at least for the purposes of this survey. However, should they need to be excluded with both $z$ and magnitude information they can be identified.

\subsection{Conclusions}

We summarize the lensing theory results below.

- The deflection (bend angle) varies inversely with impact parameter for any mass distribution which can be considered as a point mass and for the case of a circularlysymmetric mass sheet.

- Solving for the deflection geometrically introduces a new variable $\Sigma_{c r}=\frac{c^{2}}{4 \pi G} \frac{D_{S}}{D_{L} D_{L S}}$, defined in terms of the ratios of the lens and source distances. It is a measure of the mass needed to have an important lensing effect. For a massive lensing cluster a typical value is $\Sigma_{c r}=0.35 \mathrm{gcm}^{-2}$.

- The Einstein ring radius is the radius of maximum magnification, $\theta_{E}=\left[\frac{4 G M\left(\theta_{E}\right)}{c^{2}} \frac{D_{L S}}{D_{L} D_{S}}\right]^{1 / 2}$, tending to infinity for a point source behind a supercritical lens $\left(\Sigma>\Sigma_{c r}\right)$.

- Sources for which $\theta<\theta_{E}$ will be highly-magnified and likely to form multiple images, depending on the exact geometry and surface mass density. Also, multiple images will be separated by roughly $2 \theta_{E}$ for typical arrangements of source positions. 
- For a singular isothermal sphere the deflection is a constant and is equal to the Einstein ring radius: $\hat{\alpha}=\frac{4 \pi \sigma^{2}}{c^{2}}$. For a typical galaxy cluster $\hat{\alpha}=25^{\prime \prime}$, compared to $\hat{\alpha}=1.4^{\prime \prime}$ for a galaxy lens.

- A source images twice for an isothermal lens, with the magnification going inversely with the impact parameter, $\mu=\left(1 \mp \frac{\theta_{E}}{\theta_{ \pm}}\right)^{-1}$, tending to one (no lensing) at infinity and going singular at the center. Our survey data are shown to be more consistent with a model flatter than isothermal (see Chapter 5, esp. Fig. 5.23).

- The lensing potential depends on the nonlocal deflection field, but satisfies Poisson's equation, giving the solution directly. The stretch factors for the major and minor axes are $a=\frac{1}{1-\kappa-\gamma} \quad b=\frac{1}{1-\kappa+\gamma}$ and the magnification, $\mu=\frac{1}{\operatorname{det} A_{i j}}=\frac{1}{(1-\kappa)^{2}-\gamma^{2}}$, where $\gamma$ is a component of the shear and $\kappa$ is the image convergence, $\kappa=\frac{\Sigma}{\Sigma_{c r}}$. The convergence produces only an isotropic magnification while the shear introduces the anisotropy.

- Measuring the mass from image distortions provides only lower limits to the mass, as it is insensitive to lensing by constant mass-sheets.

- Measuring the magnification breaks the mass-sheet degeneracy. The observables are related uniquely to the mass as: $\kappa=1 \pm \frac{1 \pm \delta}{2 \sqrt{\mu \delta}}$. There is a 4-fold degeneracy in the imaging which can be broken with a knowledge of the image parity.

- The magnification is measured by the modification of the redshift distribution of background objects, by the effect of magnification bias. The main limitations of the method are shot noise and clustering. The shot noise can be improved by measuring more redshifts and the clustering can be taken into account with a knowledge of the redshifts. 


\section{Chapter 3}

\section{The Radio Ring Lens PKS 1830-211}

\subsection{Introduction}

The bright radio source PKS 1830-211 (Jauncey et al., 1991) is a well-studied example of a galaxy lens in which the background source, a radio-loud quasar, is nearly on-axis with the lens, producing a large magnification. The jet component of the quasar images into $\sim 1^{\prime \prime}$ diameter ring on which two hot spots fall, corresponding to the doublylensed compact radio core (Kochanek \& Narayan (1992), and Fig. 3.1). The time delay is short, 44 days as measured by Van Ommen et al. (1995), and $26_{-5}^{+4}$ by Lovell et al. (1996), and the lens geometry relatively-clean (e.g., Subrahmanyan et al., 1990), making it a good candidate for measuring $H_{0}$.

The lens, a gas rich galaxy at $z=0.89$, was discovered in the millimeter via molecular absorption (Wiklind \& Combes, 1996). Wiklind \& Combes scanned frequency space along the sight-line towards PKS 1830-211. They discovered a total of 12 molecular lines in absorption belonging to 5 different species, and all at a redshift $z=0.88582$. They found that many low-order molecular transitions had very similar absorption depths, reaching down $36 \%$ of the continuum. This is unexpected given their different $A_{\nu}$ values and differing molecular abundances. They also detected the relatively rare ${ }^{13} \mathrm{C}$ isotope in $\mathrm{H}^{13} \mathrm{CO}^{+}(2-1)$, indicating that, if the abundances are similar to those of the Milky Way, the corresponding $\mathrm{H}^{12} \mathrm{CO}^{+}(2-1)$ line is fully saturated. From their single antenna data they concluded that the absorption is confined to the SW component, so that it appears diluted at low resolution.

Spatially-resolved observations in the mm show that the absorption is seen towards only one of the two flat spectrum hot spots, as expected (Wiklind \& Combes, 1996) but that it does not reach the base of the continuum (Frye, Welch, \& Broadhurst, 1997). A simple explanation for this is that the absorption does not fully cover the source. The souce is known to be small, $\sim 8 \mathrm{pc}$, and gas density known to vary significantly on this scale within 


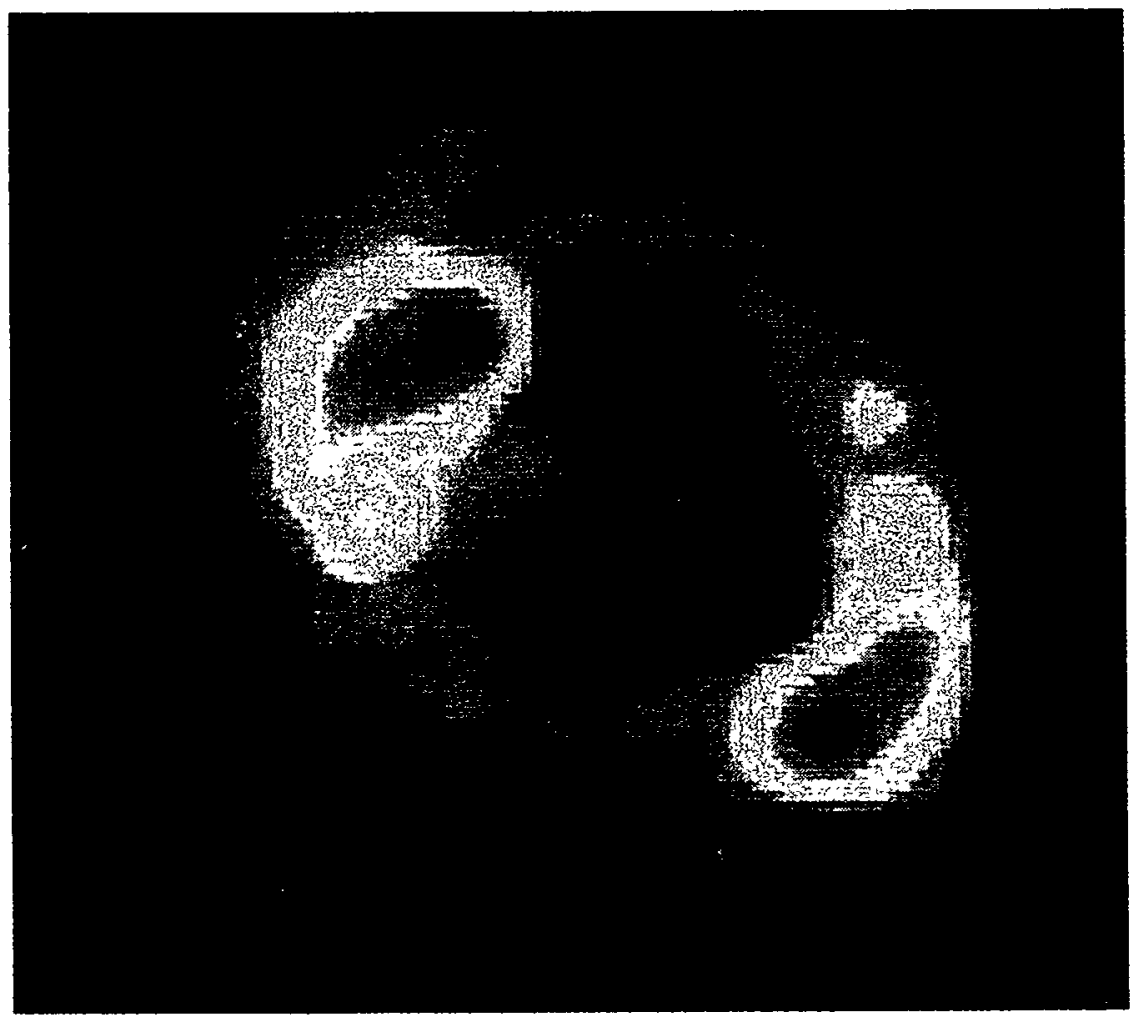

Figure 3.1: Radio image of the radio ring lens PKS 1830-211. Here a quasar with a core plus jet structure is lensed by a foreground galaxy. The two hot spots correspond to the doubly-lensed image of the core, with the jet tail forming the ring. 
spiral arms our own Galaxy (Bieging et al., 1982).

A nearby saturated M-star and heavy extinction along the line of sight $(b=-5.7$ degrees, Djorgovski et al. (1992, hereafter D92) has obscured the lens and the source from identification in the optical and infrared (see the top panel of Fig. 3.3). This limitation has been overcome by the application of a powerful new deconvolution algorithm which decomposes the image into point sources and a diffuse background. It has in this way become possible to identify the positions of the two components of the lensed radio core, the SW and NE components, and the lensing galaxy. The SW and NE components are very red, $I-K \sim 7$, suggesting strong Galactic absorption with additional absorption by the lensing galaxy at $z=0.885$, and consistent with the detection of high redshift molecules in the lens.

At lower redshift, $\mathrm{z}=0.19, \mathrm{HI}$ absorption system has been detected in the $21 \mathrm{~cm}$ line (Lovell et al., 1996). The source is well-resolved and the HI absorption is concentrated toward the NE component. Integrating over their line profile, the HI column is measured to be $N_{H} L=9.2 \times 10^{17} T_{s p i n} \mathrm{~cm}^{-2}$, typical of the disk of a large spiral galaxy (Frye, Welch, \& Broadhurst, 1997). This object may of course have a significant lensing effect, depending on the unknown degree of alignment with the radio core (Lovell et al., 1996). There may be weak absorption seen in the SW component of the $21 \mathrm{~cm}$ VLBI spectrum towards this object which is displaced only slightly in velocity from the strong absorption detected in the NE component (Lovell et al., 1996). A higher signal-to-noise spectrum may reveal a significant signal in the SW component, which would argue strongly for both lensed beams intersecting the same side of the outer disk of the galaxy, where only a small magnification is expected. Such observations would be an important step in sorting out the compound nature of the lens geometry.

Recently, the redshift of the quasar has been found to be at $z=2.507$ via infrared spectroscopy (Lidman et al., 1999). The redshift is based on the two strong emission lines of $\mathrm{H} \alpha$ and $\mathrm{H} \beta$. Using these lines to measure a Balmer decrememt shows it to be highlyreddened, suggesting that the deflector is very dusty and so consisitent with being a spiral. In this chapter the lens properties of PKS 1830-211 are discussed, using multi-wavelength observations taken at the BIMA and Keck telescopes.

\subsection{Observations}

\subsection{1 $\lambda 3 \mathrm{~mm}$}

PKS 1830-211 was observed on 01 and 20 March 1996 with the 9-antenna BerkeleyIllinois-Maryland-Association (BIMA) millimeter Array in the A configuration, with antenna separations ranging from $80 \mathrm{~m}$ to $1.3 \mathrm{~km}$ (Welch \& et al, 1996). Over the elevation angle range $15^{\circ}$ to $28^{\circ}$, single sideband system temperatures varied from $400 \mathrm{~K}$ to $1200 \mathrm{~K}$ with an average of $\sim 650 \mathrm{~K}$. The flux scale was established mainly by observations of the QSO 1730-130, whose flux was measured to be $12 \mathrm{Jy}$ to $\sim 15 \%$ accuracy in early April in 


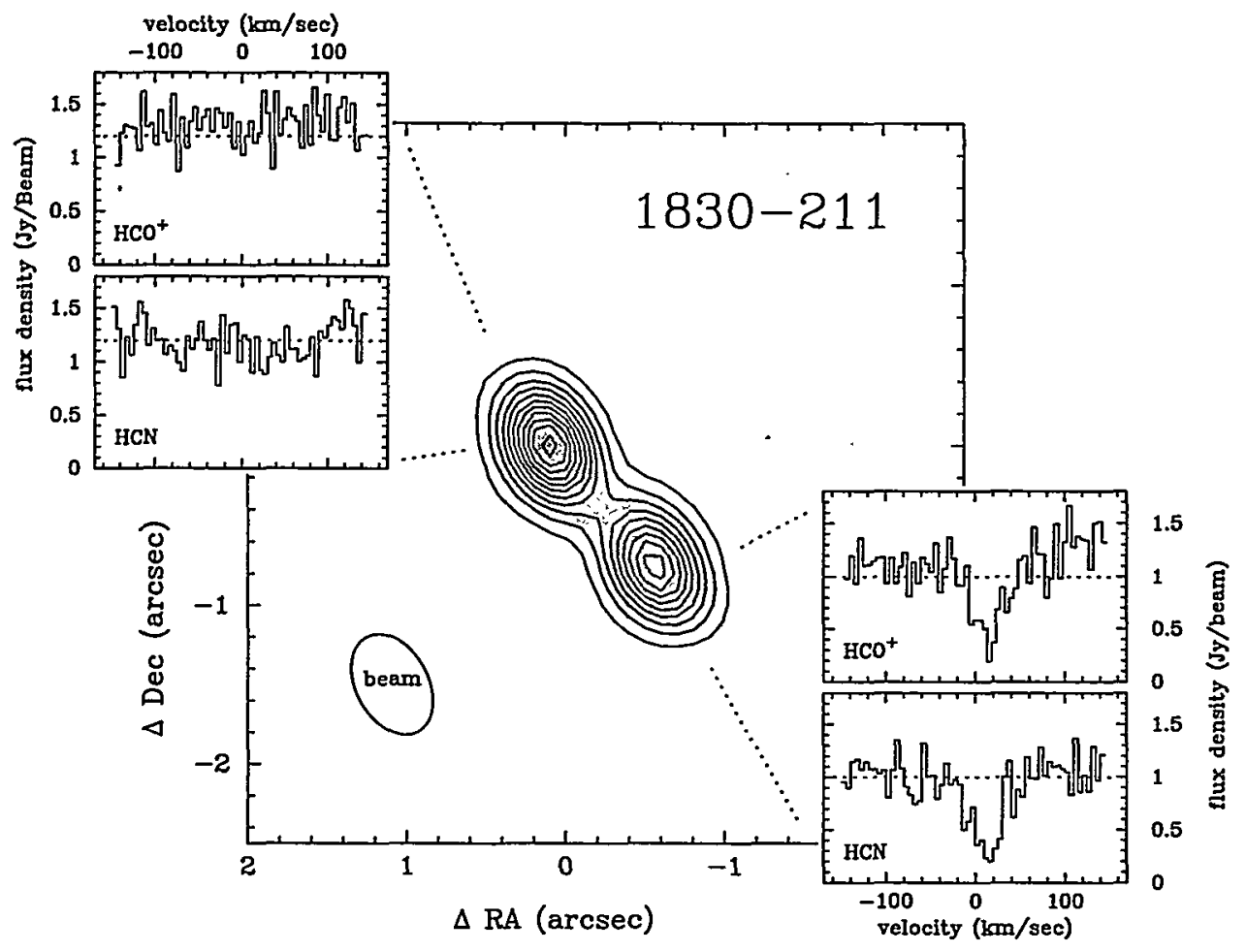

Figure 3.2: The $3 \mathrm{~mm}$ continuum map (center) showing the known double lensed structure is well resolved with a separation of $0^{\prime \prime} 98$ and a flux ratio of $1.14 \pm 0.05$. Contour levels are spaced by $0.1 \mathrm{Jy} /$ beam with the lowest value at $0.1 \mathrm{Jy} /$ beam. The synthesized beamwidth is 0 " $68 \times 0$ ". 45 (lower left), where the asymmetry is due to the low elevation of the source as seen from the northern latitude of BIMA. The spectral range covers the redshifted $\mathrm{HCN}(2$ 1) and $\mathrm{HCO}^{+}(2-1)$ molecular transitions at $5 \mathrm{~km} / \mathrm{s}$ resolution, which are shown (inset) for both images. The molecular absorption is detected only in the SW component and does not reach the base of the continuum. Since the two images are similarly bright, the lack of absorption in the NE spectrum confirms that the spill over between the two images is negligible. 
the compact C-array.

The correlator was configured to observe both the $\mathrm{HCN}(2-1)$ and $\mathrm{HCO}^{+}(2-1)$ transitions at 93.997 and $94.588 \mathrm{GHz}$, respectively, along with $400 \mathrm{MHz}$ of continuum. The spectral windows were centered on the lines, based on the published redshift of $z=0.88582$ (W\&C), with 64 channels and over $100 \mathrm{MHz}$ in each window for a resolution of about $5 \mathrm{~km} / \mathrm{s}$. The 01 March 1996 observation gave results consistent with the 20 March observation, but was only tuned to $\mathrm{HCO}^{+}$. The QSO $3 \mathrm{C} 273$ was used for calibration of the spectrometer passband.

The continuum was first reduced using self-calibration. Since atmospheric phase fluctuations rapidly de-correlate the signal on $\mathrm{km}$ length baselines, we used integration times of just 11 seconds, then self-calibrated the data on a record by record basis in order to derive the antenna-based phases. After 3 selfcal iterations, starting with a single point source model, the image converged to a double source as shown in Fig. 3.2, with fluxes in the NE and SW components of 1.20 and $1.05 \mathrm{Jy}$ respectively, to $\pm 15 \%$. The observations on both dates gave the same flux ratio, 1.14 to $\pm 5 \%$. Uniform weighting was applied with a synthesized beam of $0^{\prime \prime} 68 \times 0.45$. The source separation is $0^{\prime \prime} \cdot 98 \pm 0.01$, similar to what is observed at lower frequencies (Subrahmanyan et al., 1990). There are only two clean components in the continuum image; that is, there is no evidence of the ring structure seen at longer wavelengths, as expected because of its steep spectrum (Jauncey et al., 1991).

The spectral channels were reduced with the individual antenna gains obtained from the continuum self-calibration. The $\mathrm{HCN}$ and $\mathrm{HCO}^{+}$absorption spectra toward the $\mathrm{NE}$ and SW sources were derived from the channel maps and are shown in the insets in Fig. 3.2. Absorption is seen only toward the SW source, and is of comparable depth, $\sim 70 \%$, and velocity width, $40 \mathrm{~km} / \mathrm{s}$, for both species. This width is somewhat larger than is typical of single molecular clouds found in our own Galaxy and may imply that the sight line passes though a complex of clouds which blend in velocity, broadening the line. Adding the fluxes observed towards both sources, the resulting depth of absorption is $S_{a b s} / S_{c}=0.33 \pm 0.03$. This is similar to the $S_{a b s} / S_{c}=0.36$ measured by W\&C.

The surprising feature of the absorption is that even though the two components are spatially-separated, the absorption does not reach the zero level of the continuum of the SW component, despite the clear evidence for saturation in both species (W\&C). This argues most simply that the molecular material does not fully cover the source, as we discuss below.

Infrared spectroscopy was taken using the Near Infrared Camera (NIRC) on Keck I in June 1998. The reduction is being carried out using purpose built software similar to that described in Chapter 4 on the data reduction, and the analysis will be discussed in a future paper (Frye et al. 1999). This data will be very useful for modelling the optical rest spectrum of the lens. 


\begin{tabular}{|c|c|c|c|}
\hline & M-star & NE Comp. & SW Comp.+Lens \\
\hline$I$ & $19.3 \pm 0.1$ & $22.0 \pm 0.2$ & $22.3 \pm 0.3$ \\
$K$ & $16.6 \pm 0.2$ & $15.1 \pm 0.1$ & $18.2 \pm 0.2$ \\
$\mathrm{x}(I)$ & & $+0.08 \pm 0.01$ & $+0.48 \pm 0.1$ \\
$\mathrm{y}(I)$ & & $-0.70 \pm 0.01$ & $-1.15 \pm 0.1$ \\
$\mathrm{x}(K)$ & & $+0.06 \pm 0.01$ & $+0.59 \pm 0.05$ \\
$\mathrm{y}(K)$ & & $-0.54 \pm 0.01$ & $-1.20 \pm 0.05$ \\
\hline
\end{tabular}

Table 3.1: Summary of the photometry and astrometry the NE component and the SW component of the lensed source (plus lensing galaxy), and photometry for the M-star.

\subsubsection{Optical and Infrared}

PKS 1830-211 was imaged in the $I$ band on 15 June 1997 with the Low Resolution Imaging Spectrograph on Keck II using a 300 line grating and $1^{\prime \prime}$ slitwidth (Oke et al., 1995). The observations were taken during a full moon and with a mean seeing of $\sim 0 ! 8$. The pixel scale is 0 ' 215 . The individual exposure times were restricted to 3 minutes in order to avoid saturation of the brightest stars in this extremely-crowded field. The details of the observations and reductions are presented in Courbin et al. (1998a). In addition, a Keck $K$ band image was taken in 1997 (Courbin et al., 1998a). The limiting magnitudes of the images ( $3 \sigma_{s k y}$ integrated over the whole object) are 24.0 in $I$ and 21.3 in $K$.

The MCS deconvolution process is described in detail by Courbin et al. (1998b) and was applied to the optical and infrared data in an identical manner. As output from the procedure, one gets a deconvolved image, decomposed into point sources and a diffuse background, which is compatible with all the input images included in the data set. The photometry and the astrometry of the point sources are also obtained as byproducts of the deconvolution Courbin et al. (1998a). Objects near the frame edges are not well-fitted. (objects labelled 2,3 and 4 in Fig. 3.3). Note that object 1 is never well-fit by a point source and leaves significant residuals after deconvolution. The conclusion is that it is extended or that it is a very strong blend of point sources.

In Fig. 3.3 both the raw and deconvolved images in $I$ and $K$ are shown. In addition ESO $J$ and $K^{\prime}$ data were taken and are presented in Courbin et al. (1998a). The deconvolved images clearly show one red point source at the position expected for the $\mathrm{NE}$ radio source of PKS 1830-211. Another red object is observed close to the position of the SW radio source of the lensed system, but the extended nature of the source and the poor quality of the PSF do not allow one to sort out its morphology. The photometry and astrometry of the field are presented in Fig. 3.4.

The point source at the position of the NE radio source is likely to be the IR counterpart of the NE radio image of PKS 1830-211. At such high signal-to-noise the shape can be shown to be compatible with a point source, and its color, $I-K=6.9$, is much redder than any "normal" star (e.g., Koornneef, 1983). The $I$-band position is 

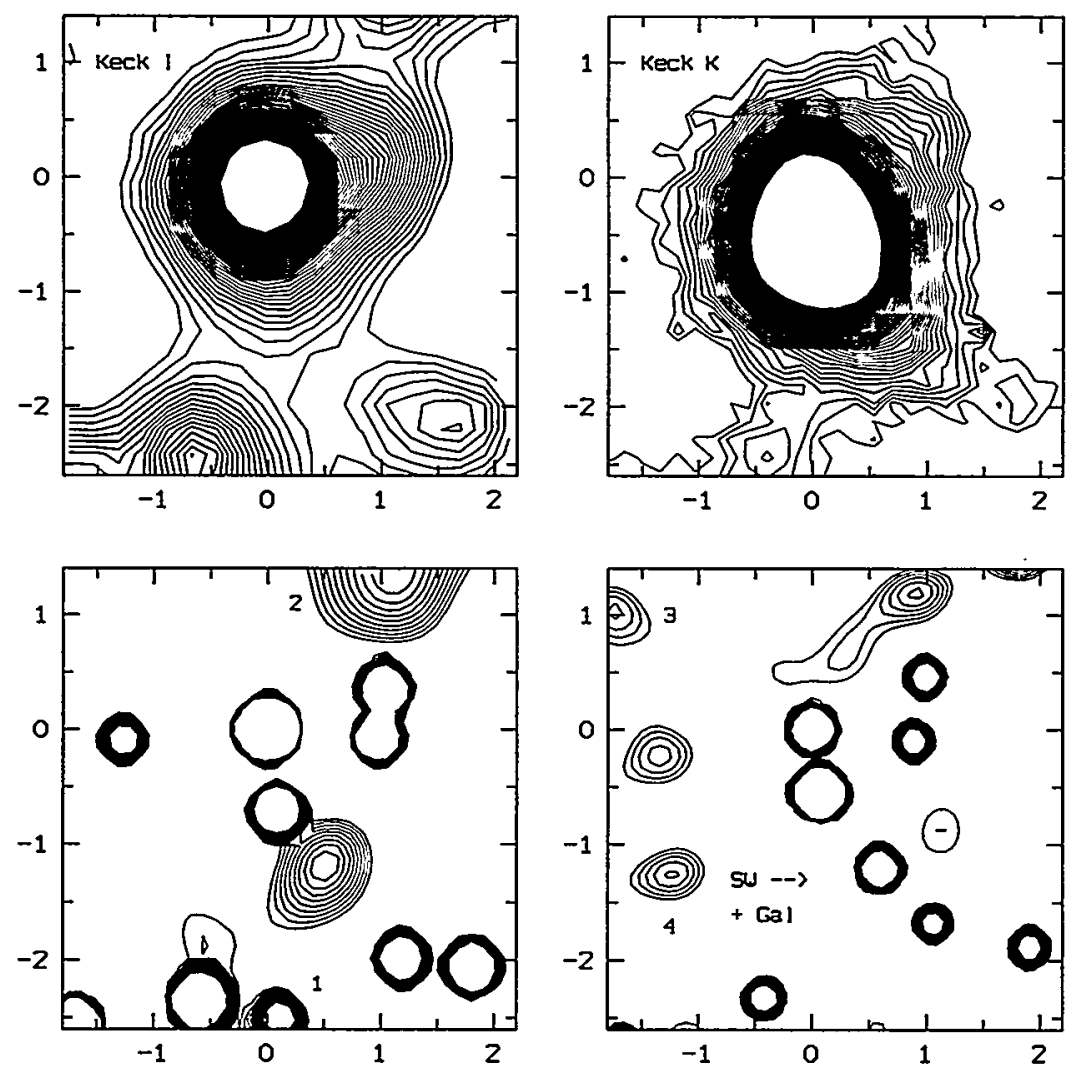

Figure 3.3: Top row, from left to right: 1. Field of 4.0" around PKS 1830-211 observed with Keck II in the I-band. This image is a stack of 6 frames with a pixel size of 0.215 and seeing of $0^{\prime \prime} 8$. 2. Mean of $5 \mathrm{~K}$-band images obtained with Keck I and NIRC. The pixel size is $0 \prime 157$ and the seeing is $0^{\prime \prime} .7$. Bottom row, from left to right: 1 . Simultaneous deconvolution of the 6 I-band frames: resolution of $0^{\prime \prime} 215$ and pixel size of $0^{\prime \prime} 1075$. 2. Deconvolution of the mean of 5 NIRC images: resolution of $0{ }^{\prime \prime} 157$ and pixel size of $0{ }^{\prime \prime} 0785$. In all the images North is up, East left. The M-star, the NE QSO component candidate and the blended SW component + lensing galaxy candidate are detected. The scale is given in arcseconds relative to the M-star. 


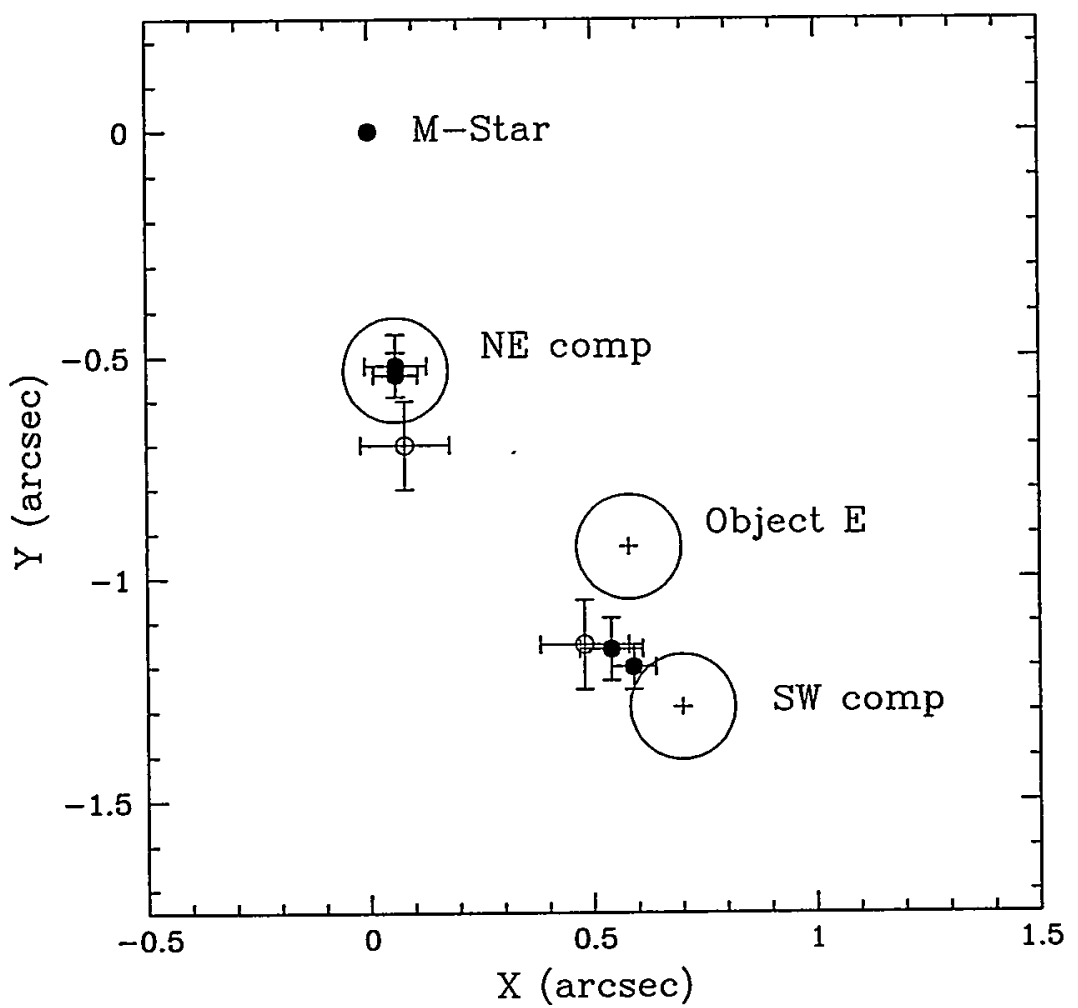

Figure 3.4: Positions observed for the different objects detected in the optical and near-IR, relative to the $\mathrm{M}$ star (Courbin et al., 1998a). The large open circles show the geometry of the system in the radio. Their radius corresponds to the error bars quoted by S90. The black dots are from the near-IR images while the open circles indicate the result obtained from the $I$ band data. 
also within the $1 \sigma$ radio error bars. In Fig. 3.4 all positions are aligned with respect to the M-star so that one can compare the different positions measured for the NE and SW components at optical and IR wavelengths.

The lensed source separation is measured in the $\mathrm{mm}$ to be $0^{\prime \prime} 98$ (Frye, Welch, \& Broadhurst, 1997). By contrast, in our optical and IR images the SW component is measured to be $0.61 \pm 0.13^{\prime \prime}$ and $0.85 \pm 0.09^{\prime \prime}$ away from the NE component respectively. A plausible explanation for the apparent positional shift between the optical, IR and radio positions is that the SW component is a blend of two objects: the lensing galaxy and the heavily reddened SW component seen in the radio images.

The flux ratio between the two lensed images of the source is 1 in the $I$ band and $<20$ in $K$. The combination of a reddened SW radio source plus blue lens can explain the large flux ratios. Both NE and SW components are reddened, making PKS 1830-211 another good example of a dusty lens, along with MG 0414+0534 (e.g., Annis \& Luppino, 1993) and MG1131+0456 (e.g., Larkin et al., 1994), the mean galactic extinction being far below the values considered for PKS 1830-211.

\subsection{Cloud Coverage}

What is the size of the molecular cloud which produces absorption in the SW component? An approximate lower limit is obtained by combining the maximum molecular density, inferred from the molecular spectrum (W\&C), and its total molecular column. The low excitation temperature of $T_{\text {exc }} \leq 6 \mathrm{~K}$, derived from the line transitions, means that the collisional excitation is much smaller than the radiative excitation by the redshifted cosmic microwave background on the low order transitions. A statistical equilibrium calculation for $\mathrm{HCO}^{+}$which assumes radiative excitation by the redshifted background radiation at $z=0.89$, collisional excitation by molecular hydrogen at a kinetic temperature of $25 \mathrm{~K}$, and high optical depth $(\tau \geq 3)$ in the low order transitions, yields a maximum ambient density of molecular hydrogen of $\sim(9 / \tau) \times 10^{4} \mathrm{~cm}^{-3}$ for $T_{e x c} \sim T_{C M B}=5.1 \mathrm{~K}$. For an optical depth of $\geq 3$, and the observed line width of $40 \mathrm{~km} / \mathrm{s}$ with an excitation temperature of $\leq 6 \mathrm{~K}$, the column depth is $\geq 2.3 \times 10^{14} \mathrm{~cm}^{-2}$ in $\mathrm{HCO}^{+}$, in agreement with estimates by W\&C. Using a typical fractional abundance of $\mathrm{HCO}^{+}$in the Milky Way of $10^{-9}$ relative to molecular hydrogen (van Dishoeck et al. 1993), yields a high molecular hydrogen column of $N_{\mathrm{H}_{2}} \mathrm{~L} \geq 2.3 \times 10^{23} \mathrm{~cm}^{-2}$, where $N_{H_{2}}$ is the molecular hydrogen volume density. Dividing this by the maximum molecular hydrogen density of $\leq 3 \times 10^{4} \mathrm{~cm}^{-3}$ gives us a minimum cloud diameter of $\sim 3 \mathrm{pc}$.

Jones et al. (1996) have recently obtained a VLBA image of PKS 1830-211 at 22 Ghz with an angular resolution of $2.2 \times 1.1$ mas and major axis position angle of $1.5^{\circ}$. Their deconvolved image size is $0.6 \times 0.2$ mas. Comparing the latter semi-minor axis dimension with longer wavelength observations, they find that the size scales as $\lambda^{1.96 \pm 0.14}$, very close to the dependence expected for interstellar scattering of point sources. A slightly less 
aggressive deconvolution would give an upper limit of about 1 mas for the larger dimension of the source. At $z=0.89$ and $q=1 / 2,1$ mas corresponds to a source dimension of $8 \mathrm{pc} / \mathrm{h}$, which must be considered an upper limit to the source size.

Strong absorption by high dipole moment gases in low density molecular clouds is also observed toward continuum sources in the Milky Way at millimeter wavelengths. For example, in an observation of the $12 \mathrm{kpc}$ distant Galactic HII region W49 in $\mathrm{HCO}^{+}$and $H C N$, Nyman(1983) detected absorption over a wide range of radial velocities. Emission and absorption in the interval -15 to $+30 \mathrm{kms}^{-1}$ is evidently associated with the W49 molecular core. In addition, there are absorptions over the range 33 to $70 \mathrm{kms}^{-1}$, mainly grouped into features around $40 \mathrm{kms}^{-1}$ and $60 \mathrm{kms}^{-1}$. There is no emission associated with these features, indicating that they are clouds of low density as is the case for the absorbing clouds in PKS 1830-211. They are interpreted as lying in the foreground Sagitarius spiral arm at a distance of about $8 \mathrm{kpc}$. At centimeter wavelengths, the HII region of W49A has a size of about 9 arc-minutes, corresponding to an extent of about $21 \mathrm{pc}$ at the distance of the absorbing Sagitarius clouds. Bieging et al. (1982) mapped the W49 region in the six $\mathrm{cm}$ line of formaldehyde with a resolution of 2.6 arc-minutes. Over both the 36 to $41 \mathrm{~km} / \mathrm{s}$ and the 51 to $66 \mathrm{~km} / \mathrm{s}$ spectral regions, they found typical variations in the line optical depth from 0 to 0.3 in an angle of 3 arc-minutes (their resolution limit), corresponding to a distance across the line of site of about $7 \mathrm{pc}$. If this Milky Way cloud structure is also typical of the cloud structure in PKS 1830-211, it is no surprise that a background source of extent 8pc (or even somewhat less) is not completely covered.

Therefore the gas making up the absorption then in PKS 1830-211 may be the superposition in velocity of cool saturated components along the sight-line which are smoothed together at this low resolution mimicking the appearance of a single broad unsaturated line.

This quasar is of course magnified substantially as it lies close to the Einstein ring, requiring an even smaller intrinsic size for the source. A magnification of $\sim 6$ has been derived for the bright SW component by Kochanek \& Narayan (1992), who find a good fit to the detailed radio-ring structure using a simple mass distribution for the lens (elliptical singular isothermal sphere). This limits the intrinsic unmagnified source size to $\sim 1 \mathrm{pc} / h$ at the cloud distance. Note, since lens magnification is just the ratio of lensed image to source image areas (surface brightness is conserved) the unknown distances do not enter in this

calculation. What little is known of the sizes of $\mathrm{mm}$ AGN's from VLBI work is consistent with such a small scale (Lerner et al., 1993).

\subsubsection{Constraints on Source Redshift}

Is the absorption local to the bright mm source? The proximity of the cloud to the source is geometrically constrained by the $\mathrm{mm}$ data because the absorption is confined to only one of the two components. As Fig. 3.5 shows, the dimension of the cloud, $R_{c}$, must be sufficiently small that it covers only one of the two lensed images of the core, which are separated by twice the Einstein ring radius, $\theta_{e}(0 ! 5$, see Fig. 3.1). As described above, the 


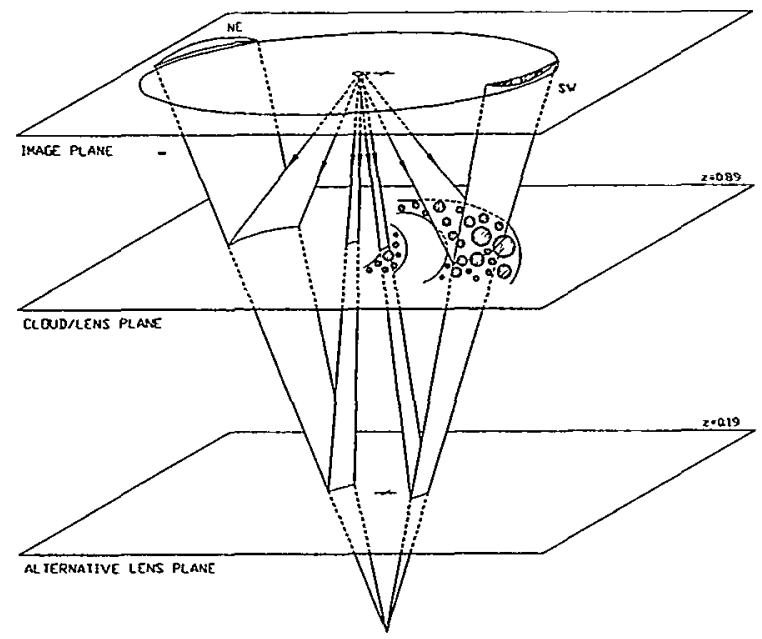

Figure 3.5: A geometric model for the lens, image and cloud planes, with two choices for the lens distance, corresponding to the distant molecular absorption at $\mathrm{z}=0.89$ and the nearby $\mathrm{HI}$ absorption at $\mathrm{z}=0.19$. The incomplete absorption of the $\mathrm{SW}$ beam is represented as a partially covered arc in the image plane, along with an unabsorbed counter arc to the NW, on either side of the Einstein ring. The spiral arm is shown at two alternative positions in the cloud plane, illustrating the two possible arrangements that give rise to the same $70 \%$ fractional coverage of the resultant lensed image. 
smallest cloud which can be tolerated physically is $R_{c} \geq 3 \mathrm{pc}$ placing a lower limit to the separation between the source and cloud of,

$$
d_{c s}>\frac{R_{c}}{\theta_{e}} \frac{d_{l s}}{d_{l}}
$$

or $d_{c s}>1.2 \mathrm{Mpc}$, adopting $d_{l s} / d_{l}$, the ratio of lens-source distance to lens distance, of 1 . Hence the conclusion is that the molecular cloud cannot reside in the same galaxy as the source but lies in the foreground.

The redshift for the source was found by infrared spectroscopy to be $z=2.507$ (Lidman et al., 1999). It was observed with the Son OF Isaac (SOFI) infrared imaging spectrometer and the $1^{\prime \prime}$ slit, giving a resolution of 700 at $2.1 \mu \mathrm{m}$. The slit was aligned to pass through both the NE and SW components of the source. Fig. 3.6 shows the fluxed spectrum over the redshift range $1.5-2.5 \mu \mathrm{m}$. The horizontal lines mark regions of significant absorption by the atmosphere.

Two emission features are especially prominent: $\mathrm{H} \alpha$ and $\mathrm{H} \beta$, which were used to determine the redshift and can also be used as a measurement of the Balmer decremement. The line-of-sight absolute extinction via the Balmer decrement is measured to be $F\left[H_{\alpha}\right] / F\left[H_{\beta}\right]=11 \pm 2$ (Lidman et al., 1999), significantly-redder than measurements towards other quasars (e.g., Hill et al., 1993)), which typically have $F\left[H_{\alpha}\right] / F\left[H_{\beta}\right]=4-5$. Assuming that the absorption takes place at the $z=0.9$ lens, the Balmer decrement intrinsic to the quasar is 5 and the galactic absorption law holds, they find that the NE component has $E(B-V) \approx 1.2$ in the rest frame of the lens. Thus the extinction towards the NE component in the rest frame of the lens is $A_{V} \approx 3.7$ magnitudes, and is $\approx 12$ magnitudes towards the SW component.

\subsubsection{Time Delay from Spectral Monitoring}

The light travel time is expected to be different between the two beams on a time scale of several weeks, depending on the detailed lensing geometry. This has been modeled for this source (Subrahmanyan et al., 1990; Kochanek \& Narayan, 1992) assuming only one deflector. W\&C point out that monitoring the absorption depth would be sufficient to establish the time delay in the millimeter source, requiring only single dish observations. This would be true if the SW component were completely covered by the absorbing gas and the absorptions were complete. However, our data show this procedure may lead to ambiguous results. The incomplete coverage of the $\mathrm{SW}$ component by the absorption means that one would need to know what part of the source is covered by the absorption. The flares responsible for brightening AGN mm sources like PKS 1830-211 must be limited to a very small scale, $\sim 0.1 \mathrm{pc}$, since they typically last only months. As the flare arrives at each image, the net absorption depth measured with low spatial resolution would change, depending on where in the absorbed image the flare takes place. So, for example, a flare occurring in the uncovered part of the SW image could not be distinguished in absorption 


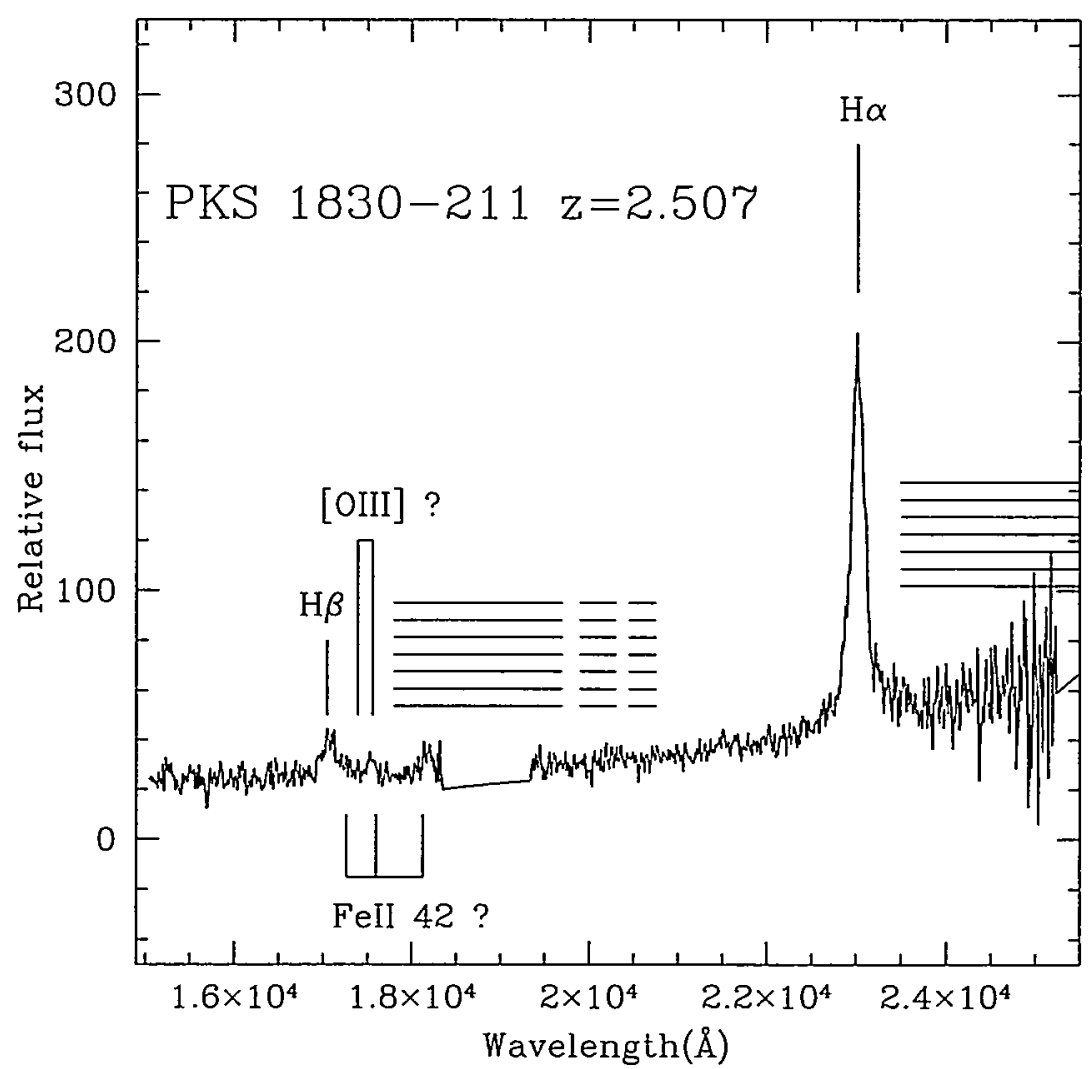

Figure 3.6: Fluxed spectrum of the source of PKS 1830-211 (Lidman et al., 1999). Regions of strong atmospheric absorption are marked by horizontal lines. They measure $z=2.507$ from the two strong emission lines of $\mathrm{H} \alpha$ and $\mathrm{H} \beta$. They measure $F\left[H_{\alpha}\right] / F\left[H_{\beta}\right]=11 \pm 2$, significantly-redder than measurements of other quasars. 
from a flare taking place in the NE component. Resolving the overall image is necessary to track the evolution of the flare if one is to make use of the time delay.

\subsection{Conclusions}

The most reasonable explanation for the near but incomplete absorption of the SW image is that the intervening molecular material is clumpy and does not cover the source. The source however is known to be small $(\leq 8 \mathrm{pc})$ but comparison with similar absorption in our own Galaxy towards small Galactic sources shows that the gas density can vary considerably on this scale in spiral arms. Tracking the depth of absorption at a resolution which does not separate out the two components will not be useful for measuring time delay.

Using deconvolution the optical and near-IR counterpart to the NE radio source of PKS 1830-211 have been detected and the lensing galaxy, or blend of the two has also been detected. The position of the SW component is in good agreement with the predictions from the models calculated by $S 90$ and Nair et al. (1993). The hypothesis of a demagnified third image of the source (S90) between the 2 main lensed images is unlikely as in such a case extinction of the lens would have made it visible in the IR. Furthermore, the IR centroid of the SW component would have been shifted towards "object E" rather than to the radio position of the SW component.

Deep, high resolution near-IR imaging is needed to reveal the exact nature of the faint SW component. However, even at the highest resolution attainable, 0.'2- 0.3 in the IR with HST (in particular in K where the SW component of PKS 1830-211 is best visible), deconvolution will be essential to discriminate between the SW component candidate, the lensing galaxy and additional faint galactic stars. The source is bright enough for selfcalibration over very long baselines at $\lambda 3 \mathrm{~mm}$, so that VLBI measurements will allow a mapping of the lensed image and a measurement of its size.

Much of the lens geometry has been discovered in the past few years. The lens and source redshifts have been determined and the source and possibly the lens has been identified in the optical and infrared. New spectroscopic data have recently been obtained using the Near Infrared Camera on Keck I and the analysis is in progress. From this high signal-to-noise spectroscopy we hope to produce a better mass model for the lens, which in combination with the known geometry and measured time delays can be used to measure $H_{0}$. 


\section{Chapter 4}

\section{Spectroscopic Data Reduction}

\subsection{Introduction}

This chapter describes the process for reducing the multislit spectroscopy data taken for this survey. It starts with a discussion of the raw data acquired at the telescope and documents every operation performed on it including producing the final spectra. The aim is to maximize the signal to noise without resampling the pixels, so that groups of pixels carrying faint continuum signal have every chance of being detected as a coherent pattern in the final reduced image.

A complete spectroscopic reduction package was constructed for this project. It performs all of the tasks expected of a standard reduction pipeline, and in addition has some unique features. For example, it can lock onto the object signal over the full dispersion range despite distortions induced by instrumental flexure, by fitting the low order polynomials to the clean intervals. It can also take out mask defects such as the complex spatial light profile or slit function, and subpixel shifts between the flatfield and the data frames, by modelling it with a dispersed flatfield.

The complete code was written up in the Interactive Data Language (IDL). The set of tasks comprising this reduction package were designed for reducing Low Resolution Imaging Spectrometer (LRIS) multislit spectroscopy data, but are general and can be applied to data from other telescopes and in longslit or multislit modes.

In addition, the IDL tasks are user-friendly, featuring cursor-interactive input and common variables stored as keywords which can be changed on the command line. The primary tasks necessary to generate the finished spectrum from the raw data will be discussed below in the order in which it makes sense to apply them. They are: the bias subtraction and gain correction, the frame combine and cosmic ray removal routines, the flatfielding, the background subtraction, the 1d extraction, the coaddition of 1d spectra, and the spectrum display software. All tasks that are applied to $2 \mathrm{~d}$ frames input fits files and operate on them directly, returning as output the reduced $2 \mathrm{~d}$ fits files. 


\begin{tabular}{cccc}
\hline Name & Service Dates & Gain (Left Amp) & Gain (Right Amp) \\
\hline CCD-2 & 24 Jun 1996 - present & $1.97 \mathrm{e}-/ \mathrm{DN}$ & $2.10 \mathrm{e}-/ \mathrm{DN}$ \\
CCD-1 & 7 Dec 1995 - 24 June 1996 & $1.83 \mathrm{e}-/ \mathrm{DN}$ & $2.16 \mathrm{e}-/ \mathrm{DN}$ \\
\hline
\end{tabular}

\subsection{The Bias and the Gain}

The CCD registers data numbers (DN) from the object, the sky, and also from the output electronics itself, called bias. The bias level must be determined separately for each readout amplifier, and in the optical is independent of signal level or integration time. One can take a 'bias frame' to measure the bias, by median-averaging a set of $0 \mathrm{sec}$ exposures. Alternatively, one can measure it directly from the overscan region, or the edge-bearing portion of the frame which is not exposed.

For most of the analysis described here we measure the bias as a single value by median-averaging the signal from a large set of pixels in the overscan region of each frame $\left(\geq 10^{4}\right.$ pixels, so that the Poisson noise is $\leq 1 \%$ ). If the variation in the zero voltage levels for different pixels is small, so that the bias frame has little noticeable structure, then the bias can be measured as a single number $b$ to be applied to each pixel of each frame $i$. Here the number $b$ is calculated for each raw frame $P_{i}$ and is subtracted off, resulting in the $2 \mathrm{~d}$ bias-subtracted frame $B_{i}$ :

$$
B_{i}=P_{i}-b_{i}
$$

Another correction related to the CCD's electronics is the gain, which is a factor that converts Data Units (DU's) into photon counts and also varies with each amplifier of each CCD (but does not vary between frames). For the two CCDs which have been in commission since this project began, the left and right amplifier gains are given in Table 1: Each bias-subtracted frame $B_{i}$ is multiplied by the constant gains $g$ for each amplifier to obtain the $2 \mathrm{~d}$ frame $G_{i}$ :

$$
G_{i}=B_{i} \times g
$$

\subsection{Flatfielding}

\subsubsection{Pixel to Pixel Variations}

CCD pixels each have slightly different electronic responses to incident light. The relative variations in pixel response constitutes a form of noise which can be removed by 'flatfielding'. The array of relative pixel responses, or flatfield, is achieved by exposing the CCD array to a uniform source of light at high signal to noise and, as there is a modest time dependence on CCD array parameters, ideally on the same night as the target observations. The flatfield is taken at high signal to noise so that when it is applied to the data it does not noticeably-degrade the data's signal to noise . 
In practice it is not easy to find a perfectly uniform source of light. Artificial lamps have edges and vary significantly in brightness from center to edge. Blank patches of sky, although uniform, can be used as sources only during the twilight minutes, a short timeframe compared to the long readout time of of a few LRIS frames (110 s each even in fast, two-amp mode), compromising on the signal to noise. Alternatively, one can collect together patches of sky from different imaging observations, as will be discussed below. The most convenient approach is to take 'dome flats', in which the telescope is trained on an illuminated spot in the closed dome. This experiment can be carried out during the daytime, but has the disadvantage that the lamp is not uniform (brighter and of higher signal to noise in the middle as compared to the edges). The effect of a nonuniform light source is to leave an imprint of the source on the frame. This source image can be taken out by dividing the dome flat into a boxcar-smoothed version of itself, and the signal-to noise at the edges can be improved on by combining several dome flats together, after first $\sigma$-clipping the high, deviant pixels (Fig. 4.1).

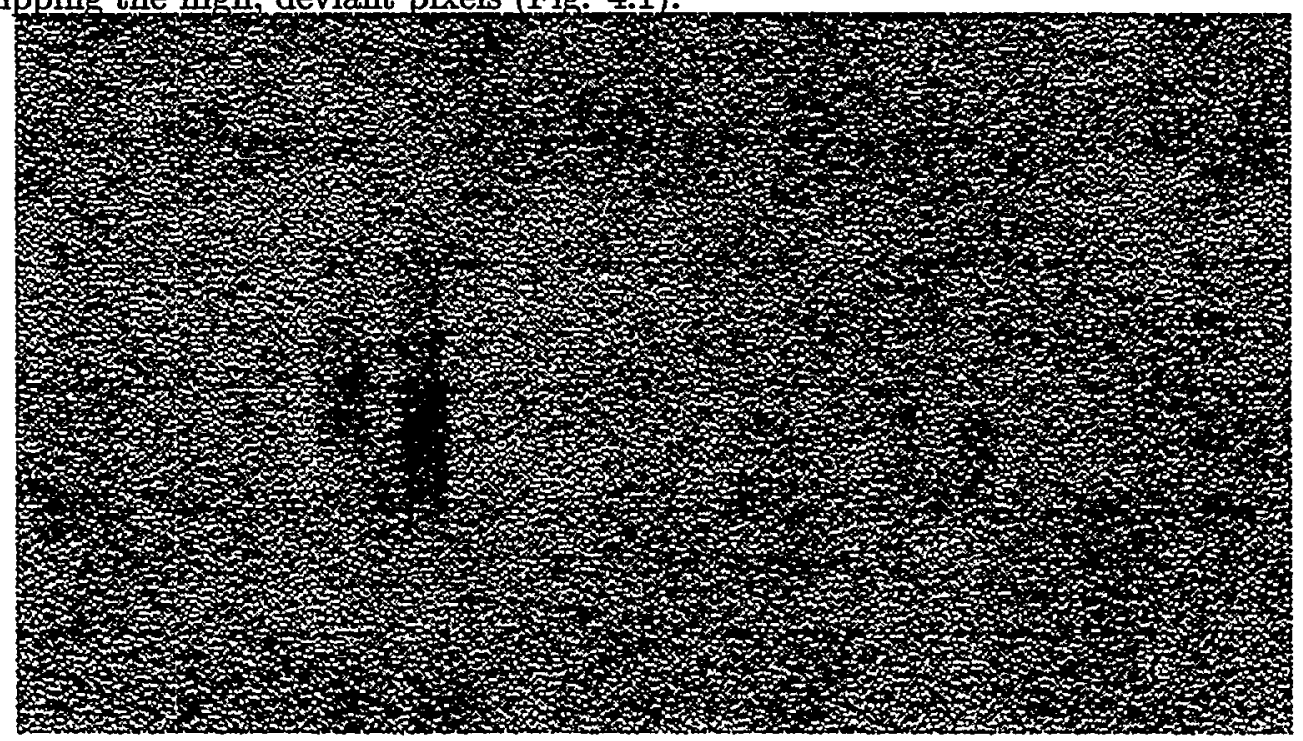

Figure 4.1: Sample section of a V-band dome flat image. The irregular blotches are CCD defects while the regular pattern of striped rows are inherent to the CCD array.

To achieve uniform illumination one can use the sky as the source of light. If imaging is a part of the planned program that same night, then for a given band, the method is simply to sum up all of the images taken over the course of a given night, after first clipping out the high, deviant pixels (objects and cosmic rays), resulting in a composite flatfield called a 'superflat'. Note the importance of clipping out the high, deviant pixels, as opposed to medianing them out, which would otherwise bias the value upwards. Ideally, superflat images should come from images of blank sky taken at different dithering positions at twilight, so that the photon count is high and their distribution uniform. A superflat may not make an ideal flatfield for spectroscopy data, as the pixel response is wavelength- 
dependent. However, for the bluer optical bands which do not suffer from fringing (BVR) it is usually a fair approximation (Fig. 4.1).

To take into account the wavelength dependence of the pixel responses one can construct an 'object flat' (Fig. 4.2). Here short exposures are taken of a halogen lamp through the slitmask and grating. The dispersed flatfield can also be used for correcting other defects such as uneven illumination of light along the slits (the slit function) and interferometric fringing. Like the dome flat, the object flat is nonuniformly illuminated, but as it is taken very close in time to the actual observations, it gives a precise, high signal to noise representation of the time-dependent effects of fringing and the slit function. Since we require the object flat to correct for the slit function and fringing anyway, and we want to limit the number of operations on the data so as not to risk introducing any extra noise, we generally favor this approach for the flatfielding.

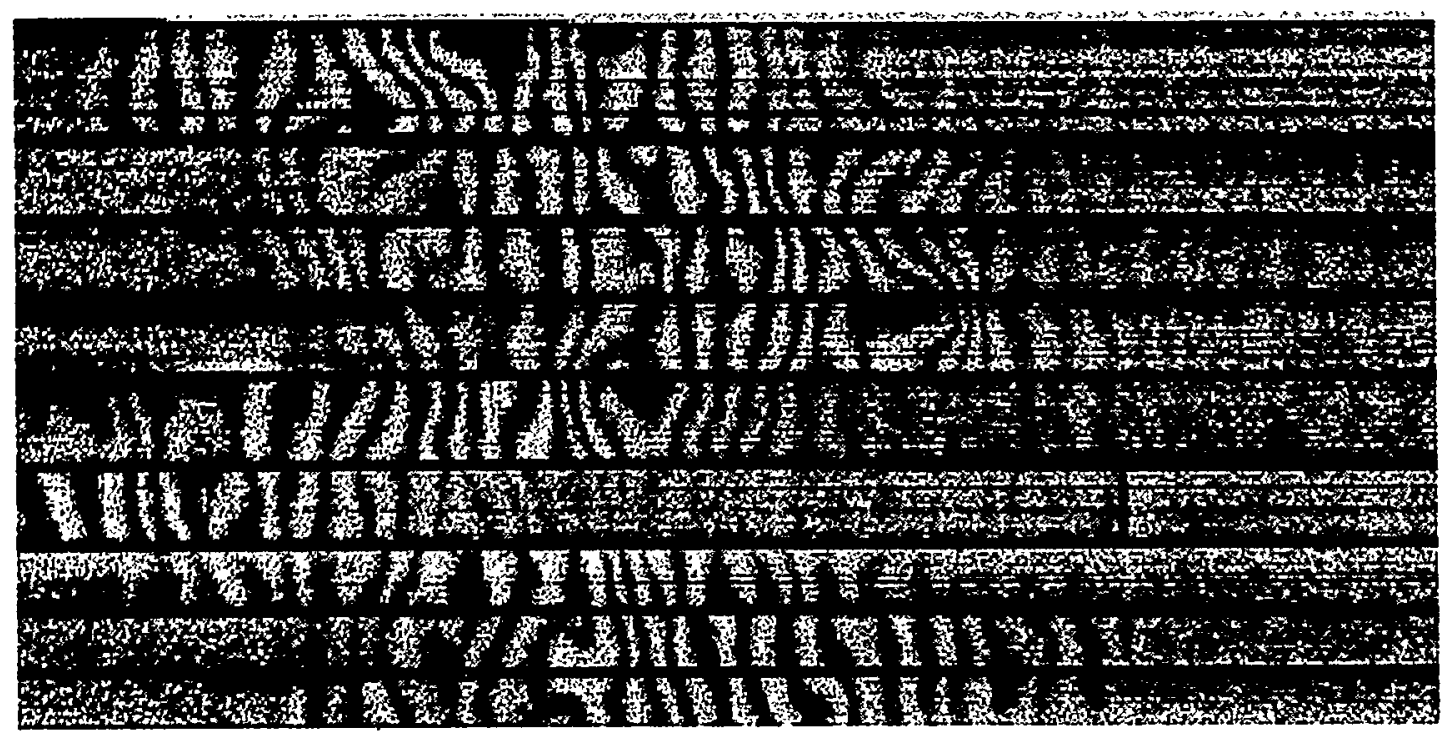

Figure 4.2: Sample section of an object flat from a multislit spectral image. Dispersion direction is left-right and the spatial direction is up-down. Note the interferometric fringes (left), and the wavelength-independent dark and bright rows of pixels, caused by nonuniform illumination of the lamp along the length of the slit. Each spectrum has a unique spatial illumination profile, and a different position in the dispersion direction at which fringing starts to become important.

\subsubsection{Fringing}

Red light has an absorption depth comparable to the thickness of the coating on the CCD chip. As a result, the longer wavelength light suffers internal reflections. In addition, the chip itself is not perfectly uniform. Gradients in its thickness cause the reflections to take 
place at slightly different depths. The resulting interference of the incident and reflected light produces fringe patterns the $2 \mathrm{~d}$ images. The fringing appears at the $\sim 5 \%$ level, as wavelength-dependent wavy bands or stripes (see Fig. 4.2). The fringe pattern can change as the CCD parameters change (on a timescale of minutes). Fringing is a multiplicative effect that can be taken out, at least to some extent, by dividing the data frames into a nearby object flat (Fig. 4.2). However, the time overhead in switching between calibration and observing modes is dear, and until recently, the positional accuracy of returning to the field uncertain, so it has not been feasible to taken object flats more often than every $\sim 2$ hours (between sets of exposures). Note that even if object flats could be taken after each 20 min exposure it would still not be frequent enough to remove the fringing completely. The flats have a different source and hence different spectrum than the sky, so the fringing may also be different. This is most significant in the red where the fringing is most pronounced.

\subsubsection{Slit Function}

Although a milling machine usually outperforms a punching machine in cutting precision slitmasks, neither one can manufacture perfectly rectangular slitlits, and dust lodged inside of the slits can further exaggerate the physical irregularities. As a result, images taken of light shining through the imperfect slitlets reveal nonuniform illumination along its extent. Shining dispersed light through an imperfect slitlet produces a series of wavelength-independent streaks of dark and bright pixels to appear at $\sim 2 \%$ level. This slit profile or slit 'function' is recorded in the object flat at high signal to noise (Fig. 4.2). If the distortions change only slowly with time (on a timescale of hours) they can be taken out, at least to some extent, in two different ways.

One can try to remove the slit function by by dividing the object flat into the data. If there is a spatial translation between the object flat and data frame the object flat may need to be translated spatially first.

A more preferred approach is to characterize the slit function in the object flat, where it is recorded at high signal to noise, and apply it to the data. The problem with this is that flexure can deform the images with repect to the fixed rectilinear CCD array, typically amounting to $\sim 3$ pixels of shift in the spatial direction from center to edge. The slit profile is a complex function and shows structure on subpixel scales, so that different representations of the same slit function are achieved for each integer shift along the dispersion direction.

If each representation of the slit function is sampled ( 3 for a 3 pixel shift from center to edge) the relevant sampling can be divided into the object flat and into the data, smoothly taking into account the box shape and the possible sub-pixel shift between the object flat and the data.

Since the intervals between adjacent spectra in the object flat are clean but fuzzy, and there are only a limited number of bright reference spectra in the data, the limitation of this method lies with finding the subpixel translation between object flat and data. Our test cases have shown noticeable improvement in some cases, but at this time we reserve 
this procedure only for spectra where the object has fallen right down a peak or valley of the slit function. Our standard procedure is to divide the object flat into the data directly and without translation.

\subsubsection{The Algorithm}

The procedure for creating the object flat is straightforward. We take a minimum of two consecutive exposures of the dispersed halogen lamp though the mask, so as to improve on the signal to noise and so that cosmic rays can be removed. We coadd the set of object flat frames, removing the high, deviant pixels, and then take out the blackbody shape of the halogen lamp by dividing it by a smoothed version of itself. We then divide the flattened, coadded object flat into the data.

The code which combines frames is a general task which can be applied to the coaddition of two to several $2 \mathrm{~d}$ fits images of any kind (i.e. object flat, dome flat, data). Starting with a set of two frames, in this case two object flats, we take out the small differences in the background level (due to small differences in exposure time or variations in brightness) by normalizing the second frame to the median of the first to obtain the normalized $2 \mathrm{~d}$ frame $G_{2}^{\prime}$ :

$$
G_{2}^{\prime}=G_{2} \times \frac{\operatorname{median}\left(G_{1}\right)}{\operatorname{median}\left(G_{2}\right)}
$$

We will work with the normalized frame for $G_{2}$ for creating the mask of bad pixels, but will construct the final, composite $2 \mathrm{~d}$ frame $R$ using the original data. With only two frames to work with the high, deviant pixels can still be clipped out by comparing the difference of the two pixel values in the stack with the $\sim 5 \sigma$ deviation from the minimum value of the stack $G_{\min }$. Note that the minimum is important here, as it is least likely to be a cosmic in short exposures $(\sim 2 \mathrm{sec})$ and so may more closely represent the actual sky value. This step is summarized below:

$$
R= \begin{cases}\frac{G_{1}+G_{2}}{2}, & \text { if }\left|G_{2}^{\prime}-G_{1}^{\prime}\right|<G_{\min }+\sigma \sqrt{G_{\min }} \\ G_{1}, & \text { if }\left|G_{2}^{\prime}-G_{1}^{\prime}\right|>G_{\text {min }}+\sigma \sqrt{G_{\text {min }}}\end{cases}
$$

For the multislit data, there are $\sim 40$ individual slitlets per $2 \mathrm{~d}$ frame. The slitlets are all cut in different positions with respect to the central axis of the mask, giving them all different central wavelengths. As a result each spectrum in an object flat exposure will record a unique wavelength range of the halogen lamp blackbody spectrum $P$. To make this a uniform flatfield, the blackbody lamp spectrum must be taken out of each spectrum box separately, by dividing it by a smoothed version of itself. A simple boxcar-smoothing algorithm is used to generate the blackbody spectrum $P$ for each pixel in the dispersion direction $k$ of a given spectrum $l$ : 


$$
P_{k}=\sum_{j=y s t}^{y f i n} \sum_{i=(k-w)}^{(k+w)} R_{i j}
$$

where $i$ and $j$ are the summation indices for the dispersion and spatial directions respectively, $w$ is half the width of the boxcar, $k-w$ and $k+w$ give the range of the boxcar about a given pixel $k$, and $y s t$ and $y$ fin record the length of the sample box in the $y$ direction. Written as a vectorized function, the boxcar-smoothed version of each spectrum, $P_{l}$, is divided into itself, $R_{l}$, to obtain the $2 \mathrm{~d}$ flattened, combined object flat $U$ :

$$
U=R / P_{l}
$$

Finally, the $2 \mathrm{~d}$ frame $U$ is divided into each of the $2 \mathrm{~d}$ data frames $G_{i}$ to obtain the $2 \mathrm{~d}$ flatfielded data frames $Q_{i}$ :

$$
Q_{i}=G_{i} / U
$$

Upon flatfielding there are noticeable improvements in the data frames. CCD blemishes are alleviated or divided out, the slit function is improved (usually), and the sky becomes less noisy and less grainy-looking.

When object flats are not available and we are faced with flattening the data using only dome flats, we carry out a similar algorithm to that described above. A set of bias-subtracted dome flats are combined after they have been multiplied by the gain. Then the combined dome flat is divided into a $2 \mathrm{~d}$ boxcar-smoothed version of itself (to take out the $2 \mathrm{~d}$ shape of the dome lamp). This flattened, combined dome flat is then divided into each of the data frames, yielding the flatfielded data frames $Q_{i}$. Regardless of the method used, once the data frames are flatfielded the sky the background can be determine dand subtracted off of them. The background subtraction code will operate on all $\sim 40$ spectra on the mask and output the fully-reduced $2 \mathrm{~d}$ fits image.

\subsection{The Background Subtraction}

\subsubsection{Signal to Noise}

Most of the spectra included in the survey are 'sky-limited'. We can see what this means by taking a look at the general signal to noise equation. Here the signal counts $O$ come from the object and the noise counts from the sum of the combination of the readout noise of the CCD $r$, the noise on the counts from the object, and the noise on the counts from the sky. Note that the sources of noise are random and independent, so that the noise terms can all be added in quadrature, and that the noise is assumed to be Poisson-limited.

Assuming that all else is perfect, the total signal to noise ratio, $\frac{S}{N}$, from McLean,p299 can be written as : 


$$
\frac{S}{N}=O \sqrt{T}\left[u_{r}^{2}+O+\sum_{i=1}^{n}\left\{\begin{array}{c}
\left(S+D+\frac{r^{2}}{t}\right)+\varepsilon_{D}\left(D+\frac{r^{2}}{t}\right) \\
+(1+f)^{2} \varepsilon_{S}\left(S+D+\frac{r^{2}}{t}\right)+(1+f)^{2} \varepsilon_{D}\left(D+\frac{r^{2}}{t}\right)
\end{array}\right\}\right]^{-\frac{1}{2}}
$$

where $t$ is the integration time for one exposure, $N_{S}$ is the number of object frames, $N_{B}$ is the number of sky/flat frames, $N_{D}$ is the number of dark frames, $T=t N_{S}$ is the total integration time, $f=O / S$ per pixel, $\varepsilon_{B}=N_{S} / N_{B}$, and $\varepsilon_{D}=N_{S} / N_{D}$.

If the calibration frames are perfectly accurate, $\varepsilon_{B}$ and $\varepsilon_{D}$ become negligible. Further, if there is no residual nonflatness $u_{r}$ is also negligible and we are left with a muchsimplified equation:

$$
\frac{S}{N}=\frac{O \sqrt{T}}{\left[O+\sum_{i=1}^{n}\left\{\left(S+D+\frac{r^{2}}{t}\right)\right\}\right]^{-\frac{1}{2}}}
$$

In the background or sky-limited case, $S \gg\left(D+r^{2} / t\right)$ and $S \gg O$. Note that the dark current $D$ refers to the unwanted CCD signal caused by atoms jostling around in the CCD. This frees up electrons which then get registered as counts. For a cooled optical CCD the dark current is negligible. Also for a high-performance optical CCD camera the readout noise is negligible, and the equation is further simplified:

$$
\frac{S}{N}=O \sqrt{T}\left[\sum_{i=1}^{n} S_{i}\right]^{-\frac{1}{2}}
$$

The signal to noise improves only as the square root of the total integration time. For a given object signal, the signal to noise increases inversely with the square root of the sky. Since the sky level increases with wavelength, emitting $\sim$ twice the number of counts in the far red compared to the blue, four times the integration time is required to achieve the same signal to noise there. This is what we are faced with in trying to detect the red, sky-limited objects which are characteristic of the survey.

\subsubsection{Spatial Distortions}

The spectra are sky limited and àlso have a limited number of sky pixels. After the cosmic rays and object have been identified, there are relatively few good sky pixels left in a given spatial column, $\sim 12-42$, with which to find the best estimate of the sky. Obviously, it is important to make use of every possible good pixel, and so to locate the spatial limits of each spectrum with precision. The problem is that instrumental flexure can curve the edges of each spectrum box into roughly quadratic shapes with respect to the fixed CCD grid, amounting to $\sim 3$ pixels from center to edge. These intervals are dark or bright compared to the neighboring spectra (depending on the CCD used), and so can be traced out in principle, but contamination from the skylines of the neighboring spectra 
makes it difficult to trace the interval all the way along the the dispersion direction of the chip.

Fortunately, the object flats are not exposed to our atmosphere and so do not record atmospheric skylines, which makes them ideal for tracing the curvature of each spectrum box. For each spectrum $k$ we read in the object flat, identify one of the two nearest box intervals and fit it with a low order polynomial (usually a quadratic). We translate this curve over to the data frames, and in this way properly 'follow the curvature' of the spectrum boxes (Fig. 4.3).

(a)

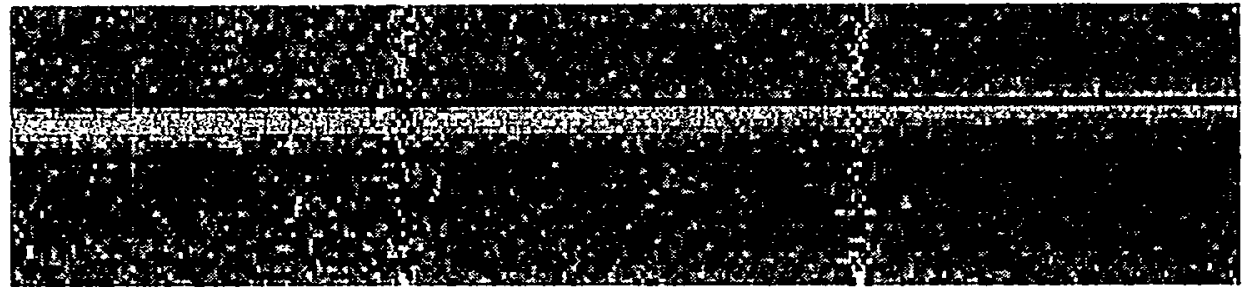

(b)

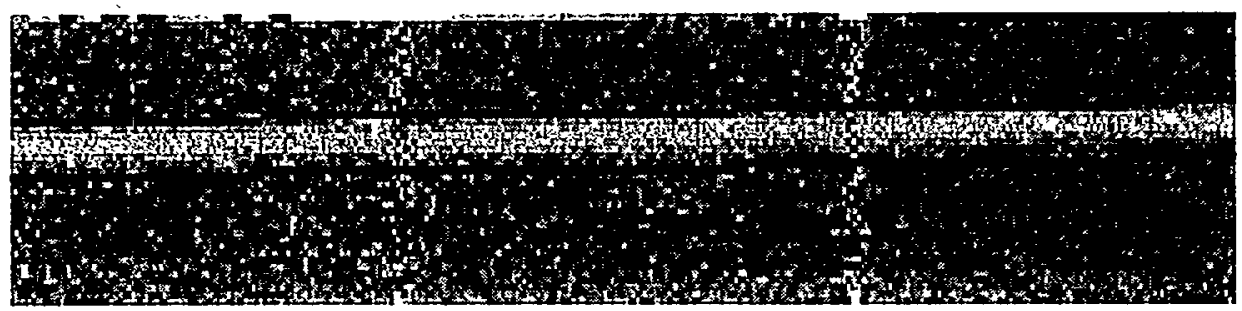

Figure 4.3: Example showing how this algorithm follows the curvature of the object. Both panels show the same patch of a fully-reduced $2 \mathrm{~d}$ spectrum, where the dispersion direction is left-right. The two bright vertical lines in each spectrum are skyline residuals. The occasional groups of bright pixels are cosmic rays, which were identified and ignored in finding the best estimate for the sky but not removed from the $2 \mathrm{~d}$ image. The bright horizontal stripe extending through the middle of each spectrum is the object continuum. The two dark lines which frame the top and bottom of the object continuum mark the sptial extraction region. In Panel (a) the curvature of the object is not followed. Note that in going from left to right, towards the blue edge of the chip, increasingly more of the object signal is lost. In Panel (b) the continuum is followed. Note that in this case the continuum signal is fully collected, maximizing on the signal to noise of the extracted object.

Note that the usual procedure for following the curvature of the object is to follow the continuum of the object directly on the data frame, but then for faint, red targets the polynomial is not very well constrained. In the red the atmospheric lines are strong and numerous, effectively hiding the faint continuum signal behind them, and the continuum is fainter because the signal to noise is lower (see Eq. $\mathrm{xxx}$ ). In the blue there are fewer strong skylines, but the continuum signal will still be difficult to identify if the object is faint and/or red.

We properly follow the curvature of the object by simply translating the box curvature onto the position of the object, assuming only that there is not a significant change in the flexure over the course of a set of exposures ( $\sim 2$ hours). We find that this is 
a fair assumption over this time interval, at least to the extent that a low order polynomial can keep track of it.

\subsubsection{Median Cosmic Ray Clipping}

Now that we know exactly where the sky and object are for each spectrum, the next step is to identify the cosmic rays. This is an iterative process, beginning with a column by column search for the especially strong cosmic rays. Without this first round of flagging, a least squares fit through a set of points with a strong cosmic ray in it would be skewed abnormally-high, making it insensitive to finding the more common, weaker hits.

Following the curvature, the set of pixels for each column $i$ of each spectrum $k$ of the flatfielded data is collected and any pixels outside of a generous $10 \sigma$ cut from the median sky count are flagged. Note that the median is used in this first round of flagging, as opposed to the mean, so that in the columns where there are strong cosmic rays the value is not drastically overestimated. The offending pixels are saved on a $2 \mathrm{~d}$ mask $M$. This step can be summarized mathematically as follows, for each column $i$ of each spectrum $k$ :

$$
\sum_{j=s t(i)}^{f i n(i)} S_{i j k}=\operatorname{median}\left(Q_{i k s t(i)}, Q_{i k s t(i)+1)} . . Q_{i k f i n(i)}\right)
$$

where $j=s t(i), f i n(i)$ is the starting and ending pixel value for column $i$ of spectrum $k$. ' $S t$ ' and ' $F i n$ ' are functions of $i$ because they follow the curvature. The number which is the median is written to every pixel of column $i$ of spectrum $k$ in the $2 \mathrm{~d}$ sky matrix $S$, as the first estimate of the sky. The first 2 d mask $M$ is then created, based on the $2 \mathrm{~d}$ frame of median data values $S$ :

$$
M= \begin{cases}0, & \text { if } 10 \sqrt{S}+S>Q \\ 1, & \text { otherwise. }\end{cases}
$$

where the subscripts are omitted because the operation is performed for each pixel of the $2 \mathrm{~d}$ array. After this round of median cuts is completed, the pixels are collected again, this time excluding the bad pixels. Instead of calculating the median, this time a low order polynomial is fit through the points of each column $i$ of each spectrum $k$ and a revised the new $S_{i j k}$ is produced:

$$
\sum_{j=s t(i)}^{f i n(i)}\left(S_{i j k}=a_{1 i k} j^{2}+a_{2 i k} j+a_{3 i k}\right)
$$

where as before, $j$ is the spatial pixel index for a given column $i$ of spectrum $k$, following the curvature, and the $a_{i k}$ 's are the coefficients of a low order polynomial fit to spatial column $i$ (typically of second order). 


\subsection{4 $\sigma$-cut Cosmic Ray Clipping}

The second round of cosmic ray cuts proceeds in much the same way as the first. We take the best estimates of the sky from the $2 \mathrm{~d}$ array $S$, and calculate the standard deviation $\sigma$ of each pixel in column $i$ of spectrum $k$ about the low order polynomial fit to the pixels in that column. We ask if any pixels are more than a strict $2 \sigma$ away from the mean, and record that information on the $2 \mathrm{~d}$ mask array $M$ as before:

$$
M_{i j}= \begin{cases}1, & \text { if } 2 \sigma+S_{i j}>Q_{i j} \\ 1, & \text { if flagged before } \\ 0, & \text { otherwise. }\end{cases}
$$

We then collect the good pixels again and fit them with a low order polynomial fit as outlined in the last section, obtaining a new array $S$ which gives our final best estimates for the sky.

\subsubsection{Background Subtraction}

Having done all the hard work, finally the best estimates for the 2d sky frame $S$ is subtracted from the $2 \mathrm{~d}$ data frame $Q$, to obtain the $2 \mathrm{~d}$ background-subtracted frame $P$ (Fig. 4.4):

$$
P=Q-S
$$

\subsection{Extraction and Wavelength Calibration}

The aims of this task are to extract the $1 \mathrm{~d}$ spectra from the $2 \mathrm{~d}$ fits images, following the curvature, and to wavelength-calibrate the data. This code requires the pre and post $2 \mathrm{~d}$ background subtracted frames, the object flat, the start and stop pixel values of the extraction in the spatial direction and the pixel position of the OI $5577 \AA$ line as input. The task is performed separately for each spectrum. Cursor-interactive options allow the user to exclude deviant pixels in the wavelength calibration and to iterate on the solution as necessary. The result is a four-column 1d data file of object counts as a function of wavelength for each spectrum. The columns are pixel number, wavelength in $\AA$, Object counts, and sky counts.

\subsubsection{The Spatial Profile}

The extent and shape of an object's spatial profile depends on the seeing and the object's size. For an unresolved source, where the spatial profile is a Gaussian, the pixels at the ends of a 'top hat' profile will contain a smaller fraction of signal relative to the central pixels, introducing excess noise into the data. The process of assigning different weights to 


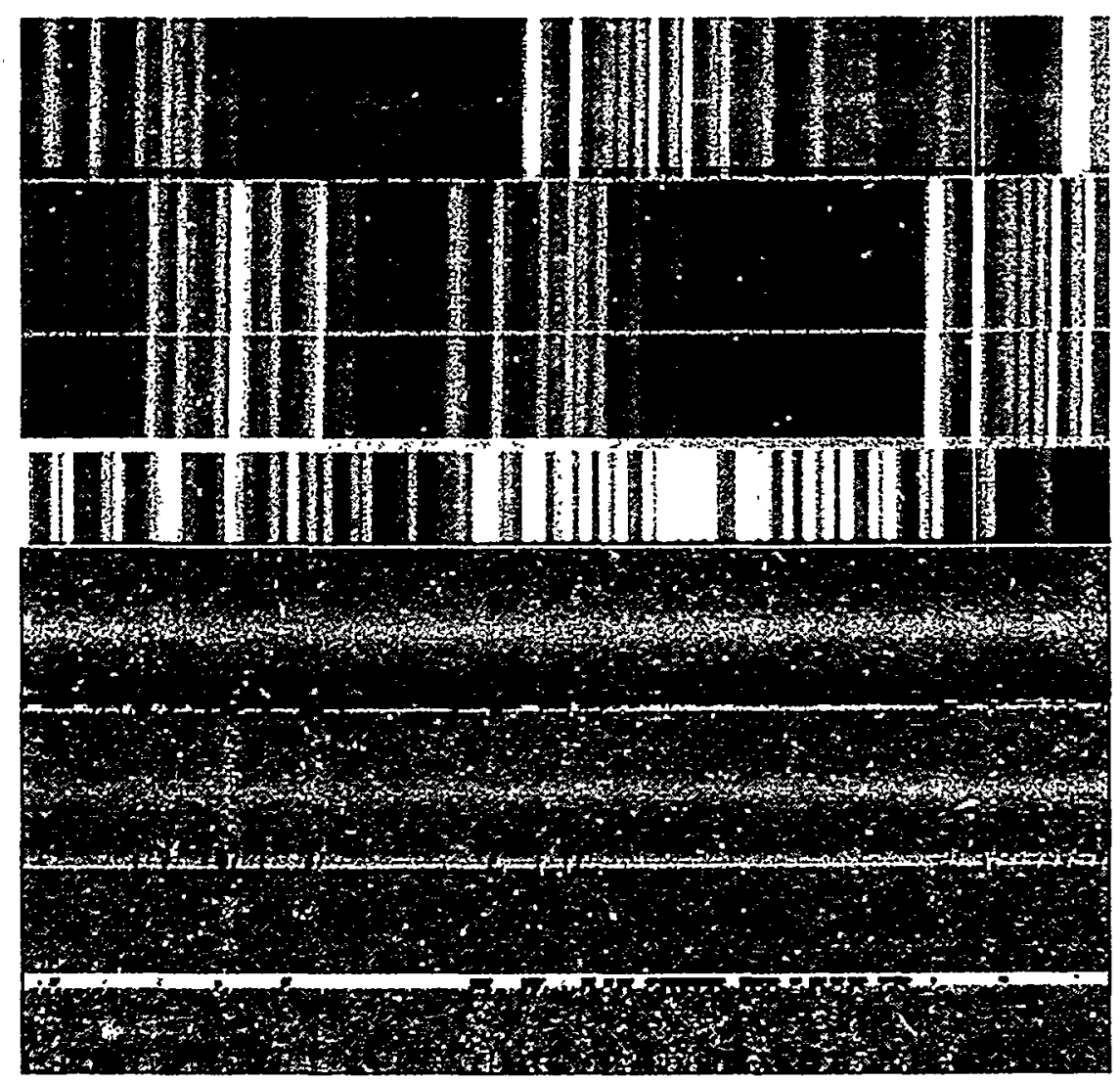

Figure 4.4: Comparison of frames before and after background subtraction over the same $2 \mathrm{~d}$ spectral region. Sample regions from four different spectra are shown here, each covering a different wavelength range. The dispersion direction is left-right and the spatial direction is up-down. Note that there is an emission line object in the lower left-hand corner which is only made obvious after the background subtraction. This is Ly $\alpha$ at 7418 Aand $z=5.12$. The redshift of the other emission line object in this field, in the upper right hand side of the image, has yet to be determined. Note the general smoothly-distributed noise of the subtracted background and the inevitable skyline residuals 
pixels in the spatial direction, while preserving spectrophotometric accuracy, can improve on the signal to noise ratio for background-limited objects by up to $70 \%$ (Horne 1986).

Will the objects in our data benefit from using this 'optimal extraction' technique?

Yes, but with LRIS $1 \sigma$ for the continuum corresponds to $\sim 1.5$ pixels, so that $95 \%$ of the signal falls over a total of only $\sim 5$ pixels, on average. This is a very small number with which to sample a Gaussian profile. We find that by including all the flux in the central pixels and $50 \%$ of the flux in the outermost pixel at each end gives a fair, although rough, representation of the real spatial profile and a corresponding improvement in the signal to noise ratio, and so we adopt this profile for the $1 \mathrm{~d}$ extraction. The full scale optimal extraction technique by Horne 1986 has not been adequately-tested and so has not yet been implemented into the code for general use.

\subsubsection{The 1d Extraction}

The 2d image spectra are of insufficient signal to noise to identify anything but the most prominent spectral features. The more common, subtle features which are normally used to confirm/identify an object's redshift can be boosted up to higher signal to noise by summing up the object pixels over the spatial direction, producing a 1d spectrum. This process is described here. The inputs to the $1 \mathrm{~d}$ extraction code are the pre and post background subtracted $2 \mathrm{~d}$ images (the former so that we can extract the sky and latter so that we can extract the object) and the range of the spatial extraction. We follow the curvature as before, by tracing the shape of the nearest spectrum edge interval in the object flat and applying the solution to the relevant spectrum in the data (Fig. 4.3). A crosssectional view of the spectrum taken at 100 pixel intervals shows how well the object is being followed along the extent of the chip. In each panel the horizontal line gives the region over which the spectrum is to be extracted. Note that despite a 7 pixel shift from center to end in this case the curvature is followed rather well. For each column $i$ in the dispersion direction of a given spectrum $k$, the pixels are summed up over the spatial limits from ymin to ymax inclusive, modulo the spatial profile weights as described in the last section:

$$
\begin{gathered}
p_{i}=\sum_{j=y \min (i)}^{y \operatorname{ymax}(i)} P_{i j} T_{j} \\
q_{i}=\sum_{j=y \min (i)}^{y \max (i)} Q_{i j} T_{j} \\
T_{j}= \begin{cases}0.5, & \text { if } j=y \min , y \max \\
1, & \text { otherwise. }\end{cases}
\end{gathered}
$$

where ymin and ymax are functions of $i$ because we are following the curvature, $Q$ and $P$ are the pre and post background subtracted $2 \mathrm{~d}$ frames respectively, $\mathcal{T}_{j}$ is the spatial profile, and $p$ and $q$ are the $1 \mathrm{~d}$ object and sky data output respectively. 
As a quality check, the functions $y \min (i)$ and $y \max (i)$ are drawn onto a copy of the background subtracted data frame, and that test frame written out to file (Fig. 4.3). Also the spatial profile of the object is displayed to the screen in real time, sampled in sets of 20 pixels every 100 pixels, with the region chosen for extraction overlayed. In this way the user can efficiently monitor the box curvature and the choice of extraction limits.

\subsubsection{The Wavelength Calibration}

The atmospheric skylines imprint directly onto the data. The positional centroid of the lines can be measured and matched up with their known wavelengths. If several of such matches can be made then a low order polynomial fit through these ordered pairs will yield wavelength as a function of pixel position, or the wavelength solution.

The wavelength calibration requires a couple of inputs. The first is the pixel position of the OI $5577 \AA$ skyline, which varies with each spectrum and depends on where the slit is punched or milled with respect to the central axis of the mask. The second is a single two column parameter file, good for all spectra, which gives the wavelength and the offset in pixels from the OI skyline for each of the skylines to be used in the calibration.

Finding the weighted centroid is a two-step process. The code first roughly identifies the centroid of a given skyline $x c_{i}$ by adding the relevant offset $o_{i}$ to the pixel position of the OI line:

$$
x c_{i}=O I+o_{i}
$$

The initial value $x c_{i}$ may be off by a few pixels. The actual offset varies with the difference of the slitlet position from the central axis of the mask. To get a better estimate of the centroid, the maximum pixel is found over a five pixel range about this initial value and, if different, replaces the inital one. In vectorized notation, for a given pixel $j$ about skyline $i$ this can be written as:

$$
\sum_{j=i-5}^{i+4}\left(x c_{i}=x c_{i}+\left[q\left(x_{j}\right)>q\left(x c_{i}\right)\right]\left(x_{j}-x c_{i}\right)\right)
$$

where $q\left(x_{j}\right)$ is the sky value at pixel $x_{j}$ and $q\left(x c_{i}\right)$ is the sky value at pixel $x c_{i}$, $x_{j}>x c$ is an implicit conditional, equalling one if true, and zero if false. The weighted centroid is computed about the maximum pixel position and that value taken to be the final, true centroid pixel position of skyline $i$ :

$$
x c_{i}=\frac{\sum_{j=x c_{i}-4}^{x c_{i}+5} x_{j} p_{j}}{\sum_{j=x c_{i}-4}^{x c_{i}+5} p_{j}}
$$

Various things are plotted up at this stage which allow the user to inspect the fit in progress and make changes to it as necessary. A colorful multi-panel plot comes up first, which shows the spatial profile for each skyline, overplotting the exact range used in the 

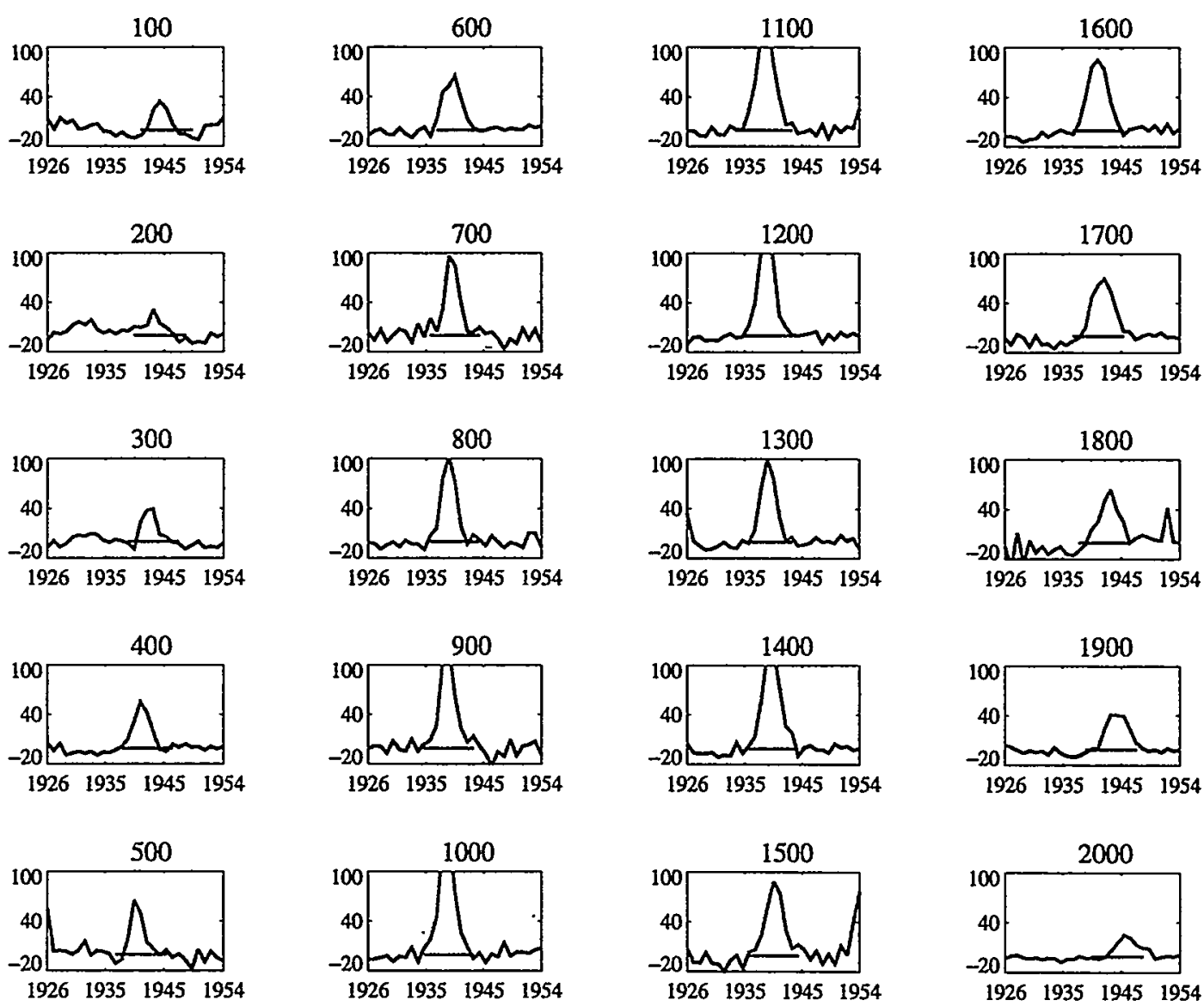

Figure 4.5: Cross-sectional view of the spectrum of a bright object sampled at 20 different positions along the dispersion direction, from pixel number 100 to 2000 . In each panel the spatial profile is plotted up as counts vs. pixels in the spatial direction centered on the object. This is the first plot which appears as part of the 'wavecal' code. Note that the algorithm does a fairly-good job of following the curvature across the full dispersion range. 
weighted centroid calculation, and also overlays a vertical line at the exact position of the final calculated centroid position (Fig. 4.6). If this position is obviously well off from the centroid position as measured by eye, the culprit may be a bright nearby cosmic ray or a bad column on the CCD. These deviant skylines can be excluded by the user in next plot that comes up, which gives the skyline residuals and rms of the fit. Here the user has the option of either accepting the fit as is or excluding deviant points and recalculating the wavelength solution. This is an iterative process that continues until the user is content with the fit. Using the 300 lines/mm grating, usually an rms of $1 \AA$ is considered to be an adequate fit. The accuracy increases with the spectral resolution of the data, i.e. with the density of grating used.

The final result is written out as a four column data file, giving the pixel number, wavelength, object counts, and sky counts.

\subsection{Coaddition of Spectra}

There is one correction to make to the 1d spectra in hand, which is to identify and remove the cosmic ray hits. Like spectra are compared over their common wavelength bins, and the high, deviant pixels are identified and eliminated. Once the cosmic rays have been removed, the cleaned data are then coadded, improving on the signal to noise as the square root of the number of equal exposures (see section $\mathrm{xx}$ ). If a subset of the spectra from a particular object are taken with different gratings, or over different wavelength ranges, then it may also be necessary to flux the data prior to the coaddition. This ensures that spectra taken under different parts of possibly different spectral sensitivity functions will be added together fairly.

The code which carries this out requires the user to input a list of the 1d spectra to be summed together. The user may also enter a value for the cosmic ray $\sigma$-clipping cutoff. Note that the cosmic rays on the object were not eliminated at the $2 \mathrm{~d}$ background subtraction stage. The object pixels, the continuum of which is higher than the sky, were masked out so as to not to skew the best estimate for the sky artificially upwards. In any event, cosmic rays were were not removed or replaced anywhere in $2 \mathrm{~d}$.

This algorithm can accommodate spectra taken over different, but overlapping, wavelength ranges. All of the spectra are read in to temporary arrays, and the minimum and maximum wavelength of the set found and recorded. Then each spectrum is interpolated onto a common wavelength grid, minding the wavelength limits unique to each spectrum in the set. The pixels outside of the common wavelength range, and/or with a data value of zero are flagged permanently.

The cosmic rays are flagged internal to each spectrum in a two-step iterative process (Fig. 4.7). The strong cosmic rays are flagged first. A boxcar-averaged version of each spectrum is created and pixels outside of a generous $\sigma$-cutoff are assigned flags. Then these bad pixels are temporarily replaced by the unflagged average of their immediate 

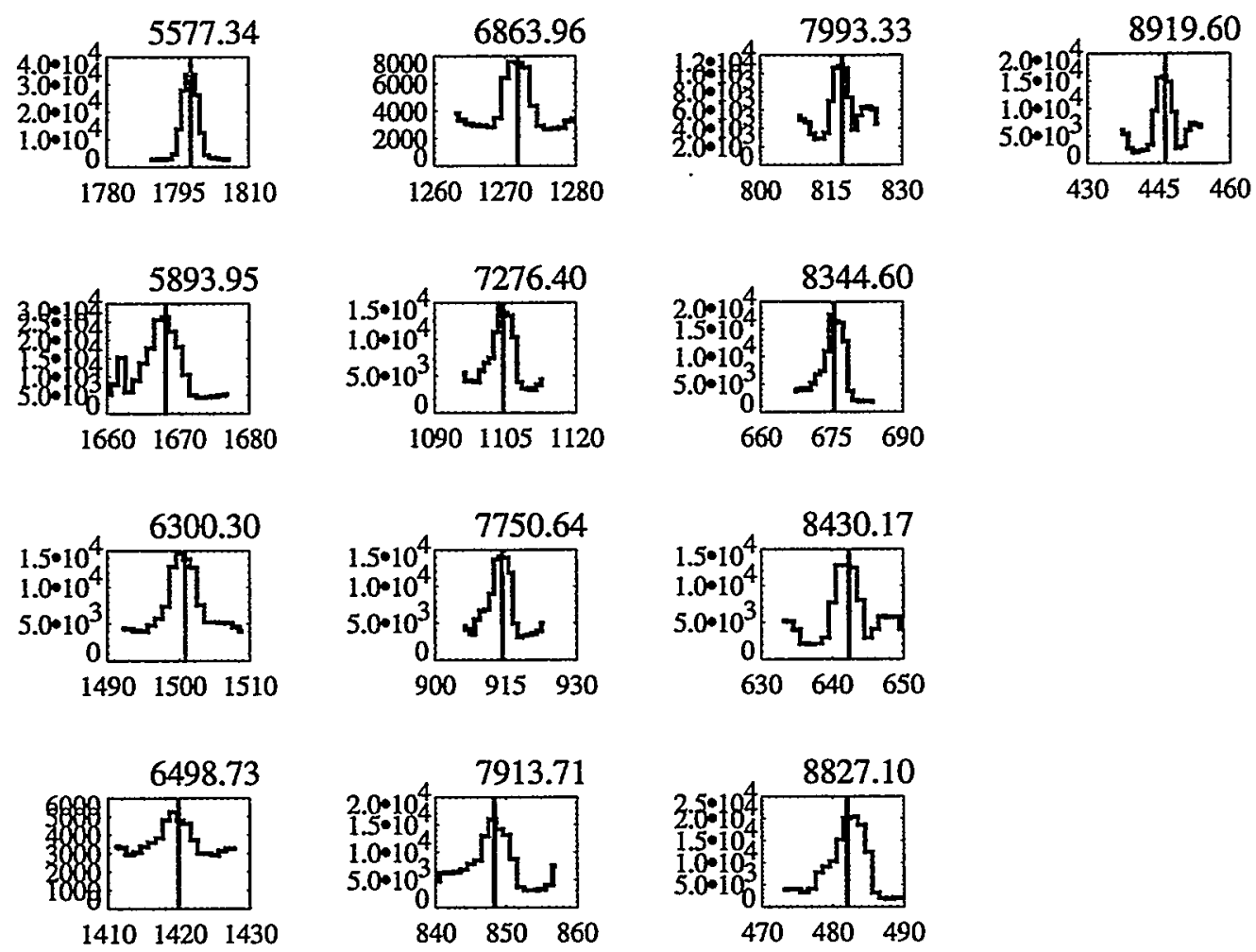

Figure 4.6: Another 'wavecal' task output. This is a plot of counts vs. pixel number in the dispersion direction for the 13 skylines which were used to wavelength calibrate this particular spectrum. The wavelengths of the skylines are given as the titles and the position of the line core is given by the solid vertical bar in each panel. 


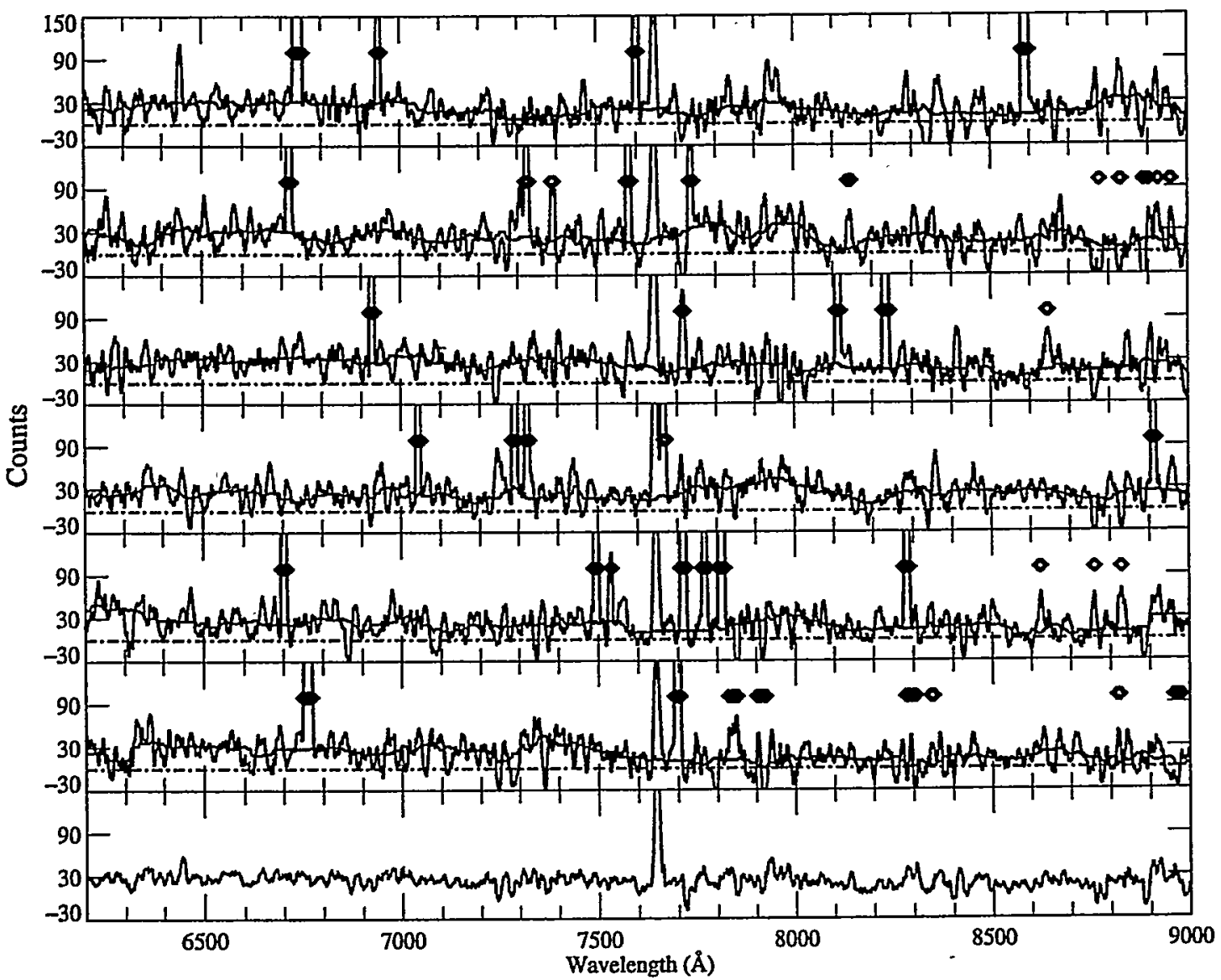

Figure 4.7: The 'spadd' spectral coaddition task output. This is a multi-panel plot of counts vs. observed wavelength for each of the 6 exposures of this particular spectrum, and the seventh panel (bottom) gives the result of the coaddition. The continuum level is marked by the solid line, against which high and low deviant points are flagged (diamond points). The dot-dashed line marks the zero level of the continuum. 
neighbors and a new boxcar-averaged spectrum is calculated for each spectrum. This new boxcar-averaged spectrum will not suffer from being artificially skewed upwards by averaging in strong cosmic rays. Now the $\sigma$-cut is repeated, forgetting the first round of flags, and new flags assigned and saved to the mask array.

After all the cosmic rays have been flagged, their wings are also flagged. Even though the cosmic ray wings fall below the critical sigma-cut threshold, their values tend to be made unnaturally-high by the typical grazing hit, and so need to be removed. This can be done in practice by removing a single adjacent pixel on either side of each cosmic ray flag.

There is a special case which needs to be treated at this point, which is where an unacceptably high number of pixels are flagged in any given pixel stack. A pixel stack is the set of $n$ pixels in any given wavelength bin. How the code works is that if $m$ or more of the pixels in a pixel stack are flagged, they will all be unflagged. This safeguards against flagging real spectral features. The value of $m$ can be set on the command line, and typically has the value $m=n-1$.

Finally, all of the good, unflagged pixels are averaged together. A 4-column data file is written out consisting of the pixel number, the wavelength (in $\AA$ ), the averaged signal (in counts), and the averaged sky (in counts). Note that the sky, which has been extracted over the same region as the object, is coadded in the same way as the object. The result is plotted up on the screen in the form of a multipanel plot of $n+1$ windows (Fig. 4.7). Each spectrum is shown as Counts/Exposure vs. Wavelength, with the boxcar-averaged spectrum overlayed and mask of flags overplotted. The final, averaged spectrum is shown at the bottom, along with the composite mask of all flags. The wavelength range, the count range, the sigma-clip factor, and number of acceptable flags in any one pixel stack $m$ may be set or changed at will on the command line.

\subsection{The Final Spectra}

The user needs a way to view and analyze the spectra that have just been harvested. The plotting software which was designed here for this task is quick and flexible, allowing for useful parameters to be set or changed on the command line.

In this task the object spectrum itself is plotted up as a two panel plot, with Counts/Exposure vs. Wavelength appearing in the upper panel with the corresponding spatially-overlapping sky spectrum plotted below for comparison. The rest wavelengths are displayed on the top axis, and the $\mathrm{x}$ pixel positions on the $2 \mathrm{~d}$ image shown on the middle axis. The redshift label appears in the upper right hand corner of the plot and the typical spectral features seen at the given redshift and over the given wavelength range are overplotted (Fig. 4.8). The parameters which may be set or changed on the command line are: the spectrum filename, the wavelength range, the counts range, the redshift, the number of pixels in a boxcar-averaged smoothing, if any, and the spectrum title. The spectra 


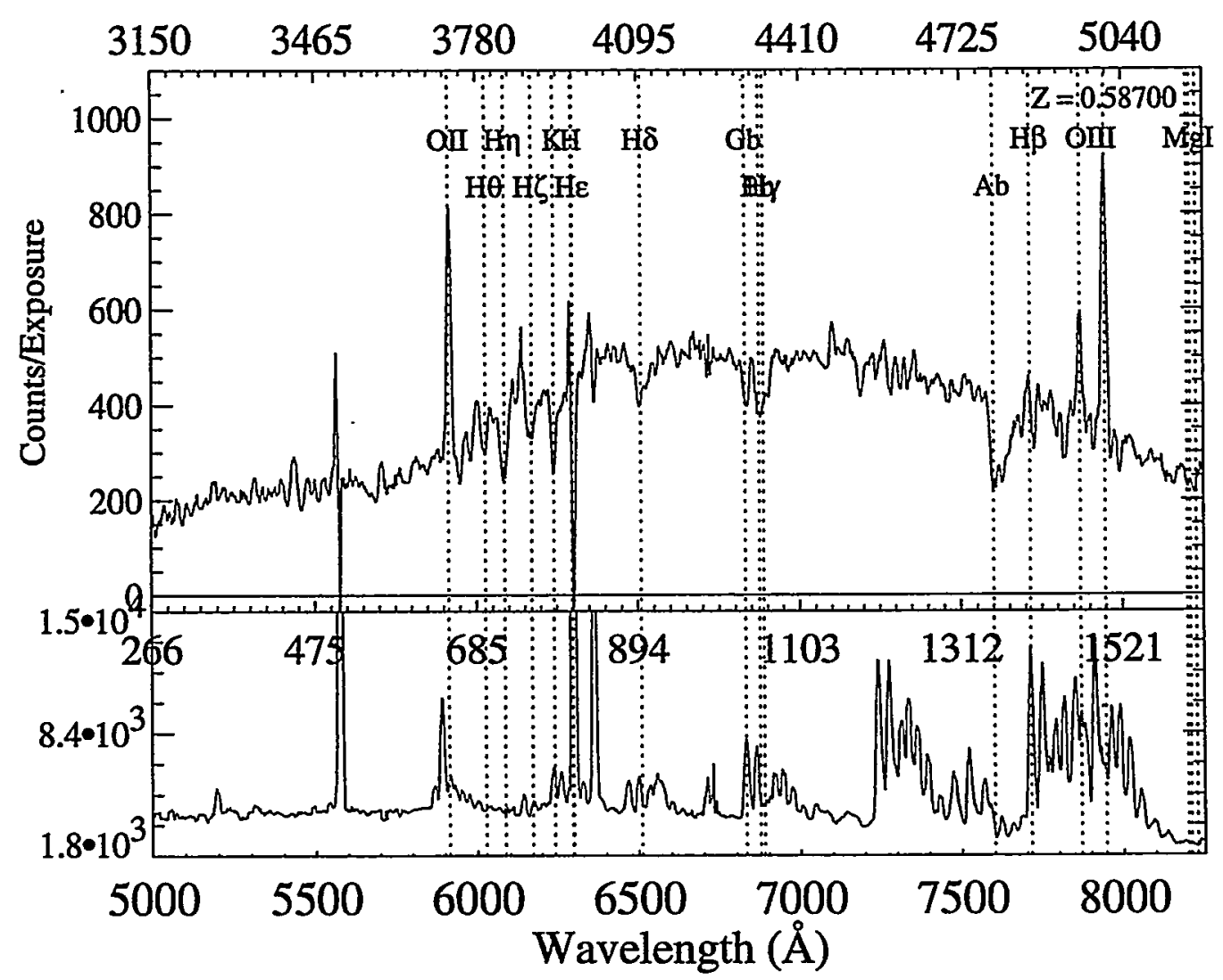

Figure 4.8: A sample coadded spectrum, in this case of 'Arc 8' in A1689. Counts are plotted against Observed wavelength on the bottom axis and rest wavelength on the top axis, with the middle axis giving the corresponding $2 \mathrm{~d}$ spectrum pixel values. For comparison, the sky spectrum is given in the bottom panel. The redshift, which can be changed on the command line, appears in the upper right-hand corner, and features are both labelled and marked with a dashed vertical line. 
in the form of Counts vs. Wavelength are adequate for finding redshifts, but eventually the counts should be converted into flux. This has been done for certain interesting targets and that process is discussed in detail in Chapter 6. 


\section{Chapter 5}

\section{Cluster Redshift Survey}

The data will be presented in two sections. In the first we present new spectra of the most interesting giant arcs. In the second the larger arclet dataset is presented and analyzed, featuring the measurement of cluster magnification.

\subsection{The Giant Arcs}

Rich, centrally-condensed galaxy clusters occasionally produce dramatic giant arcs, formed when a background galaxy is aligned with a cluster caustic. Photometry and spectroscopy of some of the more famous arcs will be presented and discussed below.

\subsection{1 $\mathbf{A 2 3 9 0}$}

An HST color image of the central arcminute of the $z=0.18$ cluster A2390 is shown in Fig. 6.10. The round, yellowish objects are cluster sequence members. They have the same color because they have roughly the same stellar content and spectrum, and therefore also have the same $K$-correction. In addition there are many blue and red arcs seen in this rather asymmetric-looking cluster. The spectra labelled $z=4.04,4.09$, and 3.75 will all be discussed in Chapter 6 . The most interesting giant arc at lower redshift is the so-called 'straight arc', appearing just below and roughly parallel to the galaxy at $z=4.04$, labelled as $A$ and $C$ in the upper right-hand panel.

The straight arc was first observed spectroscopically by Pellò et al. (1991) at $z=0.913$. The straightness and length of this arc, $13^{\prime \prime}$, led to difficulties in finding a reasonable lens model (Kassiola et al., 1992). An image of it appears in the upper righthand panel of Fig. 6.2. It appears to be disk-like, showing obvious dust lanes. We present here Keck spectra taken normal to the length of the arc at both ends, positions $A$ and $C$ in Fig. 6.2, confirming the known redshift, $z=0.913$, but also revealing that the redshift of component $A$ of this arc (in the notation of (Pellò et al., 1991) is in fact an unrelated galaxy at $z=1.033$. Thus the straight arc comprises two unrelated disk galaxies, closely-aligned 


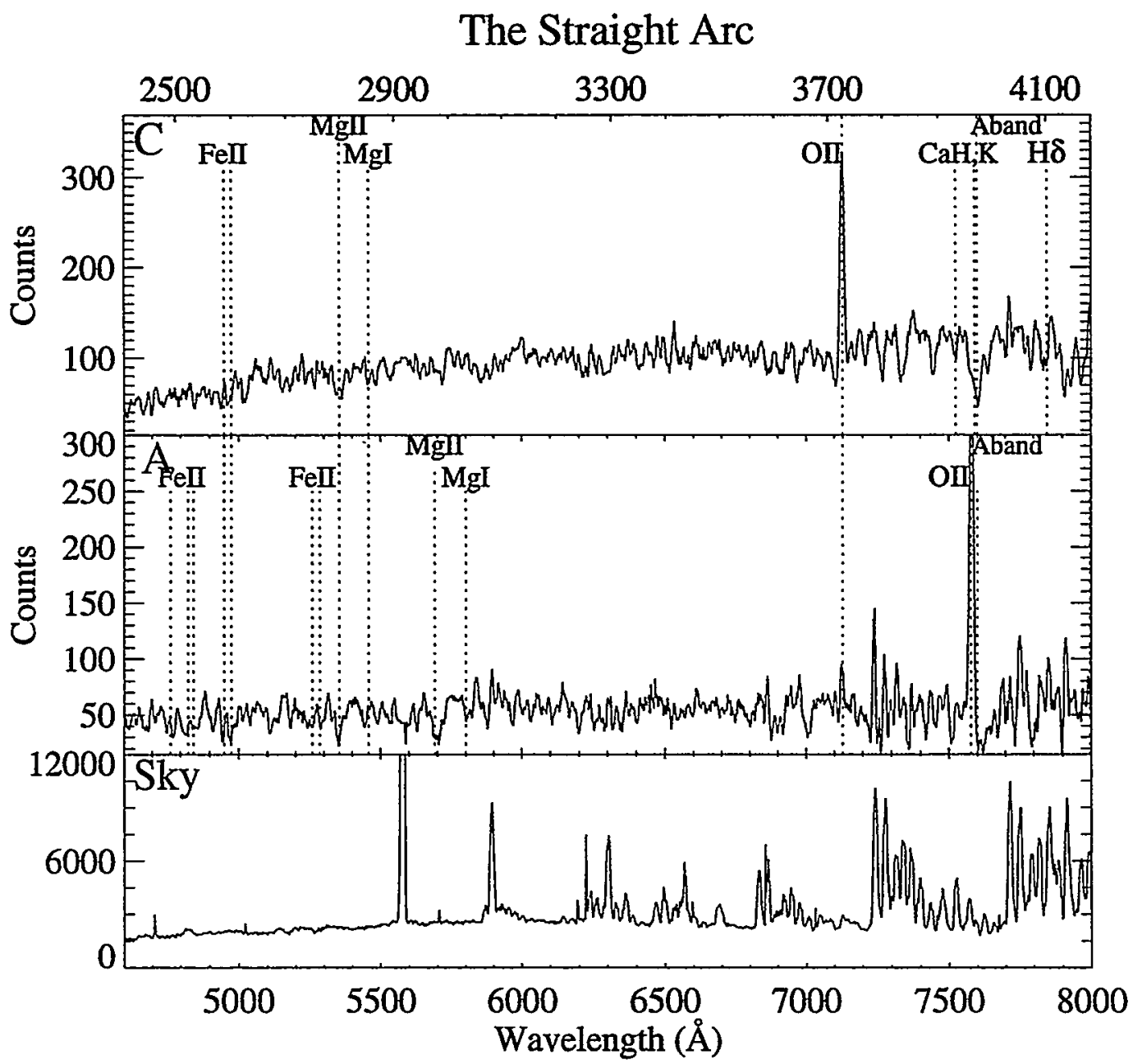

Figure 5.1: Keck Spectra taken at two different positions along the Straight Arc. Two unrelated galaxies are found at $\mathrm{z}=0.913$ and $\mathrm{z}=1.033$, corresponding to components $\mathrm{C}$ and A respectively (in the notation of Pellò et al. (1991)). In addition interstellar lines of MgII and FeII at the redshift of component $\mathrm{C}$ are seen in absorption towards the higher redshift component $A$ (indicated by dashed lines extending downward into the middle panel), evidence for an extended gaseous halo.

in projection. Interestingly, low ionization metal lines are found at $z=0.913$ against the spectrum of the higher redshift galaxy, indicating an extended gaseous halo around this lower redshift galaxy, similar to the haloes detected against bright QSOs (Bergeron et al., 1992; Steidel et al., 1994; Churchill et al., 1996), as shown by the dashed extending through both spectra. 


\subsubsection{A2218}

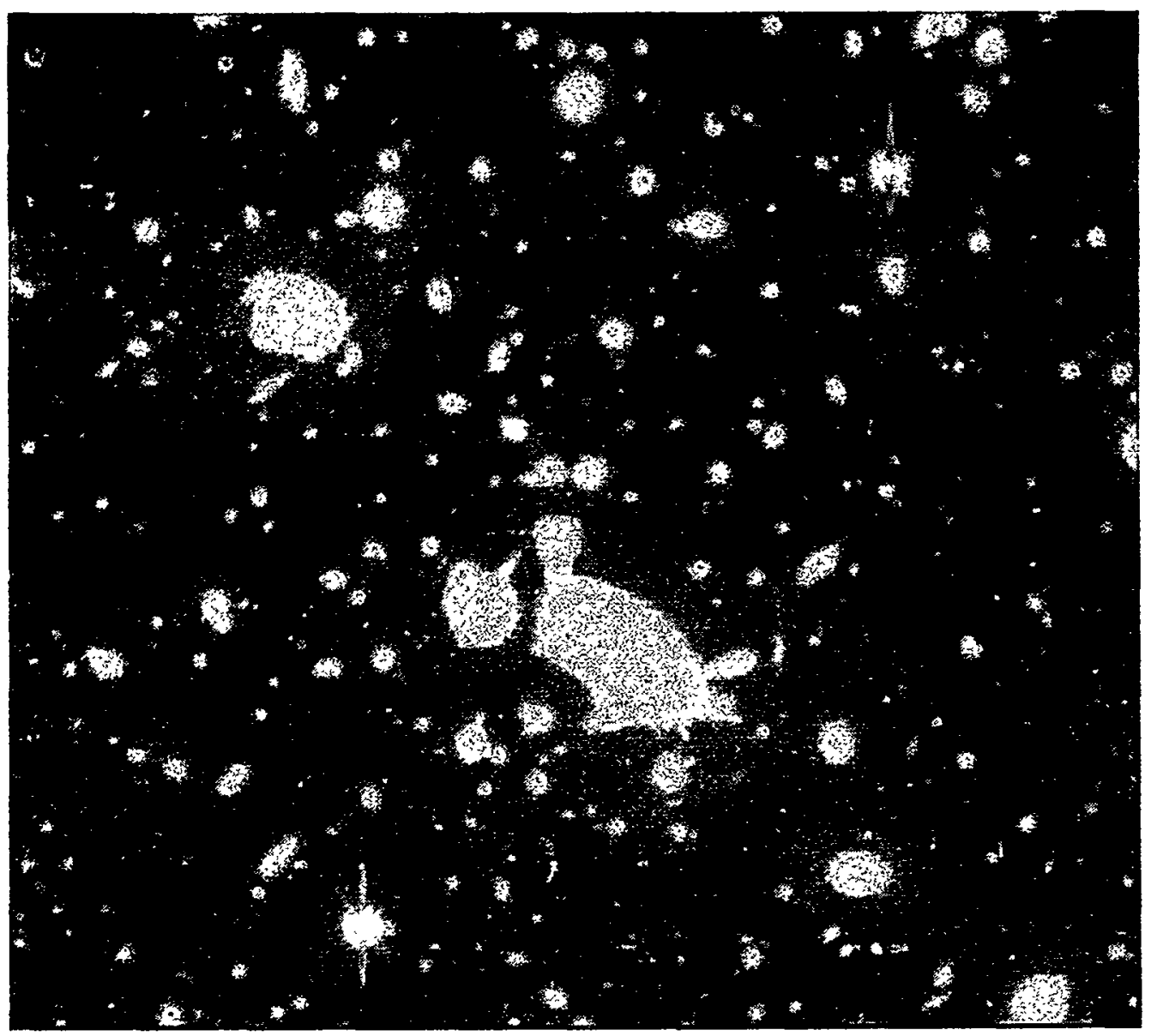

Figure 5.2: $V$ and $I$ images were taken at the CFHT and combined to make this $3^{\prime} \times 2.5^{\prime}$ color image of the cluster center of A2218, in $\sim 0.5^{\prime \prime}$ seeing. The giant blue arc seen just to the right of the central cluster galaxy, extending roughly up-down in this image orientation, is star-forming galaxy at $z=2.51$ discovered by Ebbels et al. (1996). .

A color image of the $z=0.175$ cluster A2218 is shown in Fig. 5.2. In this NTT image of the central $3^{\prime} \times 2.5^{\prime}$ the cluster members appear as the yellow spheroids, with several red and blue arcs seen about the cluster center. Perhaps the most interesting giant arc in this cluster is the bright blue arc which appears just to the right of the cluster center. It has a roughly-vertical extent as seen at this orientation (north up and east to the left) and shows interesting structure in the HST image. The redshift of this arc was predicted correctly by cluster lensing models (KESCS) and first confirmed spectroscopically by Ebbels et al. (1996) by its strong absorption features to be at $z=2.515$. It is very bright, with $V=21.4$ and $B-V=0.8$, bluer than the cluster sequence.

A Keck spectrum of this object was obtained as part of the arc survey and appears 


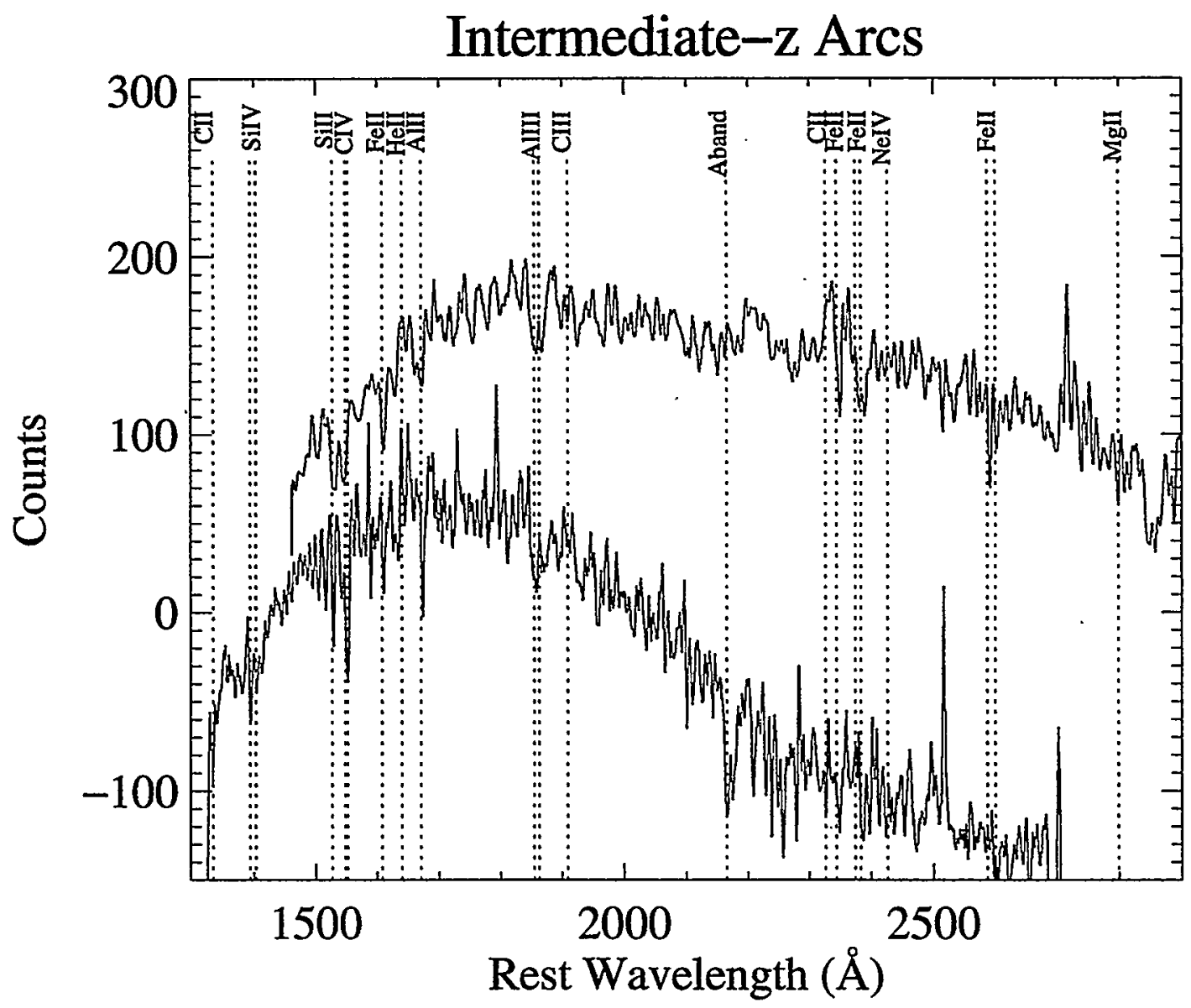

Figure 5.3: Keck spectra of two intermediate- $z$ objects. The lower spectrum is of the giant arc at $z=2.51$ in A2218, first discovered by Ebbels et al. (1996). The upper spectrum is of the giant $\theta$ arc in Cl0024, also shown in Fig. 1.4. The many strong metal lines make this a useful template for galaxies of intermediate redshift. The lower spectrum has been offset by 150 counts, so that the zero-level of the continuum is at -150 .

as the bottom curve in Fig. 5.3. Photon counts are plotted vs. observed wavelength on the bottom axis and rest wavelength on the top axis. The sky spectrum appears in the lower panel for comparison. This spectrum was taken at higher signal-to-noise than the Ebbels et al. (1996) spectrum, and extends $2500 \AA$ more redward, out to $8600 \AA$. The 
top spectrum is of the giant arc in the cluster Cl0024, which will be discussed in more detail below. The absorption lines reported by Ebbels are confirmed in this spectrum. The lines which are clearly-detected in these two examples of intermediate- $z$ objects are SiII $\lambda 1528$, CIV $\lambda 1448$ and $\lambda 1552$, FeII $\lambda 1608$, HeII $\lambda 1640$, AllI $\lambda 1671$, AlIII $\lambda 1855$ and $\lambda 1863$, FeIIlambda2344, FeII $\lambda 2374$ and $\lambda 2383$, FeII $\lambda 2587$, and $\lambda 2600$. The semi-forbidden lines of [CIII] $\lambda 1909$ and [CII] $\lambda 2326$ are labelled for reference but not detected. They would not be expected to see them in absorption. From the UV flux they estimate a star formation rate of $\approx 7-11 h_{50}^{-2} M_{\odot} y r^{-1}$ for $q_{0}=0.5$, similar to the range seen in the Steidel sample (Steidel et al., 1996a,b).

\subsubsection{Cl0024}

The $z=0.39$ rich cluster Cl0024+16 (Zwicky, 1959) is one of the finest examples of gravitational lensing. Four clearly-related images are identified around the tangential critical curve in HST WF/PC-1 data (e.g., Smail et al., 1996), and Fig. 5.5. A further radially directed image of the same source was later found in a refurbished HST WFPC-2 image by Colley et al. (1996). Our deep color Keck image of the central arcminute appears in Fig. 5.4. The four bright blue arcs are clearly-distinguished from the the redder cluster members.

For many years the redshift of this lensed source had eluded identification despite long exposures on large telescopes. The blue color and lack of optical emission lines suggested a redshift $1<z<2$ (Mellier et al., 1991). The importance of the redshift for lensing studies lies primarily in measuring the central mass and mass-to-light ratio of the lensing cluster, and in the case of $\mathrm{Cl} 0024+16$ these quantities can be measured particularly accurately because the images are so-nearly circularly-symmetric.

A spectrum of this giant arc was obtained as a part of this survey and appears as the bottom spectrum in Fig. 5.6. $F_{\lambda}$ is plotted vs. observed wavelength on the bottom axis and rest wavelength on the top axis. Two spectra are shown in this upper panel: the top one is for the giant and arc and the bottom one is of the nearby starburst galaxy NGC4214 (Leitherer et al., 1996) for comparison. Note that both spectra have a similar continuum shape and common absorption lines, which are labelled and were discussed above. From this spectrum the absorption line redshift has been measured to be $z=1.675$.

A lens model derived from assigning NFW (Navarro et al., 1995) profiles to the brightest cluster members and and minimizing the difference between locations of the three obvious features in common to the 5 total images of the source appears in Fig. 5.5. The fit favors an overall shallow profile centered on the centrally-concentrated galaxies. The best fit mass-to-light ratio is $324 h\left(M / L_{H}\right)_{\odot}$ at $z=0$ (Broadhurst et al., 1995), slightly higher than other lensing clusters (Kneib et al., 1996; Natarajan et al., 1998), but neglecting evolution reduces it by about half, and should be allowed for in making accurate comparisons between clusters. The main result of the lens model is that some degree of substructure is required in Cl0024, contrary to the conclusions of Tyson et al. (1998) It is not clear if the level of 


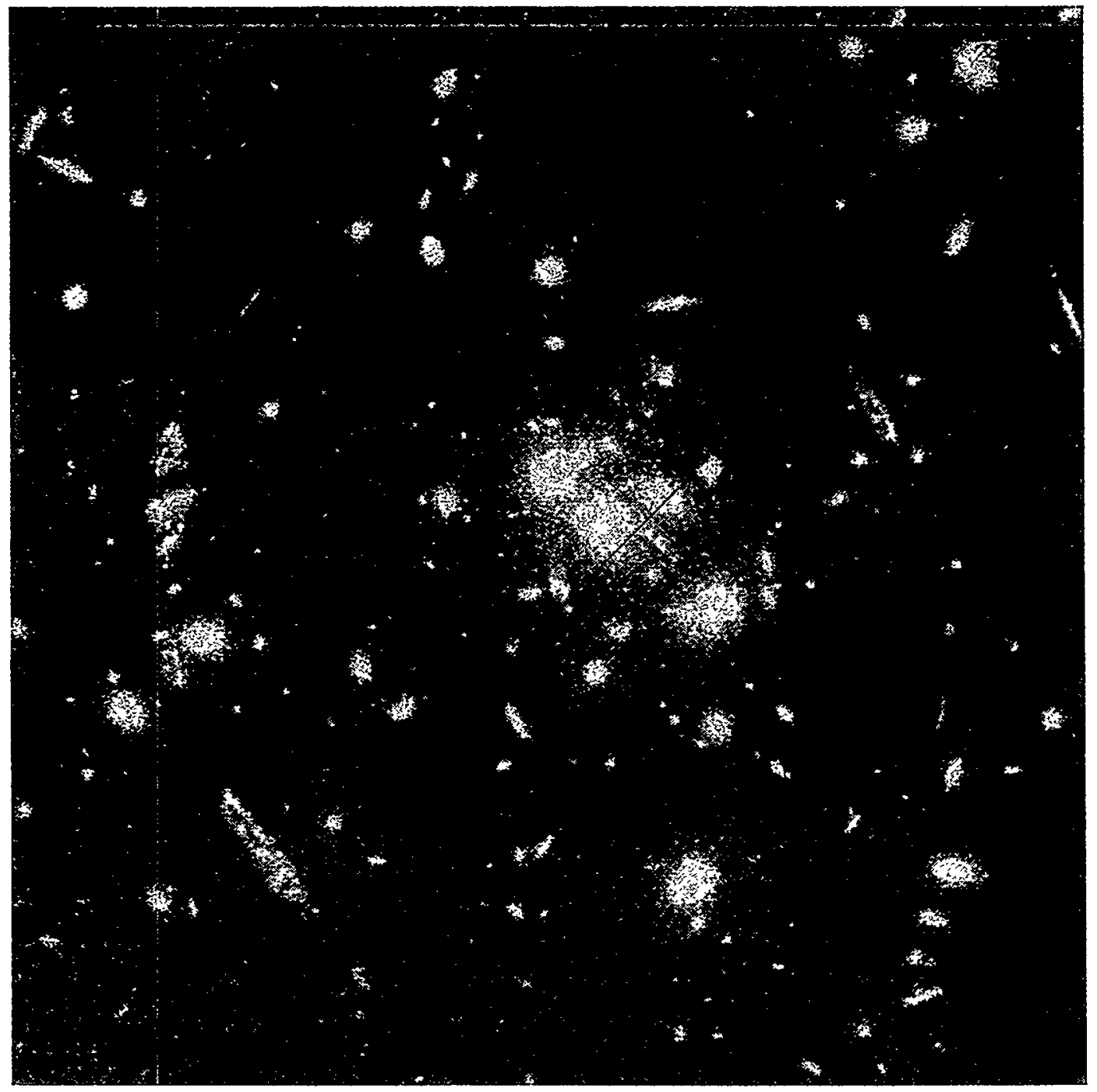

Figure 5.4: Keck color image of the cluster center of the cluster Cl0024. 


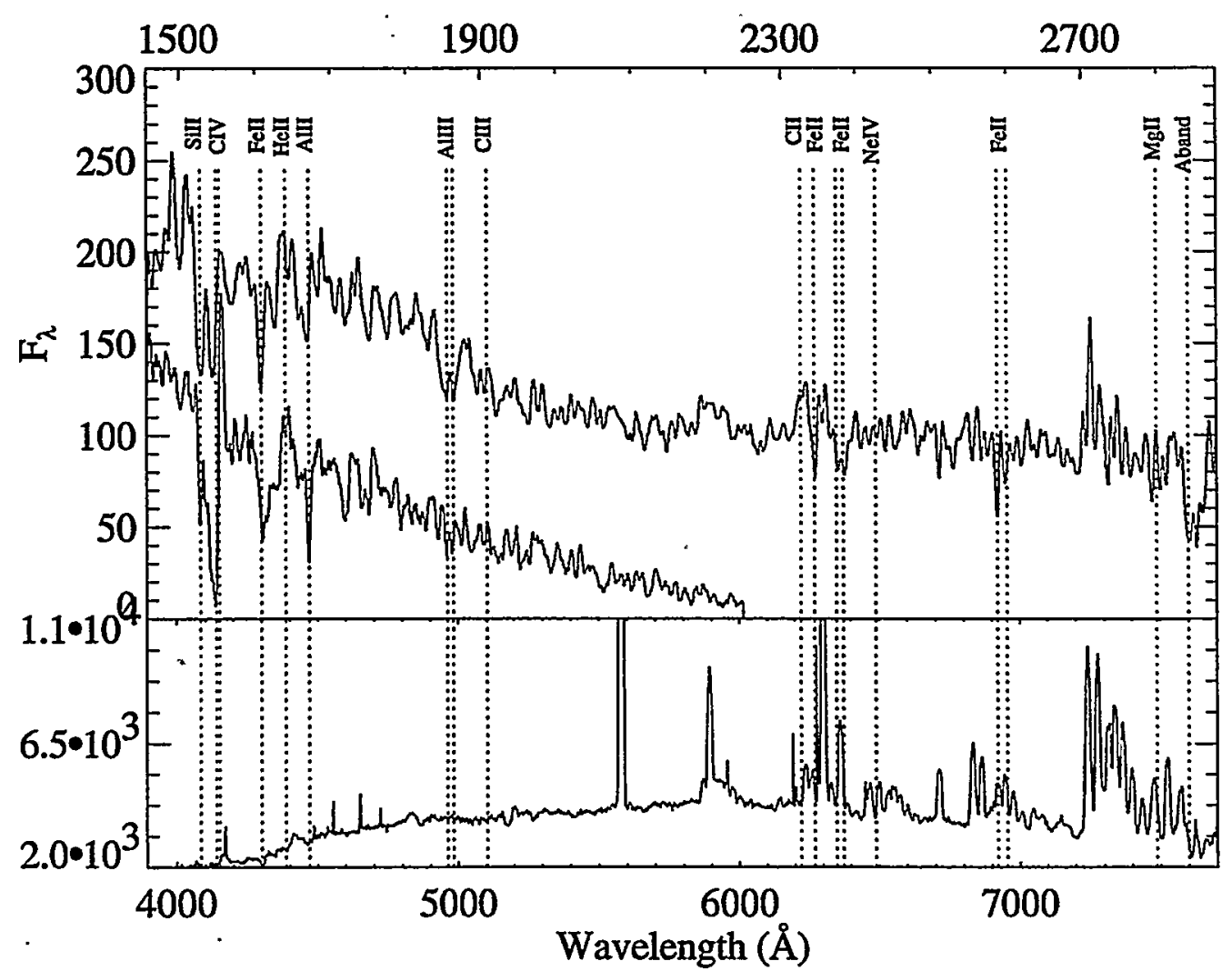

Figure 5.5: The upper curve is the fluxed spectrum of the upper HII region of arc C (fig 2b). Many weak absorption lines are visible yielding an unambiguous redshift of $z=1.675$. Note the similarity with the local "Wolf-Rayet" galaxy (lower curve) NGC4214 (Leitherer et al., 1996). The sky spectrum is also shown, in the lower panel

substructure is in excess of N-body predictions which, as Ghigna et al. (1998) point out are underestimates withint the central $50 \mathrm{kpc} / \mathrm{h}$, where the problem of 'overmerging' is still significant.

\subsubsection{MS2137}

Fig. 5.7 shows an HST $R$ band image of the central arcminute of the cluster MS2137. Note the two parallel giant arcs just below and to the left of the central cluster galaxy, and the radial arcs as well. This is probably one of the best examples of radial arcs, of interest to lens modelling for determining accurate cluster caustics (ref?).

A spectrum of this giant tangential arc appears in Fig. 5.8, where counts are plotted vs. observed wavelength on the bottom axis and rest wavelength on the top axis. The sky spectrum appears in the lower panel for comparison. The redshift fhas yet to be determined, but it is probably the highest signal-to-noise spectrum taken of this arc and so 

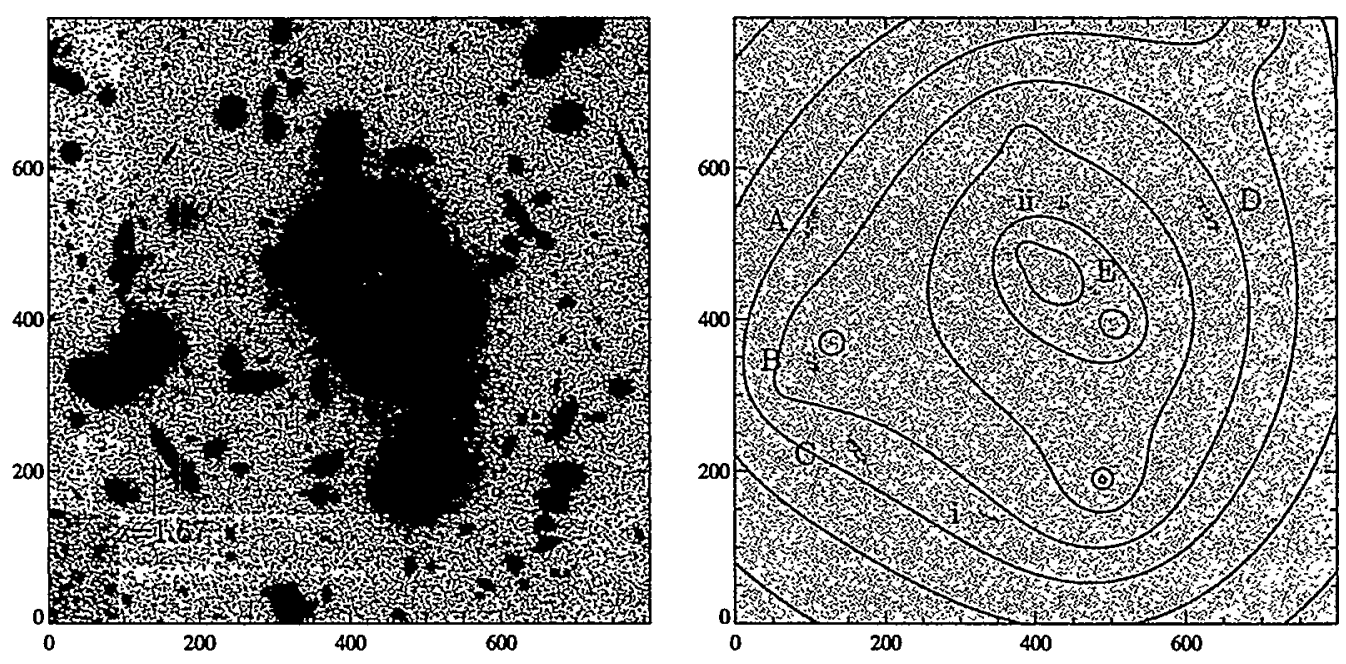

Figure 5.6: HST I band image of the central region of Cl0024 is shown on the left for comparison with the model on the right. Note the good agreement with the 5 main images (A-E). These may be improved further by hand, incorporating masses local to each image, but will not alter the conclusion that the central mass distribution traces well the location of the brightest ellipticals. A new multiple image pair is identified by the model (i,ii) at a predicted redshift of $z \simeq 1.3$. The contours represent surface mass density from $(0.6-1.2) \Sigma_{\text {crit }}$, separated by 0.1 . 


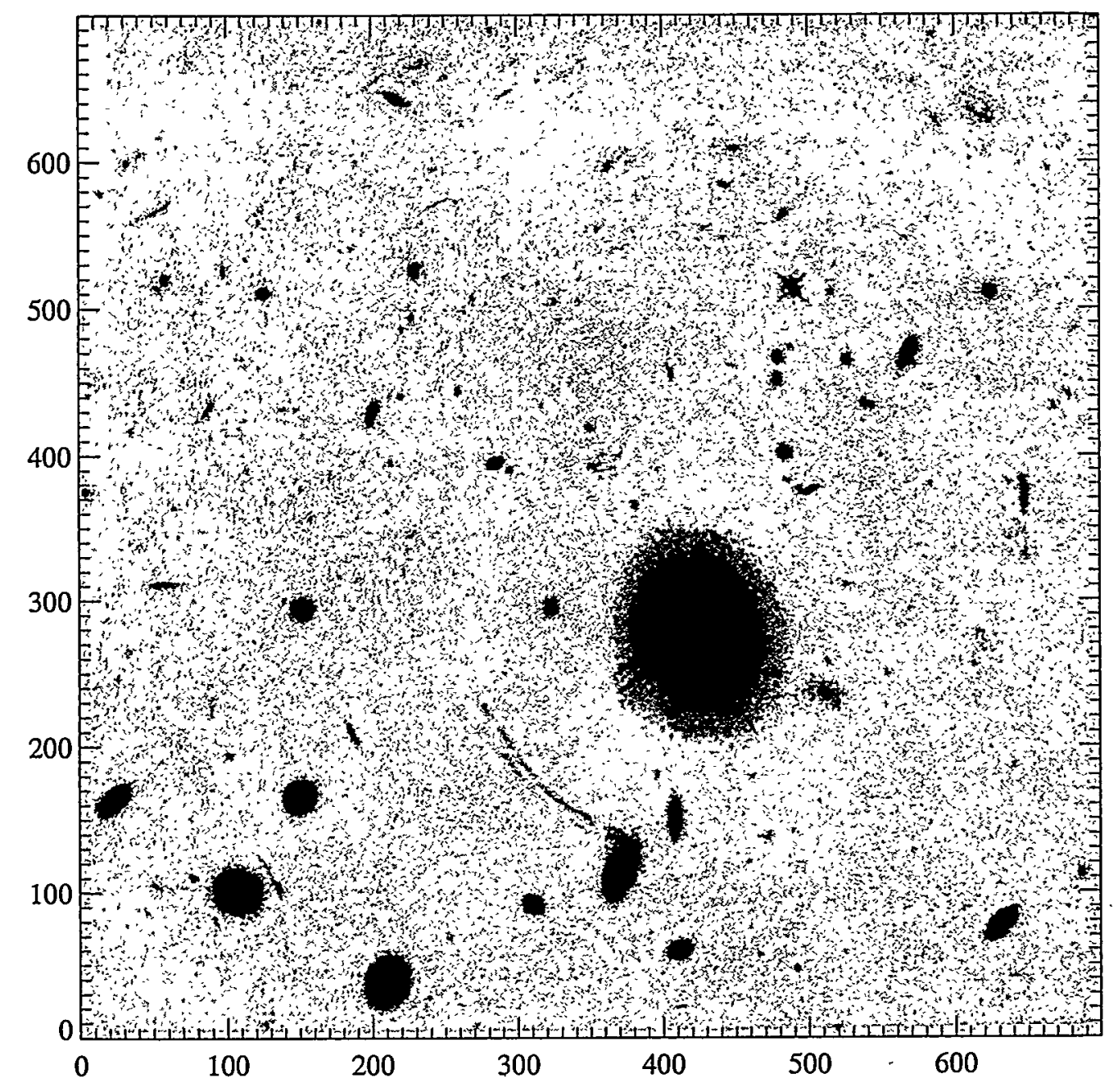

Figure 5.7: HST $R$ band image of the central arcminute of the cluster MS2137. Note the radial arcs emanating from the central cluster member, at the pixel coordinates $(380,250)$ and the set of parallel giant tangential arcs $15^{\prime \prime}$ in length centered at pixel coordinates $(320,180)$. 


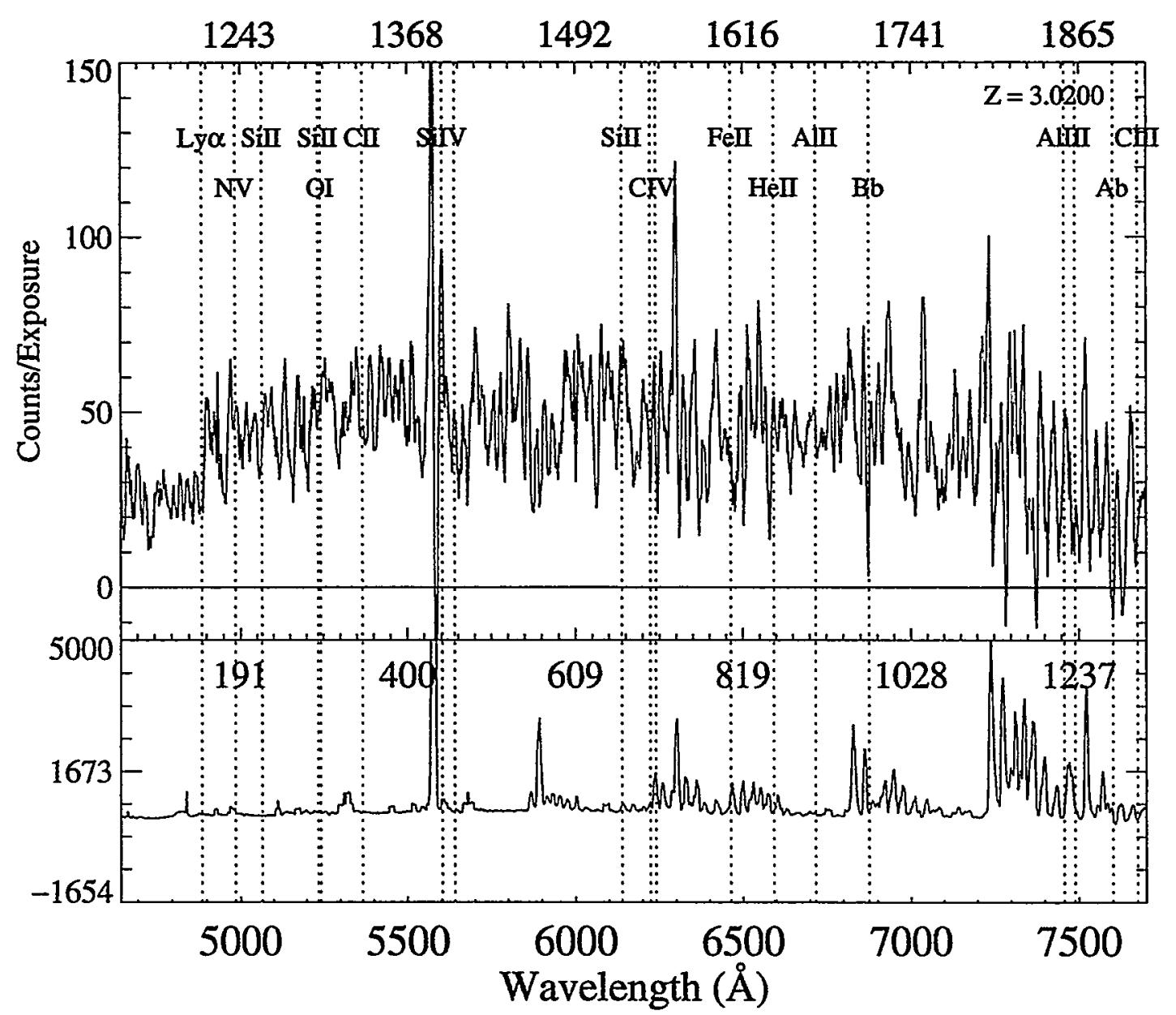

Figure 5.8: Spectrum of the giant tangential arc in MS2137. This is the first spectrum presented of this object, but there is not yet a convincing redshift for it. The best guess $z=3.02$ template laid down for inspection. Counts are plotted as a function of observed wavelength on the bottom axis and rest wavelength on the top axis. The break at $4900 \AA$ appears to be real but there are no other distinguishing features. More data is required to secure the redshift of this giant arc. The sky spectrum is also shown, in the lower panel, for comparison.

is shown out of interest. The only feature is a possible continuum break at $4900 \AA$, which would give it a best guess redshift of $z=3$. 


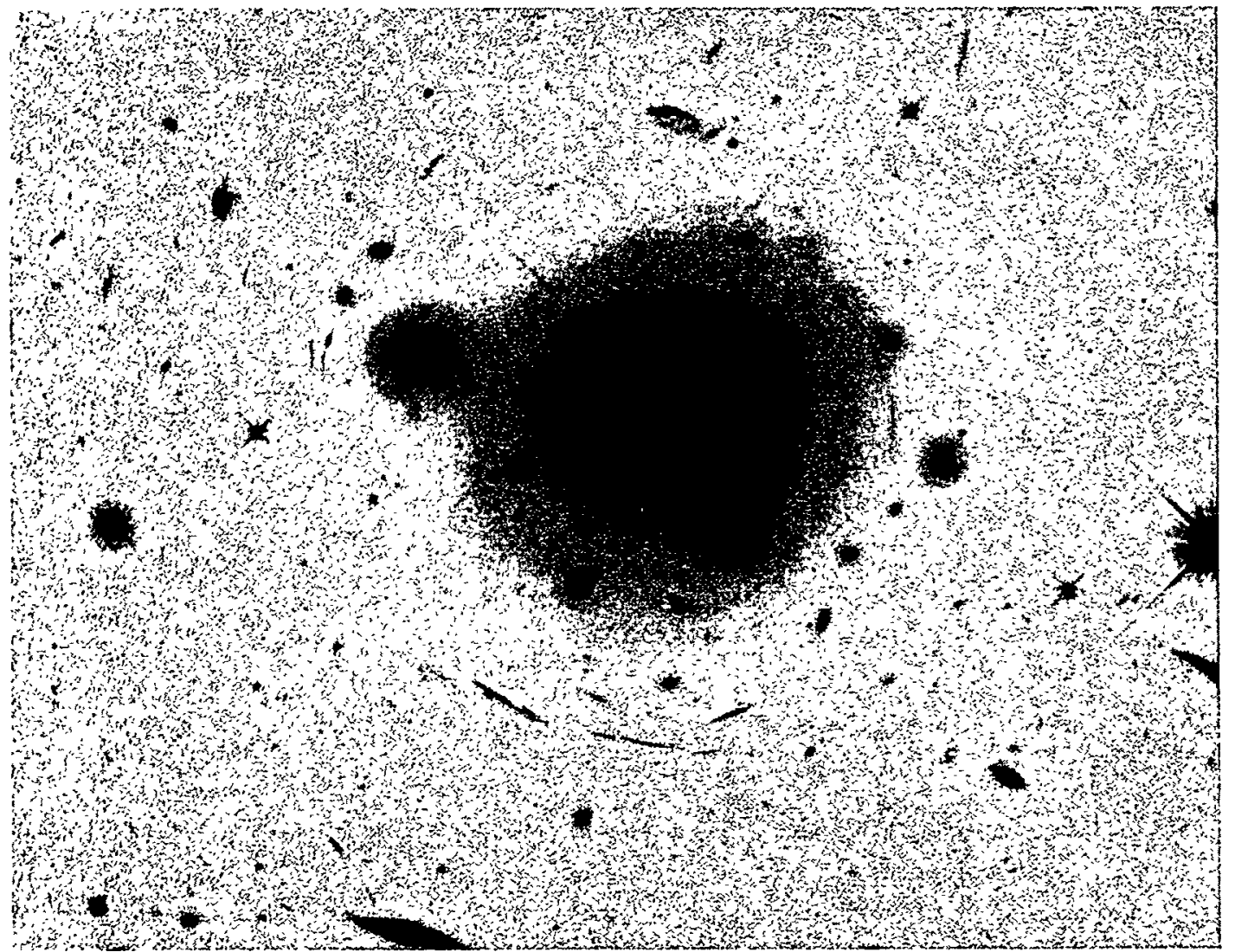

Figure 5.9: HST $R$ band image (702 nm) of the central arminute of the cluster Cl0404. 


\subsubsection{Cl0404}

Fig. 5.7 shows an $R$ band HST image of the central arcminute of the cluster Cl0404. It is a prime example of a symmetric lensing cluster. Many tangential arcs are seen about the center, some of which appear to be related. The relatively-simple structure and symmetric mass profile make this cluster a prime candidate for lens modelling.

\subsection{The Redshift Survey}

\subsubsection{The Spectra}

Every massive cluster produces many weakly-distorted images of background galaxies or arclets. If the $2 \mathrm{~d}$ projected distortion and magnification is known, then the parameterfree surface mass density can be found uniquely (Broadhurst et al., 1995). The distortion can be obtained by measuring the ellipticities of the background galaxies, which on average should trace the mass. The lensing also has an effect on the redshift distribution, through the combined effect of image brightening and field dilation. The primary aim of this thesis is to measure the magnification by the modification that it has on the number counts. To do this a redshift survey of arclets was carried out at the Keck telescope which is presented and analyzed below.

Three Abell clusters were selected for the redshift survey: A2390, A1689, and A2218. I band images of these clusters appear in Figures 4.1, 4.2 and 4.3, with the targets circled. There is a spectrum and $I$ band closeup image presented in the Appendix for every target. For readability the targets are not labelled, but for convenience the $\mathrm{x}$ and $\mathrm{y}$ coordinate values correspond to those in the image close-ups in the Appendix. Note the spatial uniformity of objects across the field, especially for A1689 and A2390.

Table 5.1 gives the log of all the spectroscopy data taken since this project began in April 1996, for these three clusters and the others which are listed. The columns are: cluster name, the date of the observing run, the mask number, the number of $20 \mathrm{~min}$ exposures (or equivalent), and the number of targets on the mask. The spectra of high- $z$ galaxies behind A2219 are shown in Chapter 6 of this thesis. A statistically-useful number of spectra (> 100) were acquired behind the lenses A1689, A2218, A2390, and C10024.

The spectra in the Appendix are organized by mask number into 11 sections and by spatial position along the mask within each section. Along with $\sim 40$ targetted objects on each mask the spectra of untargetted 'serendipitous' arcs are also shown, which may include stars, cluster members and background objects. The spectra are all plotted in the same two-panel format, as photon counts vs. observed wavelength on the bottom axis and rest wavelength on the top axis. For reference, the pixel values corresponding to the $2 \mathrm{~d}$ image appear on the middle axis. The redshift, or best-guess redshift appears in the upper right-hand corner, and the features are both labelled and marked with vertical dashed lines. The sky spectrum is plotted in the lower panel over the same spatial extraction range as 


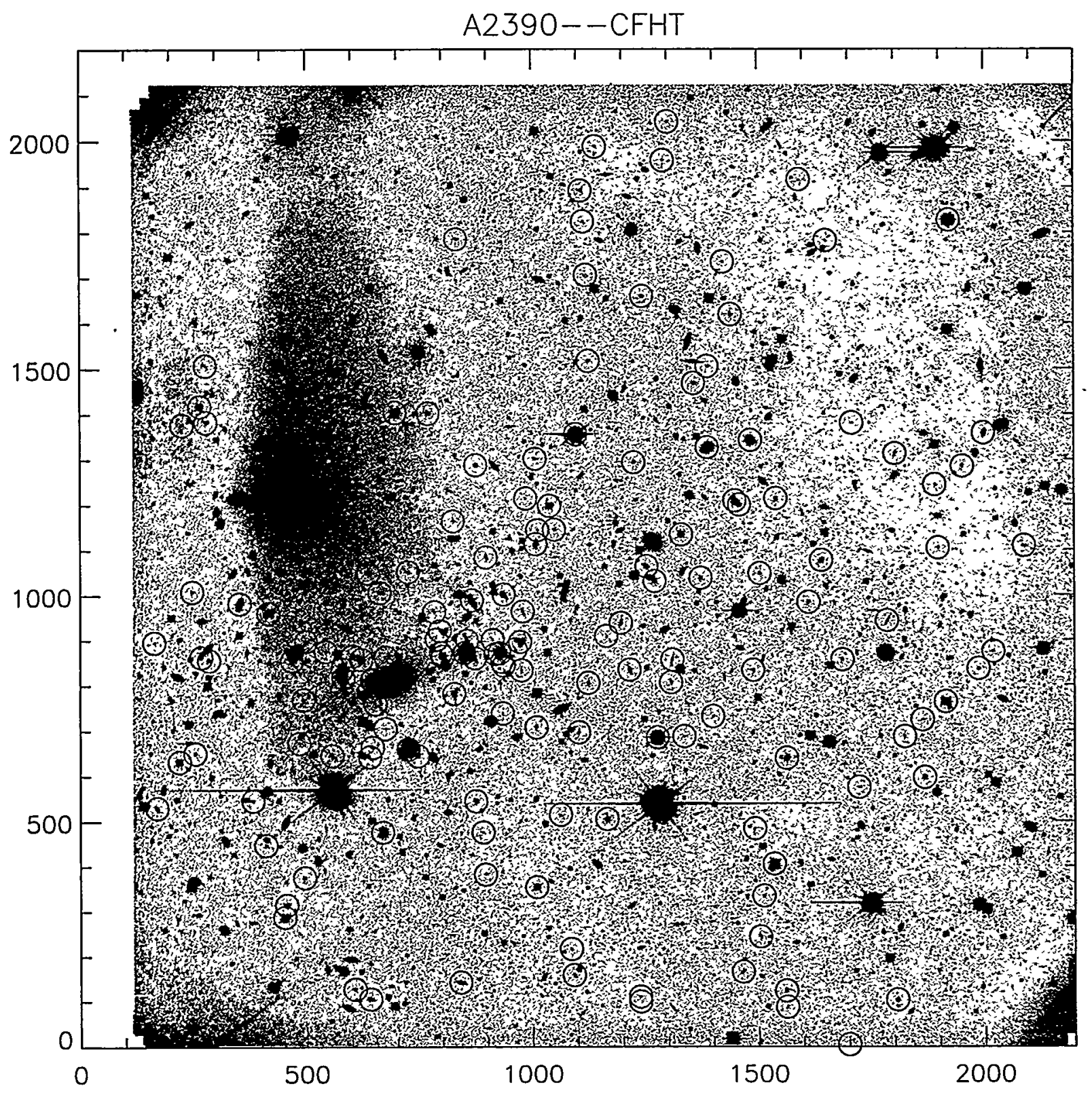

Figure 5.10: $I$ band image of A2390, taken at the CFHT. The pixel scale is 0.206 "/pix. 
A1689

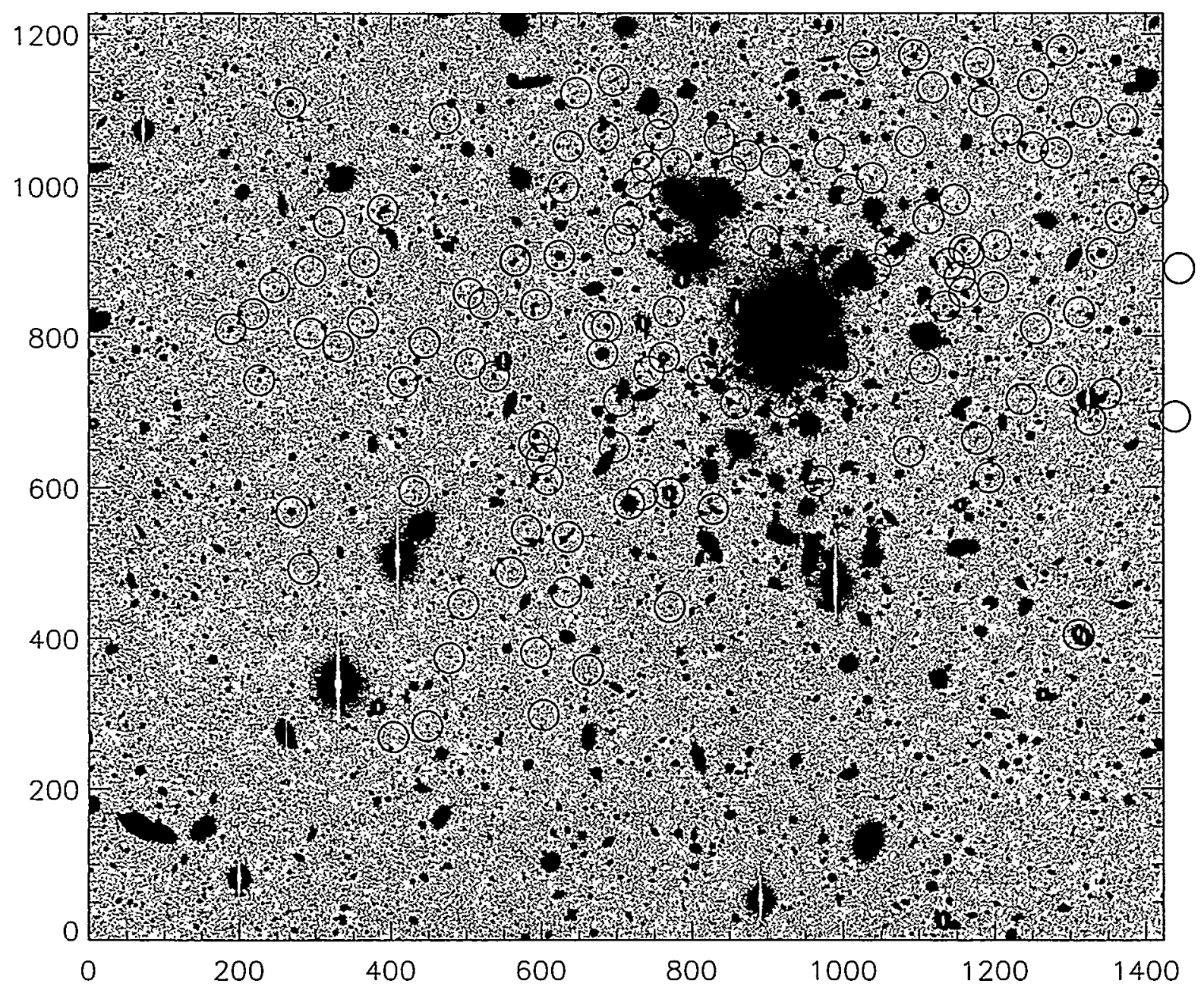

Figure 5.11: $I$ band image of A1689, taken at the NTT. The pixel scale is 0.350 "/pix. 


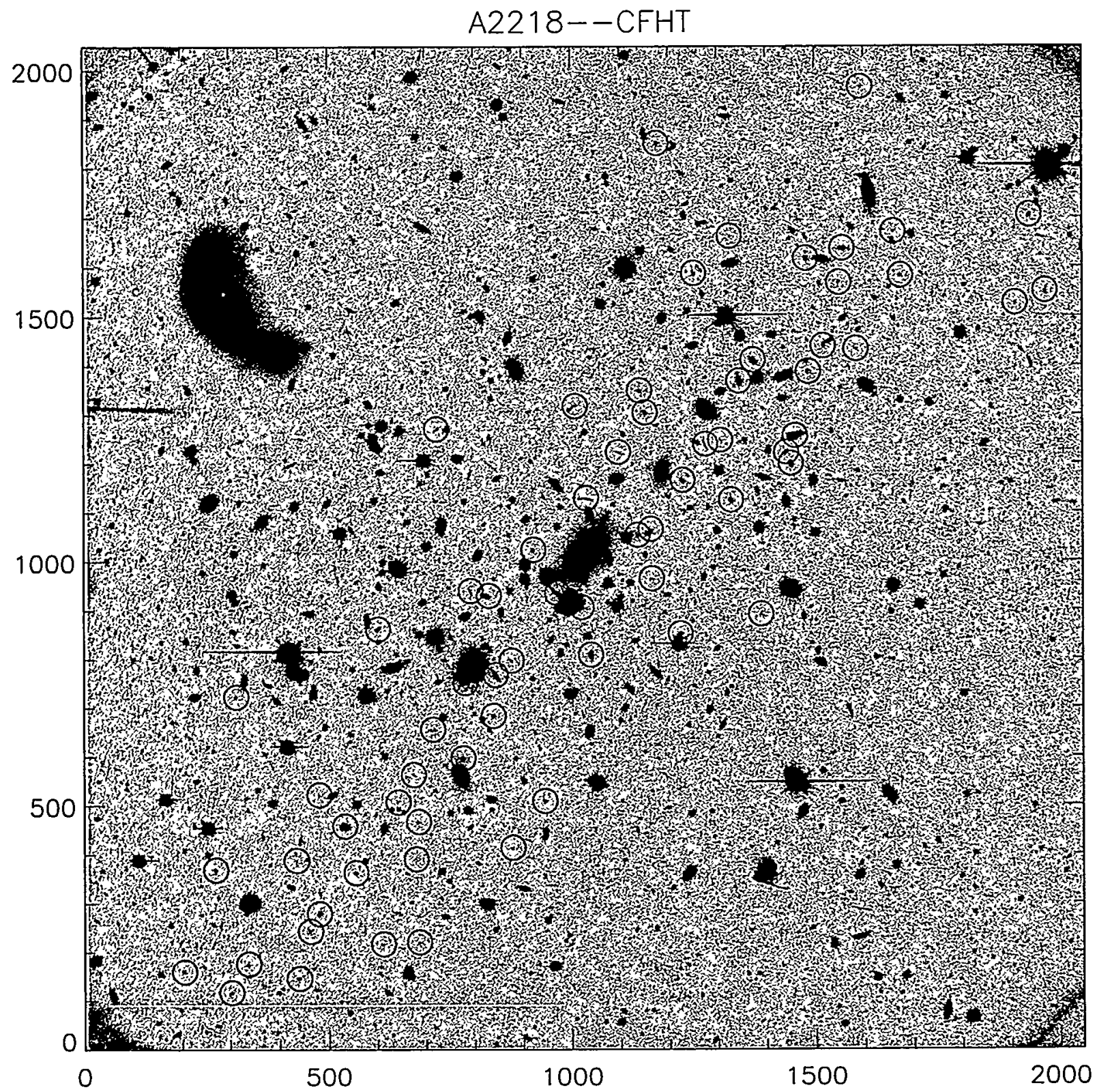

Figure 5.12: I band image of A2218, taken at the CFHT. The pixel scale is 0.206 " /pix. 


\begin{tabular}{|r|c|c|c|c|c|}
\hline Cluster & Date & Mask & \# 20min Exp. & $I_{\text {limit }}$ & \# Objects \\
\hline A1689 & $4 / 96$ & 1 & 6 & 24.5 & 40 \\
& $4 / 96$ & 2 & 6 & 24.5 & 40 \\
& $6 / 97$ & 3 & 8 & 24 & 30 \\
A2218 & $7 / 96$ & 1 & 5 & 23 & 40 \\
& $7 / 96$ & 2 & 6 & 23 & 40 \\
& $6 / 97$ & 3 & 6 & 23 & 30 \\
A2390 & $7 / 96$ & 1 & 3 & 23 & 39 \\
& $7 / 96$ & 2 & 2 & 23 & 38 \\
& $7 / 96$ & 3 & 4 & 23 & 39 \\
& $6 / 97$ & 4 & 5 & 23 & 37 \\
& $8 / 97$ & 5 & 6 & 23 & 35 \\
C10024 & $8 / 97$ & 1 & 4 & 23 & 20 \\
& $7 / 98$ & 2 & 5 & 23 & 35 \\
& $8 / 98$ & 3 & 8 & 23 & 40 \\
MS2137 & $8 / 97$ & 1 & 5 & 23 & 35 \\
& $7 / 98$ & 2 & 5 & 23 & 35 \\
A2219 & $8 / 98$ & 1 & 18 & 24 & 40 \\
AC114 & $8 / 97$ & 1 & 5 & 23.5 & 35 \\
C0152 & $8 / 98$ & 1 & 2 & 22 & 40 \\
\hline \hline
\end{tabular}

Table 5.1: Logbook of multislit Keck observations. The columns are: Cluster name, Date of the observation, mask number, number of 20 minute exposures, or equivalent, $I$ band limiting magnitude, and the total number of objects observed on the mask. 


\begin{tabular}{|c|c|c|c|l|}
\hline Position $(x, y)$ & $I$ & $V-I$ & $z$ & Comments \\
\hline$(914,1143)$ & 19.54 & 1.63 & 0.232 & \\
$(1042,542.5$ & $?$ & $?$ & 0.912 & [OII] \\
$(937,832)$ & $?$ & $?$ & 1.130 & {$[\mathrm{OII}]+[\mathrm{NeII}]$} \\
$(1060,1114.5)$ & $?$ & $?$ & 1.220 & {$[\mathrm{OII}]$} \\
\hline
\end{tabular}

Table 5.2: Longslit Data for A2390, courtesy of Hy Spinrad, Dan Stern, and Andy Bunker. The columns are: $(x, y)$ pixel position in the $2 \mathrm{~d} I$ image, $I$ magnitude, $V-I$ color, and identifying features.

the data, for inspection. A companion object image in $I$ is included with each spectrum. The slit aperture is superimposed onto the object as a dark solid line. The $I$ magnitude and $V-I$ color, if measured, are given in the upper left-hand corner. The object images for the five masks in A2390 and the two masks on A2218 is 200 pixels wide, or $\sim 41$ "on a side. The object image for Masks 1 and 2 in A1689 is 100 pixels wide, or $35^{\prime \prime}$ on a side, and for Mask 3 is 200 pixels in width, or $70^{\prime \prime}$.

Longslit observations of some objects behind the cluster A2390 were taken which compliment our survey. They are presented in Table 5.2, courtesy of Hy Spinrad, Daniel Stern, and Andy Bunker. The columns are: $(x, y)$ pixel position in the cluster image of Fig. $4.1,4.2$, or $4.3, I$ magnitude, if measured, $V-I$ color, if measured, $z$, and the identifying lines.

\subsubsection{Success Rate}

The survey generated a total of 293 objects with known redshifts and magnitudes, 269 of which have limiting apparent magnitudes of $I<23$, and 175 of them additionally are background galaxies. All this data appears in the Appendix, having been reduced to 130 pages worth of information, and it will now get further reduced to a series of a histogram plots. The first, Fig. 5.14, gives the number of objects vs. apparent $I$ magnitude as the solid line for each of the three clusters, as labelled. Those objects with a measured $I$ magnitude and a measured $z$ are given by short-dashed lines, and those objects with a measured $I$ magnitude which are behind the cluster, $z>0.3$, are given by the long-dashed lines. As can be seen from the plots, $I_{\text {lim }}=23$ for A1689 and A2390 and closer to $I_{\text {lim }}=22$ for A2218. The surpus of objects at $I=17.5$ are the cluster members, some of which were targetted for studies of internal velocity dispersion and others were obtained at no cost (serendipitous).

The next plot, Fig. 5.15, gives histograms with the same magnitude and redshift cuts as in Fig. 5.14, but for the objects in all three clusters combined. The excess of objects at $I \sim 17.5$ is more obvious in this plot. None of these bright cluster members appear in the long-dashed histogram, as expected, because they fail the $z>0.3$ criterion. Note the number counts increase steadily up to $I=22$ for the set of background objects with known redshifts and measured $I$ magnitudes (long-dashed curve). 

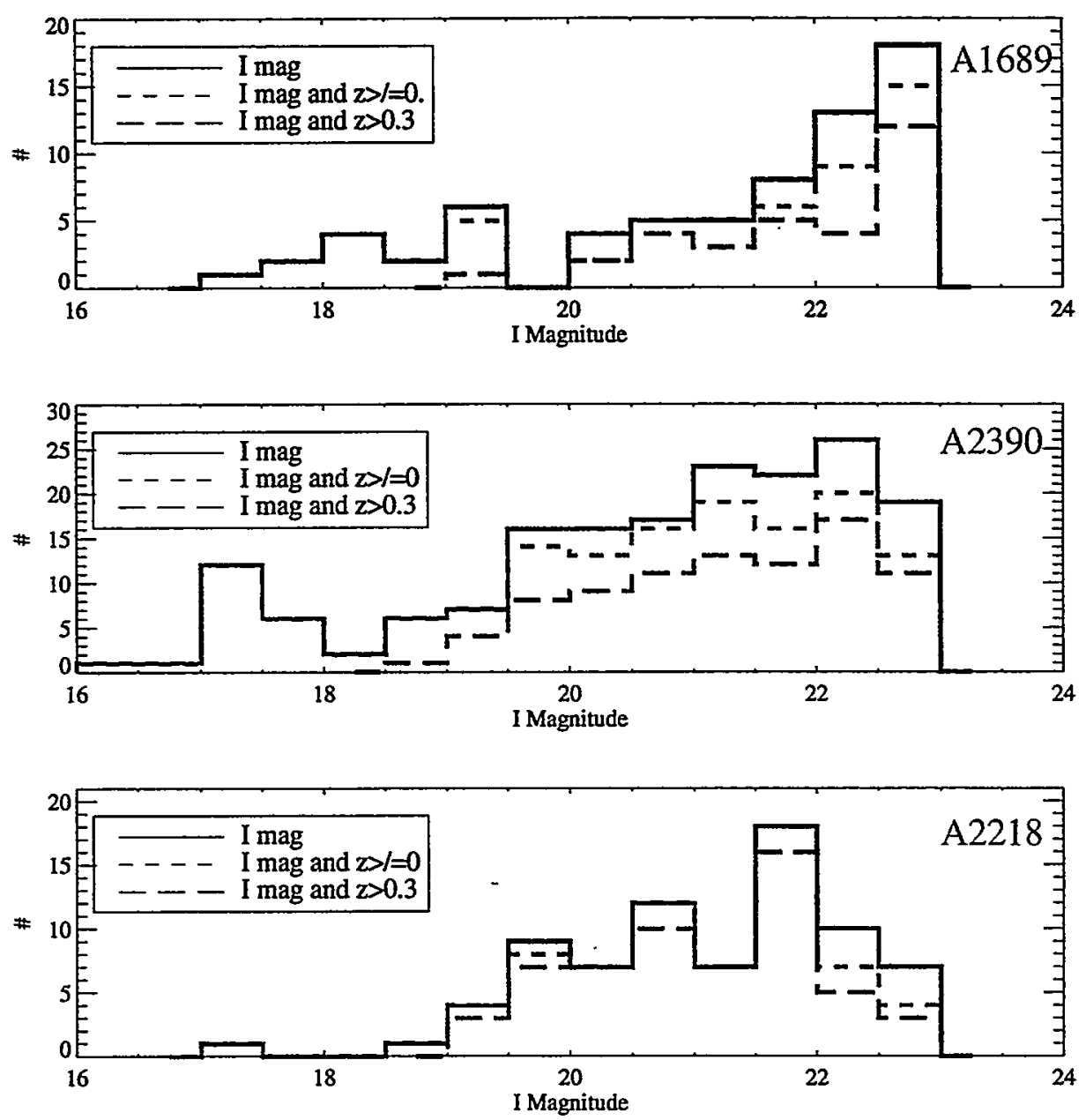

Figure 5.13: 3-panel plot showing histograms of the differential counts of the $I$ band apparent magnitude distributions for the individual clusters A1689, A2390, and A2218 . In each panel the solid histogram gives the counts for $I<23$, the short-dashed histogram gives the counts for $I<23$ and $z \geq 0$, and the long-dashed histogram gives the counts for $I<23$ and $z>0.3$. Note that all three clusters are fairly-complete down to their individual $I$ band limits, which are $I=23$ for A1689 and A2390 and $I=22$ for A2218, and that the surplus of objects at $I \sim 18$ in each panel are cluster members.

\begin{tabular}{|c|c|c|c|c|c|}
\hline Cluster & $I<I_{\text {lim }}$ & $I<I_{\text {lim }}, z \geq 0$ & $I<I_{\text {lim }}, z>0.3$ & $z$ rate (\%) & Arclet Rate (\%) \\
\hline A1689 & 68 & 56 & 31 & 0.82 & 0.46 \\
A2390 & 174 & 146 & 86 & 0.84 & 0.49 \\
A2218 & 76 & 67 & 58 & 0.88 & 0.76 \\
Total & 318 & 269 & 174 & 0.85 & 0.55 \\
\hline \hline
\end{tabular}

Table 5.3: The success rate for the spectroscopy. The columns are: Cluster name, the number of objects for which $I<I_{\text {lim }}$ the number of objects for which $I<I_{\text {lim }}$ and a redshift is known, $z \geq 0$, the number of objects for which $I<I_{\text {lim }}$ and are behind the cluster, $z>0.3$, the success rate for redshifts, or the fraction of objects with redshifts, and the arclet rate, or the ratio of background objects to the total. 


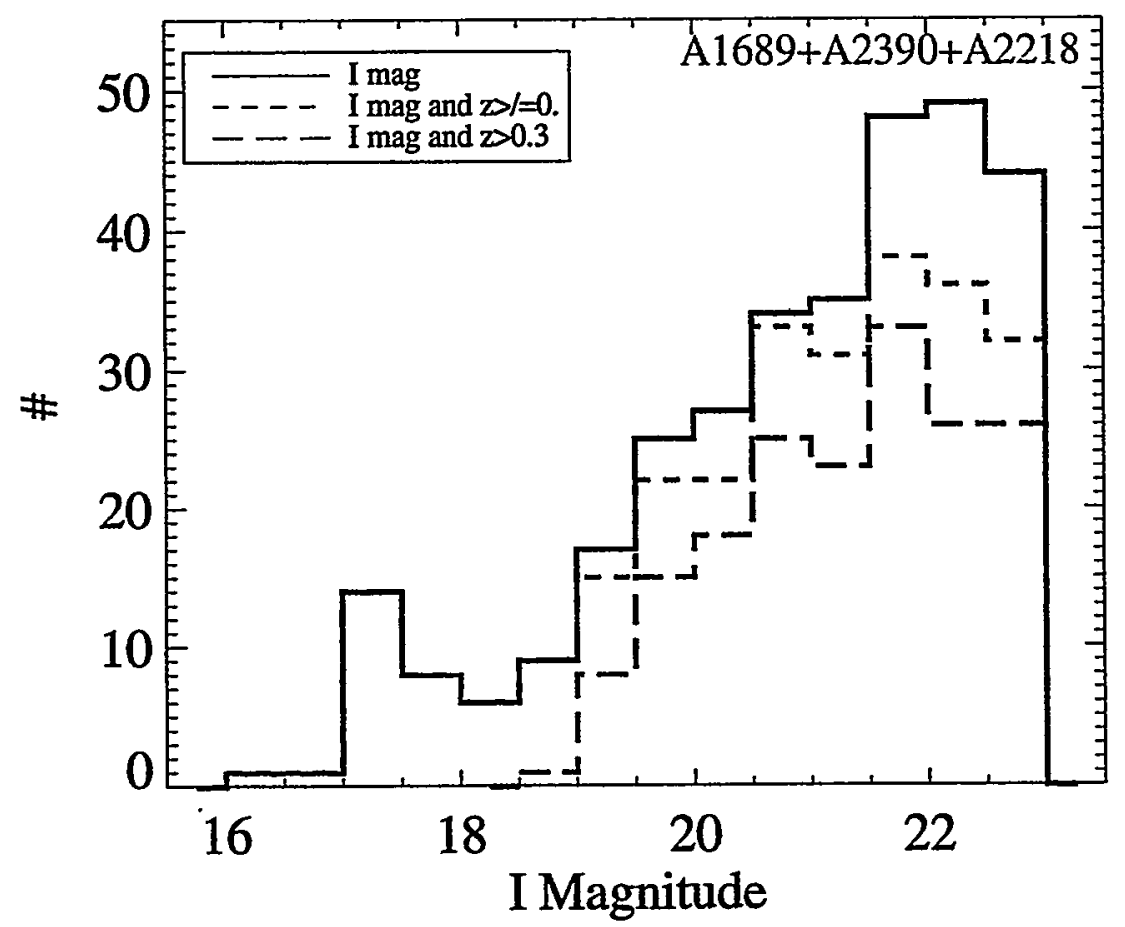

Figure 5.14: Histograms of the $I$ band apparent magnitude distributions plotted as differential counts for the sum total of all objects in the clusters A1689, A2390, and A2218. In each panel the solid histogram gives the counts for $I<23$, the short-dashed histogram gives the counts with $I<23$ and $z \geq 0$, and the long-dashed histogram gives the counts for $I<23$ and $z>0.3$. Note from this distribution that it is fairly-complete down to $I=23$ and that the surplus of objects at $I \sim 18$ are cluster members. 

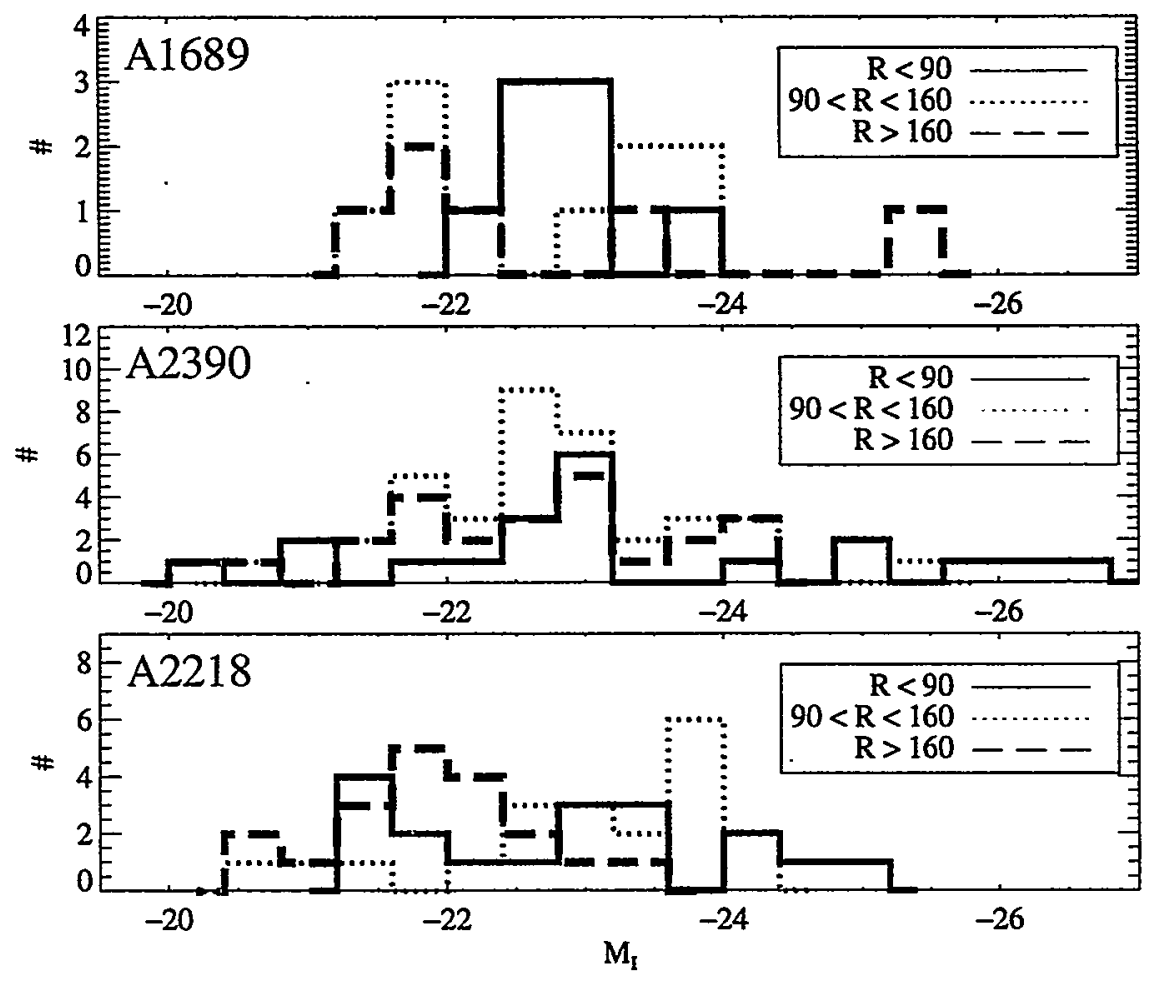

Figure 5.15: The $I$ band absolute magnitude distributions for the three clusters A1689, A2390, and A2218, plotted as differential counts. Cuts are made for different radial bins, as labelled. A systematic shift towards higher $z$ is clear for increasing radius.

Table 5.3 summarizes these results. The columns are: Cluster name, the number of objects for which $I<23$, the number of objects for which $I<23$ and a redshift is known, $z \geq 0$, the number of objects for which $I<23$ and are behind the cluster, $z>0.3$, the success rate rate for redshifts, or the fraction of objects with redshifts, and the arclet rate, or the ratio of background objects to the total. The success rates are about the same for the three clusters, $\sim 85 \%$. The highest arclet rate, however, is $76 \%$ for A2218, compared to about half for the other two clusters. As the histograms in Fig. 5.14 show this cluster had by far the brightest sample, with $I_{l i m} \approx 22$. Known cases of giant arcs were excluded from this sample, but are discussed in the first section of this chapter.

\subsubsection{Magnitude and Redshift Distributions}

The set of $I$-band apparent magnitudes, $m_{I}$, were converted to absolute magnitudes, $M_{I}$ in the following way. Given an arclet with redshift $z$, the galaxy type was found which best matches its color at that $z$, be it elliptical, spiral, or irregular. Then the $K$-correction, $K_{I}$, was applied to each $z$-galaxy type pair, which accounts for both the redshifting of the object's SED through the passbands and the cosmological expansion of the wavelength scale. Then the absolute magnitude was calculated using the formula for 


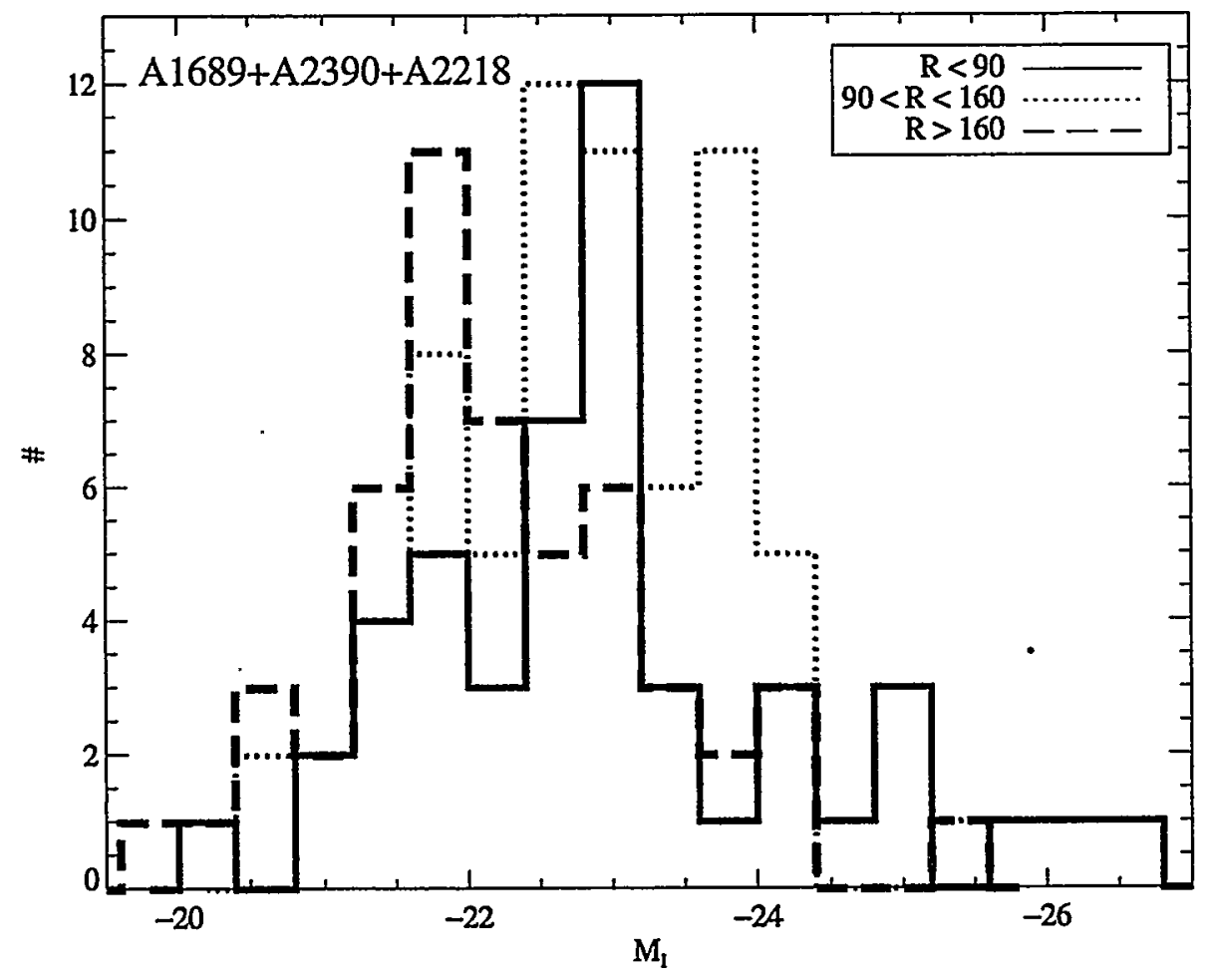

Figure 5.16: The $I$ band absolute magnitude distributions for the three clusters A1689, A2390, and A2218 combined, plotted as differential counts. Cuts are made for different radial bins, as labelled. A systematic shift towards higher $z$ is clear for increasing radius. 


\begin{tabular}{|c|c|c|c|c|}
\hline Radius (") & A1689 & A2390 & A2218 & Total \\
\hline$R<90$ & $-22.7 \pm 0.2$ & $-23.2 \pm 0.4$ & $-22.8 \pm 0.3$ & $-23.0 \pm 0.2$ \\
$\#$ & 10 & 20 & 18 & 48 \\
$90<R<160$ & $-22.5 \pm 0.3$ & $-22.8 \pm 0.2$ & $-23.0 \pm 0.2$ & $-22.8 \pm 0.1$ \\
$\#$ & 11 & 36 & 20 & 67 \\
$R>160$ & $-22.4 \pm 0.6$ & $-22.4 \pm 0.2$ & $-21.9 \pm 0.2$ & $-22.2 \pm 0.2$ \\
$\#$ & 7 & 25 & 19 & 51 \\
\hline
\end{tabular}

Table 5.4: $I$ band absolute magnitude distributions for the three radial bins listed, and the total number of arclets in each bin.

absolute magnitude:

$$
M_{I}=m_{I}-5 \log \left(D_{L} / 10\right)-K_{I}
$$

where $D_{L}$ is the luminosity distance for $\Omega=0.2$, and $H_{0}=50 \mathrm{~km} / \mathrm{s} / \mathrm{Mpc}$. The absolute magnitude distributions for the three clusters appears in Fig. 5.17. Histograms are given for each of three different radial bins, $R<90^{\prime \prime}, 90<R<160^{\prime \prime}$, and $R>160^{\prime \prime}$. Table 5.4 records the mean absolute magnitude $\bar{I}$ over these same radial bins. Fig. 5.18 gives the distribution for the three clusters combined. It is reasonable to do this, even though the clusters all have different individual masses and critical radii because to the level at which trends can be measured in the data the limiting factor is the total number of objects. Until the survey becomes larger there is not the sensitivity to distinguish between a critical curve at $50^{\prime \prime}$, as for A1689, and critical curves at $25^{\prime \prime}$, as for A2390 and A2218.

From Fig. 5.17, 5.18 and Table 5.4 there is a trend seen in increasing flux towards the cluster center. This is the result of image magnification, such that at fixed redshift one seens further down the luminosity function. It becomes more pronounced for the total on account of the larger numbers, where the distributions for $R<90^{\prime \prime}$ and $R>160^{\prime \prime}$ have a two-parameter K-S statistic, or maximum deviation of the two distributions, of 0.29 .

The redshift distributions for each cluster appears in Fig. 5.15. Histograms are plotted up in three different radial bins: $R<90^{\prime \prime}, 90<R<160^{\prime \prime}$, and $R>160^{\prime \prime}$. In addition, histograms of the sum of the three clusters is plotted in Fig. 5.16, over the same radial bins. The two sets of dashed lines represent the near-field, $R<90^{\prime \prime}$, and the far-field, $R>160^{\prime \prime}$, with the dotted-line giving redshift distribution of objects in the intermediate annulus. There is a clear trend in that the redshift increases towards the center, where the distributions for $R<90^{\prime \prime}$ and $R>160^{\prime \prime}$ have a two-parameter K-S statistic, or maximum deviation of the two distributions, of 0.39 . The two curves are model number density distributions.

The low- $z$ curve represents the unlensed redshift distribution while the higher- $z$ one shows the distribution for the best-fit magnification of $\mu \approx 4$. The mean redshifts, $\bar{z}$ are recorded in Table 5.4, for each of the three radial bins for each cluster, and for the total. 

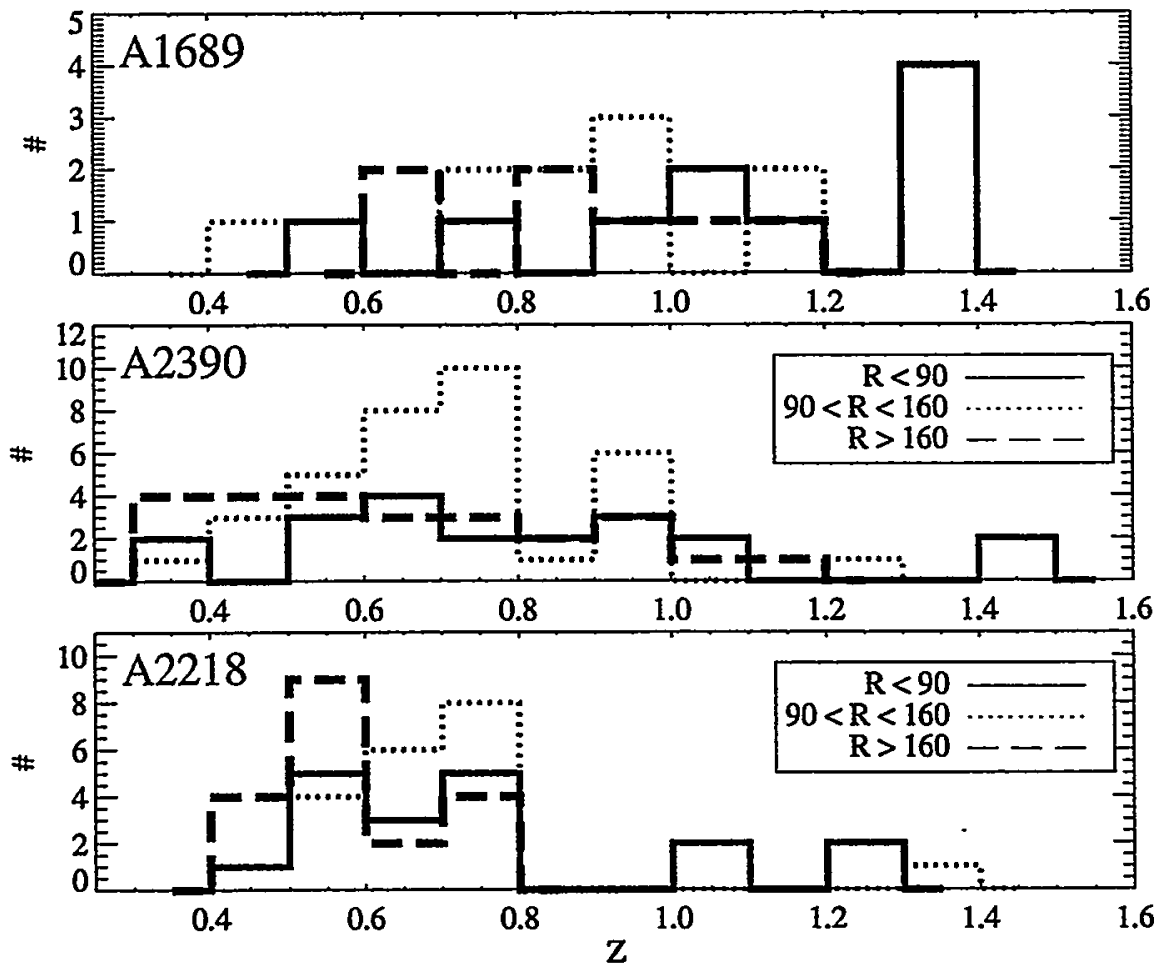

Figure 5.17: The $z$-distributions for the three clusters A1689, A2390, and A2218. Cuts are made for different radial bins for each cluster, as labelled, in arcseconds. 


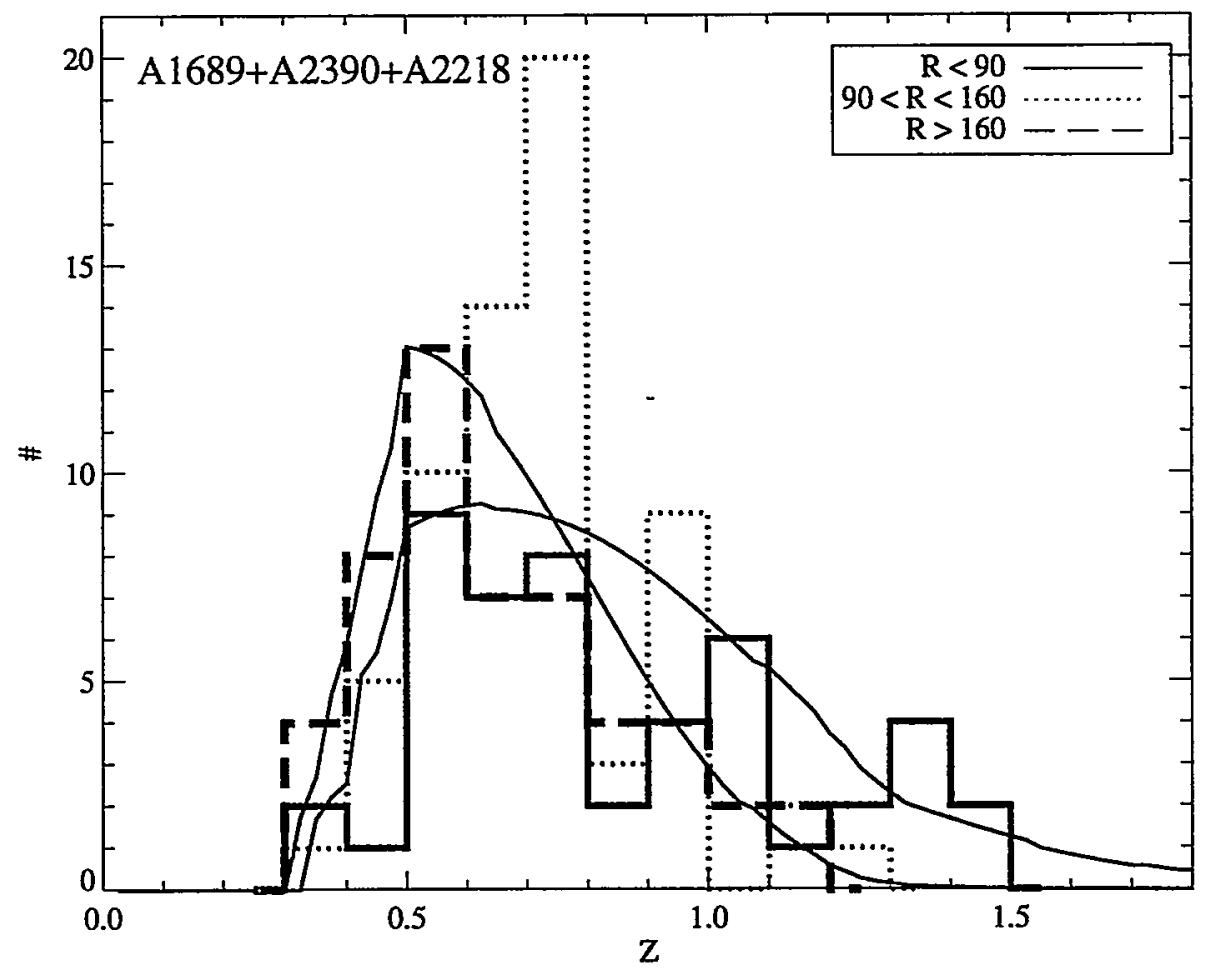

Figure 5.18: Histograms of the $z$-distributions for the three clusters A1689, A2390, and A2218 combined. Cuts are made for three different radial bins, as labelled, in arcsec. The two solid curves represent the model field redshift distributions. The low- $z$ curve gives the unlensed redshift distribution and the high- $z$ curve gives the redshift distribution for the best-fit magnification of $\mu \approx 4$. A shift is found behind the cluster relative to the field. 
The measurement of the magnification will be discussed in the next section.

The radial behavior of redshift, flux, and color is shown for each cluster in Fig. 5.20. The top row gives redshift vs. radial distance in arcseconds. The solid curve was created from a running boxcar mean fit to the points. A clear trend is seen for each cluster in that $z$ rises towards the center, as expected from magnification. The middle row gives absolute magnitude vs. radial distance. A trend is seen here too for each cluster, such that the flux increases towards the center, as expected from magnification. Finally, in the bottom row the $V-I$ color is plotted vs. radial distance. Here there is not a clear trend seen for every cluster. In A1689, where there are the fewest number of objects, a trend is seen in that the colors become bluer towards the center, as expected if the blue number counts rise more steeply than the red ones. In this case the effective increase in $L_{\text {lim }}$ caused by the magnification pulls into the sample an excess of blue objects, with the amount depending on the slope of the number counts. Note that the field dilation does not have an effect on the color, but pushes object radially outward indiscriminantly.

The combined histograms are shown in Fig. 5.18, with the running boxcar mean plotted as the solid line. In this 3-panel plot the trends of increasing redshift and flux toward the center are clearly seen, with the 'feature' near the center of each cluster in each panel not related to any particularly-interesting radial distance but just arising from the binning.

\subsubsection{Measuring Cluster Magnification}

The increase in redshift and flux towards cluster centers discussed in the previous section is new observational evidence for image magnification, clearly showing that at fixed redshift one sees further down the luminosity function behind a cluster. Here we use this information to study the lens, by calculating the magnification $\mu$ in different radial bins and comparing it with different mass models. The gradient of the magnification is sensitive to the mass profile. We measure the magnification profile here, and compare it to both the pure isothermal and NFW profiles.

As a review, the modification in the redshift distribution is related to the magnification through the magnification bias. Two opposing effects make up the bias, the magnification boosting and the field dilation, with the magnitude of the effect depending on the slope of the luminosity function at the flux limit, $L_{l i m}$. From Section 2.9 the lensed redshift distribution $N^{\prime}(z)$ can be written down as:

$$
N^{\prime}(z)=\frac{d V(z)}{\mu} \int_{L_{l i m}(z) / \mu}^{\infty} \phi(L) d L
$$

For a given $L_{\text {lim }}, \mu$ can be varied until it matches the measured value for $\bar{z}$, yielding the magnification. We measured the magnification for they survey data in this way. As a preliminary test of this model, starting with the the unlensed case where $\mu=1$, models were fit to the redshift distribution of the Canada-France Redshift Survey (CFRS). The 

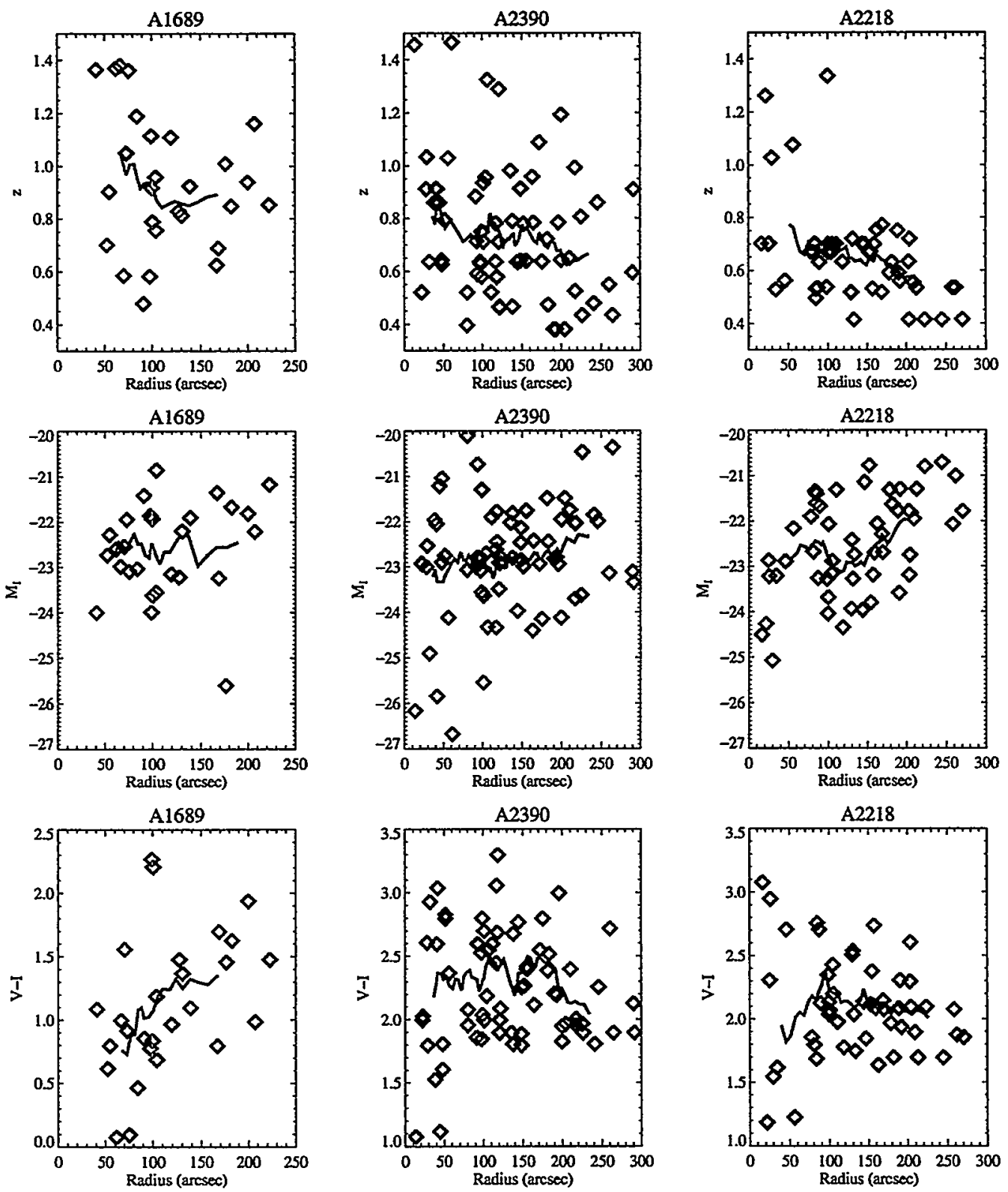

Figure 5.19: This 9-panel plot shows the redshift, absolute $I$-band magnitude, $M_{I}$, and $V-I$ color as a function of radial distance in arcseconds, for the three clusters A1689, A2390, and A2218. The set was selected to have $0.3<z<2$, and to have $I<23$. Overplotted is a boxcar mean. An object with a given $z$ and $M_{I}$ is systematically at higher $z$ and brighter towards the cluster center, showing evidence for cluster magnification. 

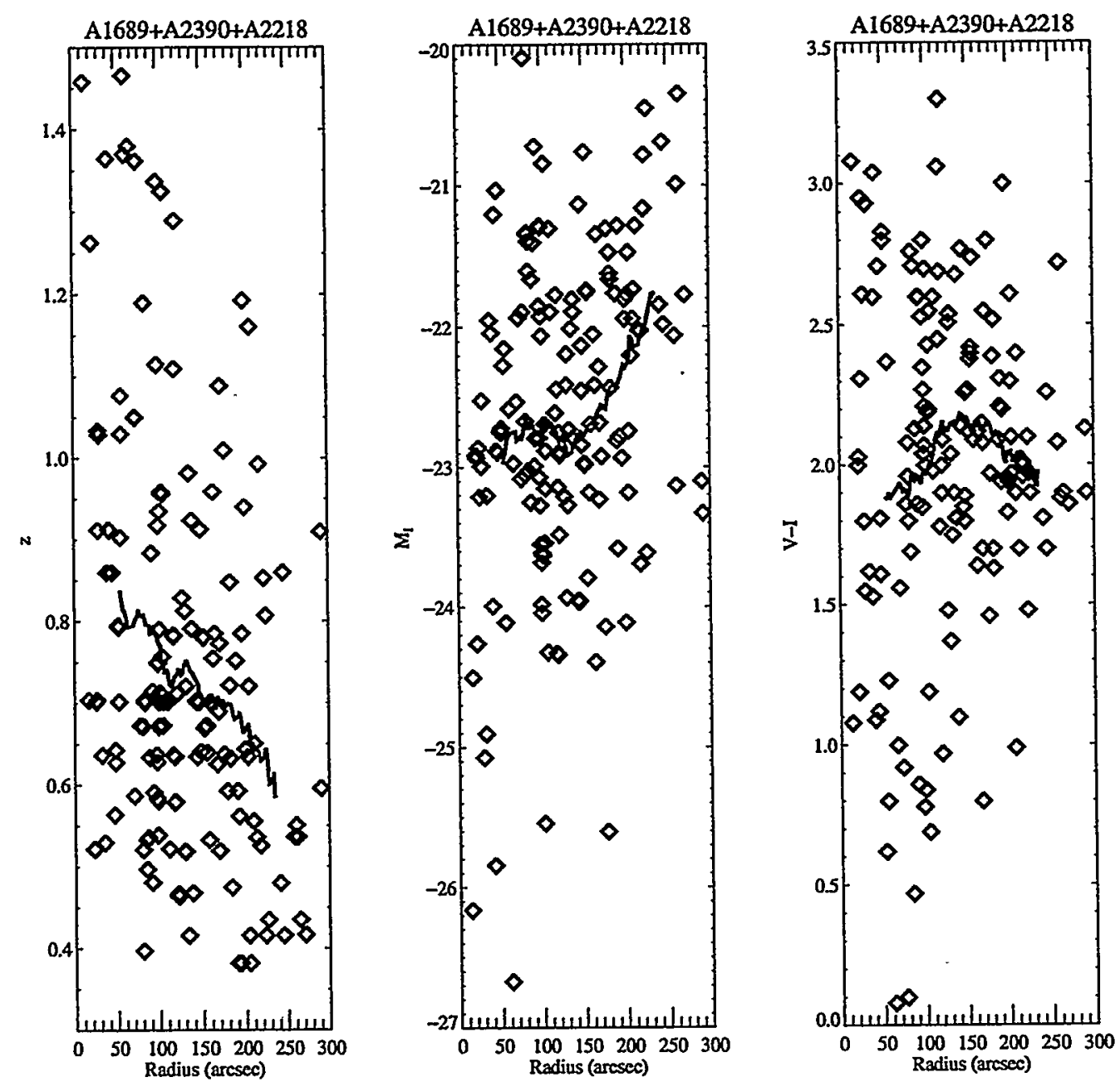

Figure 5.20: This 3-panel plot shows the redshift, $I$ band absolute magnitude $M_{I}$, and $V-I$ color as a function of radial distance in arcseconds, for the points in the three clusters $\mathrm{A} 1689, \mathrm{~A} 2390$, and A2218 combined. The set was selected to have $0.3<z<2$, and to have $I<23$. Overplotted is a boxcar mean. An object with a given $z$ and $M_{I}$ is systematically at higher $z$ and brighter towards the cluster center, showing evidence for cluster magnification. 


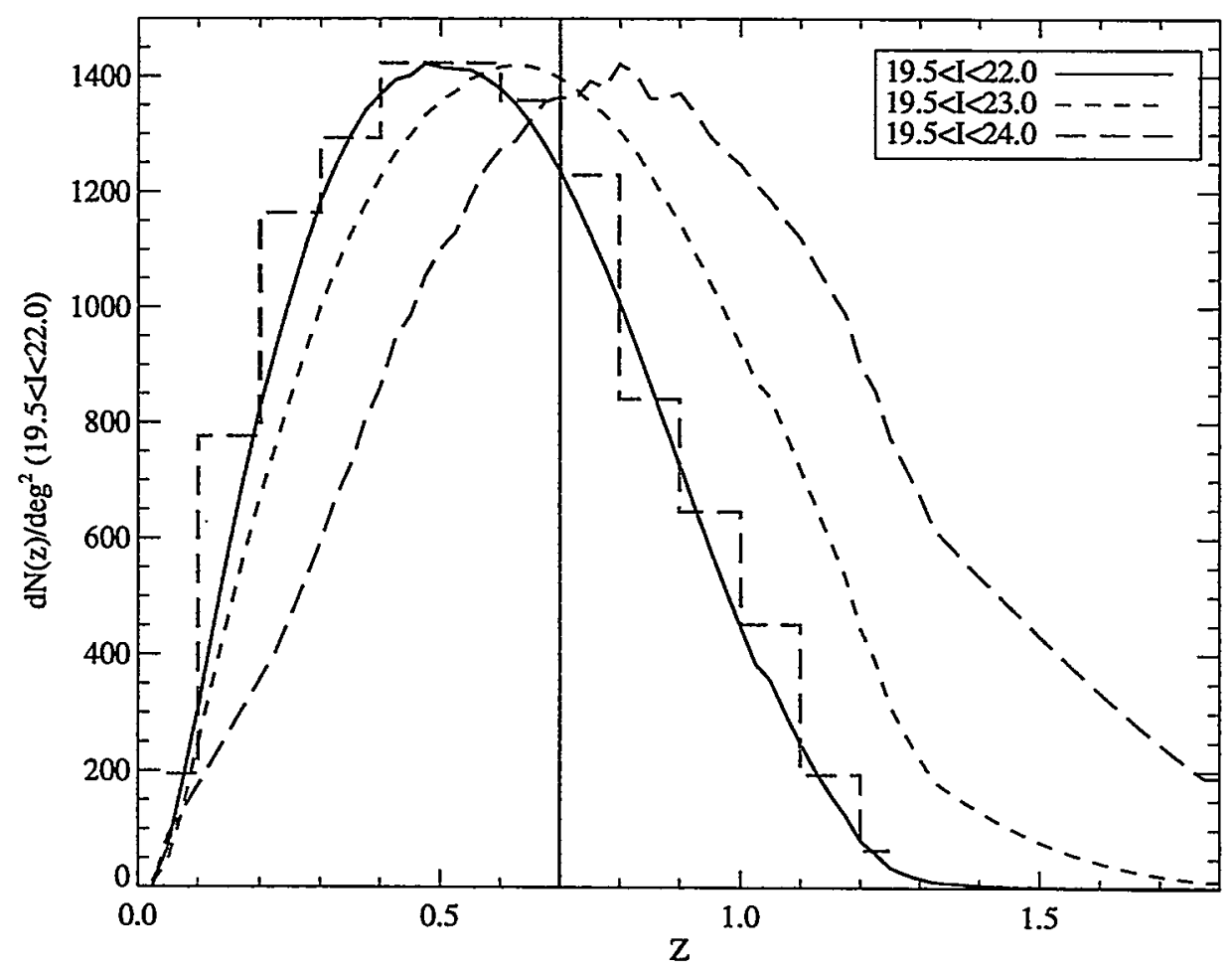

Figure 5.21: The Canada-France Redshift Survey (CFRS) is plotted as the histogram, with the three model curves showing the expected redshift distributions for all morphological types and in certain $I$ band absolute magnitude ranges, as labelled. 
result appears in Fig. 5.13, where the histogram gives the data and the curves the model fits for three different absolute magnitude ranges, as labelled. The best fit to the data is for $L_{l i m}=22$ (solid curve), matching their flux limit. The short-dashed curve gives the model for $L_{l i m}=23$, the flux limit of our survey, for which $\bar{z}=0.67$.

Only one of the three individual cluster were selected for making comparisons with the models, A2390, which has the largest number of arclets with measured redshifts. The result appears in Fig. 5.22. The left panel shows the redshift distribution vs. radial distance in arcseconds. The points give the mean redshift and standard deviation in each of three radial bins at $0<R \geq 90,90<R \geq 160$, and $R>160$. The right-hand panel gives the magnification $\mu$ and standard deviation corresponding to these mean redshifts vs. radial distance in arcseconds. The solid curve shows the magnification profile for the pure isothermal case and the dashed curve shows the power law profile with an index of -0.6 , flatter than isothermal, and indistinguishable from NFW profile at the level at which this can be measured so far for our survey. The diamond-shaped points give the mean values for the power law model of $n=-0.6$ summed up over the same intervals as the data, for comparison. The data show convincingly that magnification increases towards the center, but without more data cannot be used to distinguish between the two models.

Note that the first point, at $R=45^{\prime \prime}$, is far different from the integrated model value. This is related to the binning and the assumption of circular symmetry. The binning is finite, such that the integrated value will depend on the bin size, with smaller binning resulting in higher mean values. Also the source sizes are finite and so the magnification never unreasonably-high. By excluding a small region about the Einstein ring radius it should ease the brunt of both of these problems and improve the fit near the position of the Einstein ring radius. Also the cluster mass distribution is not perfectly circular, and from distortion measurements in the cluster A2390 is known to be highly-elliptical (Frye \& Broadhurst, 1998; Pellò et al., 1998). For this reason the circular models are a poor approximation at radii close to the position of the Einstein ring, but for larger radii this assumption becomes much less important. Recall that the bending of light is more or less circular even for elliptical mass profiles, because it is a convolution of the mass with $\frac{1}{R}$ (See Theory Chapter), which tends to smooth out the distribution. Therefore we expect agreement with the model only exterior to the Einstein rind radius, in other words only for the last two points of this plot.

The magnification profile for the sum of the data from all three clusters appears in Fig. 5.24, over 6 different radial bins. Once again the first point does not show agreement with the integrated value, for the reasons explained above. This dataset is most consistent with the power law model with $n=-0.6$, although more data is required to establish this trend. If the data really is more consistent with the flatter profile, then this implies more generally that the dark matter distribution is shallow unlike the light.

Tables 1.6 and 1.7 summarize the results, giving $\bar{z}, \mu$, and the number of objects in each radial bin for each of the three clusters and for the total. This technique has a great 

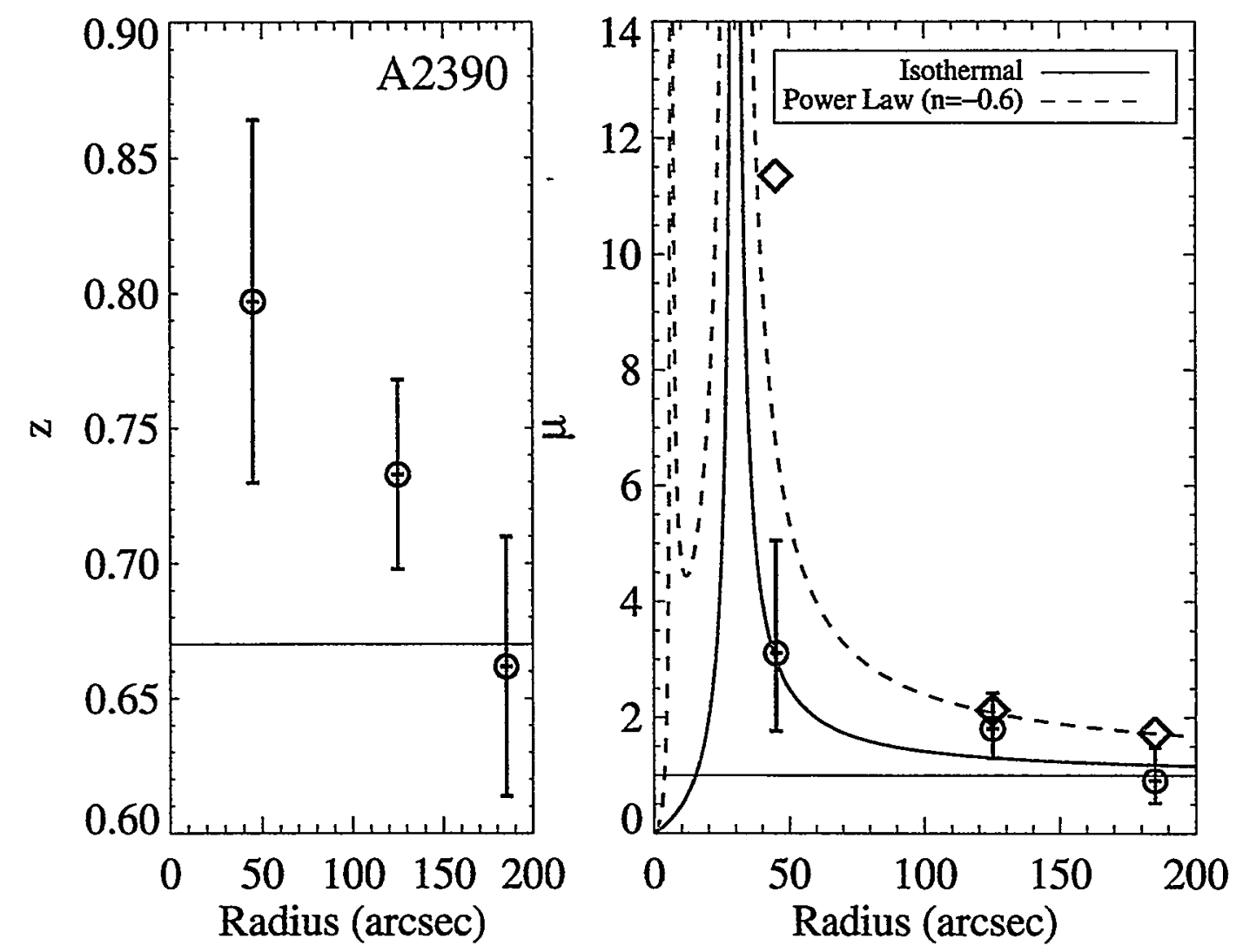

Figure 5.22: The mean $z$ as a function of distance in arcsec away from the cluster centerof-mass appears on the left and the corresponding magnification profile on the right for the cluster A2390. Two models are overlaid for comparison with the data points. They are an isothermal profile with an Einstein radius of $30^{\prime \prime}$ (solid line), and an isothermal model with a radius of $30^{\prime \prime}$ and a power law slope of -0.6 (dashed line). The mean values of the isothermal model summed up over the same intervals as the data is shown by the diamonds. 

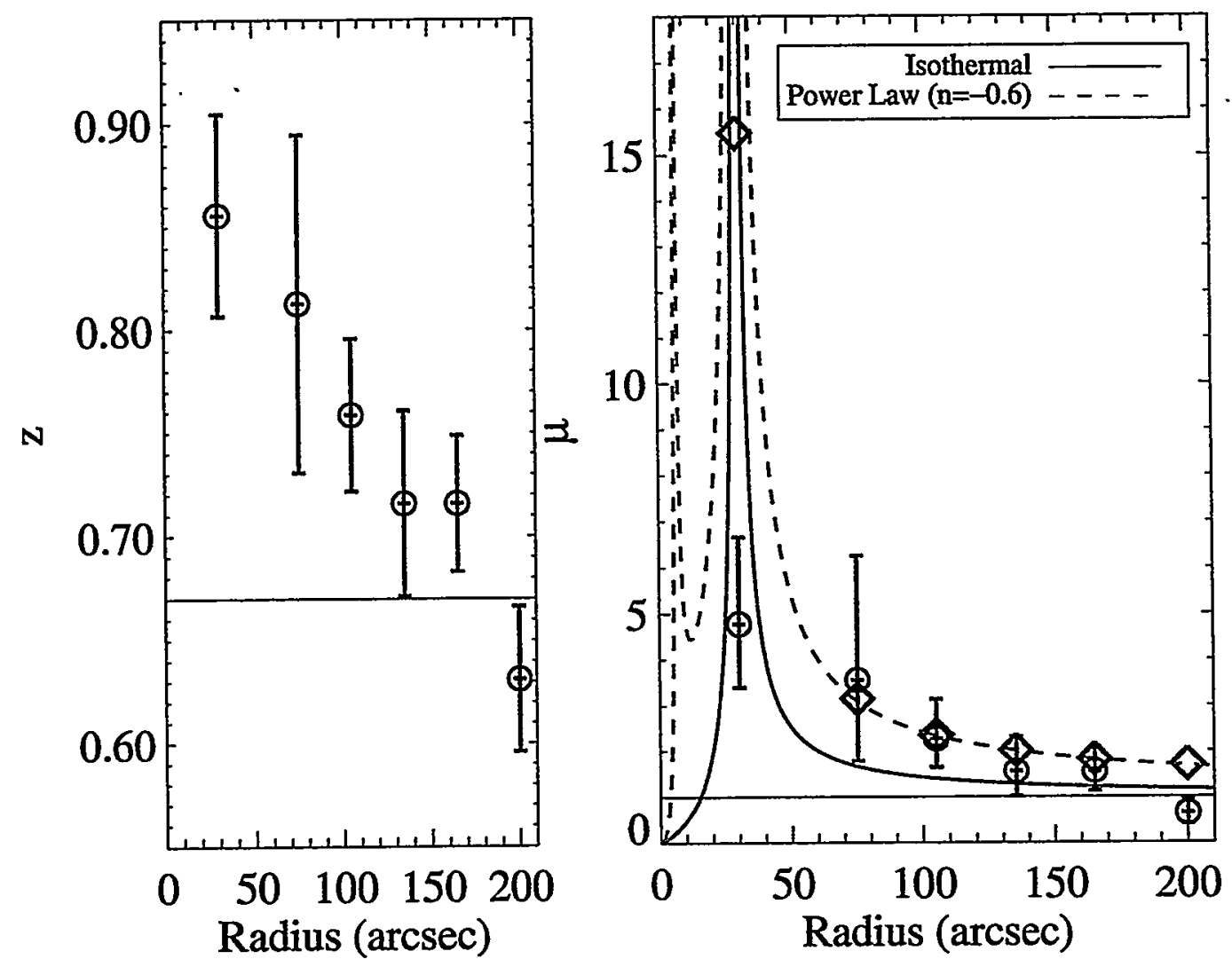

Figure 5.23: The mean $z$ as a function of radial distance in arcsec away from the cluster center-of-mass appears on the left and the corresponding magnification profile on the right. Two models are overlaid for comparison with the data points. They are an isothermal profile with a mean Einstein ring radius of $30^{\prime \prime}$ (solid line), and a power law model with a radius of $30^{\prime \prime}$ and a power law slope of -0.6 (dashed line). For reference, the integrated model mean in the same radial bins as the data is indicated by the diamond-shaped symbols. 


\begin{tabular}{|c|c|c|c|c|c|}
\hline & Radius (") & A1689 & A2390 & A2218 & The Total \\
\hline $\bar{z} \pm \Delta \bar{z}$ & $0<R<90$ & $1.10 \pm 0.09$ & $0.80 \pm 0.07$ & $0.74 \pm 0.06$ & $0.84 \pm 0.04$ \\
$\bar{\mu} \pm \Delta \bar{\mu}$ & $0<R<90$ & $26.5 \pm 25.5$ & $3.1 \pm 1.9$ & $2.0 \pm 1.1$ & $4.2 \pm 1.2$ \\
$\#$ & $0<R<90$ & 10 & 20 & 18 & 48 \\
$\bar{z} \pm \Delta \bar{z}$ & $90<R<160$ & $0.84 \pm 0.06$ & $0.73 \pm 0.04$ & $0.68 \pm 0.04$ & $0.73 \pm 0.03$ \\
$\bar{\mu} \pm \Delta \bar{\mu}$ & $90<R<160$ & $4.4 \pm 2.2$ & $1.8 \pm 0.6$ & $1.05 \pm 0.5$ & $1.85 \pm 0.4$ \\
$\#$ & $90<R<160$ & 11 & 36 & 20 & 67 \\
$\bar{z} \pm \Delta \bar{z}$ & $R>160$ & $0.88 \pm 0.07$ & $0.66 \pm 0.05$ & $0.57 \pm 0.03$ & $0.66 \pm 0.03$ \\
$\bar{\mu} \pm \Delta \bar{\mu}$ & $R>160$ & $5.5 \pm 3.0$ & $0.9 \pm 0.6$ & $0.35 \pm 0.15$ & $0.9 \pm 0.3$ \\
$\#$ & $R>160$ & 7 & 25 & 19 & 51 \\
\hline \hline
\end{tabular}

Table 5.5: The mean redshifts, $\bar{z}$, magnifications $\mu$, are given for each radial bin of each cluster and for the total. The associated errors are also recorded, as is the number of objects in each bin.

\begin{tabular}{|c|c|c|}
\hline & Radius $\left(^{\prime \prime}\right)$ & A1689 + A2390+A2218 \\
\hline $\bar{z} \pm \Delta \bar{z}$ & $0<R<60$ & $0.86 \pm 0.05$ \\
$\bar{\mu} \pm \Delta \bar{\mu}$ & $0<R<60$ & $4.8 \pm 1.9$ \\
$\#$ & $0<R<60$ & 28 \\
$\bar{z} \pm \Delta \bar{z}$ & $60<R<90$ & $0.81 \pm 0.08$ \\
$\bar{\mu} \pm \Delta \bar{\mu}$ & $60<R<90$ & $3.6 \pm 2.7$ \\
$\#$ & $60<R<90$ & 20 \\
$\bar{z} \pm \Delta \bar{z}$ & $90<R<120$ & $0.76 \pm 0.04$ \\
$\bar{\mu} \pm \Delta \bar{\mu}$ & $90<R<120$ & $2.3 \pm 0.9$ \\
$\#$ & $90<R<120$ & 36 \\
$\bar{z} \pm \Delta \bar{z}$ & $120<R<150$ & $0.72 \pm 0.05$ \\
$\bar{\mu} \pm \Delta \bar{\mu}$ & $120<R<150$ & $1.6 \pm 0.8$ \\
$\#$ & $120<R<150$ & 22 \\
$\bar{z} \pm \Delta \bar{z}$ & $150<R<180$ & $0.72 \pm 0.03$ \\
$\bar{\mu} \pm \Delta \bar{\mu}$ & $150<R<180$ & $1.6 \pm 0.5$ \\
$\#$ & $150<R<180$ & 21 \\
$\bar{z} \pm \Delta \bar{z}$ & $R>180$ & $0.63 \pm 0.04$ \\
$\bar{\mu} \pm \Delta \bar{\mu}$ & $R>180$ & $0.7 \pm 0.3$ \\
$\#$ & $R>180$ & 39 \\
\hline \hline
\end{tabular}

Table 5.6: The mean redshifts, $\bar{z}$, magnifications $\mu$, are given for 6 different radial bins for the sum total of all three clusters. The associated errors are also recorded, as is the number of objects in each bin. 
deal of potential, and is most consistent with the power law profile, but awaits more data to hone the results.

\subsection{Conclusions}

We summarize the results of the survey below.

- The giant straight arc in A2390 is not a single $23^{\prime \prime}$ long arc but rather a chance superposition of two galaxies, at $z=0.913$ and $z=1.033$ respectively (Fig. 5.1).

- The giant blue arc in A2218 and the theta arc in Cl0024 both show many strong interstellar metal lines, similar to those seen in local starbursting galaxies (Fig. 5.3,5.5).

- The redshift survey has an $85 \%$ success rate for finding redshifts and a $55 \%$ success rate for finding redshifts of objects behind clusters. To give the numbers, there are 318 objects with measured redshifts and $I<23$ (see Appendix), with 174 of them being behind the clusters.

- The redshift and magnitude distributions both show a trend of increasing redshift and flux towards the center. This behavior is an effect of cluster magnification, such that we are seeing further down the luminosity function to fixed redshift (Fig. 5.16, 5.18, 5.20).

- The magnification profile for the cluster A2390, collected into 3 radial bins, shows a trend of decreasing magnification with radial distance, but is not good enough to distinguish clearly between a pure isothermal vs. a power law model with $n=-0.6$ (Fig. 5.22). The shotnoise is the main limitation.

- The magnification profile was also calculated for all three clusters summed together. This increased the sample size by $\sim 2 \times$. The result is a profile which is consistent with a power law model with $n=-0.6$, similar to the NFW profile to within the errors (Fig. 5.23). 


\section{Chapter 6}

\section{High- $z$ Galaxies}

\subsection{Introduction}

The search for distant galaxies using color selection and large telescopes have now made the discovery of $z>3$ galaxies routine. Despite the sensible idea that Ly- $\alpha$ photons produced by the first generation of stars would be free to propagate in the absence of dust, making the Ly- $\alpha$ line an enormously-bright beacon, early searches for this elusive emission line were unsuccessful.

Galaxies at $\mathrm{z} \sim 3$ were first found by photometric selection using the Lyman limit as a discriminator (Steidel et al., 1996a,b). This technique proved to be highly-successful and still drives the search for high $z$ galaxies, although at higher redshift, $z>4$, photometric selection is made more difficult by the reduced flux between the Lyman limit and the Lyman- $\alpha$ line, and objects become fainter making spectroscopy less practical. The first discoveries of galaxies with $z>4$ with spectroscopic confirmations were (Dey et al., 1998a; Franx et al., 1997; Frye \& Broadhurst, 1998; Trager et al., 1997).

In this chapter new spectra and rest-UV properties of six lensed high- $z$ galaxies are presented, which were discovered as a part of the larger survey in a search for distant forest-depressed objects behind massive lensing clusters. Their spectra are of remarkablygood quality owing to the high magnification. Although this is not a requisite feature in high- $z$ spectra (Bunker et al., 1999) the ones in this sample with confirmed redshifts all show Ly- $\alpha$ in emission, shifted redward in velocity by $200<v<750 \mathrm{~km} / \mathrm{s}$ from the $\mathrm{z}$ of the interstellar gas, indicating substantial gas outflow. In addition, two of them show spatially-resolved Ly $\alpha$. This chapter is divided into four sections, which are the properties of the lens, the color-selection of background targets, the results, and a discussion section will address the implications of these findings on galaxy evolution and the evolution of the IGM. 


\subsection{The Lens}

Lensing by clusters provides a means to access the highest redshift galaxies, aided by fortuitously large magnifications. Fluxes enhanced by magnification effectively increase the magnitude limit, allowing us to sample to higher $z$ behind the cluster than in the field to a fixed flux limit. On average, a given target is boosted by $\sim 3$ magnitudes within the inner arcminute of a massive cluster. By providing such a large magnification boost over such a wide area cluster lensing gives us a viable route for obtaining useful spectroscopic samples of high- $z$ galaxies.

The large magnification generated by clusters is responsible for the discovery of some of the most distant galaxies known (Trager et al., 1997; Franx et al., 1997; Frye \& Broadhurst, 1998), and section 6.4 of this Chapter. The lensing clusters are all X-ray bright but have different concentrations centrally-dominant cluster members. Do lensing clusters have common mass profiles which are conducive to producing large magnifications? What is the mass profile of massive lensing clusters?

Consider how the magnification $\mu$ scales with radius $R$ in the simple case of an isothermal lens:

$$
\mu=\frac{1}{\left|1 \pm \theta_{E} / \theta_{I}\right|}
$$

where $\theta_{E}$ is the Einstein ring radius and $\theta_{I}$ is the angular distance to the image position. The magnification falls off on either side of $\theta_{E}$, approaching 1 for large $R$. We find from our imaging data that the number counts of background galaxies falls off with angular separation less steeply than isothermal, consistent with $r^{-1.5}$ slope for $r<0.5 M p c$. A profile flatter than isothermal would make the magnification higher for a given $R$. Do most lensing clusters have a common mass profile?

Flatter slopes are also predicted by N-body simulations (Navarro et al., 1995, hereafter NFW). They ran standard CDM N-body simulations, fitting mass profiles to condensations over a whole range of scales from individual galaxies to galaxy clusters. A flexible fitting function was found which is flatter than isothermal but still singular. It features a flattening off in the middle, the region of interest. The profile has the form:

$$
\frac{\rho(r)}{\rho_{c r}}=\frac{\delta_{c}}{\frac{R}{R_{S}}\left[1-\frac{R}{R_{S}}\right]^{2}}
$$

where $R_{S}=3-400 \mathrm{kpc}, \delta_{c}=10^{4}$, and $\rho_{c r}=\frac{3 H_{0}^{2}}{8 \pi G}$. The mass density $\rho(r) \sim R^{-1}$ out to a few hundred kpc, making the surface mass density is flat. The table below summarizes the expected magnification for the isothermal and NFW profiles, given a couple of typical values for $R$ and and for an Einstein ring radius of $\theta_{E}=25^{\prime \prime}(100 \mathrm{kpc}$ ).

Work is in progress to determine the mass profile of the lensing cluster Cl0024 (see Fig. 5.4, Broadhurst etal 1999, (Tyson et al., 1998). Cl0024 features a dramatic 


\begin{tabular}{|c|c|c|}
\hline Model & $R(\mathrm{Mpc})$ & $\bar{\mu}$ \\
\hline NFW & 0.2 & 30 \\
& 0.5 & 3 \\
Isothermal & 0.2 & 2 \\
& 0.5 & $5 / 4$ \\
\hline
\end{tabular}

Table 6.1: Table showing the expected magnification for the isothermal and NFW profiles, given a couple of typical values for $R$ and for an Einstein ring radius of $\theta_{E}=25^{\prime \prime}(100 \mathrm{kpc})$.

case of a multiple image where 5 images punctuate the Einstein ring radius. In addition HST spatially-resolves 3 obvious bright features in common to each image, probably HII regions. Broadhurst, et al. 1999 assigned NFW profiles to the brightest cluster members, by minimizing the difference between the model predicted locations of the images and HII regions. They find that the lensed images are reproduced most accurately by a projected mass distribution which traces the locations of the brightest cluster ellipticals, suggesting that the most significant minima of the cluster potential are not fully-erased. This is in contrast to earlier work, where accurate lensed images were obtained by a smooth, dominant central potential, with the cluster members playing only a minor role through dark deflecting 'mascons' which make adjustments to the location and shape of the images through particles called mascons (Tyson et al., 1998).

The geometry is also important in producing large magnifications, as it depends on the reduced distance $D$, or the ratio of lens and source distances. For an isothermal mass profile the magnification is maximized if the lens is placed halfway between the observer and the source, where $\mu \propto \frac{D_{L} D_{S}}{D_{L S}}$. For a cluster with a NFW profile, the lens will want to be closer to the observer (Huang et al. 1999).

How does one select a lensing cluster? Many are X-ray selected and confirmed in the optical by the presence of lensed features such as giant arcs and multiple images. These arcs betray the presence of a large underlying surface mass density with $\Sigma>\Sigma_{c r}$. The relative magnification power between clusters is more difficult to measure, as this depends on the gradient of the surface mass density, which is the subject of this thesis.

\subsection{The Targets}

Target selection is based on color. The $V$ and $I$ bands are the most important here because for galaxies with redshifts between 4 and 5 the wavelength of the Ly- $\alpha$ emission falls between the two bands (Fig. 6.1,6.2). At such redshifts we expect a significant continuum depression amounting to $\sim 2$ magnitudes in $V$.

Objects are not selected on the basis of morphology because not all giant arcs have an arc-like appearance. In particular, small lensed images which are poorly-resolved can be confused with that of red stars. An object with an intrinsic size larger than the seeing 

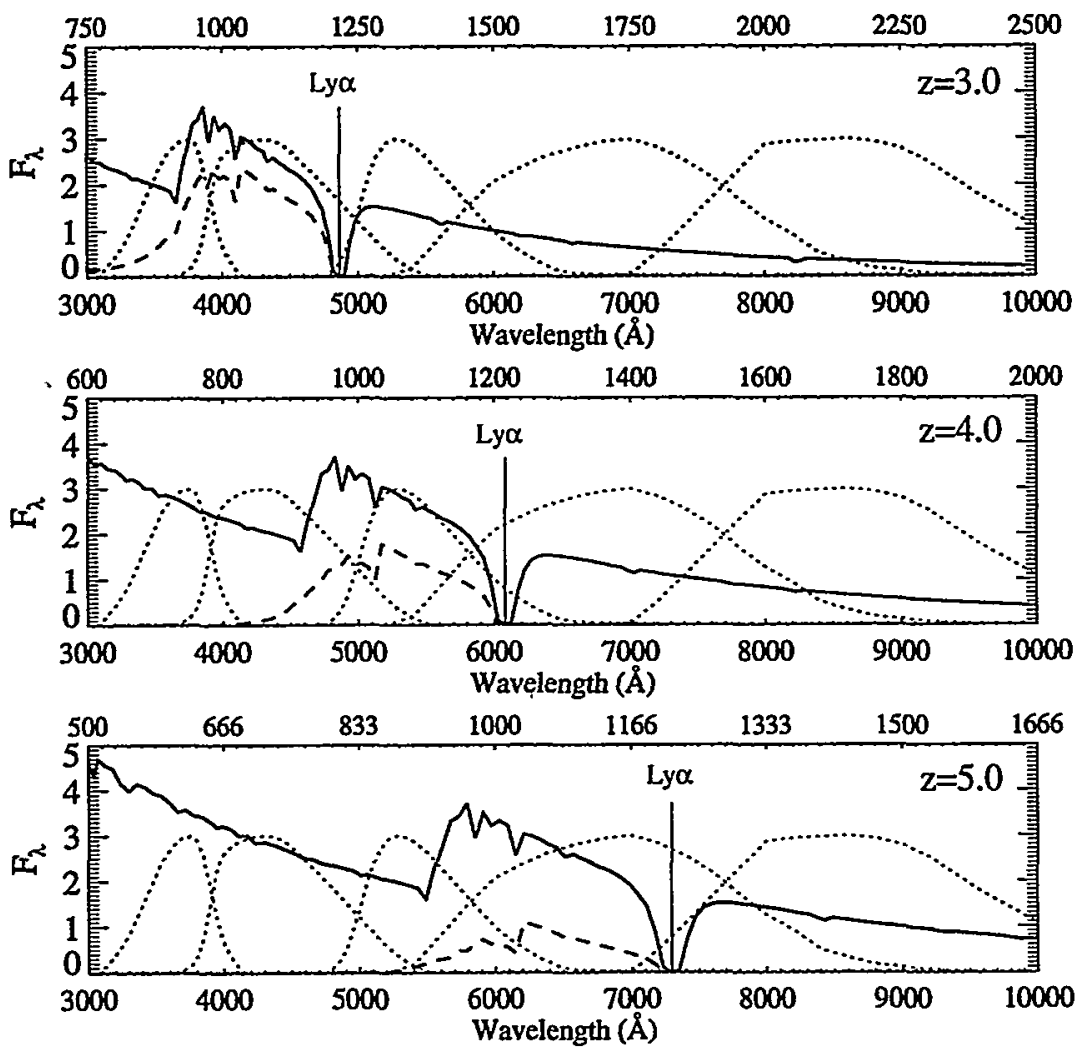

Figure 6.1: Model spectra of the $z=4$ galaxy for a stellar population dominated by B3 stars for three different $z$ 's. $F_{\lambda}$ is plotted against observed wavelength on the lower axis and rest wavelength on the upper axis. The solid line gives the model spectrum, including $\mathrm{HI}$ absorption at the source. The model given by the dashed line shows the effect of the $z$-dependent Lyman-series forest opacity. The five dotted continuous curves give the transmission curves for the $U B V R I$ filters, in order of increasing wavelength. 
disk will become elongated on the image plane and get recognized as an arc. However, an object which is intrinsically very small, so small that it's image size is smaller than the seeing disk, will appear round and deceivingly star-like in appearance. Also in general mass sub-structure can create apparently undistorted images. Fig. 6.7 shows the spectrum of a galaxy at $z=4.868$ along with its ground-based $U B V I J$ images. This giant arc is hugely magnified, by $\sim 30 x$, but only marginally-resolved even on HST images due to its intrinsically small size. Note that it looks round and star-like.

What values of $V-I$ are favored for color-selecting high- $z$ galaxies? The dependence of a high- $z$ galaxy's color on $z$ can be obtained by modelling its SED and then redshifting the spectrum through the different filter passbands, taking into account the $z$ dependent opacity of the Lyman-series forest. Fig. 6.11 shows a spectrum of the high- $z$ lensed galaxy in A2390 which served as a basis for our model spectrum.

The model spectrum has two main components. The first is the stellar spectrum, which is best fit by a stellar population dominated by hot $\mathrm{O}$ and $\mathrm{B}$ stars and wellapproximated by the blackbody of a single $\mathrm{O}$ or $\mathrm{B}$ type star. The second is the Lyman-series opacity, which attenuates the spectrum between the Lyman limit and Ly- $\alpha$ at the source $z$. It arises from the combined effect of $\mathrm{H}$ absorption along the line of sight from Ly- $\alpha$ clouds, Lyman-limit systems and the occasional damped Ly $\alpha$ system. This Lyman-series opacity, $\tau_{e f f}$, can be well-approximated analytically by a power law in $z$, Madau (1995):

$$
\tau_{e f f}=\sum_{j=2, i} A_{j}\left(\frac{\lambda_{o b s}}{\lambda_{j}}\right)^{3.46}
$$

where $A_{j}=\left(1.7 \times 10^{-3}, 1.2 \times 10^{-3}, 9.3 \times 10^{-4}\right)$ and $\lambda_{j}=(1026,973,950 \AA)$ for $\mathrm{Ly}-\beta, \mathrm{Ly}-\gamma$, and Ly- $\delta$ respectively. It is a fairly steep power law which is a significant source of opacity on a galaxy spectrum by $z=4$. Fig. 6.2 shows this model spectrum for two different stellar types. Here the solid line is the Kurucz blackbody spectrum for the labelled spectra type star redshifted out to $z=4$, with the dashed curve giving the attenuated spectrum.

The purpose of constructing a model spectrum is to study the effect of the high- $z$ forest on the colors. The $V R I$ bands are the most useful here since the Lyman-series forest redshifts into the $V$ band by $z=3.3$, as can be seen in Fig. 6.1. The $U B$ bands are not particularly useful, since the Lyman-series forest redshifts past these bands by $z \sim 2.5$. Model spectra were constructed for two different stellar types, a B0 star at $T=30000$ $\mathrm{K}$ and a B3 star at $T=19000 \mathrm{~K}$. The B3 star was chosen because it was the best fit to the $z=4.040$ spectrum in A2390 on which the model was based, and the B0 star is used for comparison. The model spectra were constructed over a whole range of redshift, $0<z<5.5$.

Since hot B stars seem to give a best fit to the data in many cases, it is interesting to ask if there are sufficiently-many ionizing photons to explain the large equivalent widths seen in the high- $z$ spectra. For the 6 high- $z$ spectra in this survey this would be difficult 

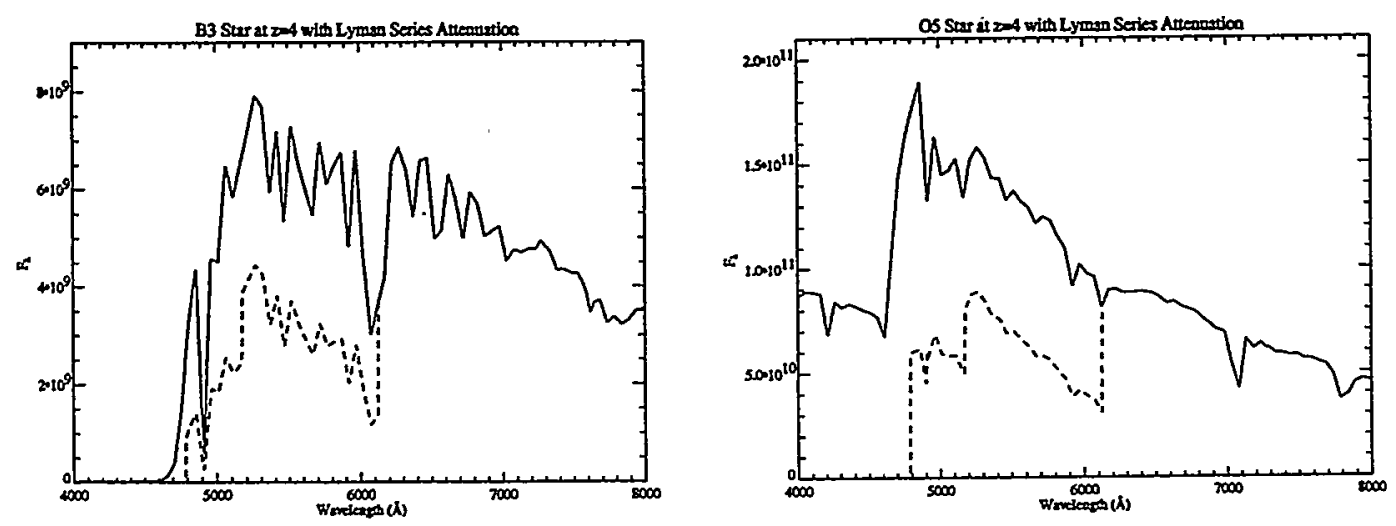

Figure 6.2: Model spectra of a $z=4$ galaxy for a stellar population dominated by B3 stars (left-hand panel) or OV stars (right-hand panel). In each case the stellar blackbody (solid line) is attenuated by HI absorption from the z-dependent Lyman series (dashed line). Note the increased absorption at the observed wavelength of Ly- $\alpha, 6130 \AA$, from $\mathrm{H}$ absorption intrinsic to the B3 star.

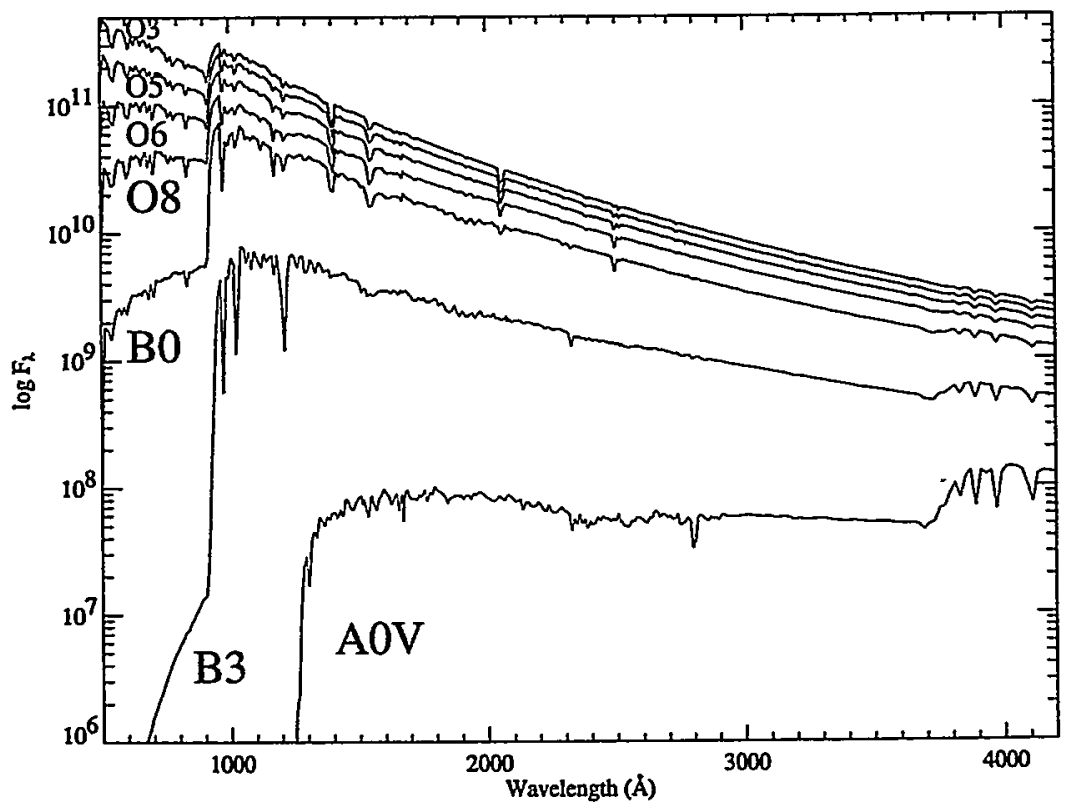

Figure 6.3: The blackbody spectra of hot stars with spectral types A0 through O3. Note the increasing strength of Ly- $\alpha$ absorption in the transition from $O$ to the B type stars. 
to measure, as no spectrophotometric standard stars were observed which could be used to convert the flux measurement to an absolute scale. If this were the case then one could calculate the equivalent width of Ly $\alpha$ by comparing the UV continuum flux for Kurucz stellar models of different stellar types with the flux in the Ly $\alpha$ line in the observed spectra. The coolest possible stellar type which reproduces the observed equivalent width would give the answer.

A complicating issue is that B stars show an intrinsic Ly $\alpha$ absorption which can dilute an otherwise strong Ly $\alpha$ emission. This becomes increasingly more pronounced for late B-stars, in which the aborption increases exponentially with decreasing temperature. Valls-Gabaud computed the total rest equivalent width of Ly $\alpha, W_{L y \alpha}$ in the Bruzual-Charlot model for a starburst with a Salpeter IMF (Bruzual \& Charlot, 1993). He found $W_{L y \alpha}=$ $250 \AA$ during the first $10^{6.5}$ years and decreases sharply after that, so that by $10^{7}$ years it is negligible or in absorption. The $O$ stars and hot $B$ stars have turned off the main sequence by this time. Although the calculation was not performed on single O or B stars, it would appear from the timescales involved that in the absence of dust hot B stars would be capable of producing the observed equivalent widths, all of which are on the order of $\sim 10 \AA$, less than the predicted value. With the exception of one case, the galaxy in A2390 at $z=4.04$, an example of spatially-resolved Ly $\alpha$, they all show the general trend of increasing $W_{L y \alpha}$ with $z$, possibly related to decreasing dust extinction.

Given the chosen models of a B0 and B3 star, one can calculate their colors at different $z$ 's by redshifting the HI-attenuated model spectrum through the bandpasses. For a given $z$ the color is calculated as follows. The difference in magnitudes between any two bands 1 and $2, M_{1}-M_{2}$ is calculated by measuring magnitudes in the model with respect to an AOV star:

$$
M_{1}-M_{2}=\left(M_{1 m}-M_{1 A 0}\right)-\left(M_{2 m}-M_{2 A 0}\right)
$$

where the subscripts $m$ and $A 0$ refer to the model and A0V star respectively. The SED of the model, $I_{m}$, the SED of the AOV star, $I_{A 0}$ and the filter transmission function $T$ are the observables. $M$ can be written in terms of these observables:

$$
\begin{gathered}
M=-2.5 \log F+C \\
F=\int_{0}^{\mathrm{inf}} I T d \lambda
\end{gathered}
$$

The resulting color- $z$ and color-color diagrams appear in Fig. 6.4 and 6.5. The Color- $z$ dependence for the $V-R$ and $R-I$ colors is shown in Figure 6.4 as a two panel plot. Here the stellar population dominated by B0 stars is given by a solid line and the one dominated by B3 stars as a dashed line. The curves all have the same general shape, that is fairly-flat until $z \sim 4$ at which point they both turn upward. The upward turn is caused by the Lyman-series forest redshifting into the $R$ band (see Fig. 6.1). The redshifts at which major features in the model spectra inhabit the various bands are marked by features in 
the model. For example, the bump seen in the B3 stellar model at $z \sim 1$ in both panels can be explained by the Balmer discontinuity redshifting through the $R$ and $I$ bands. The detailed behavior of the curves will be discussed below.

While a B0 star is relatively featureless a B3 star has a non-negligible Balmer discontinuity extending shortward of roughly $3700 \AA$, resulting in a bump seen in both panels in the blue part of the color-color plane. The rise in the bump takes place over the $z$ interval where the Balmer discontinuity first redshifts into the $R$ band, $0.5<z<1$, causing the measured $R$ band flux to go down relative to the as yet unabsorbed $I$ band flux, making $R-I$ increasingly redder. Then for $z \sim 1$ the $R$ and $I$ bands straddles the Balmer discontinuity (at $\sim 3700 \AA$ ) and the bump turns over. For $1<z<1.4$ the $I$ band redshifts past this discontinuity, with the already absorbed $R$ band staying relatively constant, causing $R-I$ to become bluer. Finally, for $z>1.4$ both $R$ and $I$ have redshifted well below $3700 \AA$, and the spectrum remains more or less flat until the Lyman-series forest redshifts through the various bands. The bump seen in the blue in the $V-R$ color- $z$ diagram is also caused by the Balmer discontinuity but is smaller overall and peaks at $z=0.5$ where it straddles the $V$ and $R$ bands. The difference in size and amplitude is caused by the much narrower width of the $V$ band, making $V-R$ less sensitive.

The next major feature in the model spectrum is the Lyman-series forest. $R$ redshifts into the forest starting at $z \sim 4$, causing the measured $R$ band flux to go down relative to $I$, making $R-I$ redder (Fig. 6.1). For $z \sim 5 I$ redshifts into the forest, but that the curve does not turnover as it did for the Balmer discontinuity. This is because at the same time the Lyman-limit starts to redshift into $R$, making $R$ drop precipitously. What is crucial here is that $R$ is dropping off faster than $I$, keeping $R-I$ red and rising.

Fig. 6.5 shows color-color diagrams of the model high- $z$ spectra for the underlying B0 stellar population (left-hand side) and the underlying B3 stellar population (right-hand side). For each plot the $V-R$ is plotted up against $R-I$ in Vega magnitudes, with redshifts labelled on the plot at useful intervals. The model curves for both sets of models have a fairly similar shape, that is fairly flat in the color-color plane with a slope of $\sim 2$ and a dip in the blue. The added structure in the B3 model curve is the result of the more complex B3 stellar spectrum (see Fig. 6.2).

The shape of the curve for the B3 stellar model can be explained as follows. The Balmer discontinuity starts redshifting into $V$ at $z \sim 0.3$, causing $V-R$ to become red and the curve to steepen. For $z \sim 0.5$ the Balmer discontinuity starts redshifting into $R$, making $V-R$ to flatten out and $R$ to become depressed relative to $I$. As a result the curve moves red in $R-I$. For $0.9<z<1.5$, the Balmer discontinuity passes through $I$, making $R-I$ become less red with no associated change in $V-R$ because it is still on a relatively flat part of the spectrum, and the curve moves blueward. The 'retrograde' motion is a natural consequence of the Balmer discontinuity moving through the passbands, with the size of the resulting 'loop' maximized for the star with the most pronounced Balmer discontinuity, the A0 star (see Fig. 6.3). 

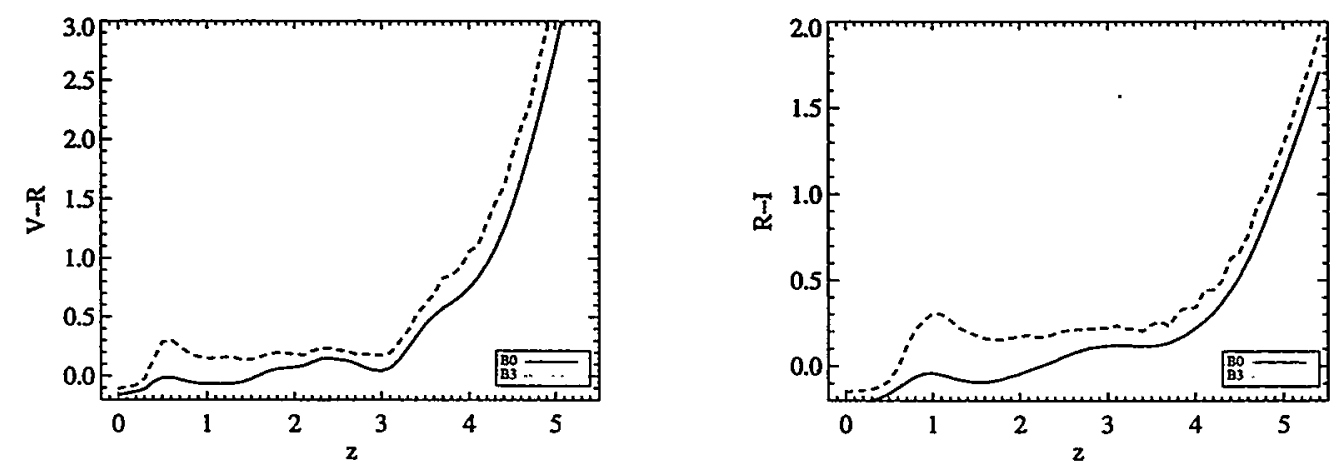

Figure 6.4: Color- $z$ plots of high-z spectra, plotted in Vega magnitudes. In both panels models with a stellar population dominated by $\mathrm{BO}$ stars appears as the solid line and the one dominated by B3 stars with a dashed line. In the panel on the right-hand side $V-R$ is plotted against $z$ and in the left-hand panel $R-I$ is plotted against $z$. All four curves have the same general shape, which is roughly-flat until $z=4$, when the Lyman-series forest has redshifted into both the $V$ and $R$ bands. A B3 star has a non-negligible Balmer discontinuity, causing the bump seen in the B3 model curve at $z \sim 1$ in both panels.

The curve emerges again when $V$ redshifts into the Lyman-series forest at $z \sim 3$ (Figure 6.1). The drop $V$ between $3<z<4$ causes $V-R$ become redder while $R-I$ remains constant, and the curve steepens sharply. $R$ reaches the Lyman-series forest at $z \sim 3.5$, making $V-R$ less red and $R-I$ redder. The model loses sensitivity for $z>4.5$, when the Lyman-limit redshifts past $V$. The forest-attenuated $V R I$ magnitudes are all so large beyond this redshift that they no longer have a pronounced effect on the colors.

The $\mathrm{B} 0$ model curve has a much more simple shape because the spectrum is less complex. Unlike the B3 star, there is relatively little change across the Balmer discontinuity and so there is a relatively- minimal effect on the colors. The main feature is the Lymanseries forest, which redshifts into the $V$ band at $z \sim 3$, causing the only major feature in the curve, a sharp redward turn in $V-R$ relative to $R-I$, and from there onward curve evolves similarly to that just described for the B3 model curve.

In summary, the redder optical bands $V R I$ can be used to identify high- $z$ galaxies through the effect that the Lyman-series forest opacity has on the colors. The bluer bands $U B$ redshift into the Lyman-series forest at too low of a $z$ to be of interest for this project.

\subsection{Observations and Results}

All spectroscopic observations were carried out on the Keck telescopes using the Low-Resolution Imaging Spectrometer (LRIS). Instrument flexure and fringing required a purpose-built code for efficient and accurate reduction. This code and all details of the data 

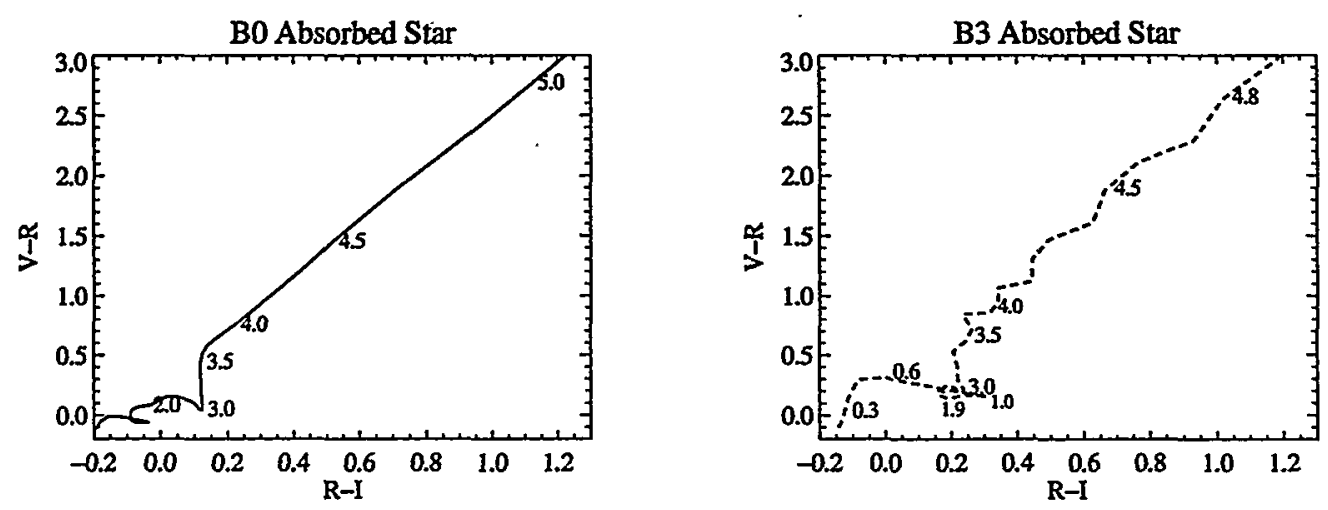

Figure 6.5: Color-color diagrams of model high- $z$ spectra. The set of models with a stellar population dominated by B0 stars appears on the left-hand side one dominated by B3 stars on the right-hand side. For each plot the Johnson $V-R$ is plotted up against Johnson $R-I$ and redshifts labelled alongside the curves. The general shape of the curves is the same, with the sudden rise in the curve in the blue part of the color-color plane caused by the drop in $V$ band flux when the $V$ band redshifts into the Lyman-series forest. Note that $V-R$ is fairly steep with respect to $R-I$ for $z>4$ but is still useful if the photometry can be measured with reasonably-good accuracy.

reduction are presented in Chapter 3 of this thesis. The observations were carried out on three different observing runs and each will be discussed in turn.

\subsubsection{A1689}

A1689 is arguably the best cluster for this work by virtue of its large critical radius of $\theta_{E} \sim 50^{\prime \prime}$, about twice that of any other lens. The giant arcs are best seen in Fig. 6.6a,b. One thin blue arc is seen in the lower left hand corner of both panels subtending $\mathbf{3 0}$ degrees about the cluster center, with many other giant arcs visible at this radius. The image in Fig. 6.6a was taken with the NTT in $V$ and $I$ in 0.8 seeing (see Taylor et al. 1998). Fig. 6.6b shows a color HST WFPC2 image of the inner 50 arcsec of the cluster, taken in the F606W and F814W bands. The superior angular resolution of the HST is crucial for identifying the numerous thin, tangentially and radially-stretched arcs, while the deep, ground-based images are helpful for locating the very faint objects. Our highest redshift galaxy in this field at $z=5.12$ (Fig. 6.8) is very close to the critical curve but just off of the color image in Fig. 6.6a. The second-highest redshift object in the field, at $z=4.868$ (Fig. 6.7), is much brighter and is labelled in both panels of Fig. 6.6.

The object at $z=4.868$ is not spatially-resolved from the ground. It is the reddest object with a measured color in the field, $V-I=3.1$, and redder than any passivelyevolving elliptical is expected to reach at their maximum near $z=1$. Another high- $z$ object is labelled by its redshift, $z=3.85$ in Fig. 6.5a. In the relatively-shallow image of Figure 

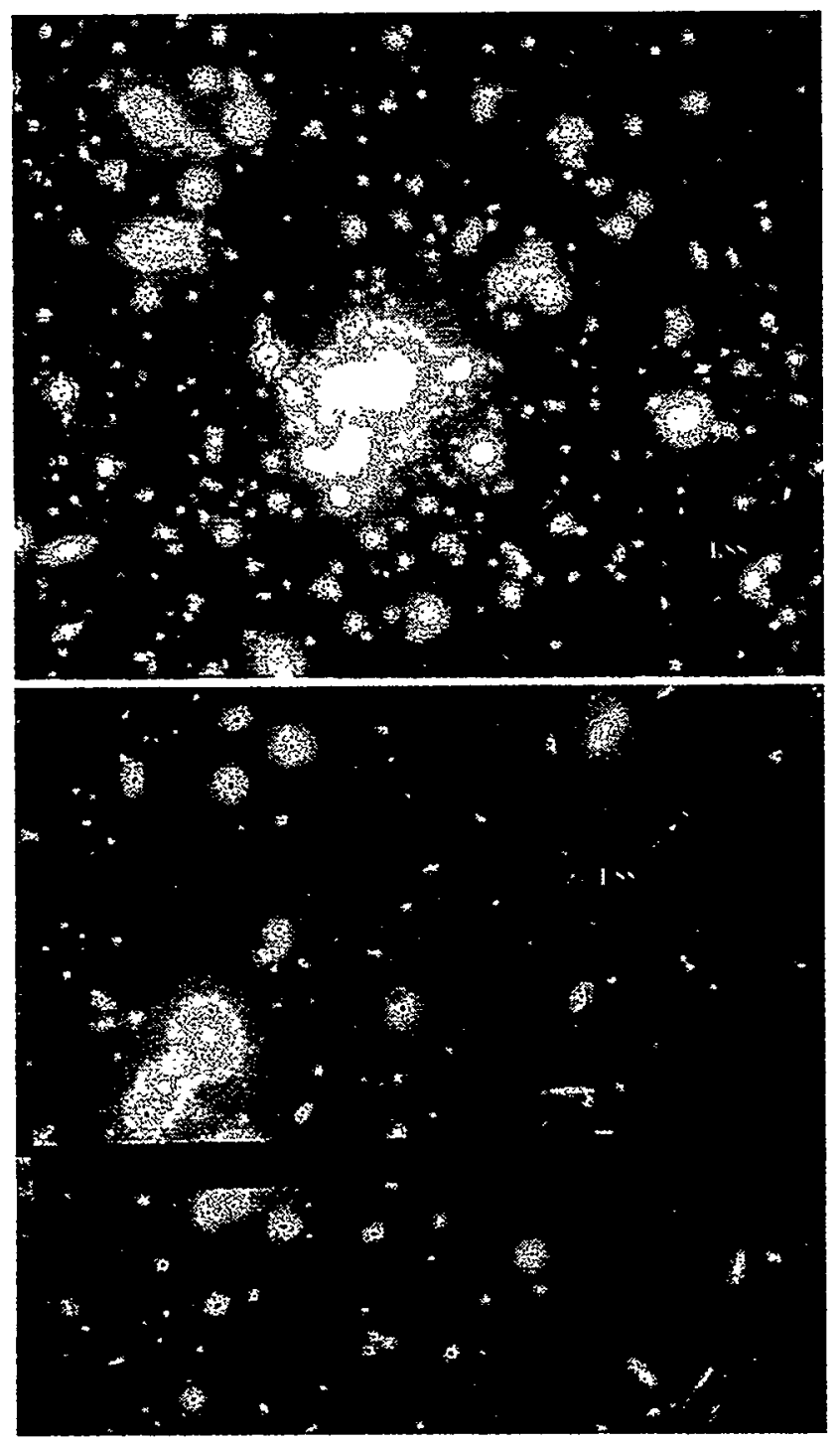

Figure 6.6: Color images of the center of A1689 $(z=0.18)$ with high- $z$ galaxies labelled. Panel (a) is a ground-based color NTT image of the inner $2^{\prime} \times 3^{\prime}$. Note in the lower left of the image a blue arc subtending 30 degrees, with many other giant arcs visible at this radius. Both of the high- $z$ galaxies on this plot are small, red and unresolved at this resolution $\left(0.85^{\prime \prime}\right)$. Panel (b) shows a fairly shallow, high angular resolution Hubble Space Telescope color image of the inner $50^{\prime \prime}$. The galaxy labelled $z=4.88$ is the same galaxy as the one in Panel (a), and is marginally-resolved at this resolution $\left(\sim 0.1^{\prime \prime}\right)$. The J2000 coordinate of the cluster center is $\alpha=13^{h} 11^{m} 28^{s}, \delta=-01^{\circ} 20^{\prime} 48^{\prime \prime}$. 
$6.5 \mathrm{~b}$ (one orbit in $I$ ) the object at $z=4.868$ is barely-resolved with an elongation in the tangential direction to the cluster of $0^{\prime \prime} .25$ or $1 . \mathrm{kpc}$ for $\Omega=1$. Its placement relative to the critical curve gives it an intrinsic source size of $\sim 0.1-1 \mathrm{kpc}$ and an unlensed apparent magnitude of only $I \sim 26$, depending on the gradient of the cluster mass profile.

The spectroscopic observations for the galaxy at $z=4.868$ were carried out on the Keck II telescope in June 1997, totaling $180 \mathrm{~min}$ of dark time through a slit of width of $1^{\prime \prime} 0$ in seeing of $0^{\prime \prime} \cdot 7-0^{\prime \prime} 8$. The 300 line/mm grating was used blazed at $5000 \AA$, resulting in a resolution of $12 \AA$ at $6000 \AA$, determined from unblended sky lines. Adequate relative spectrophotometry is obtained by comparison of the spectrum of an early-type cluster member at $z=0.175$ observed simultaneously through the multislit mask, with the standard empirical E/SO spectrum of Kennicutt (1992). The observations for the galaxy at $z=5.120$ were carried out on the Keck II telescope in March and April of 1999, totaling 210 min. The 400 line/mm grating was sued blazed at $8500 \AA$, giving a resolution of $8.75 \AA$ at $6000 \AA$.

The flux-calibrated spectrum for the object at $z=4.868$ appears in Fig. 6.7, where $F_{\lambda}$ is plotted vs. observed wavelength on the lower axis and rest wavelength on the upper axis. It is of fairly-high quality. The emission line is readily identified as Ly- $\alpha$ at $z=4.875$, and as can be seen from the close-up spectrum of this object centered on Ly $\alpha$ in Fig. 6.7, has an obviously-asymmetric profile. The distinctive Lyman-series break seen blueward of Ly- $\alpha$ is caused by numerous $H$ absorbers along the line of sight. The interstellar SiII $\lambda 1260$ absorption line is clearly-detected at $z=4.868$. Ly- $\alpha$ is shifted redward in velocity from this $\mathrm{z}$ by $360 \mathrm{~km} / \mathrm{s}$, similar to other high-z objects and local starburst galaxies, indicating gas outflow. The hot stellar continuum rises up towards the Lyman-series break from the long wavelength side of $L y-\alpha$, with a distinctive wedge like step at the Ly- $\beta$ edge. The continuum is well-fitted by unreddened B0 stars, with forest blanketing at the level observed in high-z QSO spectra. The sky spectrum is included in the lower panel for comparison.

Below the galaxy and sky spectrum are a series of $U B V I J$ images, with the object circled in each one. No flux is seen in the spectrum below the Lyman-limit and no emission is detected in the deep $U$ or $B$ band images, with $2 \sigma$ upper limits of 26.5. The lack of flux below the Ly-limit is not unexpected assuming most reasonably that the HII region surrounding the $\mathrm{O}$-stars is density bounded, although a modest column lower redshift intervening forest cloud in the range $4.3<z<4.88$ could also lead to a non-detection in these bluer bands.

The flux-calibrated spectrum for the object at $z=5.12$ appears in Fig. 6.8, where the counts averaged per exposure is plotted vs. observed wavelength on the lower axis and rest wavelength on the upper axis. It is of relatively-high quality. The emission line is readily identified as Ly- $\alpha$ at $z=5.120$, and as can be seen from the close-up spectrum of this object centered on Ly $\alpha$ in Fig. 6.9, has an obviously-asymmetric profile and the largest total rest equivalent width of the high- $z$ objects in the sample. The distinctive Lyman-series break is readily-seen. The interstellar SiII $\lambda 1260$ absorption line is detected at the same redshift at $\sim 2.5 \sigma$ level. Below the galaxy and sky spectrum are $U B V I J$ images 

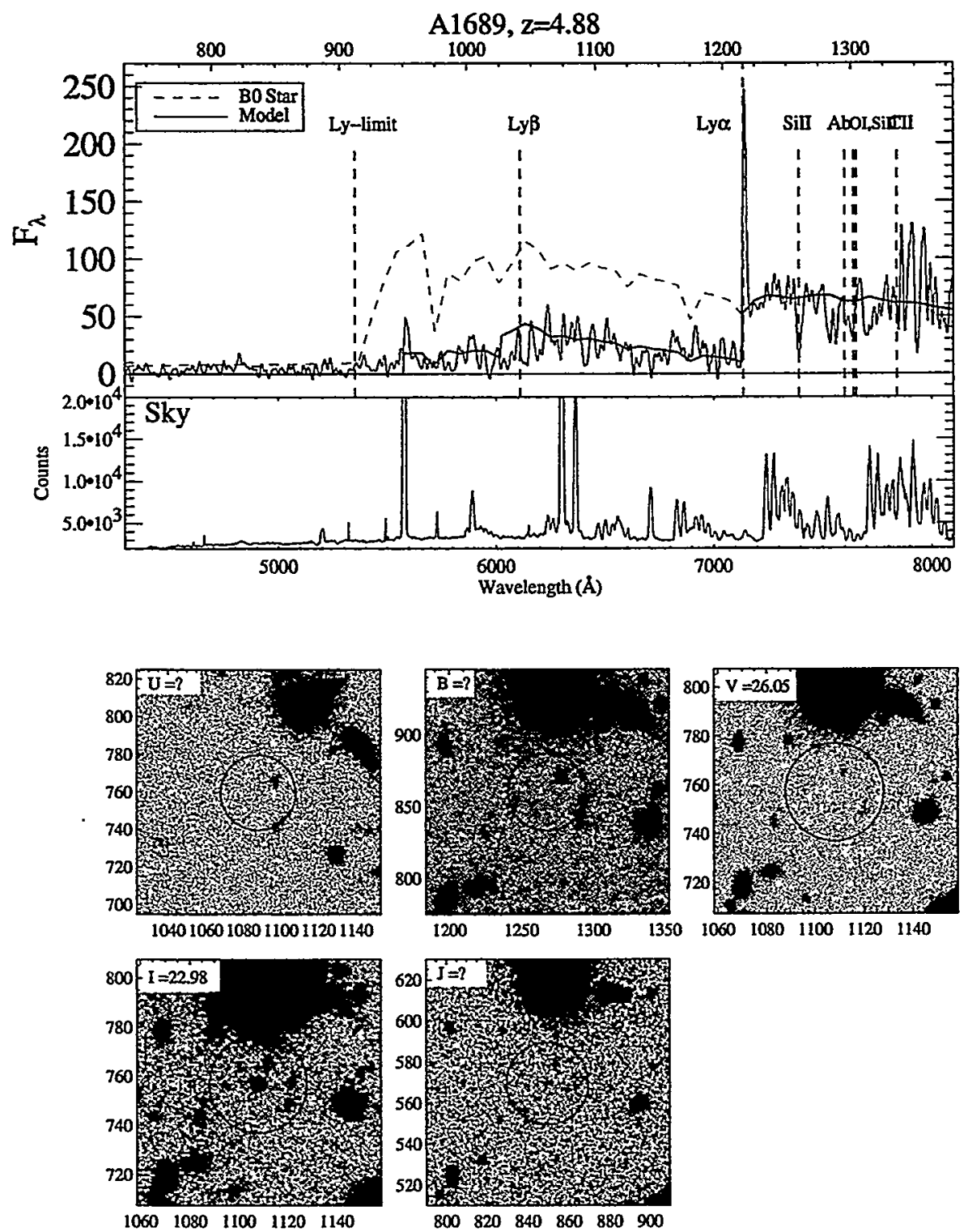

Figure 6.7: Imaging and Spectroscopy of a high-z galaxy in A1689 at $z=4.88$. The spectrum is plotted as $F_{\lambda}$ vs. observed wavelength on the lower axis and rest wavelength on the upper axis. The distinctive features are Ly- $\alpha$ in emission, the Ly- $\alpha$ break, the Ly- $\beta$ break, and the SiII $\lambda 1260$ metal line. Ly- $\alpha$ is shifted redward from SiII by $360 \mathrm{~km} / \mathrm{s}$. The continuum (dashed line) is best fitted by a hot $\mathrm{O}$ stellar population, with attenuation by the Lyman-series forest becoming severe by this $z$ (solid line). The sky spectrum is shown in the lower panel for comparison. Also included are five ground-based images of this galaxy (circled) in $U B V I J$. There is no emission from this object in $U$ and $B$. 

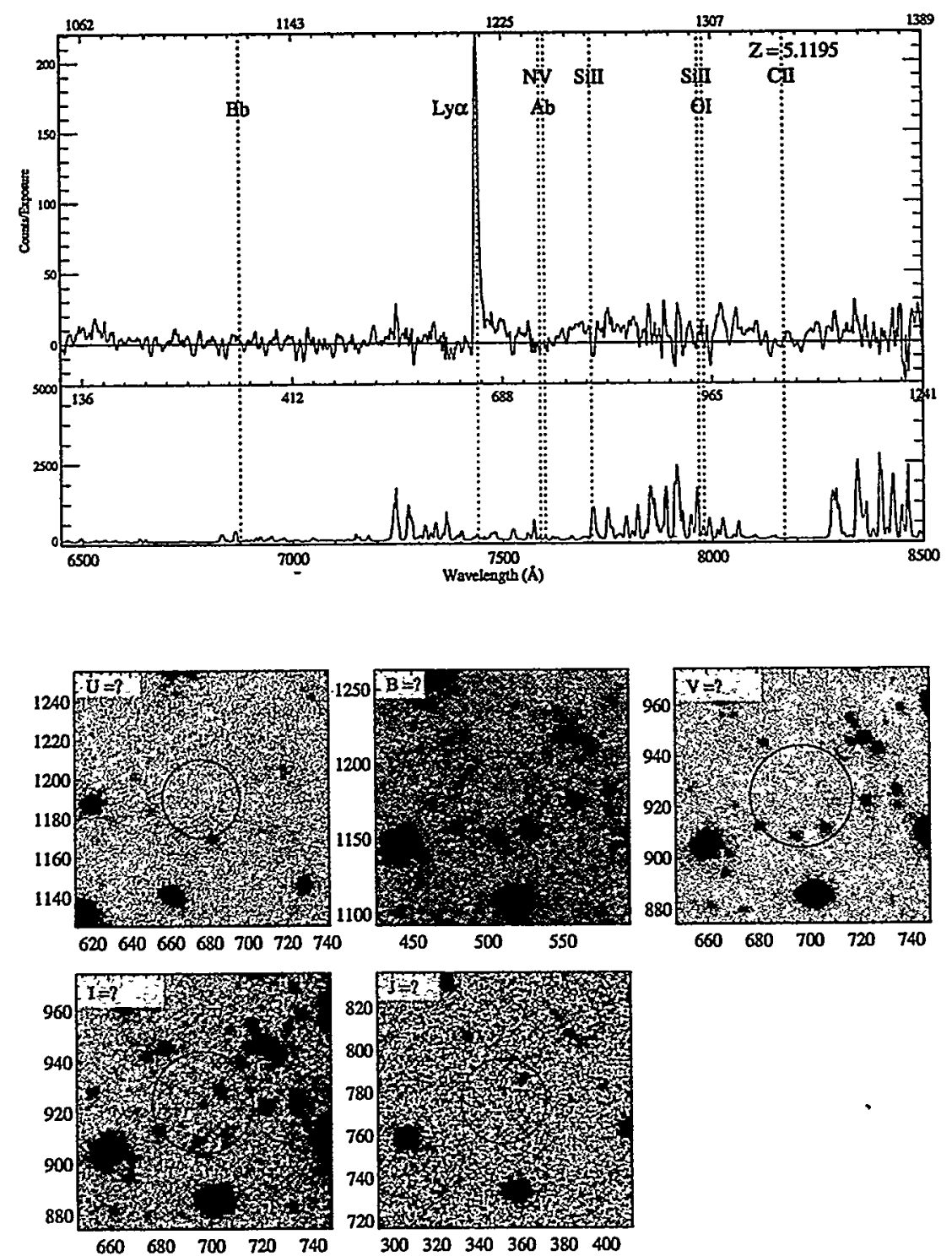

Figure 6.8: Imaging and Spectroscopy of a high- $z$ galaxy in A1689 at $z=5.12$. The spectrum is plotted as Counts vs. observed wavelength on the lower axis and rest wavelength on the upper axis. Ly- $\alpha$ is seen strongly in emission and has an asymmetric profile. The Ly- $\alpha$ break is also clearly-detected. The sky spectrum is shown in the lower panel for comparison. Also included are five ground-based images of this galaxy (circled) in UBVIJ. There is emission seen only in the $I$ band. 


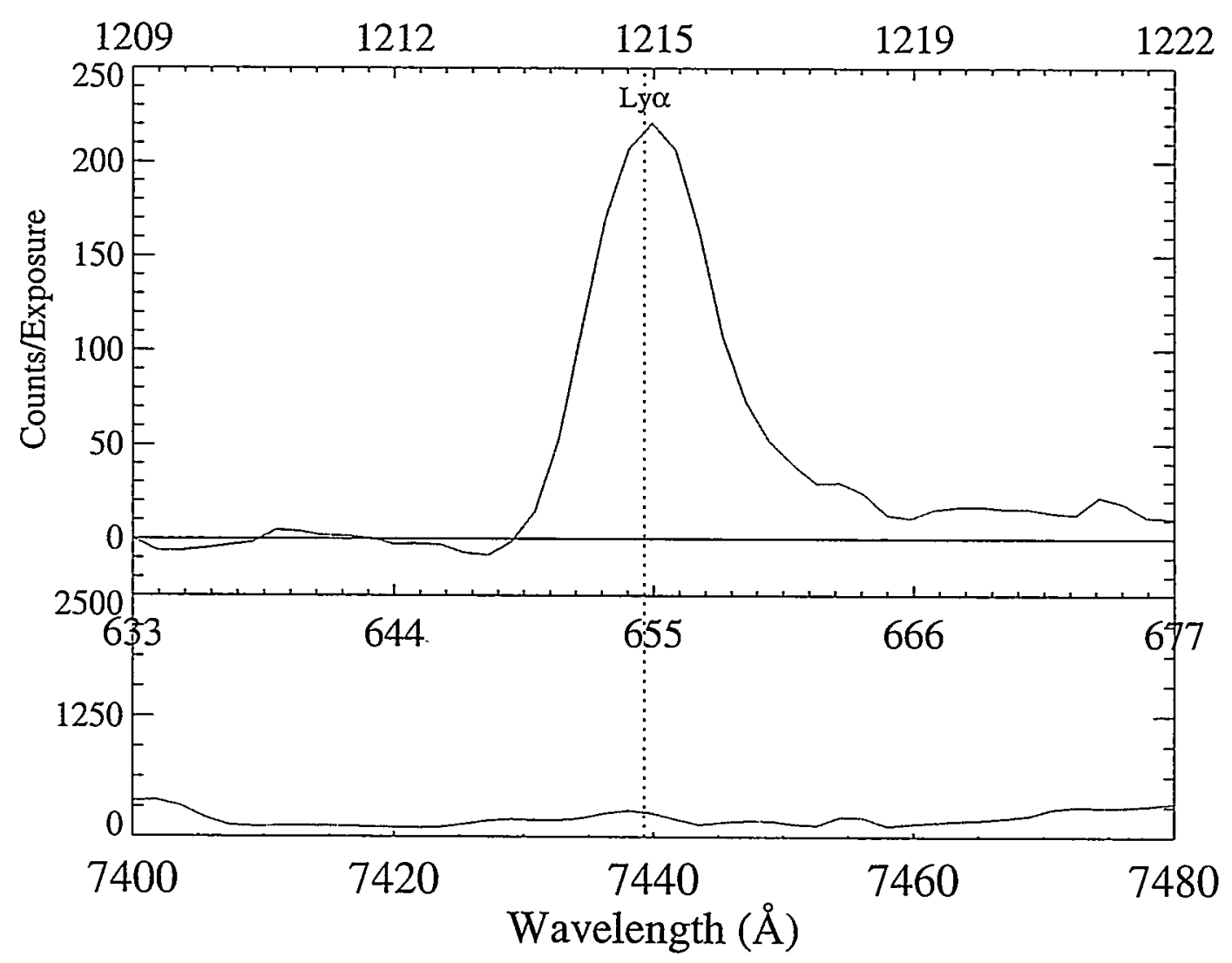

Figure 6.9: Imaging and Spectroscopy of a high- $z$ galaxy in A1689 at $z=5.12$, centered on the Ly- $\alpha$ emission line. The spectrum is plotted as Counts vs. observed wavelength on the lower axis and rest wavelength on the upper axis. Ly- $\alpha$ is seen strongly in emission and has an asymmetric profile. The sky spectrum is displayed in the lower panel for comparison. 
centered on this object and with it circled in each one. No flux is seen in the spectrum below the Lyman-limit and in these relatively-deep NTT images emission is only detected in the $I$ band.

\subsection{2 $\quad \mathbf{A 2 3 9 0}$}

HST images of A2390 were used to select galaxies redder than the cluster sequence for spectroscopy with the Keck. The imaging observations (courtesy of B. Fort) comprise four long exposures totaling 160 minutes in each of the F555W and F814W bands, corresponding roughly to $V$ and $I$. These images were aligned to the nearest integer and then cosmic rays rejected separately in each passband. Fig. 6.10 shows a color representation of the center of the cluster. A striking lensing pattern is visible, including the famous straight arc (Mellier et al., 1990; Pellò et al., 1991). A very bright and dusty red object is also seen lying along the critical curve, as well as several lensed background elliptical galaxies which are redder than the cluster $\mathrm{E} / \mathrm{SO}$ 's due simply to their larger k-corrections.

The multiply lensed high redshift images reported here are visible as relatively red and thin arcs with similar substructure along their lengths, centered on a bright cluster elliptical (Fig. 6.10), as first pointed out by Bézecourt \& Soucail (1997) in their analysis of the HST images. Removal of this elliptical by ellipse subtraction reveals four similar images forming a set of stretched arcs, as indicated in Fig. 6.10. The vertical bars in the upper panel of Fig. 61.0 makes the locations of the slitlet positions. Fig. 6.10 (lower right panel) shows a simple lens model for the source. Both the central elliptical and the cluster potential were found to be important in creating the general image configuration, as will be discussed below. The parity of the images is also displayed in the model such that the brighter part of the images is chosen to match the spatial location of the observed Ly $\alpha$ emission in the $\mathrm{N}$ and $\mathrm{S}$ arcs. These images are overlayed on the lens magnification field, showing clearly the bright asymmetric critical curve.

Spectra of the larger of the two red arcs at $z=4.04$, labeled $\mathrm{N}$ and $\mathrm{S}$ in Fig. 6.11 , were taken on two different observing runs. For the first set of observations the slits were aligned normal to the long axes of the two brightest arcs. A one hour exposure was taken through the 300 line grating in July 1996. A slit width of $1.5^{\prime \prime}$ was used, resulting in a resolution of $15.6 \AA$ and dispersion of $2.4 \AA$ per pixel. These spectra are shown in Fig. 6.11a,b and are plotted as $F_{\lambda}$ vs. observed wavelength on the bottom axis and rest wavelength on the top axis. The slit width is narrower than the length of these arcs, $5^{\prime \prime}$ and $3^{\prime \prime}$, and oriented nearly perpendicular the their long axes, hence these spectra are not expected to correspond to same spatial section of the source, and their spatial locations are indicated in Fig. 6.11c.

Ly $\alpha$ emission is seen only towards the southern arc in this first set of observations and is clearly-asymmetric. Absorption is seen at the position of Ly- $\alpha$ towards the northern arc, with the HI absorption best fit by a column density of $N=3 \times 10^{21} \mathrm{~cm}^{-2}$. Several interstellar metal lines have been identified in both spectra. They are: SiII $\lambda 1260$, OI/SiII 


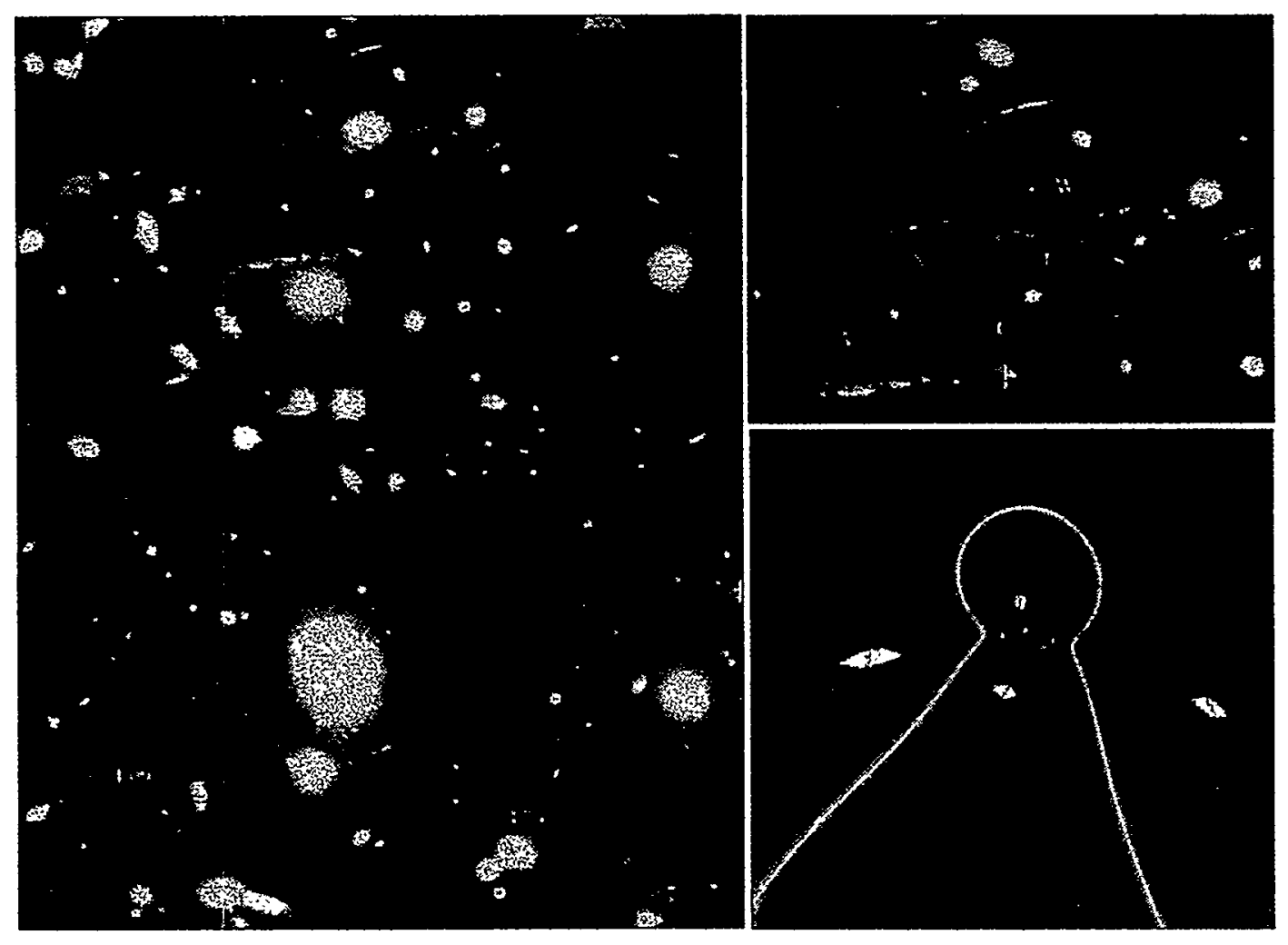

Figure 6.10: Panel (a) shows the many red and blue arcs around the center of A2390, with the elliptical galaxy centered on the $z=4.04$ arcs indicated. (b) A blow up of the region containing the 4 lensed images of the $z=4.04$ galaxy, with the central elliptical galaxy removed. The locations of the slitlet positions corresponding to Fig. 6.10 are indicated. Also shown is the "straight arc" which spectroscopy resolves into two galaxies: component $\mathrm{A}$ (in the notation of Pellò et al. (1991) at $z=1.033$ and component $\mathrm{C}$, at the known redshift of $z=0.913$ (see Fig. 6.11 for the spectra). (c) A simple lens model for the source showing the importance of both the central elliptical and the cluster potential in creating the general image configuration. The parity of the images is also displayed in the model: the brighter part of the images is chosen to match the spatial location of the observed Ly $\alpha$ emission in the $\mathrm{N}$ and $\mathrm{S}$ arcs. These images are overlayed on the lens magnification field, showing clearly the bright asymmetric critical curve. 


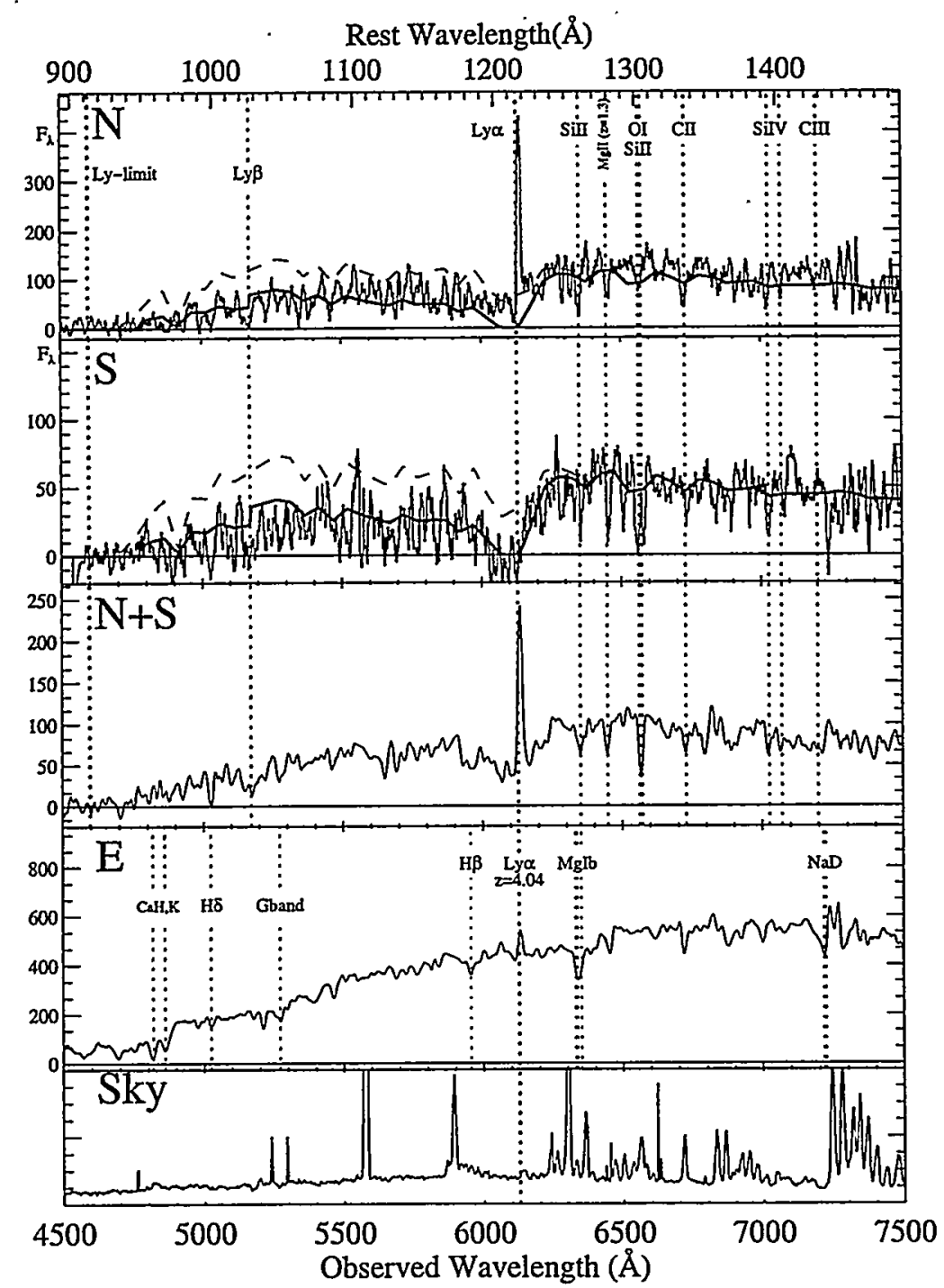

Figure 6.11: Keck spectra of the arcs at $z=4.04$. The upper two panels show fluxed spectra of the Northern and Southern arcs taken with slits aligned normal to the long axes of these arcs. Overlayed is the best fitting B3-star continuum, with the opacity of the forest included, together with Ly $\alpha$ absorption at the galaxy redshift, but no reddening. The middle panel shows the sum of both these arcs with slits aligned along their lengths, revealing clearly metal lines at $z=4.04$, on which the redshift is based. These lines lie blueward of the centroid of the asymmetric Ly $\alpha$ emission by $300 \mathrm{kms}^{-1}$, as discussed in the text. Below this is shown a spectrum centered on the Eastern arc, revealing Ly $\alpha$ emission and the central elliptical $(z=0.223)$. The sky is shown for comparison at the bottom of the panel. 


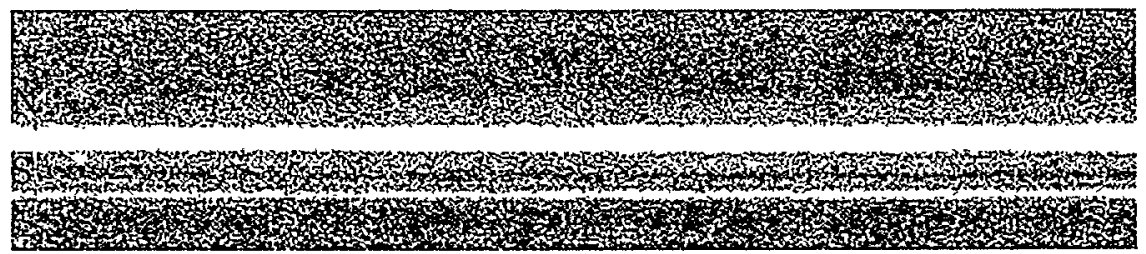

Figure 6.12: Two-dimensional spectrum of two arcs at $z=4.04$, taken along the long axis of the arcs. The northern and southern components are labelled as $\mathrm{N}$ and $\mathrm{S}$ corresponding to the image presented in Frye \& Broadhurst 1997. The dispersion direction is in the $\mathrm{x}$ direction with the blue at the left-hand side. The spatial direction is up-down and the pixel scale is 0.21 "/pix, with the northern and southern components extend 5 and $3^{\prime \prime}$ vertically on each side of the central cluster elliptical (the bright white strip). Ly $\alpha$ emission is seen in each component as a bright spot in the blue, and is spatially-separated from the broad clearly visible stellar continuum.

at $\lambda 1302 \AA$ and $\lambda 1304 \AA$, CII $\lambda 1336$, and SiIV $\lambda 1398 \AA$ and $\lambda 1402 \AA$. The total rest equivalent width of Ly $\alpha$ is $W_{L y \alpha}=12.7 \AA$, slightly larger than others at this redshift, with the difference possibly related to the fact that this galaxy is spatially-extended. The redshift is determined from these metal lines to be $z=4.040$. Ly- $\alpha$ is shifted redward in velocity by $200 \mathrm{~km} / \mathrm{s}$ from this $z$, indicating gas outflow. The Lyman- $\alpha$ break is also clearly seen against the strong stellar continuum in both spectra, caused by HI absorption along the line of sight to the galaxy.

A second set of data were taken of this multiply-imaged galaxy in June 1997, using the same instrumental configuration but with slits aligned along the arcs, totaling 2.8hrs of exposure (Fig. 6.11c,d). In this spatially-resolved dataset Ly $\alpha$ emission is seen at the northern end of both arcs. Fig. 6.12 shows the two-dimensional LRIS spectrum. The northern and southern components are labelled as $N$ and $S$ corresponding to the two galaxy images of Fig. 6.10. The dispersion direction is in the x-direction with blue at the left-hand side and the spatial direction is up-down. The northern and southern components extend in the spatial direction 5 and $3^{\prime \prime}$ on each side of the central cluster elliptical (the bright white strip). Ly $\alpha$ emission is seen in each component as a bright white spot in the blue, and is spatially-separated from the broad clearly visible stellar continuum.

Fig. 6.11c shows this higher quality spectrum. The exposure time has been tripled and the spectra from the northern and southern arcs have been combined in this panel, improving on the signal to noise by $\sim 2.5$ over the signal to noise of the first set of spectra (Fig. 6.11a,b). This spectrum is similar in general appearance to the lower signal to noise data and confirms the presence of all of the metal lines listed above. In addition a 'mystery' line is identified at $6440 \AA$, possibly from an intervening MgII system at $z=1.3$. A third arc labeled $\mathrm{E}$ in Fig. 6.10 was also observed to investigate the lens geometry and found to share the same redshift as the two bright arcs. Its spectrum is shown in Fig. 6.11d. Although contaminated by the central elliptical galaxy's continuum, Ly- $\alpha$ is clearly-seen in 
emission. The fourth image, $W$, is too faint for useful spectroscopy. The sky spectrum is shown in the last panel for comparison with the data.

\subsubsection{A2219}

Keck LRIS images of A2219 were taken on 19 April 1996 on Keck I in good seeing, $\sim 0 ! 7$. The observations totalled $1200 \mathrm{sec}$ in $V$ and $600 \mathrm{sec}$ in $I$. The fairly-deep $I$ band image appears in Fig. 6.13, which shows numerous arcs. The circled objects identify the three high- $z$ galaxies with $z>4$ which we have discovered behind this cluster.

The spectroscopy were taken in August 1998 in 2.3 hours of exposure using LRIS with the 400 line grating on Keck II. The slit width was $1^{\prime \prime}$, resulting in a resolution of $10 \AA$ and a dispersion of $1.8 \AA /$ pix. We observed of the spectrophotometric standard G24-9. The standards were divided into our observations of the standards to obtain the transmission function, which was then divided into our galaxy spectra to obtain the relative flux calibrations.

The high- $z$ spectra appear in Fig. 6.14, 6.15, and 6.16, plotted as $F_{\lambda}$ vs. observed wavelength on the lower axis and rest wavelength on the upper axis. The middle axis gives the pixel values on the $2 \mathrm{~d}$ spectral image. Close-up spectra of these objects centered on the Ly $\alpha$ line appear in Fig. 6.17. The solid line shows a model for the stellar population and the dashed line includes Lyman-series forest opacity and damped HI aborption, if any, at $z_{e m}$. The sky spectrum appears in the lower panel for comparison with the data. To the right of each spectrum is a $V$ and $I$ band image centered on the object. The $\mathrm{x}$ and $\mathrm{y}$ axis labels refer to the coordinates of the larger image of Fig. 6.13. For $z>4$ the Lyman-series redshifts into the $V$ band, suppressing $V$ relative to $I$ and giving these high- $z$ galaxies their characteristically-large $V-I$. All three high- $z$ galaxies have many interesting features and each will be discussed in turn.

The spectrum of the arc at $z=4.654$ is shown in Fig. 6.14. The signal to noise is reasonably-good, reaching $\sim 4$ in the continuum. Ly- $\alpha$ is seen in emission and has a clearly-asymmetric shape (see Fig. 6.15 for a close-up spectrum centered on Ly $\alpha$ ). There is a sharp Lyman-series break shortward of Ly $\alpha$, caused by $\mathrm{H}$ absorption in the intervening Lyman-series forest. Somewhat surprisingly, the stellar continuum rises redward of the Ly$\alpha$ emission line instead of declining as would be expected from on $\mathrm{O}$ star population with no absorption at the source. This feature is also seen in the slightly-higher signal to noise spectrum at $z=4.445$ and may be caused entirely by HI absorption at the emission redshift or by the combined effect of HI opacity and the stellar population. The best fitted model is a B3 stellar population (solid line) which has been attenuated by the Lyman-series forest and damped HI absorption at $z_{e m}$ with column density $\log N=21.5$. The interstellar metal line SiII $\lambda 1260$ has been clearly-detected in this spectrum. The centroid of the Ly- $\alpha$ emission line is shifted towards the red by $425 \mathrm{~km} / \mathrm{s}$ with respect to these lines and has a total rest equivalent width $W_{L y \alpha}$ of $16.1 \AA$. The $V$ and $I$ band images at the position of this arc are shown on the right side of Fig. 6.14. There is no emission in $V$, and the apparent size in $I$ 


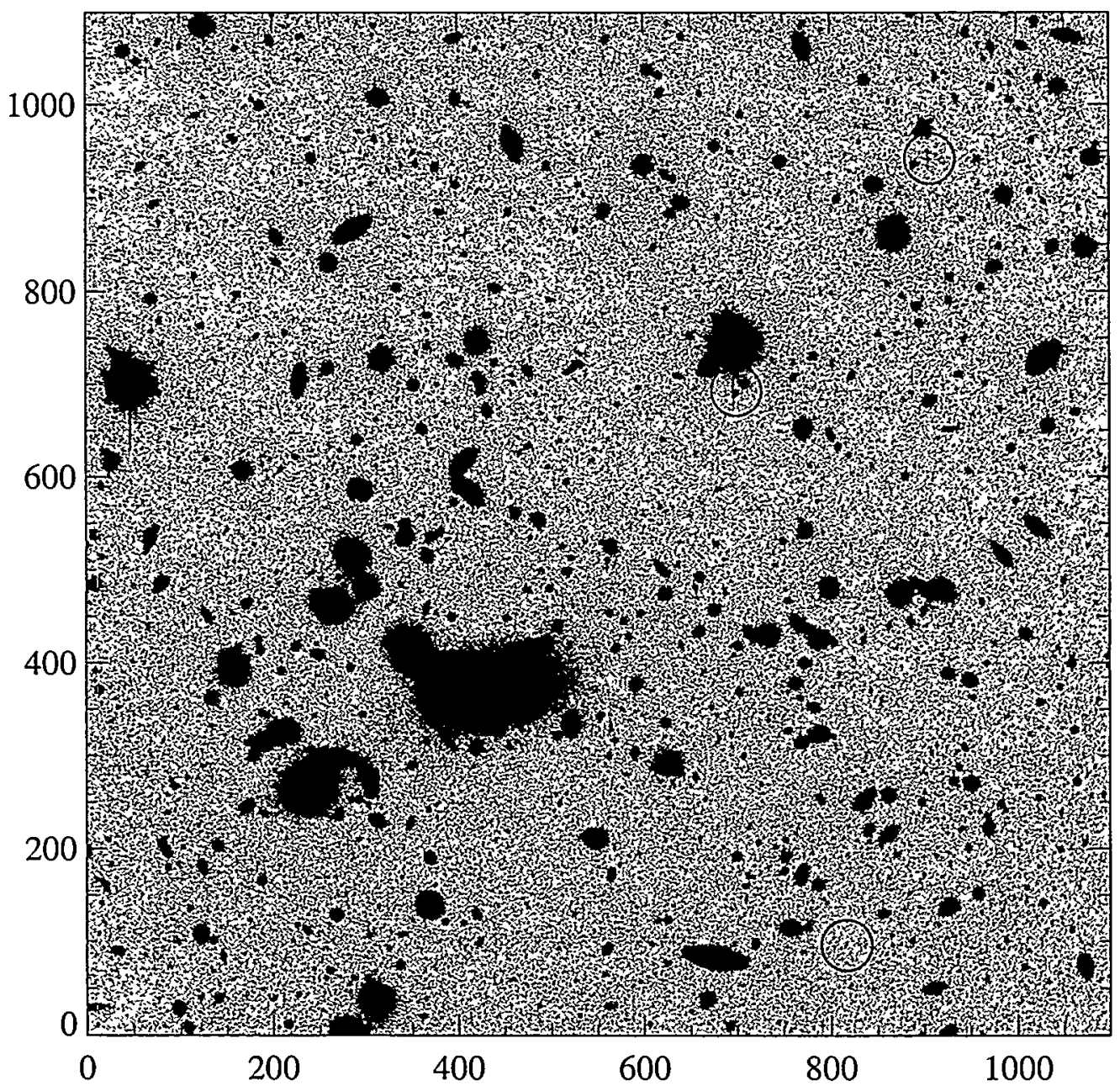

Figure 6.13: Keck $I$ band image of A2219, taken in in good seeing, 0 't7. The three high- $z$ galaxies marked on the plot were discovered as a part of our survey of faint, red galaxies behind this cluster. The pixel scale is 0.21 "/pix. The cluster center has a J2000 coordinate of $\alpha=16^{h} 38^{m} 54.0^{s}, \operatorname{dec}=46^{\circ} 47^{\text {arcmin }} 00^{\prime \prime}$. 

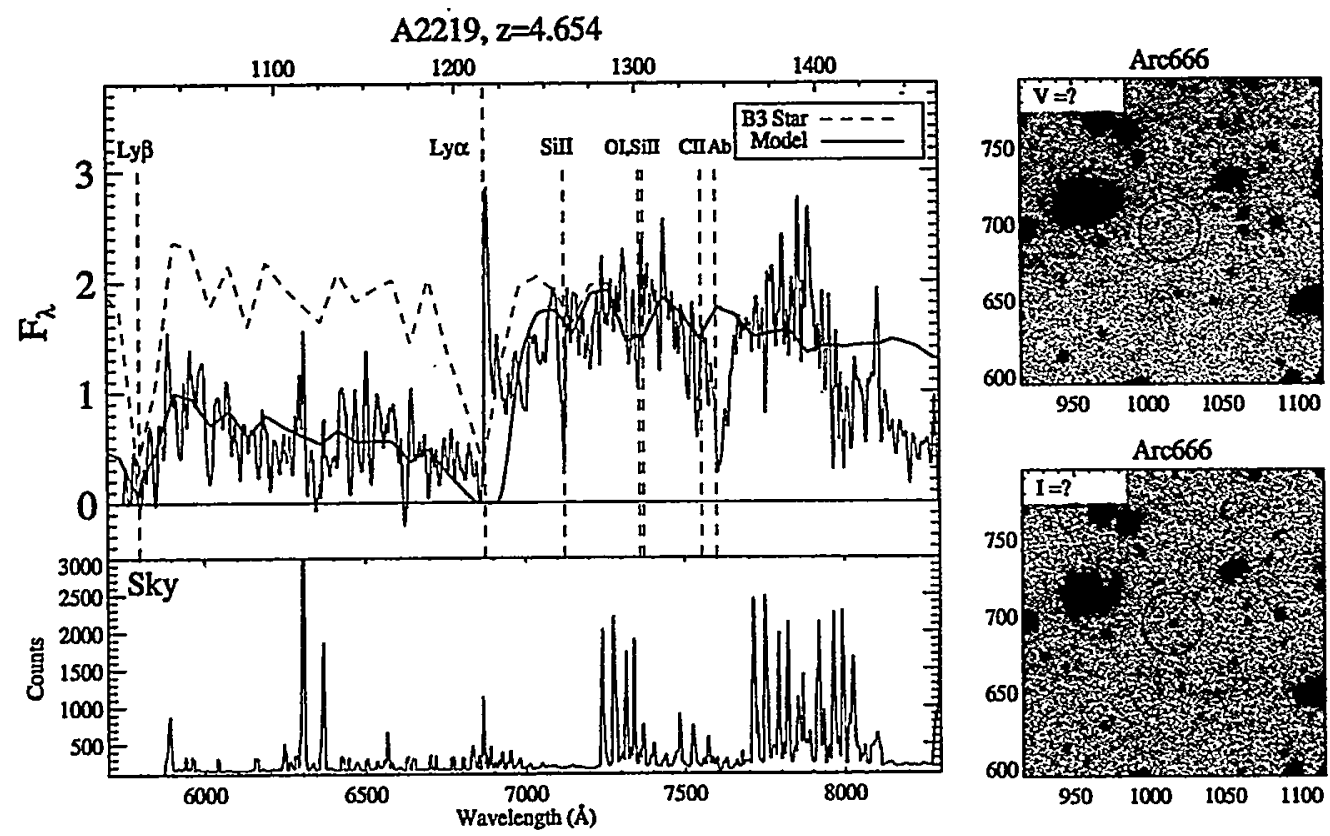

Figure 6.14: Spectrum and images of a high- $z$ galaxy at $z=4.67$ seen behind A2219. $F_{\lambda}$ is plotted against observed wavelength on the lower axis and rest wavelength on the upper axis. For reference, the middle axis gives the pixel scale for the $2 \mathrm{~d}$ spectrum. the spectrum shows a strong stellar continuum, strong metal lines, Ly- $\alpha$ emission, and a clearly-detected break shortward of Ly- $\alpha$. The dashed line shows the spectrum of a B3 stellar population and the solid line shows the model, which includes Lyman-series opacity plus an HI absorption component at $z_{e m}$ with a column density of $\log N=21.5$. The sky spectrum is plotted in the lower panel for comparison with the data. Two images of this galaxy, in $V$ and $I$ appear on the right-hand side of the spectrum. The $\mathrm{x}$ and $\mathrm{y}$ coordinates refer to the coordinates of the larger image of Fig. 6.13. The object is undetected in $V$ and resolved but small in $I$, despite its expected large magnification.

is small, as is typical for the high- $z$ galaxies in our sample.

Fig. 6.15 shows the flux-calibrated spectrum of the object at $z=4.445$, which is a truly beautiful example of a high quality spectrum of a high- $z$ galaxy. The signal to noise in the continuum is high, reaching $\sim 7$ in the continuum. The most striking features are probably the strong, but not visibly-saturated metal lines and the clear-cut Lymanseries break. As in most of our certain cases, Lyman- $\alpha$ is seen in emission and has its the characteristically-asymmetric shape, truncated at the blue end by $\mathrm{HI}$ aborption at the source and from $\mathrm{HI}$ aborption by the Lyman-series forest. The stellar continuum is strong and rises just redward of Ly $\alpha$. Many metal lines are clearly-detected. They are SiII $\lambda 1260$, OI and SiII at $\lambda 1302$ and $\lambda 1304$ respectively, CII $\lambda 1336$, and SiIV $\lambda 1398$ and $\lambda 1402$. The 

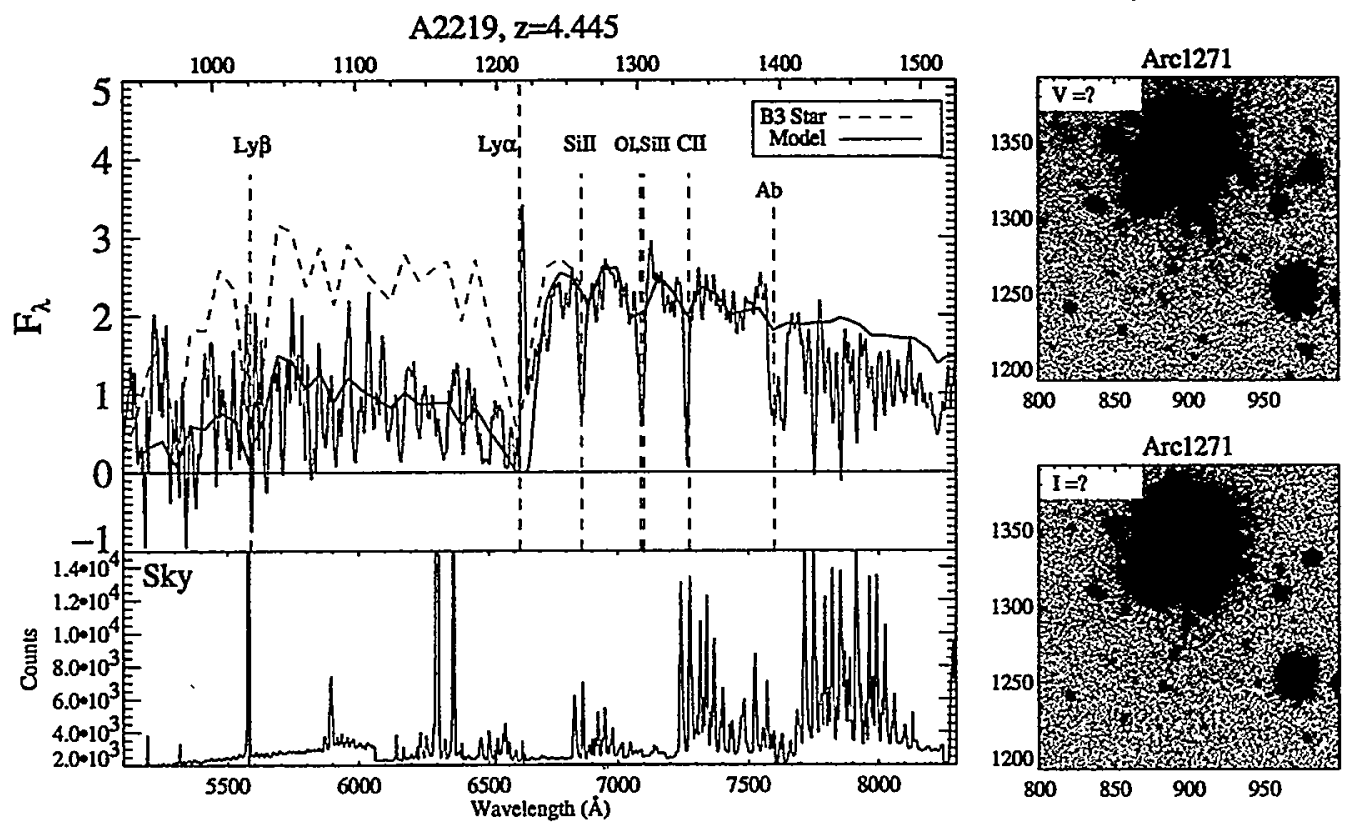

Figure 6.15: Spectrum and images of a high- $z$ galaxy at $z=4.45$ seen behind A2219. $F_{\lambda}$ is plotted against observed wavelength on the lower axis and rest wavelength on the upper axis. For reference, the middle axis gives the pixel scale for the $2 \mathrm{~d}$ spectrum. This is a high quality spectrum with strong stellar continuum, saturated metal lines, and a distinctive Lyman-series break. Ly- $\alpha$ is seen in emission and is asymmetric. The numerous Ly- $\alpha$ is shifted redward from the $z$ given by the metal lines by $690 \mathrm{~km} / \mathrm{s}$. The dashed line shows the spectrum of a B3 stellar population and the solid line shows the model, which includes Lyman-series opacity plus a damped HI absorption component at $z_{e m}$ with a column density of $\log N=21.1$. The sky spectrum is plotted in the lower panel for comparison with the data. Two images of this galaxy, in $V$ and $I$ are shown to the right of the spectrum. The $\mathrm{x}$ and $\mathrm{y}$ coordinates refer to the coordinates of the larger image of Fig. 6.13. The object appears at the center of the circle, just to the right of the stellar diffraction spike. It is faint in $V$ and resolved but small in $I$, despite its expected large magnification.

redshift determined from these lines is $z=4.445$.

The centroid of Ly- $\alpha$ is shifted redward of this $z$ by a whopping $690 \mathrm{~km} / \mathrm{s}$. This velocity shift is present in all high- $z$ galaxies of sufficiently-high quality for which it can be measured, and correlates with the equivalent width of the interstellar lines, as will be discussed below. The total rest equivalent width of $\operatorname{Ly} \alpha$ is $W_{L y \alpha}=9.6 \AA$, slightly smaller than for the galaxy at $z=4.654$, and consistent with the trend seen in this survey of equivalent width increasing with redshift. The best fitted model is for a B3 stellar population (solid line) with Lyman-series forest attenuation along the line of sight and HI absorption 

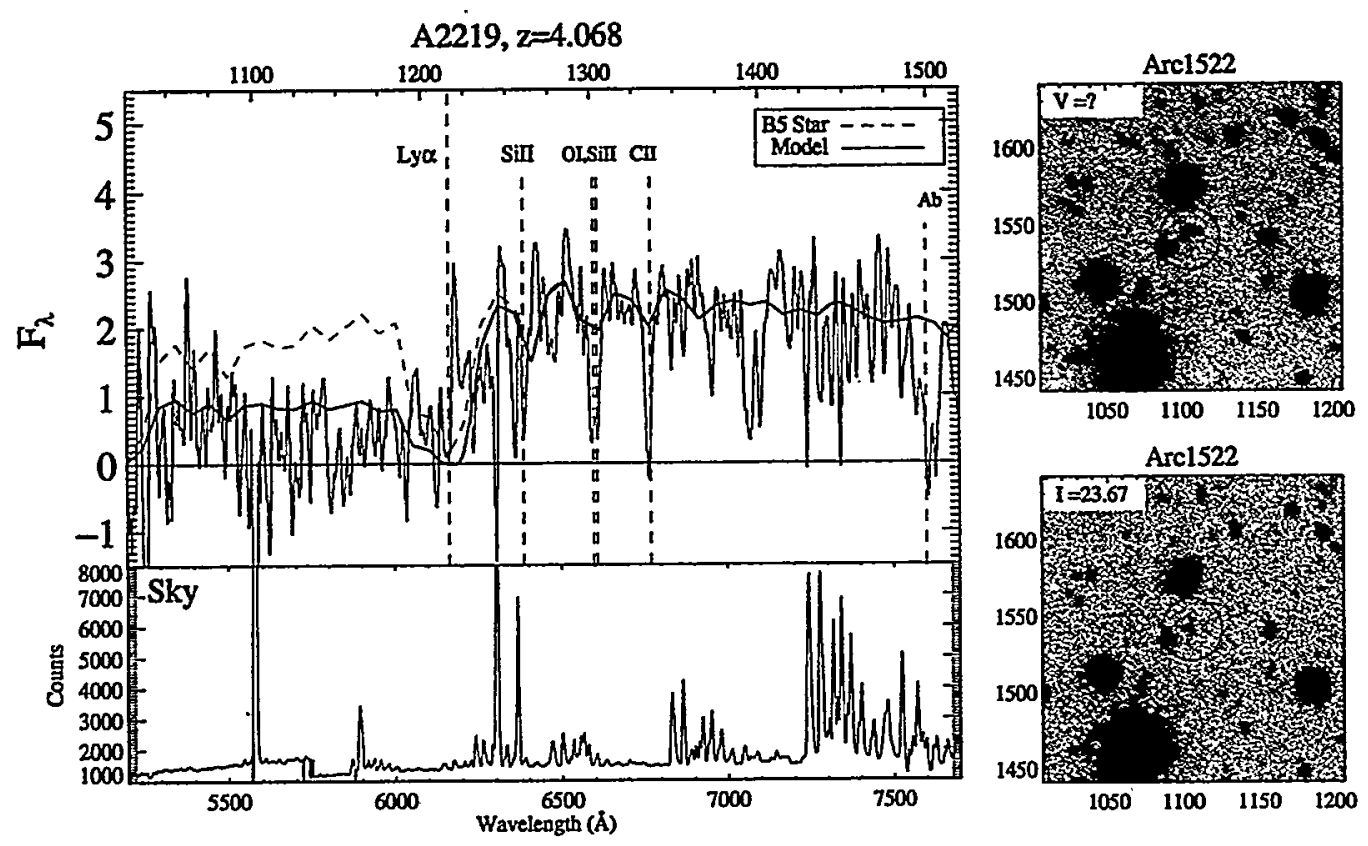

Figure 6.16: Spectrum and images of a high-z galaxy at $z=4.068$ behind A2219. $F_{\lambda}$ is plotted against observed wavelength on the lower axis and rest wavelength on the upper axis. For reference, the middle axis gives the pixel scale for the $2 \mathrm{~d}$ spectrum. Interestingly, this galaxy displays fully-saturated metal absorption lines. Ly- $\alpha$ is seen in emission above the underlying stellar continuum, and is shifted by $830 \mathrm{kms}^{-1}$ towards the red with respect to the redshift of the metal lines. The Lyman-series break is clearly-detected extending shortward of Ly- $\alpha$. The dashed line shows the spectrum of a B5 stellar population and the solid line shows the model, which includes Lyman-series opacity plus a damped HII absorption component at $z_{e m}$ with column density $\log N=21.1$. The sky spectrum is shown in the lower panel for comparison with the data. Two images of this galaxy, in $V$ and $I$, are shown to the right of the spectrum. The $\mathrm{x}$ and $\mathrm{y}$ coordinates refer to those of the larger image of Fig. 6.13. The object centered in the circle. It is very faint in $V$ and resolved but small in $I$.

both stellar and interstellar at $z_{e m}$. The object is detected in both the $V$ and $I$ images shown to the right of the spectrum in Fig. 6.15.

The third high- $z$ spectrum in this cluster is at $z=4.068$, which interestingly has a set of visibly-saturated metal lines. These are: SiII $\lambda 1260$, OI and SiII at $\lambda 1302$ and $\lambda 1304$ respectively, CII $\lambda 1336$, and SiIV $\lambda 1398$ and $\lambda 1402$. Ly- $\alpha$ is seen in emission above the underlying stellar continuum, although faint. The centroid of Ly- $\alpha$ is shifted by $830 \mathrm{~km} / \mathrm{s}$ redward from the redshift determined by the metal lines, indicating a substantial amount of gas outflow. The total rest equivalent width for Ly $\alpha$ is $W_{L y \alpha}=7.74$, the smallest of the 

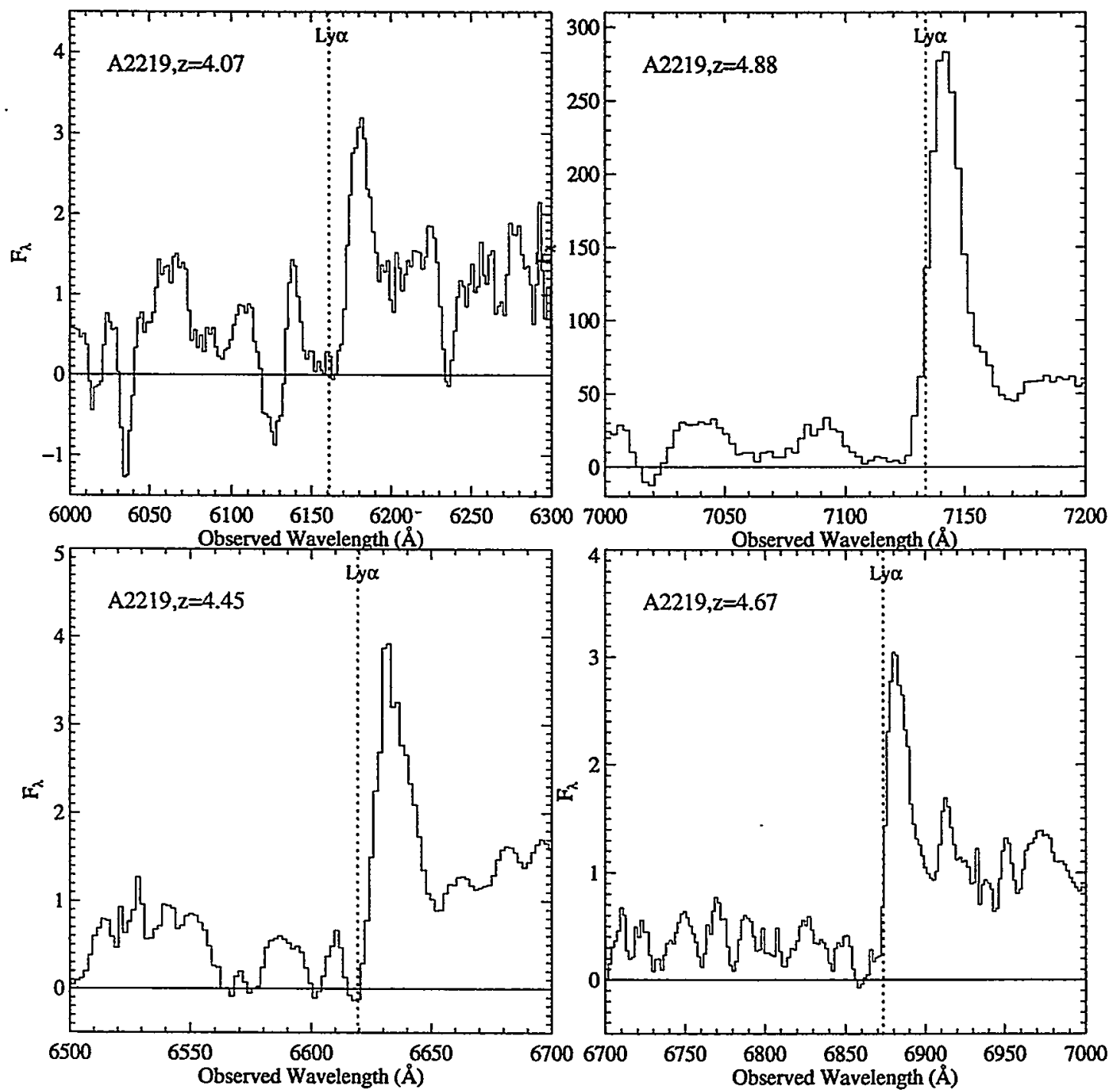

Figure 6.17: Spectra of the three high- $z$ galaxies in A2219 and the second highest redshift galaxy in $A 1689$, as labelled. $F_{\lambda}$ is plotted in arbitrary flux units against observed wavelength. Ly- $\alpha$ is seen in emission above the underlying stellar continuum, and is shifted by varying amounts with respect to the interstellar metal absorption lines (see text).

three in this cluster, and also the smallest redshift. The Ly-series break is seen shortward of Ly- $\alpha$. Two images of this galaxy appear to the right of the spectrum. The $I$ band flux is 23.67 , and the object is very faint possibly undetected in $V$. There is another object towards the upper part of the circle which should not be confused with the high- $z$ galaxy, at the expected position of which the circle is centered. 
OTHER LYMAN-SERIES BREAK GALAXIES

\begin{tabular}{|c|c|c|c|l|}
\hline Cluster & $z$ & Page \# & Name & Features \\
\hline A1689 & 4.18 & 147 & $1.40 \mathrm{~b}$ & B drop-out, metal lines \\
A1689 & 3.85 & & 24 & Ly- $\alpha$ line, $V-I=1.8, \operatorname{SiII} \lambda 1260$ \\
A1689 & 3.83 & & $7 \mathrm{a}$ & Very red, $V-I=2.61$. \\
A1689 & 3.044 & 169 & $31 \mathrm{a}$ & Ly- $\alpha$ in absorption, metal lines \\
A2390 & 4.09 & & A63 & Lyman-series break only. \\
A2390 & 3.75 & 57,91 & $22,1 \mathrm{a}$ & Very red giant arc. \\
A2390 & 3.60 & & red_56 & Red, $V-I=1.85$ \\
\hline
\end{tabular}

\subsubsection{The Leftovers}

Galaxies with interesting spectra at $z<4$ or galaxies at high $z$ with fairly-low signal to noise are listed in the table below. They are all red and have pronounced Lymanseries breaks, amongst other features. The columns are: Cluster name, $z$, page number in the Appendix on which the object's spectrum plus $I$ band image can be found, the object name, and spectral features.

The object at $z=3.044$ in A1689 is of fairly low redshift but is an interesting object with a high quality spectrum. Many metal lines appear in absorption against the strong underlying stellar continuum (Appendix, p. 222). In addition, two intervening aborption systems are seen towards this object, each identified by its own set of very strong metal lines at $z=2.87$ and $z=2.53$. All three systems show Ly- $\alpha$ in absorption and most or all of the following metal lines: SiII $\lambda 1260, \mathrm{OI}$ and SiII at $\lambda 1302$ and $\lambda 1304$ respectively, CII $\lambda 1336$, SiIV $\lambda 1398$ and $\lambda 1402$, SiII $\lambda 1527$, and SiII at $\lambda 1548$ and $\lambda 1552, \operatorname{AIII} \lambda 1671$, and AlIII $\lambda 1855$ and $\lambda 1863$. The object at $z=4.18$ in A1689 has a $V-I=2.03$ and no flux in deep $B$ band images. The spectrum shows a Lyman-series break and the metal lines of SiII $\lambda 1260$, OI and SiII at $\lambda 1302$ and $\lambda 1304$ respectively and SiIV $\lambda 1398$ and $\lambda 1402$.

A giant red arc at $z=3.75$ in A2390 subtends a full 30 degrees about a giant cluster elliptical. Fig. 6.10a shows a color HST image of the central part of A2390 with this arc labelled on it. As can be seen from the image it is very red and highly-magnified but of low surface brightness, making the acquisition of a high signal to noise spectrum a challenge. There were two attempts made and both give consistent results (Pages 51 and 97 of the Appendix). The distinguishing feature for this object is the Lyman-series break. The object at $z=4.09$ in A2390 is also labelled on the HST color image of Fig. 6.10a. Its distinguishing feature is also the Lyman-series break. See Table 6.4 in the discussion for a talley of Ly $\alpha$ emission in $z \gtrsim 4$ galaxies.

\subsubsection{The Reddest Elliptical Galaxies}

Distant ellipticals provide a means of studying what appear to be evolved stellar populations, and their ages can help set lower limits on the formation redshift of galaxies, and so are of great interest. The reddest galaxy at $z>1$ is 53W091 at $z=1.55$, which has 


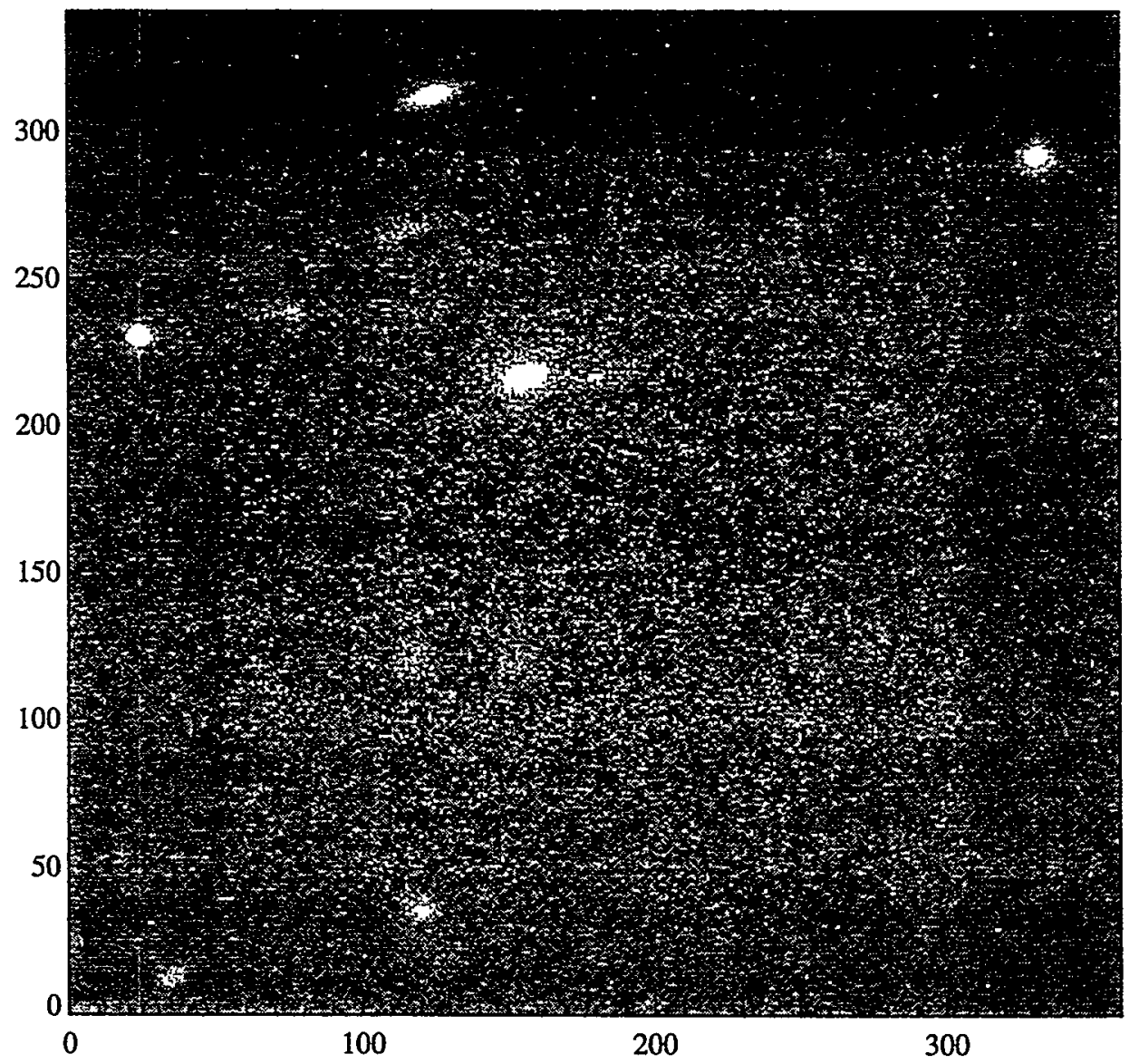

Figure 6.18: Near infrared image of the elliptical galaxy at $z=1.155$ in A1689, taken with NIRC on Keck 1 in June of 1998. This 30 min exposure in $K$ shows that this galaxy is a spheroidal. 


\begin{tabular}{|c|c|c|c|c|c|}
\hline Cluster & Name & $z$ & $I$ & $V-I$ & Age \\
\hline A1689 & Arc 21 & 1.155 & 21.4 & 2.21 & 8 \\
A2390 & red 120 & 0.791 & 22.0 & 2.68 & 8 \\
A2390 & red 122 & 0.783 & 20.4 & 3.06 & 10 \\
\hline \hline
\end{tabular}

Table 6.2: Properties for three of the best cases for distant ellipticals. The columns are: Cluster, Object Name, $z, I$ band magnitude, $V-I$ color, and age since the last starburst event.

a minimum age of 3.5 Gyr (Spinrad et al., 1997; Dunlop et al., 1996). These estimates are based on a standard single-burst model where the entire stellar population is assumed to form at a single epoch, the stellar population passively evolves, and the age of the stellar population is determined by the age of the stars at the turn-off of the main sequence. These estimates are lower-limits, since age is anti-correlated with metallicity in these determinations. A caveat is that the mass function of stars in the burst is unknown, so a relatively young stellar population with a truncated mass function could not be distinguished from an older stellar population with a more standard IMF.

The most distant ellipticals observed in this survey are easily-distinguished by their large $V-I$ colors. Table 6.2 summarizes the properties for three of the best cases in this survey. They have absorption line redshifts measured at $z=1.155, z=0.793$, and $z=0.783$.

Photometry was taken for the reddest elliptical galaxy to be discovered so far in the survey, at $z=1.155$, using the Near Infrared Camera (NIRC) on Keck II in June, 1998. $J$ and $K$ band images were obtained under conditions of $\sim 0.5^{\prime \prime}$ seeing. In $K$, three sets of twelve $5 \mathrm{~s}$ coadded exposures were obtained in each of nine dithering positions in a standard 8 "dithering sequence, resulting in a total of $27 \mathrm{~min}$ of exposure. In $J$, three sets of twelve $10 \mathrm{~s}$ coadded exposures were taken in each of nine dithering positions, resulting in a total integration time of $54 \mathrm{~min}$. The data were reduced using the purpose-built code described in the Data Reduction Chapter of this thesis. The approach, however, is somewhat different for infrared data. First, the bias was subtracted off of each frame, and then the sky. In order to account for the time-varying sky, a different flatfield was created for each exposure, taking the median of the 8 surrounding frames in the given dithering sequence. The individual flatfields were then normalized to the median of their corresponding data frames before subtraction. The reduced $K$ band image appears in Fig. 6.18. The elliptical galaxy appears as the bright, spheroidal blob just above and slightly to the left of center.

The spectroscopy for the reddest elliptical in the survey, at $z=1.155$ (Fig. 6.19), was taken using LRIS on Keck II in June 1997, totalling $180 \mathrm{~min}$ of dark time through a slit of width of $1{ }^{\prime \prime} 0$ in seeing of $00^{\prime \prime} 7-0.8$. The 300 line/mm grating was used blazed at $5000 \AA$, resulting in a resolution of $12 \AA$ at $6000 \AA$, determined from unblended sky lines. The spectroscopy for other two galaxies at $z=0.791$ and $z=0.783$ respectively were also taken 


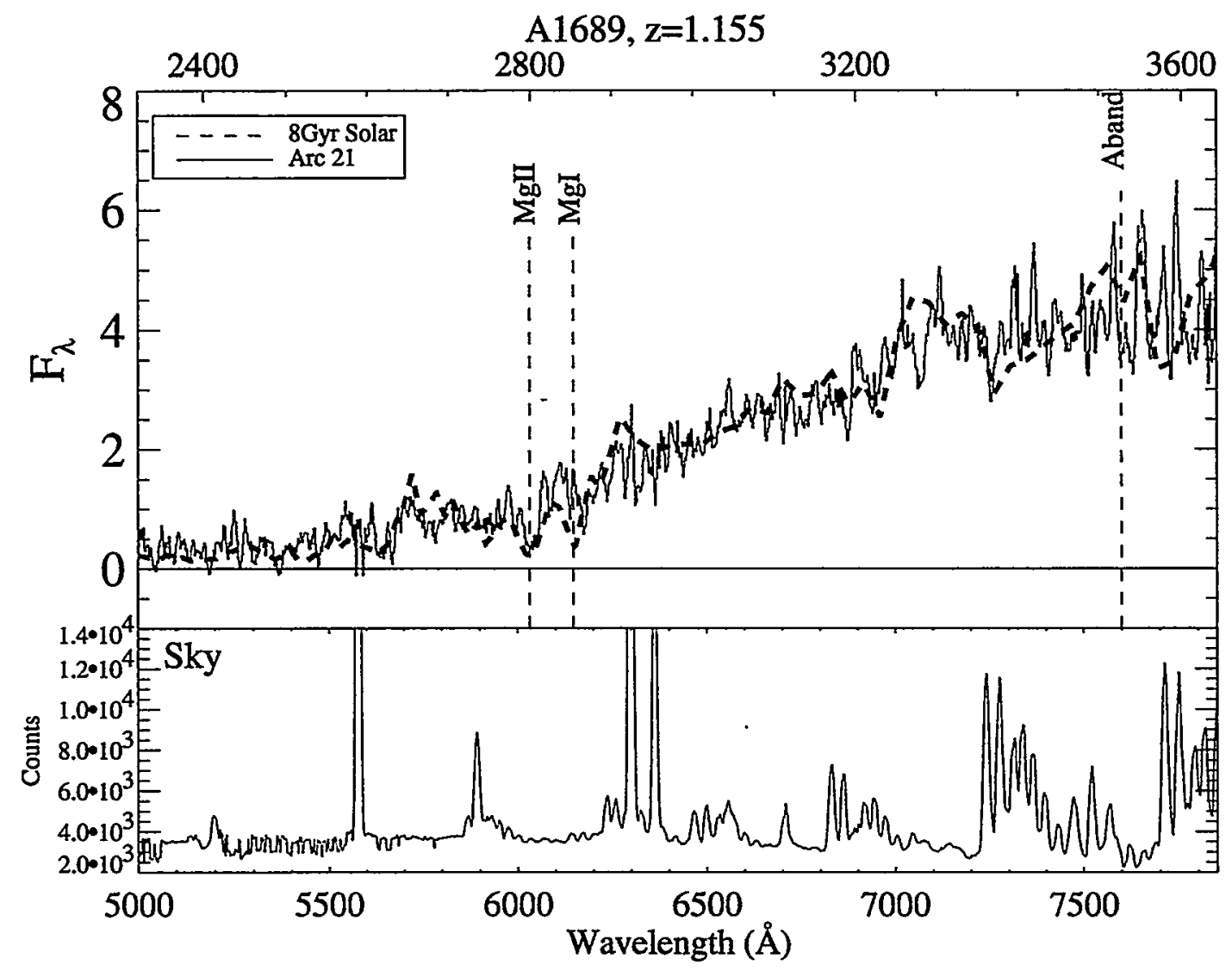

Figure 6.19: Spectrum of a elliptical at $z=1.155$ in A1689 with Bruzual-Charlot model overlayed. $F_{\lambda}$ is plotted against observed wavelength on the lower axis and rest wavelength on the upper axis. The model is for a passively-evolved $8 \mathrm{Gyr}$ galaxy with solar metallicity. MgI and MgII are clearly seen. The sky spectrum appears on the bottom panel for comparison. 


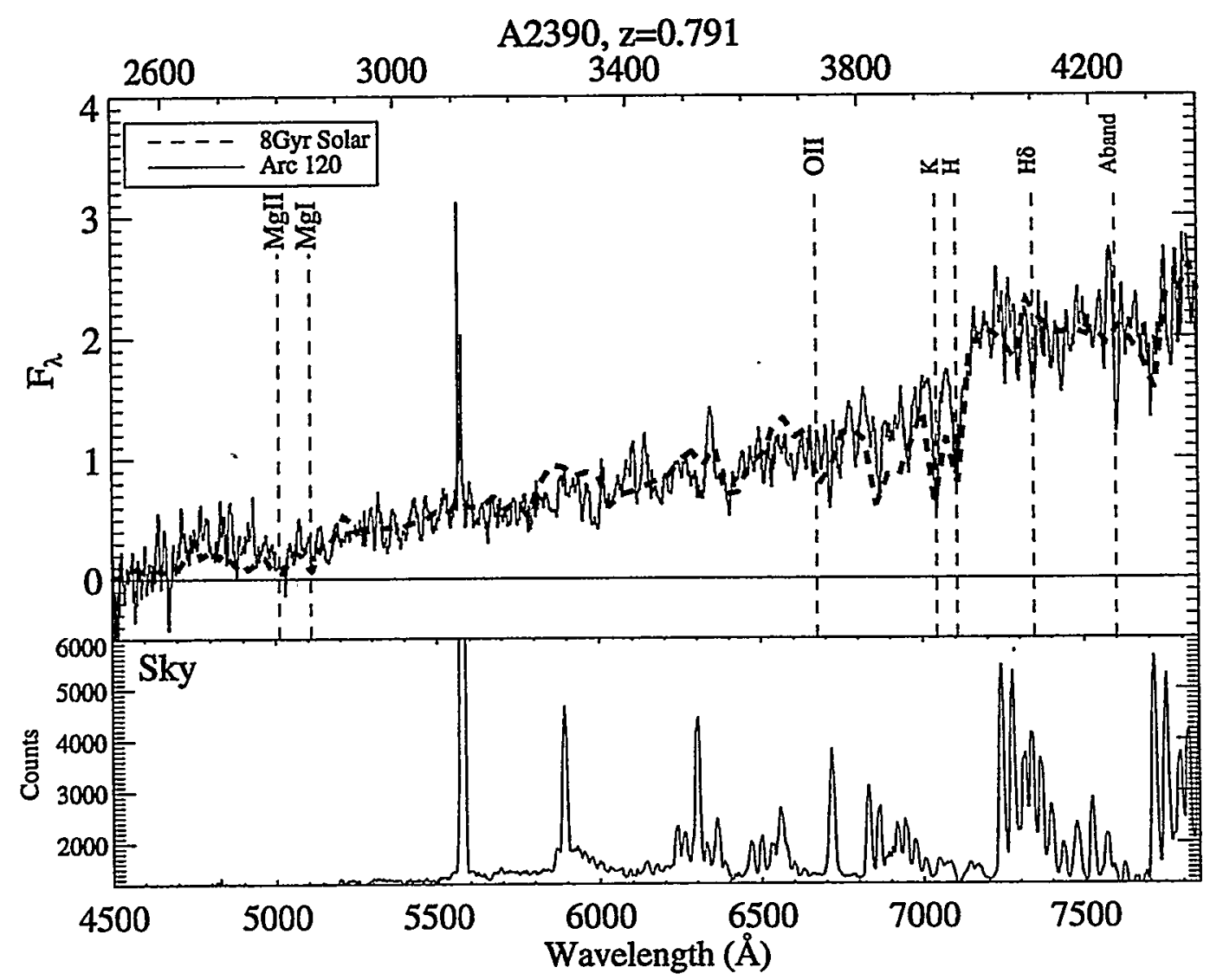

Figure 6.20: Spectrum of an elliptical at $z=0.791$ in A2390 with Bruzual-Charlot model overlayed. $F_{\lambda}$ is plotted against observed wavelength on the lower axis and rest wavelength on the upper axis. The model is for a passively-evolved $8 \mathrm{Gyr}$ galaxy with solar metallicity. $\mathrm{MgI}, \mathrm{MgII}, \mathrm{CaH} \& \mathrm{~K}$, and $\mathrm{H} \delta$ are clearly seen. The sky spectrum appears on the bottom panel for comparison. 


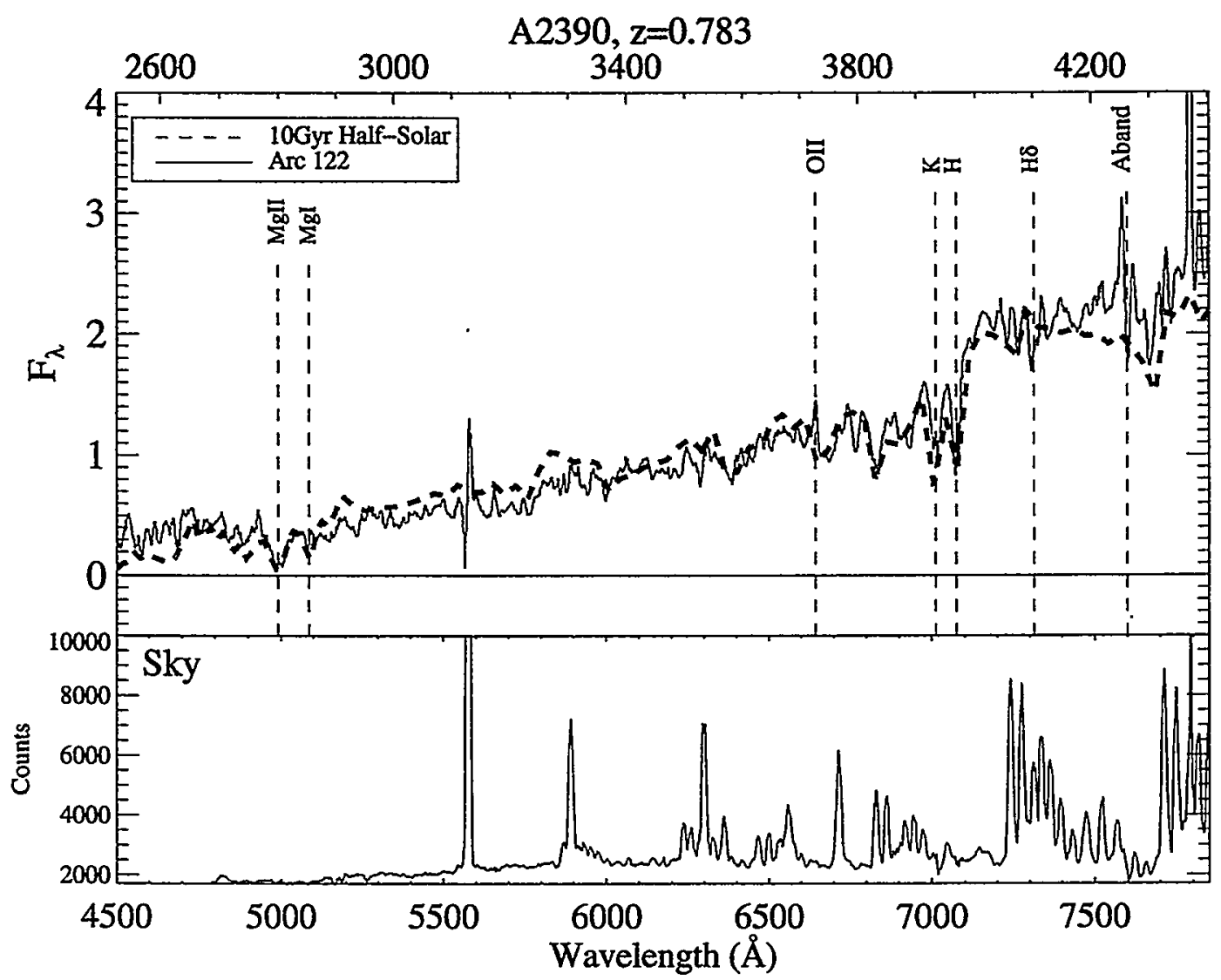

Figure 6.21: Spectrum of an elliptical at $z=0.783$ in A2390 with Bruzual-Charlot model overlayed. $F_{\lambda}$ is plotted against observed wavelength on the lower axis and rest wavelength on the upper axis. The model is for a passively-evolved $10 \mathrm{Gyr}$ old galaxy with $[\mathrm{Fe} / \mathrm{H}]=$ -0.33. $\mathrm{MgI}, \mathrm{MgII}$, weak $\mathrm{OII}, \mathrm{CaH} \& \mathrm{~K}$, and $\mathrm{H} \delta$ are clearly seen. The sky spectrum appears on the bottom panel for comparison. 
Elliptical and Template
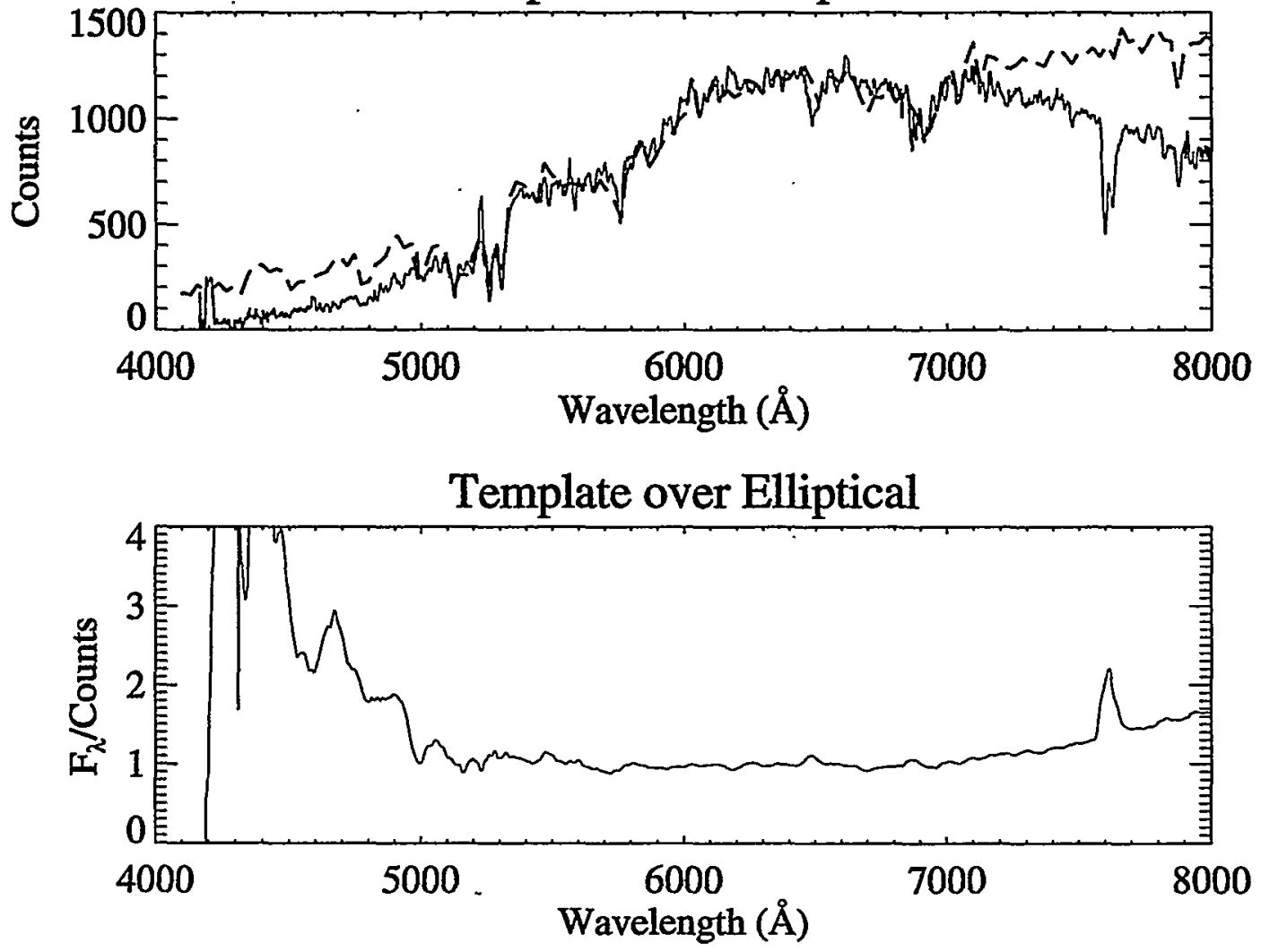

Figure 6.22: Generating the fluxing curve. The upper panel shows the spectrum of an early-type cluster member at $z=0.175$ observed simultaneously through the multislit mask (solid line), with the standard empirical E/SO spectrum of Kennicutt (1992) (dashed line). Note that the flux from the template spectrum rises above that of the data at both ends of the spectrum, which if there are minimal differences between the two galaxy spectra are due to the response function of the CCD. The bottom panel shows the ratio of the model template to the cluster elliptical. The 'feature' at $7600 \AA$ is produced by A-band atmospheric absorption.

in June 1997, using LRIS on Keck II. The spectra were taken through the 300 line/mm grating in several sets of $20 \mathrm{~min}$ exposures, with a total exposure time of $2.8 \mathrm{hr}$. A slit width of $1.5^{\prime \prime}$ was used, resulting in a resolution of $15.6 \AA$ and dispersion of $2.4 \AA$ per pixel. These spectra are shown in Figures 6.20, and 6.21, plotted as $F_{\lambda}$ vs. observed wavelength on the bottom axis and rest wavelength on the top axis. The best-fitting Bruzual-Charlot models are overlayed as a dashed line.

Adequate relative spectrophotometry was obtained by comparison of the spectrum of an early-type cluster member at $z=0.175$ observed simultaneously through the multislit mask, with the standard empirical E/SO spectrum of Kennicutt (1992). The fluxing procedure was as follows. First an empirical spectrum is laid down on the data. This is shown in Fig. 6.22, upper panel, where the solid line is the cluster member spectrum and the dashed line is the empirical E/S0 spectrum. The empirical spectrum is normalized to the data. Note that the match is good in the mid-optical, and becomes increasingly less 

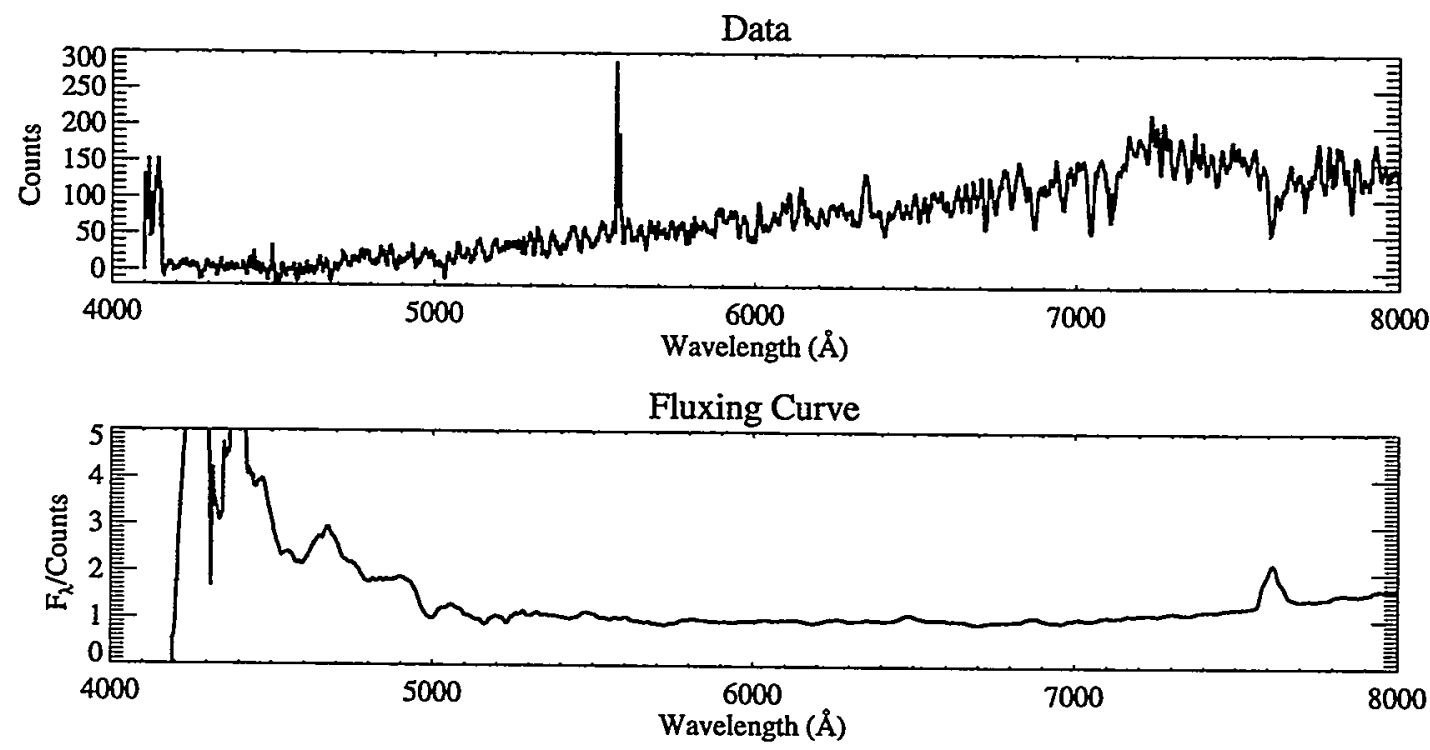

Fluxed Data

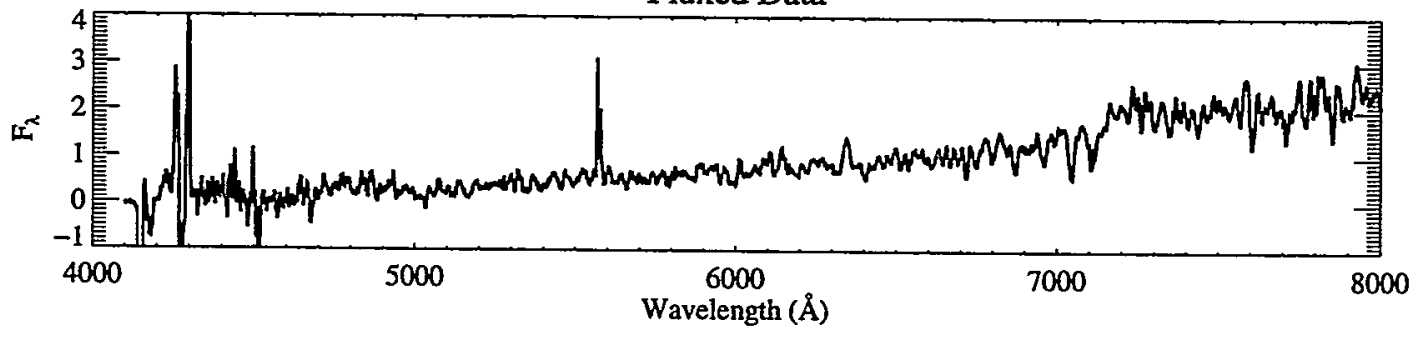

Figure 6.23: Example of fluxing. The upper panel shows the spectrum of the elliptical at $z=0.791$. The middle panel shows the fluxing curve used to flux this spectrum and the bottom panel shows the resulting spectrum. Note that the spectrum has been 'pulled up' at the edges to correct for the declining detector response in the near UV and far red, and made slightly more noisy especially at the blue end, where there were fewer counts. 

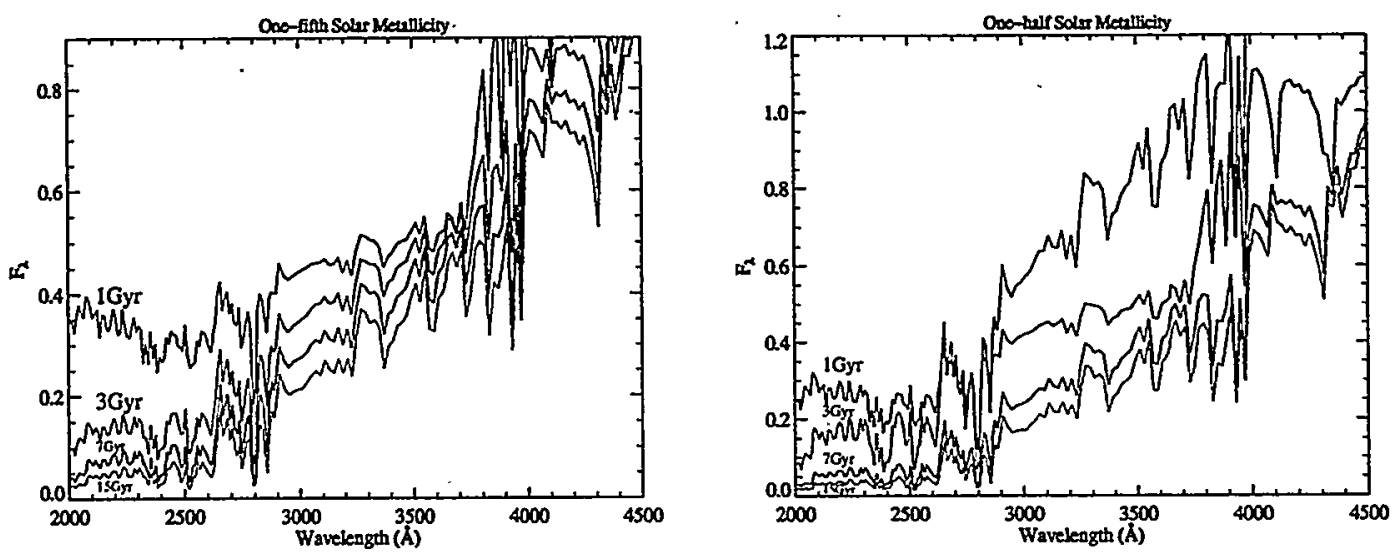

Figure 6.24: The left-hand panel shows the family of Bruzual-Charlot spectra for passivelyevolving elliptical galaxies with $[\mathrm{Fe} / \mathrm{H}]=1.0089$ (ten times solar metallicity) and the righthand panel shows the same for ellipticals with $[\mathrm{Fe} / \mathrm{H}]=-0.3300$ (half solar metallicity). The ages in both cases are are $1 \mathrm{Gyr}, 3 \mathrm{Gyr}, 7 \mathrm{Gyr}$, and $15 \mathrm{Gyr}$.

well-matched to the data at both ends of the spectrum, in the sense that there is more flux in the model relative to the data. Assuming that the differences are not intrinsic to the galaxies, which may be a fair assumption given that they are so well-matched in the mid-optical, the difference to assumed to arise from the response function of the CCD. The ratio of model template to data appears in the lower panel. The curve rises up at either end, as expected, and the 'feature' at $7600 \AA$ is due to atmospheric A-band absorption. This is the fluxing curve, in units of flux in $F_{\lambda}$ over counts, a more smoothed version of which will is used to flux the data.

Fig. 6.23 illustrates how the fluxing curve is applied to the data. The upper panel shows the spectrum of the elliptical at $z=0.791$. The middle panel gives the fluxing curve which was just discussed above, and the bottom panel shows the resulting fluxed data, obtained by multiplying it by the fluxing curve. Note that the spectrum has been 'pulled up' at the edges to correct for the declining detector response in the near UV and far red, and made slightly more noisy especially at the blue end, where there were fewer counts.

The flux in the rest frame near-UV spectrum of an elliptical galaxy is dominated by starlight from the population of stars at the main sequence turnoff. Thus the age of these stars give a fair estimate of the age of the most recent starburst galaxy, and so a lower limit on the age of the galaxy. The new 1998 Bruzual-Charlot models were chosen to make fits to the data to estimate this age. They offer a choice of metal abundances, from one-tenth to $10 x$ solar. Spinrad et al. (1997) have found that Bruzual-Charlot models fit to the overall spectral shape tended to underestimate the age when compared to local counterparts such as M32. Rather, they find more reliable results by fitting the spectral break amplitudes at $2640 \AA$ at $2900 \AA$. 

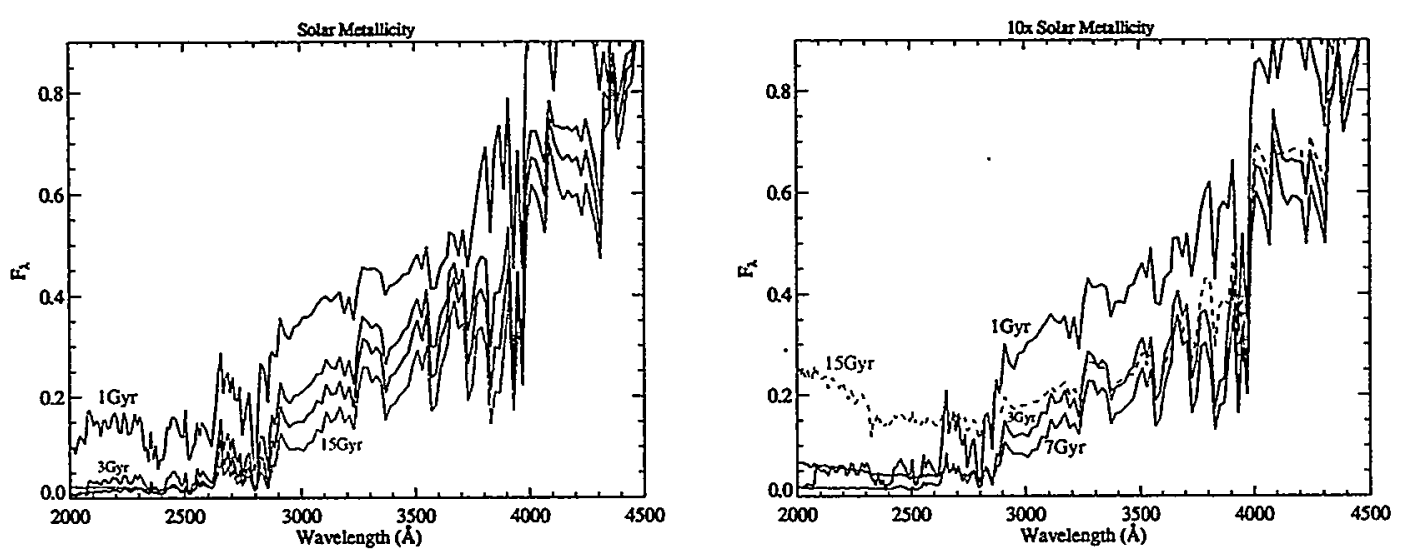

Figure 6.25: The left-hand panel shows the family of Bruzual-Charlot spectra for passivelyevolving elliptical galaxies with $[\mathrm{Fe} / \mathrm{H}]=0.0932$ (solar metallicity) and the right-hand panel shows the same for ellipticals with $[\mathrm{Fe} / \mathrm{H}]=1.0089$ (ten times solar metallicity). The ages in both cases are $1 \mathrm{Gyr}, 3 \mathrm{Gyr}, 7 \mathrm{Gyr}$, and 15Gyr.

As Fig. 6.24,25 show, the spectral break amplitudes of the Bruzual-Charlot models increase with increasing metal abundance and age, so that there is an age-metallicity degeneracy. This means, for example, that an old, metal abundant galaxy cannot be distinguished from a younger, more metal-poor galaxy on the basis of spectral shapes and break amplitudes alone. No models were capable of reproducing both the detailed spectral features and the spectral shape in the three distant ellipticals in this survey. Table 6.3 lists the ages measured for these three galaxies. The single starburst $8 \mathrm{Gyr}$ solar models were found to be adequate in reproducing the spectral breaks and continuum shape for the galaxies at $z=1.155$ and $z=0.791,4.5$ Gyr older than 53W091, or 3.5 Gyr older given the $\sim 1$ Gyr difference in lookback time for $H_{0}=50 \mathrm{kms}^{-1}$ and $q_{0}=0$. The galaxy at $z=0.783$ was best-fitted by a 10 Gyr model with half-solar abundance.

A more careful study must be carried out to test the robustness of this result, as there are several possible caveats, which are listed below. Different evolutionary synthesis models have been shown to result in different ages when fitted to the same optical spectra, due largely to the relatively-unknown rest-UV stellar spectrum. This can be improved with better template UV data and by fitting the data with more than one evolutionary synthesis model. The age-metallicity degeneracy is always of concern, but this can be at least somewhat broken by using Balmer lines and other metal lines as metallicity diagnostics (Worthey, 1994; Gorgas et al., 1993). Fourthly, dust-reddening has not been considered at all, but as Spinrad et al. (1997) have pointed out, stellar populations with ages $\sim 2-3$ Gyr have a difficult time reproducing the rest frame UV spectral features. There could be more attention paid to the fluxing for the spectra in our survey, and the result of the cluster elliptical method using to flux the spectra compared to that of fluxing by a standard star, 
since proper fluxing is obviously a crucial factor in determining these ages. The method used here was certainly adequate to show that these are bonified distant ellipticals, with the one at $z=1.55$ possibly meriting further study.

\subsection{Discussion}

\subsubsection{Continuum fits}

The continua of the high- $z$ galaxies are fitted by models consisting of hot B stars, Lyman-series banketing and HI at the source $z$ (Fig. 6.2). These simple models are sufficient for obtaining a rough idea of the intrinsic continuum shape. A good fit is found to early $B$ type stars, B0-B3.

The contribution to the continuum light is of course dominated by the hottest stars for any reasonable IMF, so that comparison with a single stellar type is reasonable. Reddening by dust is expected to be important at such short wavelengths, as is underscored by the presence of metal lines in all high redshift galaxies, so that the best fit temperatures should be considered lower limits. Reddening and/or age variations of the stellar population may both be expected to generate variations in the colors and spectral continua, leading to an age-metallicity degeneracy which can be marginally-separated out with IR colors and spectroscopy. Note that detailed continuum fitting has not been possible before for comparably high redshift galaxies (Dey et al., 1998a; Hu et al., 1998), with the stellar continuum being only marginally-detected at best, or in the case of the $z=4.92$ lensed galaxy of Franx et al. (1997), the continuum was contaminated by light from a nearby cluster member.

\subsubsection{Gas Outflows}

Ly- $\alpha$ emission line galaxies with equivalent widths $W_{L y \alpha}>20 \AA$ show up in about half of the $\sim 10^{2}$ galaxies at $z \sim 3$ (Steidel et al., 1996a,b; Lowenthal et al., 1997) and in less than half of the galaxies at $z>4$ in the HDF. They appear in about half of the galaxies in our survey with $z>4$, see Table 6.3 , and in all of the highest redshift galaxies, at $z>5$ (Fig. 6.8, this chapter, Dey et al. (1998b), and Chapter 6 of this thesis. This line may be favored in the most distant and faint examples if it distinguishes the differing properties of starbursting vs. quiescent galaxies. It is also possible that the strength of this line is related to dust content and that the epoch $4<z<5$ marks a transition before which Ly $\alpha$ propagated less-opposed by dust. More spectra of galaxies at $z>5$ are required to give us more clues as to whether $z>5$ is truly a special epoch or just another hurdle.

Our high quality spectra allow us to study some properties in detail. Fig. 6.26 shows a six panel plot of the spectra of the interstellar metal lines for the five high quality high- $z$ spectra in our sample which show both Ly- $\alpha$ in emission and metal absorption lines. In the sixth panel is a spectrum of the Franx et al galaxy at $z=4.92$ (lower right-hand 

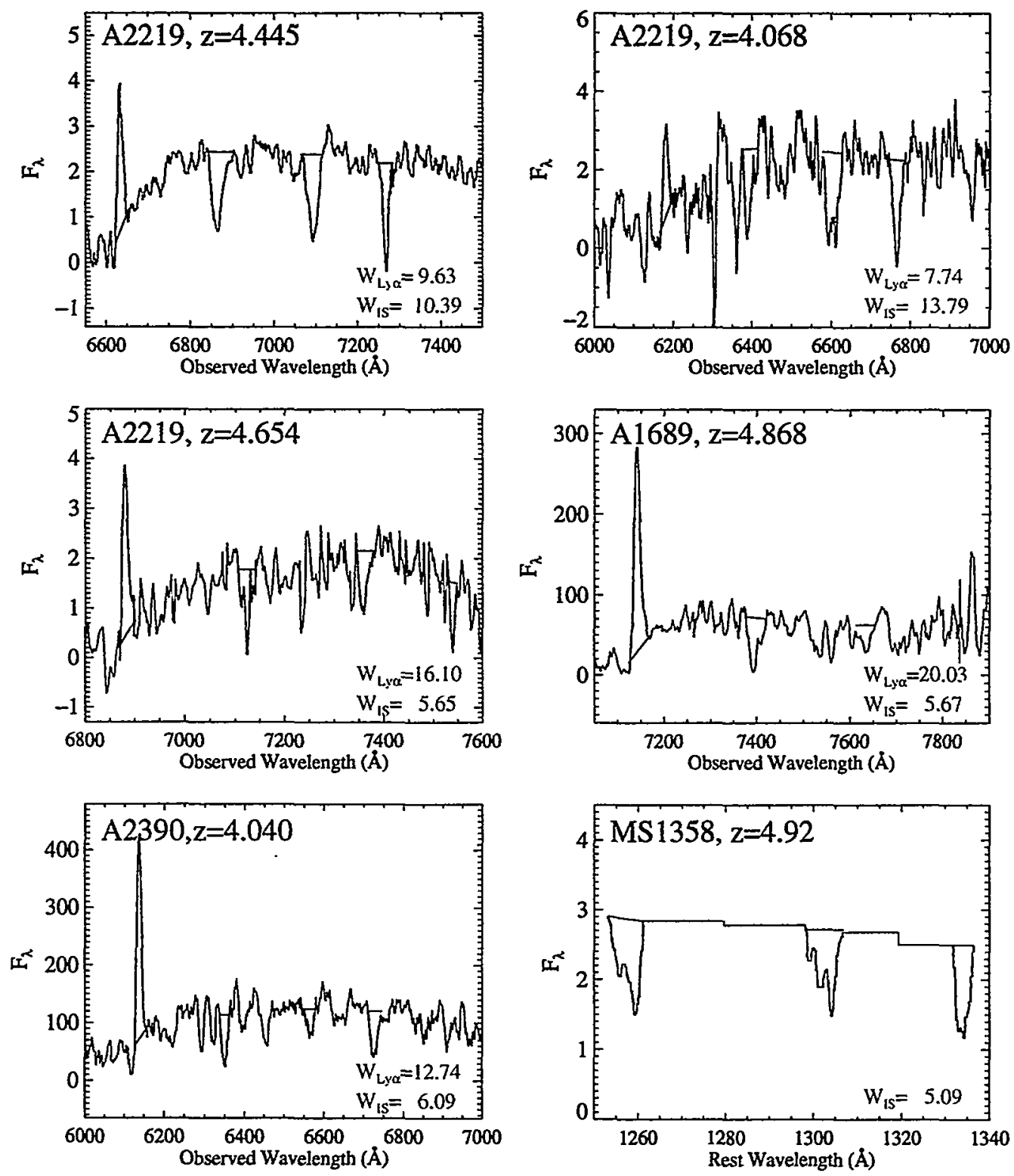

Figure 6.26: Equivalent width measurements of interstellar metal lines in six high- $z$ galaxies. The rest equivalent widths were measured for the lines SiII $1260 \lambda$, OI $1302 \lambda$, SiII1304 $\lambda$, and CII 1336 $\lambda$ and the total given in the lower right corner of each box. The dashed lines highlight the line profiles and the solid horizontal lines at the top end of each absorption feature show the extent in wavelength and the continuum level over which the equivalent width was calculated.

corner). The dashed line profiles and solid horizontal bars above each line in each spectrum mark the measuring boundries. The rest equivalent widths were totalled for the lines of 

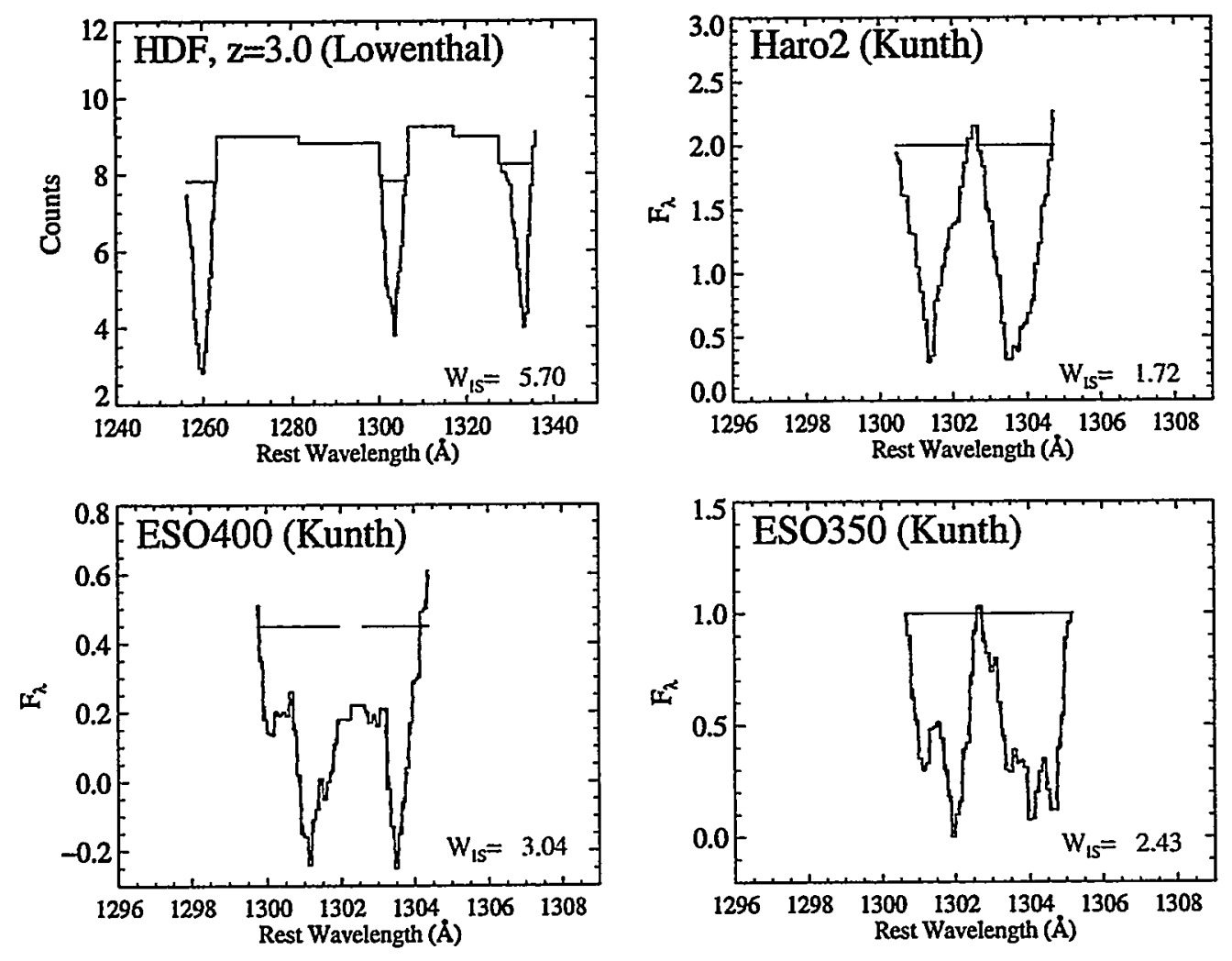

Figure 6.27: Equivalent width measurements for interstellar metal lines in high- $z$ galaxies and local starbursting galaxies. The rest equivalent widths were measured for the lines Sill $1260 \lambda$, OI $1302 \lambda$, SiII1304 $\lambda$, and CII 1336 $\lambda$ in NGC 1741 (Conti et al., 1996) and in lines OI $1302 \lambda$, SilI1304 $\lambda$ for the other panels. The total rest equivalent width is given in the lower right corner of each box. The dashed lines highlight the line profiles and the solid horizontal lines at the top end of each absorption feature show the extent in wavelength and the continuum level over which the equivalent width was calculated.

SiII1260 $\lambda$, OI $1302 \lambda$, SiIV $1304 \lambda$, and CII $1336 \lambda$, and for Ly $\alpha$ and the result given in the lower right-hand corner of each panel. Fig. 6.27 shows a four-panel plot with the composite spectrum of the $U$-band dropouts in the HDF appearing in the upper left (Lowenthal et al., 1997). The brightest $U$-band dropout in this sample, the so-called 'hot dog' galaxy with a redshift measured by absorption lines to be $z=2.8$, is interesting for showing strong metal absorption lines with P-Cygni profiles and has only modest gas outflow velocities compared to the local starbursts (Bunker et al., 1998b). In this galaxy Ly $\alpha$ emission is completely suppressed. 


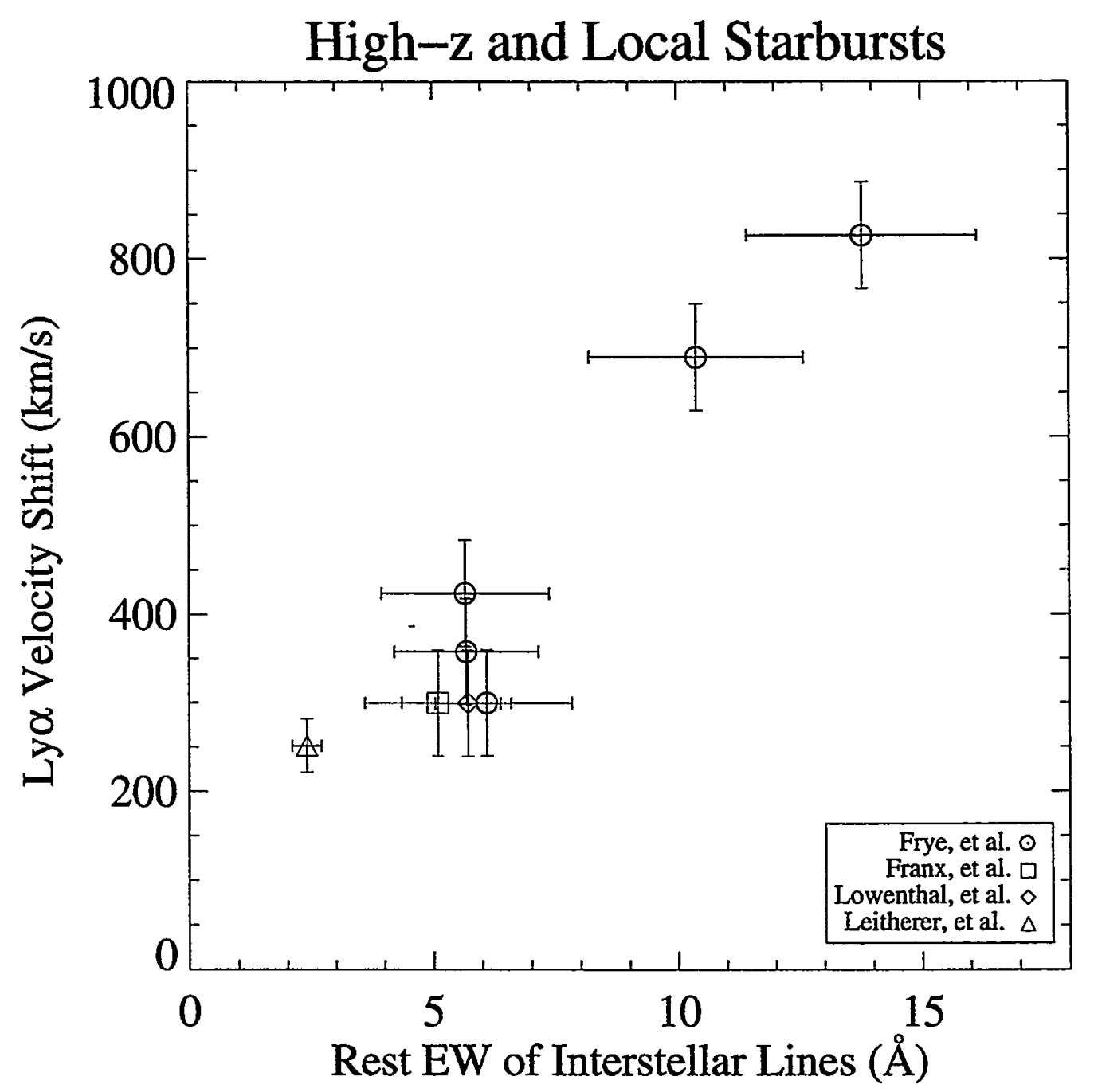

Figure 6.28: The redward velocity shift between the centroid position of Ly- $\alpha$ and the $z$ determined by the metal lines is plotted against the total rest equivalent width of the metal lines. Four different ISM metal lines were summed together. They are: SiII $1260 \lambda$, OI $1302 \lambda$, SiIV $1304 \lambda$, and CII $1336 \lambda$. The different symbols correspond to different sets of data, which are given in the legend in the lower right-hand corner. The high- $z$ points are in general agreement with the local measurement of Heckman et al. (1998)

The remaining three panels show spectra of the ISM metal lines taken from local examples of starbursting galaxies (Kunth et al., 1998). For these last three objects only two of the four metal lines were observed, so the equivalent widths of only these two lines, OI $1302 \lambda$ and SirV $1304 \lambda$, were summed up. For all the galaxies in Fig. 6.26 and 6.27 which were not a part of the sample the absorption line data were entered in by hand, ignoring the irrelevant surrounding continuum. This is why the space between metal lines appears 


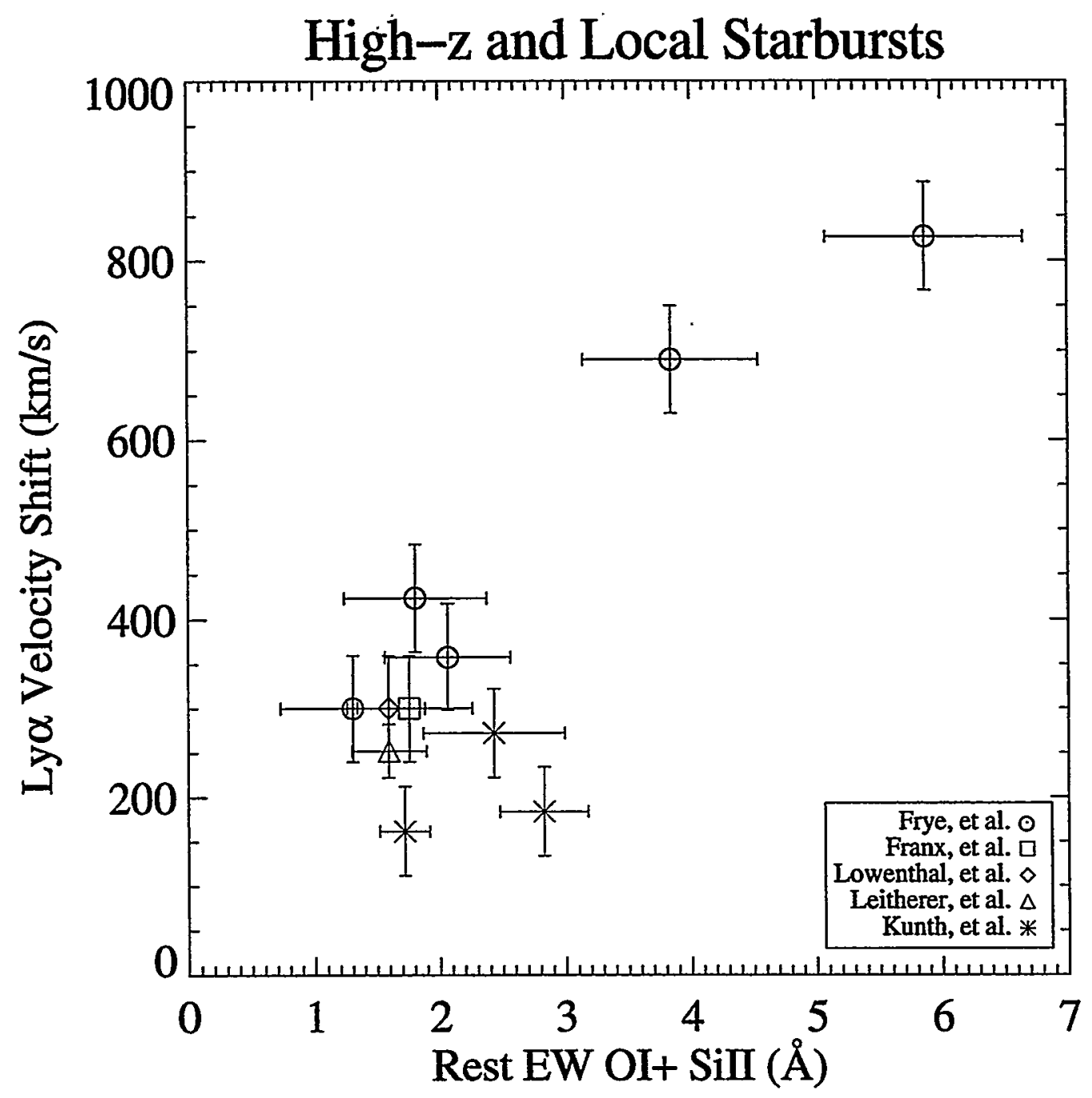

Figure 6.29: The redward velocity shift between the centroid position of Ly- $\alpha$ and $z$ determined from the metal lines is plotted against the total equivalent width of the metal lines. Two different metal lines were summed together. They are: OI $1302 \lambda$ and SiIV $1304 \lambda$. The different symbols correspond to different sets of data, which are given in the legend in lower right-hand corner.

to be absent of information.

The velocity shift between the centroid of Ly $\alpha$ and the metal lines was measured or derived from the literature for each object in Fig. 6.28 and 6.29. This shift is assumed to give the velocity between the systemic redshift and the outflowing gas, but there is some concern over whether the metal lines are entirely uncorrelated with the outflowing gas (Heckman et al., 1998). A test would to compare this redshift with that measured 
from forbidden lines observed in the infrared, say, for example [OII] $3727 \lambda$. Such infrared spectroscopy could be carried out on the Near Infra-red Spectrometer (NIRSPEC) on the Keck telescopes or with ISAAC on the VLT.

The results of the velocity shift and $W_{L y \alpha}$ measurements appear in Figure 6.28, in which the the total rest equivalent width of the four metal lines SiII1260 $\lambda$, OI $1302 \lambda$ and SiIV $1304 \lambda$, and CII $1336 \lambda$ were used. The different symbols refer to objects found by different groups, and are explained in the legend in the lower right-hand corner of the plot. Fig. 6.29 shows the result including the local examples of starbursts, where the velocity shift is plotted up against only the sum of OI $1302 \lambda$ plus SiIV $1304 \lambda$ for each galaxy. Note that there is a significant shift in velocity seen in these local examples as well. There is clearly a trend in that higher velocity shifts result in larger total equivalent widths over a very large range in redshift, $0<z<4.9$.

Doppler-broadening alone, caused by the combined effect of gas motion over a whole of velocities, would leave the equivalent width unaltered for the optically-thin case, revealing first of all that the lines are saturated. If this is true, then why do only some appear that way, such as two galaxies in this survey, $z=4.068$ and $z=4.445$ in A2219 (Fig. 6.15,16), the bright 'hot dog' galaxy in the HDF at $z=2.9$ (Bunker et al., 1998b), and $\mathrm{cB58}$ at $z=2.72$ (Ellingson et al., 1996). The answer is partly-related to the spectral resolution, because the average flux in the line core may be diluted by flux from the wings, resulting in an underestimate of its depth. Also, the HII region generally does not fully cover the source. The photons which get around the absorber can dilute the signal, thereby 'emission filling' the line.

The presence of the Ly $\alpha$ emission line appears to be connected to the dynamical state of the galaxy. A static, density-bounded HII region provides an effective barrier against the escape of Ly $\alpha$ photons by the entrapment process of resonant scattering. Even if there are just small amounts of dust present, after hundreds or even thousands of scatterings most of the receding and approaching Ly $\alpha$ photons eventually strike dust grains and get reprocessed. The result is Ly $\alpha$ absorption at the source redshift. However, the few Ly $\alpha$ photons which don't encounter dust grains and get backscattered can escape through the wings of the line if there is substantial outward gas motion.

In this scenario, the photons back-scattered off of the receding part are Dopplershifted towards the red and can escape through the red part of the line. The photons scattered off of the approaching side get shifted towards the blue in the frame of the observer and escape into the anti-direction of the observer and so get lost. Finally, the few photons which try to penetrate straight through the approaching side get blue-shifted to wavelengths with an even higher absorption cross-section, making them hopelessly-trapped and dependent on other processes for escape (e.g the 2-photon process). Thus the Ly $\alpha$ line which is seen in about half of the high- $z$ galaxies is probably only the red wing of an intrinsically much larger Ly $\alpha$ line. In fact in the absence of dust the rest $W_{L y \alpha}$ should be $\sim 10 x$ stronger than what has been seen so-far, so it appears that the era of the first epoch 
of star formation in galaxies is likely to bet at $z>5$.

\subsubsection{Lensing by $\mathrm{A} 2390$}

It is relatively straightforward to reproduce the basic properties of the lensed image configuration for the arcs at $z=4.040$ (Fig. 6.10). For generating four images stretched along the major axis of the central elliptical member requires a significant deflection from the cluster potential, so that the elliptical member galaxy must lie close to the critical curve of the cluster for a source at $z \approx 4$. Fig. 6.10 , bottom right panel, shows a reasonable model of the data, where the deflection field is calculated numerically. The critical curve of the cluster joins with that of the elliptical galaxy at $30^{\prime \prime}$ from the cluster center. The absolute image separation is set mainly by the cluster mass, and the relative separations of the N-S over the E-W images pairs constrains the ratio of elliptical/cluster masses to be $\sim 0.03$, depending somewhat on the ellipticity of the cluster. We fix the ellipticity of the elliptical galaxy to equal that of its light profile, $b / a=0.7$, resulting in a relatively large ellipticity, $b / a=0.5$, for the cluster mass so that the largest arc, $N$, can lie closer to the central elliptical than arc S. In addition, an offset of $\sim 30$ degrees is needed between the major axis of the cluster east of a line connecting the cluster center through the elliptical member. This asymmetry also helps somewhat to reproduce the straightness of the $\mathrm{N}$ and $\mathrm{S}$ arcs. For fixed ellipticities, the relative image intensities are most sensitive to the gradient of the cluster mass profile, preferring a shallower than isothermal projected slope, $\theta^{-0.8}$, at the critical radius, helping to generate the fairly lengthy arc $\mathrm{E}$ as observed.

This result is shown in Fig. 6.10, bottom right panel. It shows the parity of the images for a simple circular source, where the lighter and darker regions of the arcs are adjusted to correspond to the regions of the observed Ly $\alpha$ emission and the stellar continuum respectively, matching the parallel slit spectroscopy. Also shown is the magnification field with the critical curve, demonstrating the asymmetry required of the mass distribution. The source magnification in this model is $\sim 20$. Taking the Northern arc alone and subtracting its magnification from the observed magnitude $I_{A B}=23.0$, yields $I_{A B} \sim 25.5$ for the intrinsic apparent magnitude, where the largest uncertainty is the slope of the mass profile - the flatter the profile the larger the magnification. The observed $I_{A B}$ magnitudes of the other arcs labelled $\mathrm{S}, \mathrm{E}, \mathrm{W}$ are $I_{A B}=23.6,24.5,26$, respectively. The angular diameter of the source in this model is $\sim 0.2^{\prime \prime}$, or between $0.7 \mathrm{kpc} / \mathrm{h},(\Omega=1)$ and $1.4 \mathrm{kpc} / \mathrm{h}(\Omega=0)$, assuming the source is roughly symmetric. This is consistent with the poorly-resolved width of the arcs in the HST images (Fig. 6.10).

Our simple lens model is similar but not as extreme as those explored in the analysis of the notorious straight arc in this cluster, which has proven difficult to reproduce (Kassiola et al., 1992). Interestingly this arc lies very close to our high redshift system, shown in Figure 6.10, and is well resolved in HST images (see Figure 6.10, upper right panel, where the adjacent elliptical is removed). This difficulty is alleviated somewhat by the results of our spectroscopy, which show unambiguously that component $A$ (in the 


\begin{tabular}{|c|c|c|c|c|c|}
\hline Cluster & $z$ & $W_{I S}(\AA)$ & $\Delta V\left(\mathrm{kms}^{-1}\right)$ & $W_{\text {Ly } \alpha}(\AA)$ & $I$ \\
\hline A2390 & 4.040 & 6.09 & 300 & 12.74 & 22.5 \\
$\mathrm{~A} 2219$ & 4.068 & 13.79 & 827 & 7.74 & 23.67 \\
$\mathrm{~A} 2219$ & 4.445 & 10.39 & 690 & 9.63 & \\
$\mathrm{~A} 2219$ & 4.654 & 5.65 & 424 & 16.10 & \\
$\mathrm{~A} 1689$ & 4.868 & 5.67 & 358 & 20.03 & 22.98 \\
$\mathrm{~A} 1689$ & 5.120 & N/A & N/A & 52.57 & \\
\hline \hline
\end{tabular}

Table 6.3: Particulars for the high- $z$ galaxies discovered in the survey. The columns are: galaxy cluster, $z, W_{I S}$, the rest equivalent width of SilI $1260 \lambda$, OI $1302 \lambda$, and SiIV $1304 \lambda$, $\Delta V$, the velocity difference between the $z$ of Ly- $\alpha$ and the $z$ of the interstellar lines, $W_{L y \alpha}$, the rest equivalent width of OI $1302 \lambda$, and SiIV $1304 \lambda$, and the $I$ band magnitude, if known.

notation of Pellò et al. (1991) lies at higher redshift, $z=1.033$, than component $\mathrm{C}$, which we confirm to be at $z=0.913$ (Frye et al. (1998), and Fig. 5.1). Hence in our lens modeling we do not invoke an additional dark mass (Kassiola et al., 1992) but instead prefer a large ellipticity for the cluster mass distribution. Having said that, it seems clear that this model does not produce the alignment of the nearby $\mathrm{N}$ and $\mathrm{S}$ arcs, indicating the need for additional tangential shear. A comparison of the lens models with the weak shear analyses of this cluster by Pierre et al. (1996), and Squires et al. (1996) will be important in constraining further the mass distribution.

\subsubsection{A1689}

Using NFW models we conclude that the true extent of the source at $z=4.868$ is $\sim 0.1 \mathrm{Kpc}$, about the size of a giant HII region and half the size of others at $z>4$, most of which are only marginally-resolved with HST in the radial direction (Fig. 6.6). The small size of this galaxy and other very red objects identified in the HDF (Bouwens et al., $1998 \mathrm{~b}, \mathrm{a})$ bears a qualitative resemblance to the early evolution expected in the hierarchical models for structure formation, in which increasingly more massive bound objects collapse and merge over time. It seems that both the high redshift Universe and these simulations have difficulty forming normal gas rich disks. The detailed numerical simulations show that gas collapse is too efficient in standard dark-matter haloes to produce large rotationallysupported disks (Steinmetz \& Muller, 1995), motivating discussions of 'feedback'. Perhaps the observed high velocity gas flows generate the required heating, but more plausibly they may help explain the widespread distribution of metals in the IGM at high redshift (Lu et al., 1999; Cowie \& Songaila, 1998), requiring significant gas loss from the haloes. 


\begin{tabular}{|c|c|c|}
\hline Group & \# at $z \gtrsim 4$ & \# with Ly $\alpha$ emission \\
\hline Frye (confirmed) & 6 & 6 \\
Frye (unconfirmed) & 5 & 1 \\
Dey, et al. 1998 & 1 & 1 \\
\hline \hline
\end{tabular}

Table 6.4: The presence of the Ly $\alpha$ line in high- $z$ galaxies. the columns are: the observing group, the number of high- $z$ objects at $z \geq 4$, and the number of those objects which show Ly $\alpha$ in emission.

\subsubsection{Summary of High- $z$ Properties}

Tables 6.3 gives some measurable properties of the 6 confirmed high- $z$ galaxies in the survey. The total rest equivalent width of $\operatorname{Ly} \alpha, W_{L y \alpha}$, increases with $z$, with the exception of the one at $z=4.04$, which is $\sim 2 x$ higher and the only example of a spatiallyresolved image. The overall correlation may be due to decreasing dust content with redshift. Although Ly $\alpha$ is not a requisite feature in high- $z$ galaxies, as Table 6.4 shows it is relatively common at $z \gtrless 4$, but this is probably just a selection effect.

\subsection{Conclusion}

The inferred small sizes of the six $z>4$ galaxies found in this survey fit into the general picture now emerging of galaxy formation by a piecemeal growth of sub-galactic sized objects. The deepest images of the sky display a surprisingly high surface density of tiny galaxies (Williams et al., 1996) and may be interpreted in a model-independent way as evidence for growth on small scales (Bouwens et al., 1998b,a) Little is known about the process by which galaxies emerge from the neutral epoch although clearly the bulk of this transition takes place before $z \sim 1$, below which the density of $\mathrm{L}>\mathrm{L}^{*}$ galaxies varies little (Lilly et al., 1995; Ellis et al., 1996).

The Steidel population of Ly-limit selected galaxies found in the range $2.5<z<3.5$ (Steidel et al., 1996a,b; Lowenthal et al., 1997) have compact sizes and their rest UV images display far more sub-structure than expected of redshifted spiral galaxies (Bouwens et al., $1998 \mathrm{~b}, \mathrm{a})$. Individual star-formation rates are uncertain, but several 10's of solar masses per year have been inferred for the brightest cases (Pettini \& Et Al., 1998). Their evolution and relation to low-redshift galaxies is still unclear. By integrating their UV luminosities, Madau et al. (1998) infer a fairly-level integrated rate of star-formation for $z>2$, relying on little evolution in the shape of luminosity function. In the context of a hierarchical scheme, this results from the continuous decrease in mean galaxy size and hence luminosity expected with increasing redshift, naturally resulting in fewer galaxies detected above the limiting luminosity. Indeed, a flat or even increasing mean star-formation rates at $z>2$ may be accommodated by the observed dropout populations (Bouwens et al., 1998b,a) in the range 
$2<z<6$.

The lensed galaxies presented here provide interesting information on the earliest known optically-selected galaxies. The cluster magnification has fortuitously afforded us detailed spectral and spatial information of a very faint and presumably otherwise typical examples of galaxies at $z>4$. We have obtained higher resolution longslit spectra for one of these objects, revealing spatial and spectral detail of the Ly $\alpha$ and metal lines along the lengths of the $\mathrm{N}$ and S arcs (Frye et al., 1998; Bunker et al., 1998a) and also near-IR Keck images useful for exploring the role of dust and for a good estimate of the luminosity (Bunker et al., 1998a). The rarity of luminous high redshift galaxies and the requirement of a high magnification for useful spectroscopic follow-up means that such searches will be slow, but well rewarded by detailed information on galaxies at otherwise inaccessibly-early times. 


\section{Bibliography}

Annis, J. \& Luppino, G. A. 1993, ApJ, 407, L69

Audouze, J. \& Tinsley, B. M. 1974, ApJ, 192, 487

Begelman, M. C. \& Blandford, R. D. 1987, Nature, 330, 46

Bergeron, J., Cristiani, S., \& Shaver, P. A. 1992, A\&A, 257, 417

Bézecourt, J. \& Soucail, G. 1997, A\&A, 317, 661

Bieging, J. H., Wilson, T. L., \& Downes, D. 1982, A\&AS, 49, 607

Bouwens, R., Broadhurst, T., \& Silk, J. 1998a, ApJ, 506, 557

-. 1998b, ApJ, 506, 579

Broadhurst, T. J., Taylor, A. N., \& Peacock, J. A. 1995, ApJ, 438, 49

Bruzual, A. G. \& Charlot, S. 1993, ApJ, 405, 538

Bunker, A., Moustakas, L., Davis, M., Frye, B., Broadhurst, T., \& Spinrad, H. 1998a, in ASP Conf. Ser. 146: The Young Universe: Galaxy Formation and Evolution at Intermediate and High Redshift, 182+

Bunker, A. J., Moustakas, L. A., \& Davis, M. 1999, ApJ, ???, ???

Bunker, A. J., Stern, D., Spinrad, H., Dey, A., \& Steidel, C. C. 1998b, American Astronomical Society Meeting, 192, 7008+

Churchill, C. W., Steidel, C. C., \& Vogt, S. S. 1996, ApJ, 471, 164+

Clowe, D., Luppino, G. A., Kaiser, N., Henry, J. P., \& Gioia, I. M. 1998, ApJ, 497, L61

Colley, W. N., Tyson, J. A., \& Turner, E. L. 1996, ApJ, 461, L83

Conti, P. S., Leitherer, C., \& Vacca, W. D. 1996, ApJ, 461, L87

Courbin, F., Lidman, C., Frye, B. L., Magain, P., Broadhurst, T. J., Pahre, M. A., \& Djorgovski, S. G. 1998a, ApJ, 499, L119 
Courbin, F., Lidman, C., \& Magain, P. 1998b, A\&A, 330, 57

Cowie, L. L. \& Songaila, A. 1998, Nature, 394, 44

Dey, A., Spinrad, H., Stern, D., Graham, J. R., \& Chaffee, F. 1998a, ApJ, 498, L93

Dey, A., van Breugel, W., Vacca, W. D., \& Antonucci, R. 1998b, ApJ, 490, 698

Dickinson, M. 1999, in The Hubble Deep Field (STScI Symp.), ed. M. Livio, M. Fall, \& P. Madau, ???, in press

Djorgovski, S., Meylan, G., Klemola, A., Thompson, D. J., Weir, W. N., Swarup, G., Rao, A. P., Subrahmanyan, R., \& Smette, A. 1992, MNRAS, 257, 240

Dunlop, J. S., Peacock, J. A., Spinrad, H., Dey, A., Jimenez, R., Stern, D., \& Windhorst, R. A. 1996, Nature, 381, 581

Ebbels, T. M. D., Le Borgne, J. F., Pèllo, R., Ellis, R. S., Kneib, J. P., Smail, I., \& Sanahuja, B. 1996, MNRAS, 281, L75

Ellingson, E., Yee, H. K. C., Bechtold, J., \& Elston, R. 1996, ApJ, 466, L71

Ellis, R. S., Colless, M., Broadhurst, T., Heyl, J., \& Glazebrook, K. 1996, MNRAS, 280, 235

Epstein, R. L., Arnett, W. D., \& Schramm, D. N. 1976, ApJS, 31, 111+

Fort, B., Prieur, J. L., Mathez, G., Mellier, Y., \& Soucail, G. 1988, A\&A, 200, L17

Franx, M., Illingworth, G. D., Kelson, D. D., van Dokkum, P. G., \& Tran, K.-V. 1997, ApJ, $486, \mathrm{~L} 75$

Frye, B. \& Broadhurst, T. J. 1998, ApJ, 499, 115

Frye, B., Welch, W. J., \& Broadhurst, T. 1997, ApJ, 478, L25

Frye, B. L., Broadhurst, T. J., Spinrad, H., \& Bunker, A. 1998, in ASP Conf. Ser. 146: The Young Universe: Galaxy Formation and Evolution at Intermediate and High Redshift, $504+$

Fukuda, Y. \& et al. 1999, Phys.Rev.Lett, ???, ???, submitted

Ghigna, S., Moore, B., Governato, F., Lake, G., Quinn, T., \& Stadel, J. 1998, MNRAS, 300,146

Gorgas, J., Faber, S. M., Burstein, D., Gonzalez, J. J., Courteau, S., \& Prosser, C. 1993, ApJS, 86, 153

Gunn, J. E. \& et al. 1998, AJ, 116, 3040 
Heckman, T. M., Robert, C., Leitherer, C., Garnett, D. R., \& Van Der Rydt, F. 1998, ApJ, $503,646+$

Hewitt, J. N., Turner, E. L., Schneider, D. P., Burke, B. F., \& Langston, G. I. 1988, Nature, 333,537

Hill, G. J., Thompson, K. L., \& Elston, R. 1993, ApJ, 414, L1

Hu, E. M., Cowie, L. L., \& McMahon, R. G. 1998, ApJ, 502, 99

Jauncey, D. L., Reynolds, J. E., Tzioumis, A. K., Murphy, D. W., Preston, R. A., Jones, D. L., Meier, D. L., Hoard, D. W., Lobdell, E. T., \& Skjerve, L. 1991, Nature, 352, 132

Jones, D. L., Preston, R. A., Murphy, D. W., Jauncey, D. L., Reynolds, J. E., Tzioumis, A. K., King, E. A., MCCulloch, P. M., Lovell, J. E. J., Costa, M. E., \& Van Ommen, T. D. 1996, ApJ, 470, L23

Kaiser, N. 1995, ApJ, 439, L1

Kaiser, N. \& Squires, G. 1993, ApJ, 404, 441

Kassiola, A., Kovner, I., \& Blandford, R. D. 1992, ApJ, 396, 10

Kennicutt, R. 1992, ApJ, 388, 310

Kneib, J. P., Ellis, R. S., Smail, I., Couch, W. J., \& Sharples, R. M. 1996, ApJ, 471, 643+

Kneib, J. P., Mellier, Y., Fort, B., \& Mathez, G. 1993, A\&A, 273, 370+

Kneib, J. P., Mellier, Y., Pello, R., Miralda-Escude, J., Le Borgne, J. F., Boehringer, H., \& Picat, J. P. 1995, A\&A, 303, 27+

Kochanek, C. S. \& Narayan, R. 1992, ApJ, 401, 461

Koornneef, J. 1983, A\&A, 128, 84

Kunth, D., Mas-Hesse, J. M., Terlevich, E., Terlevich, R., \& Fall, S. M. 1998, A\&A, 334, 11

Lanzetta, K., Yahil, A., \& Fernàndez-Soto, A. 1996, Nature, 381, 759

Larkin, J. E., Graham, J. R., Matthews, K., Soifer, B. T., Beckwith, S., Herbst, T. M., \& Quillen, A. C. 1994, ApJ, 420, 159

Lattimer, J. M. \& Yahil, A. 1989, ApJ, 340, 426

Leitherer, C., Vacca, W. D., Conti, P. S., Filippenko, A. V., Robert, C., \& Sargent, W. L. W. 1996, ApJ, 465, 717+ 
Lerner, M. S., Baath, L. B., Inoue, M., Padin, S., Rogers, A. E. E., Wright, M. C. H., Zensus, A., Backer, D. C., Booth, R. S., Carlstrom, J. E., Emerson, D. T., Hirabayashi, H., Hodges, M. W., Jewell, P., Kobayashi, H., Kus, A. J., Moran, J. M., Morimoto, M., Plambeck, R. L., Rantakyro, F. T., \& Woody, D. 1993, A\&A, 280, 117

Lidman, C., Courbin, F., Meylan, G., Broadhurst, T., Frye, B., \& Welch, W. J. W. 1999, ApJ, 514, L57

Lilly, S. J., Tresse, L., Hammer, F., Crampton, D., \& Fevre, O. L. 1995, ApJ, 455, 108

Lovell, J. E. J., Reynolds, J. E., Jauncey, D. L., Backus, P. R., MCCulloch, P. M., Sinclair, M. W., Wilson, W. E., Tzioumis, A. K., King, E. A., Gough, R. G., Ellingsen, S. P., Phillips, C. J., Preston, R. A., \& Jones, D. L. 1996, ApJ, 472, L5

Lowenthal, J. D. et al. 1997, ApJ, 481, 673

Lu, L., Sargent, W. L. W., \& Barlow, T. A. 1999, in Highly Redshifted Radio Lines, ASP Conf. Series Vol. 156, Ed. by C. L. Carilli, S. J. E. Radford, K. M. Menten, \& G. I. Langston. ISBN 1-886733-76-7, p. 132., 132+

Lynds, R. \& Petrosian, V. 1989, ApJ, 336, 1

Madau, P. 1995, ApJ, 441, 18

Madau, P., Pozzetti, L., \& Dickinson, M. E. 1998, ApJ, 498, 106

Maddox, S. 1998, in ASP Conf. Ser. 146: The Young Universe: Galaxy Formation and Evolution at Intermediate and High Redshift, 198+

Mellier, Y. 1999, ARA\&A, 37, ???, in press, astro-ph/9812172

Mellier, Y., Fort, B., \& Kneib, J. P. 1993, ApJ, 407, 33

Mellier, Y., Fort, B., Soucail, G., Mathez, G., \& Cailloux, M. 1991, ApJ, 380, 334

Mellier, Y., Soucail, G., Fort, B., Le Borgne, J. F., \& Pèllo, R. 1990, in Gravitational Lensing, 261-270

Nair, S., Narasimha, D., \& Rao, A. P. 1993, ApJ, 407, 46

Narayan, R. \& Bartelmann, M. 1995, in Proc. 1995 Jerusalem Winter School, ed. ???, ???

Natarajan, P., Kneib, J. P., Smail, I., \& Ellis, R. S. 1998, ApJ, 499, 600+

Navarro, J. F., Frenk, C. S., \& White, S. D. M. 1995, MNRAS, 275, 720

Oke, J. B. et al. 1995, PASP, 107, 375

Paczynski, G. 1987, Nature, 325, 572+ 
Pellò, R., Kneib, J. P., Bruzual, G., \& Miralles, J. M. 1998, in The Birth of Galaxies 10th Rencontres de Bloi, ed. ???, 257

Pellò, R., Sanahuja, B., Le Borgne, J. F., Soucail, G., \& Mellier, Y. 1991, ApJ, 366, 405

Perlmutter, S. \& et al. 1999, ApJ, ???, ???, submitted, astro-ph/9812133

Pettini, M. \& Et Al. 1998, in ASP Coṇf. Ser. 148: Origins, 67+

Pierre, M., Le Borgne, J. F., Soucail, G., \& Kneib, J. P. 1996, A\&A, 311, 413

Reeves, H. 1972, in Age des Etoiles, Proceedings of IAU Colloq. 17, held in Paris, France, 18-22 September, 1972. Edited by G. Cayrel de Strobel and A. M. Delplace. Observatoire de Paris-Meudon, 1972., p.32, 32+

Riess, A. G. \& et al. 1998, AJ, 116, 1009

Sadoulet, B. 1997, in Dark matter in the Universe and its Direct Detection. Edited by M. Minowa. Tokyo : Universal Academy Press, 1997., p.1, 1+

Schechter, P. 1976, ApJ, 203, 297

Schneider, D. P., Schmidt, M., \& Gunn, J. E. 1989, AJ, 98, 1951

Seitz, S. \& Schneider, P. 1996, A\&A, 305, 383+

Smail, I., Dressler, A., Kneib, J. P., Ellis, R. S., Couch, W. J., Sharples, R. M., \& Oemler, A., J. 1996, ApJ, 469, 508+

Soucail, G., Fort, B., Mellier, Y., \& Picat, J. P. 1987a, A\&A, 172, L14

Soucail, G., Mellier, Y., Fort, B., Mathez, G., \& Cailloux, M. 1987b, The Messenger, 50, $5+$

-. 1988, A\&A, 191, L19

Spinrad, H., Dey, A., Stern, D., Peacock, J. A., Dunlop, J., Jimenez, R., \& Windhorst, R. A. 1997, ApJ, 484, 581

Squires, G. \& Kaiser, N. 1996, ApJ, 473, 65+

Squires, G., Kaiser, N., Fahlman, G., Babul, A., \& Woods, D. 1996, ApJ, 469, 73+

Steidel, C. C., Pettini, M., Dickinson, M., \& Persson, S. E. 1994, AJ, 108, 2046

Steidel, C. S., Giavalisco, M., Dickinson, M., \& Adelberger, K. L. 1996a, AJ, 112, 352

Steidel, C. S., Giavalisco, M., Pettini, M., Dickinson, M., \& Adelberger, K. L. 1996b, ApJ, 462,17 
Steinmetz, M. \& Muller, E. 1995, MNRAS, 276, 549

Storrie-Lombardi, L. J., MCMahon, R. G., \& Irwin, M. J. 1996, MNRAS, 283, L79

Subrahmanyan, R., Narasimha, D., Pramesh-Rao, A., \& Swarup, G. 1990, MNRAS, 246, $263+$

Subramanian, K. \& Cowling, S. A. 1986, MNRAS, 219, 333

Trager, S. C., Faber, S. M., Dressler, A., \& Oemler, A., J. 1997, ApJ, 485, 92+

Tyson, J. A., Kochanski, G. P., \& Dell'Antonio, I. P. 1998, ApJ, 498, L107

Tyson, J. A., Wenk, R. A., \& Valdes, F. 1990, ApJ, 349, L1

Tytler, D. \& Burles, S. 1997, in Origin of matter and evolution of galaxies in the universe '96. Proceedings of an international conference held in Atami, Japan, 18-20 January 1996, Singapore: World Scientific, —c1997, edited by T. Kajino, Y. Yoshii, and S. Kubono, p. $37 ., 37+$

Van Ommen, T. D., Jones, D. L., Preston, R. A., \& Jauncey, D. L. 1995, ApJ, 444, 561

Welch, W. J. \& et al. 1996, PASP, 108, 93+

Wiklind, T. \& Combes, F. 1996, Nature, 379, 139

Wiklind, T. \& Combes, F. 1998, ApJ, 500, 129+

Williams, R. E. et al. 1996, AJ, 112, 1335

Worthey, G. 1994, ApJS, 95, 107

Zwicky, F. 1933, Helv. Phys. Acta, 6, 10

—. 1937, Phys.Rev., 51, 290

-. 1959, Handbuch der Physik, 53, 390 


\section{Appendix A}

A1689:

A.1 Mask 1 

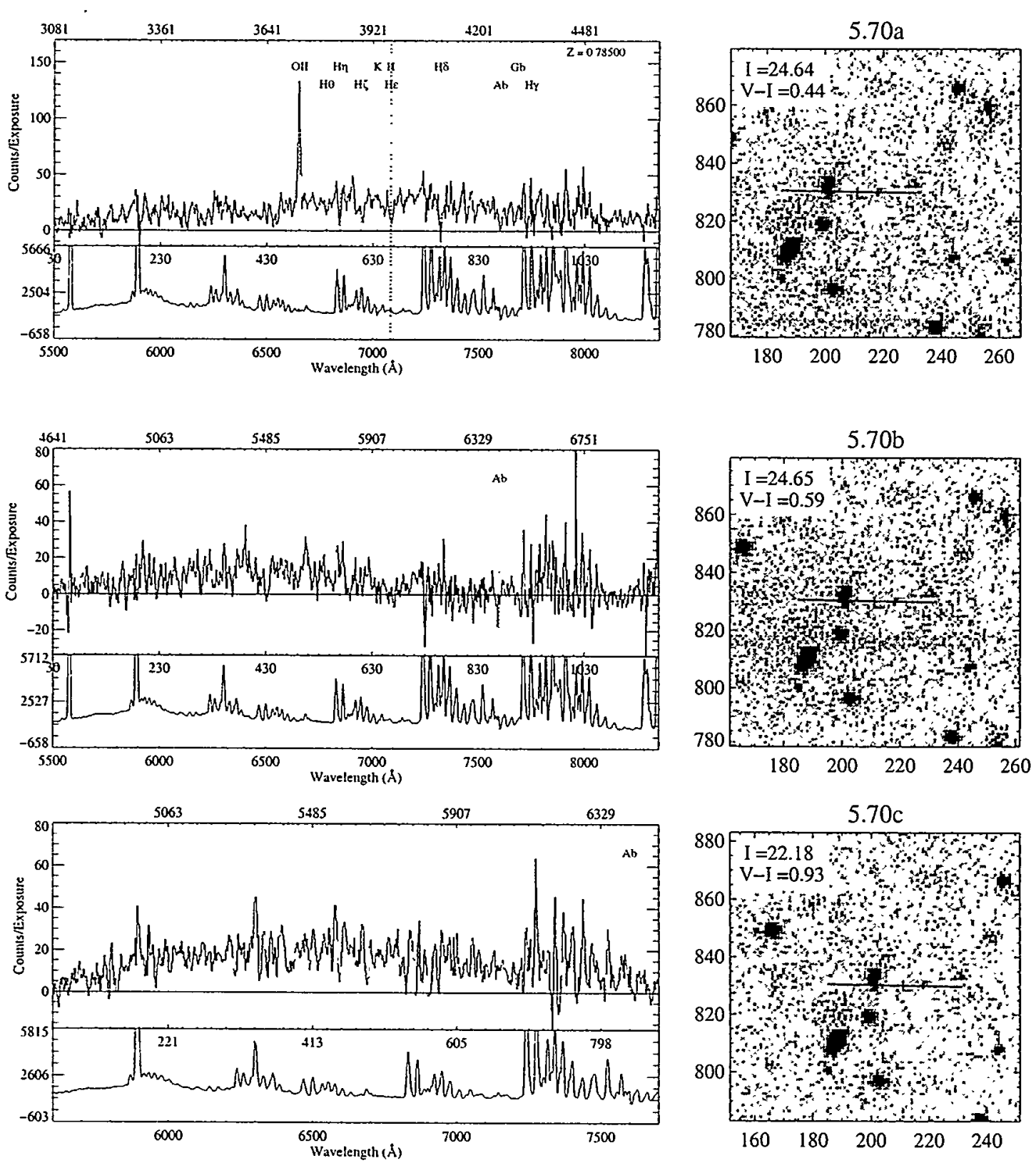

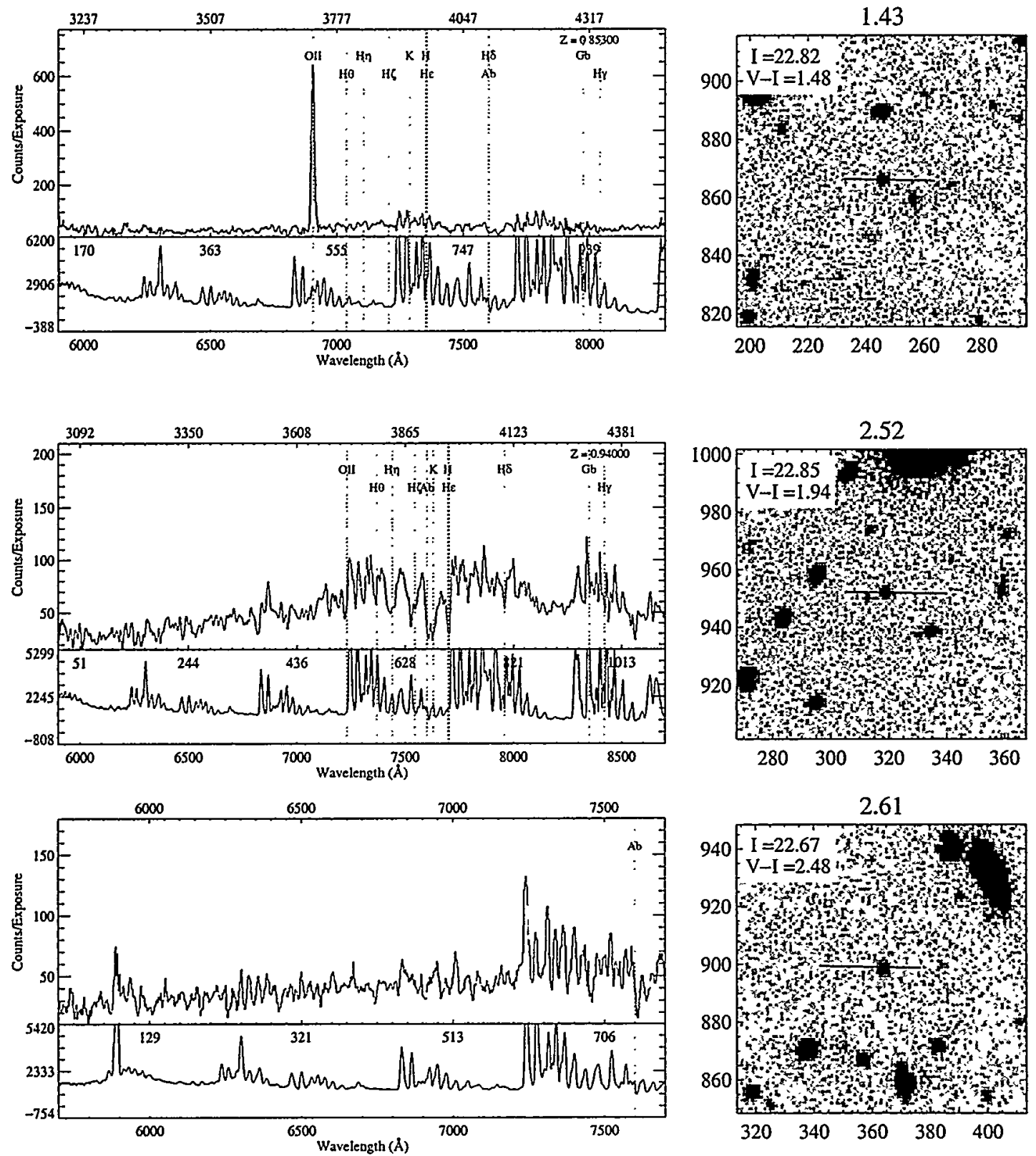

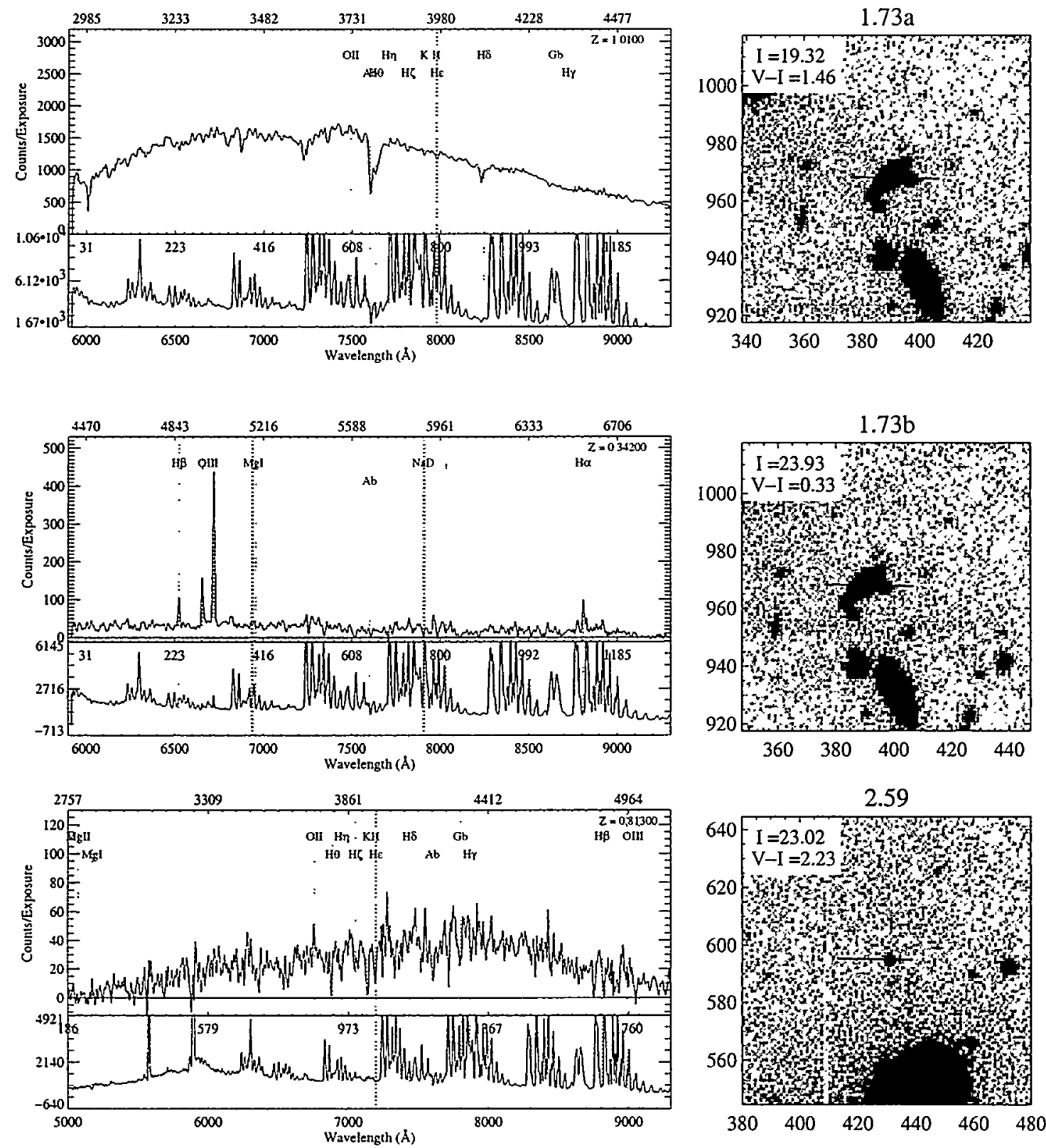

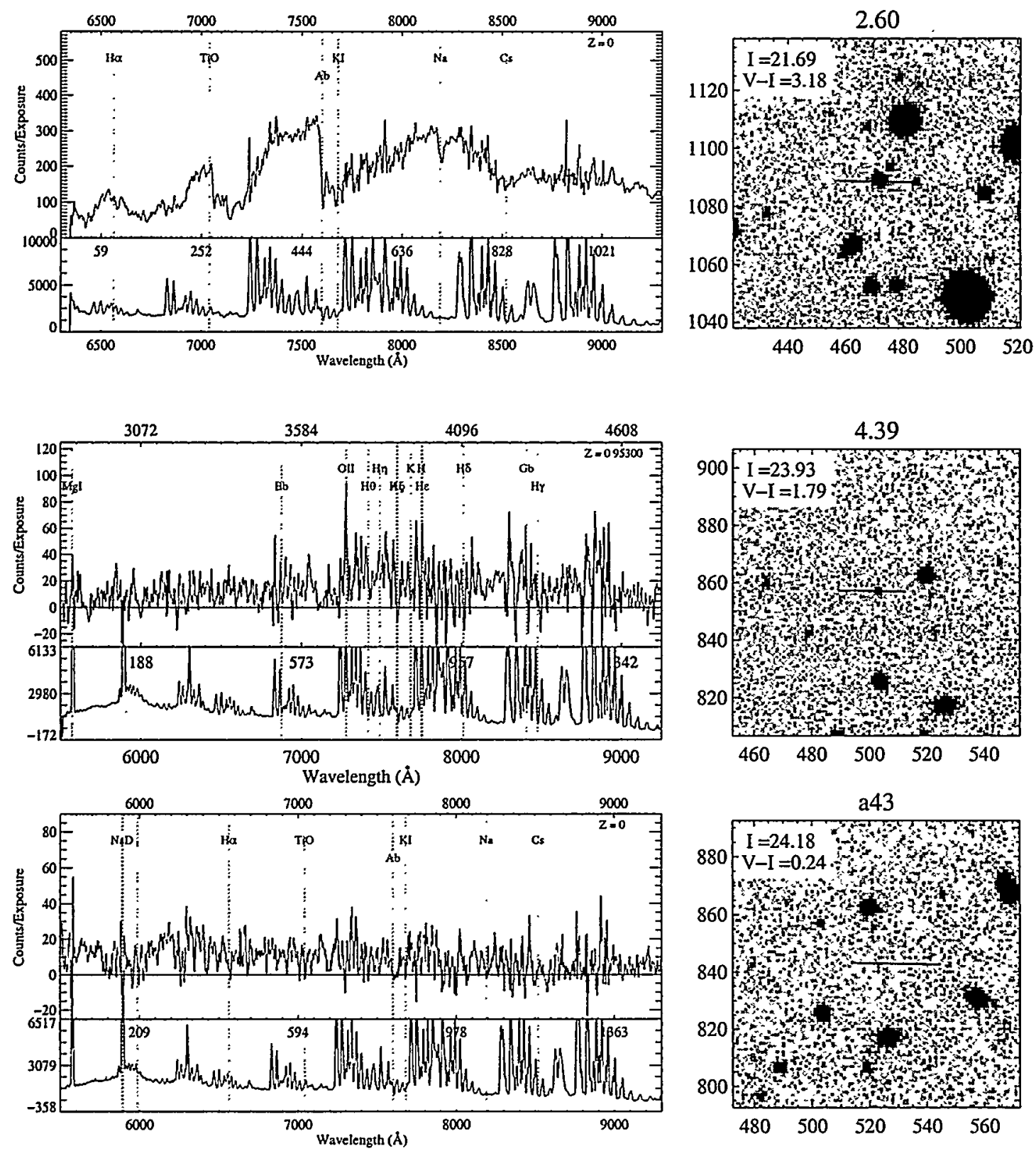

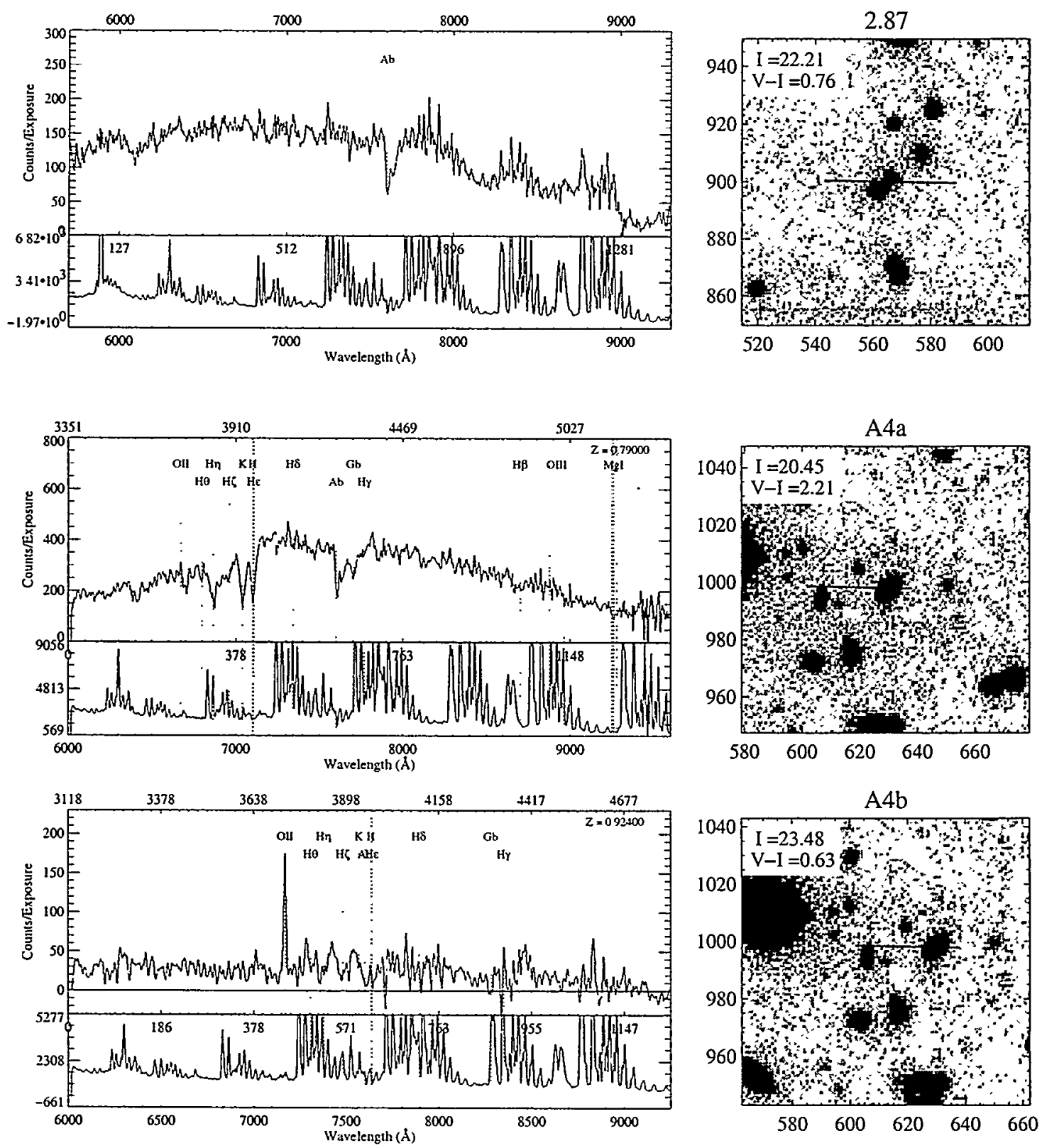

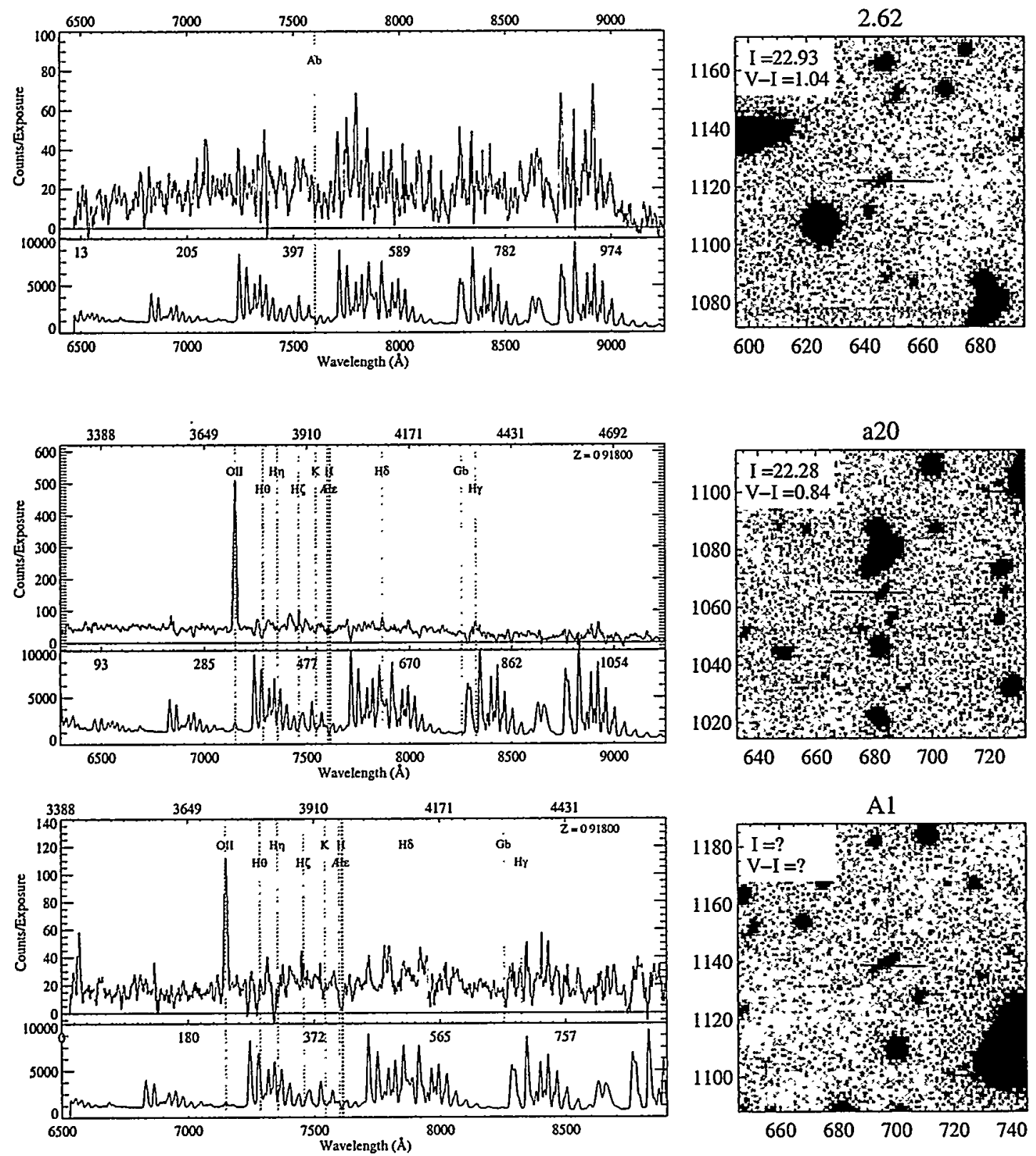

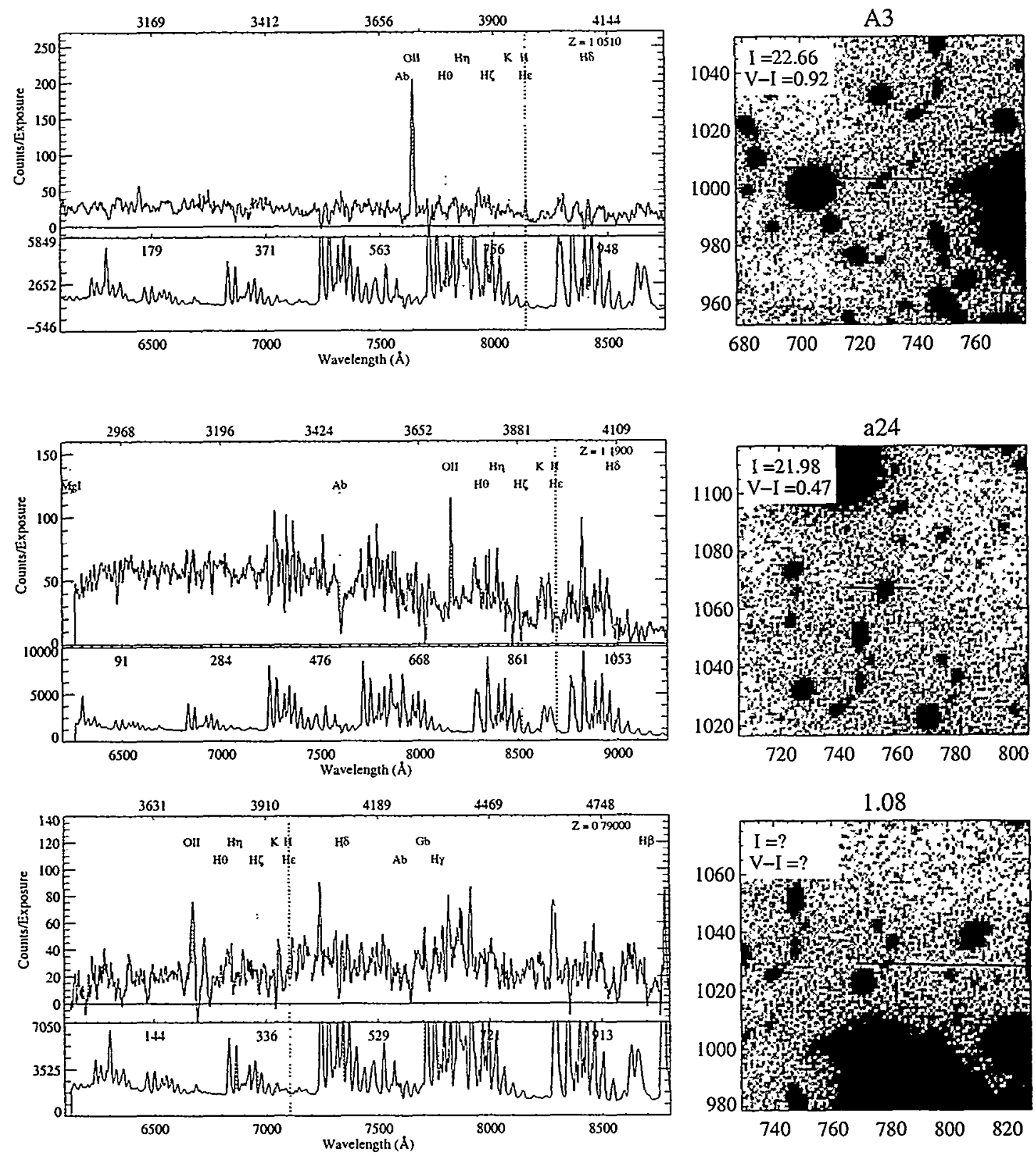

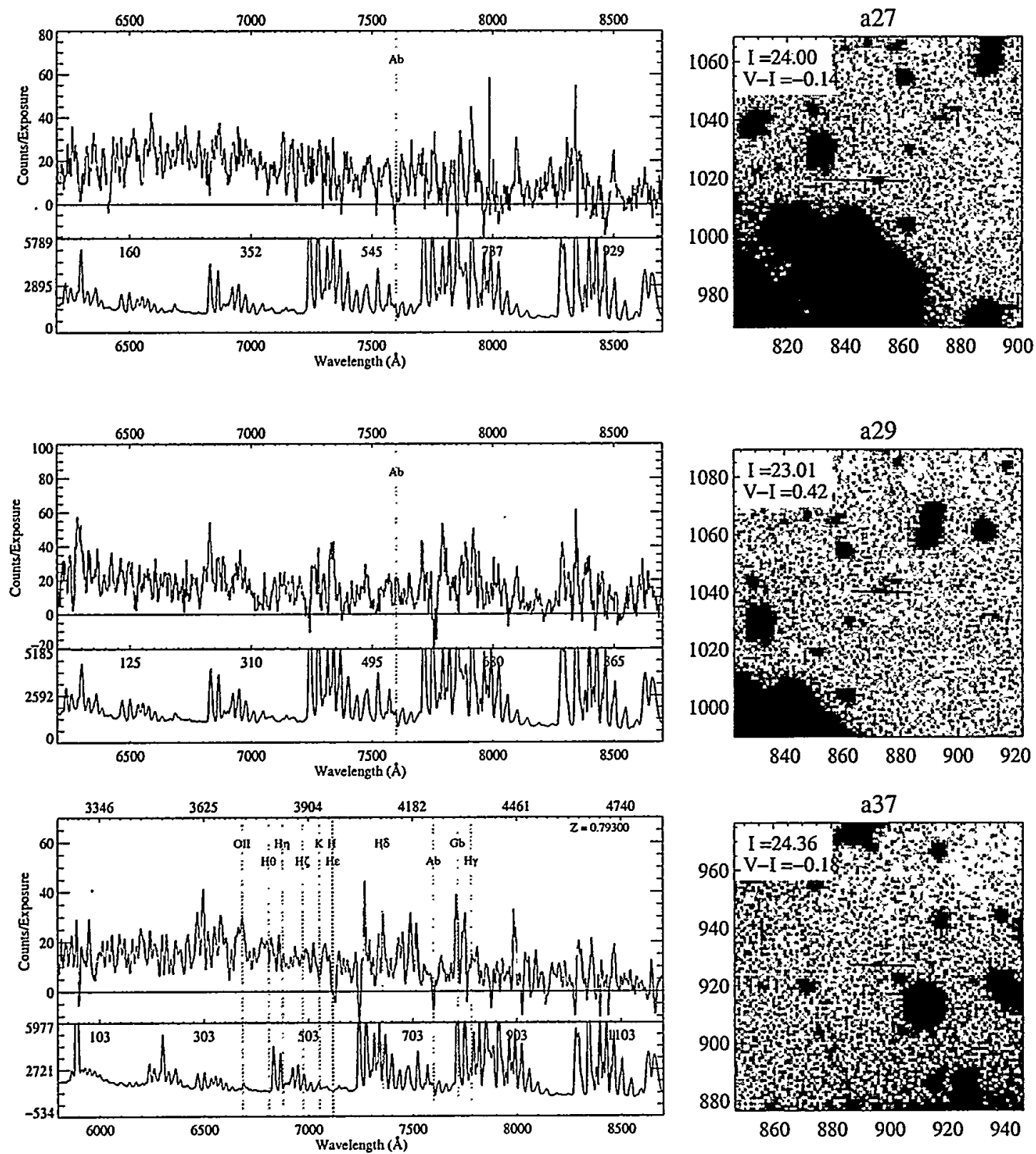

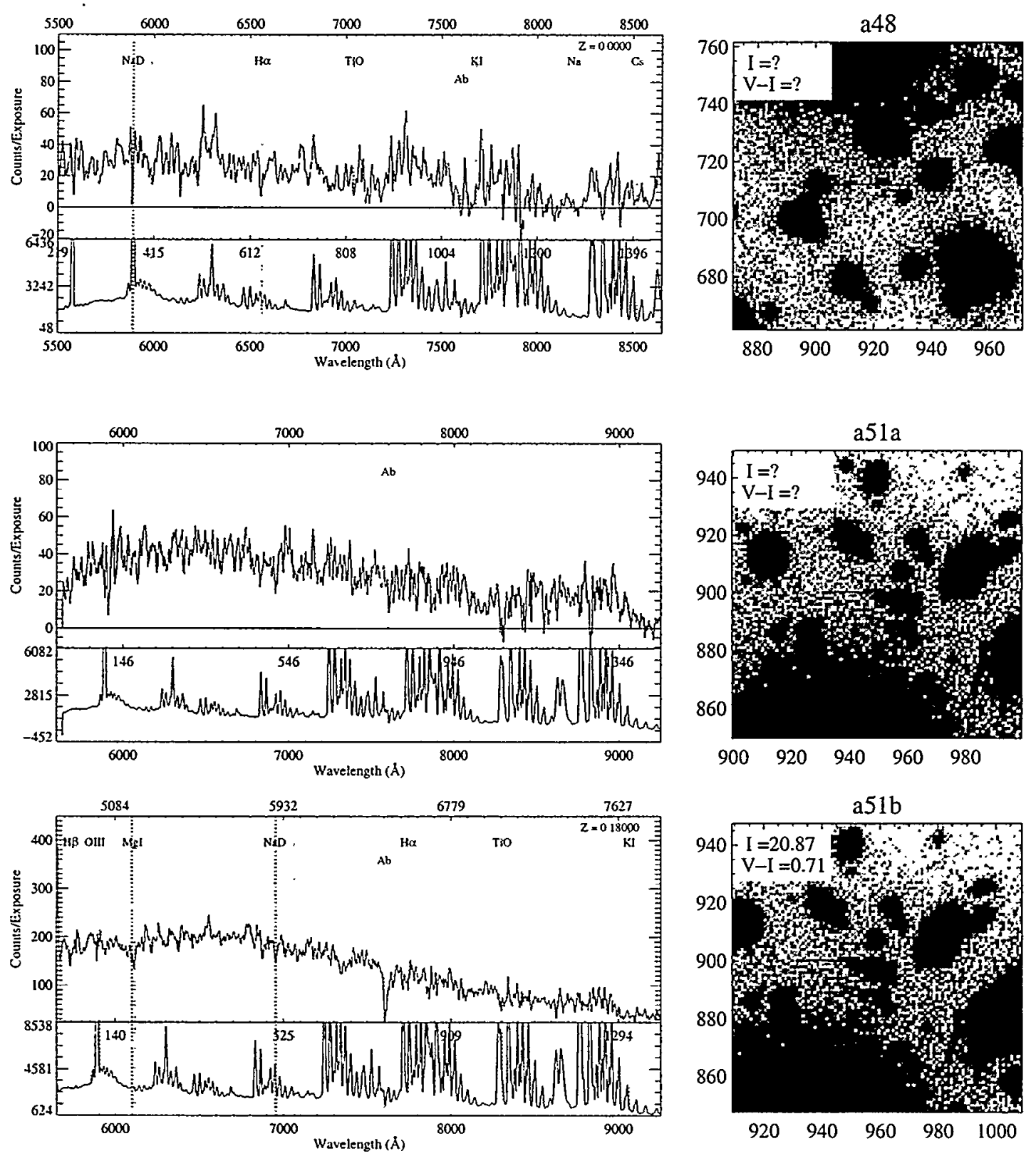

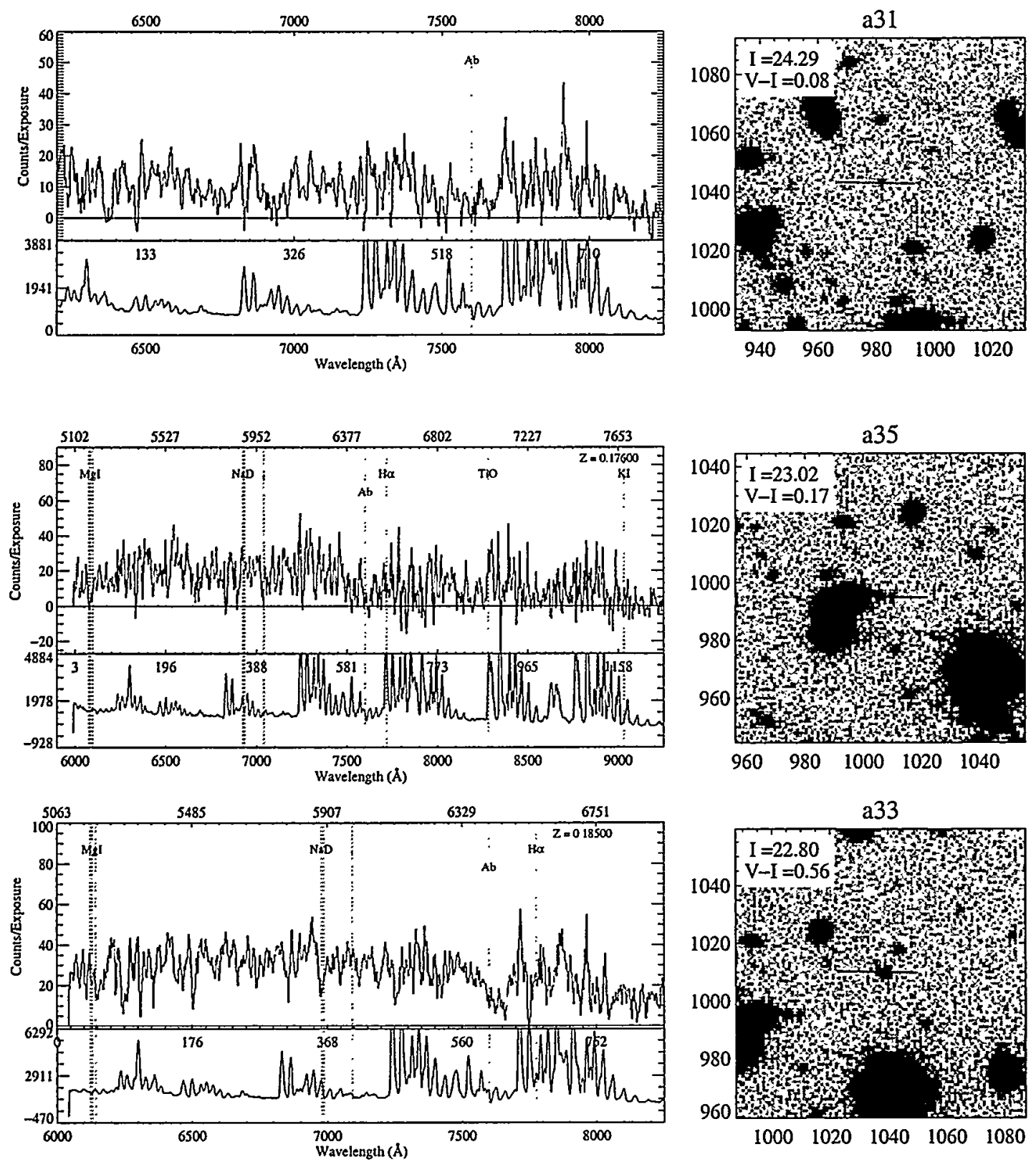

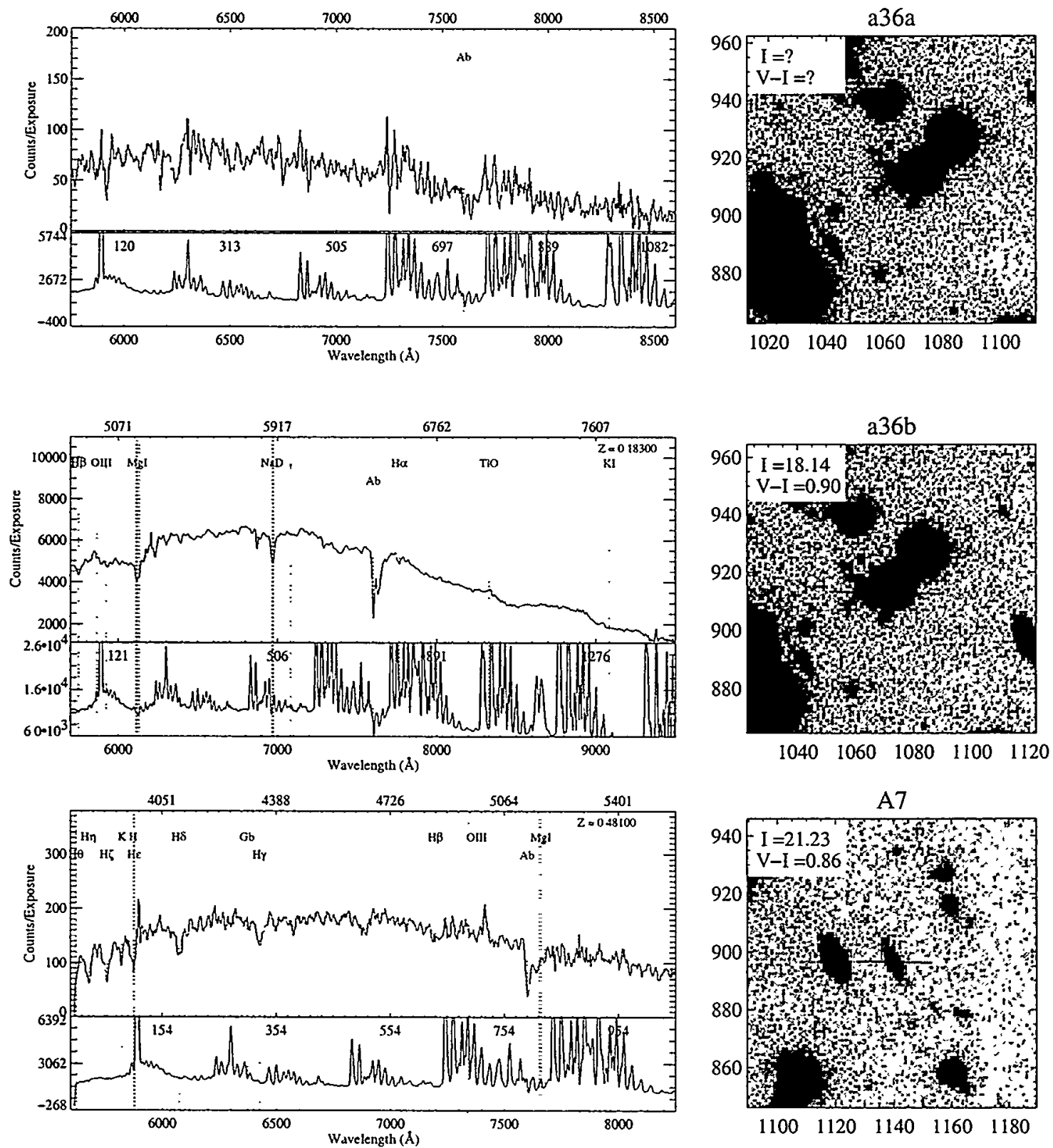

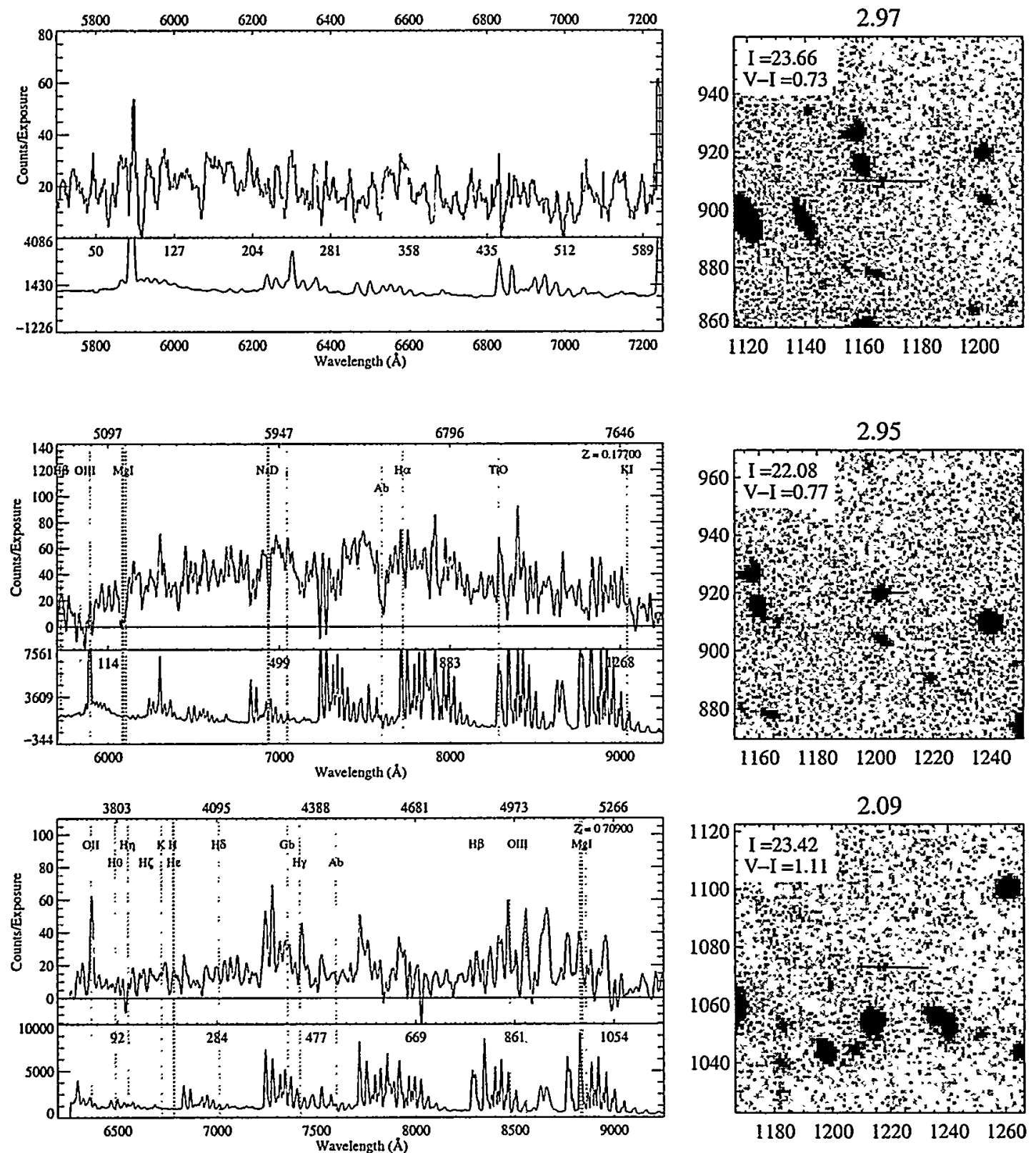

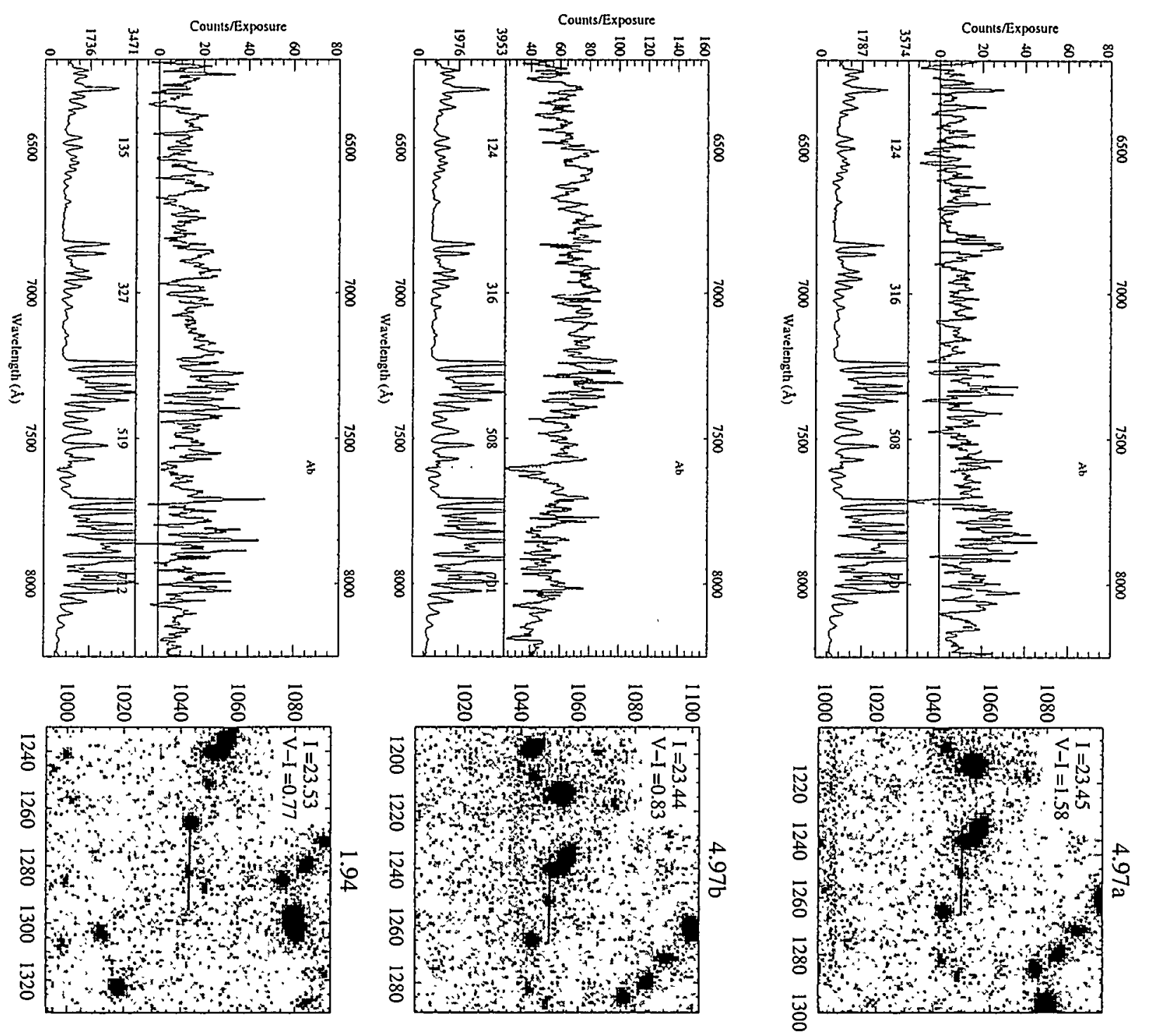

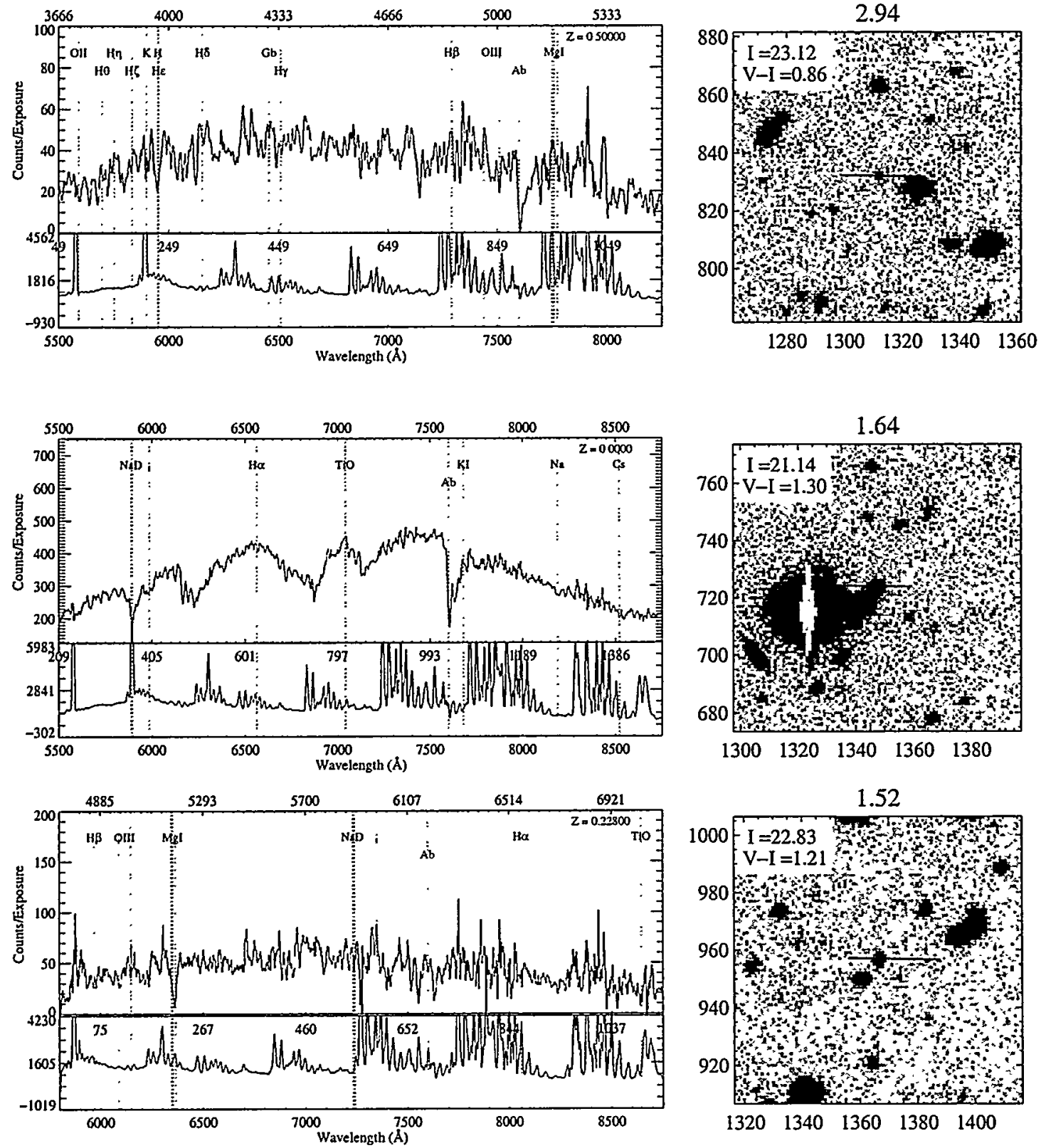
A.2 Mask 2 

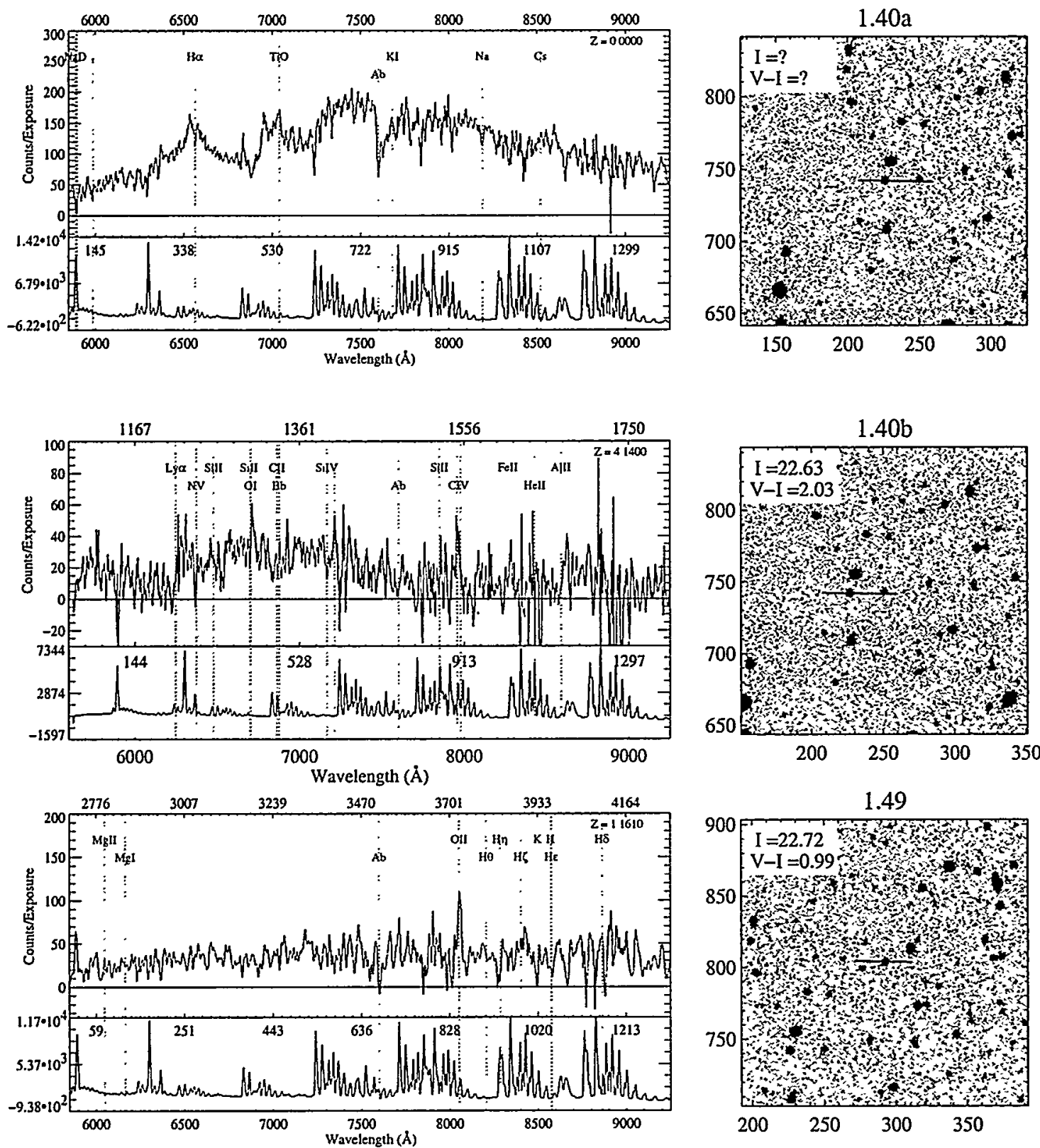

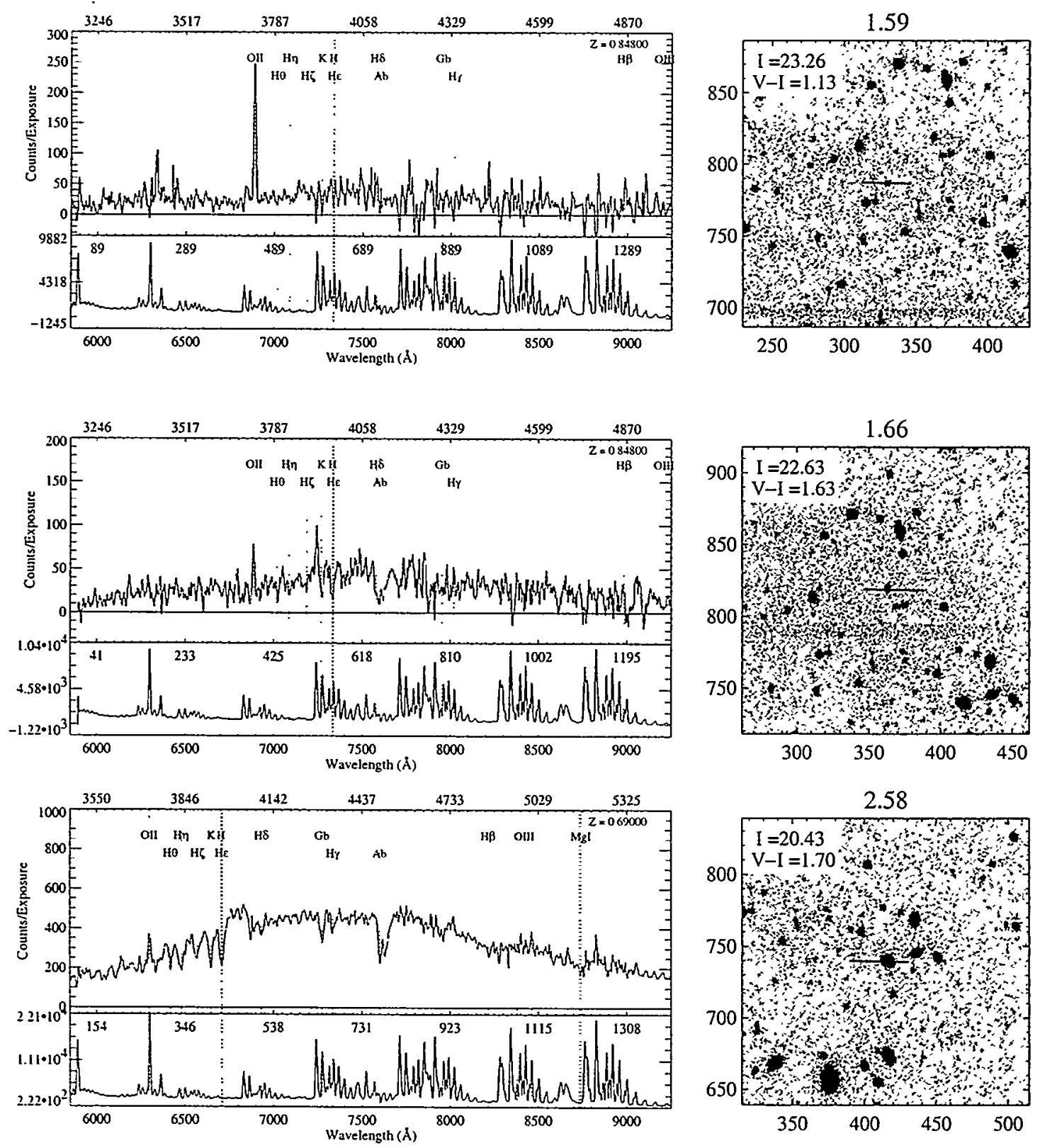

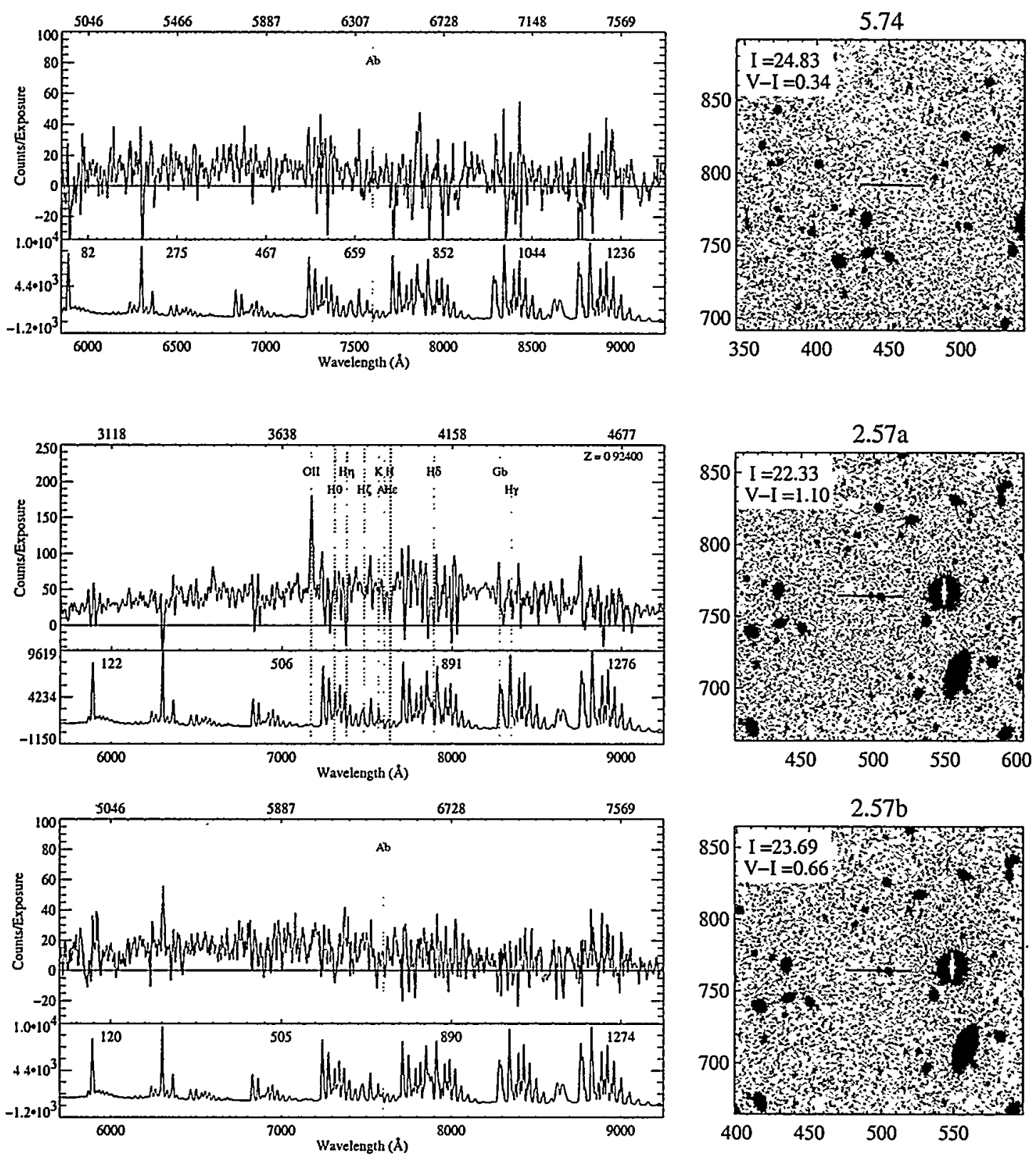

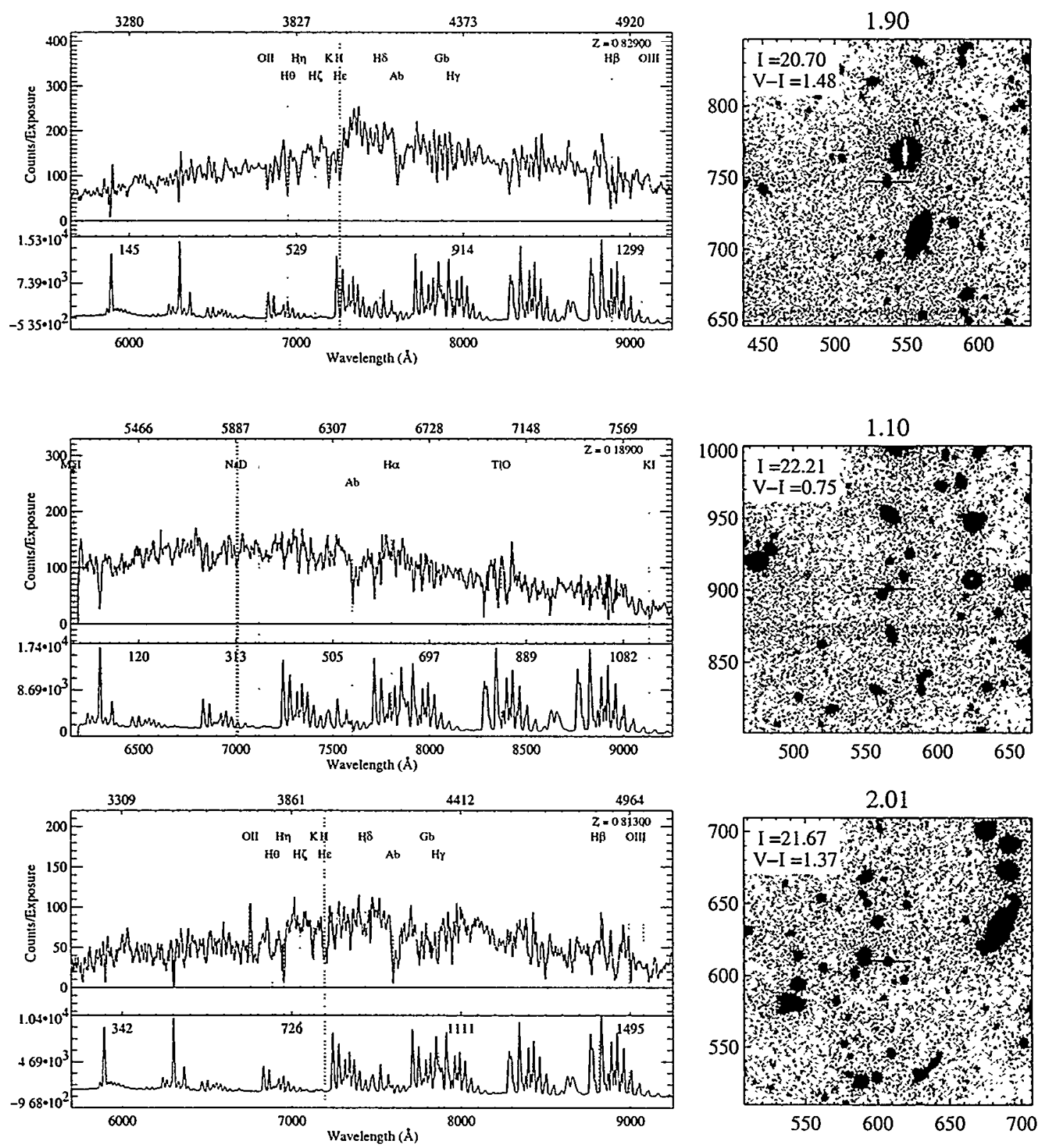

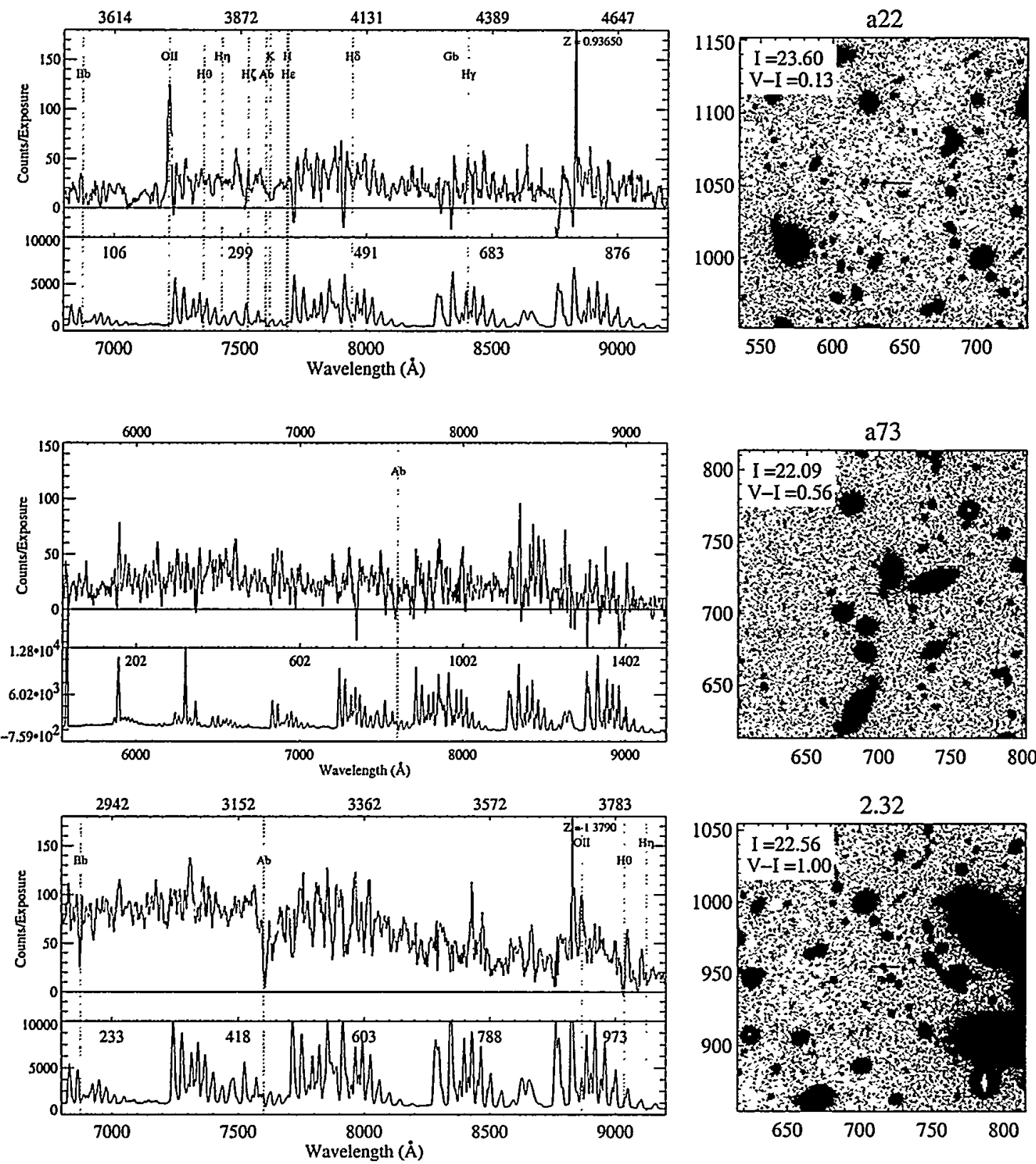

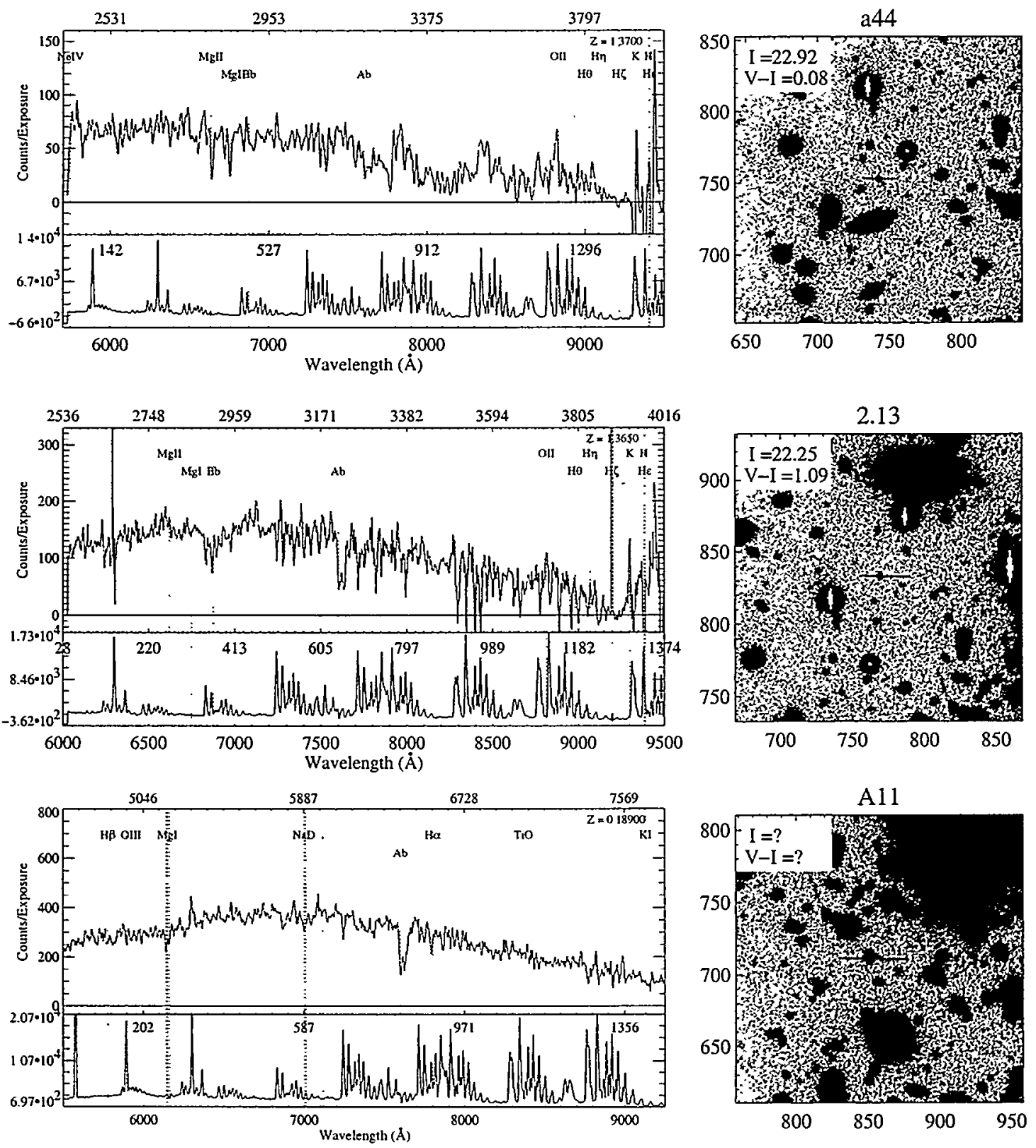

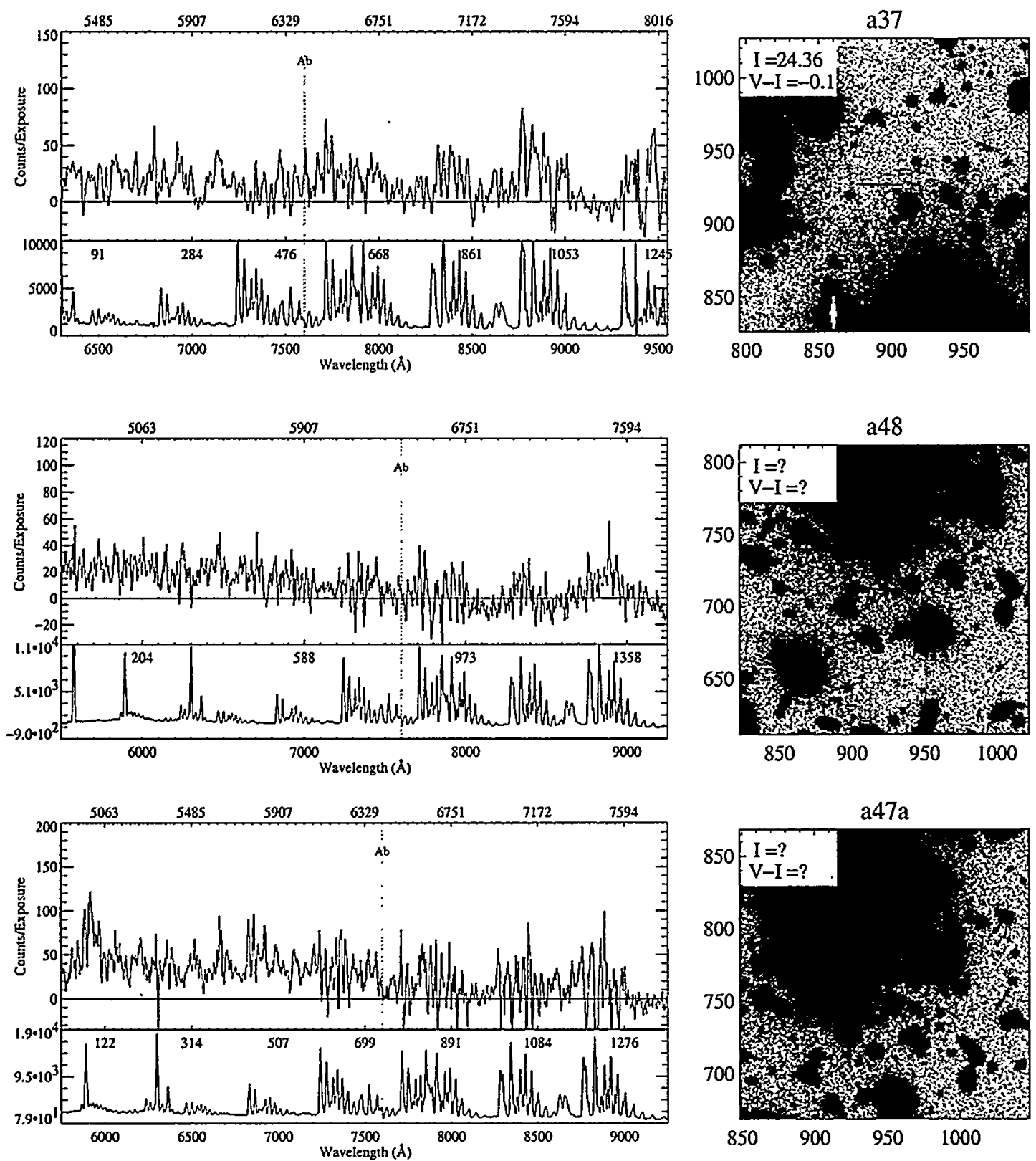

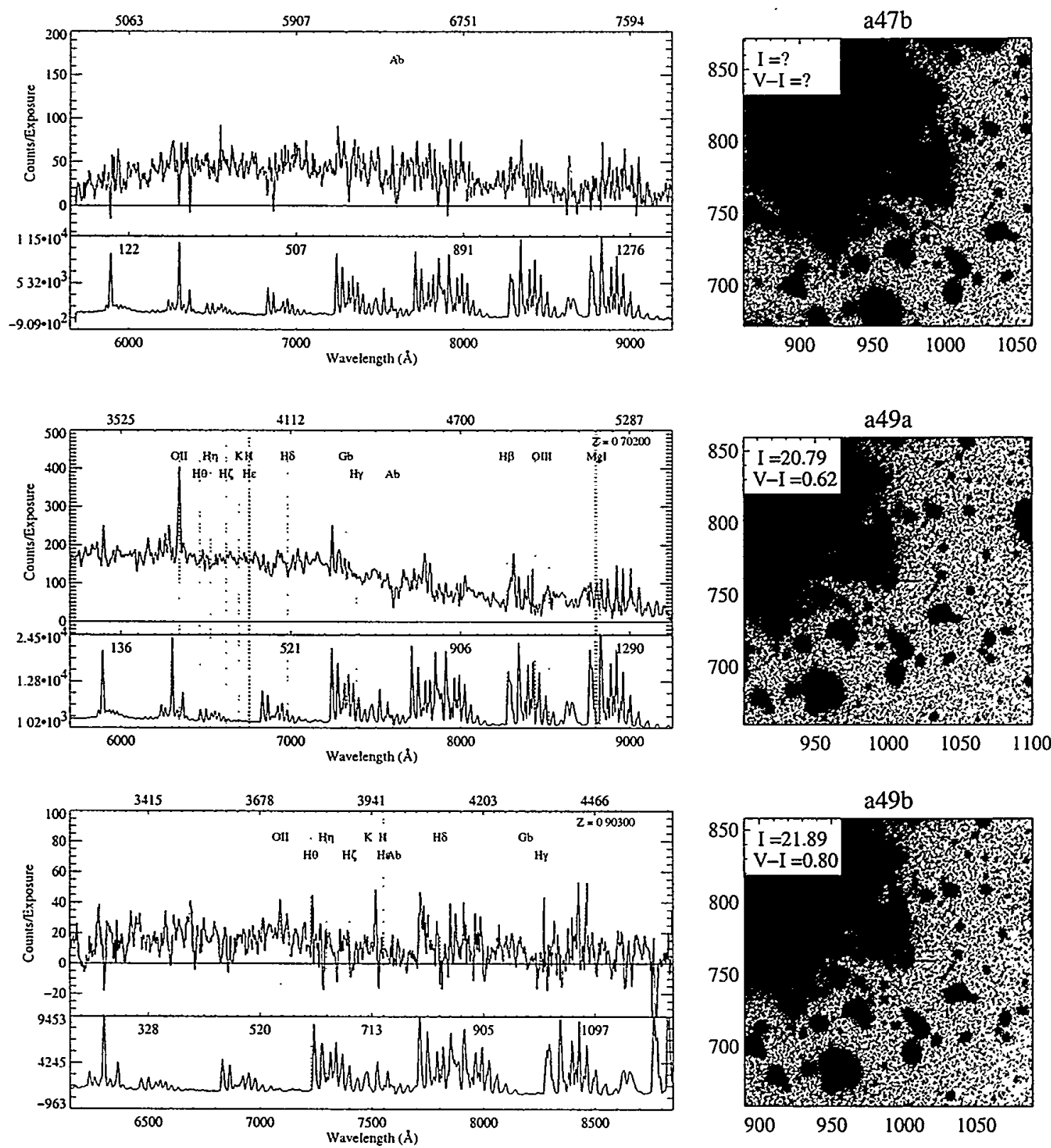

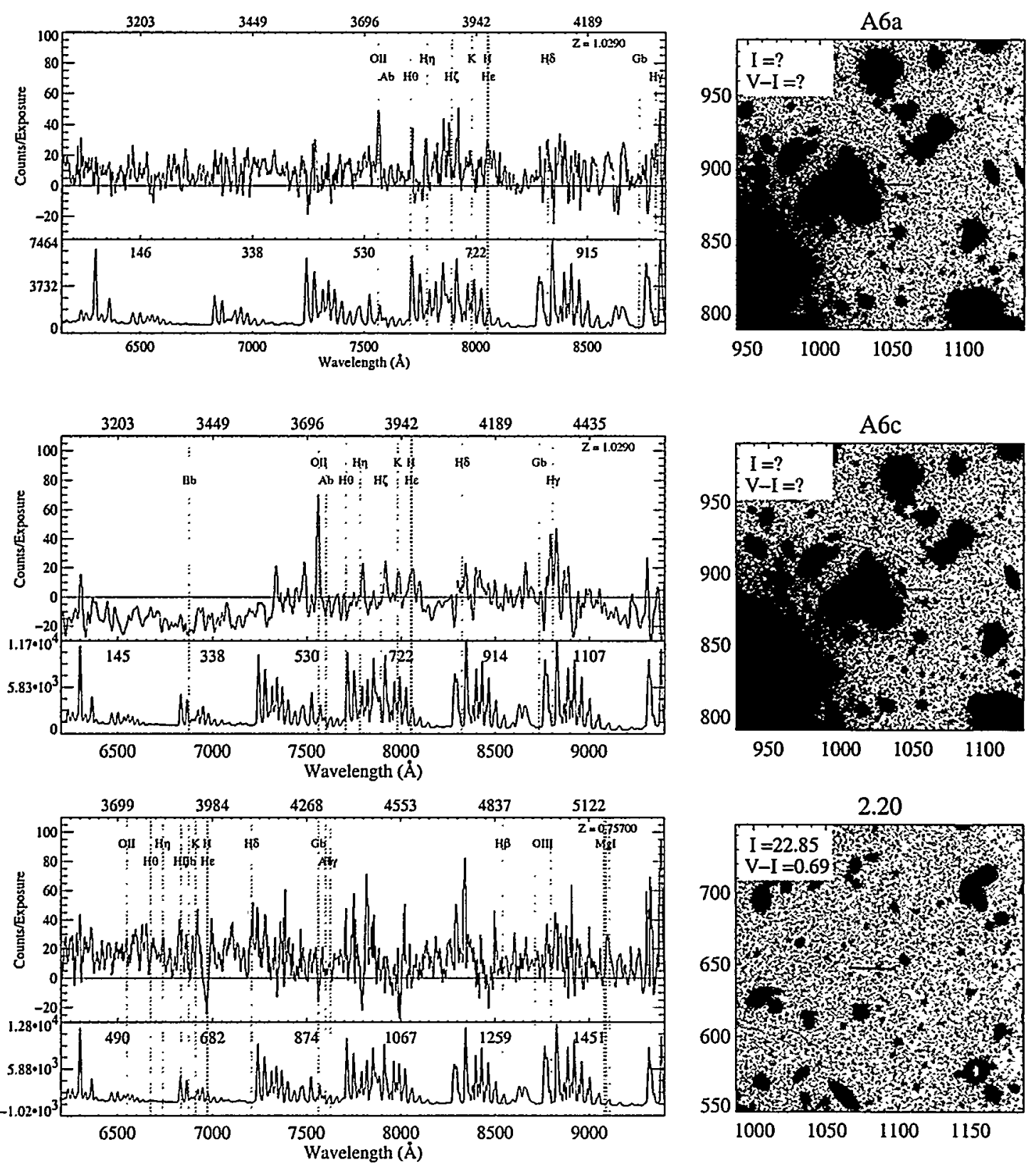

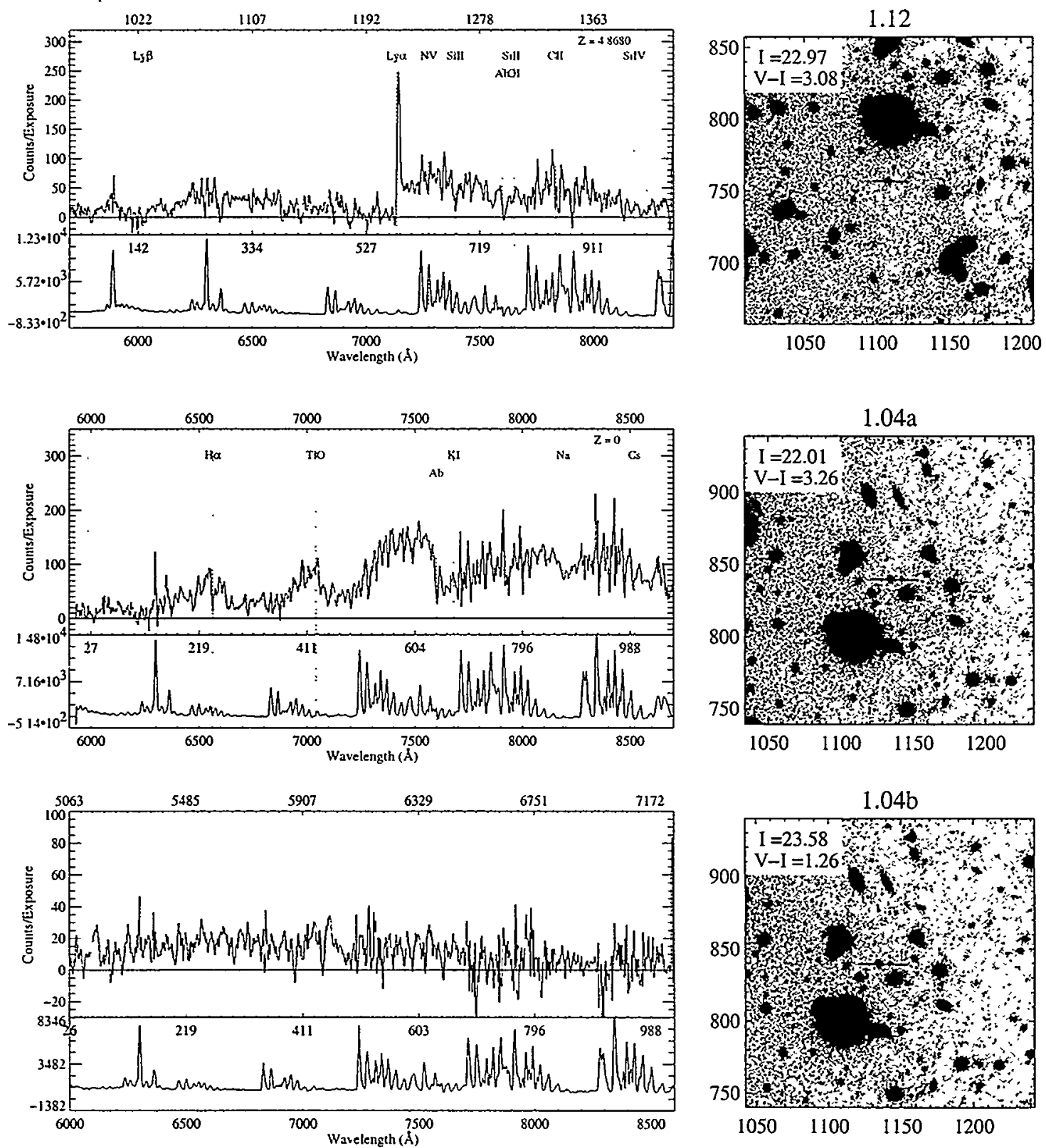

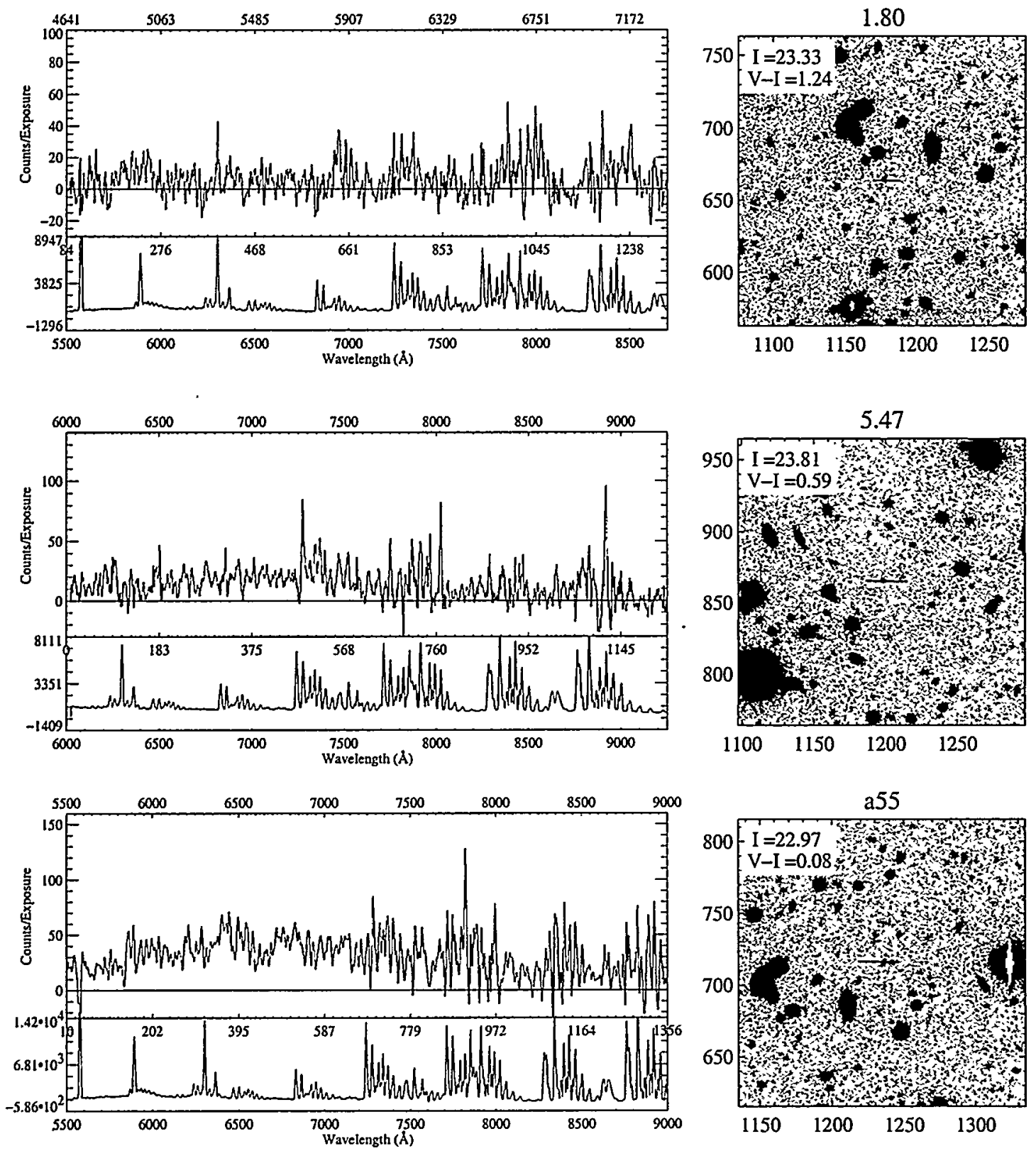

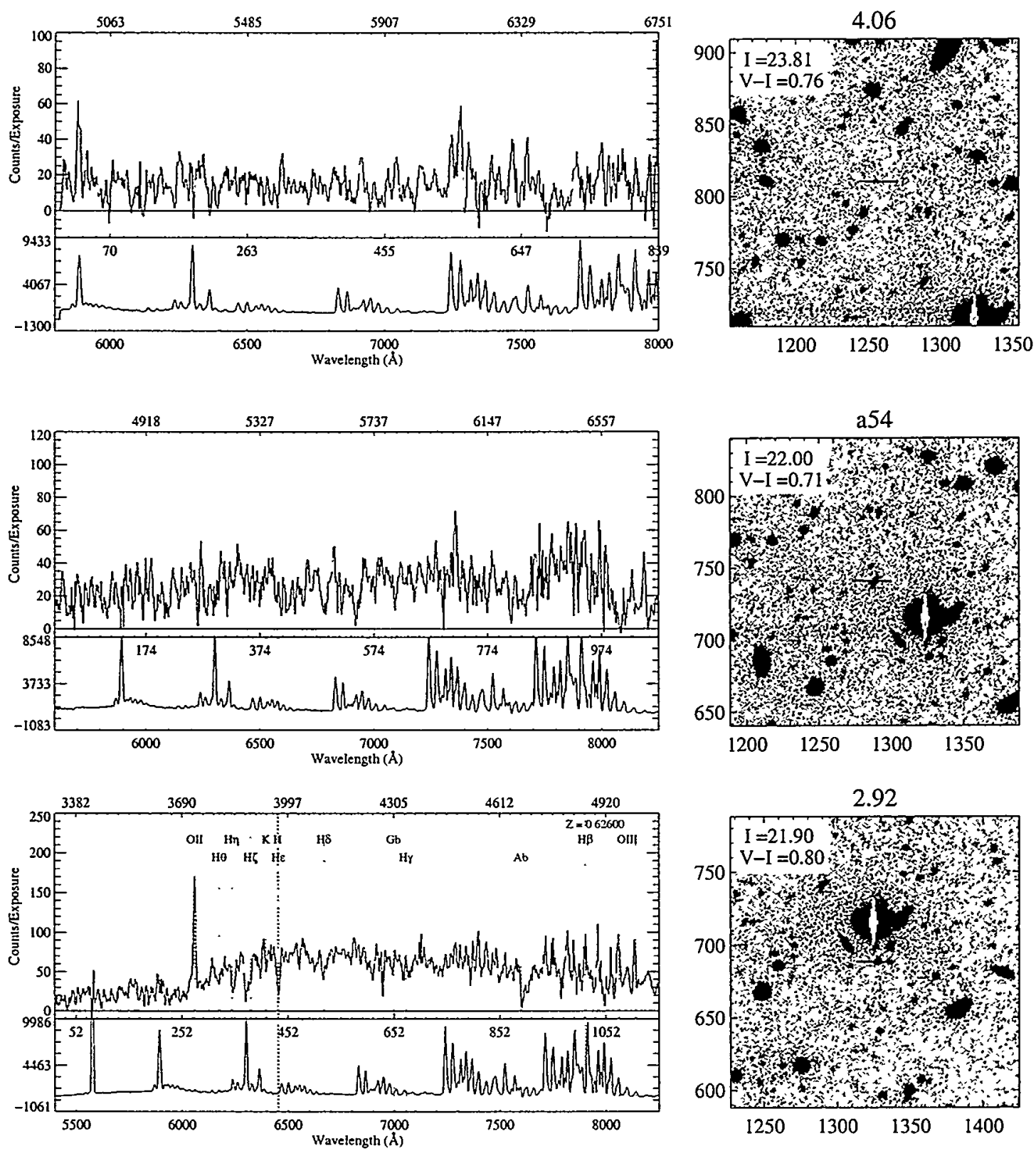

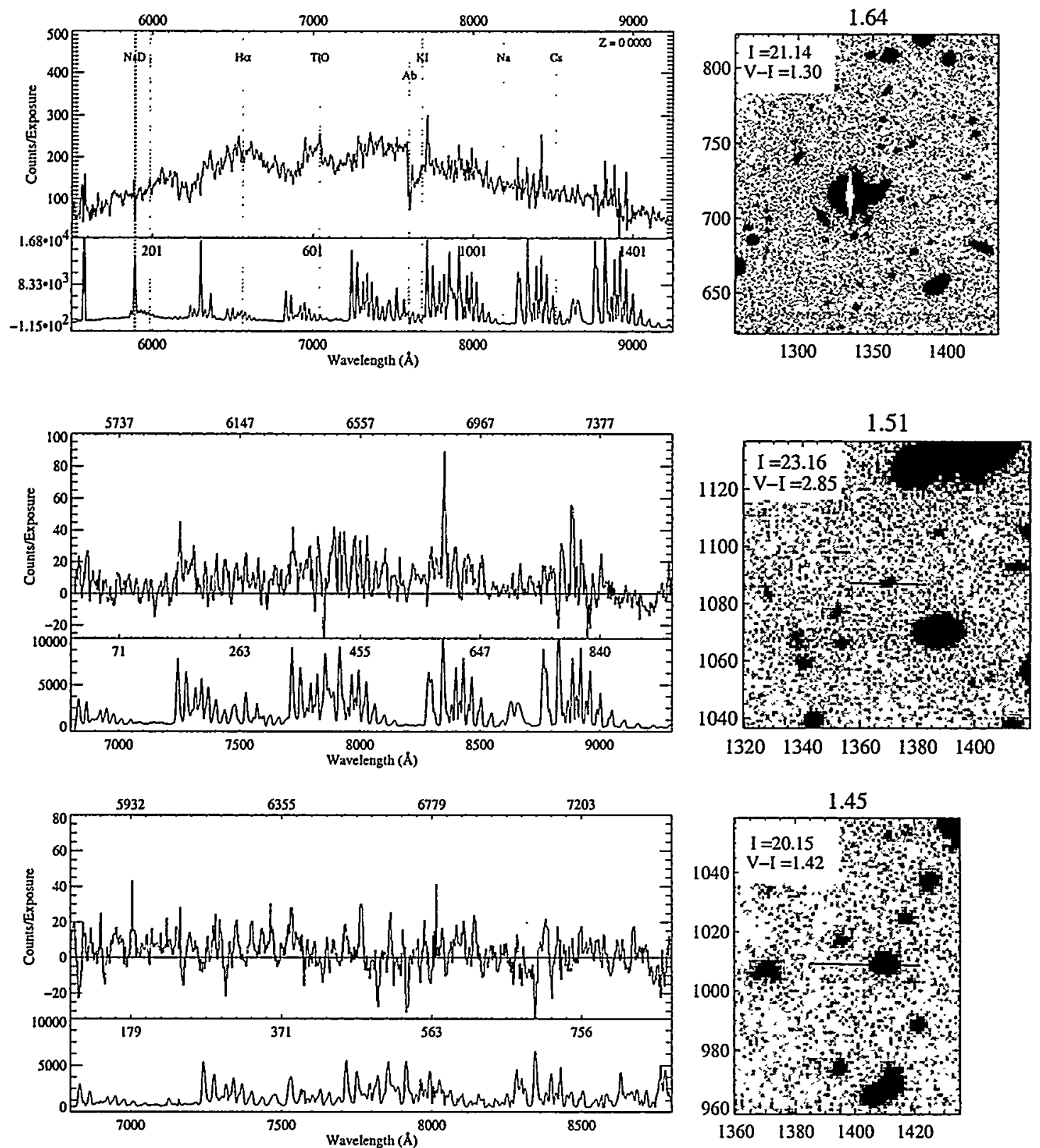
A.3 Mask 3 

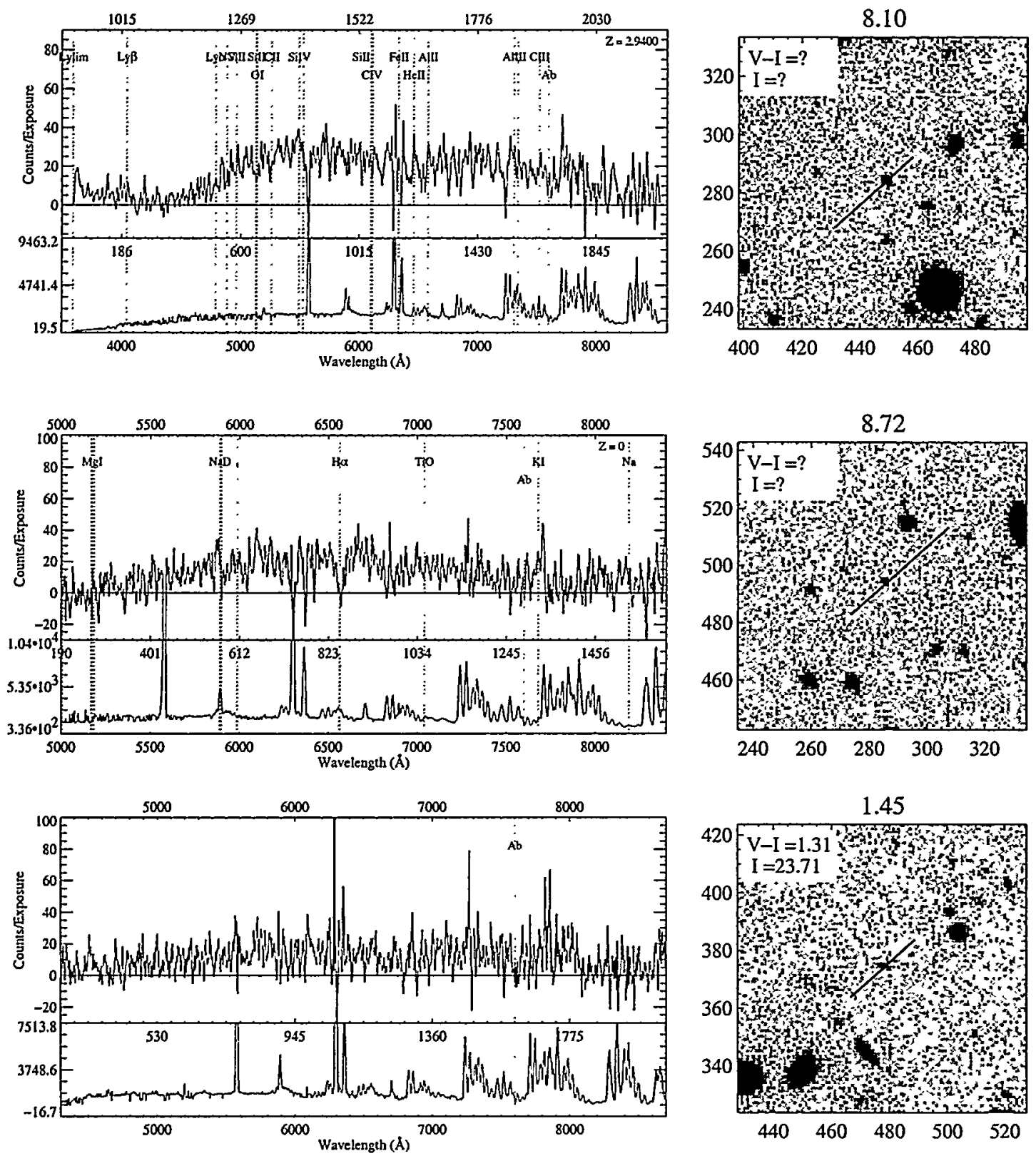

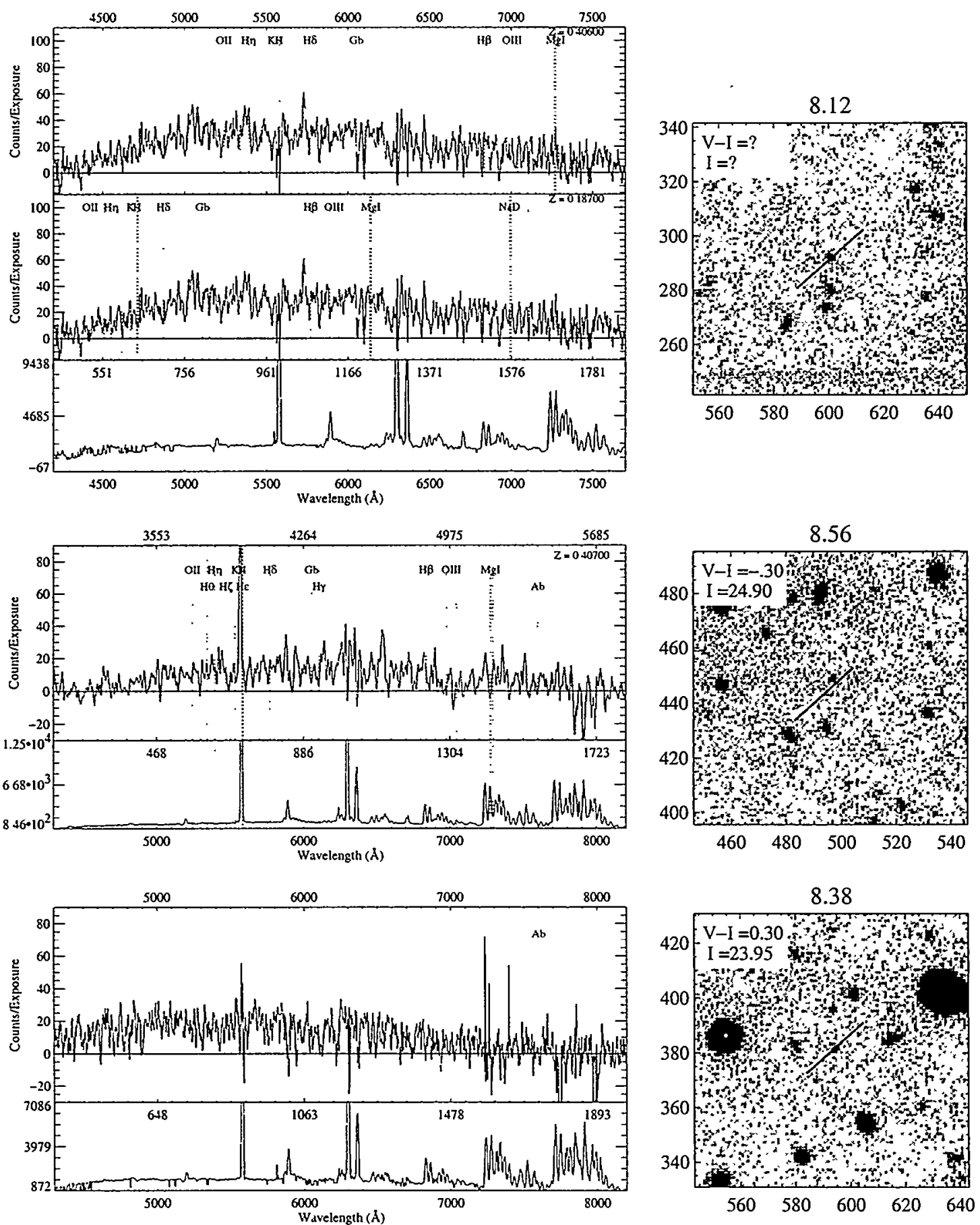

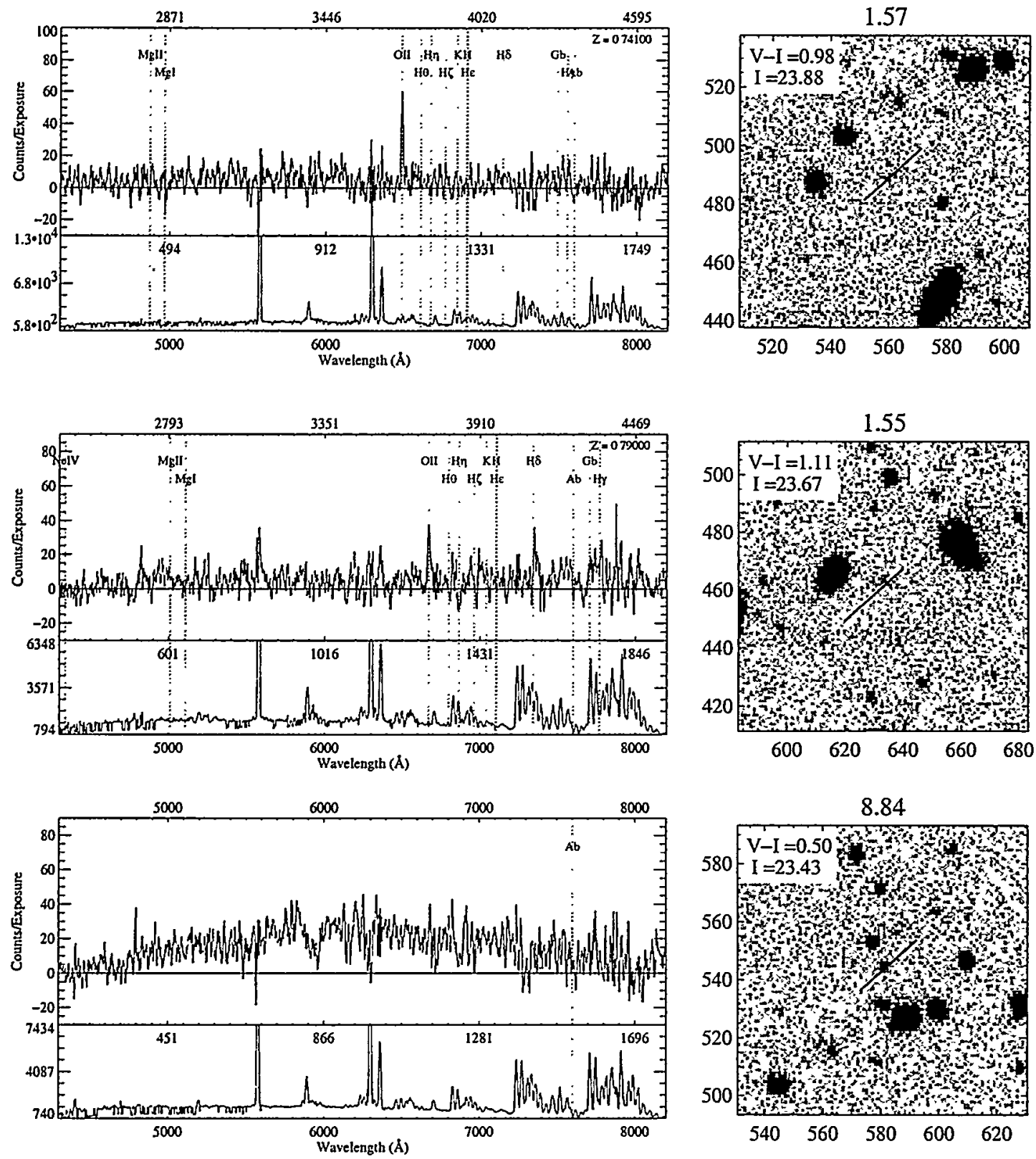

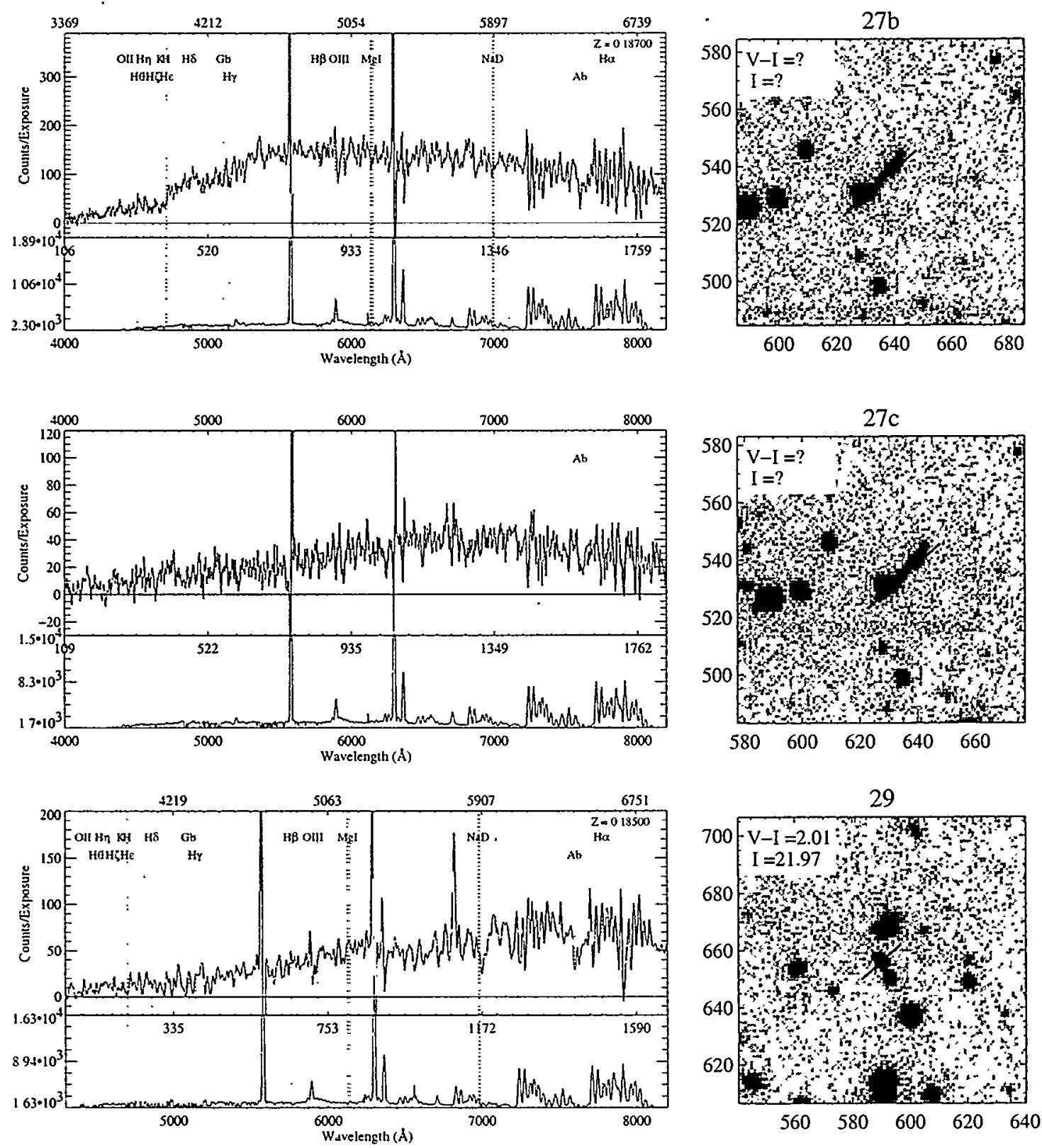

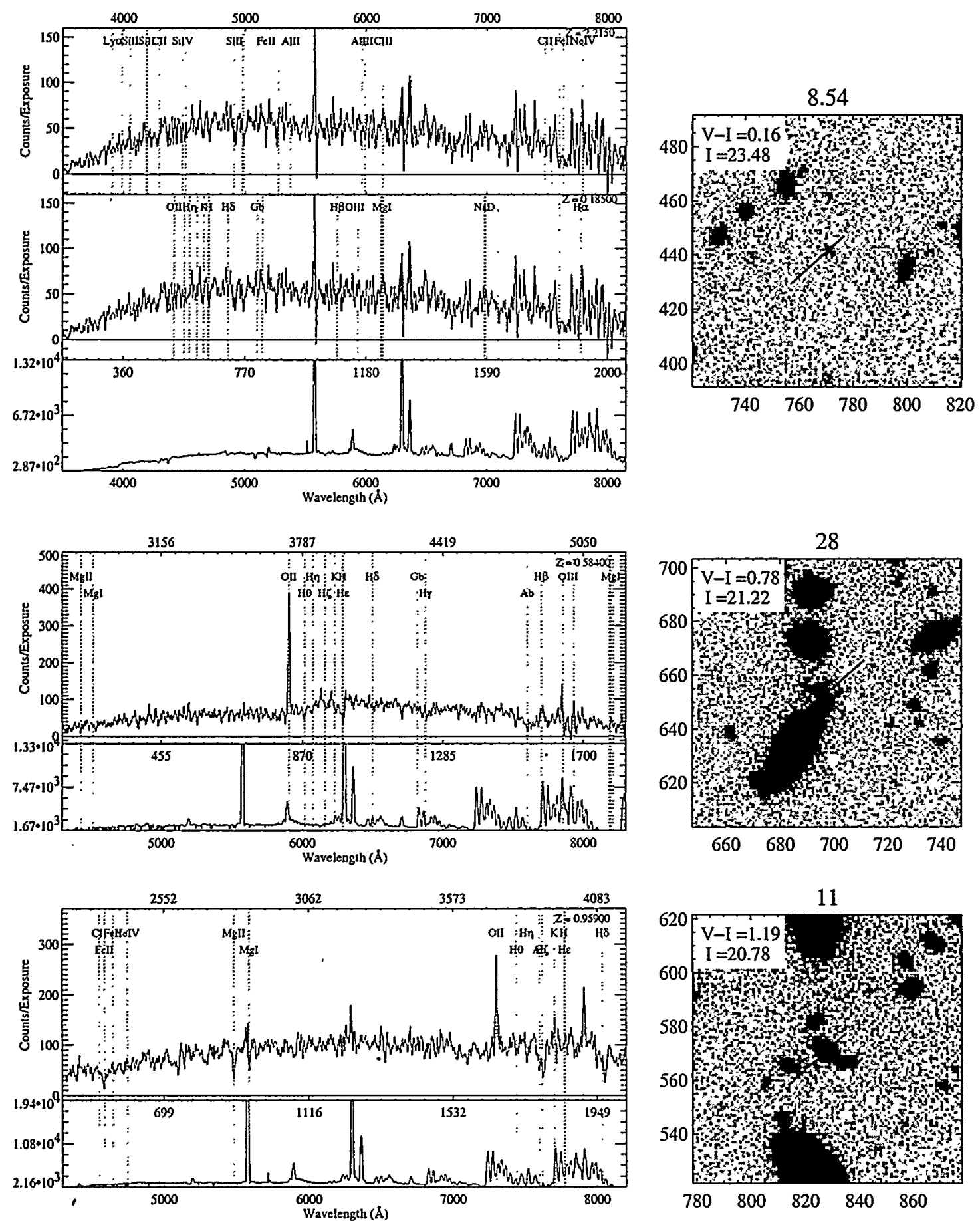

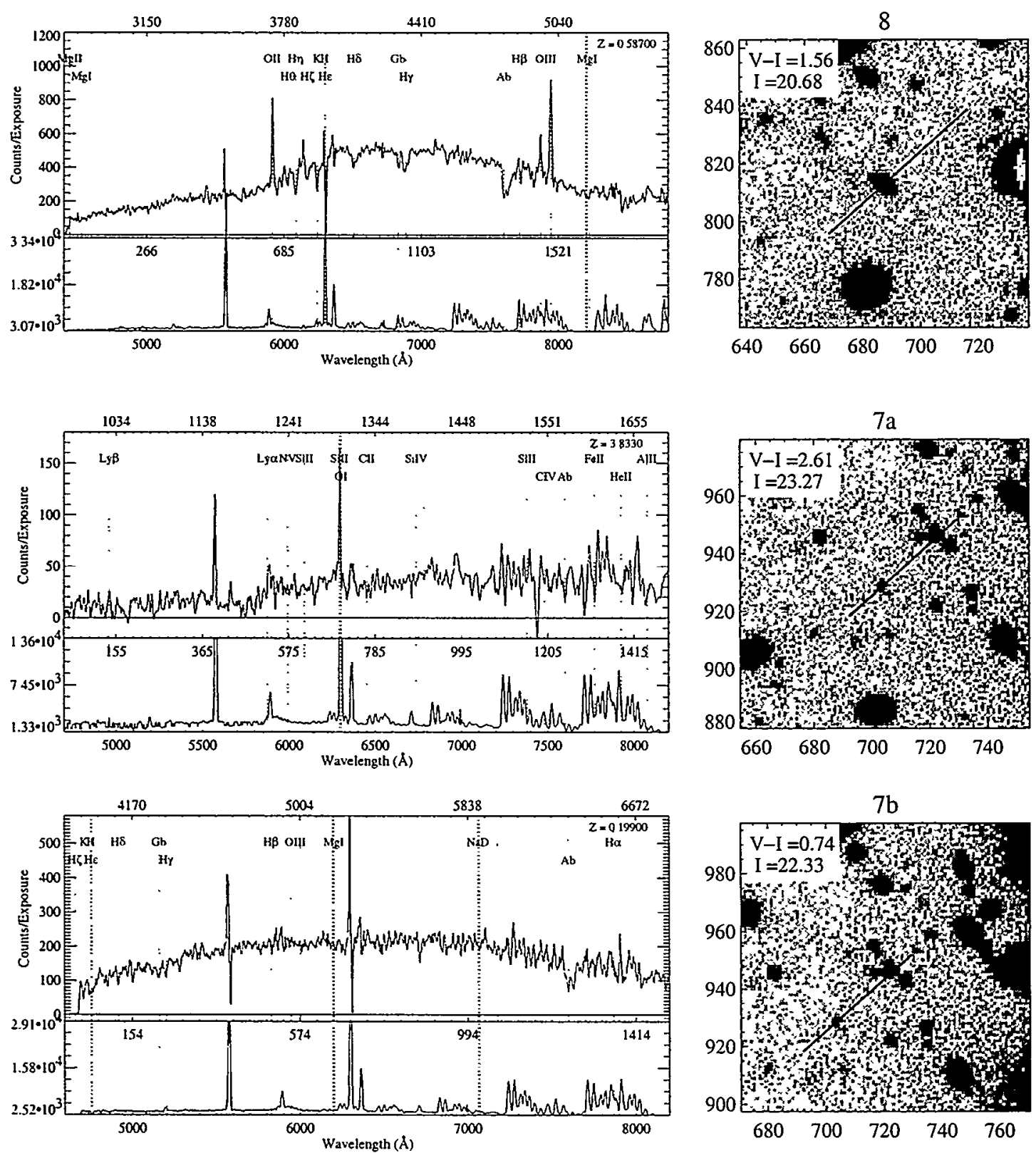

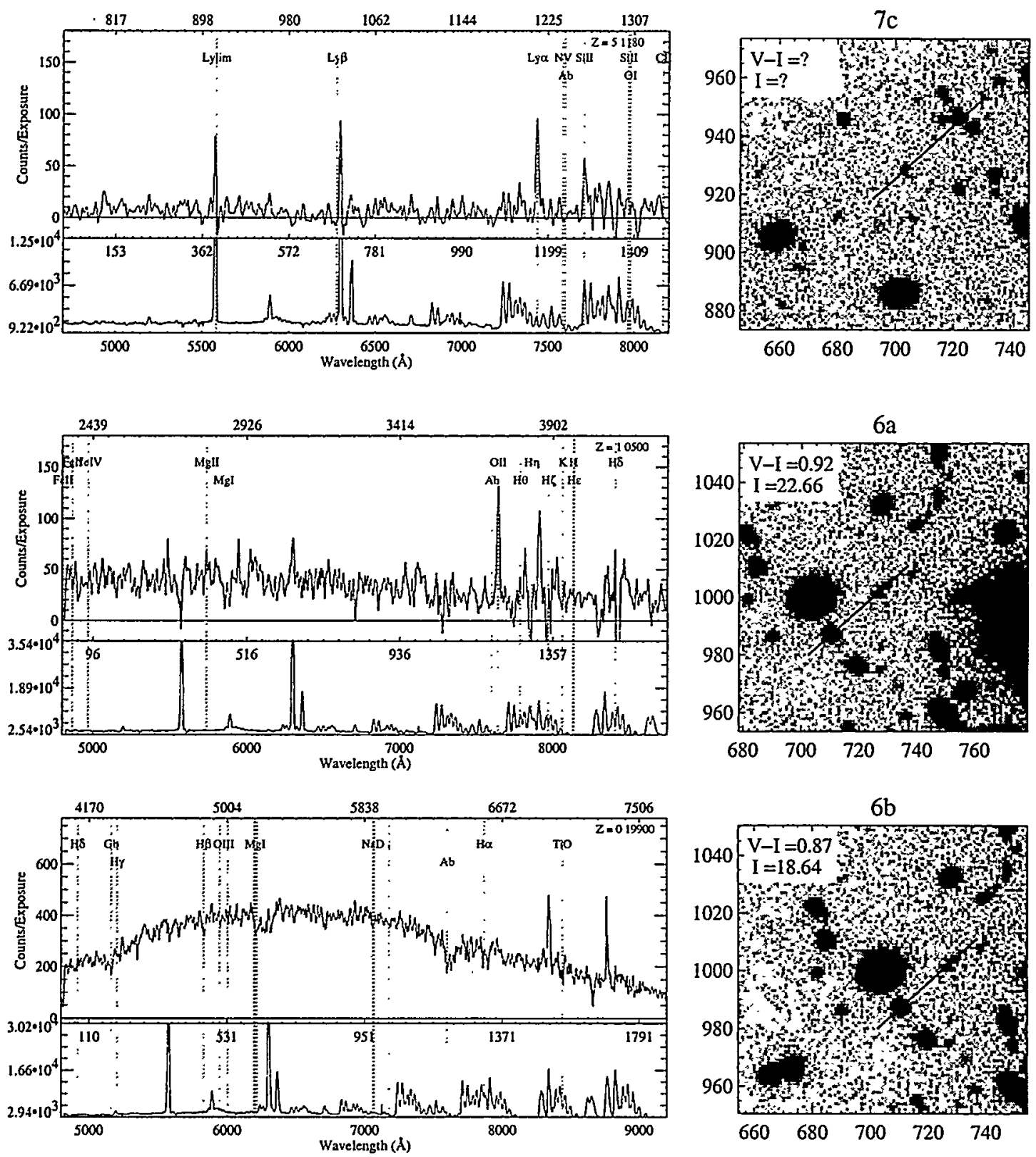

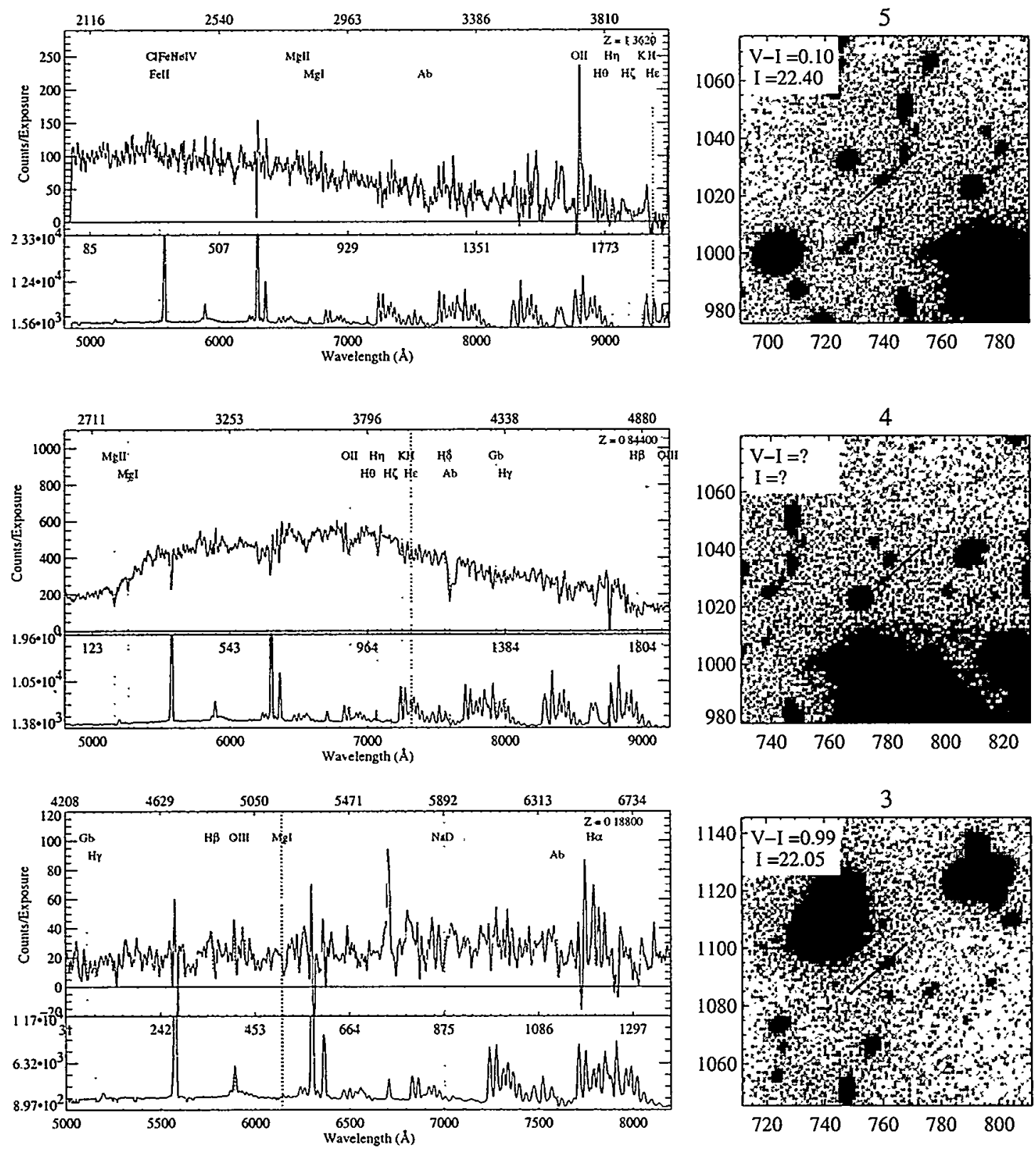

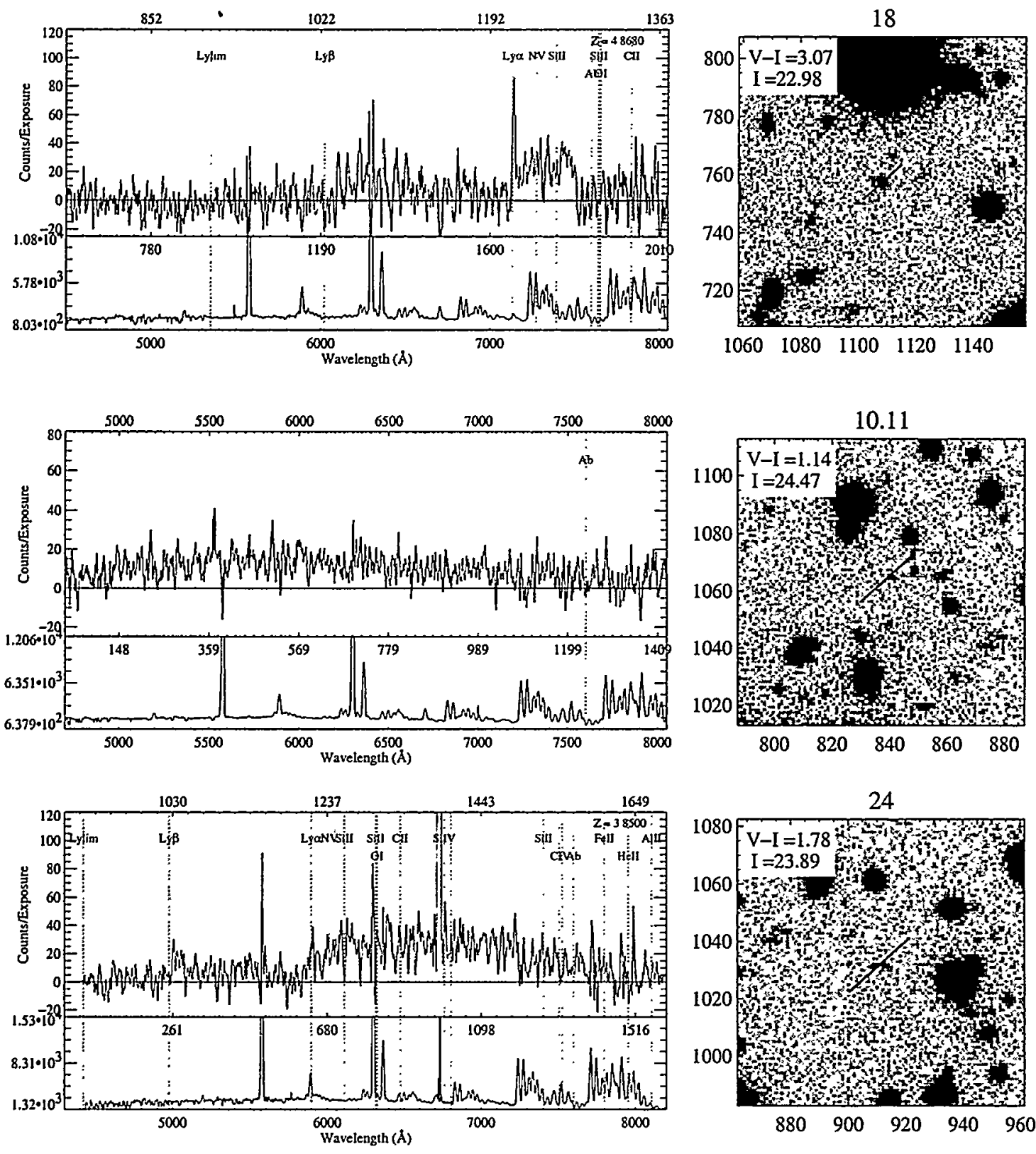

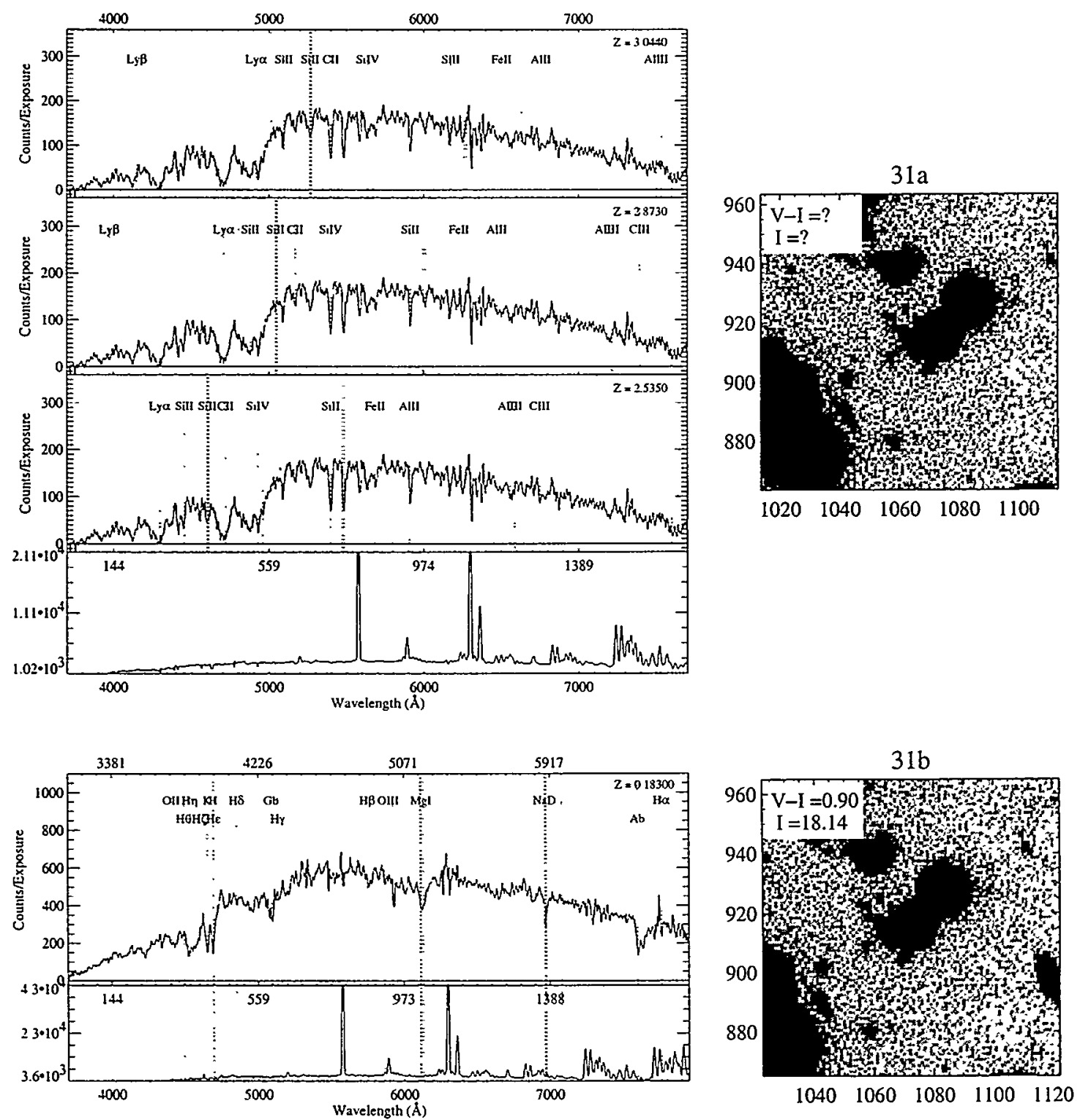

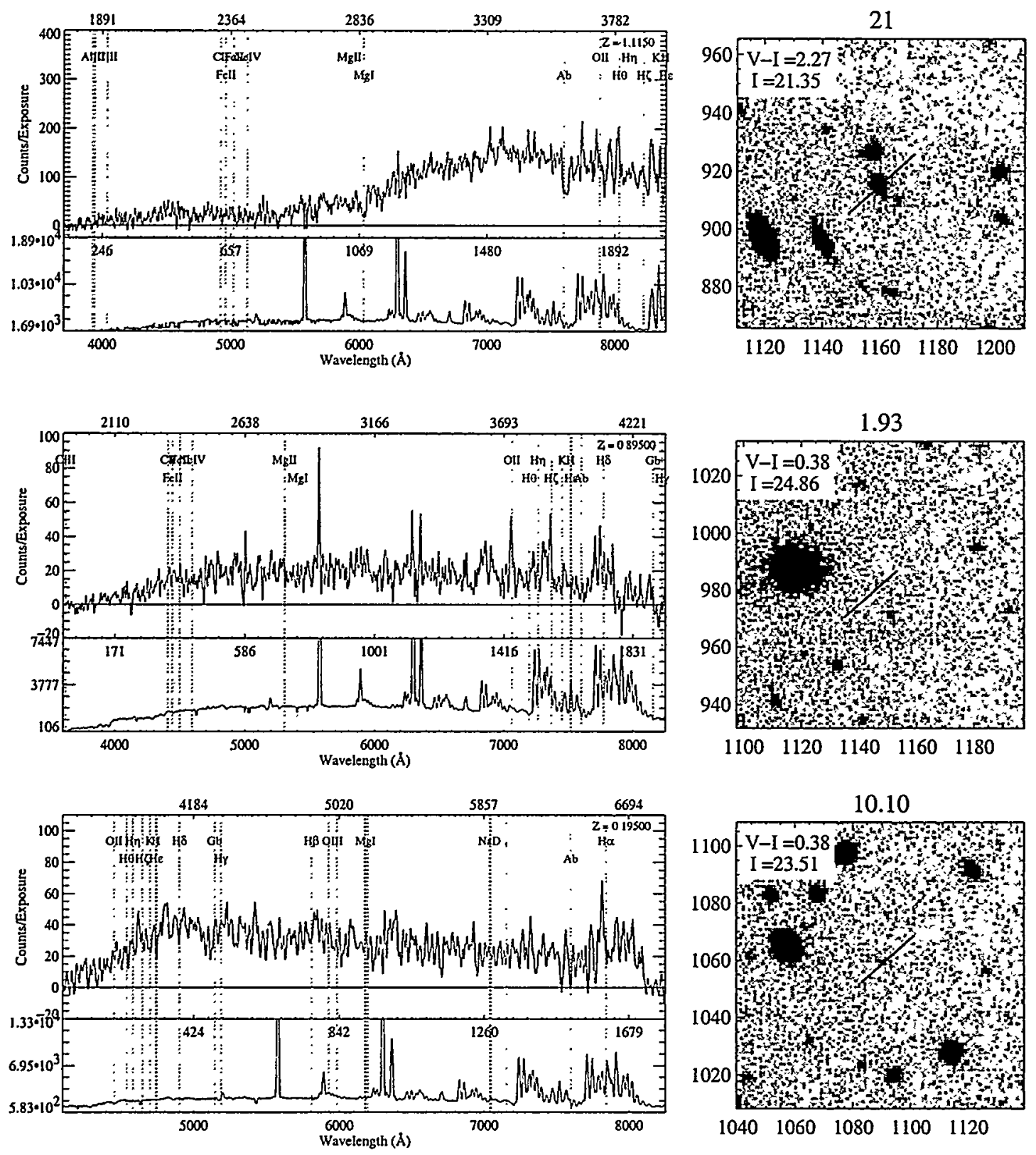

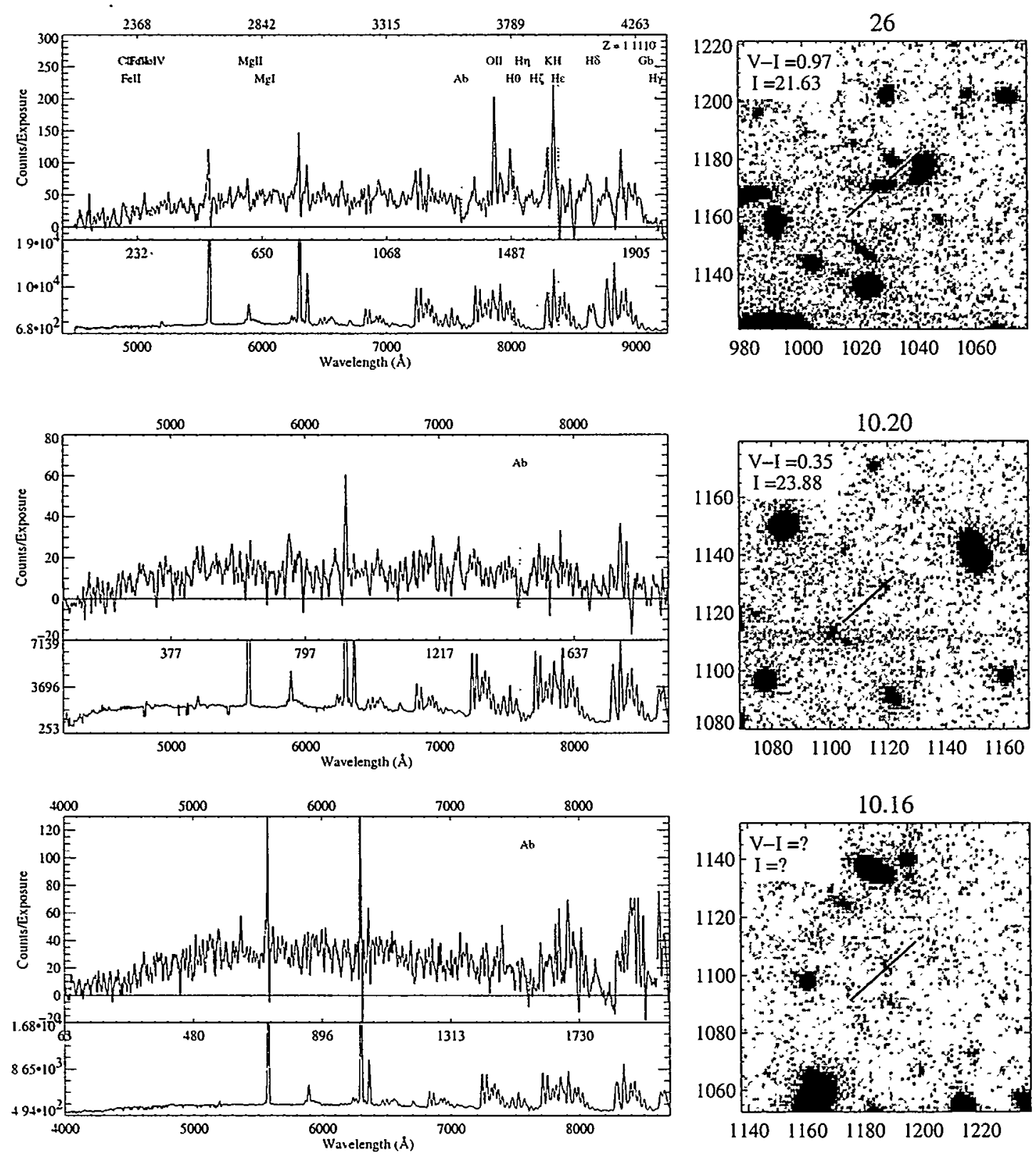

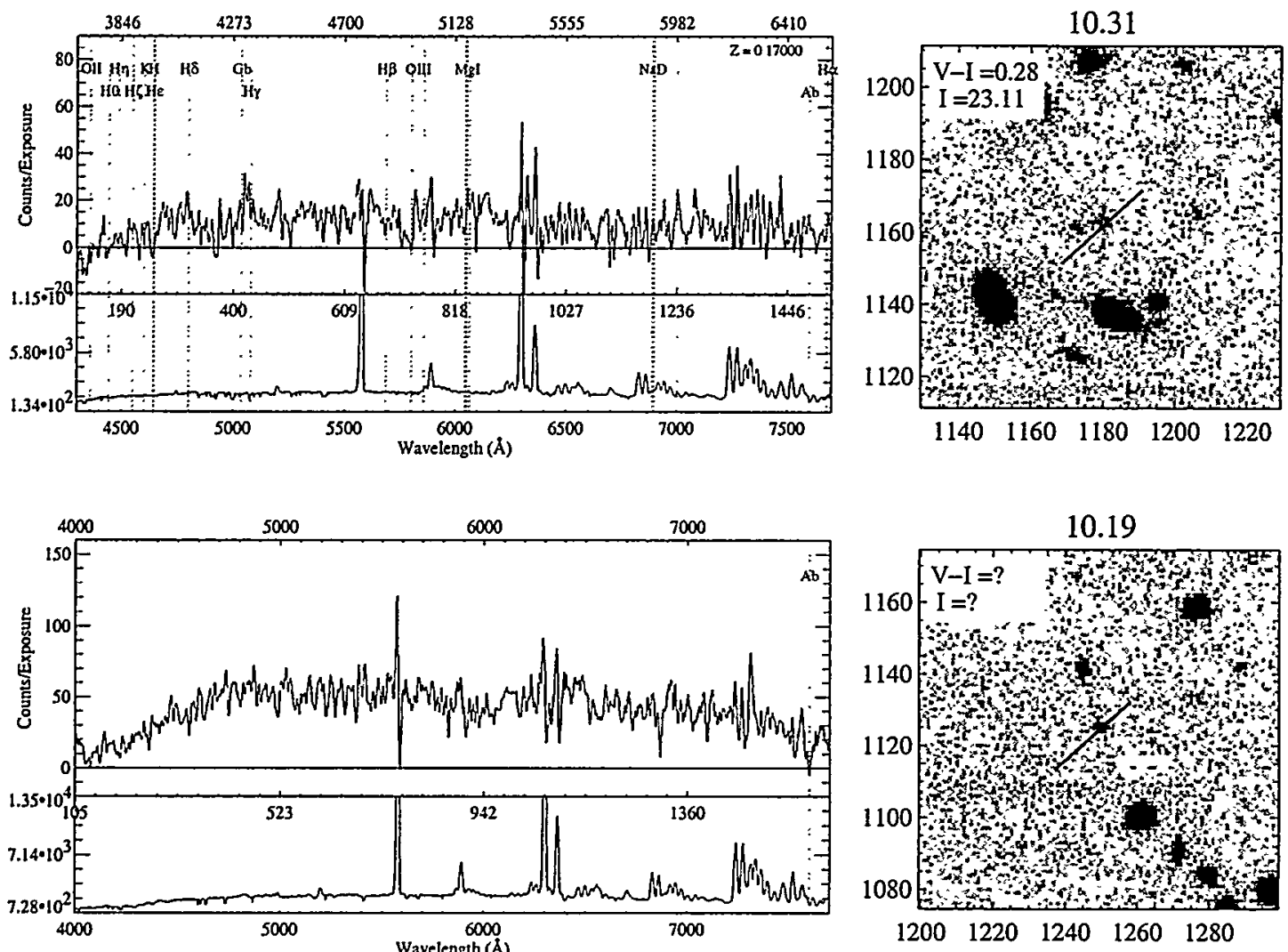
Appendix B

A2390:

B.1 Mask 1 

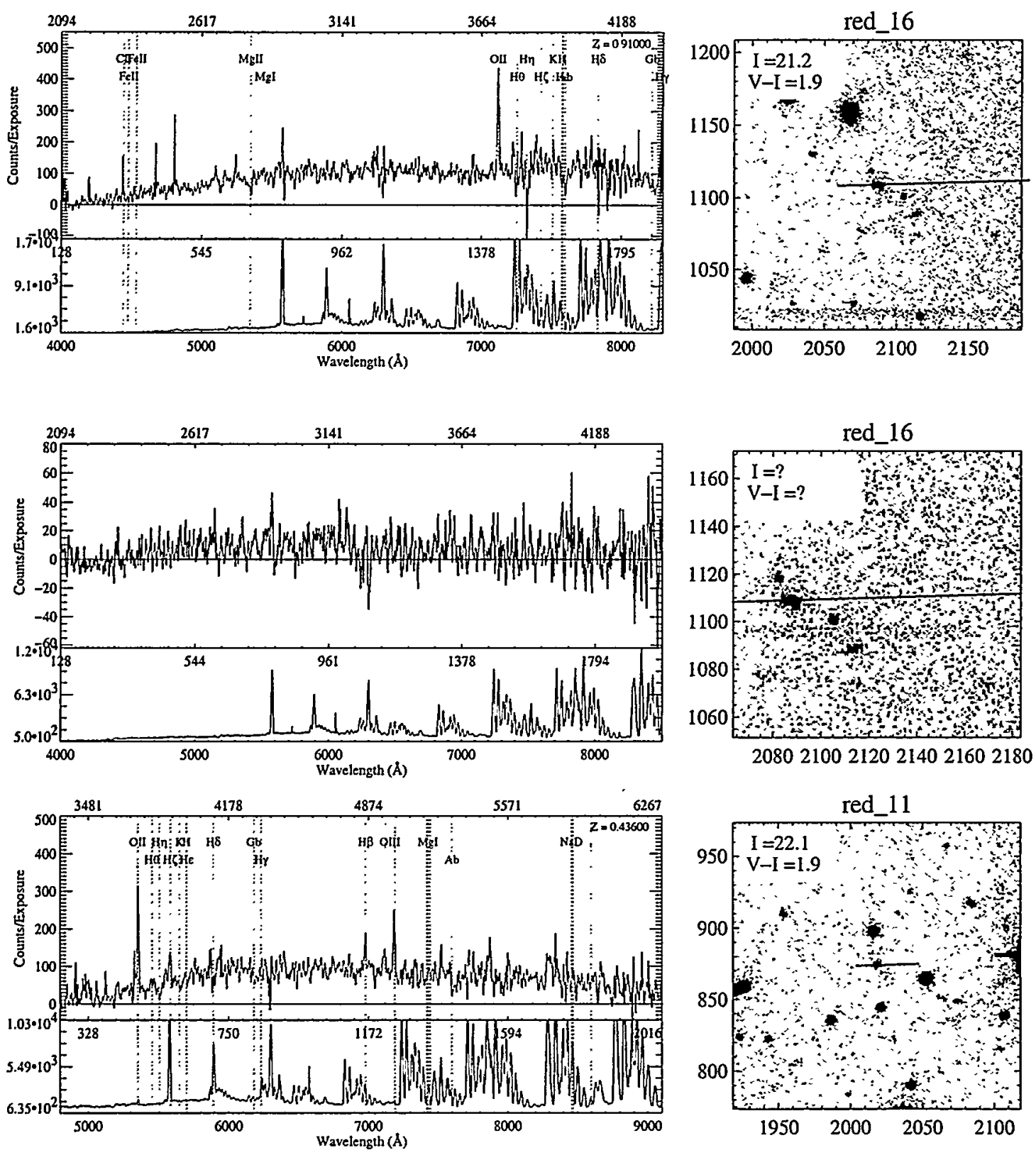

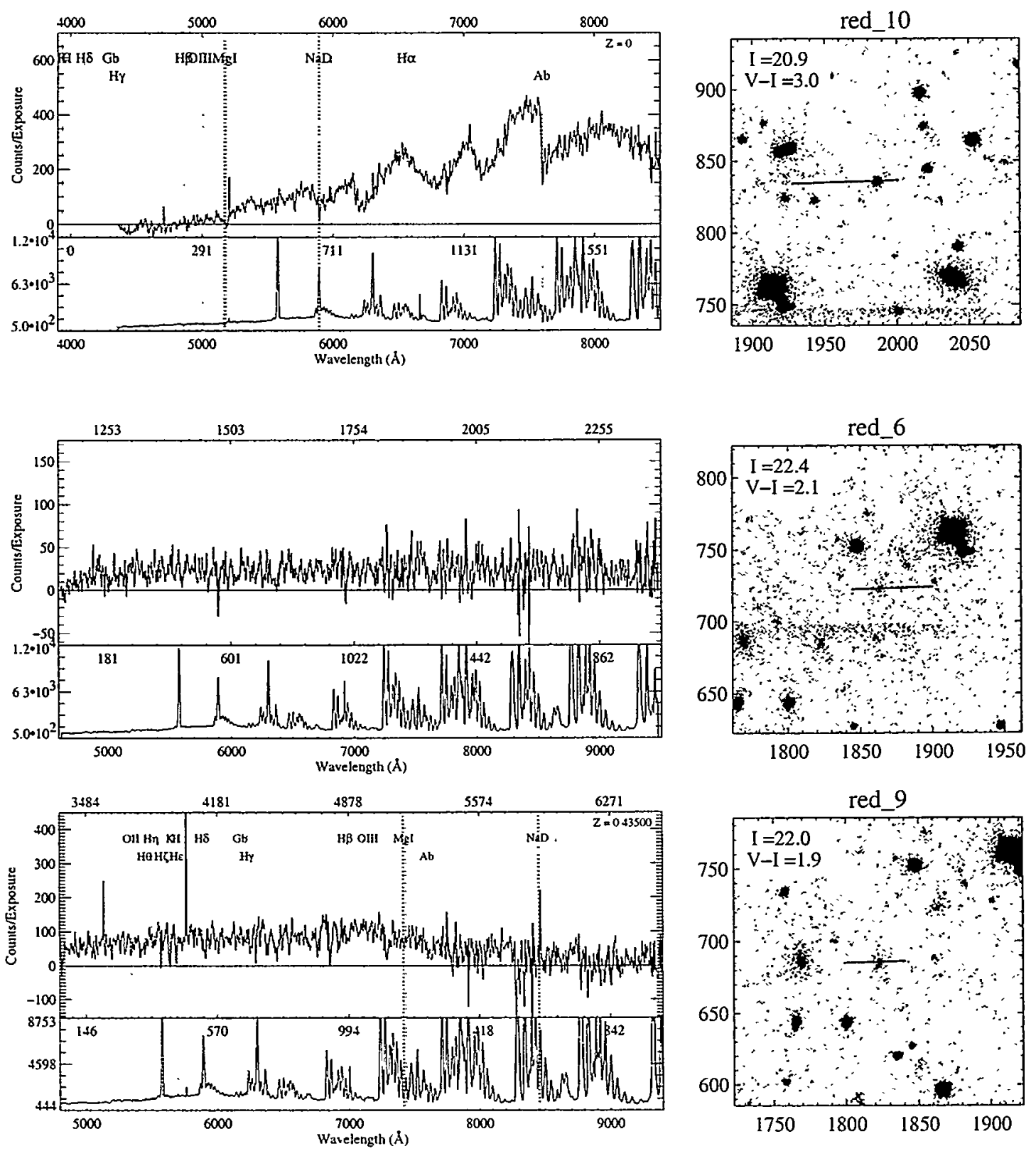

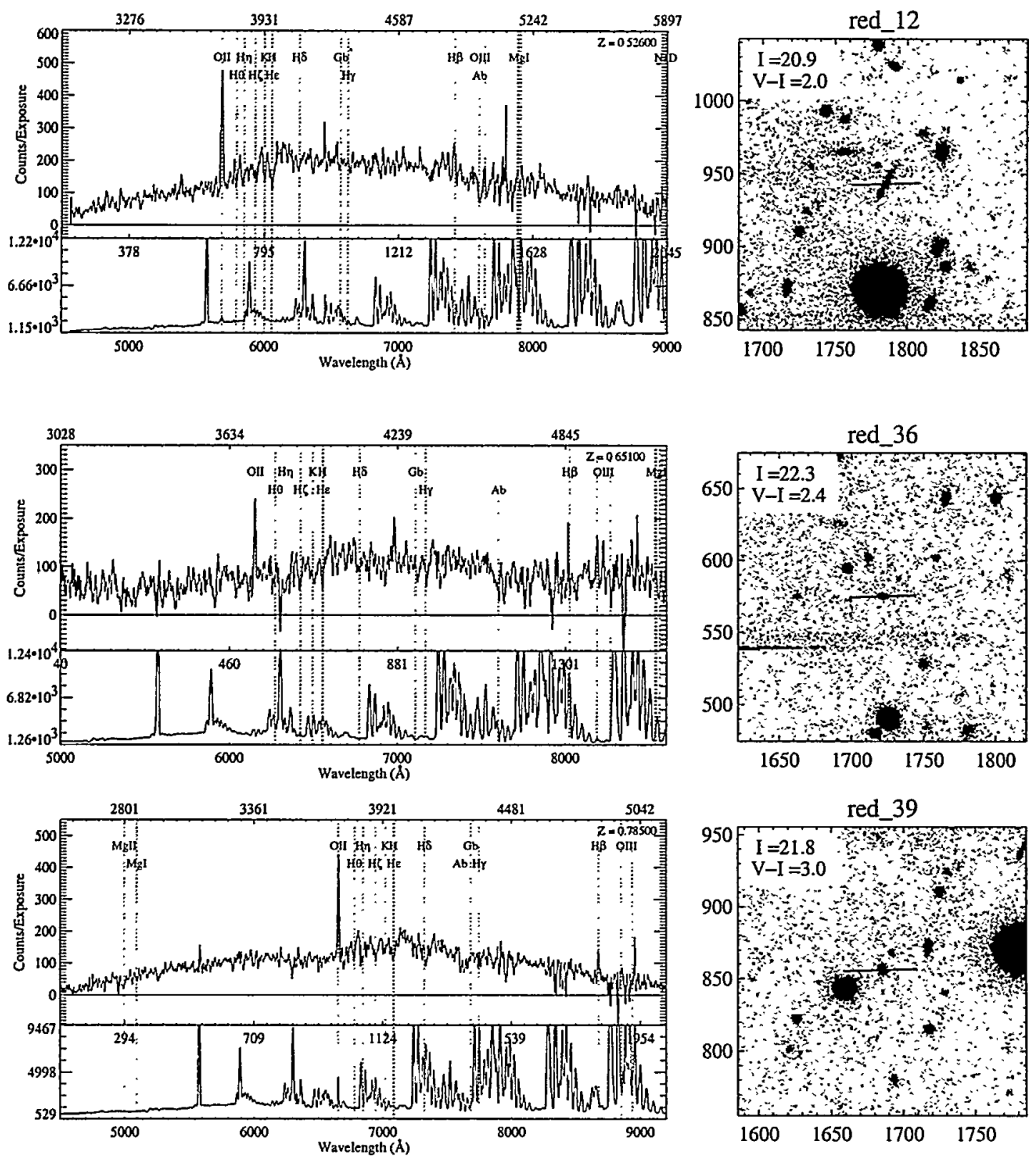

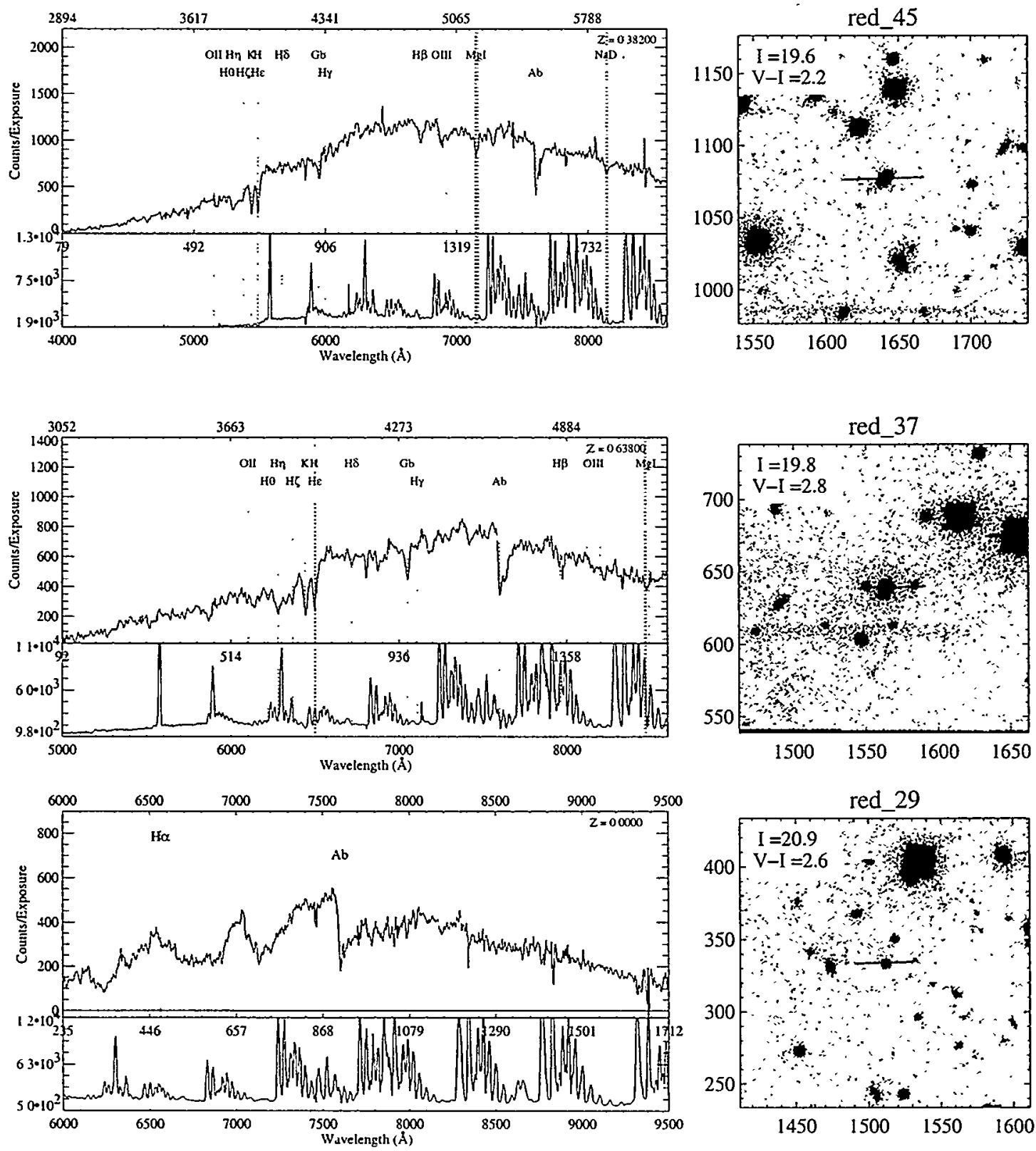

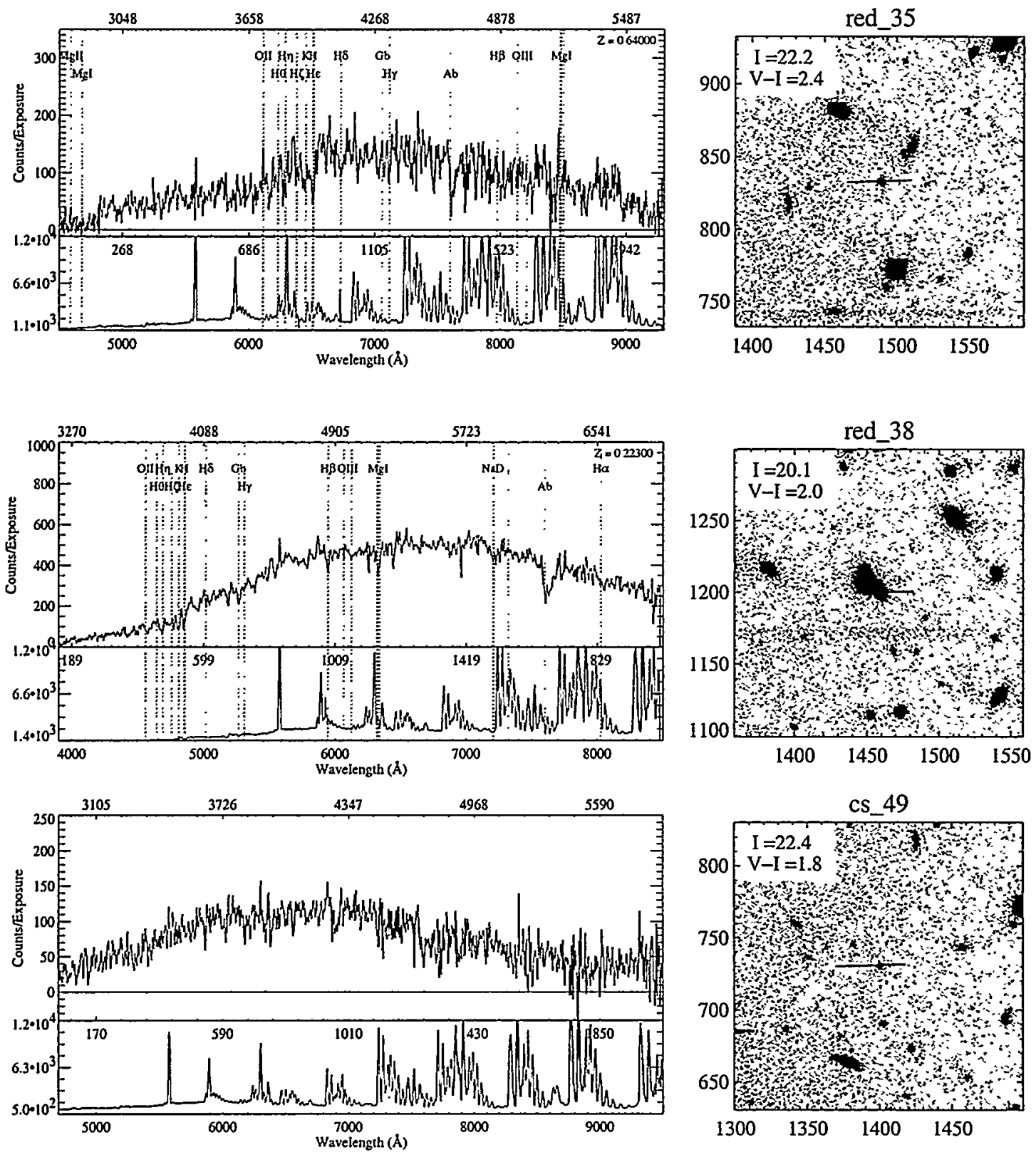

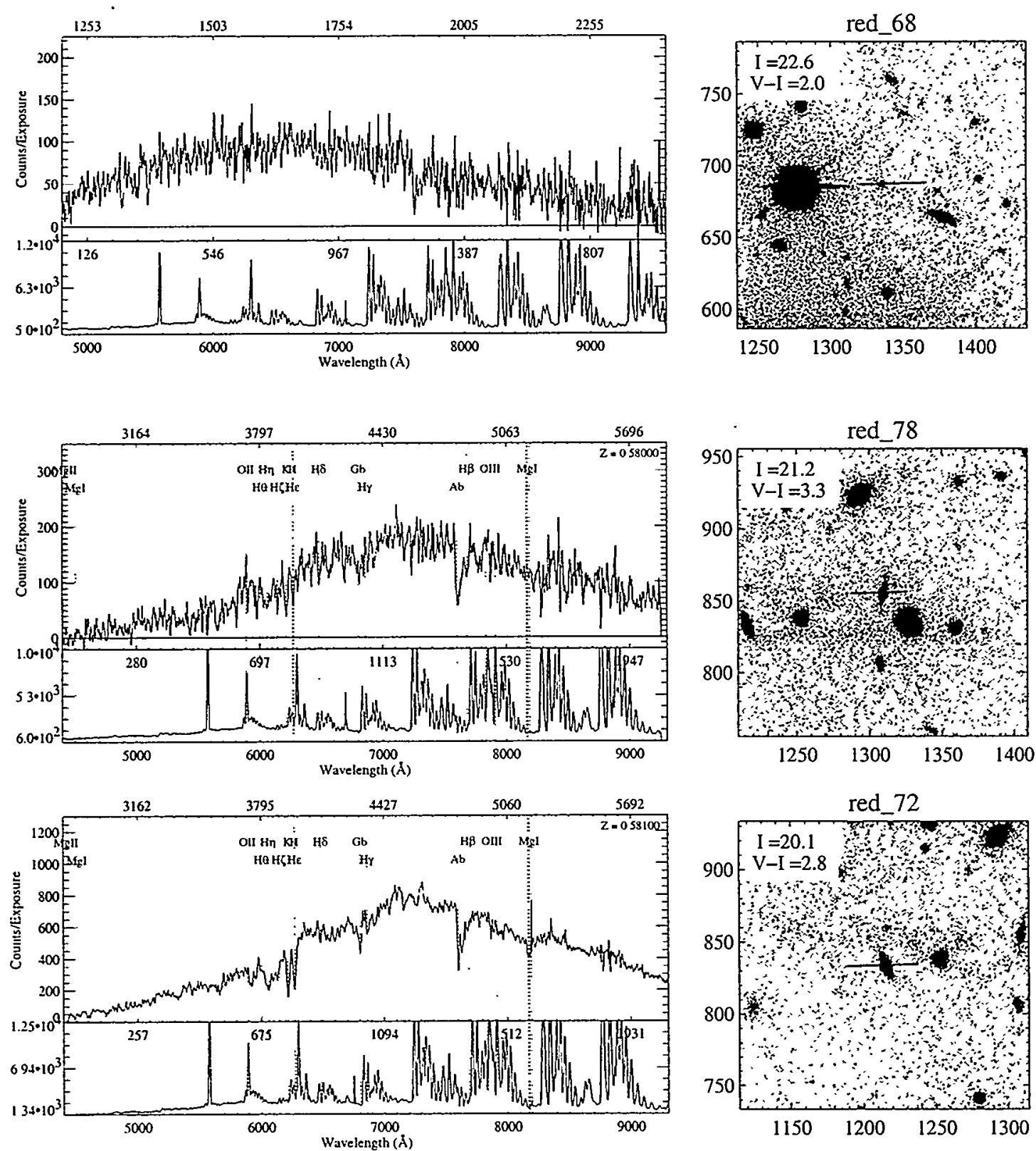

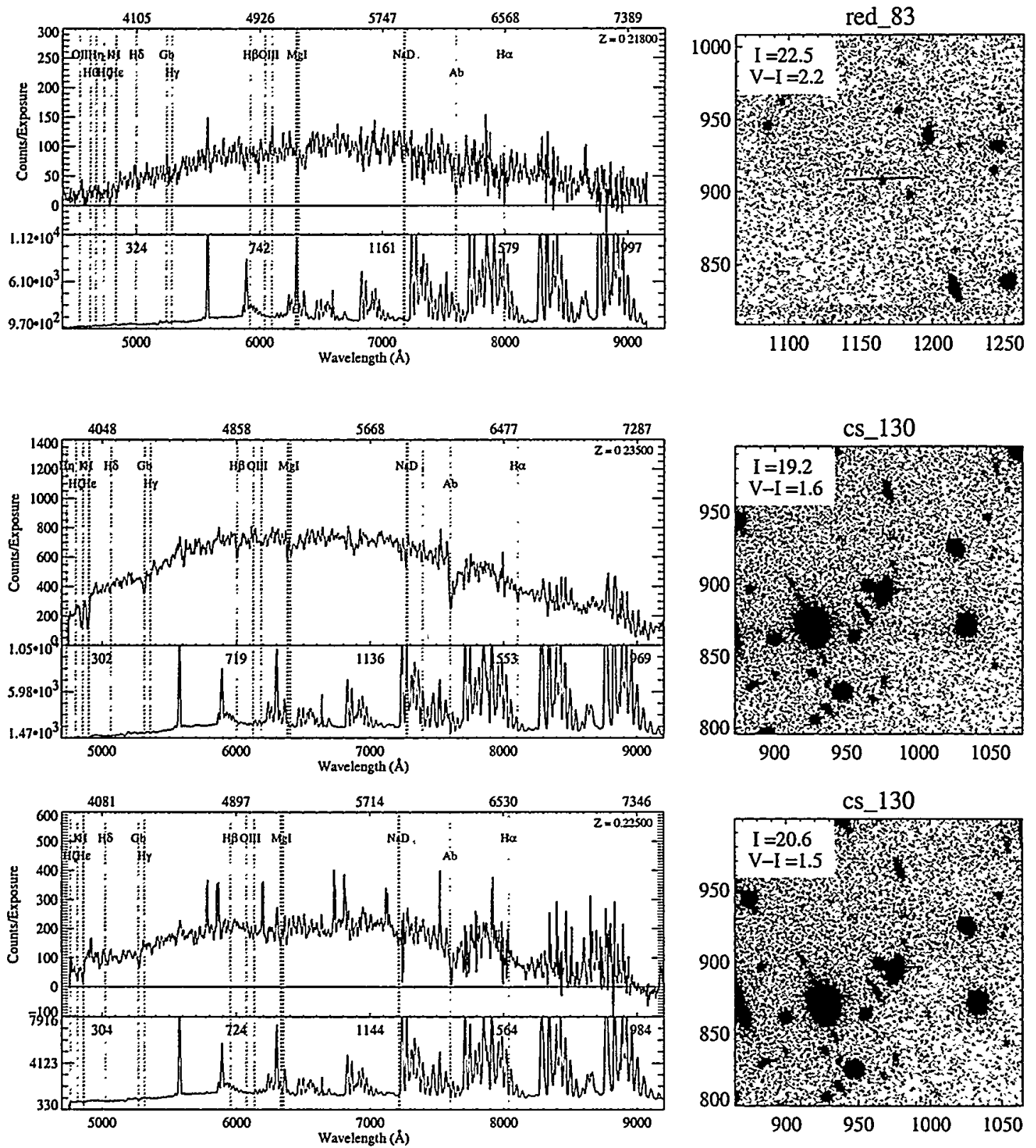

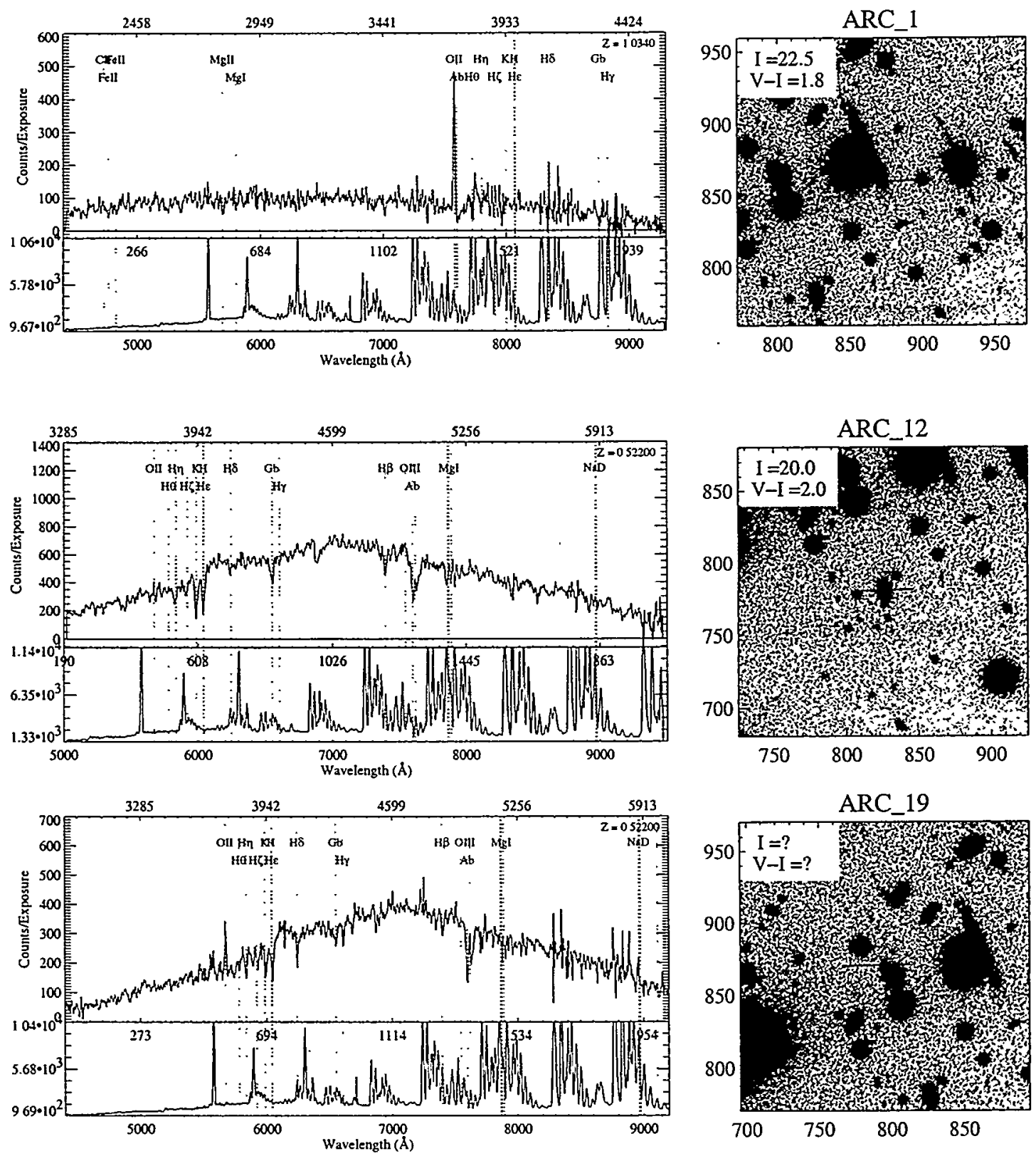

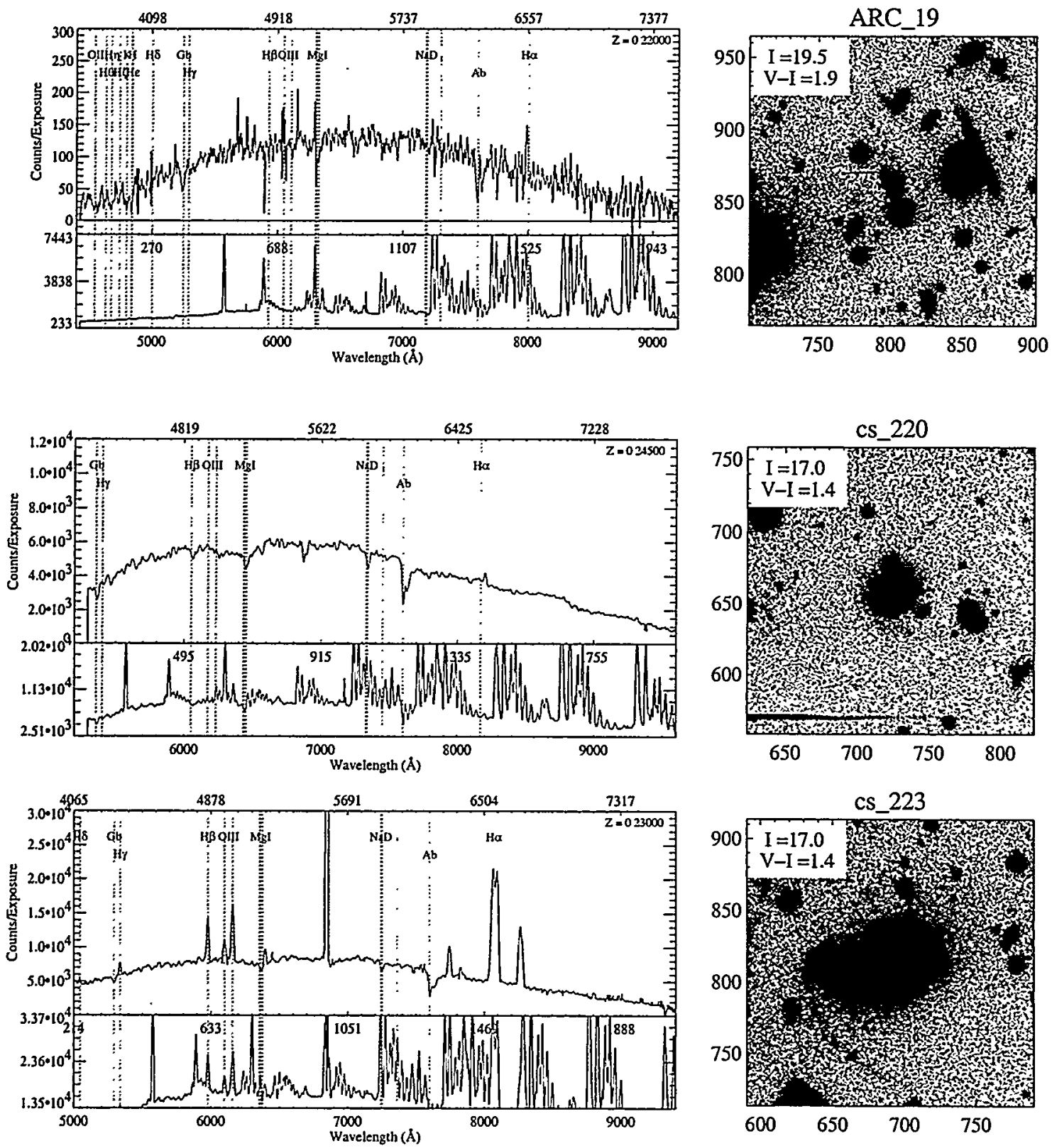

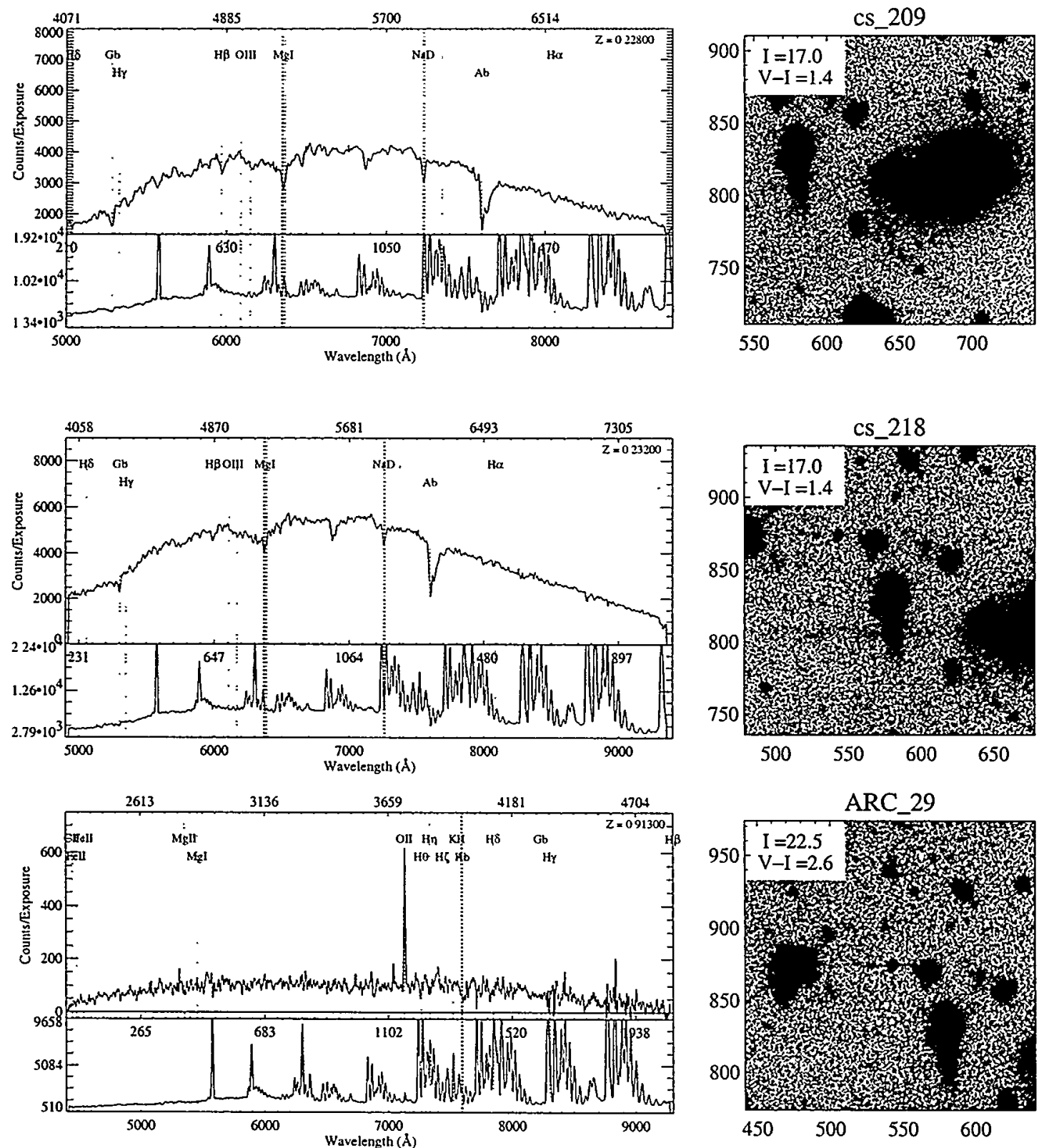

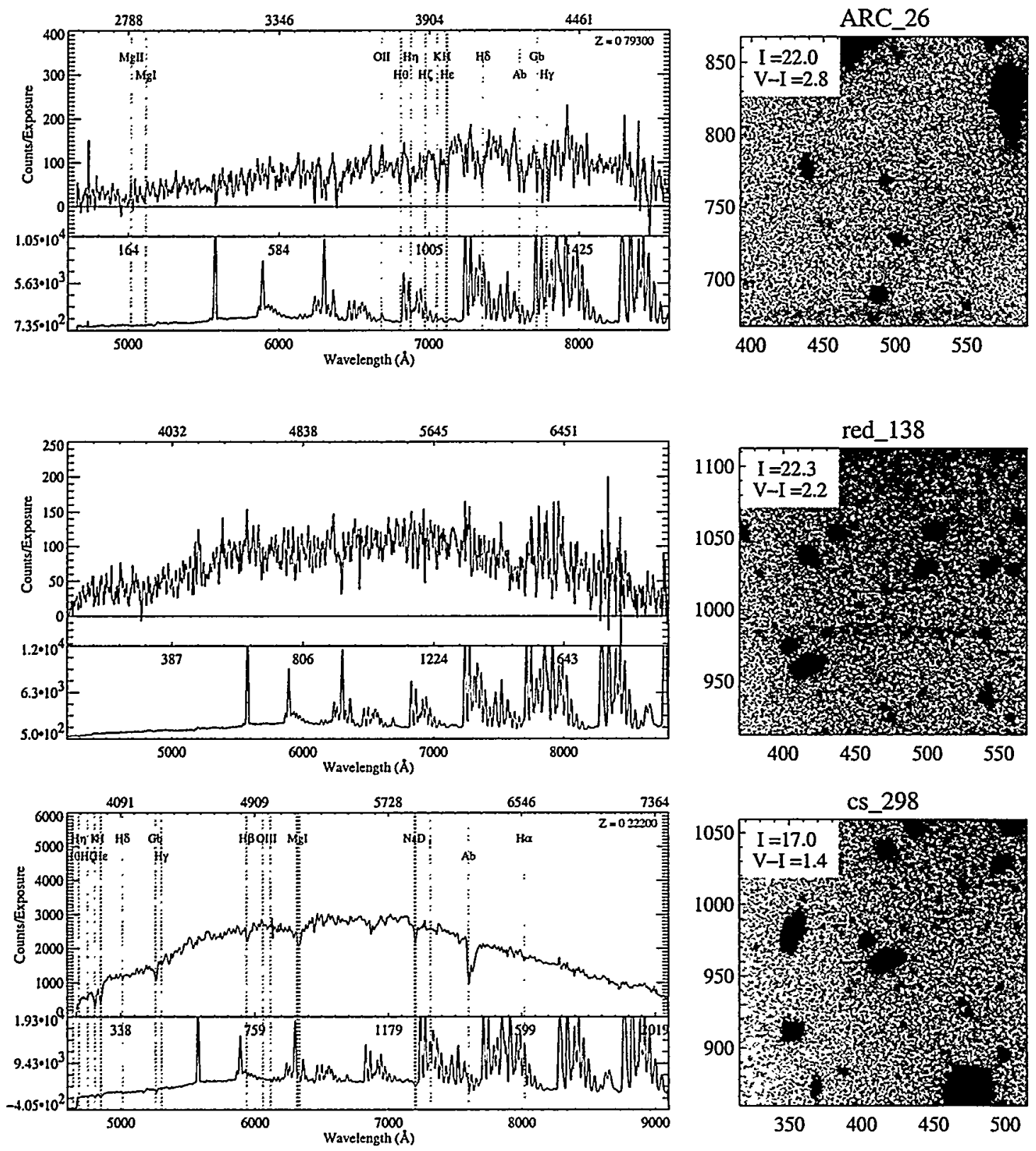

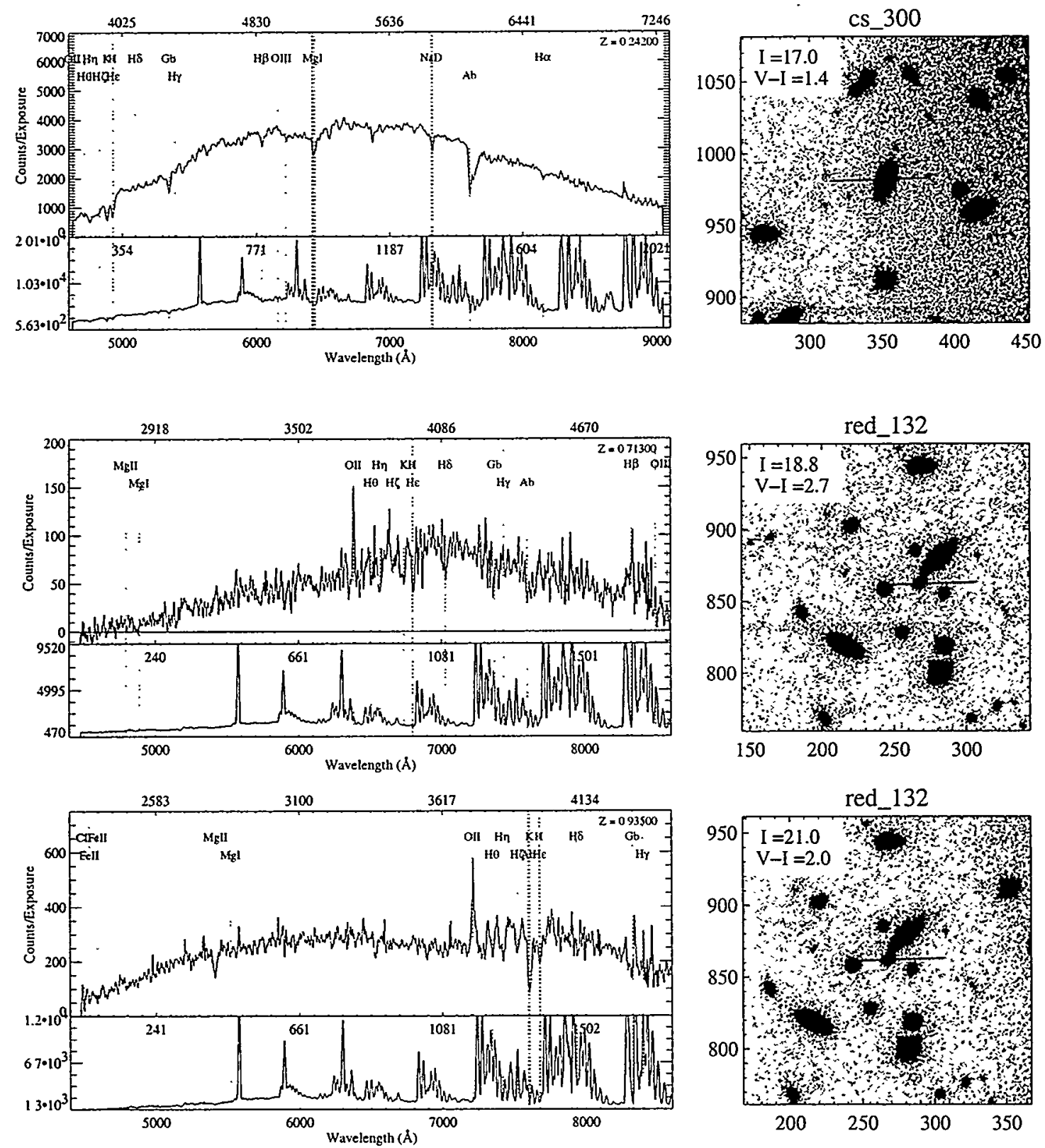

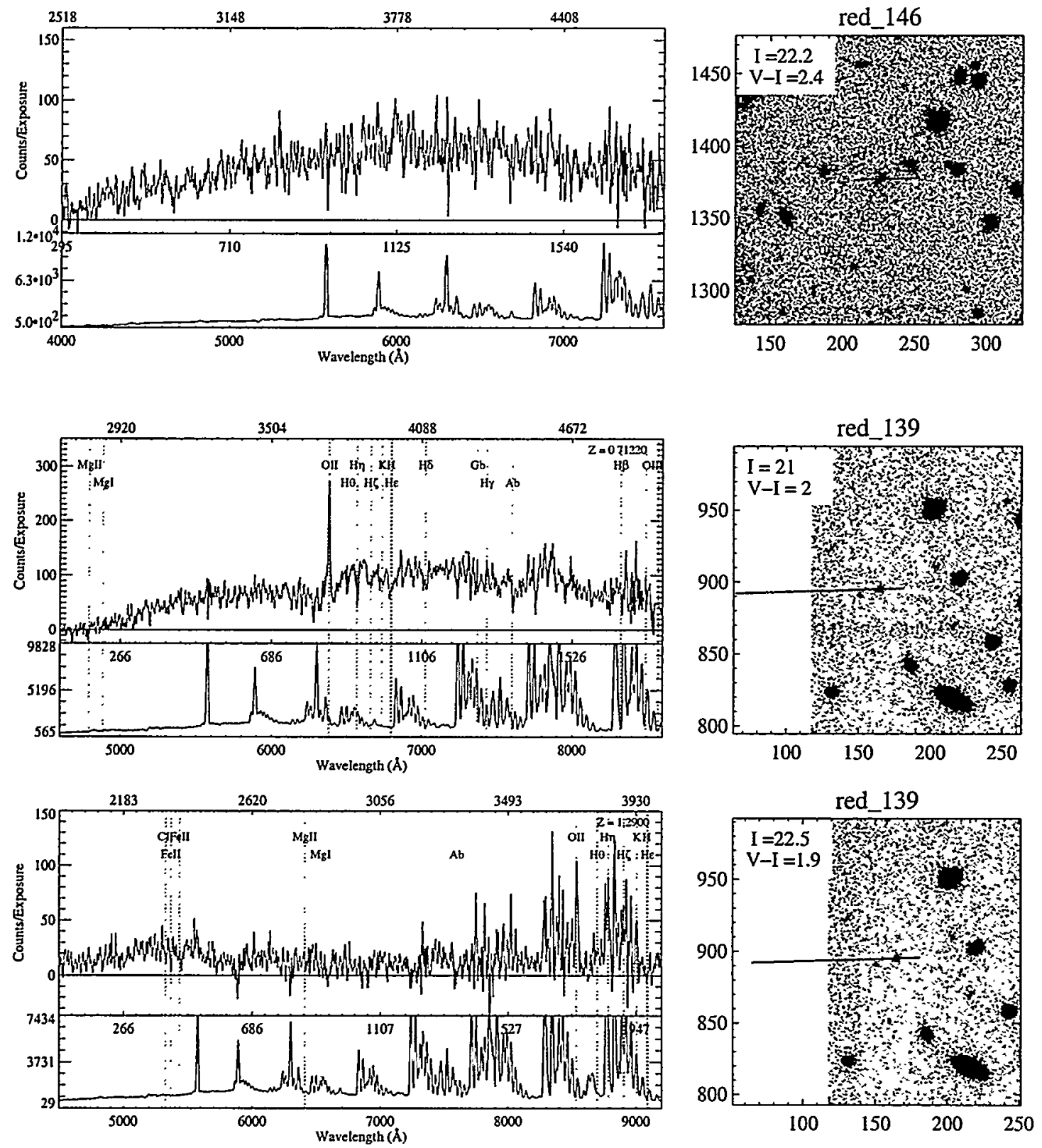
B.2 Mask 2 

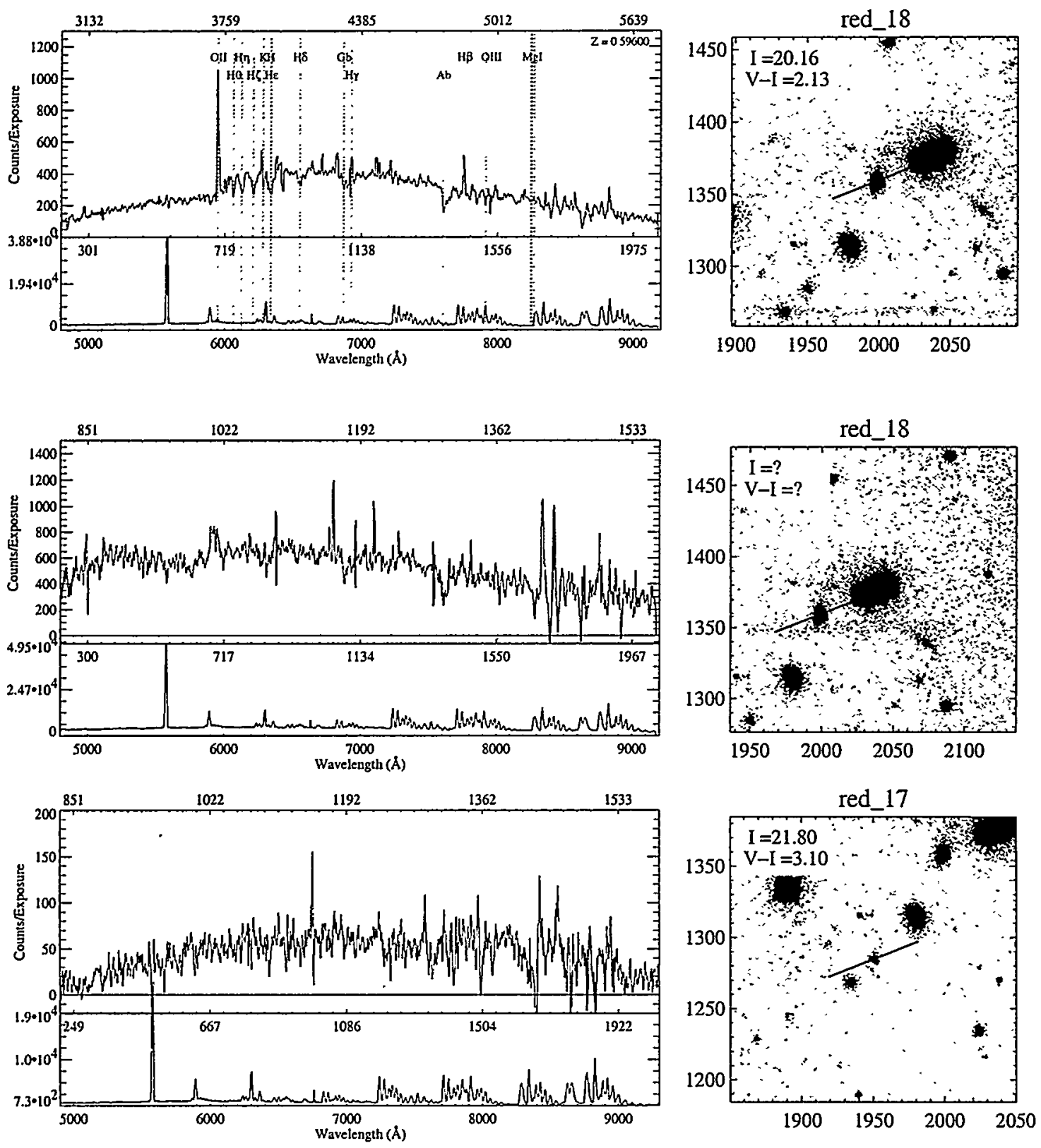

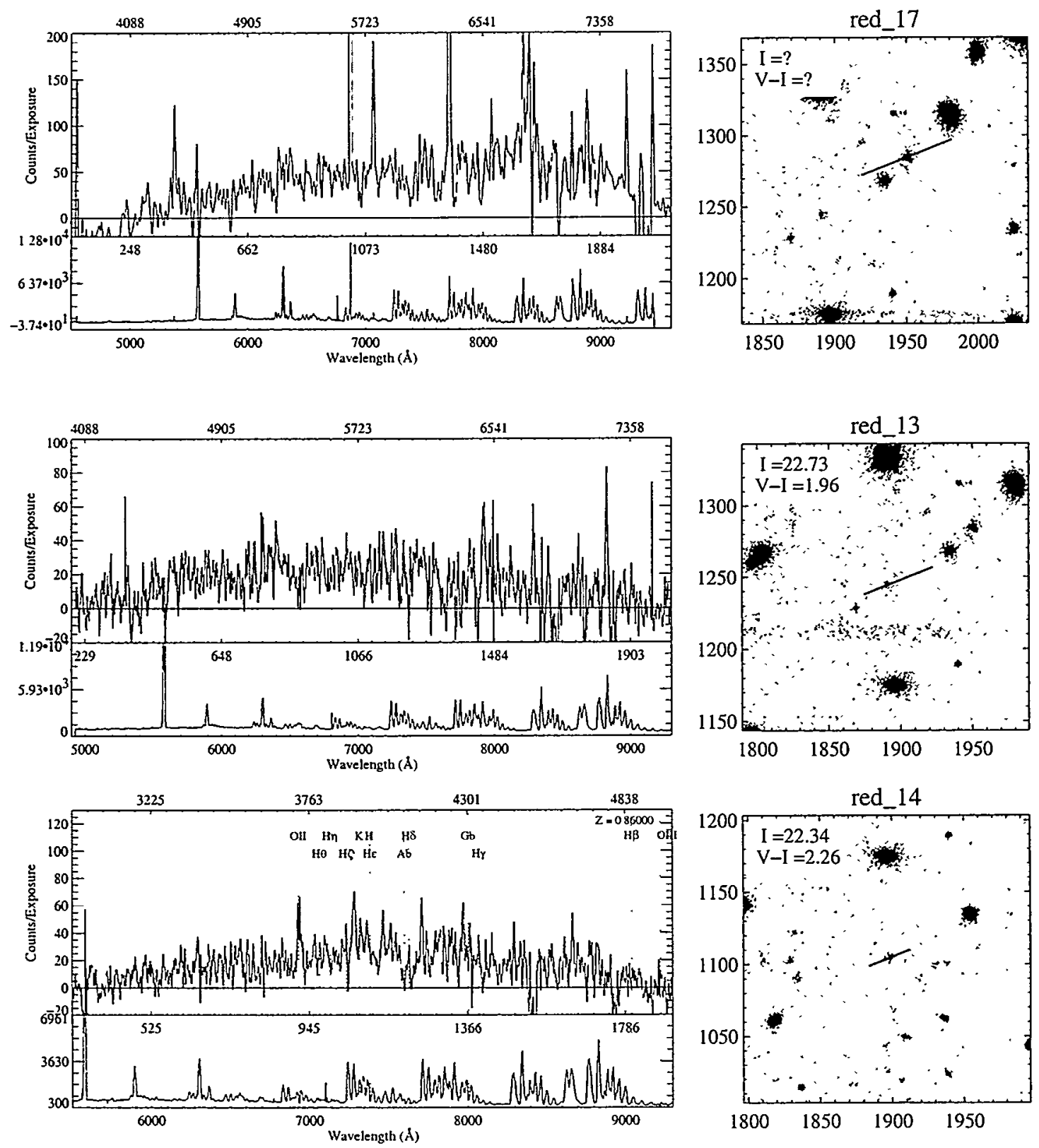

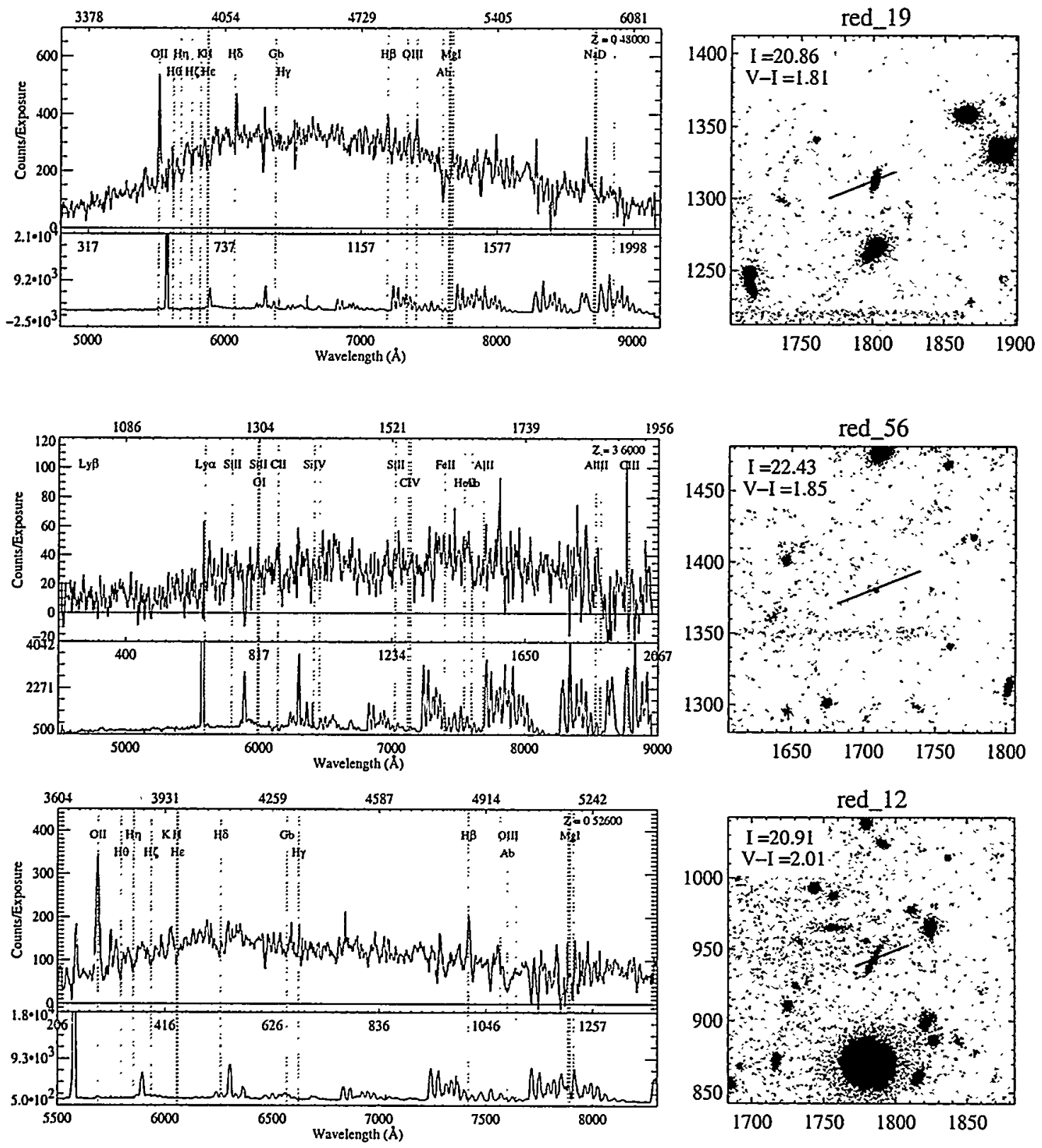

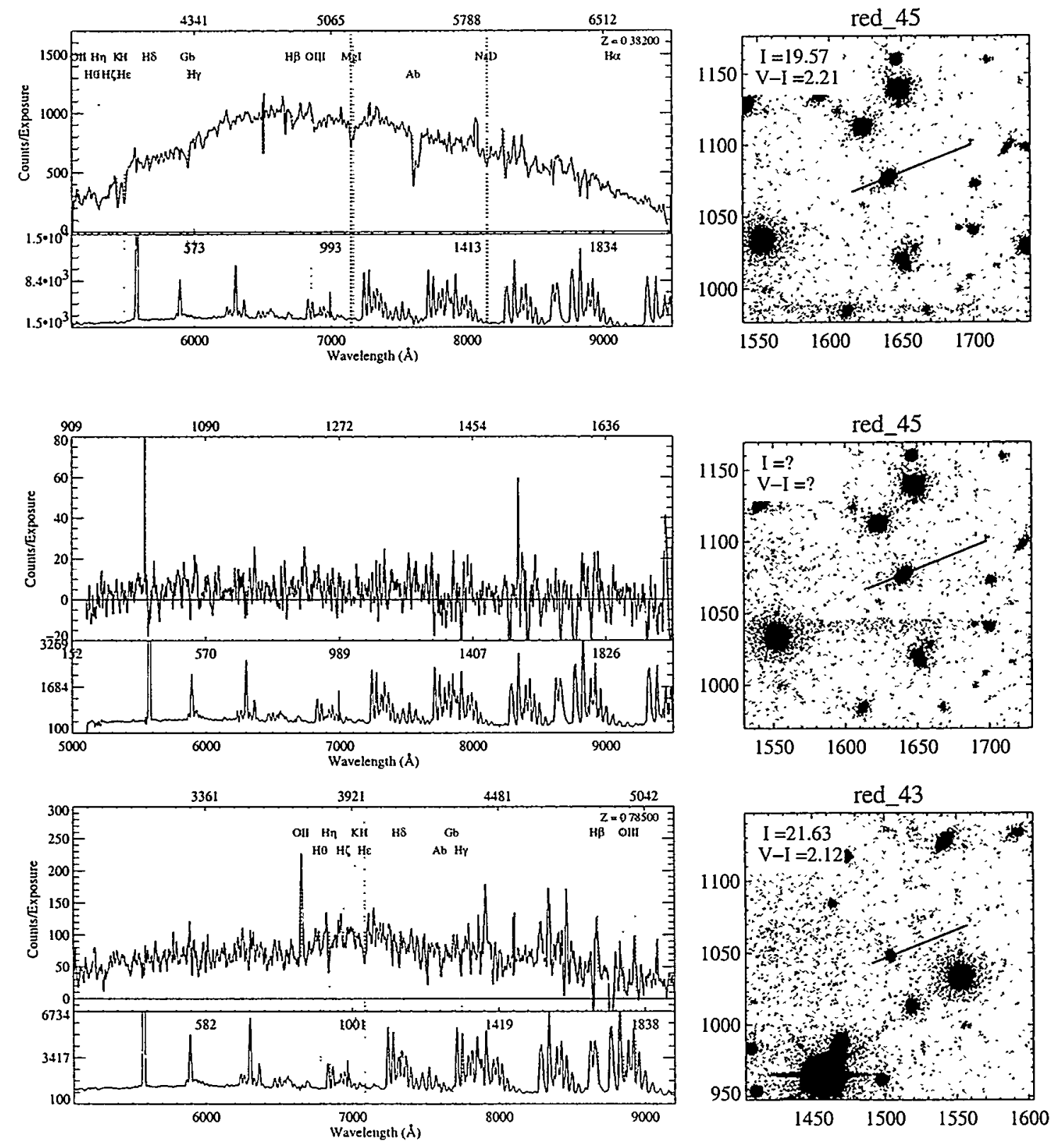

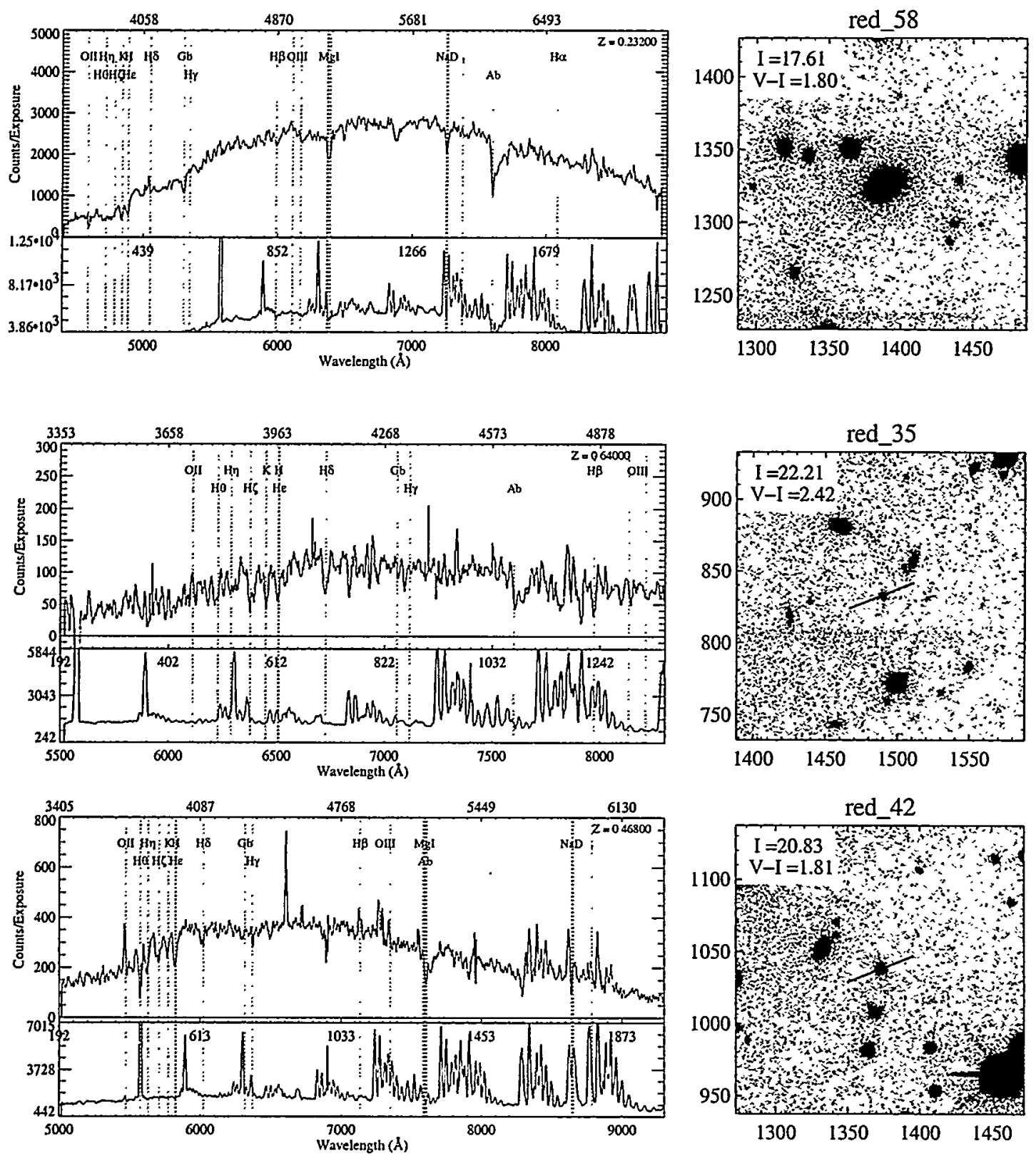

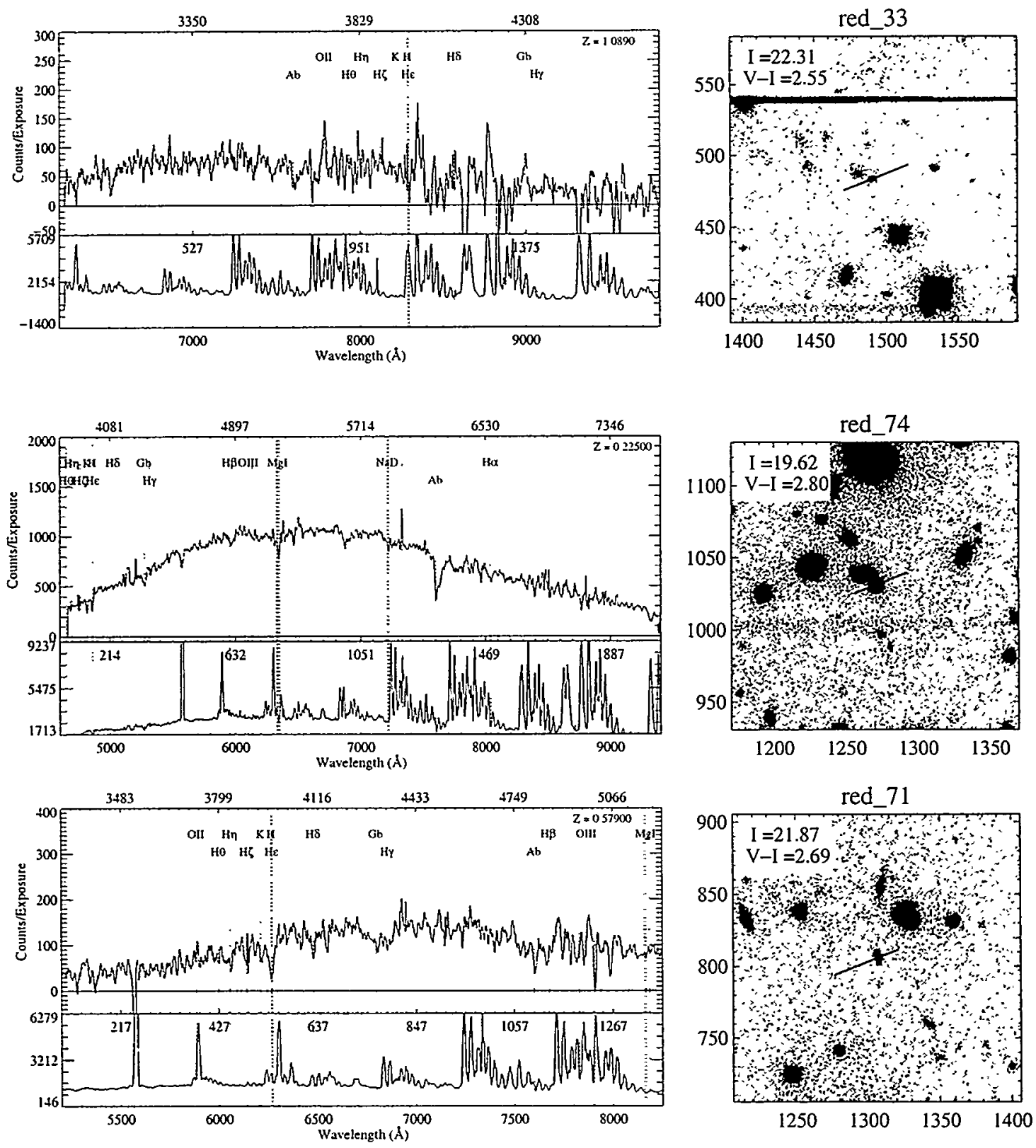

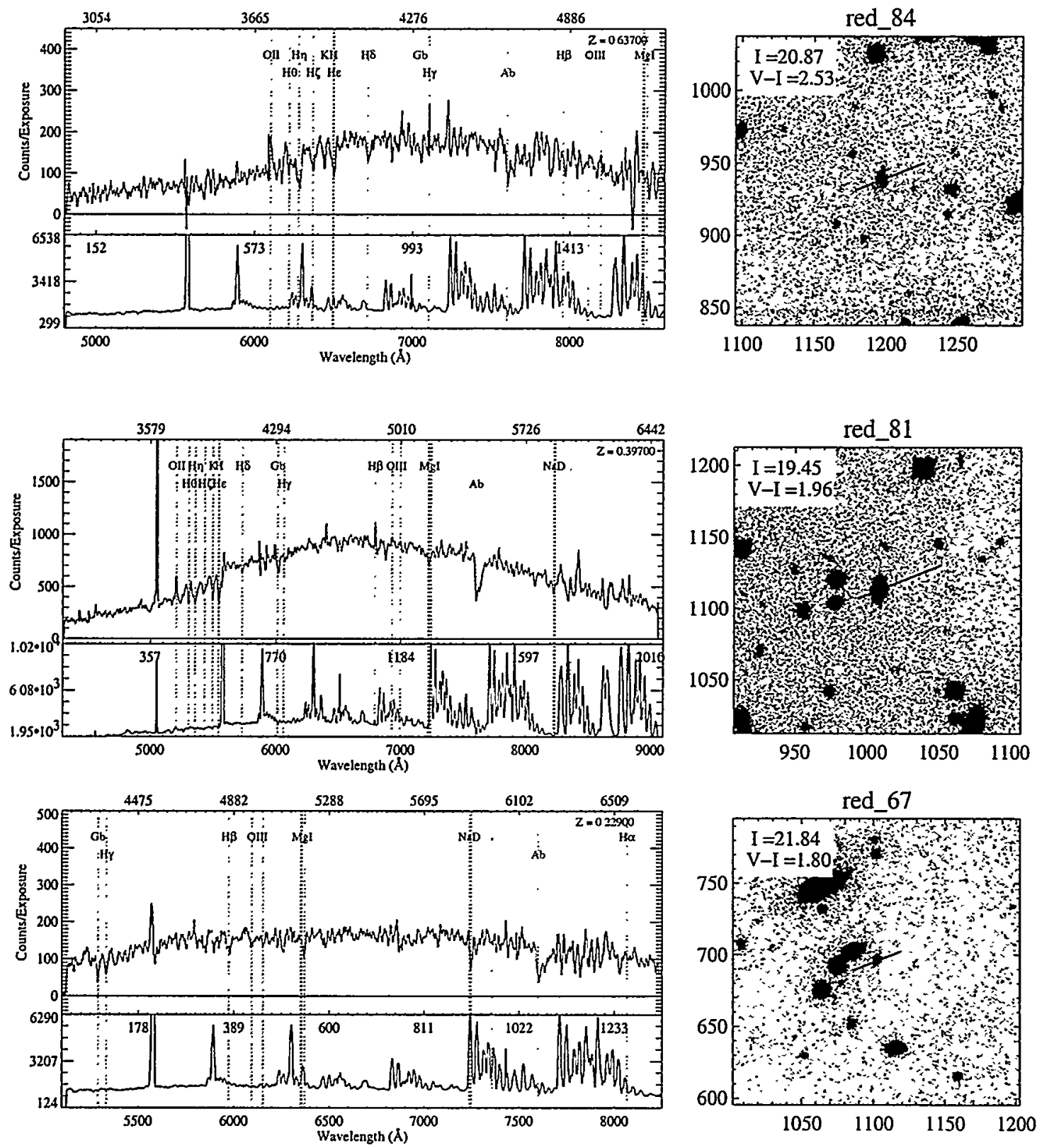

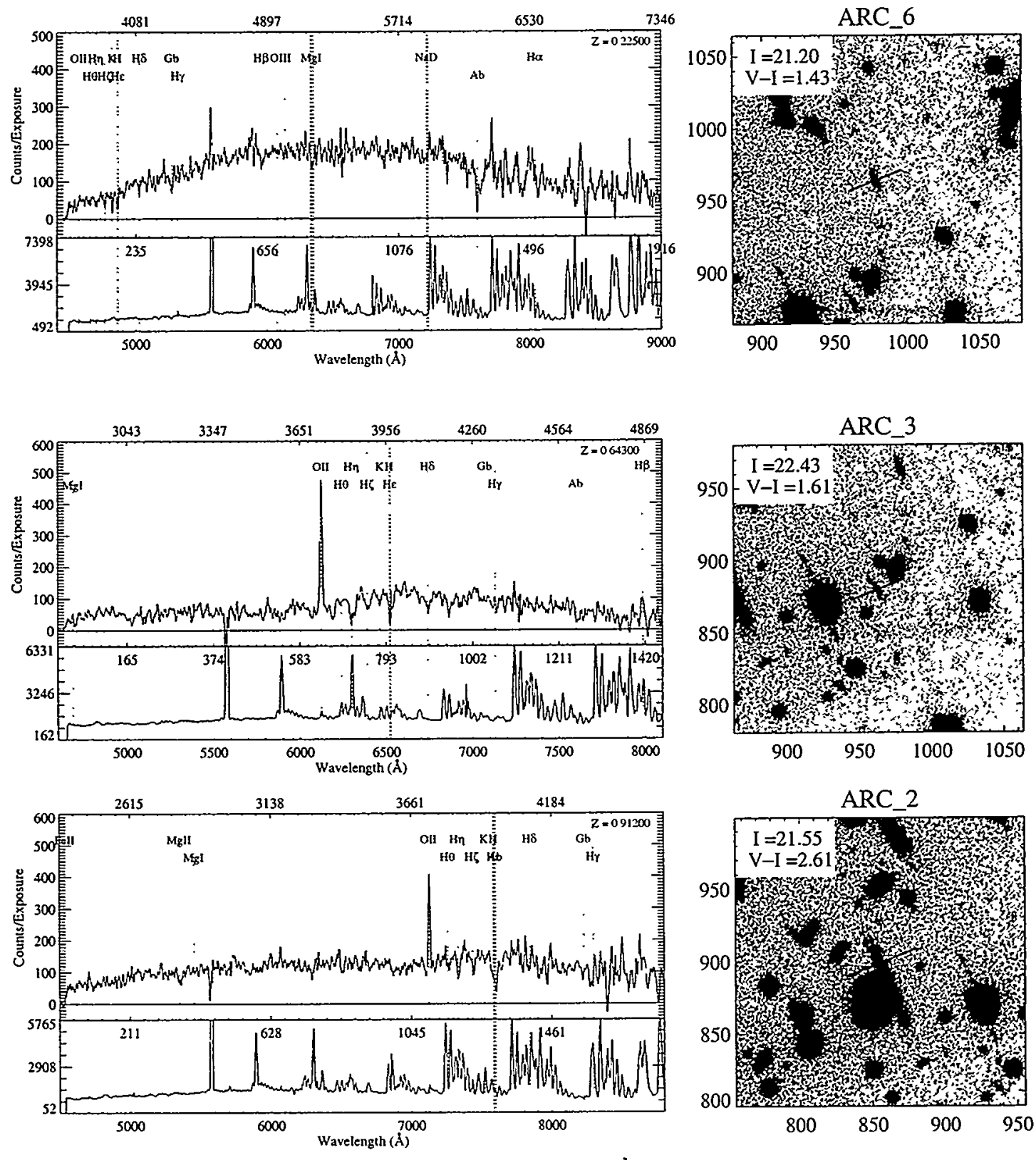

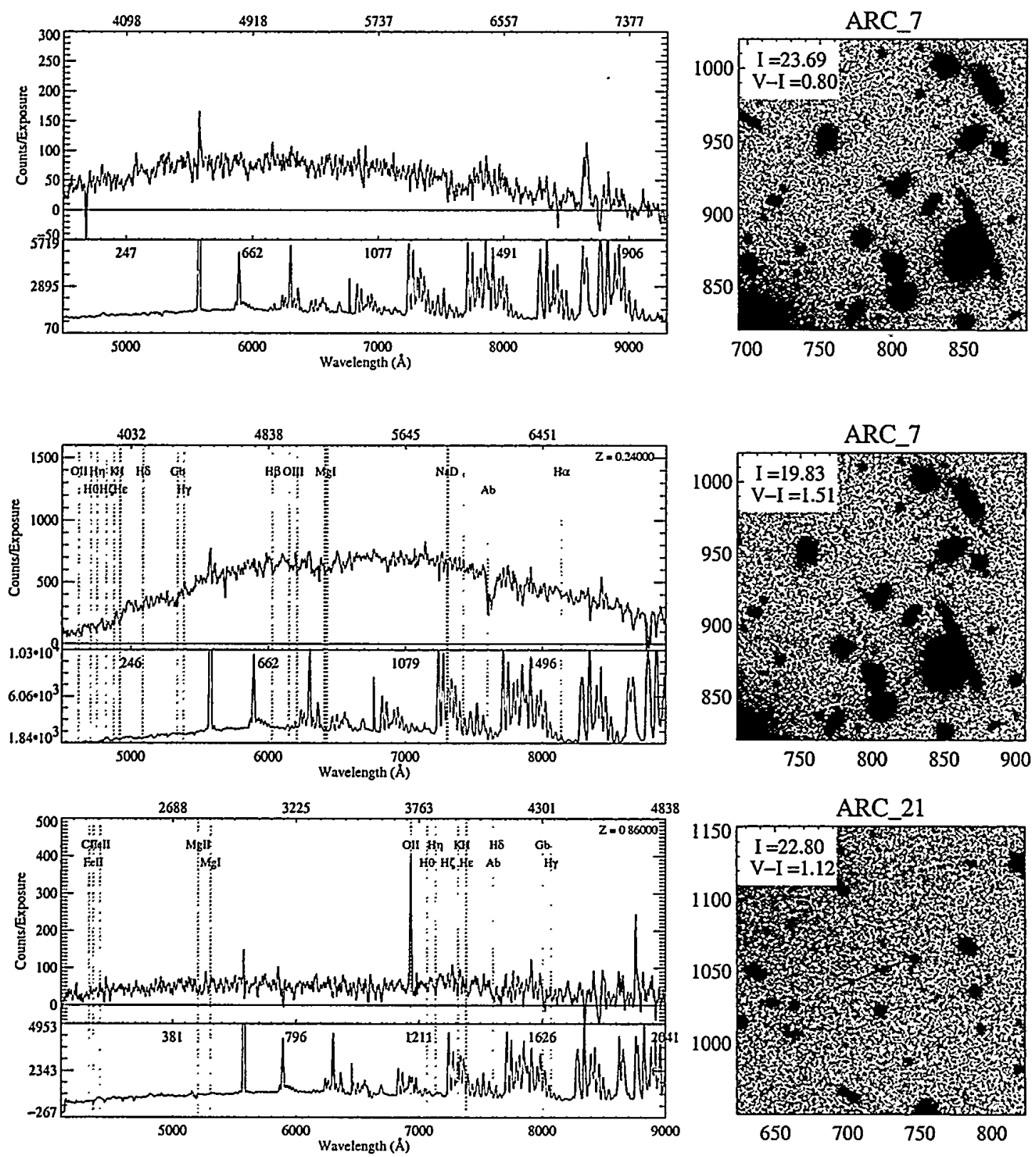

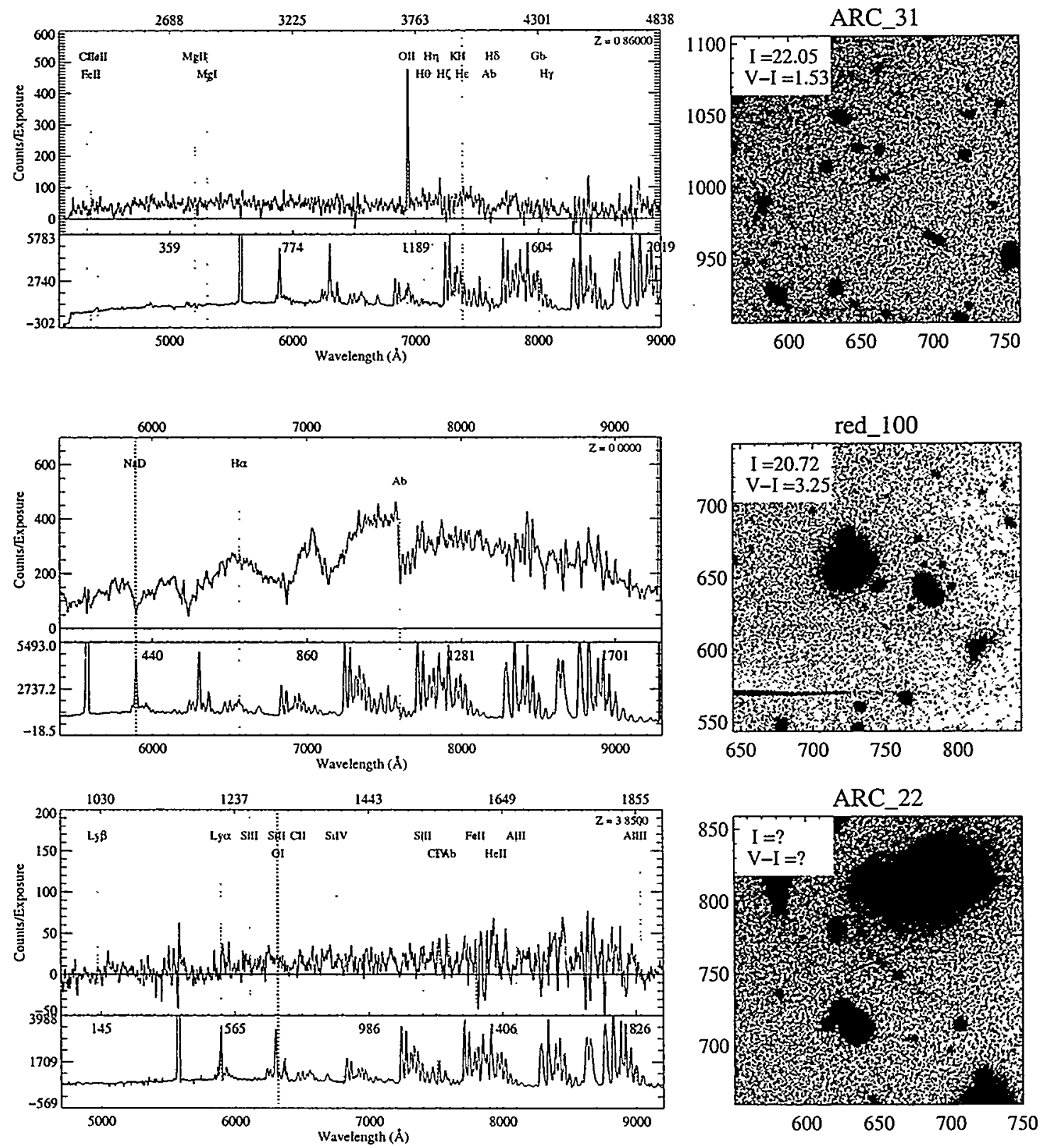

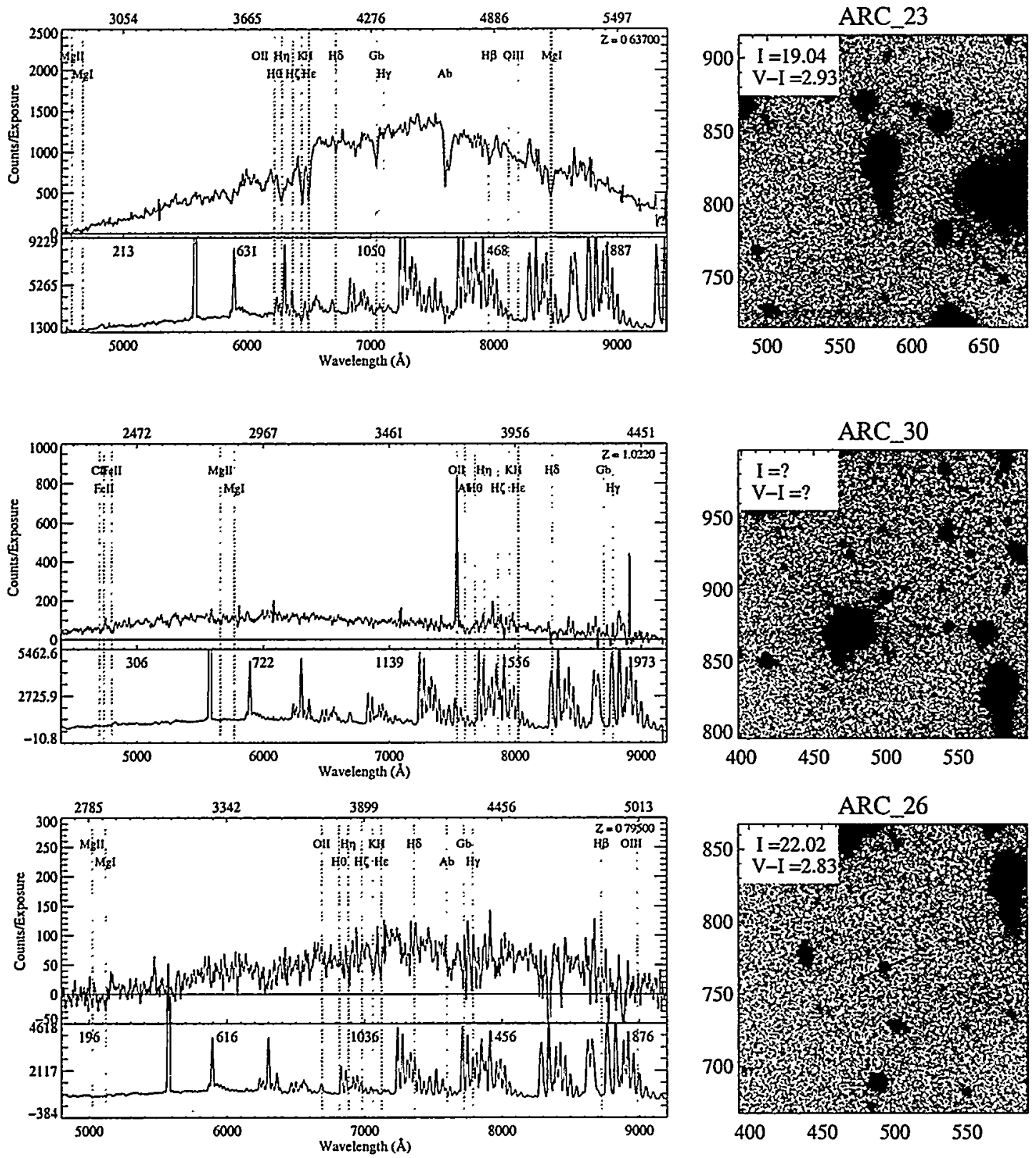

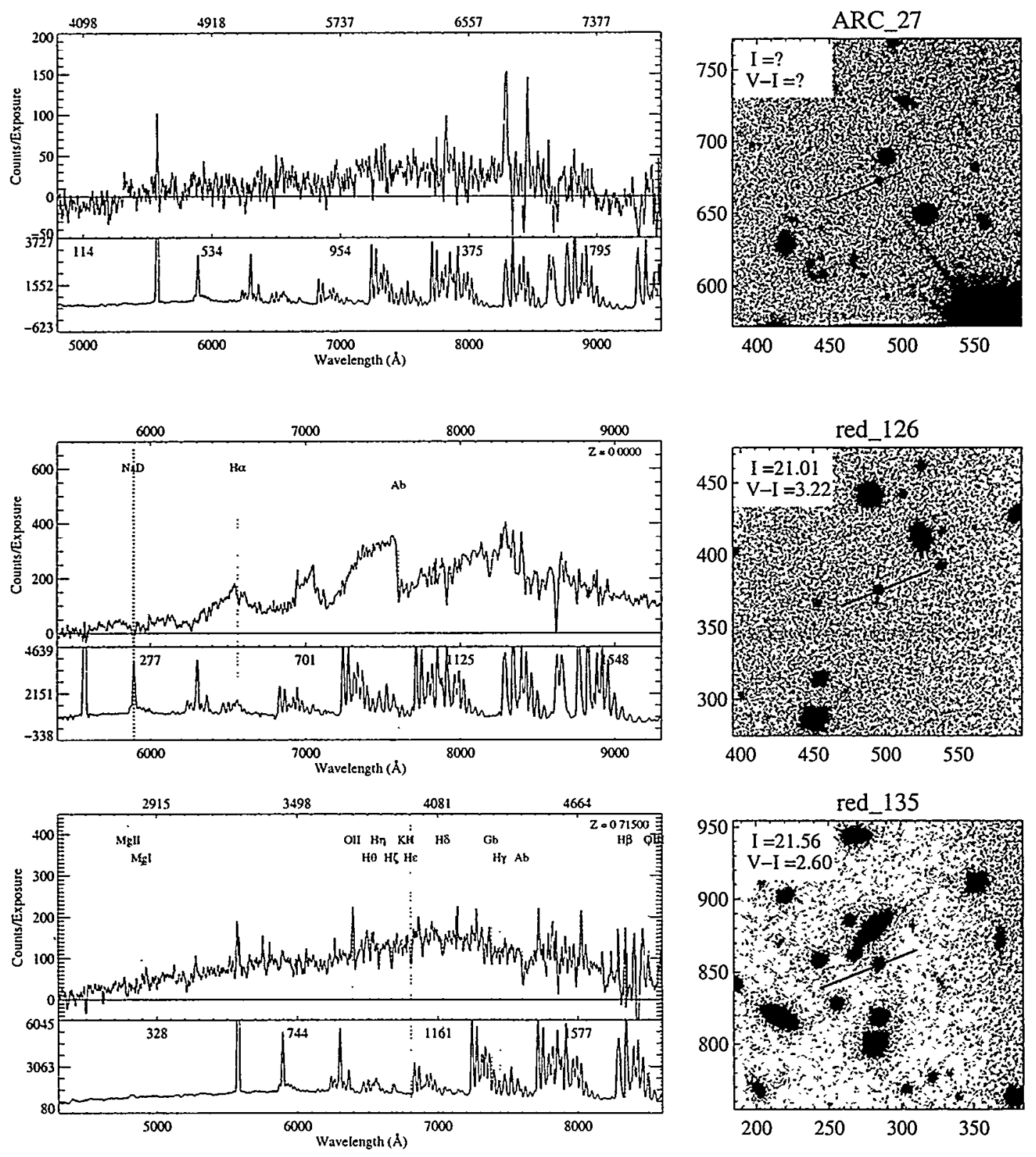

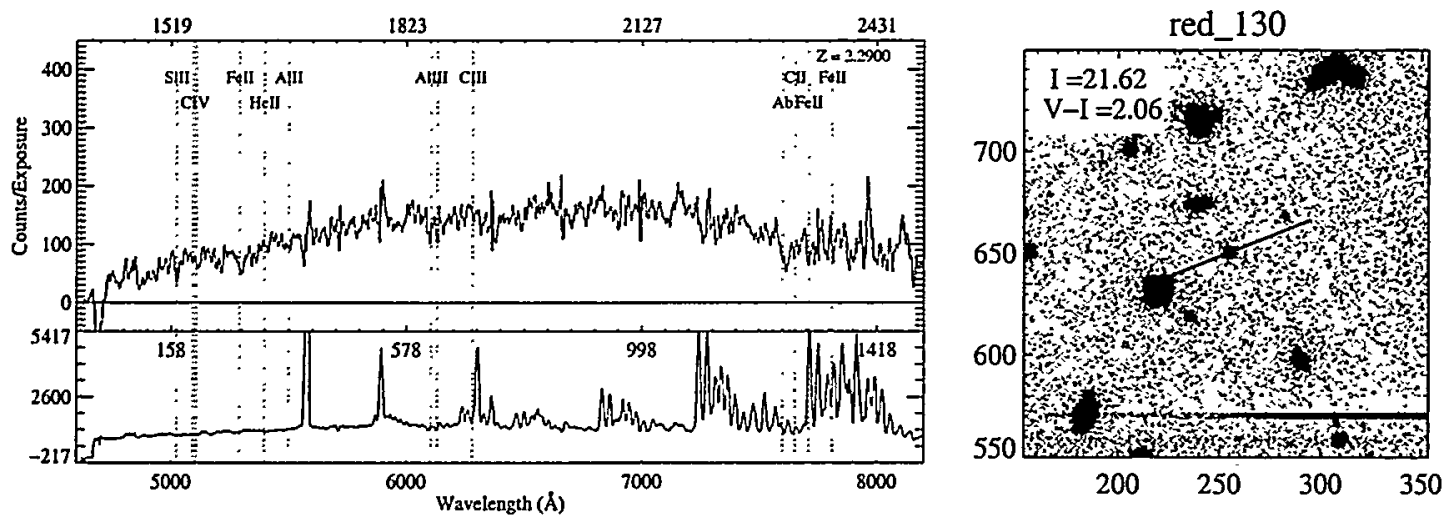


\section{B.3 Mask 3}



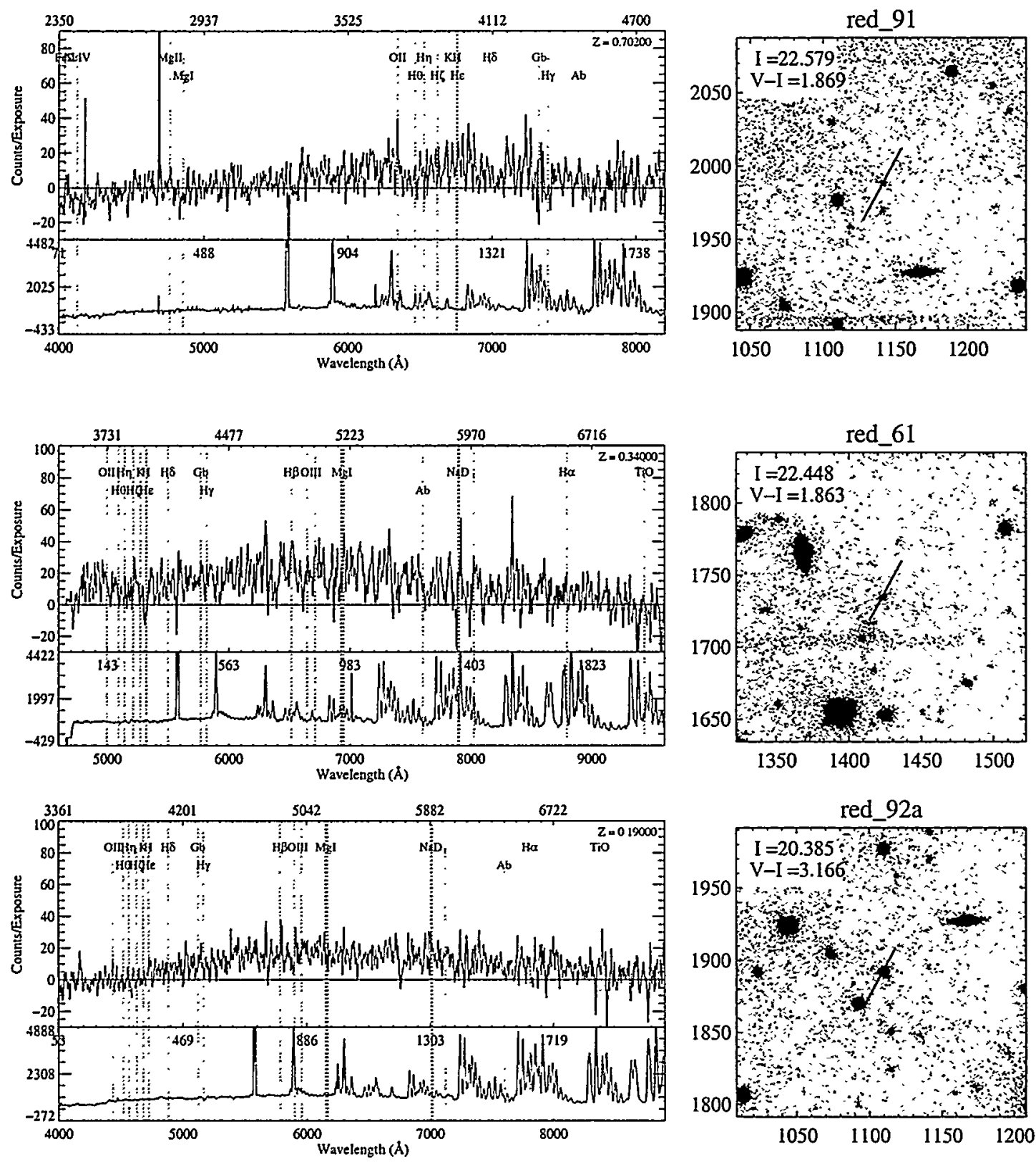

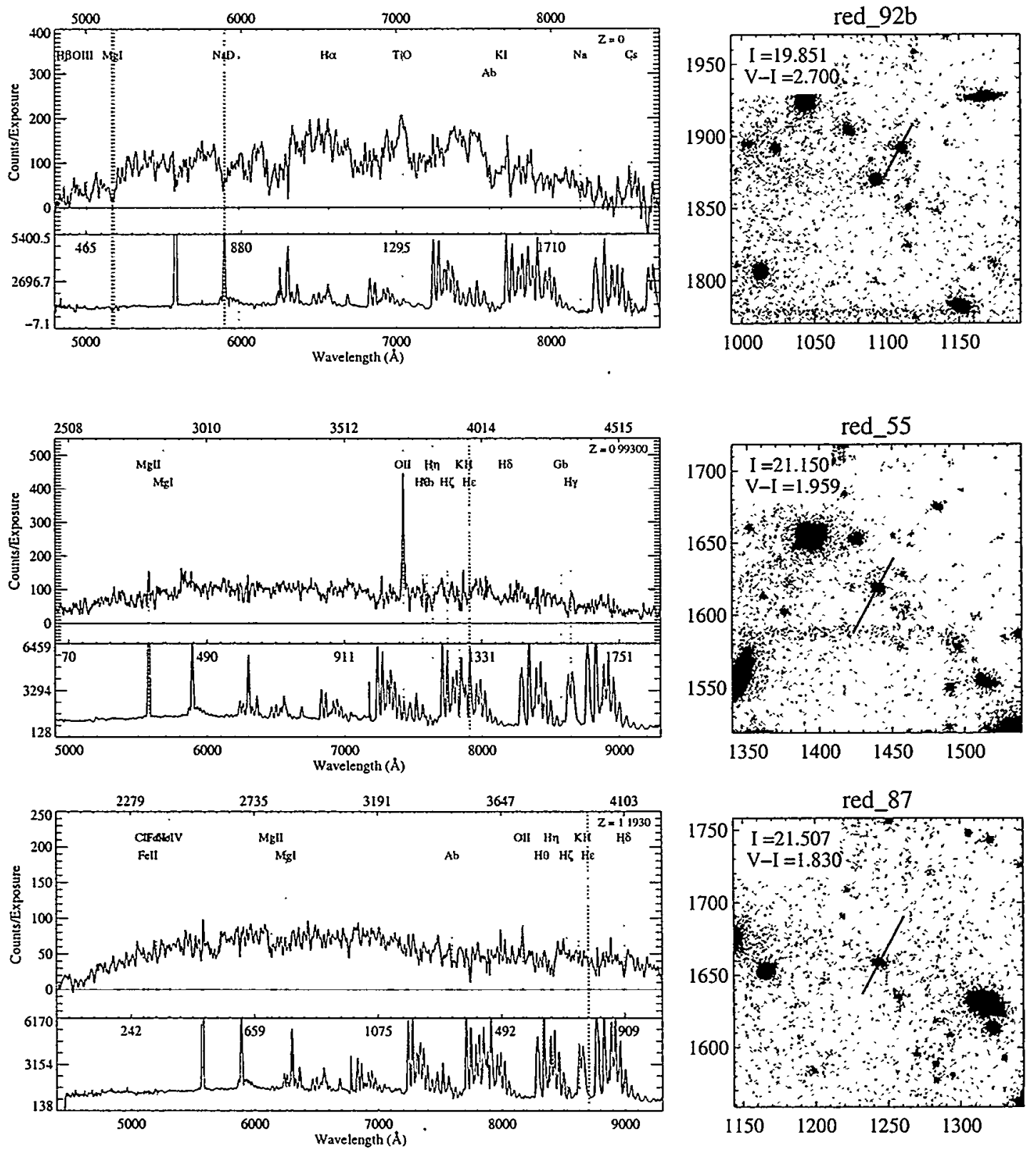

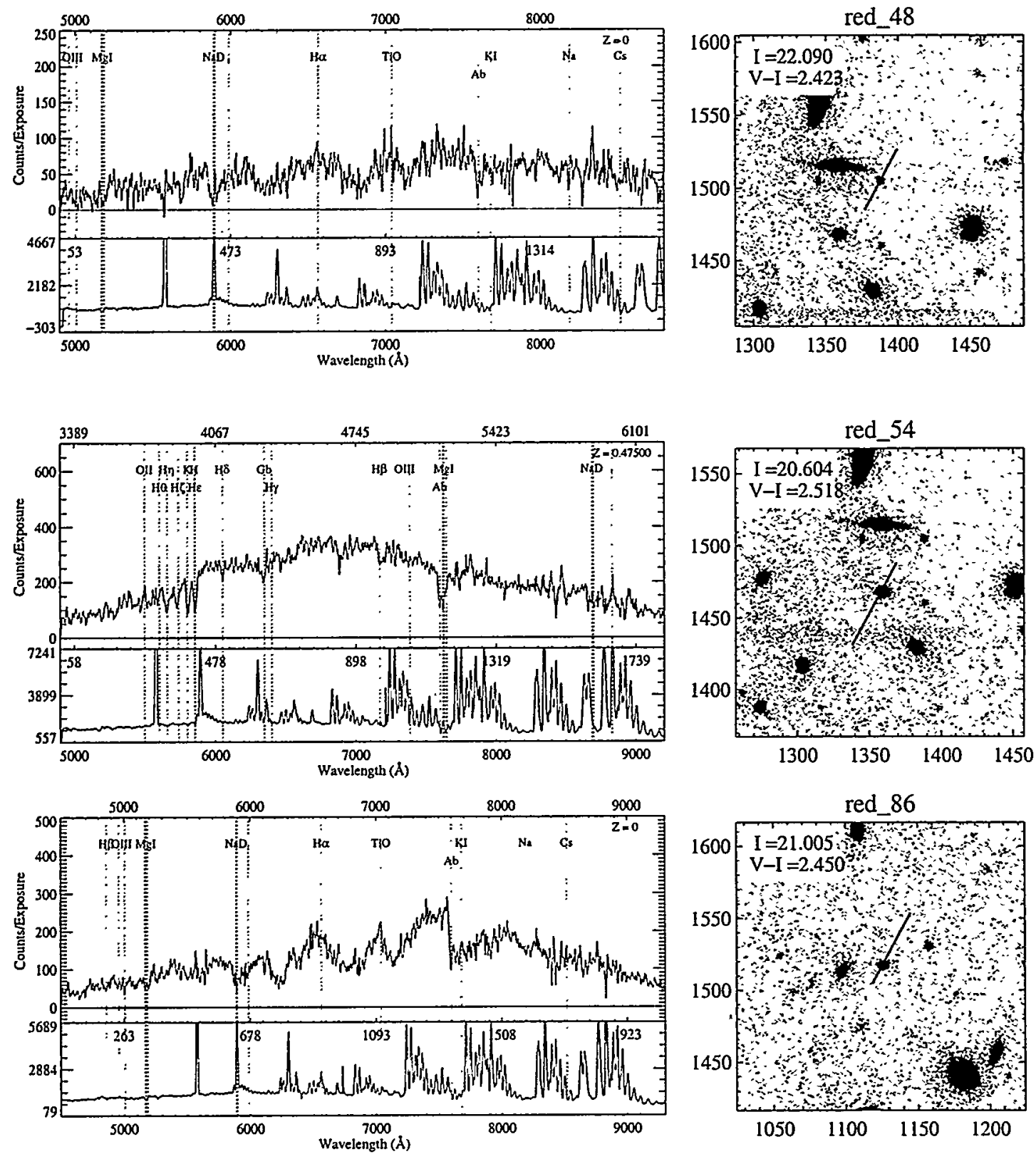

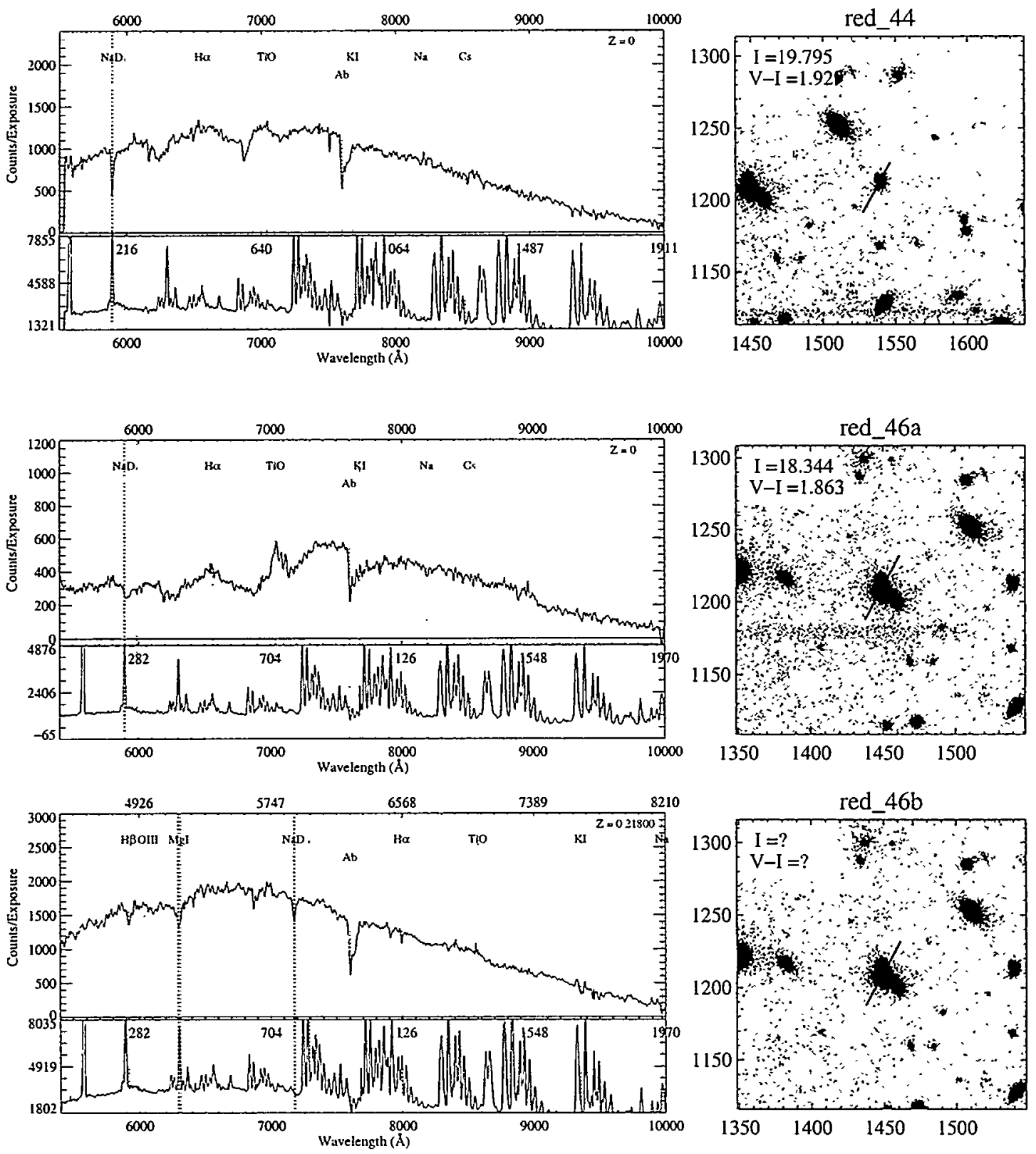

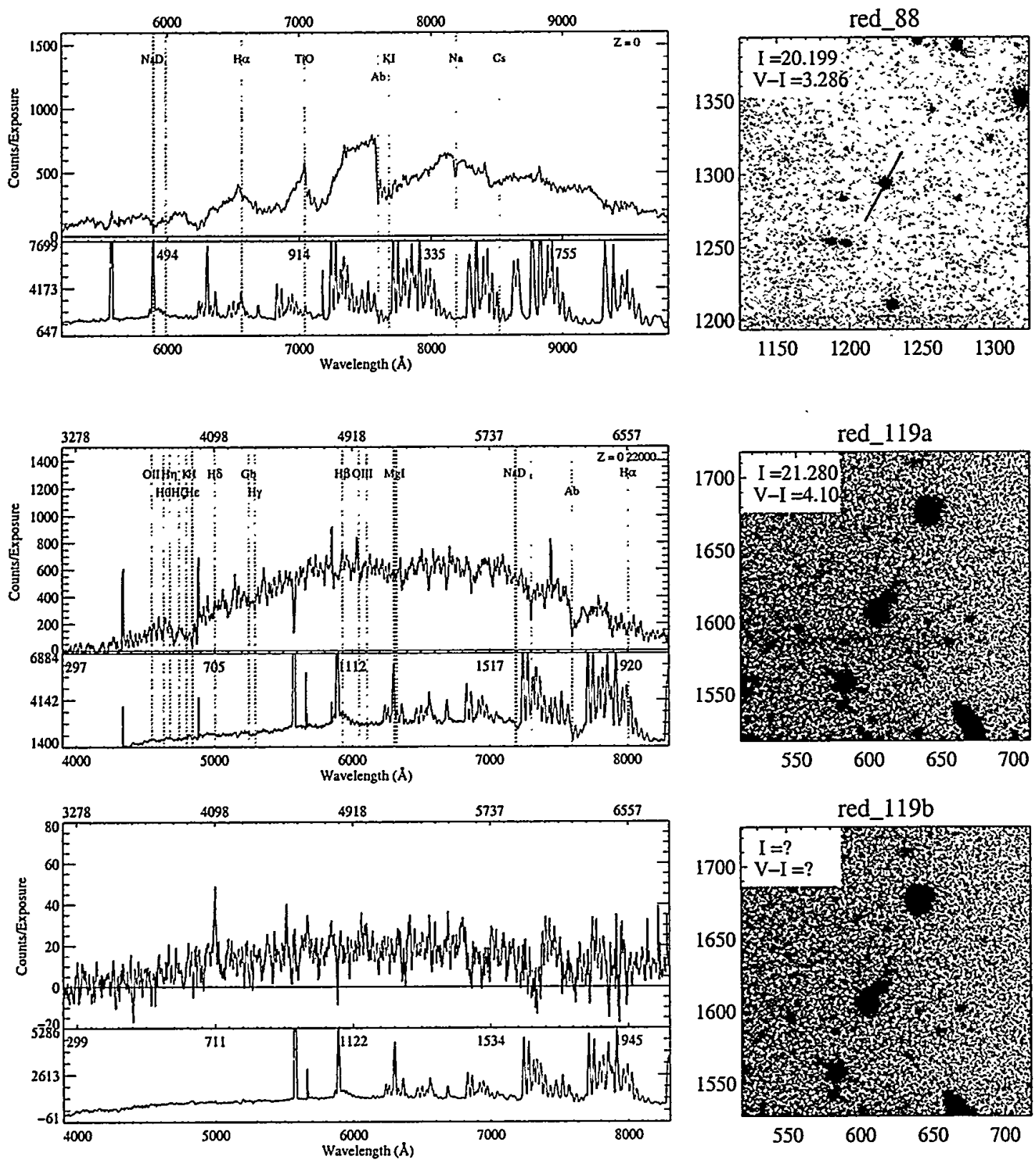

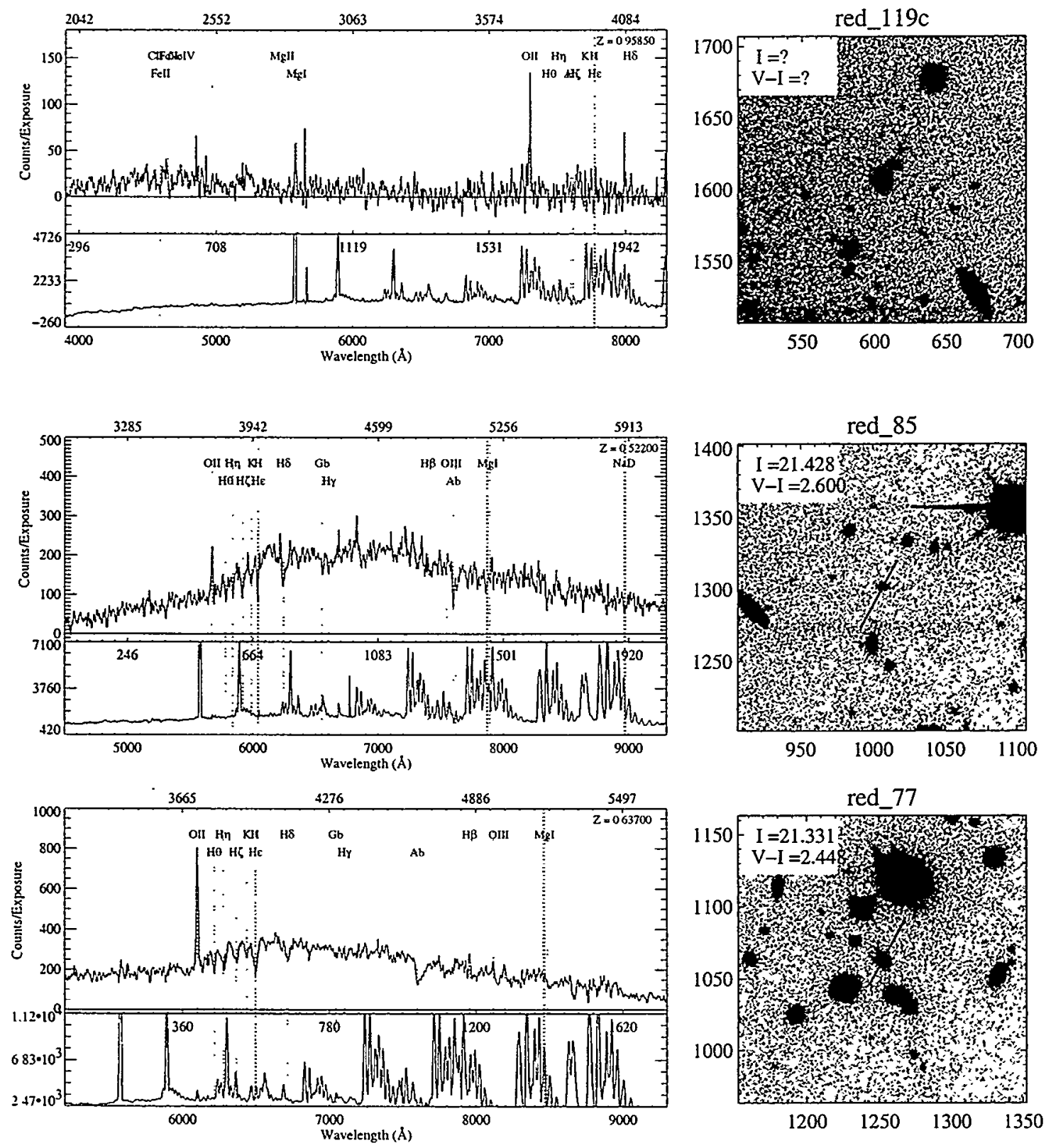

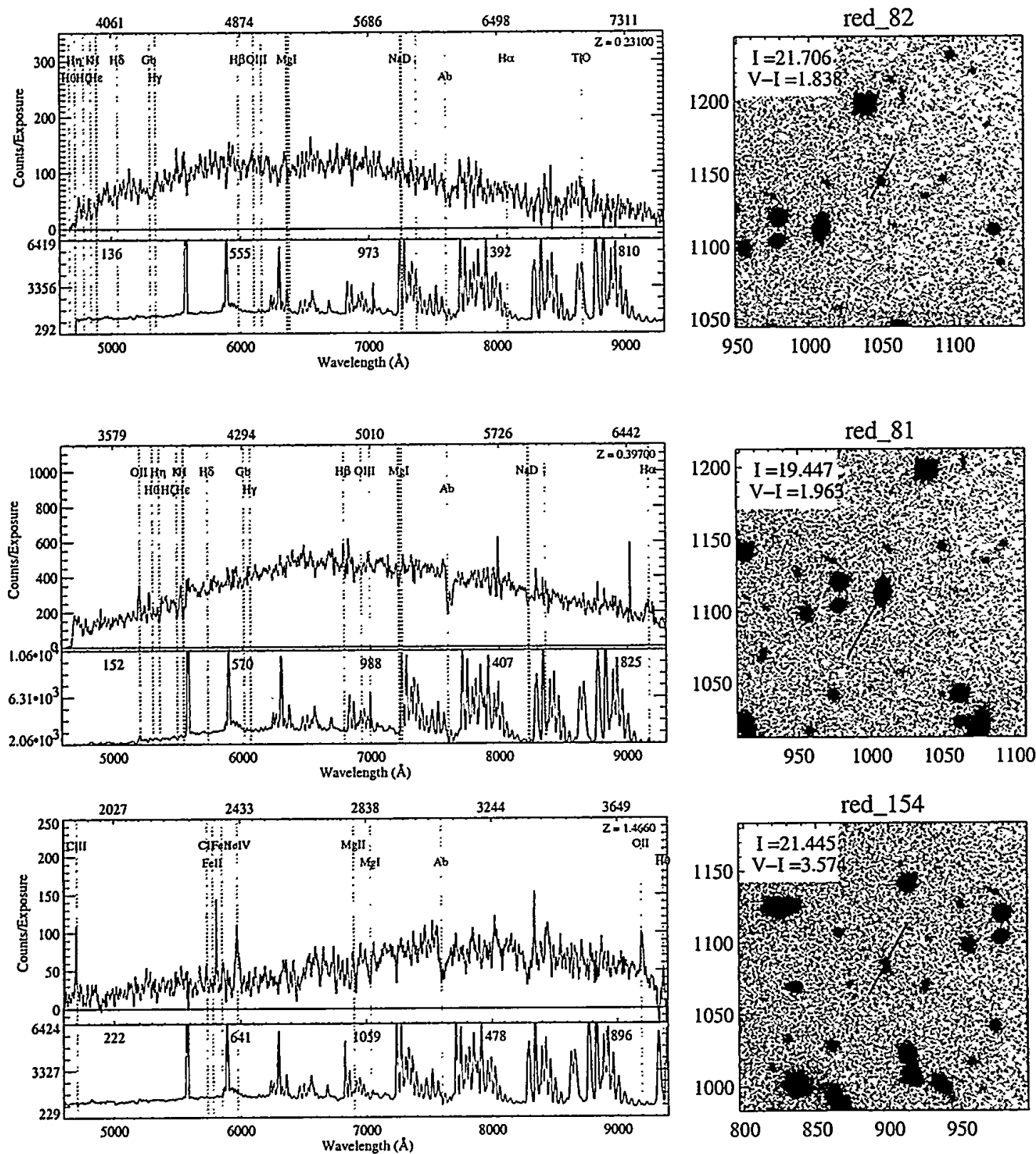

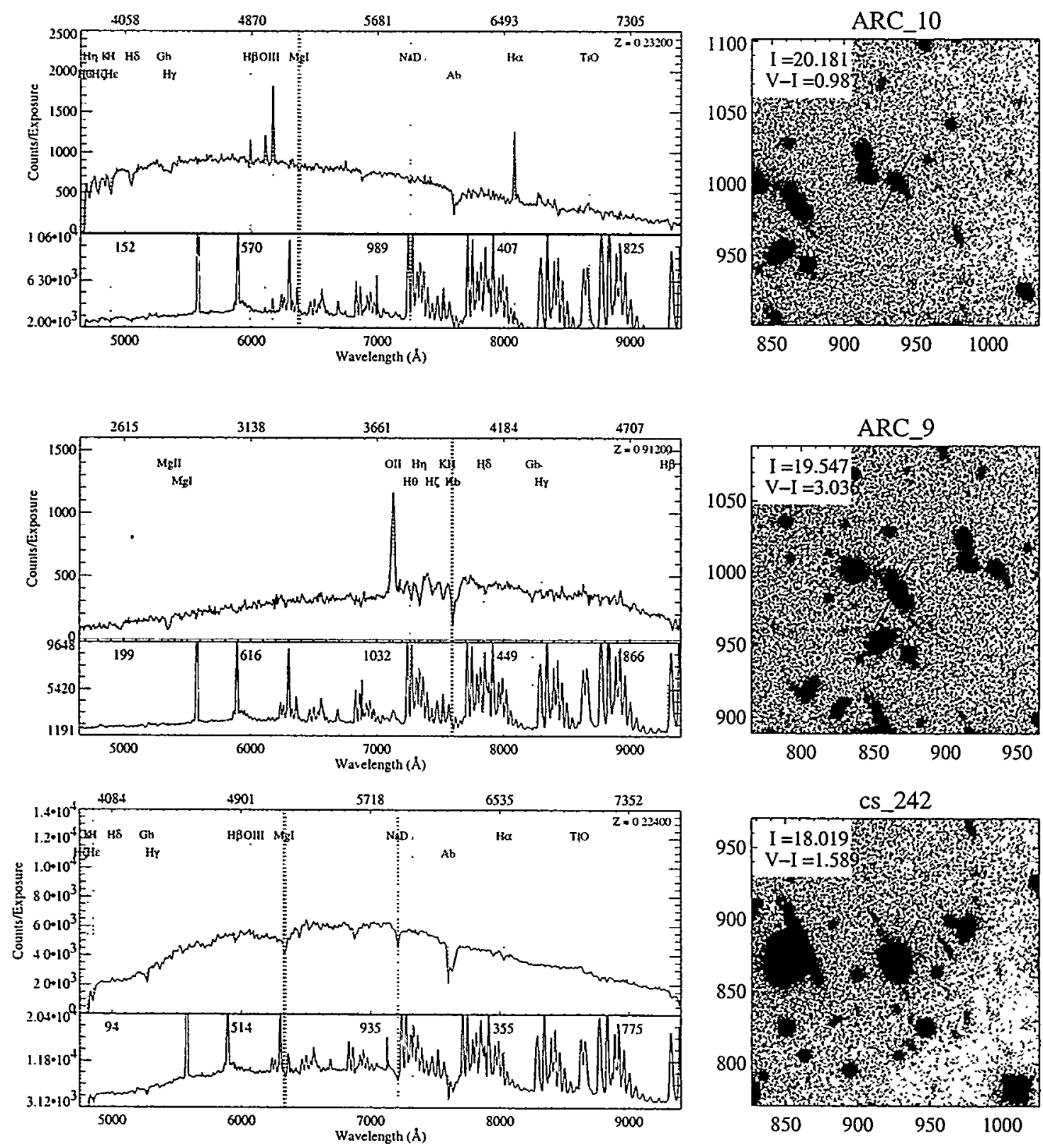

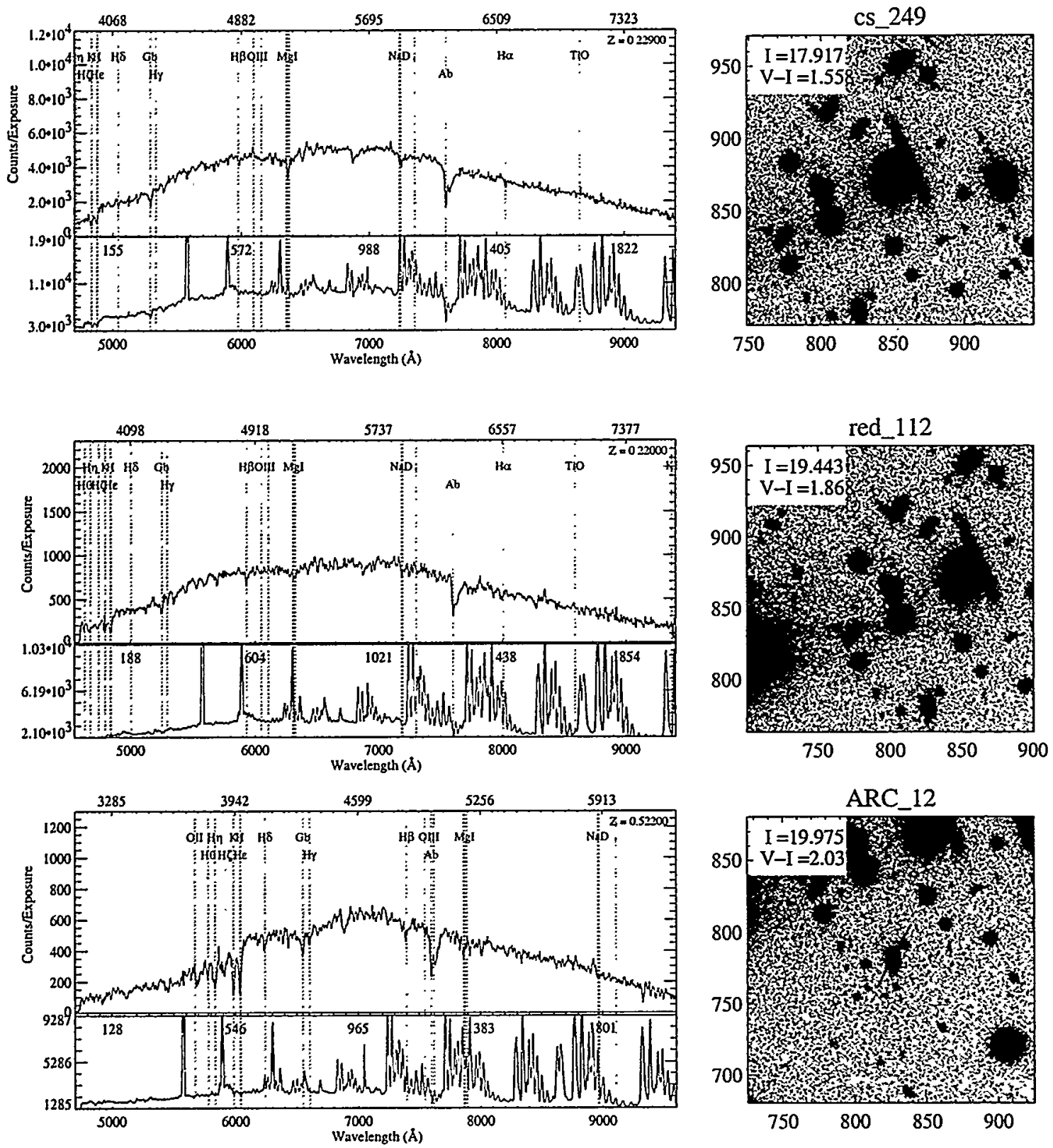

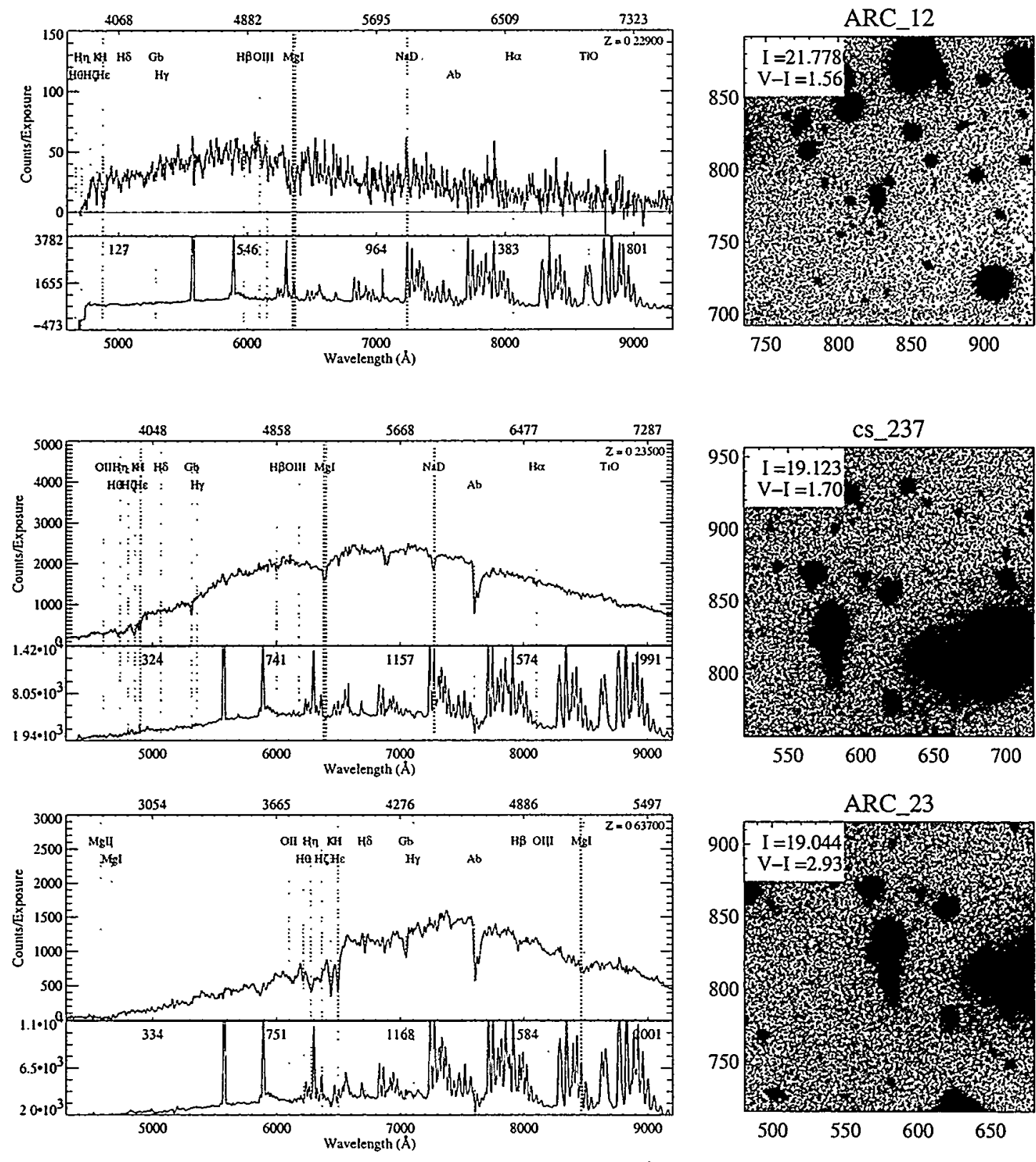

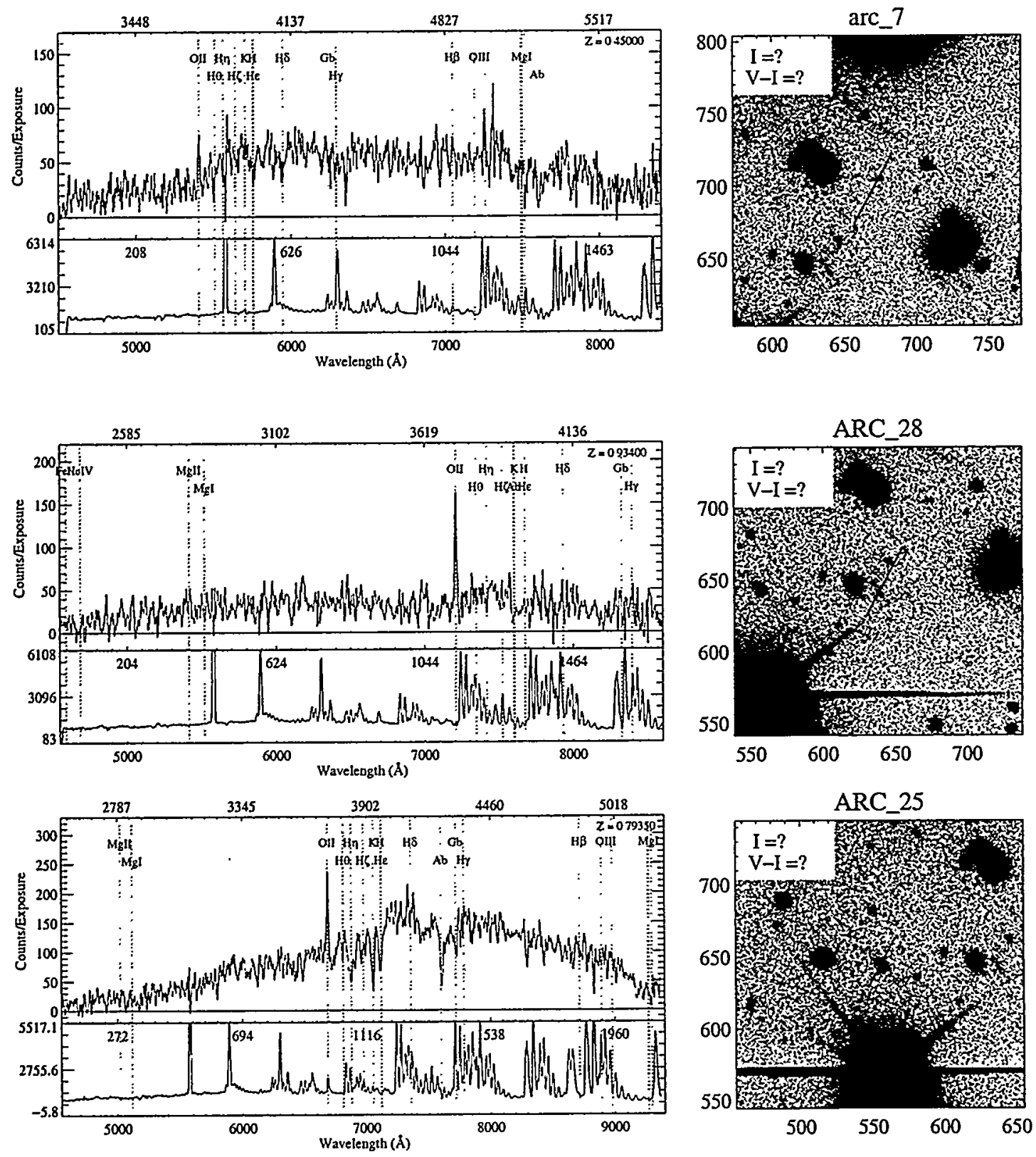

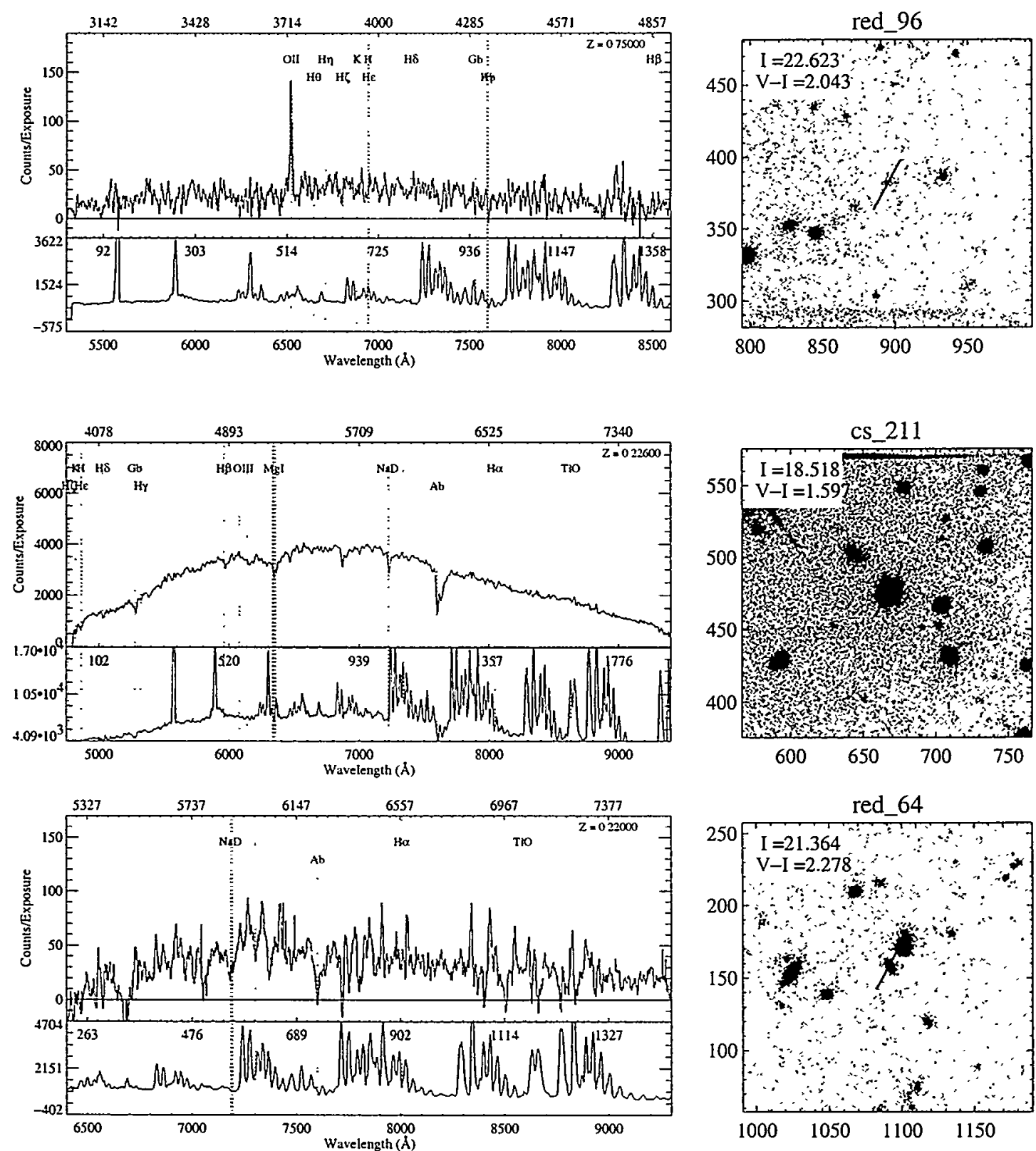

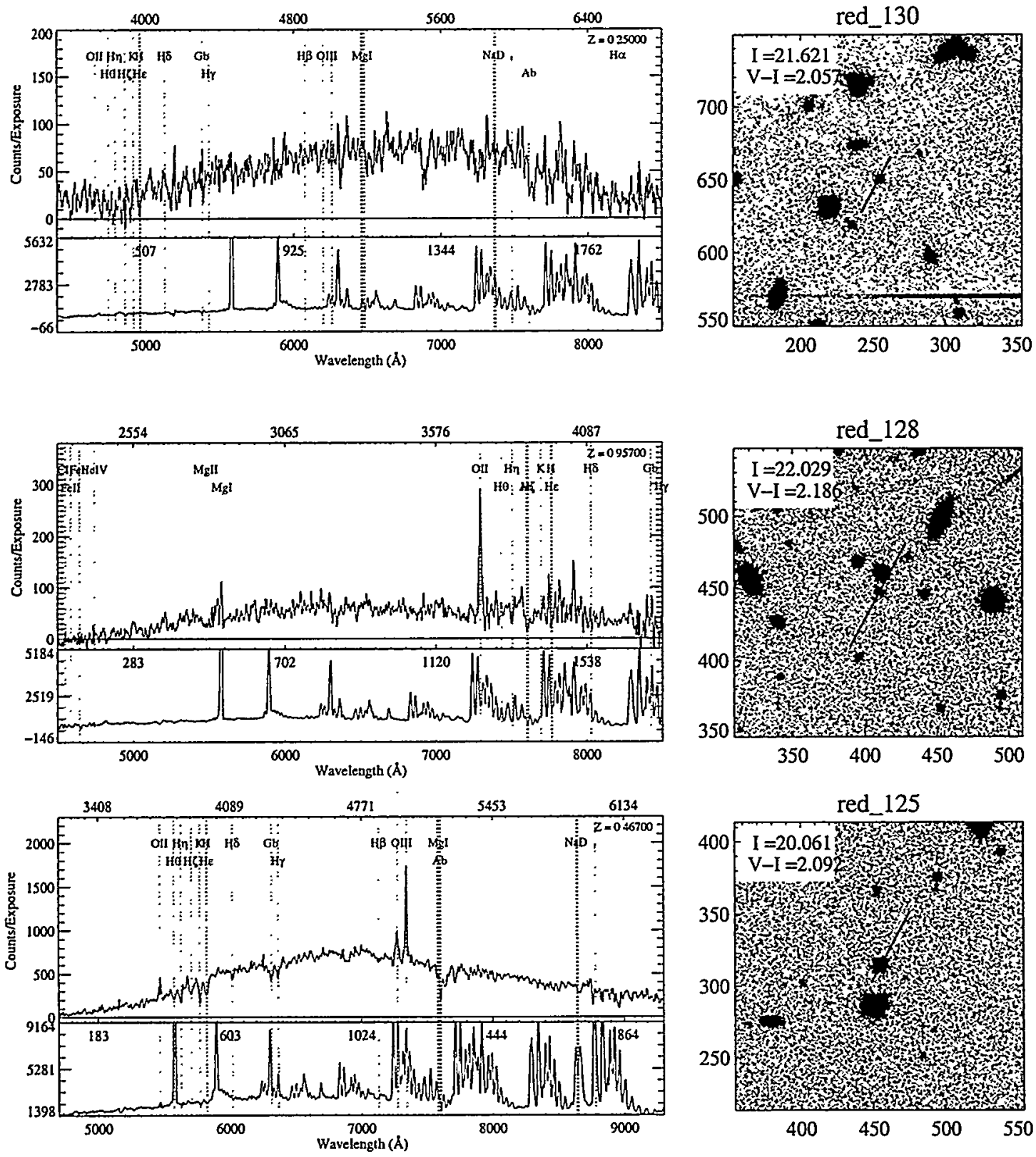
B.4 Mask 4 

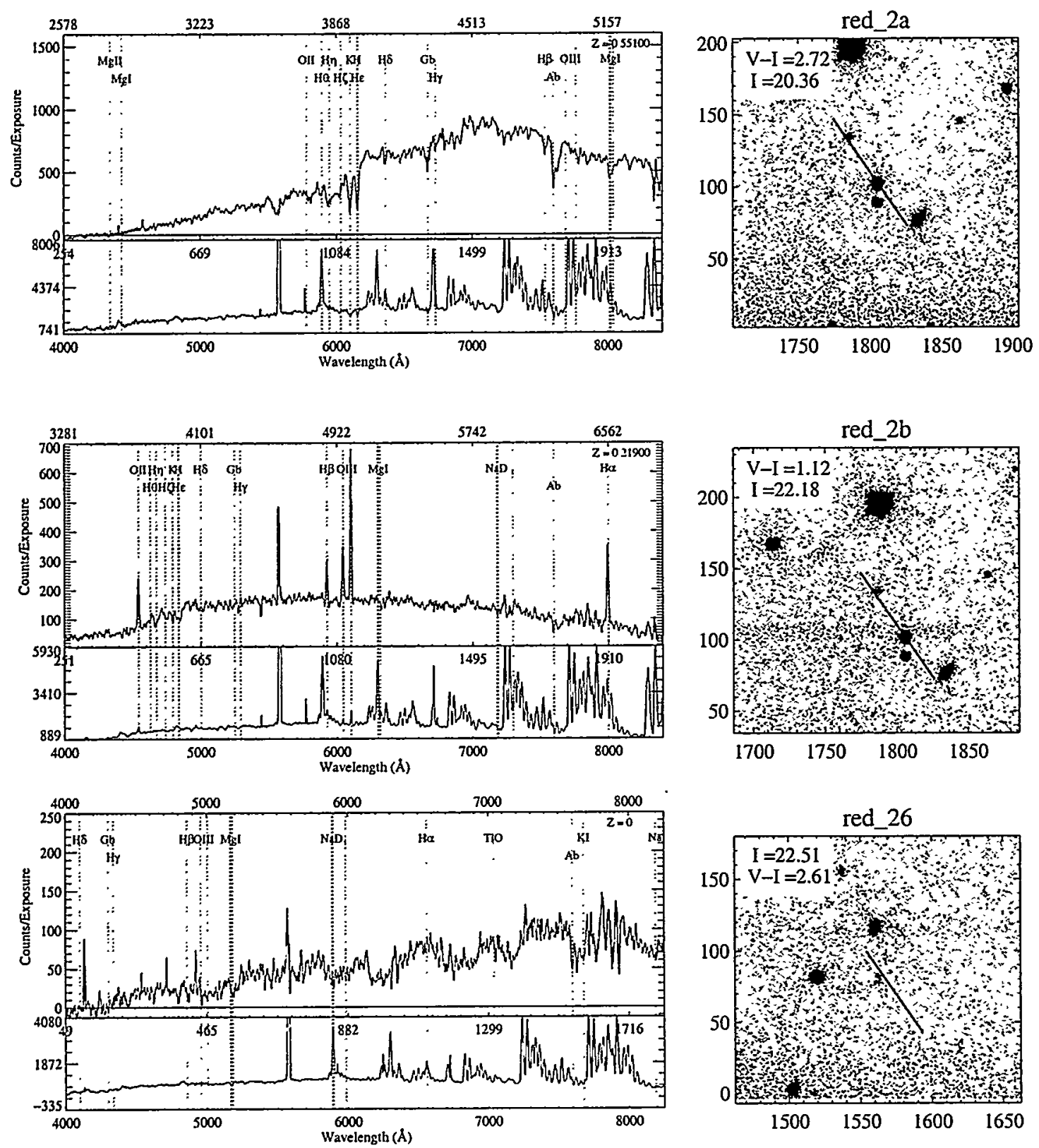

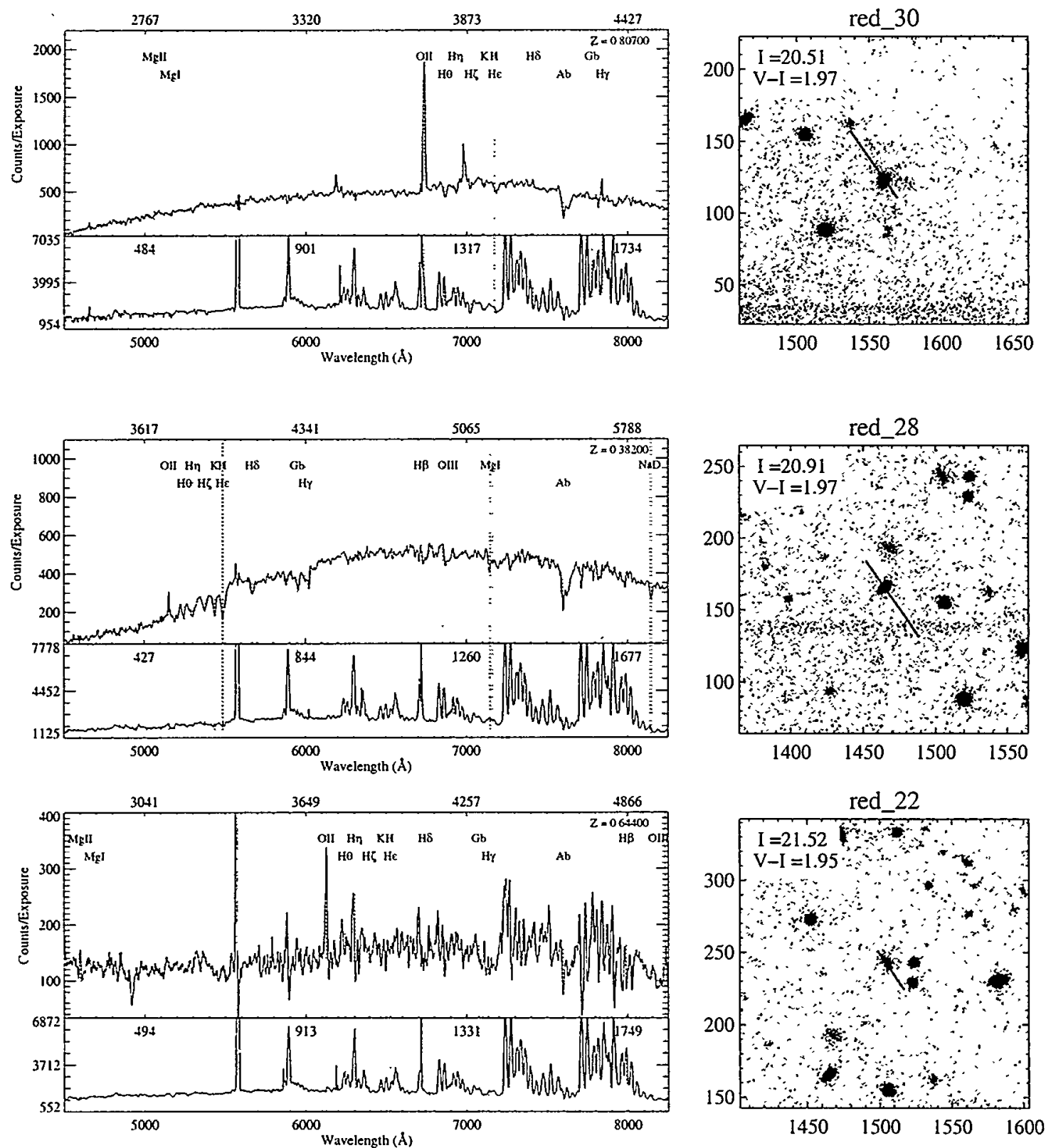

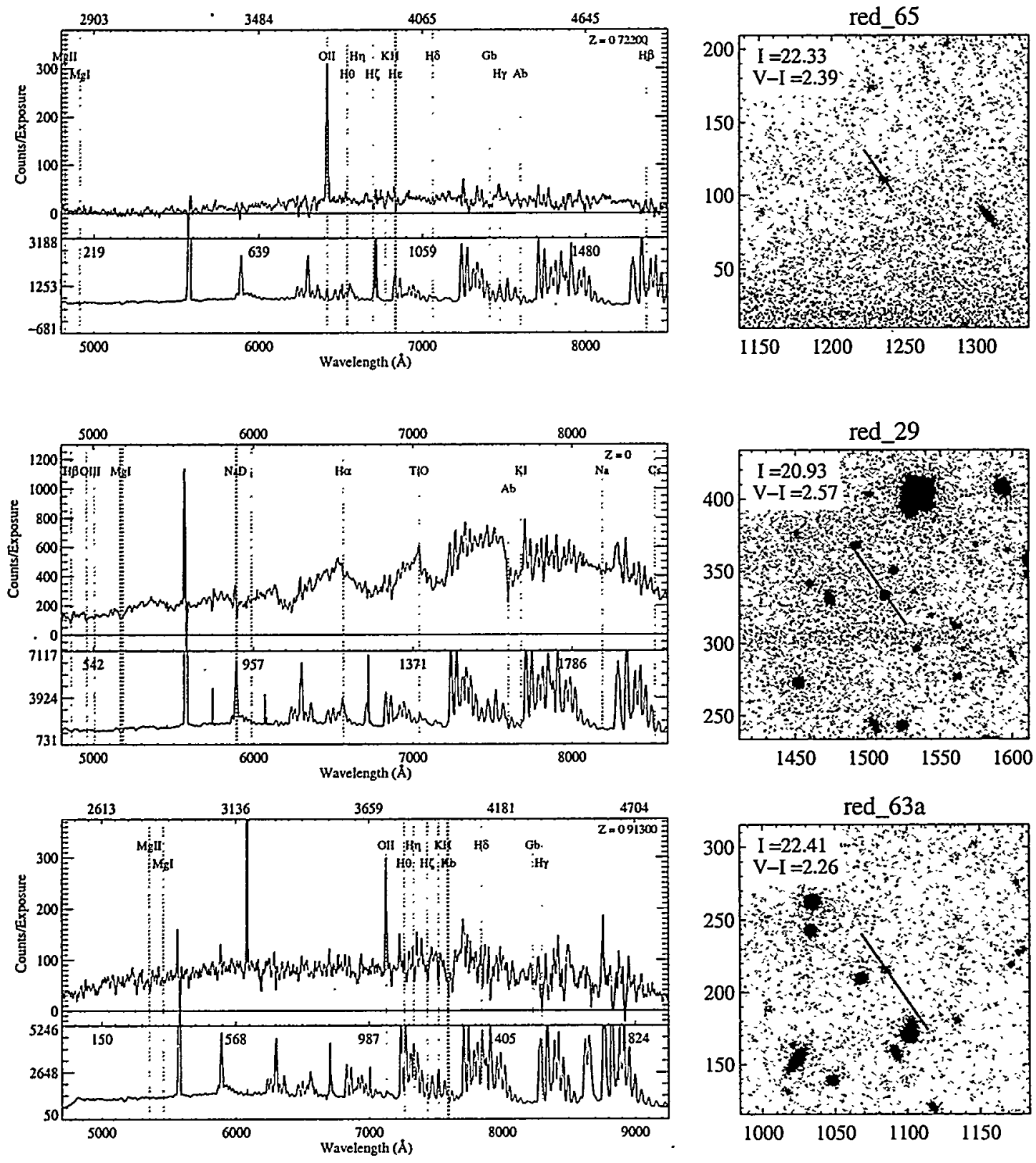

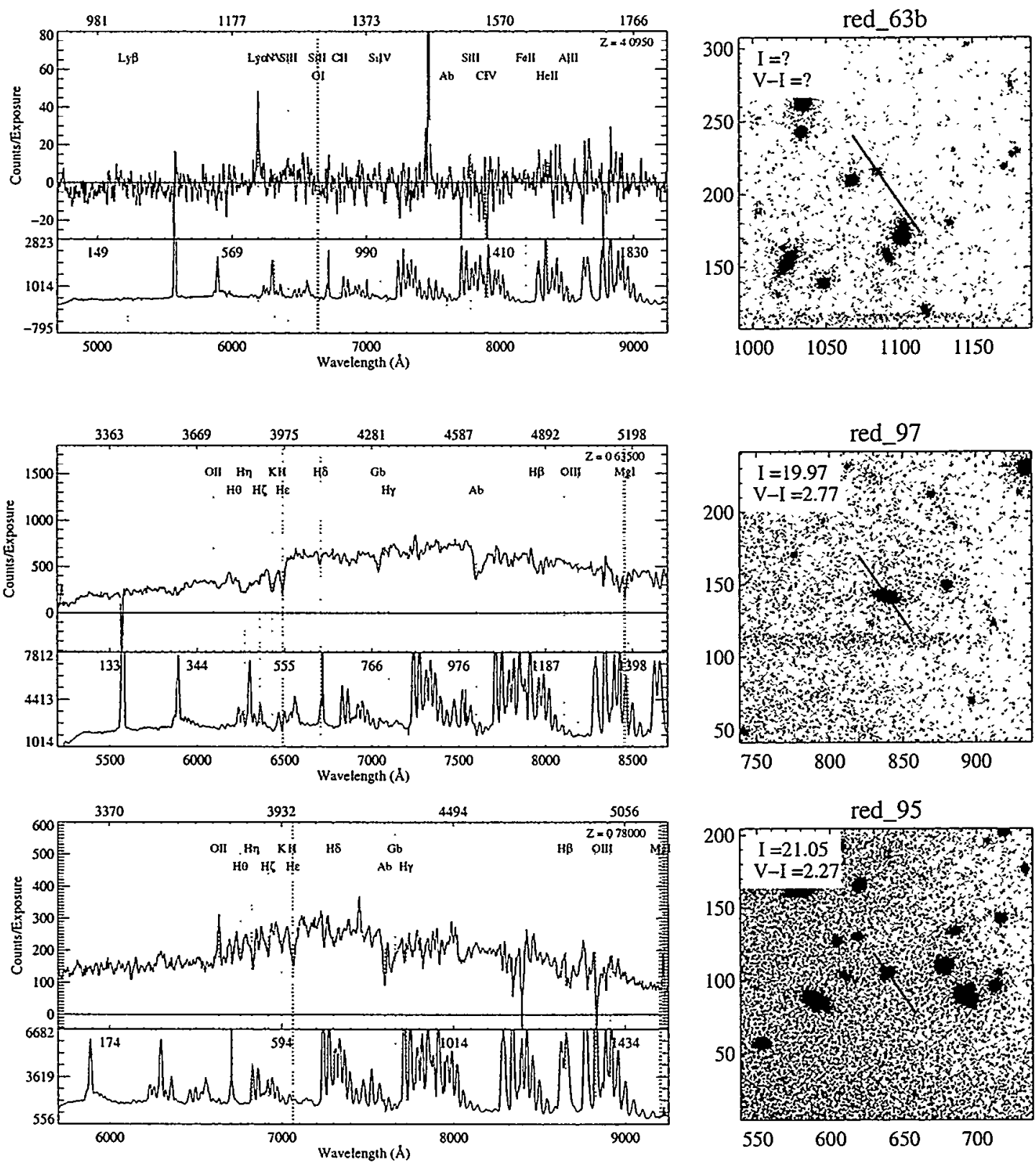

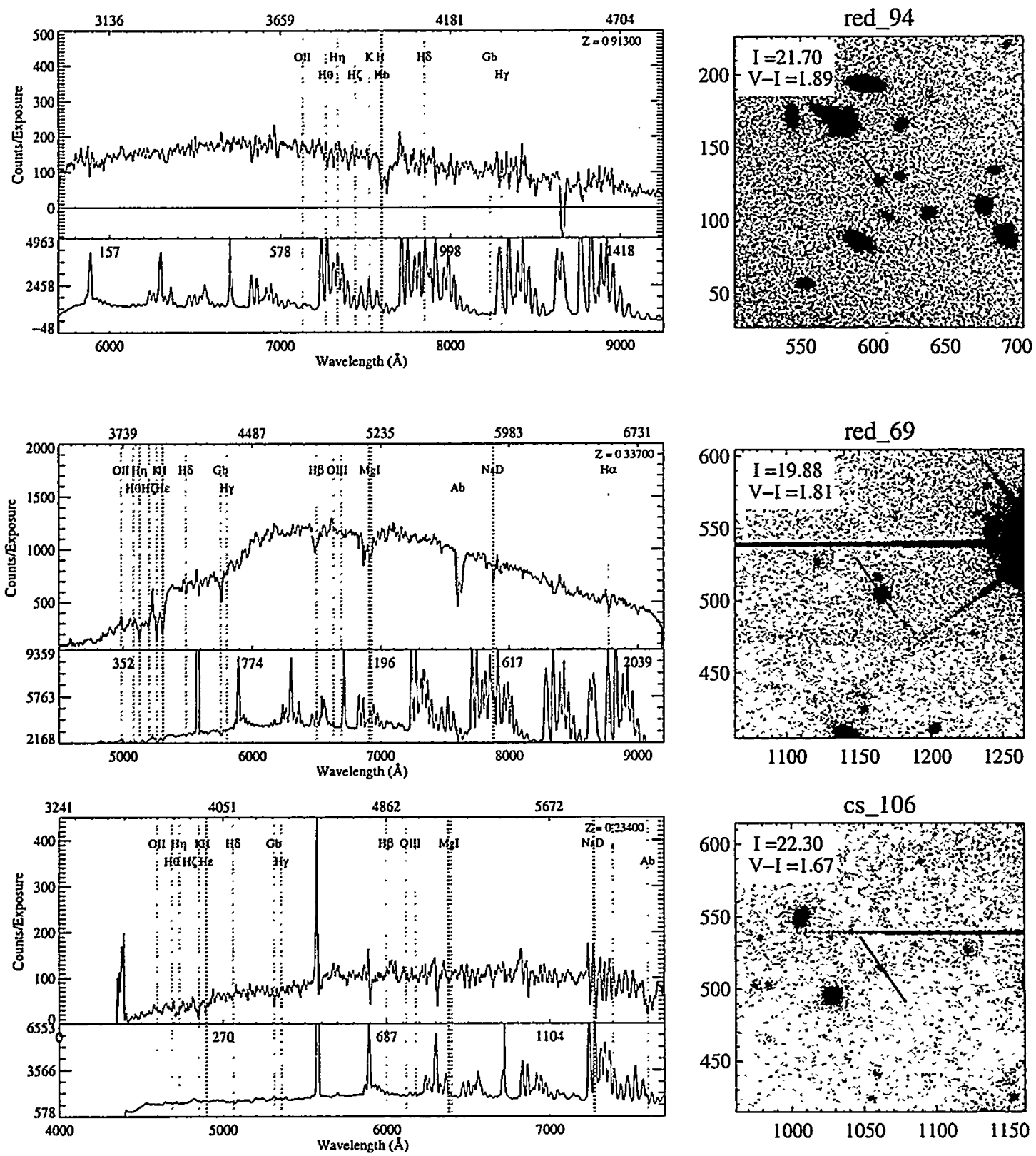

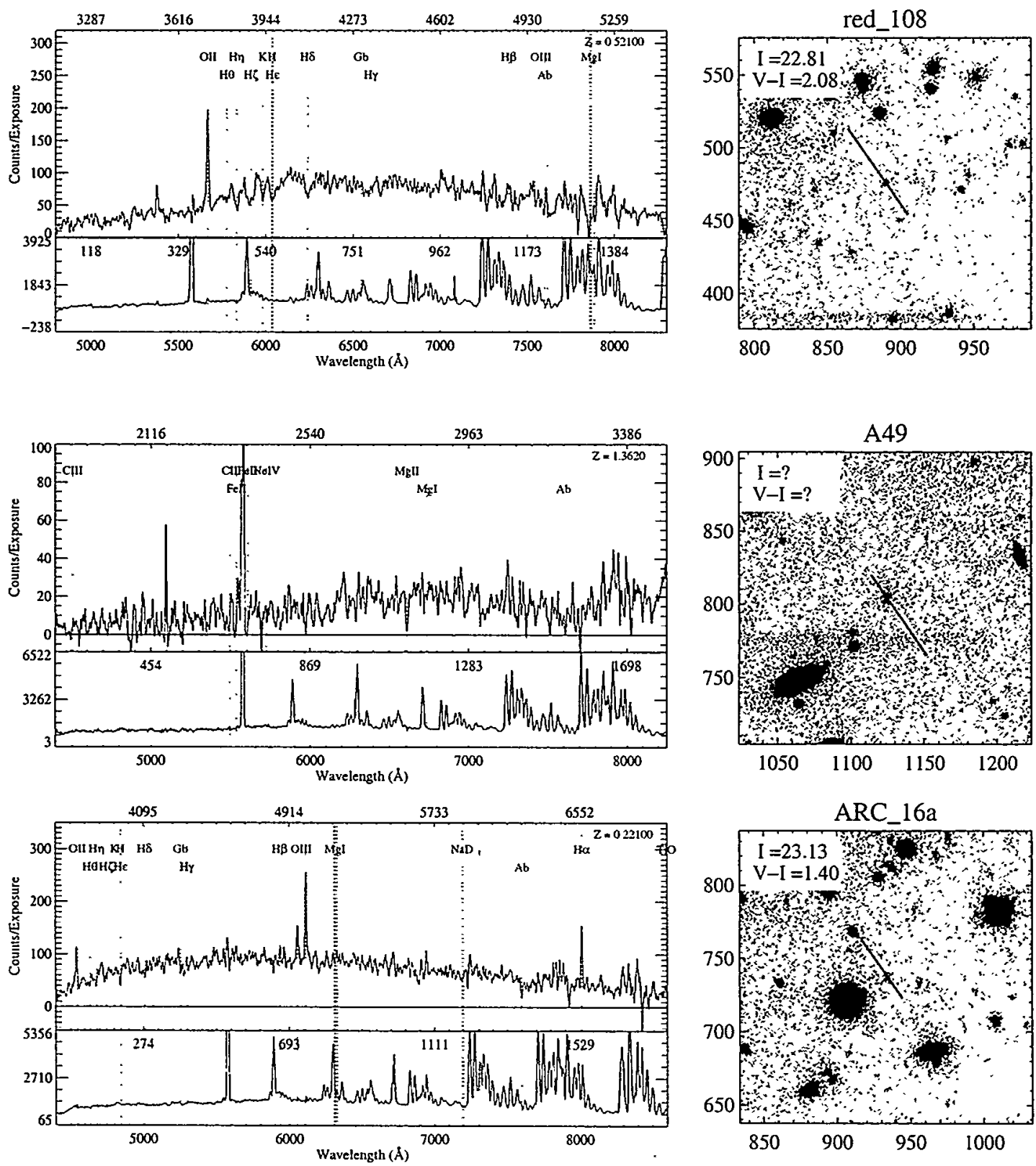

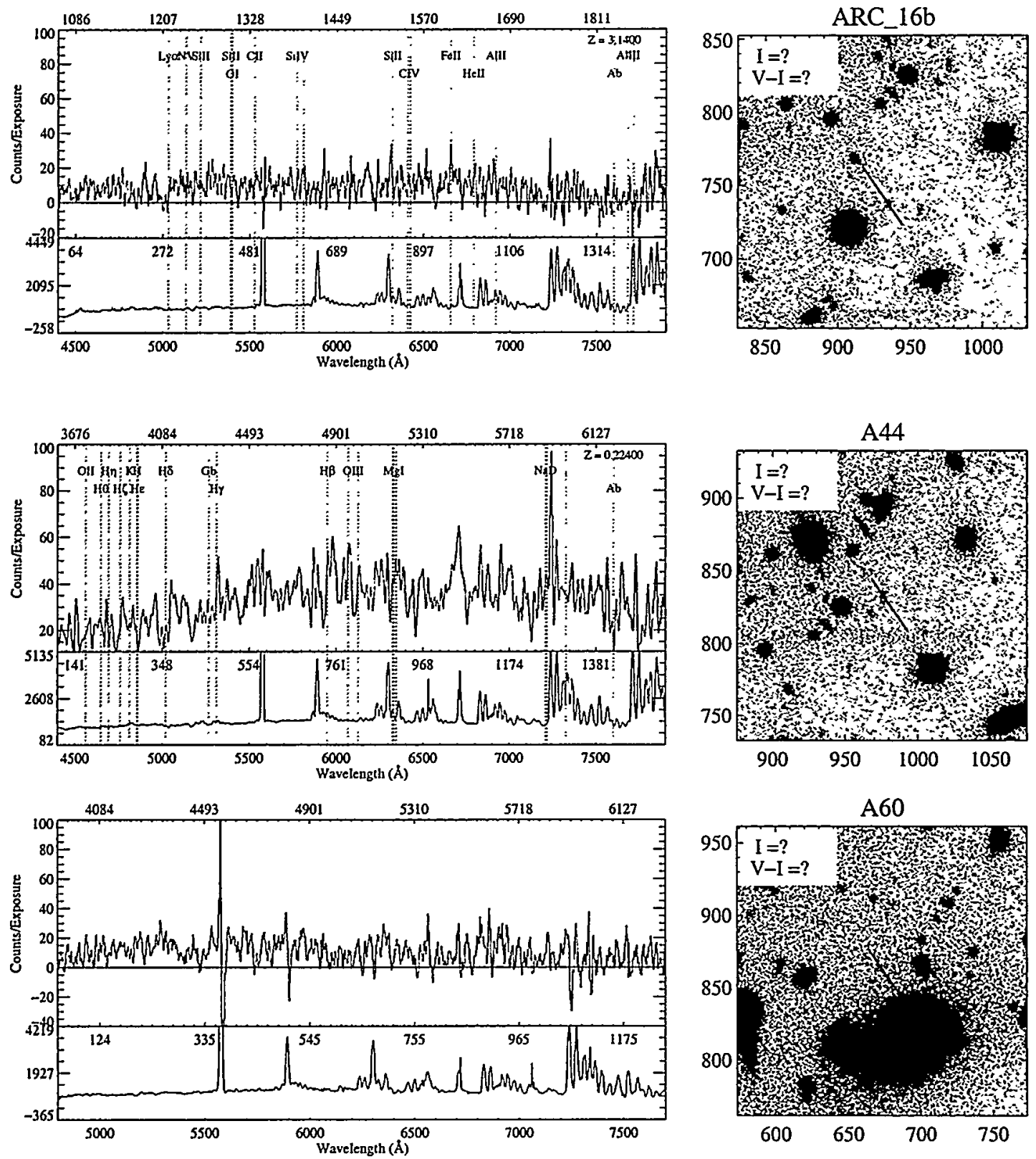

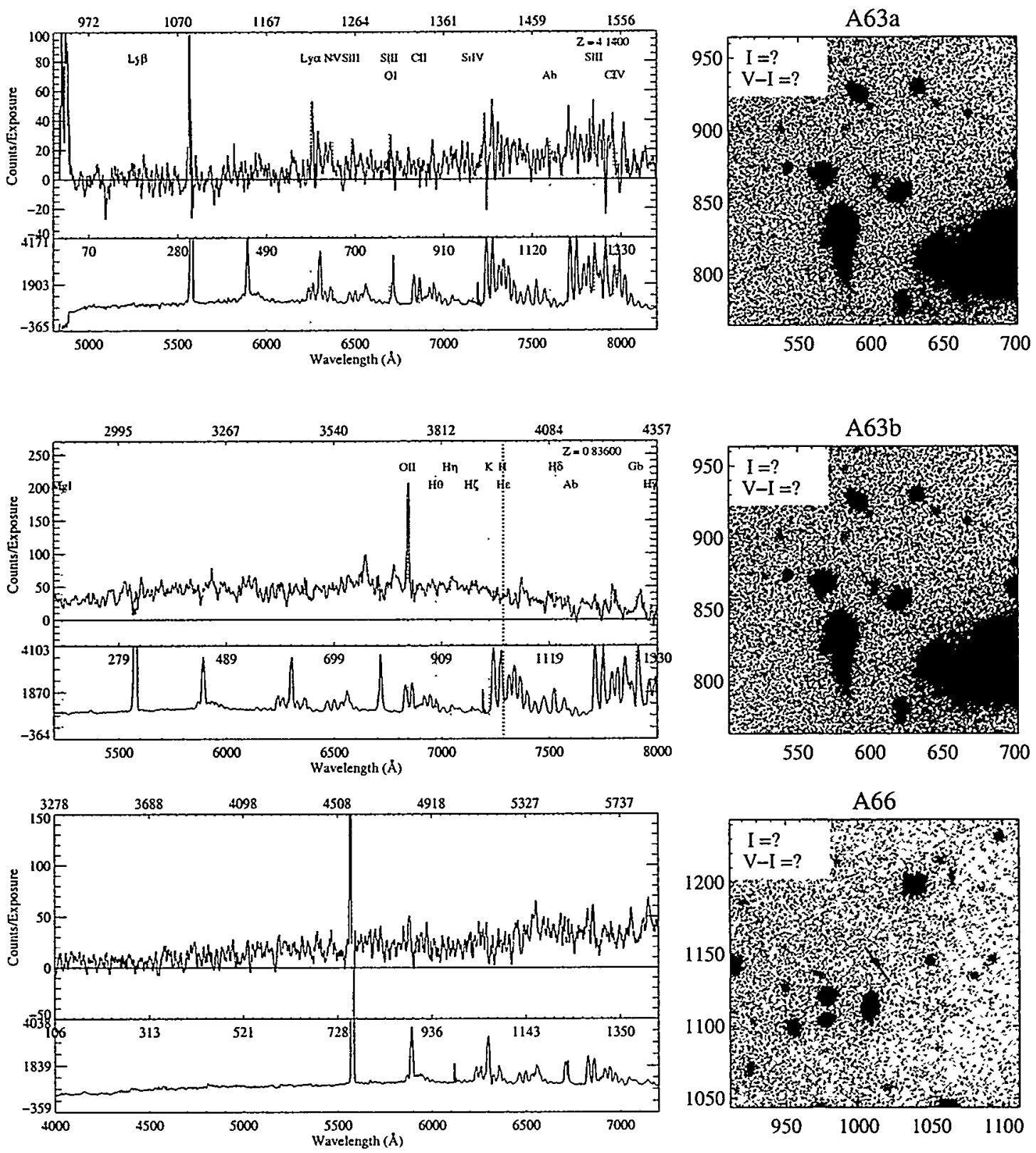

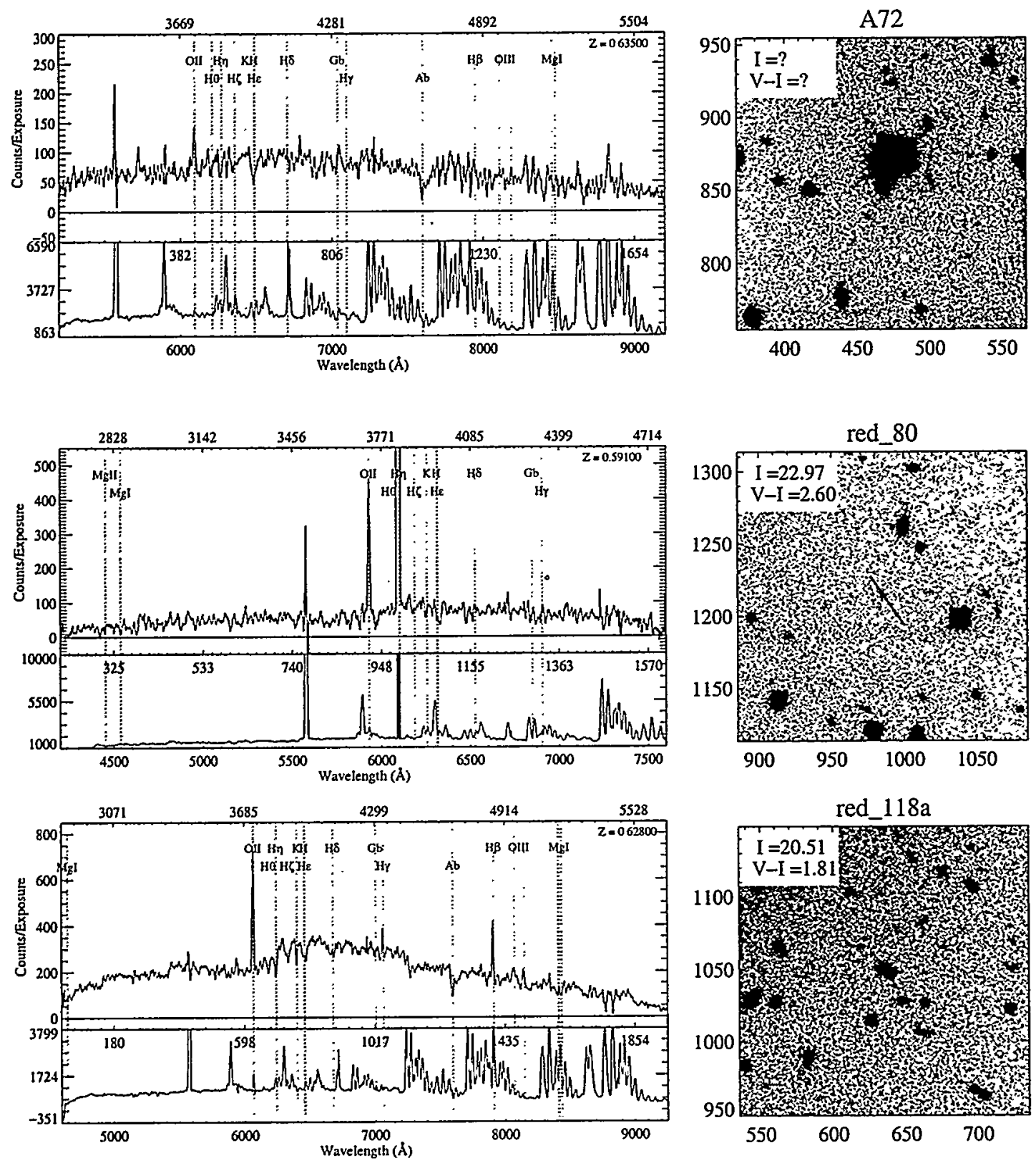

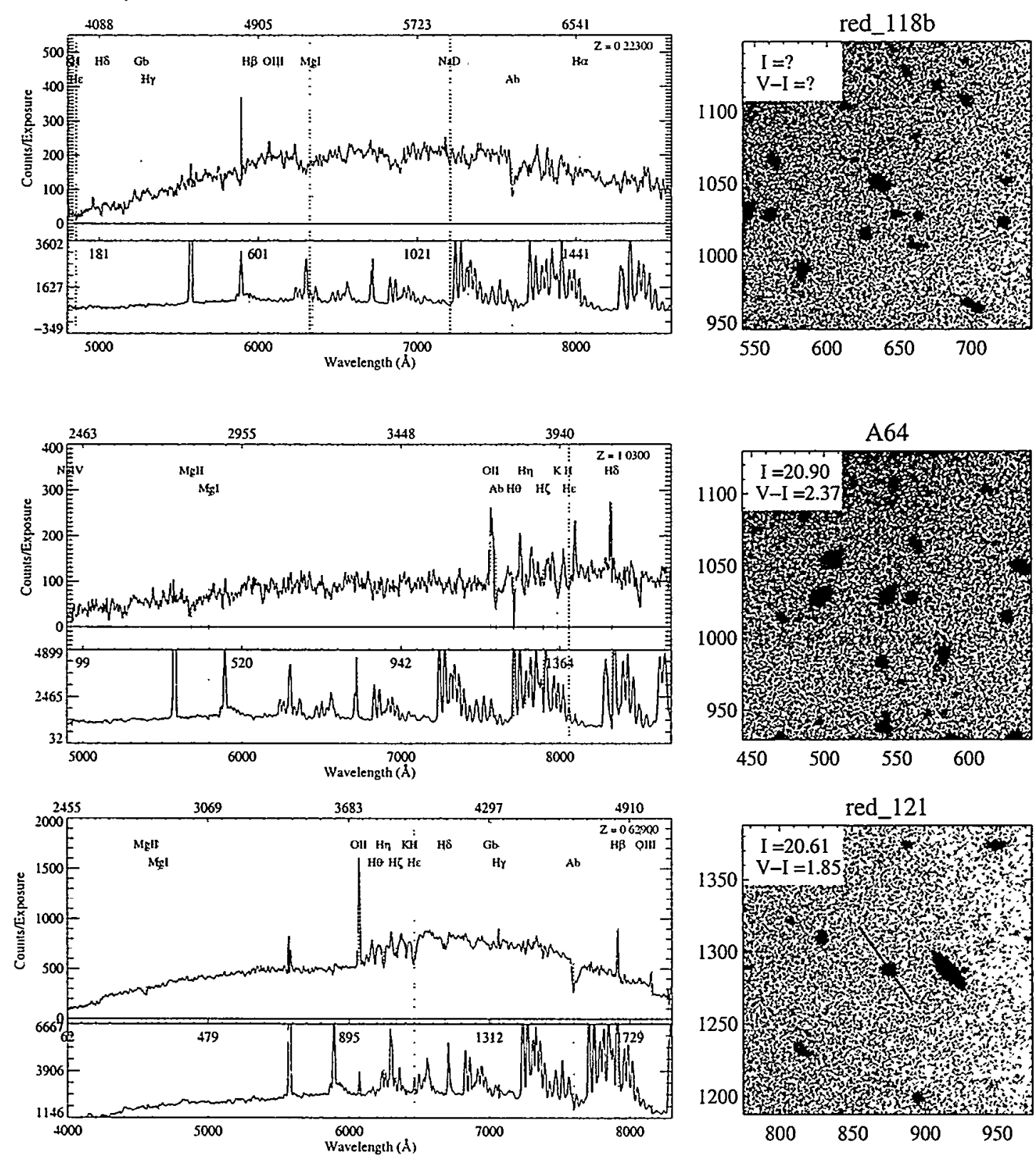

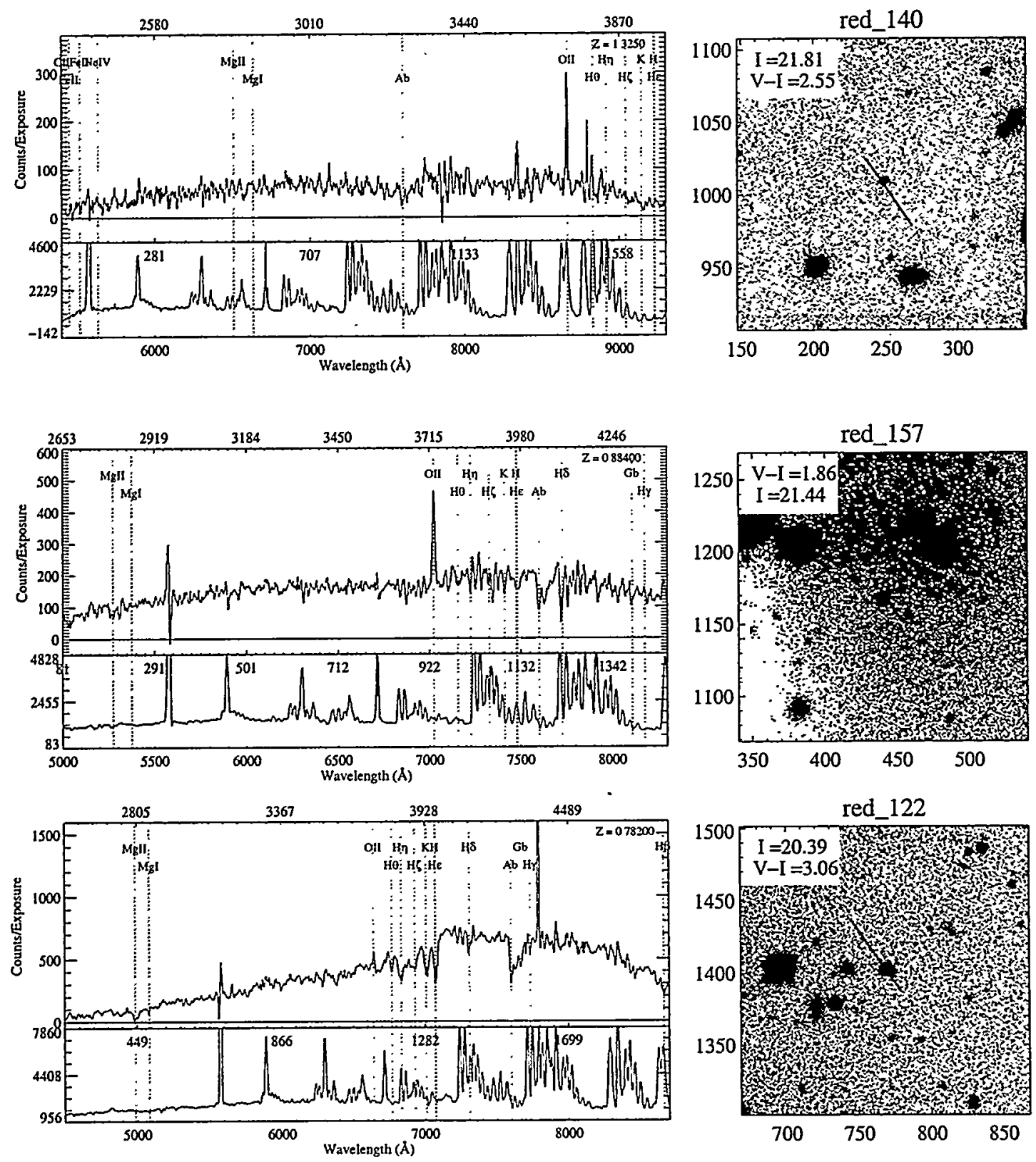

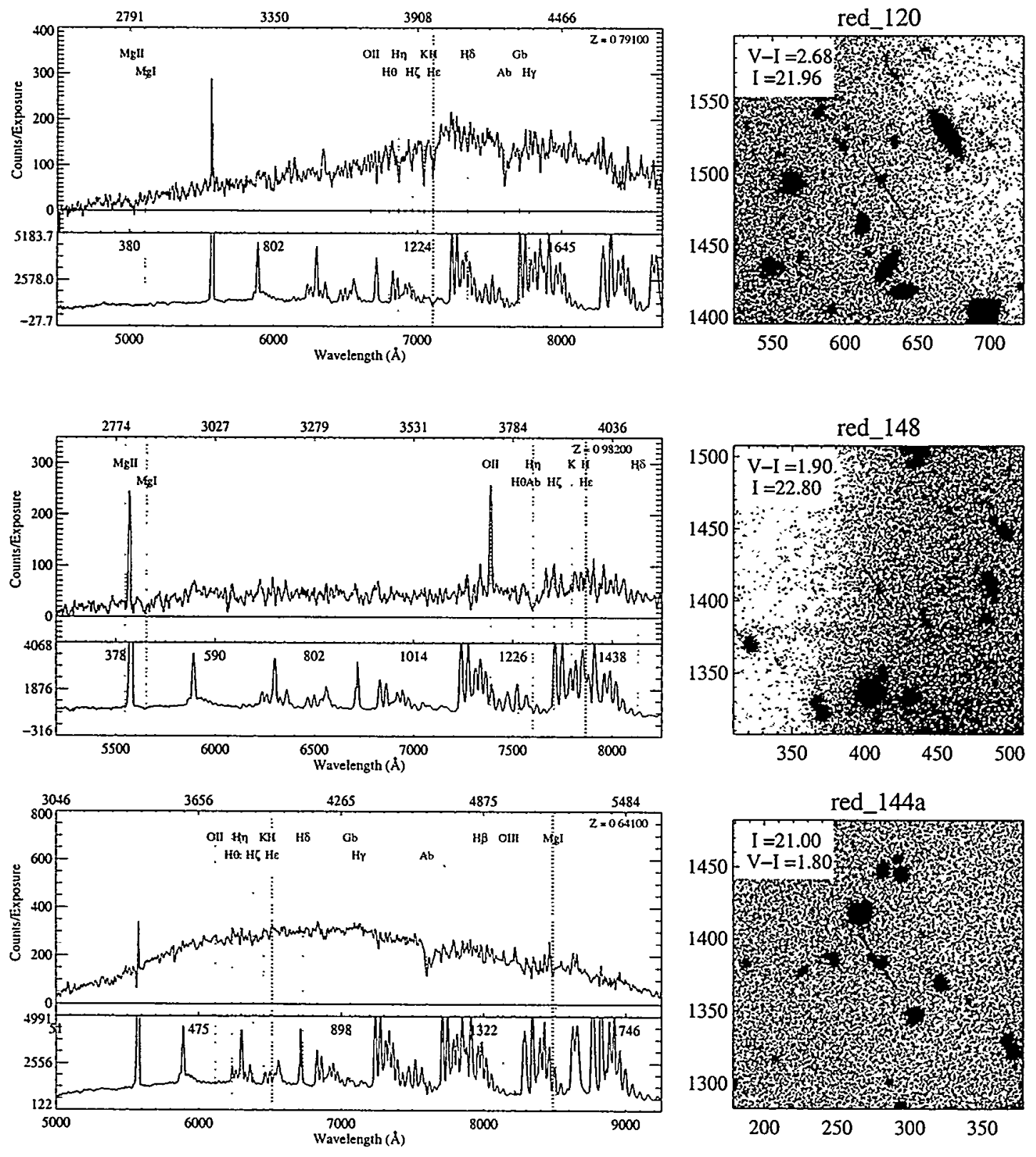

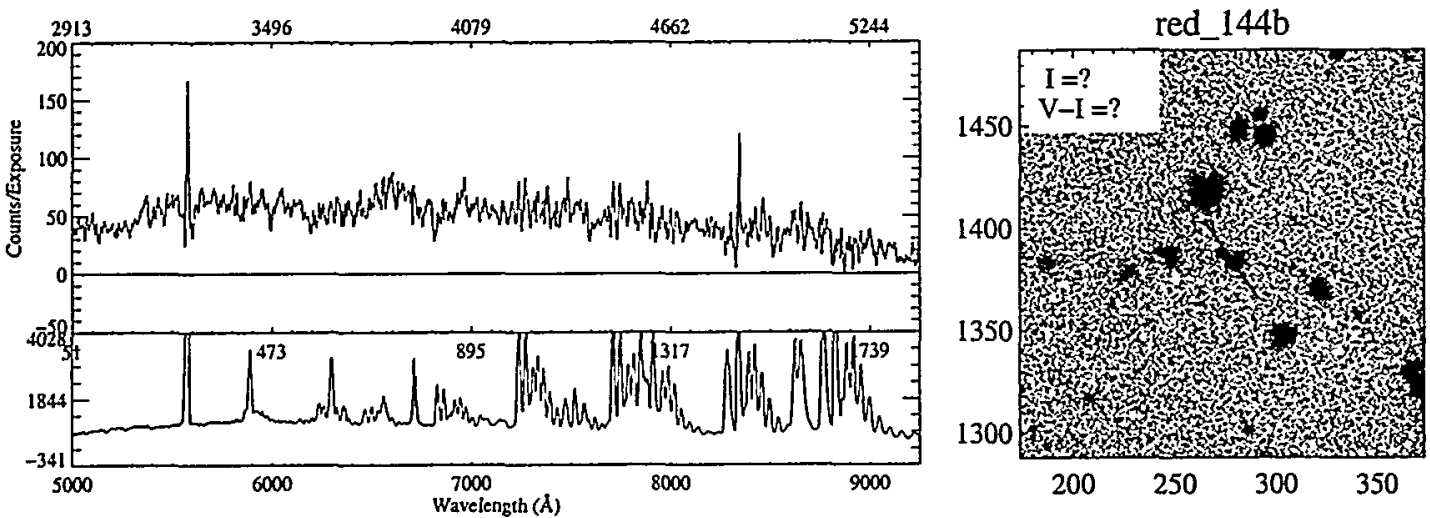
B.5 Mask 5 

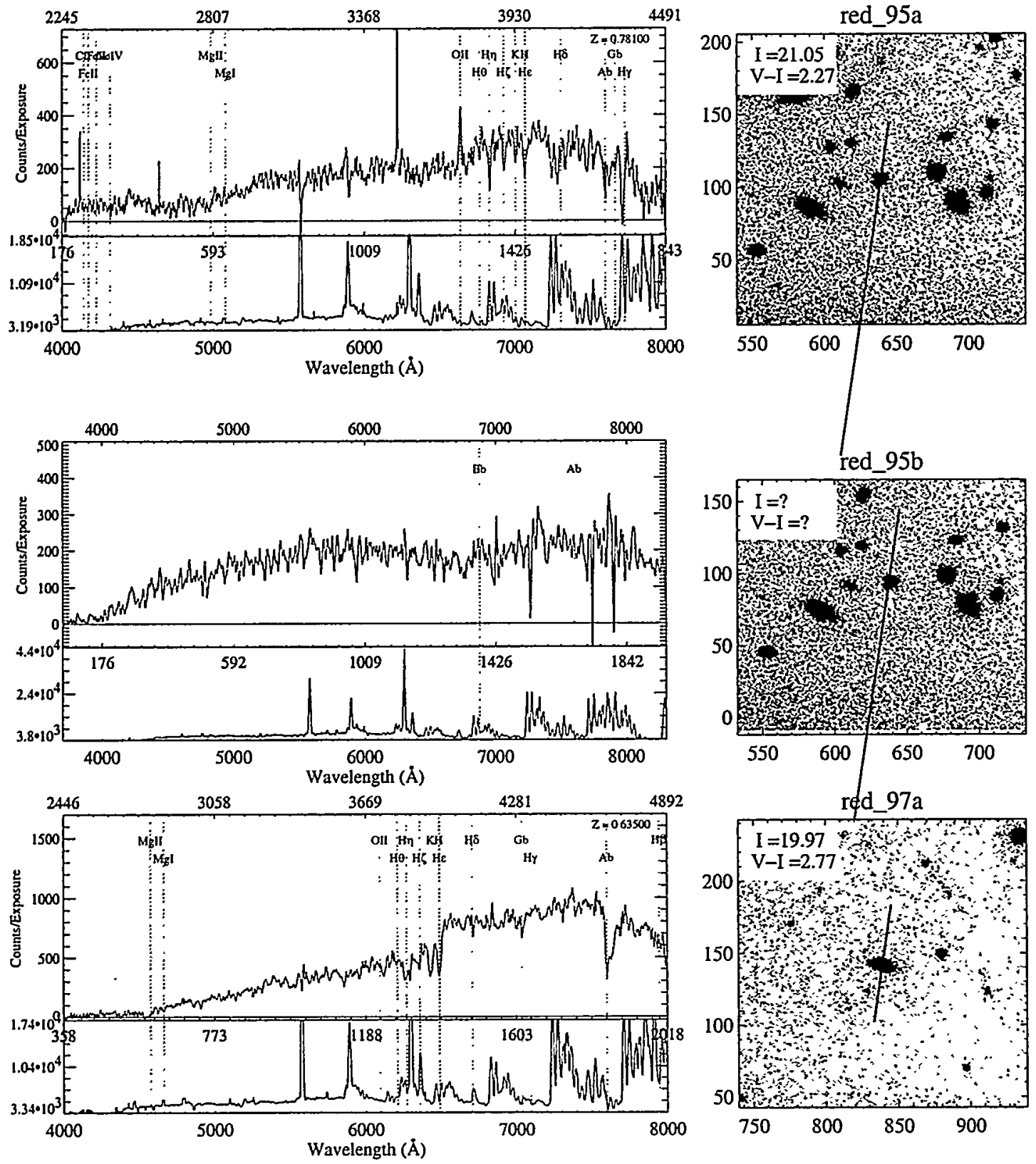

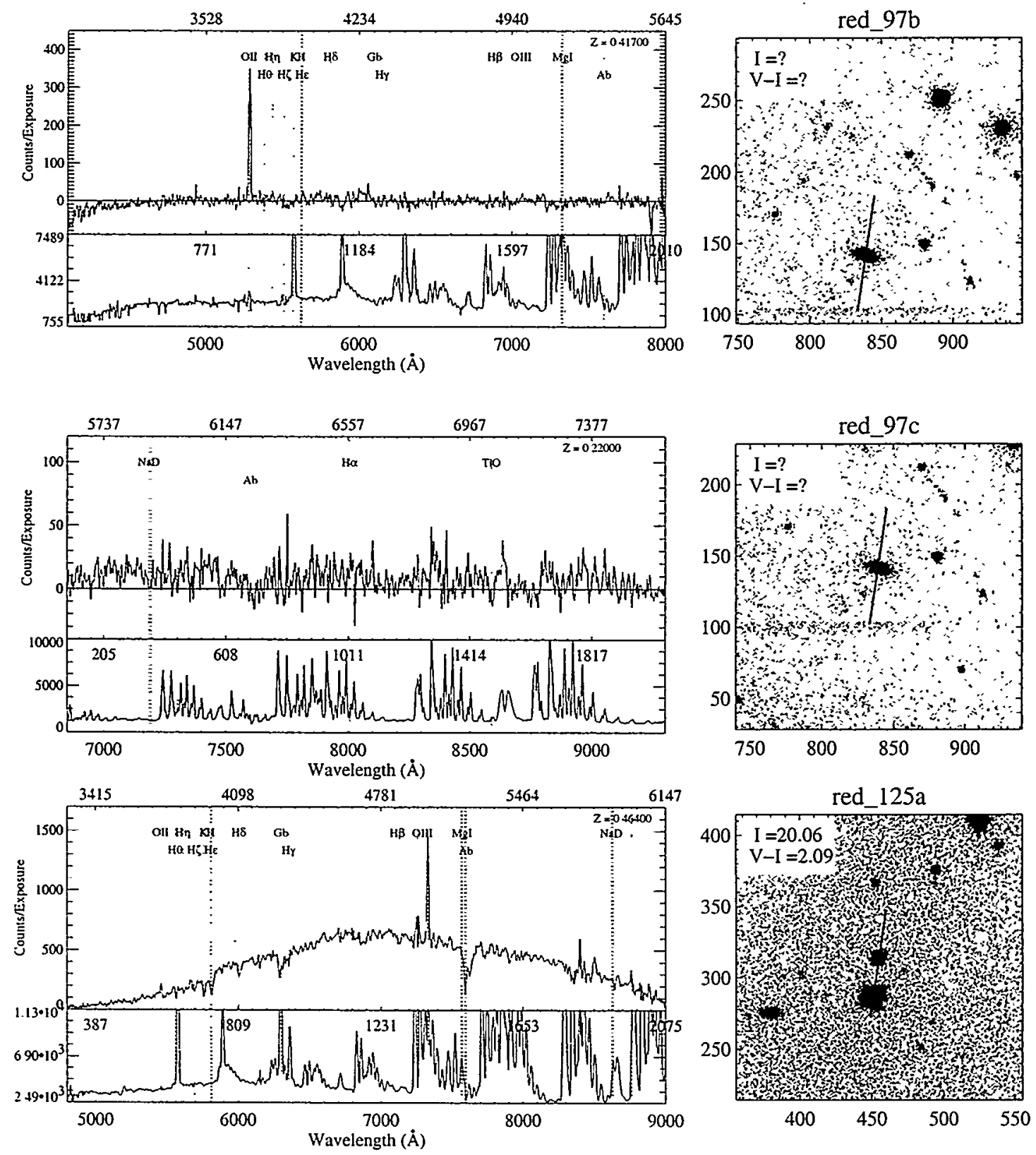

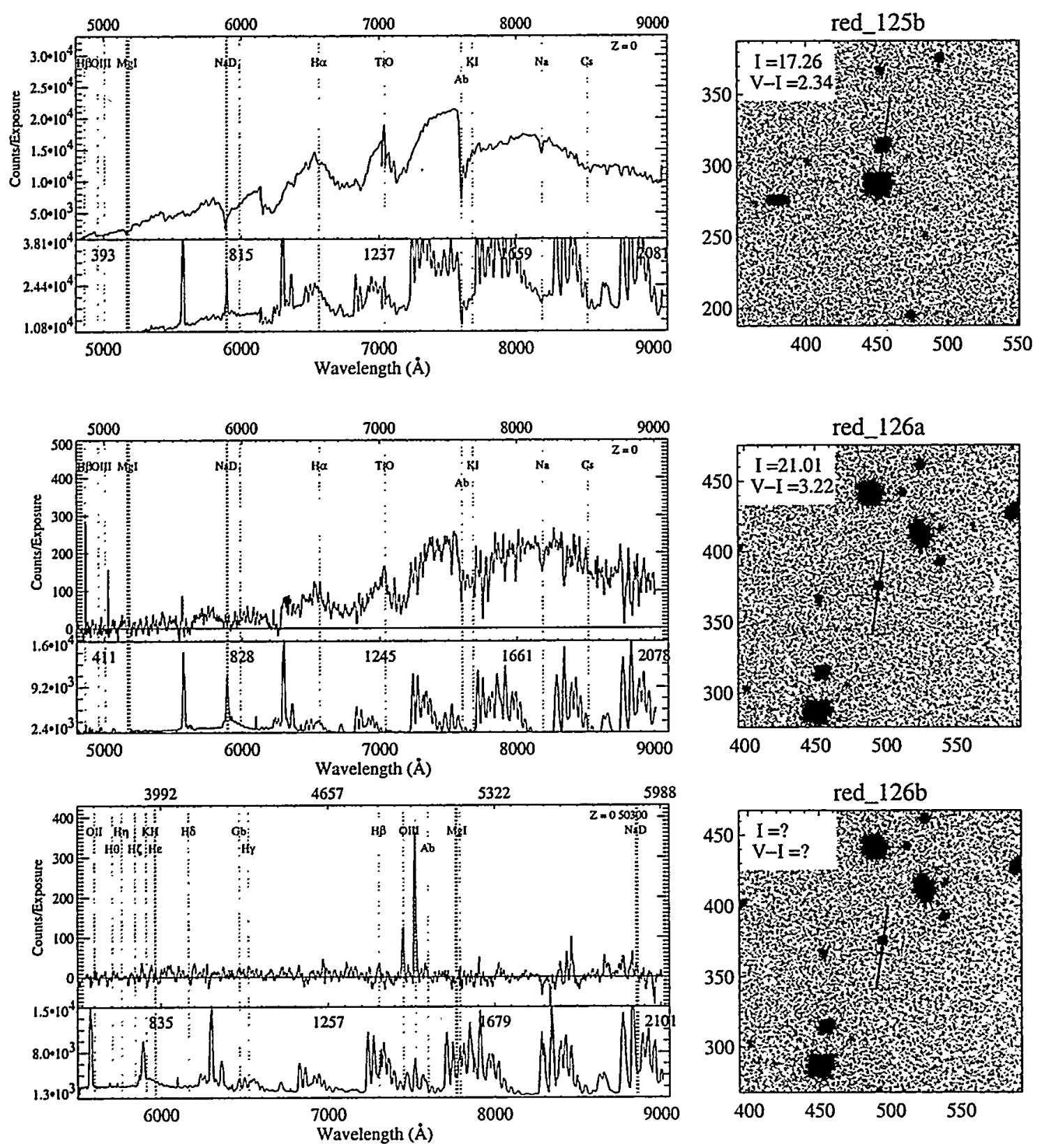

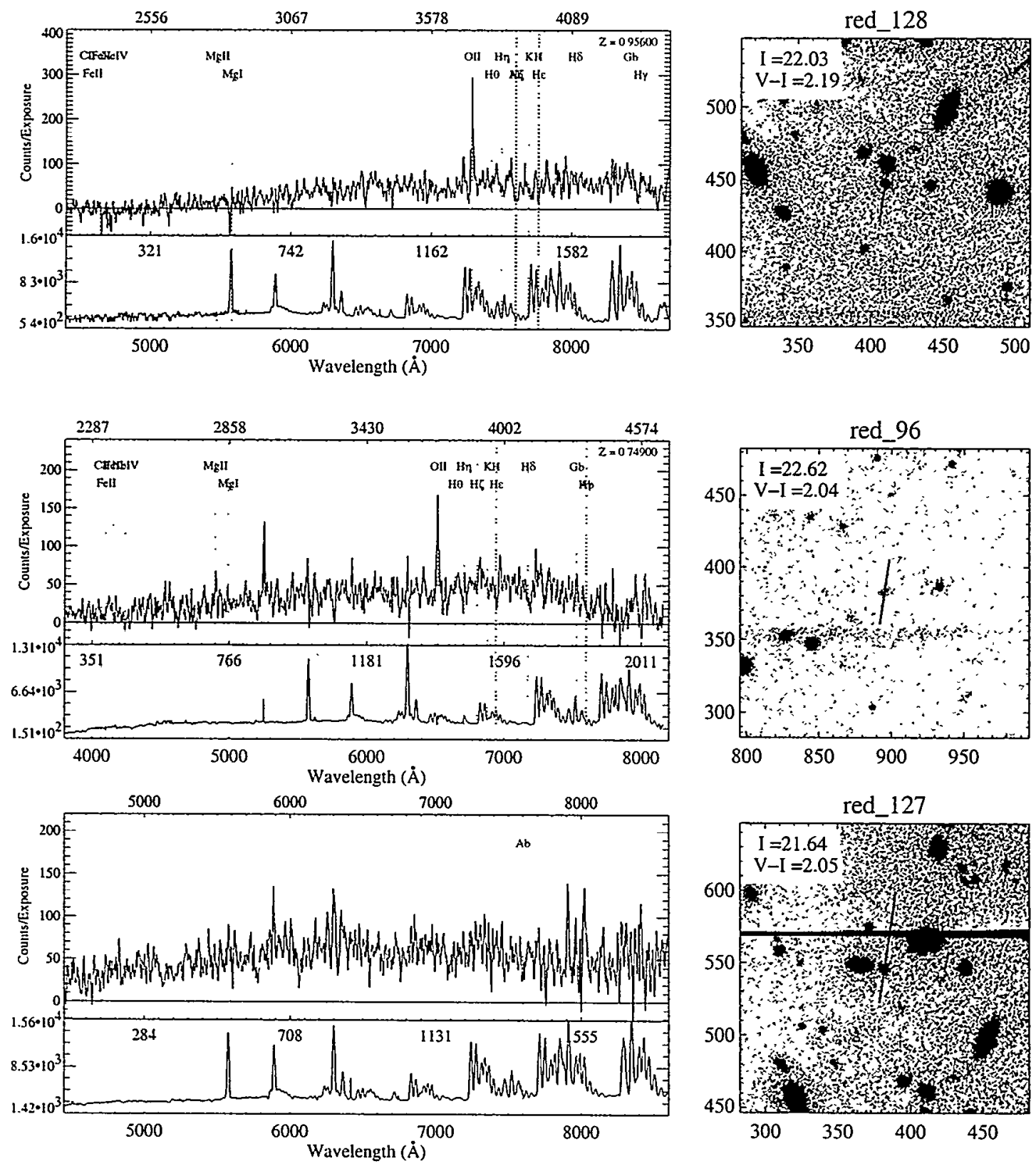

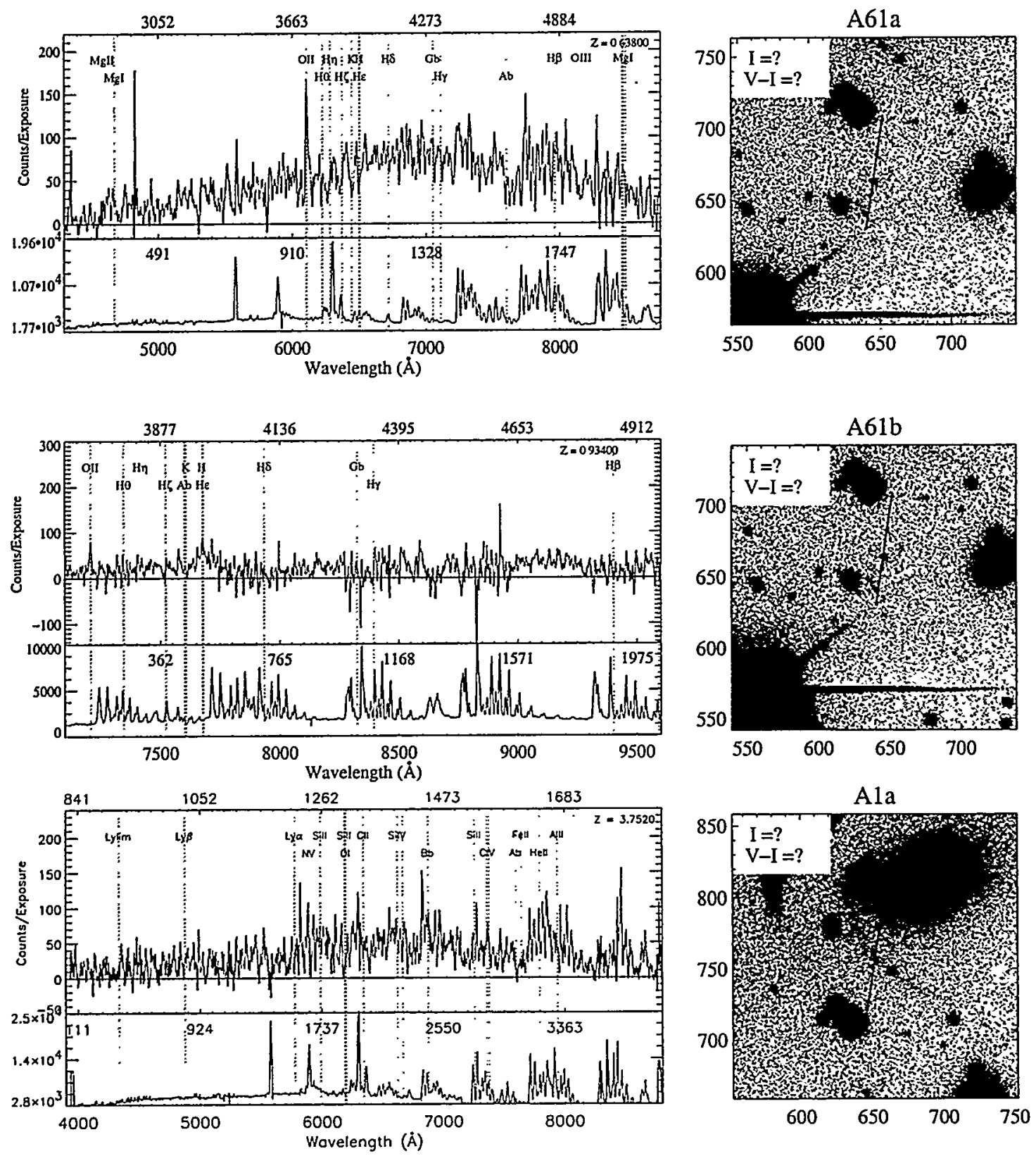

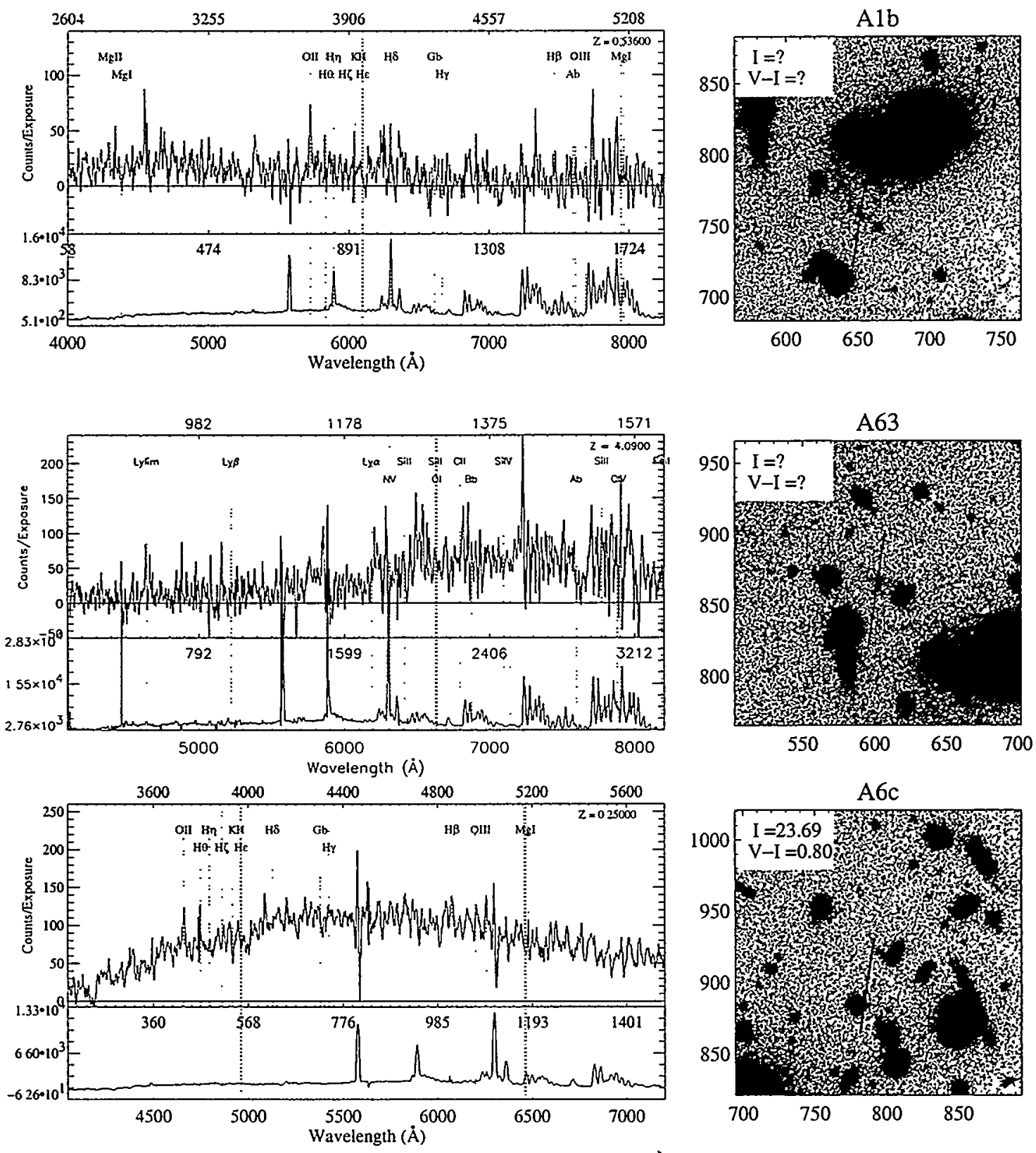

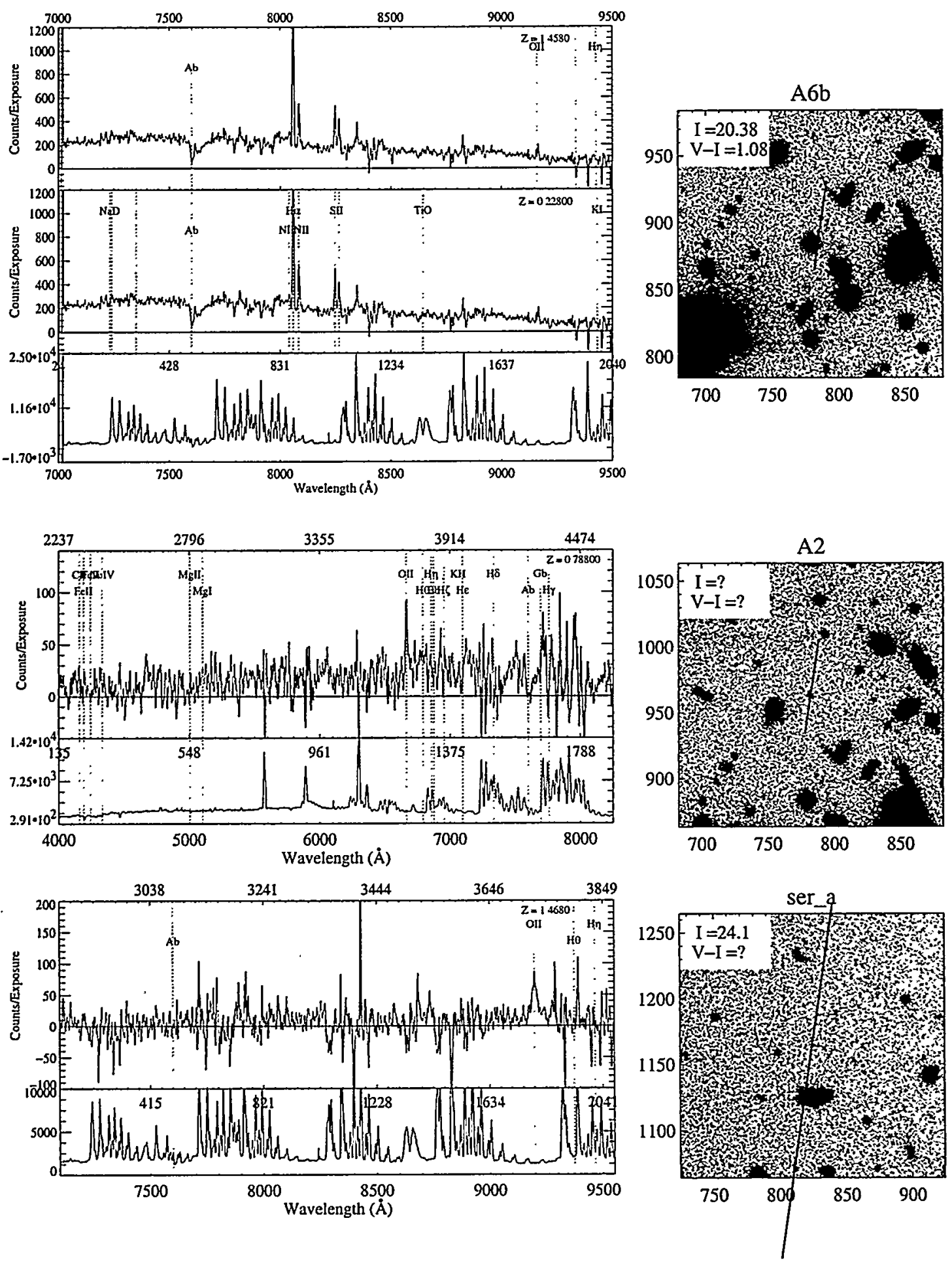

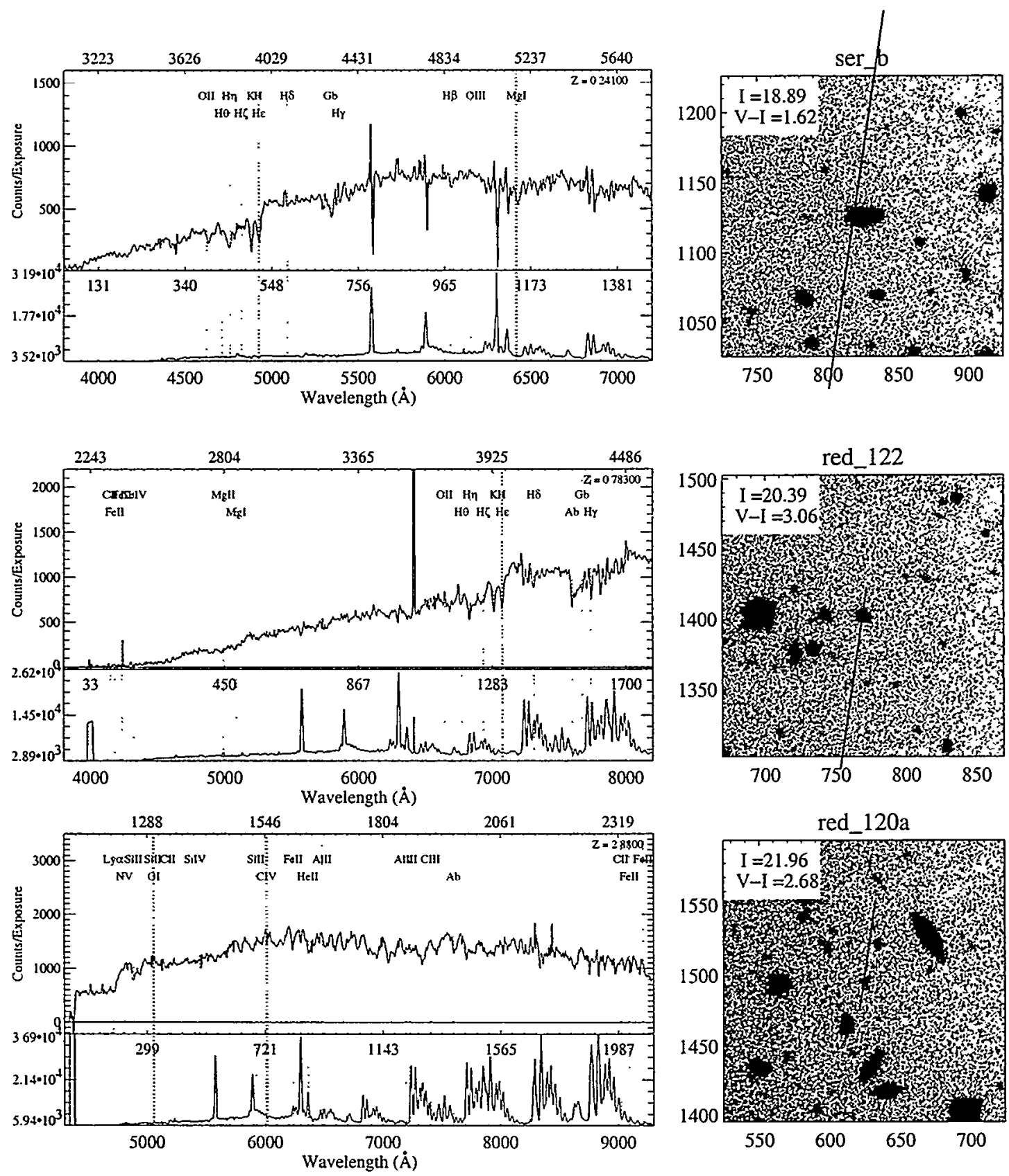

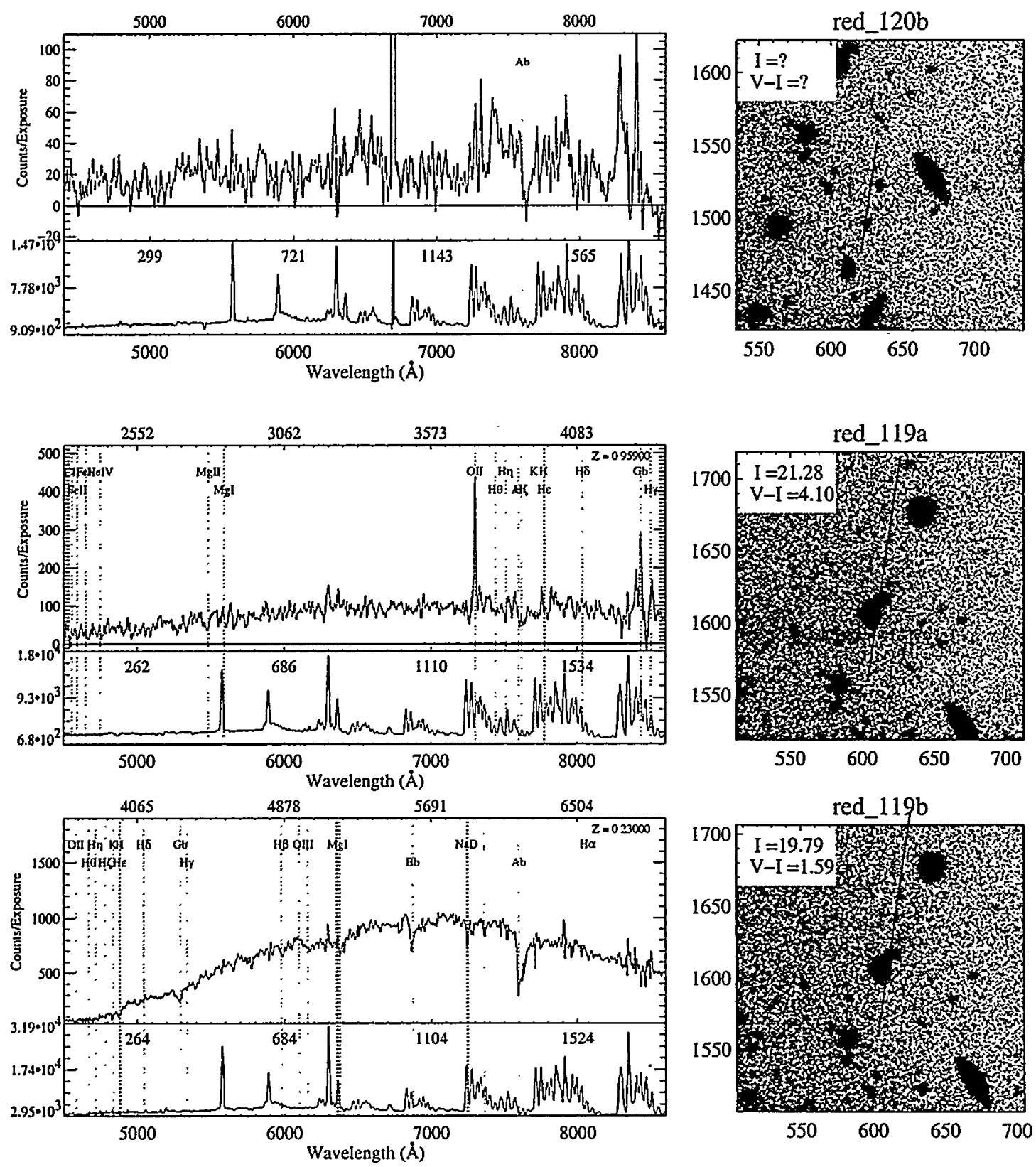

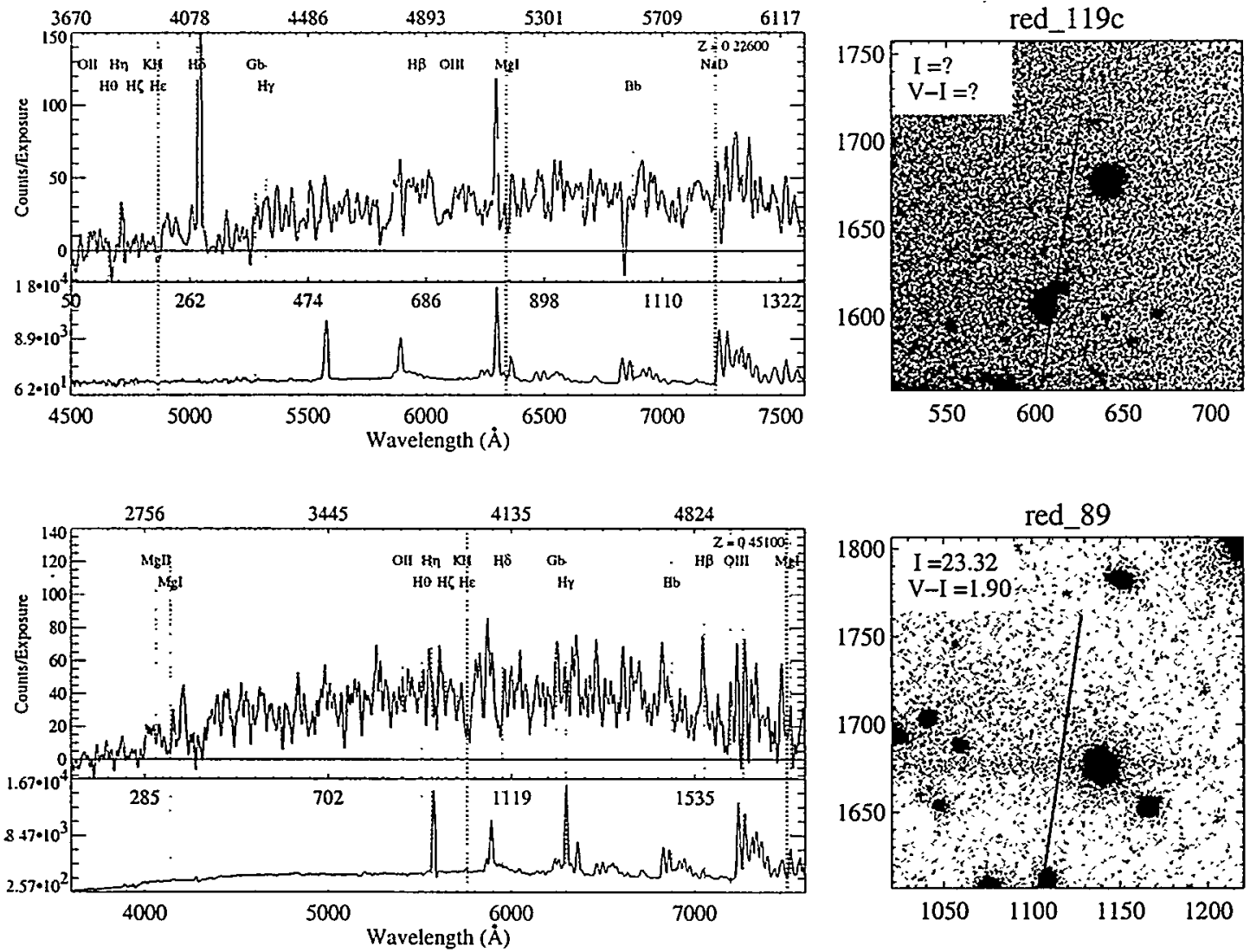


\section{Appendix $\mathrm{C}$}

A2218:

C.1 Mask 1 

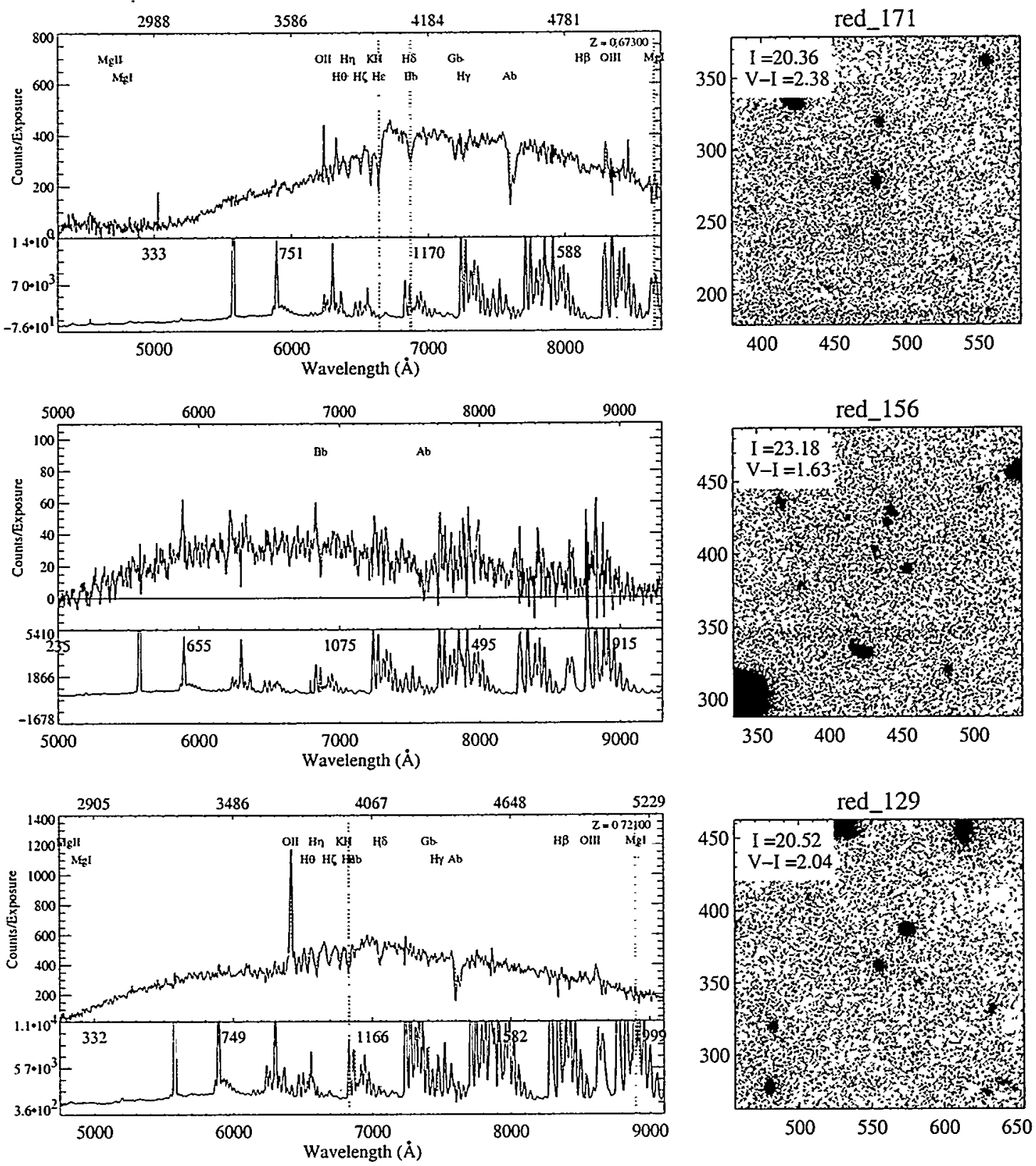

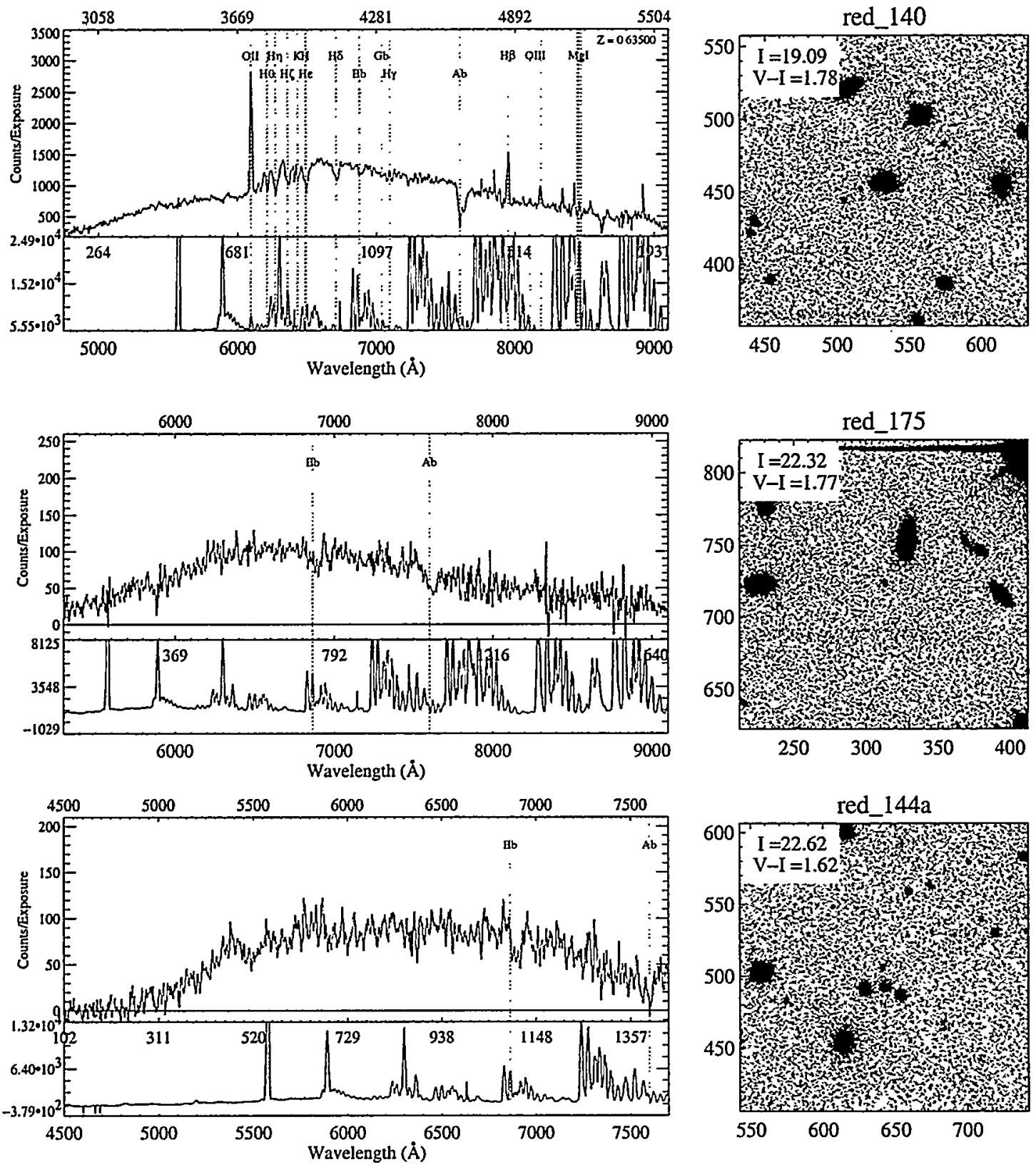

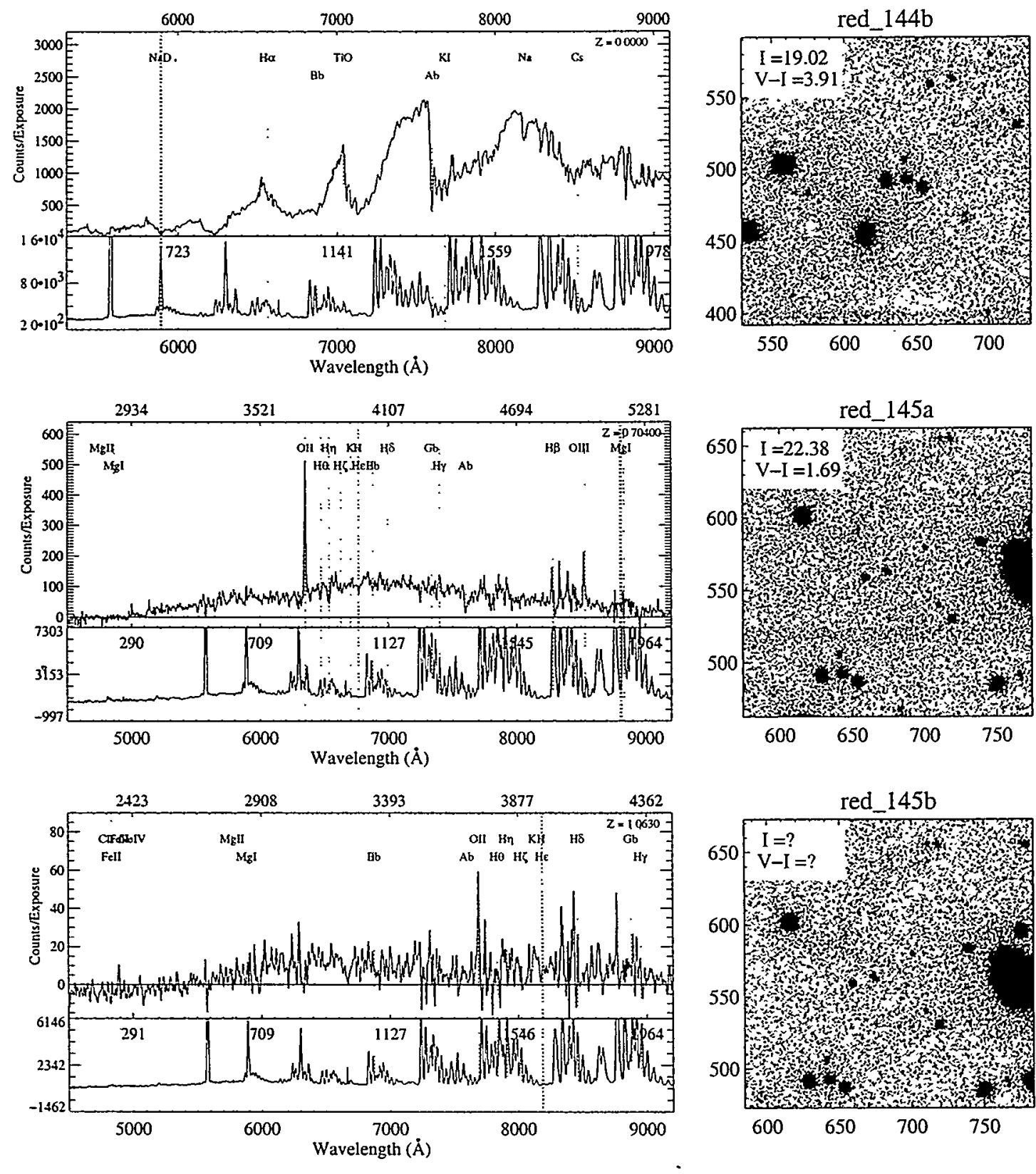

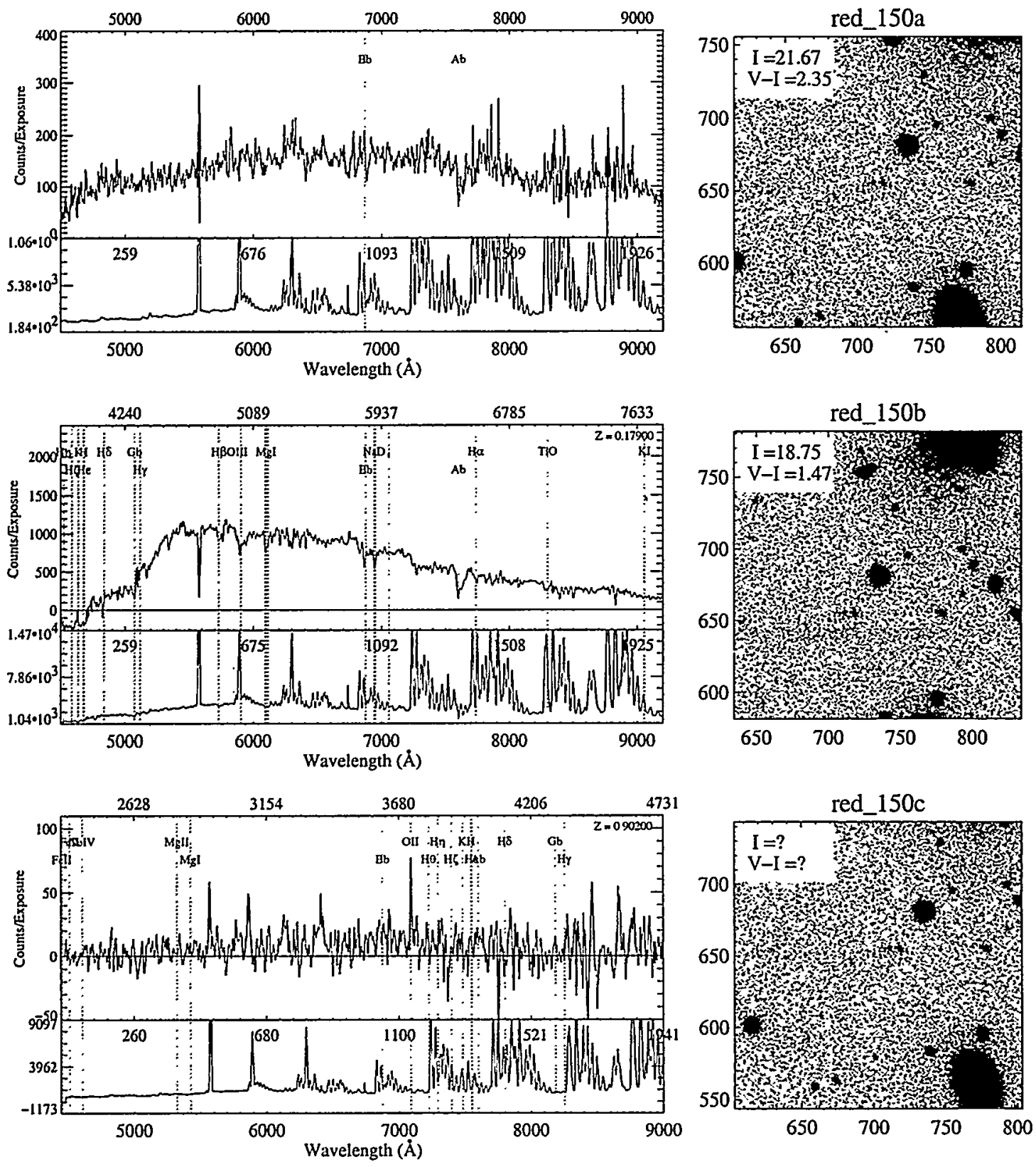

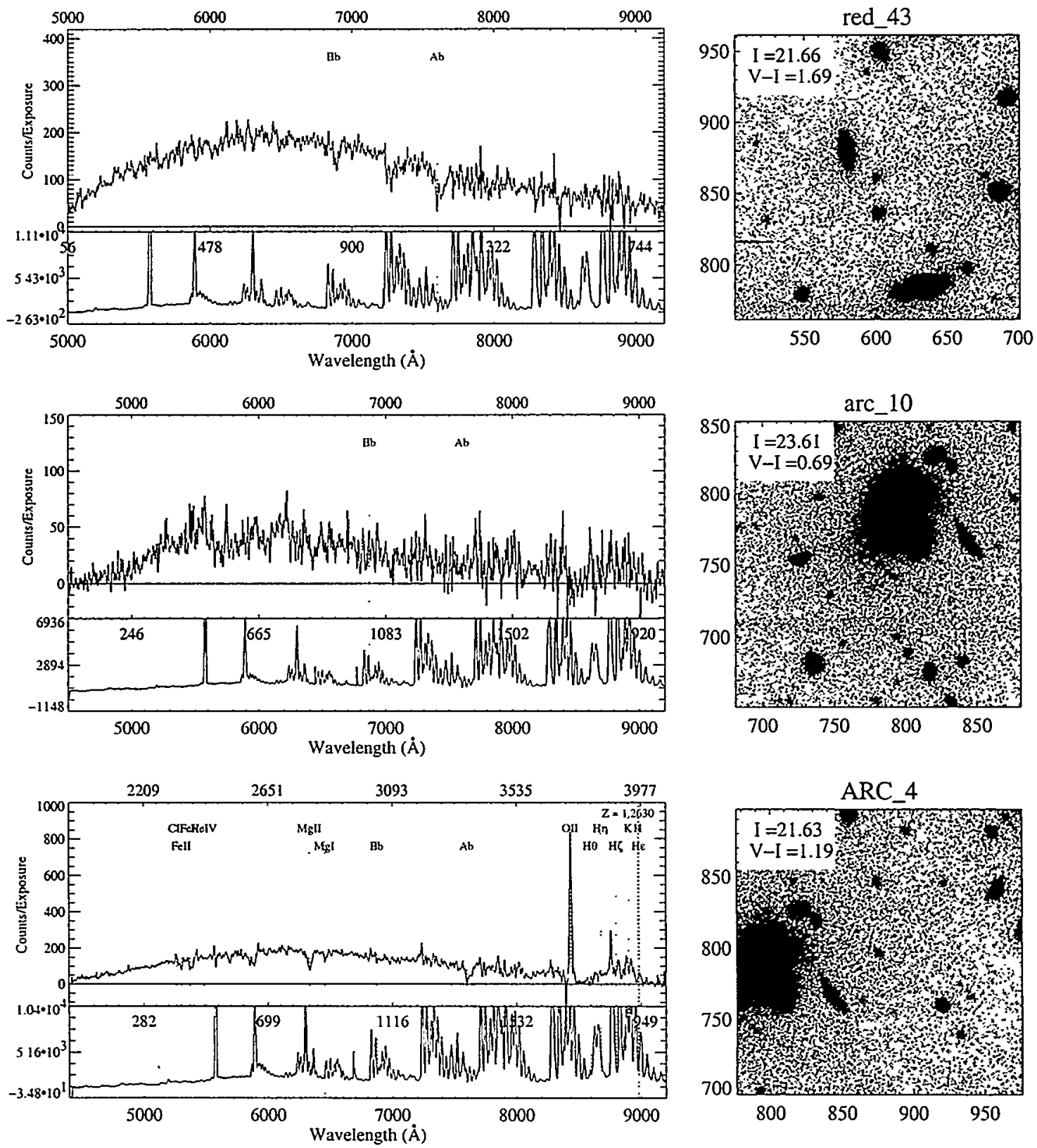

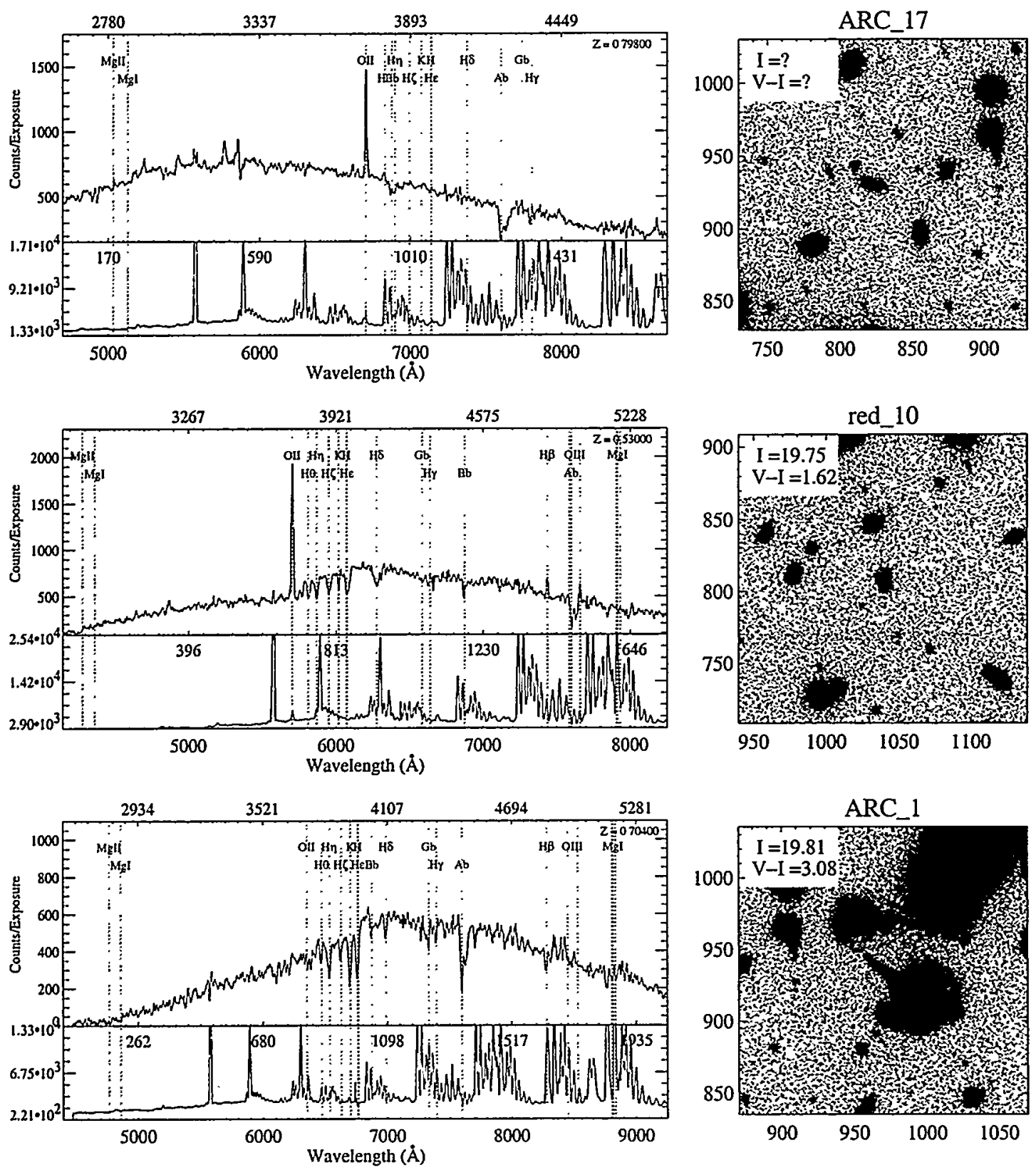

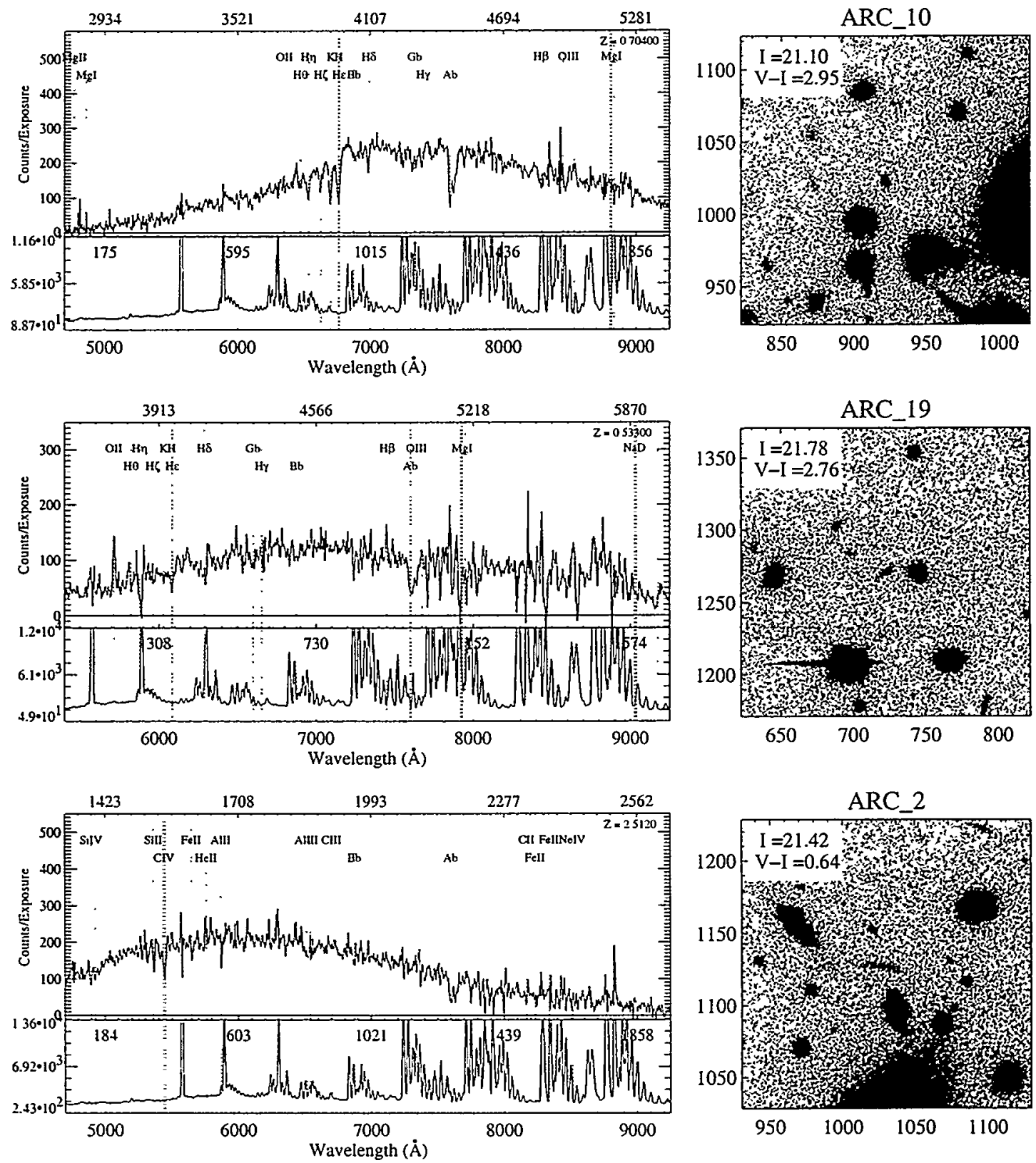

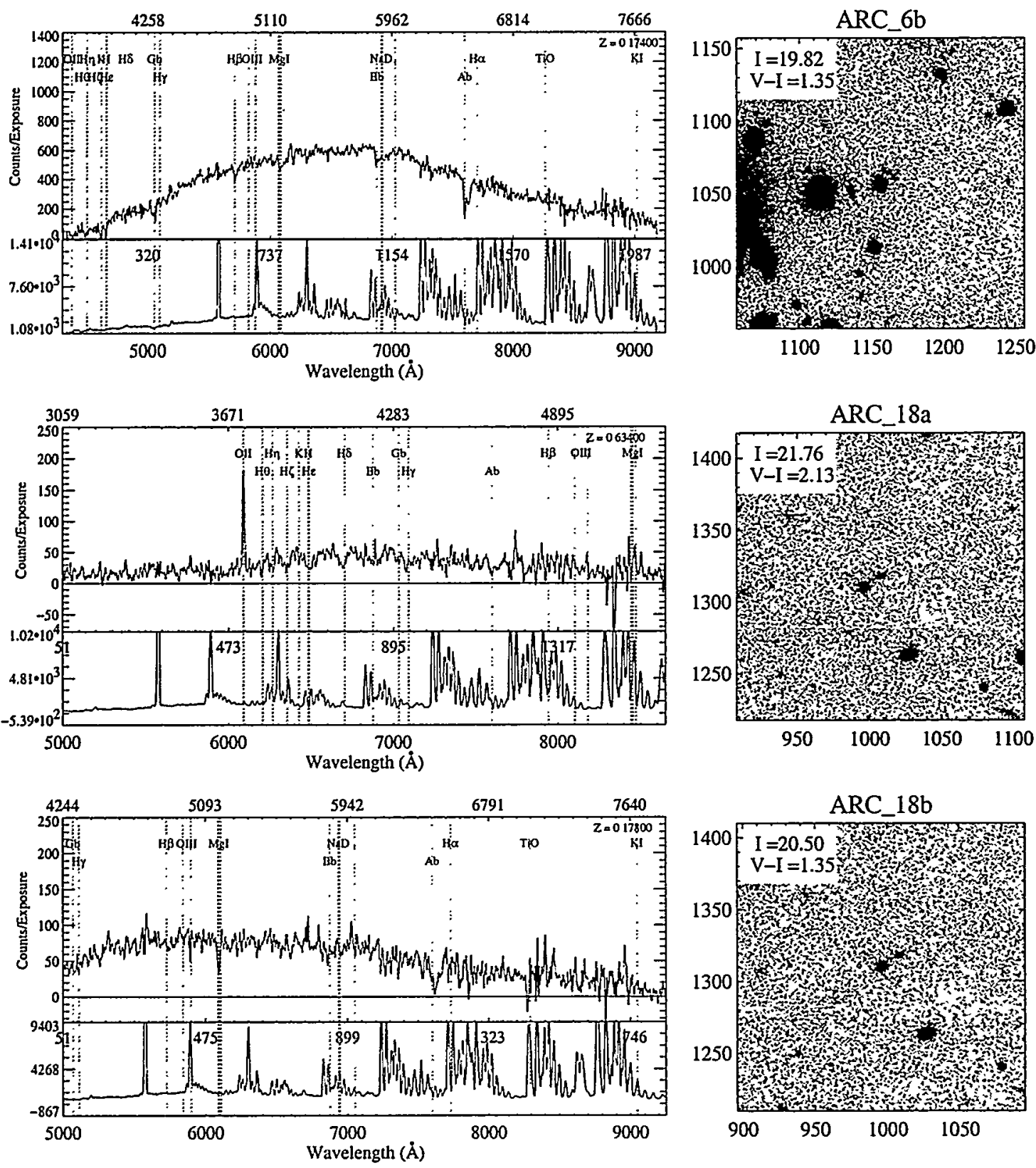

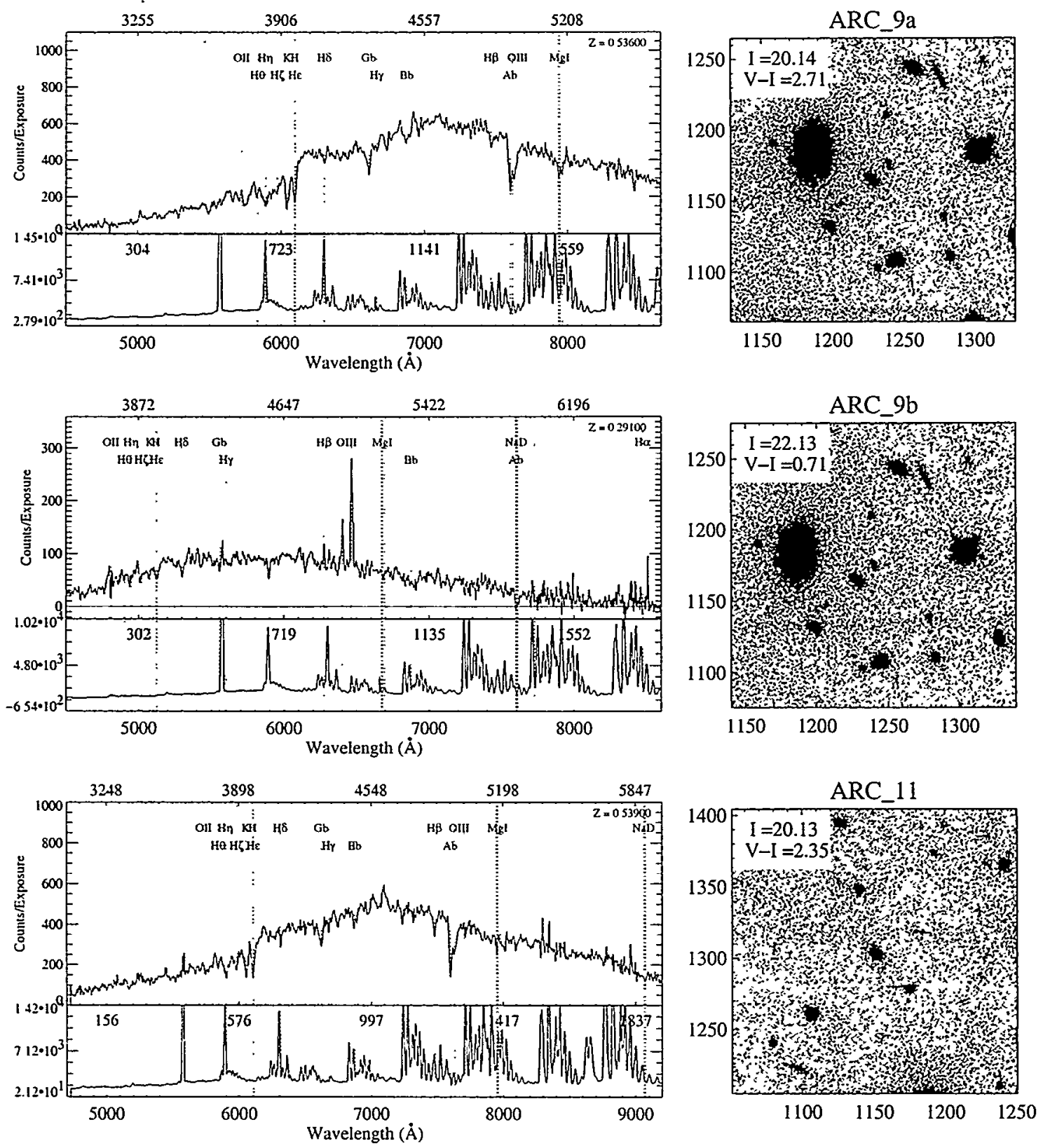

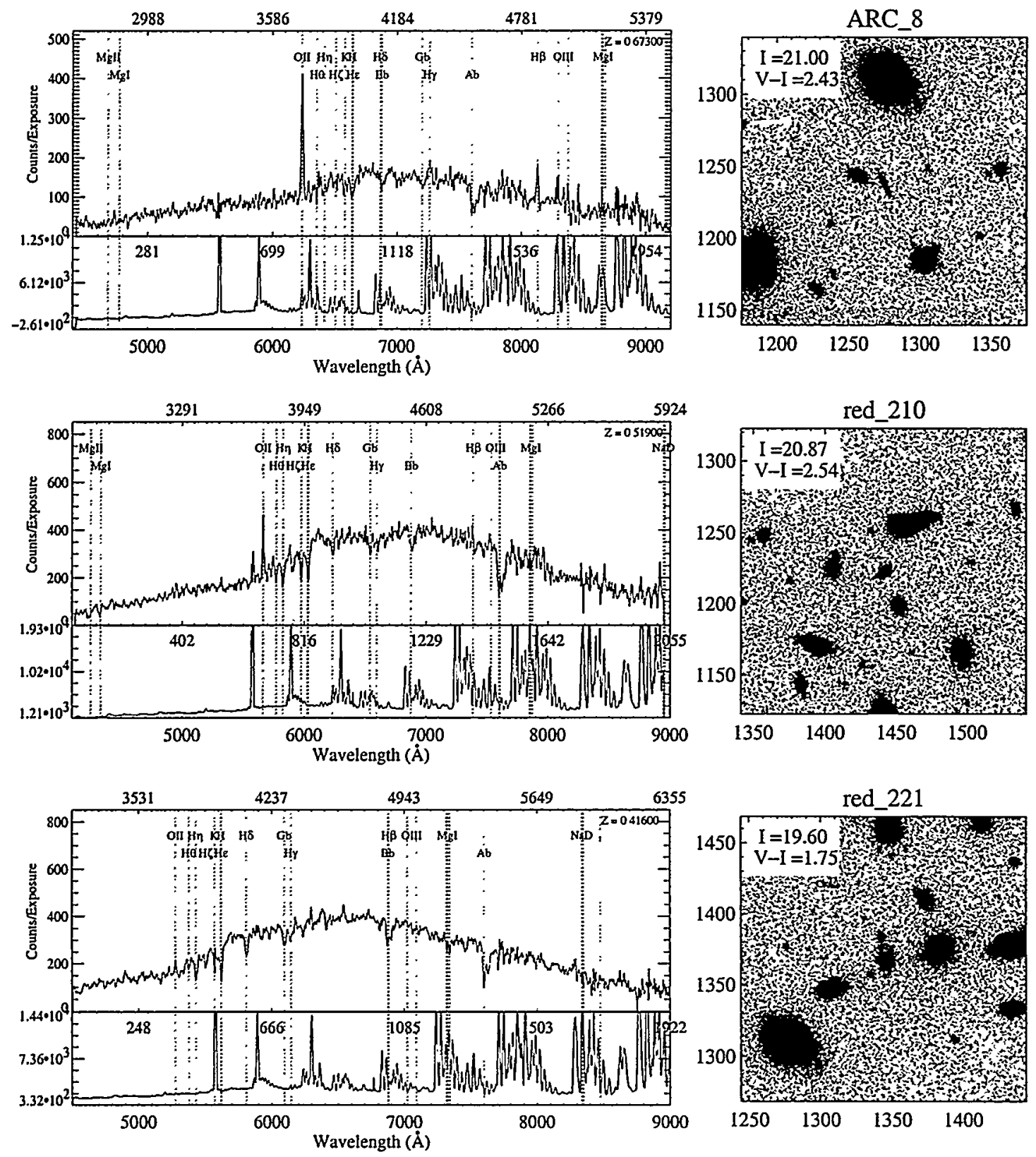

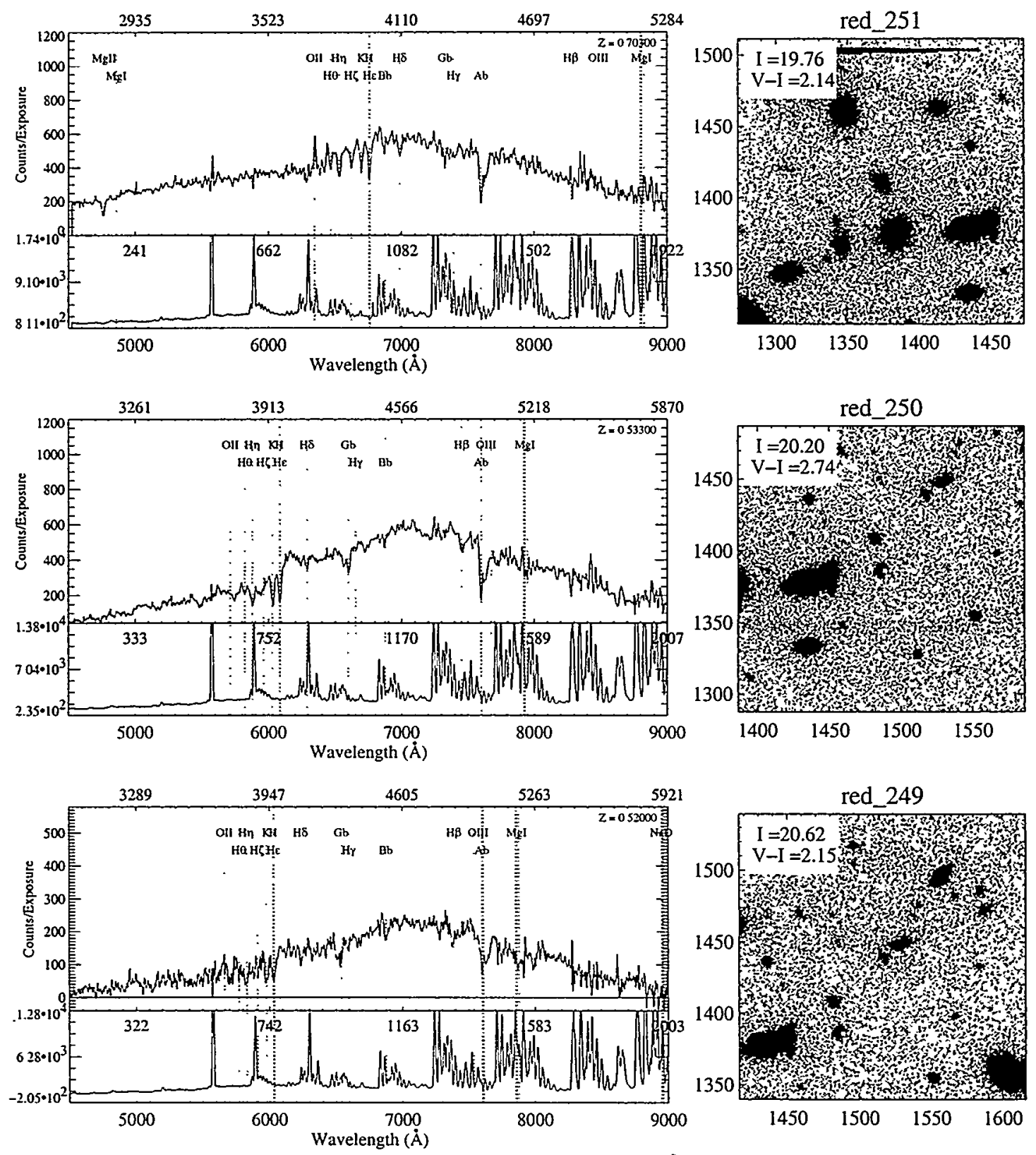

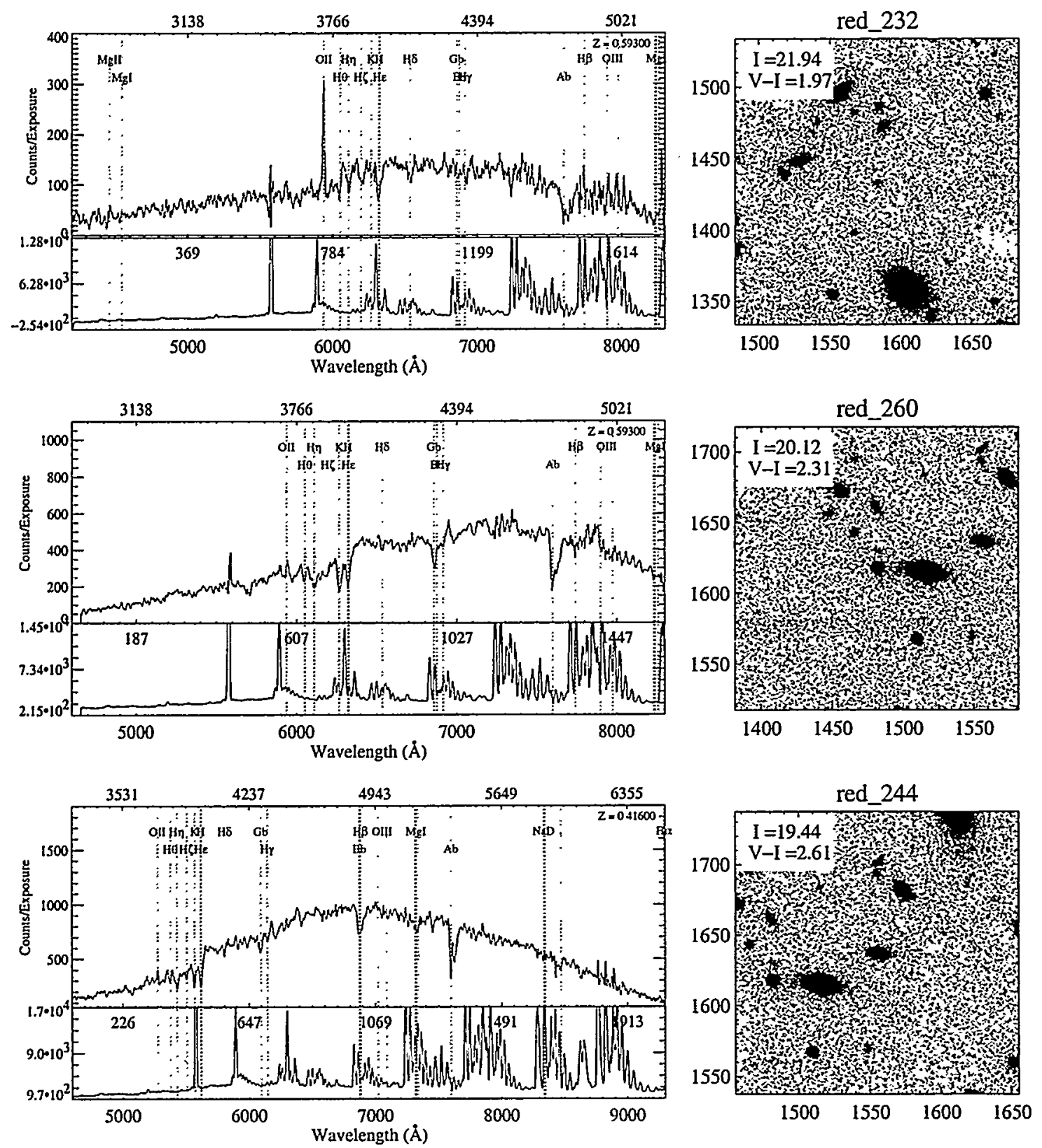

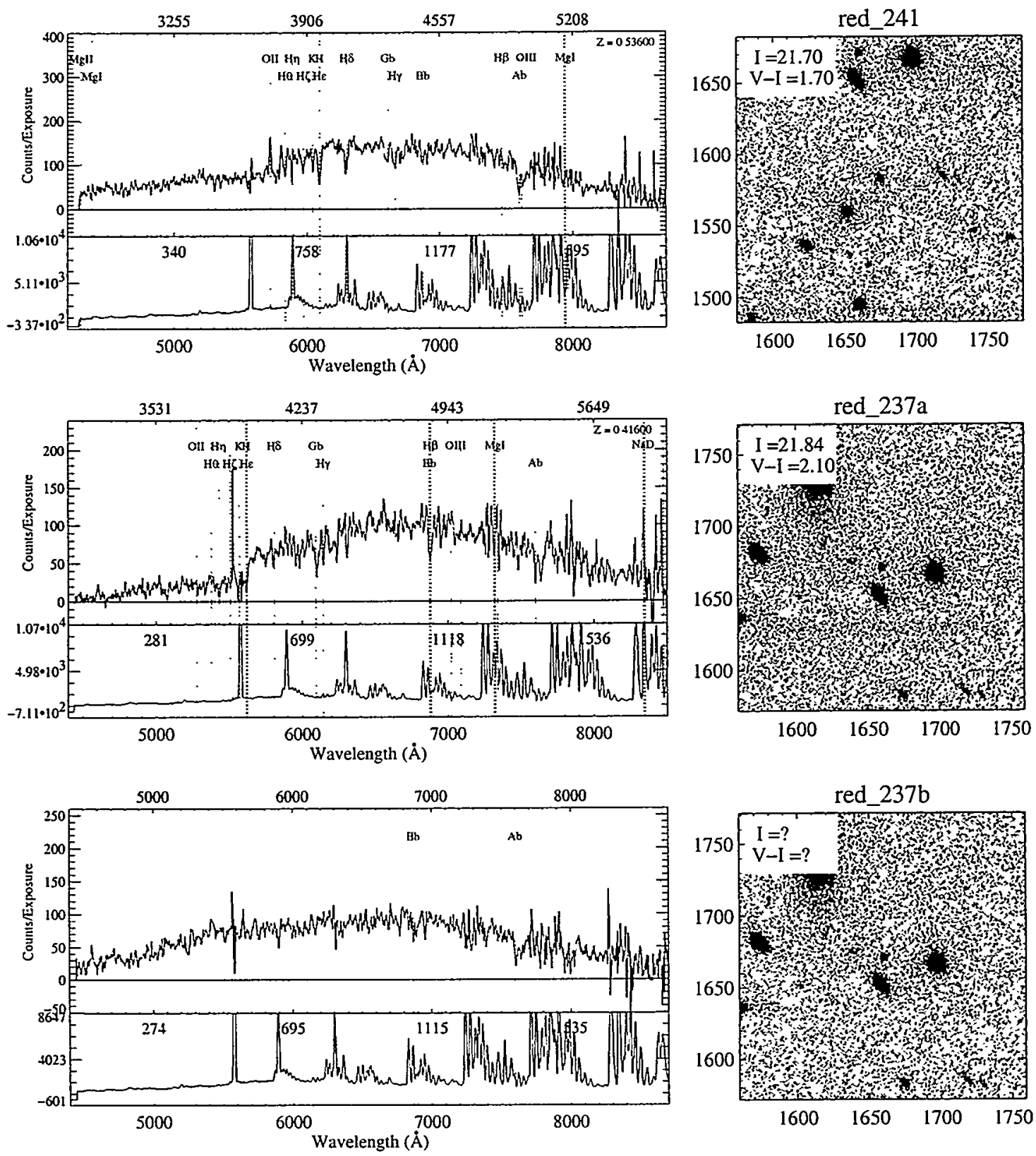

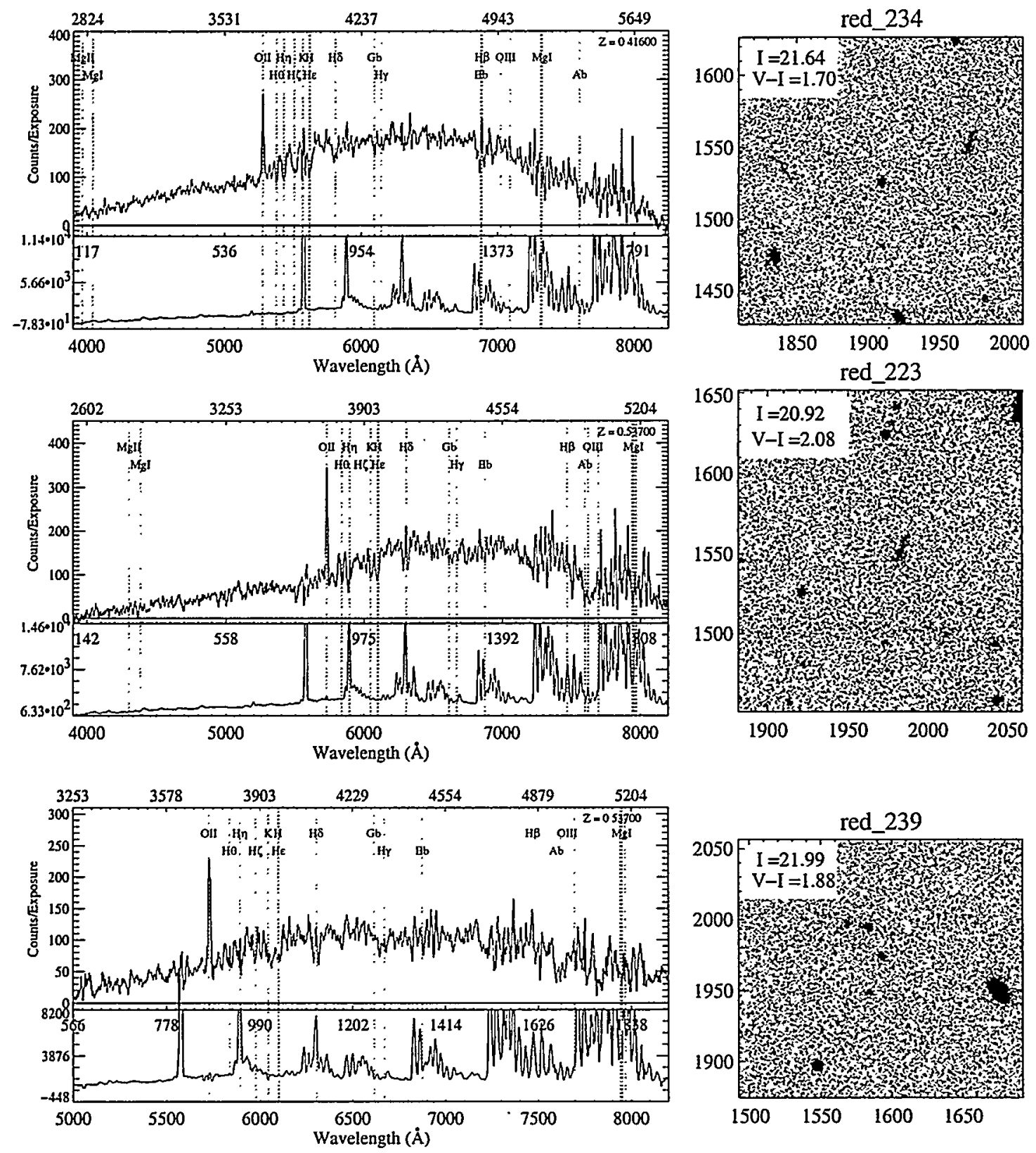

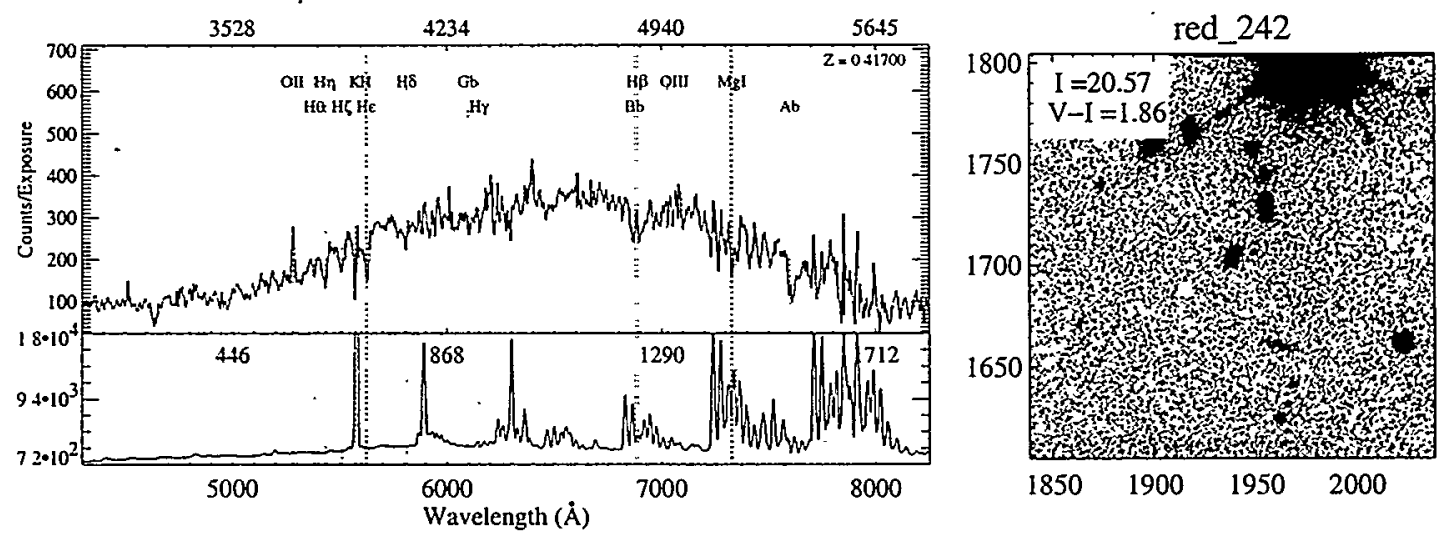


\section{C.2 Mask 2}



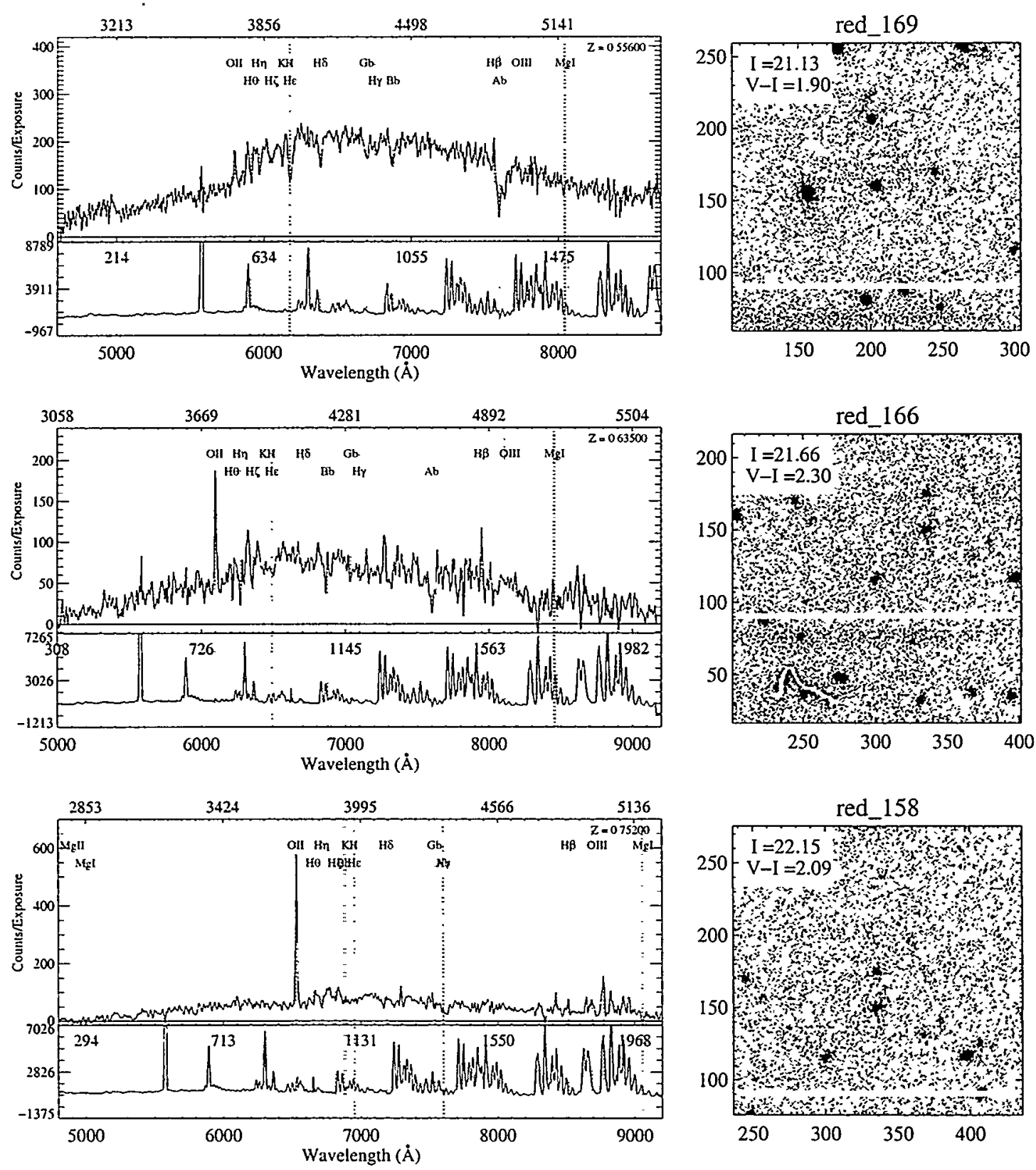

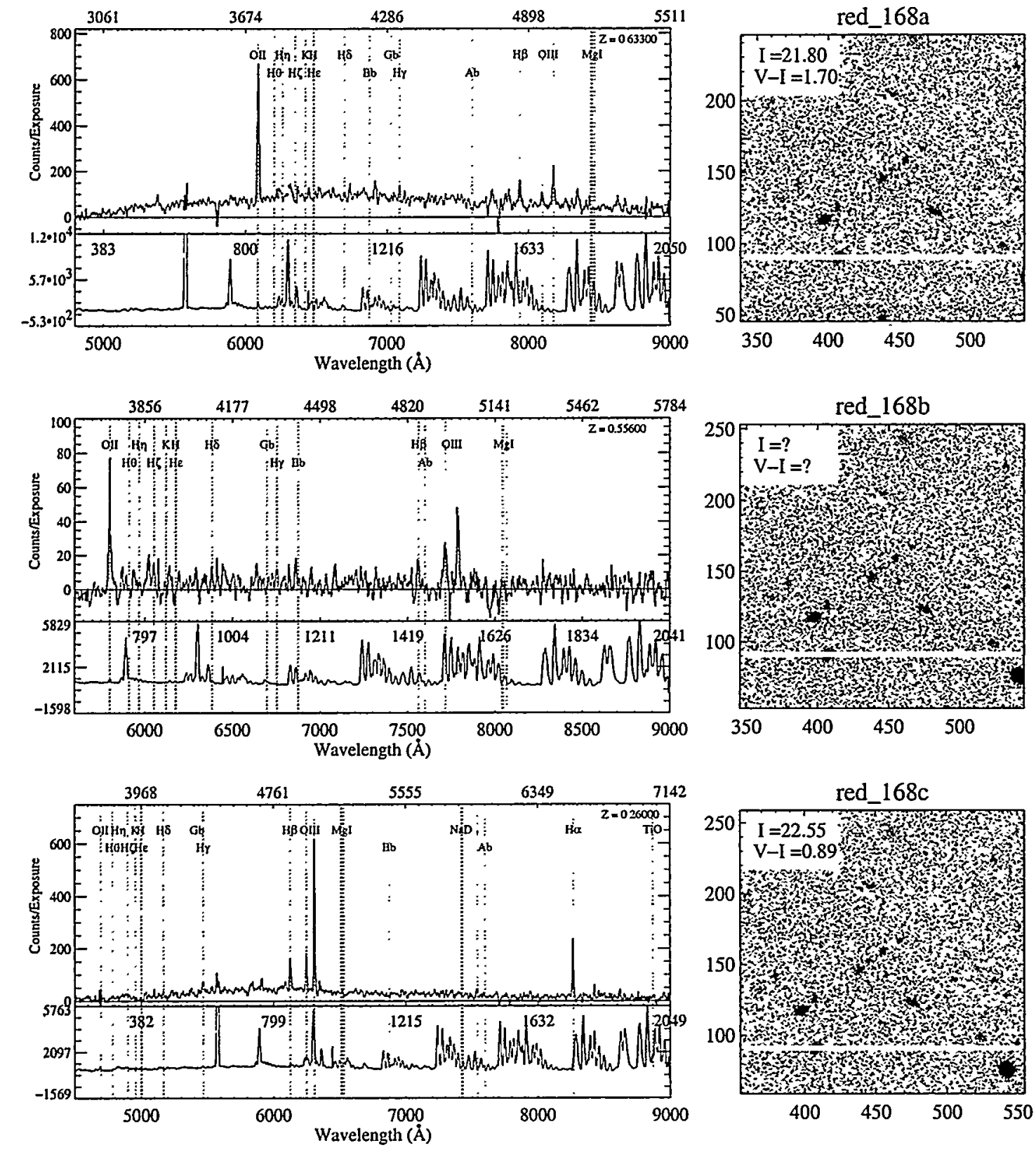

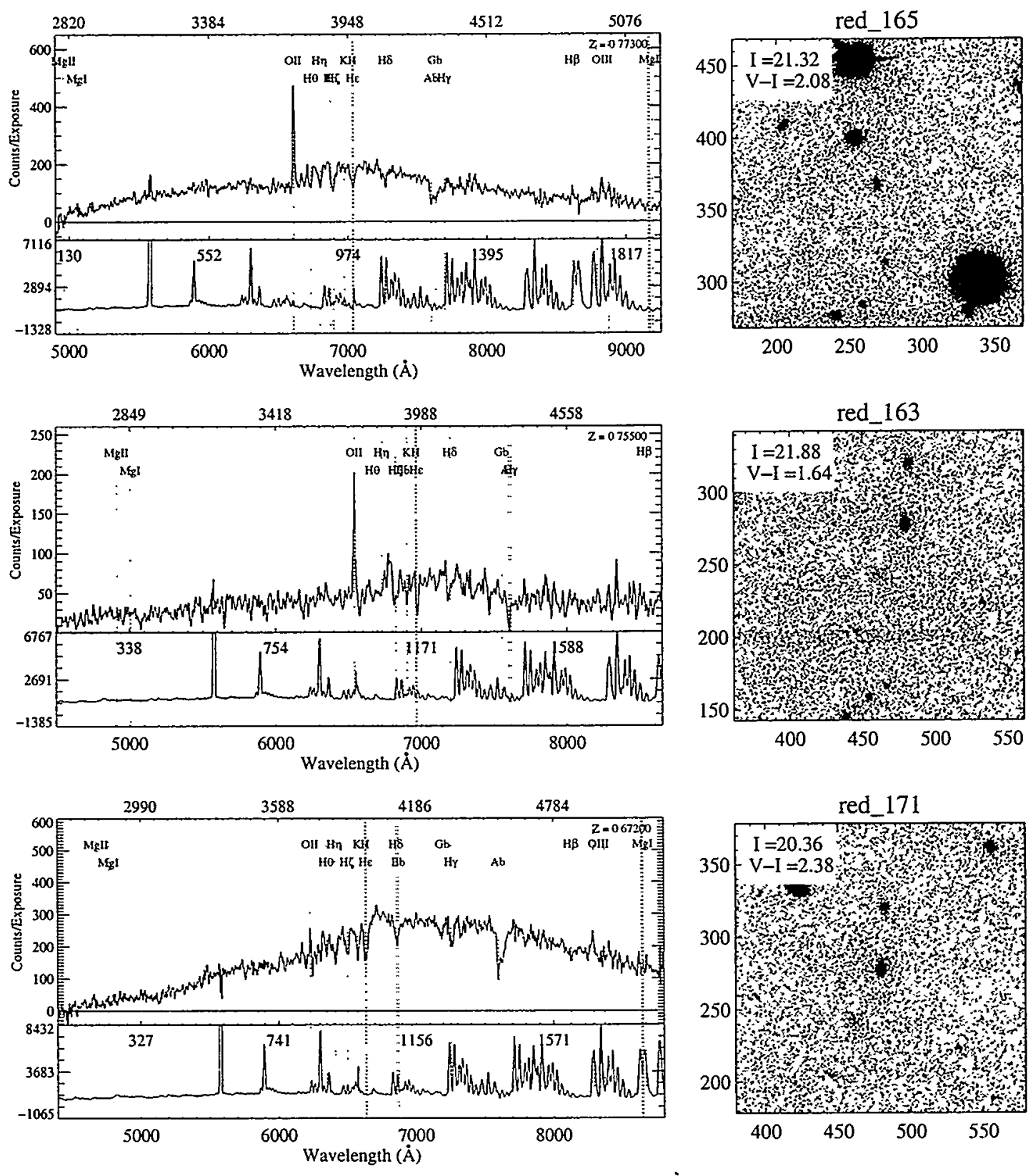

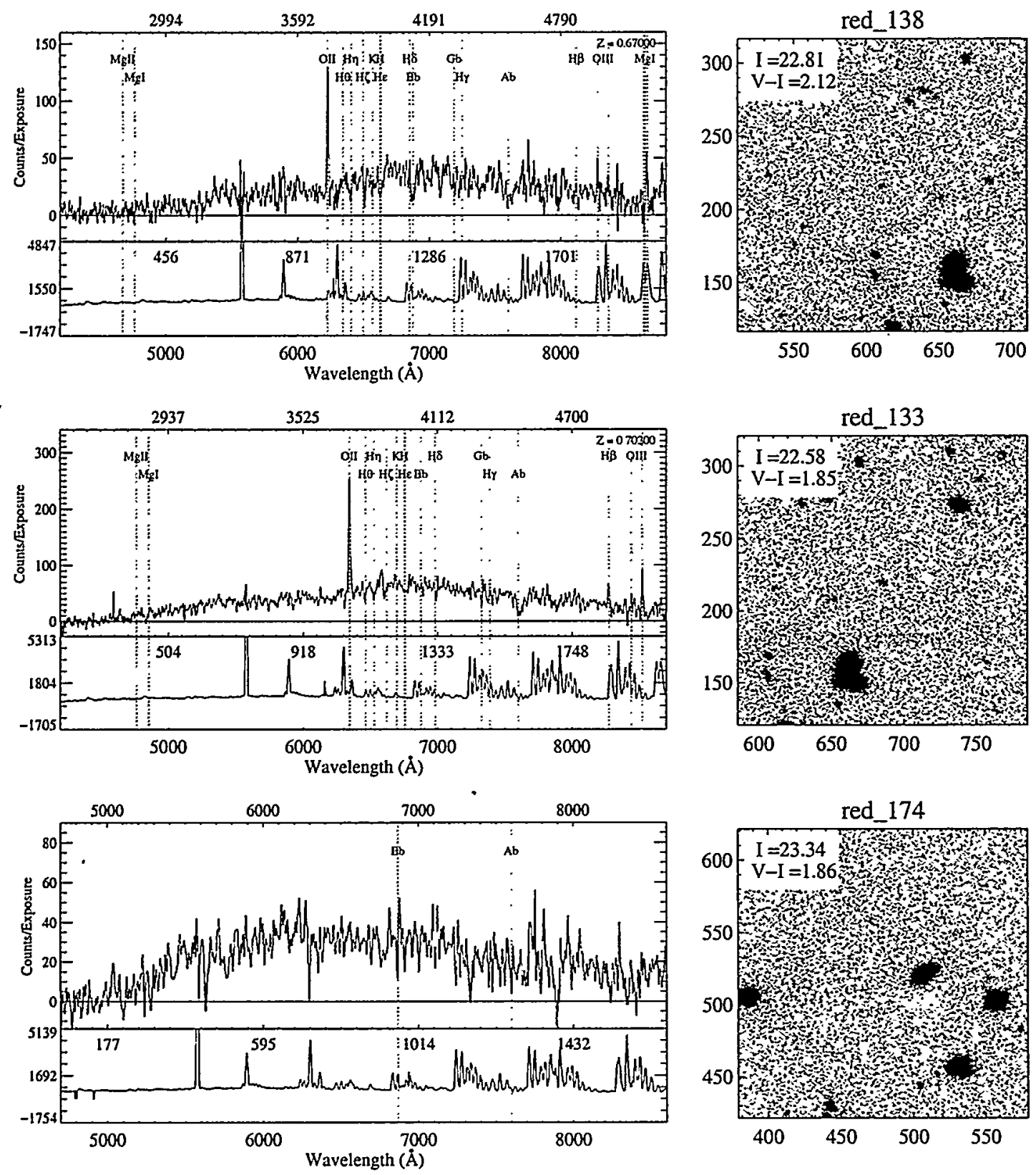

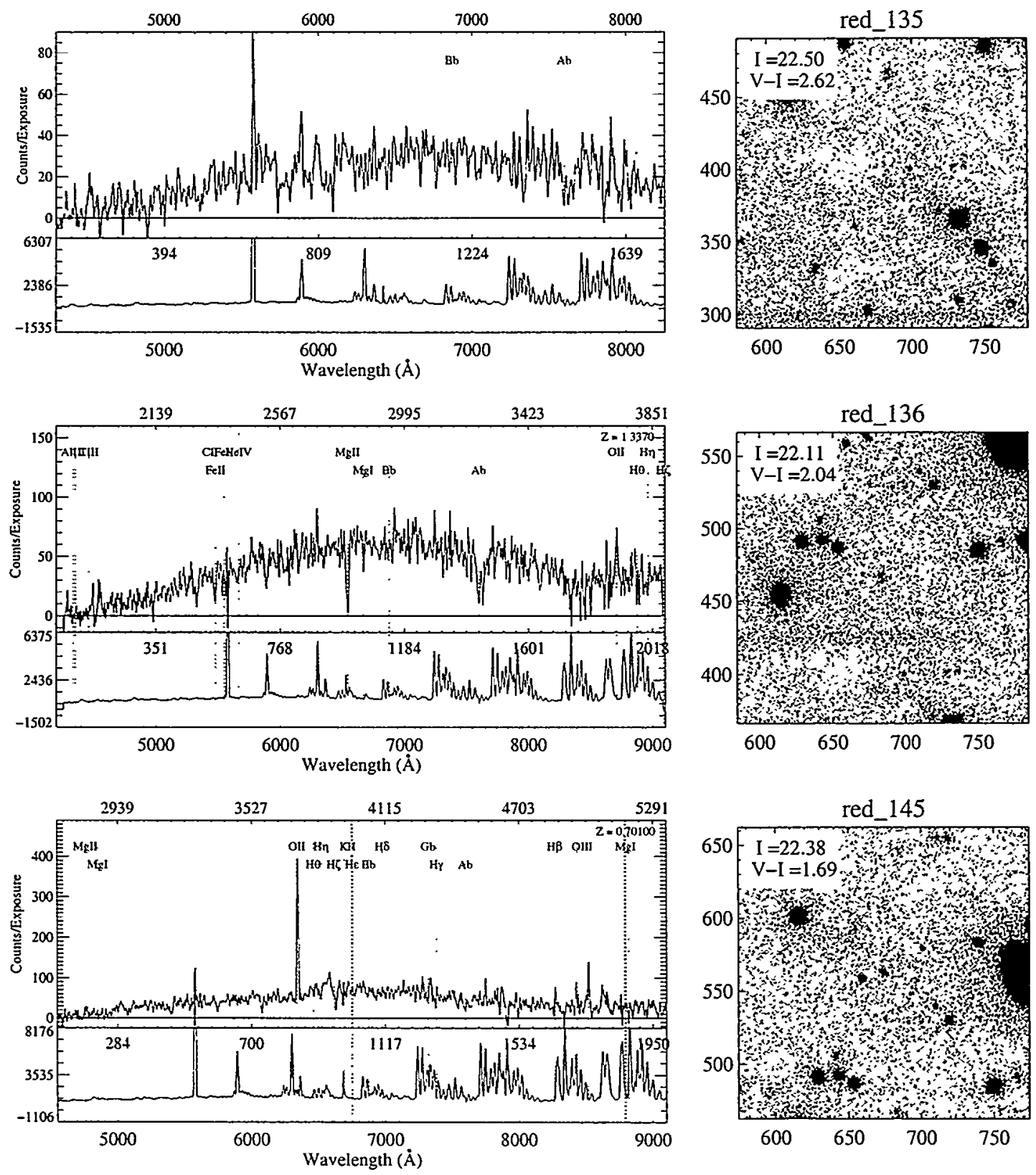

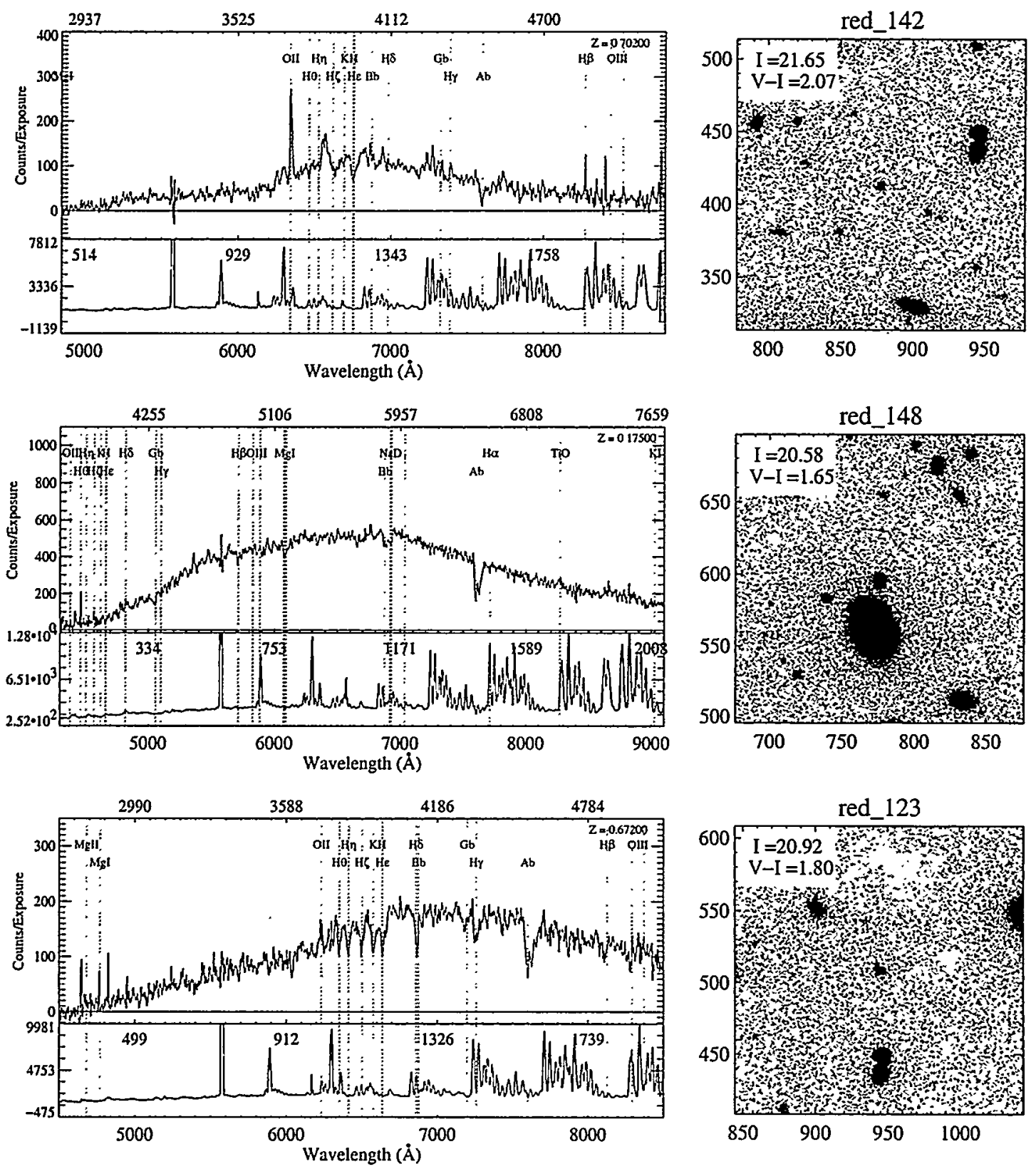

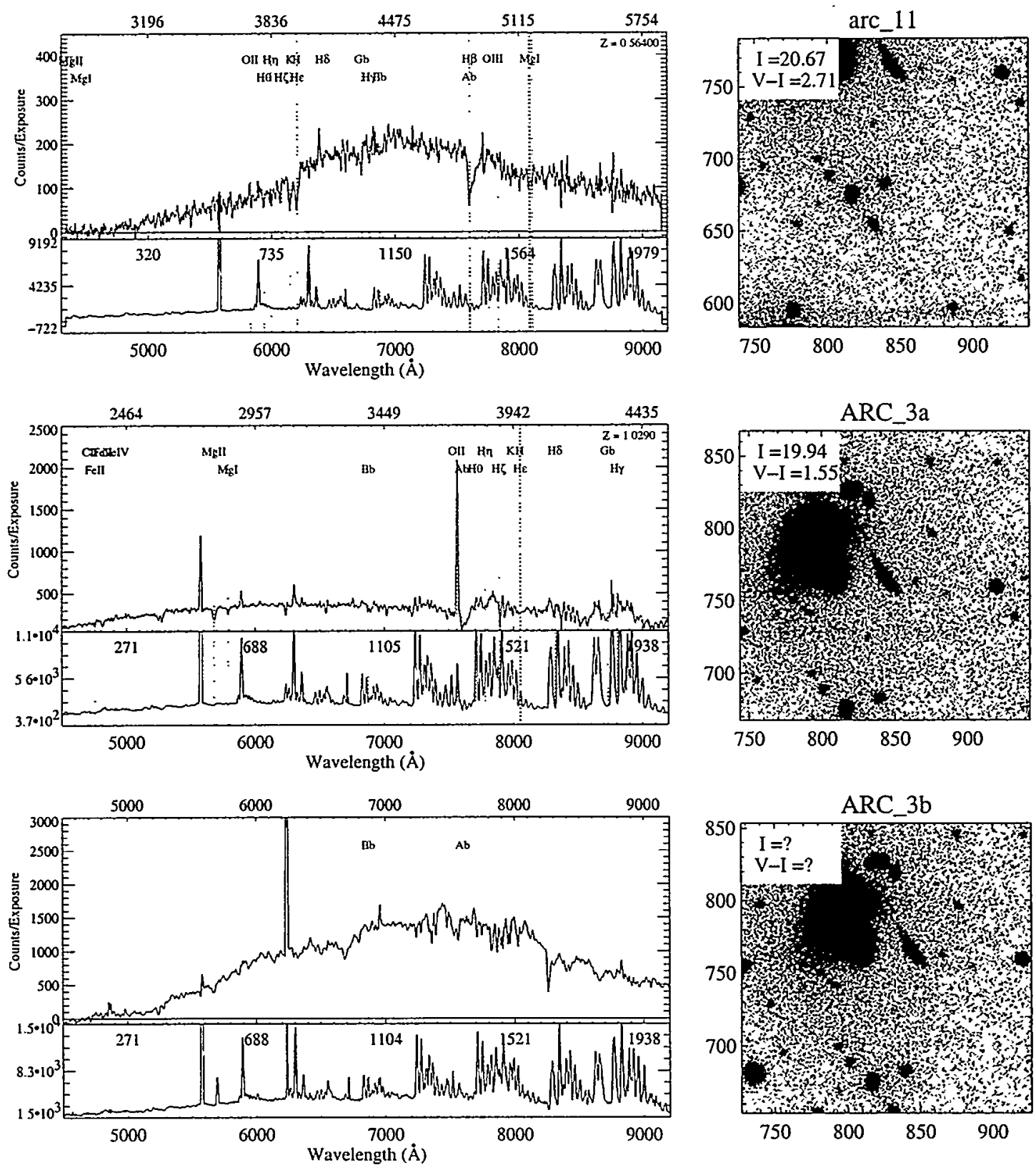

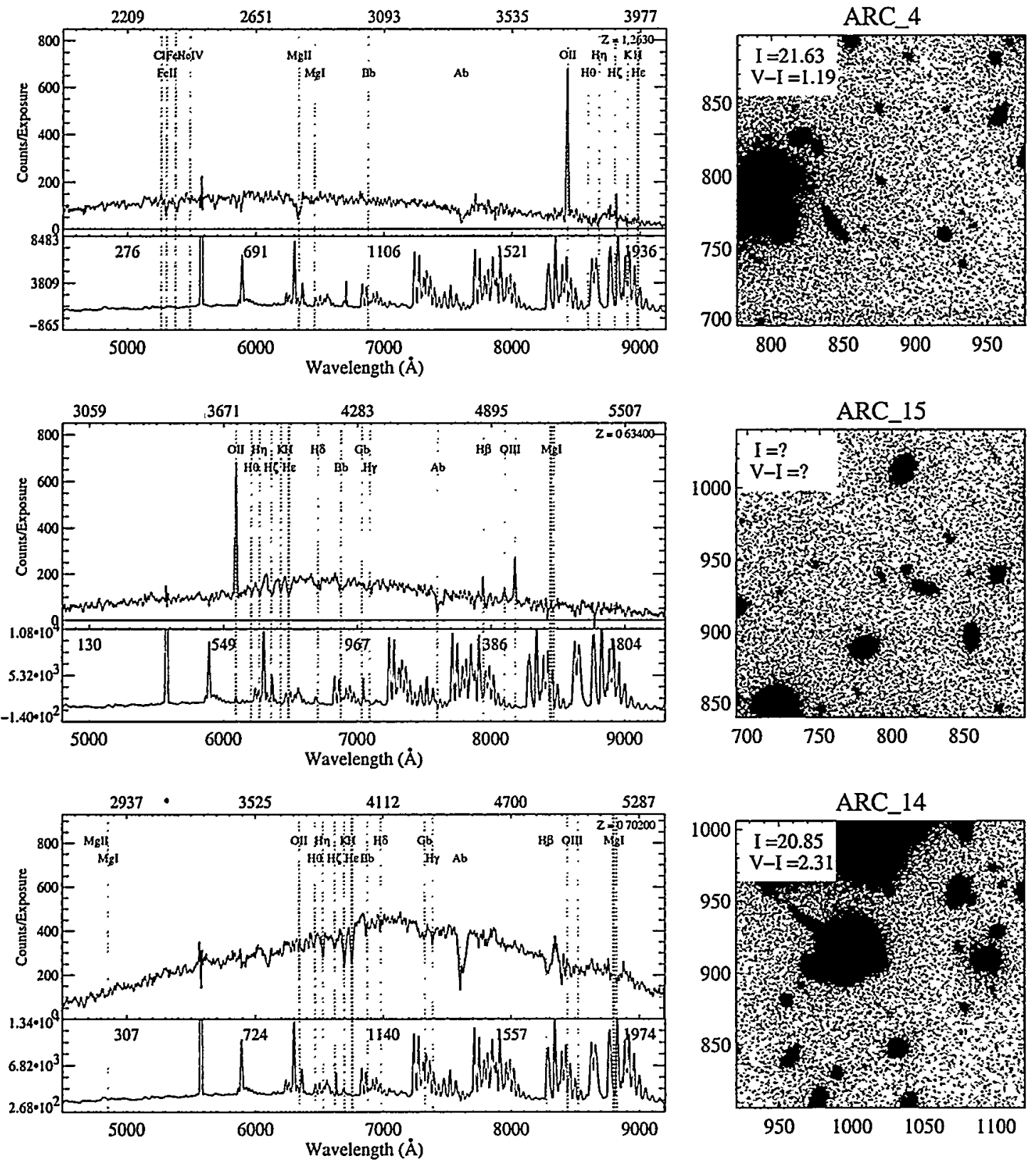

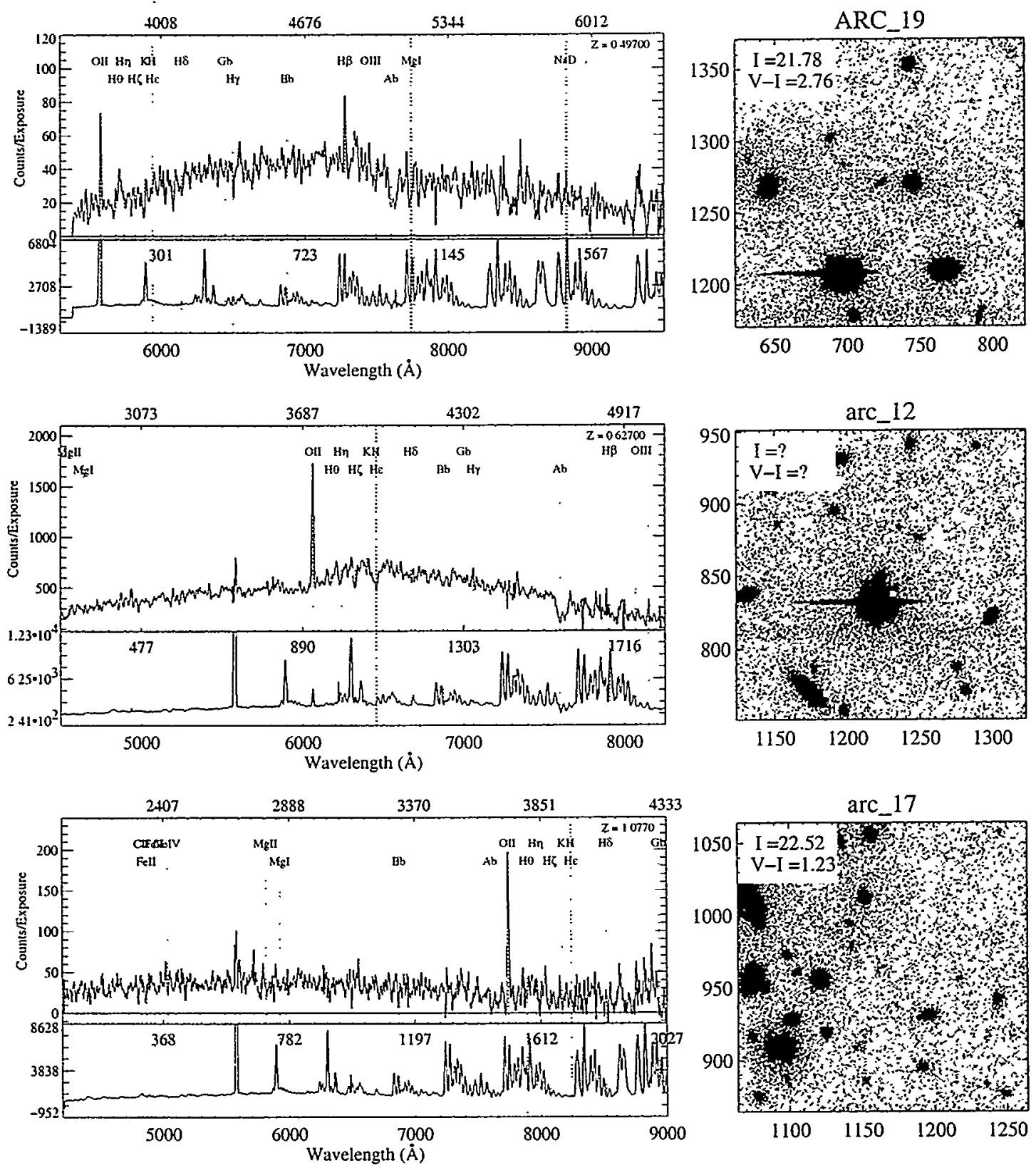

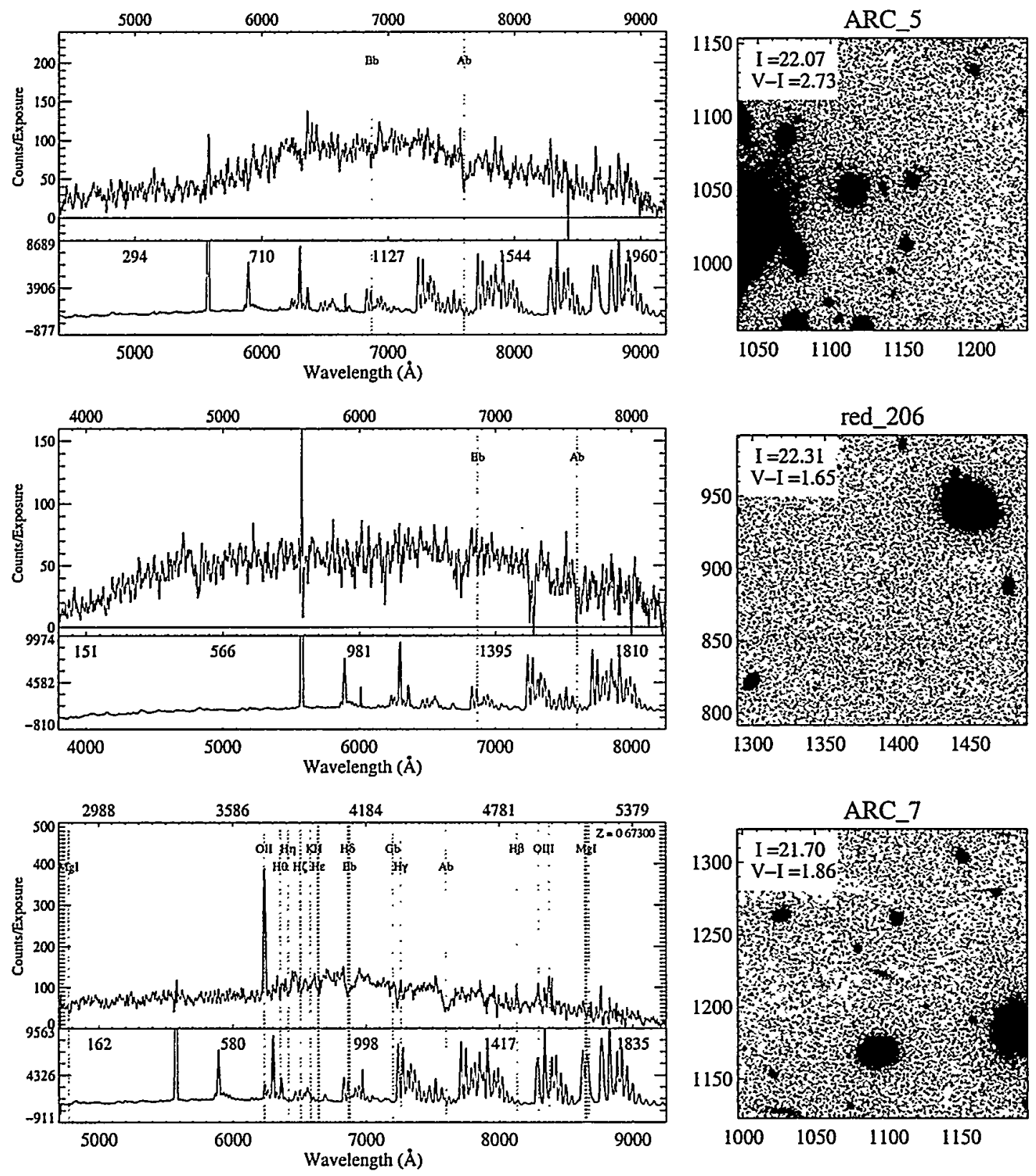

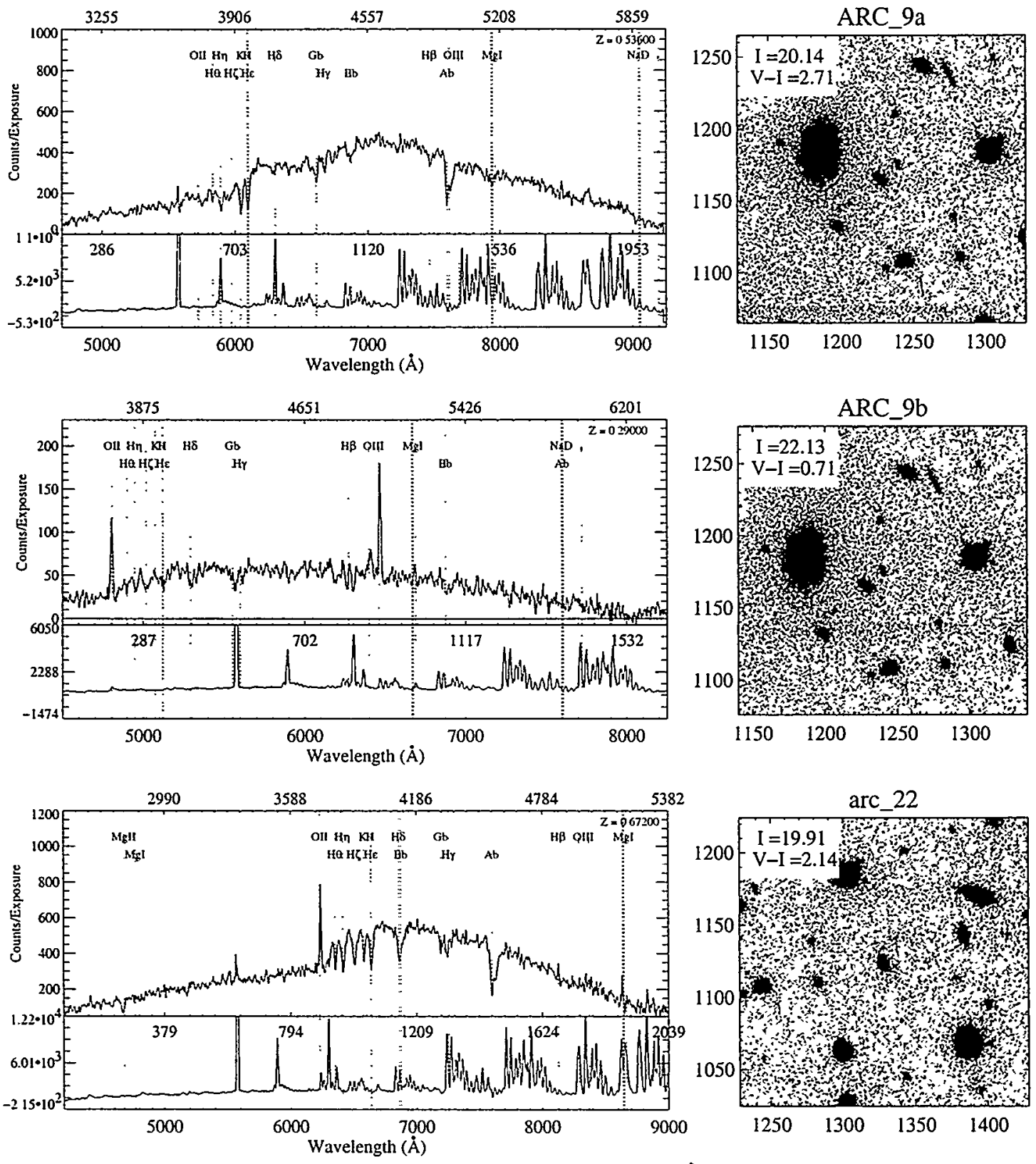

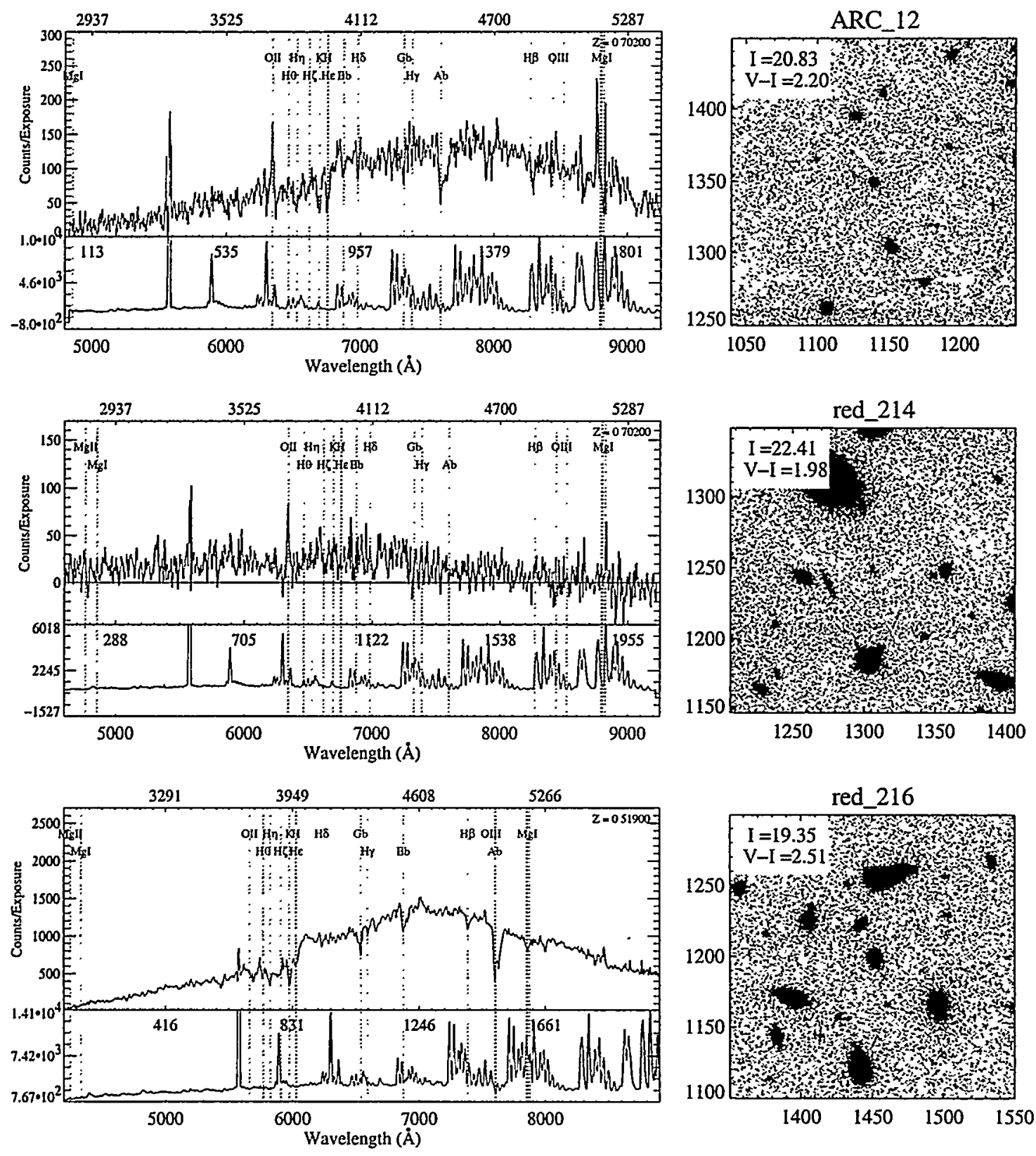

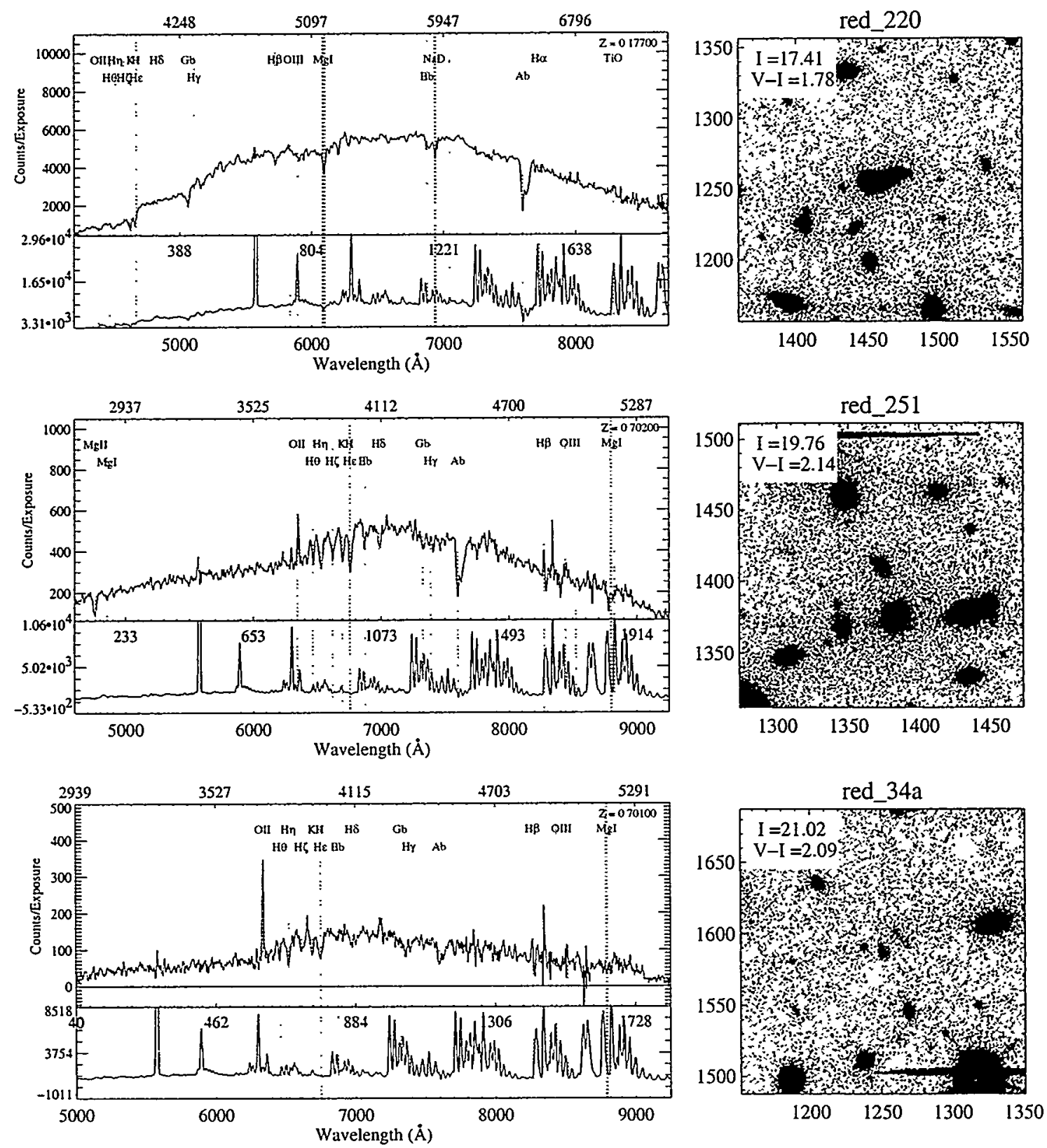

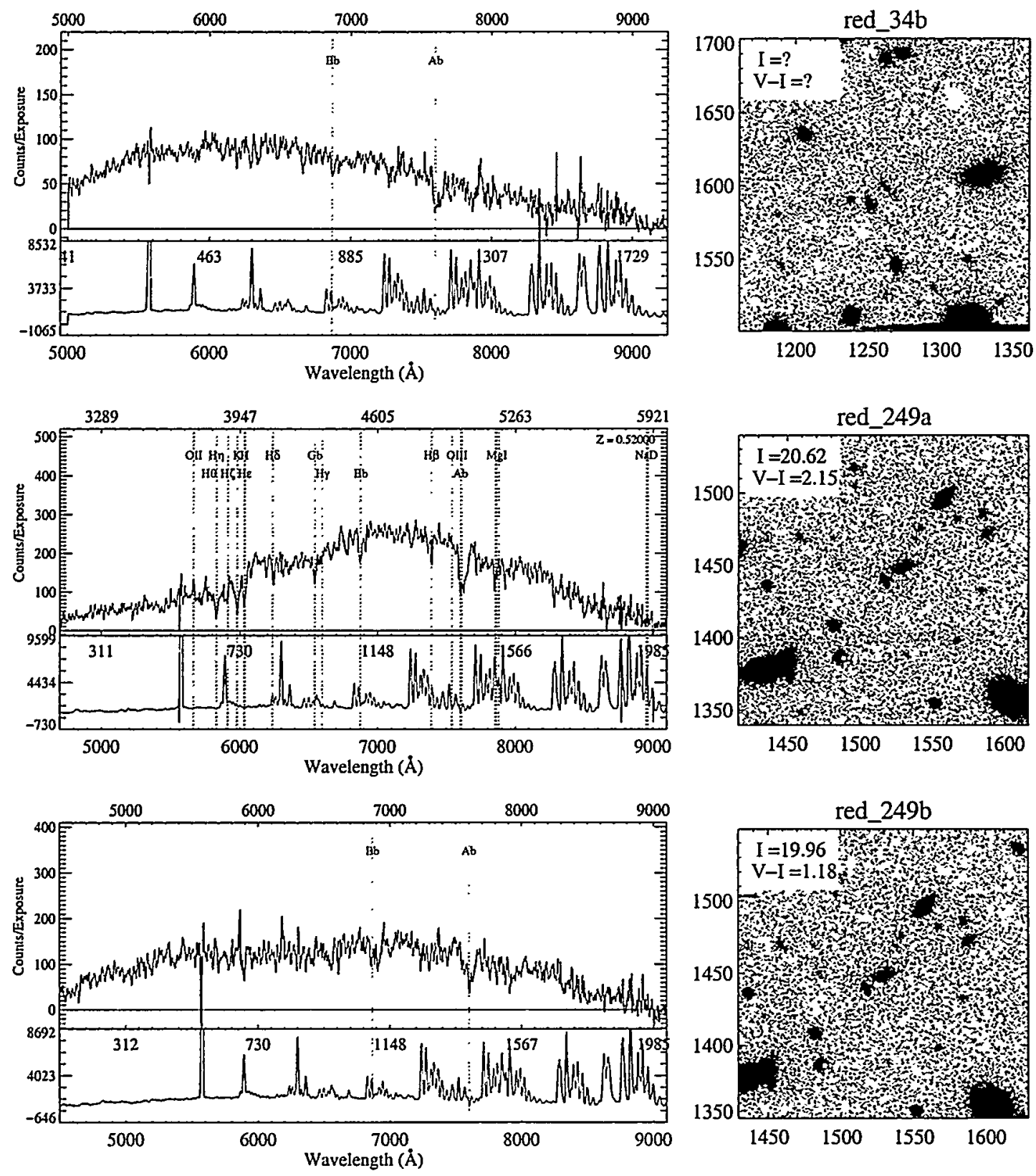

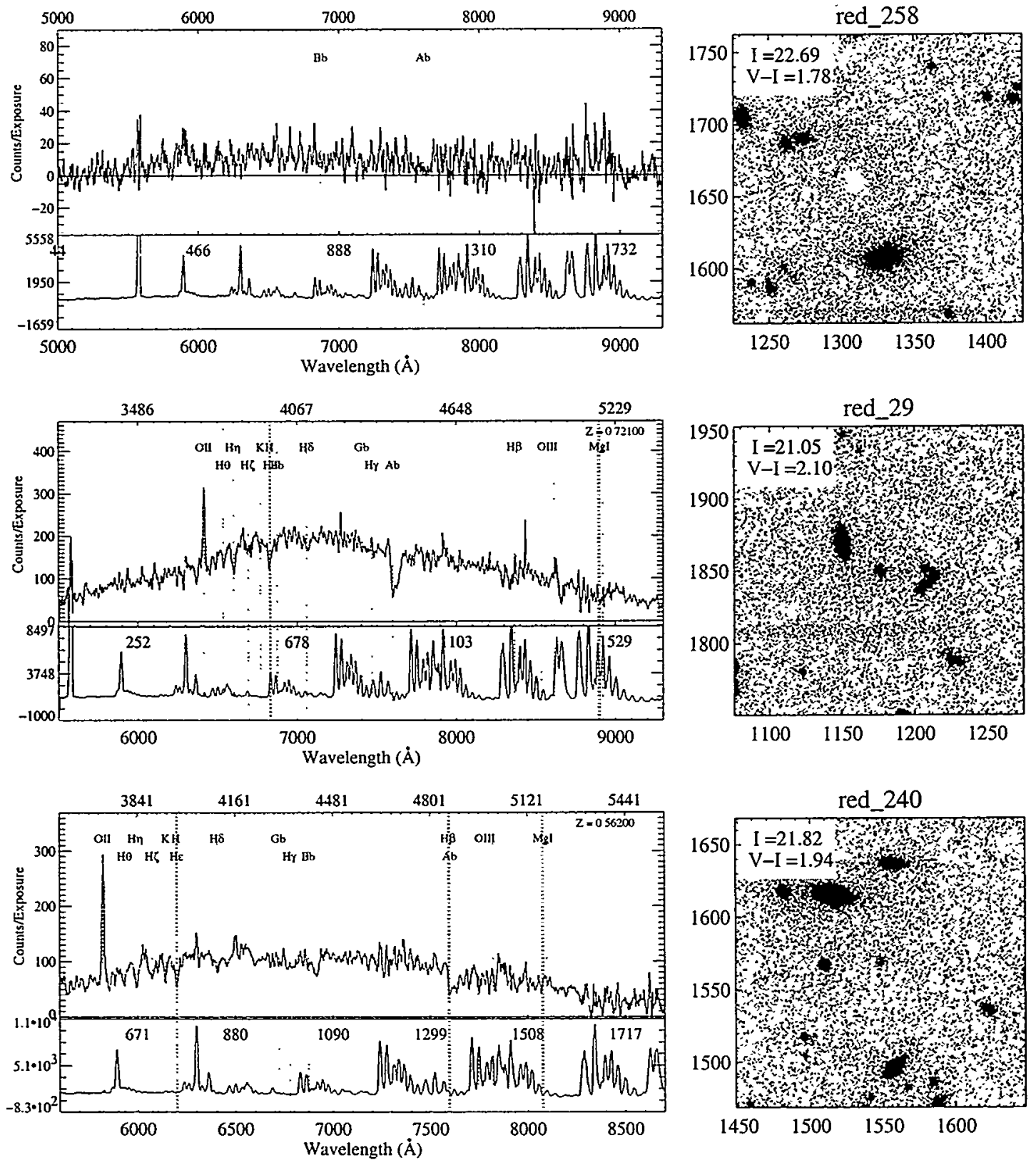